\title{
58th Annual Conference of Indian Society of Hematology \& Blood Transfusion (ISHBT) November 2017, Guwahati, India
}

(C) Indian Society of Hematology and Blood Transfusion 2017

001

Experience on Use of Oral Metronomic Therapy in Lymphoma Patients from a Tertiary Cancer Centre

Sharada Mailankody ${ }^{\#}$, Prasanth Ganesan, TS Ganesan, Venkatraman Radhakrishnan, Manikandan Dhanushkodi, Krishnarathinam Kannan, Thanda Joshua, Tenali Gnana Sagar

Email ID for Correspondence: sharadajayaram27@gmai.com Cancer Institute (WIA), Adyar, Chennai

Introduction: Oral Metronomic chemotherapy (OMC) is used in patients who may not tolerate intravenous chemotherapy or have refractory disease, or in those who have exhausted other treatment options. It is cheaper, less toxic and easy to administer. Objectives: To determine the efficacy of OMC in patients with lymphoma as assessed by response rates, progression free survival and overall survival. Material and Methods: Adult patients with lymphoma who received OMC [Cyclophosphamide $50 \mathrm{mg}$ BD (10-14 days), Etoposide $50 \mathrm{mg}$ OD (7-10 days), and Prednisolone $20 \mathrm{mg}$ BD (1014 days)] regardless of the subtype were included in this retrospective analysis. Cycles were given Q 3-4 weekly depending on tolerance and continued till progression or intolerance. Response assessment was clinical with limited use of radiology. Progression free and overall survival (PFS and OS) were calculated from the time of start of OMC until documentation of disease progression or death. Results: Between Jan 2007 and Dec 2008, 84 patients received OMC [median age: 62 years (23-83); male sex $(\mathrm{n}=55,65 \%)$; Stage III/IV $(\mathrm{n}=55$, $65 \%)$ and high grade lymphoma $(\mathrm{n}=68,81 \%)]$. OMC was used in first line $34(41 \%)$ due to poor general condition, older age, multiple comorbidities or patient preference. Others $(n=50,59 \%)$ received OMC after failure of first line therapy. Overall response rates of $64 \%$ and $24 \%$ were seen in the first line and second line settings respectively. Complete response (CR) was seen in 15 patients $(17.8 \%)$. Median PFS was 5.7 months (95\% CI 1.7-9.6) and median OS was 18.8 months (95\% CI 9.09-28.). High LDH and poor performance status were associated with inferior OS. Duration of use of OMC more than 3 months was associated with better OS. Conclusions: Though OMC is used in many centers in the country, there is very little published information on its efficacy in lymphoma. In this analysis, we demonstrate its activity in a subset of patients with predominantly high grade and advanced stage NHL. OMC is a useful option in frail patients and a small proportion can achieve deep and long lasting responses. Combination with rituximab may improve response rates without increasing the toxicity.

\section{2}

Daratumumab, Bortezomib and Dexamethasone (DVD) vs Bortezomib and Dexamethasone (VD) in Relapsed or Refractory Multiple Myeloma (RRMM): Efficacy and Safety Update (CASTOR)

Katja Weisel $^{1}$, Suzanne Lentzsch ${ }^{2}$, Maria-Victoria Mateos ${ }^{3}$, Vania Hungria $^{4}$, Markus Munder ${ }^{5}$, Ajay K. Nooka ${ }^{6}$, Tomer Mark ${ }^{7}$, Hang Quach ${ }^{8}$, Emma C. Scott ${ }^{9}$, Je-Jung Lee ${ }^{10}$, Pieter Sonneveld ${ }^{11}$, Tineke Casneuf ${ }^{12}$, Christopher Chiu ${ }^{13}$, Xiang Qin ${ }^{13}$, Himal Amin ${ }^{14}$, Piruntha Thiyagarajah ${ }^{15}$, Jordan M. Schecter ${ }^{14}$, Ming Qi ${ }^{13}$, Andrew Spencer ${ }^{16}$,

${ }^{1}$ Universitaetsklinikum Tuebingen der Eberhard-KarlsUniversitaet, Abteilung fuer Innere Medizin II, Tuebingen, Germany; ${ }^{2}$ Division of Hematology/Oncology, Columbia University, New York, NY, USA; ${ }^{3}$ University Hospital of Salamanca/IBSAL, Salamanca, Spain; ${ }^{4}$ Irmandade Da Santa Casa De Misericordia De São Paulo, São Paulo, Brazil; ${ }^{5}$ University Medical Center of the Johannes GutenbergUniversity, Third Department of Medicine, Mainz, Germany; ${ }^{6}$ Winship Cancer Institute, Emory University, Atlanta, GA, USA; ${ }^{7}$ Weill Cornell Medical College, New York, NY, USA; ${ }^{8}$ University of Melbourne, St. Vincent's Hospital, Victoria, Australia; ${ }^{9}$ Knight Cancer Institute, Oregon Health and Science University, Portland, OR, USA; ${ }^{10}$ Department of Hematology-Oncology, Chonnam National University Hwasun Hospital, Hwasun, Jeollanamdo, Korea, Republic Of; ${ }^{11}$ Department of Hematology, Erasmus MC, Rotterdam, The Netherlands; ${ }^{12}$ Janssen Research \& Development, Beerse, Belgium; ${ }^{13}$ Janssen Research \& Development, Spring House, PA, USA; ${ }^{14}$ Janssen Research \& Development, Raritan, NJ, USA; ${ }^{15}$ Janssen Research \& Development, High Wycombe, UK; ${ }^{16}$ Malignant Haematology and Stem Cell Transplantation Service, Alfred Health-Monash University, Melbourne, Australia

Email ID for Correspondence: dnagrale@ITS.JNJ.COM; Dinesh Nagrale (Presenting Authors) 
Janssen India

Introduction: Daratumumab (DARA), a human, CD38-targeting antibody, is well tolerated and induces deep and durable responses in patients (pts) with RRMM. Objectives: To provide an update on the phase 3 study of DVd vs Vd (CASTOR [NCT02136134]) in RRMM. Material and Methods: Bortezomib-refractory pts were ineligible and all pts had received $\geq 1$ prior line of therapy (LOT). Pts received Vd $\left(1.3 \mathrm{mg} / \mathrm{m}^{2}\right.$ bortezomib on Days $1,4,8$, and $11 ; 20 \mathrm{mg}$ dexamethasone on Days 1-2, 4-5, 8-9, and 11-12) \pm DARA (16 mg/kg IV weekly in Cycles 1-3, every 3 weeks for Cycles 4-8, then every 4 weeks until progression) for 8 21-day cycles. Minimal residual disease (MRD) was evaluated at sensitivities of $10^{-4}, 10^{-5}$, and $10^{-6}$ using the ClonoSEQ assay at suspected complete response (CR) and at 6 and 12 months after the first dose of study treatment. Results: Pts had received a median (range) of 2 (1-10) prior LOTs. $21 \%$ of pts were refractory to lenalidomide as their last prior LOT and $66 \%$ of pts had prior bortezomib exposure. At a median follow-up of 19.4 months, there was a significant benefit to progression-free survival (PFS) with DVd versus Vd (median: 16.7 vs 7.1 months; HR, $0.31 ; 95 \% \mathrm{CI}, 0.24-0.39 ; P<0.0001)$, regardless of number of prior LOTs received. However, the greatest benefit to PFS occurred in pts with 1 prior LOT (median: not reached vs 7.9 months; HR, 0.19; 95\% CI, 0.12-0.29; $P<0.0001)$. Regardless of number of prior LOTs, the overall response rate was significantly higher with DVd vs Vd $(84 \%$ vs $63 \%)$, along with very good partial response or better $(62 \%$ vs $29 \%)$ and CR or better (29\% vs $10 \% ; P<0.0001$ for all). At all sensitivities, MRD-negative rates were $\geq 4$-fold higher with DVd vs Vd $\left(12 \%\right.$ vs $2 \%$ at $\left.10^{-5}\right)$. Prolonged PFS was observed in pts achieving MRD negativity versus those that remained MRD-positive. Thrombocytopenia was the most common grade 3/4 TEAE and occurred in $46 \%$ and $33 \%$ of pts who received DVd and Vd, respectively. Conclusions: Significant benefits to PFS, response, and MRD-negativity were observed in pts who received DVd compared with Vd. No new safety signals were reported. These data support the use of DVd in pts with RRMM, particularly those with 1 prior LOT.

\section{$\mathbf{0 0 3}$}

A Systematic Literature Review and Network Meta-Analysis Evaluating the Efficacy of Daratumumab-Based Regimens in Patients with Relapsed/Refractory Multiple Myeloma
Meletios Athanasios Dimopoulos ${ }^{1}$, Katja Weisel ${ }^{2}$, Jonathan Kaufman $^{3}$, Pieter Sonneveld ${ }^{4}$, Maria Rizzo ${ }^{5}$, Yingxin $\mathrm{Xu}^{6}$, Kyle Fahrbach ${ }^{6}$, Maren Gaudig ${ }^{7}$, Mary Slavcev ${ }^{8}$, Lindsay Dearden ${ }^{8}$ and Annette Lam ${ }^{8}$

${ }^{1}$ National and Kapodistrian University of Athens, Athens, Greece; ${ }^{2}$ Universitaetsklinikum Tuebingen der Eberhard-KarlsUniversitaet, Abteilung fuer Innere Medizin II, Tuebingen, Germany; ${ }^{3}$ Hematology and Medical Oncology, Winship Cancer Institute, Emory University, Atlanta, GA, USA; ${ }^{4}$ Department of Hematology, Erasmus MC, Rotterdam, Netherlands; ${ }^{5}$ Evidera, London W68DL, United Kingdom; ${ }^{6}$ Evidera, Waltham, MA, USA; ${ }^{7}$ Janssen-Cilag, Neuss, Germany; ${ }^{8}$ Janssen Global Services, Raritan, NJ, USA

Email ID for Correspondence: ps10@its.jnj.com; S. Prudwidhar (Presenting Authors)

Janssen India

Introduction: Daratumumab (DARA) is a monoclonal antibody for the treatment of relapsed or refractory multiple myeloma (RRMM). Two randomized controlled trials (RCTs) demonstrated that addition of DARA to standard of care significantly reduced risk of progression or death (POLLUX: DARA plus lenalidomide and dexamethasone [DRd] vs Rd; CASTOR: DARA plus bortezomib and dexamethasone [DVd] vs Vd). Objective: To compare DRd and DVd with relevant treatment options using a network meta-analysis (NMA). Material and Methods: In order to identify and assess RCTs of treatments for RRMM, a systematic literature review (SLR) was conducted. Data from trials that met the SLR's inclusion criteria and the most recent data from POLLUX and CASTOR were extracted and included in a Bayesian NMA to allow for indirect comparisons. Results: Data from RCTs identified by the SLR allowed formulation of two evidence networks. Network 1 included DRd and other immunomodulatory agent (IMiD)-containing regimens and Network 2 contained DVd and other IMiD-free regimens. Analysis using a fixed-effects model found that, among patients RRMM, DRd and DVd prolonged PFS compared with regimens in Networks 1 and 2, respectively, and there was a trend toward prolonged OS with both DRd and DVd (see Table 1). Conclusions: This NMA suggests that the combinations of DRd and DVd improve PFS in patients with RRMM when compared with other established and novel regimens; similar trends were found for OS.

Table 1 NMA Efficacy Results

\begin{tabular}{|c|c|c|c|c|c|}
\hline \multirow[t]{2}{*}{ Network } & \multirow[t]{2}{*}{ Comparison } & \multicolumn{2}{|l|}{ PFS } & \multicolumn{2}{|l|}{ OS } \\
\hline & & HR (95\% CrIs) & $\begin{array}{l}\text { Prob DRd/DVd better } \\
\text { than comparator* }\end{array}$ & HR (95\% CrIs) & $\begin{array}{l}\text { Prob DRd/DVd better } \\
\text { than comparator* }\end{array}$ \\
\hline \multirow[t]{4}{*}{ IMiD-containing } & DRd vs. Rd & $0.37(0.27,0.51)$ & $100.0 \%$ & $0.63(0.42,0.95)$ & $98.7 \%$ \\
\hline & DRd vs. KRd & $0.54(0.37,0.78)$ & $100.0 \%$ & $0.80(0.50,1.28)$ & $83.1 \%$ \\
\hline & DRd vs. ERd & $0.54(0.37,0.80)$ & $99.9 \%$ & $0.82(0.51,1.30]$ & $80.0 \%$ \\
\hline & DRd vs. NRd & $0.50(0.33,0.74)$ & $100.0 \%$ & $0.70(0.42,1.15)$ & $91.9 \%$ \\
\hline \multirow[t]{4}{*}{ IMiD-free } & DVd vs. Vd & $0.33(0.26,0.42)$ & $100.0 \%$ & $0.63(0.42,0.95)$ & $98.6 \%$ \\
\hline & DVd vs. Kd & $0.62(0.45,0.86)$ & $99.8 \%$ & $0.80(0.48,1.34)$ & $80.5 \%$ \\
\hline & DVd vs. FVd & $0.48(0.35,0.65)$ & $100.0 \%$ & $0.67(0.43,1.05)$ & $95.9 \%$ \\
\hline & DVd vs. CVd & $0.46(0.26-0.82)$ & $99.6 \%$ & $0.54(0.24,1.23)$ & $92.9 \%$ \\
\hline
\end{tabular}

$* 100 \%$ probability represent any value above $99.951 \%$ 


\section{4}

Daratumumab, Lenalidomide, and Dexamethasone (DRD) vs Lenalidomide and Dexamethasone (RD) in Relapsed or Refractory Multiple Myeloma (RRMM): Update on POLLUX

Nizar J. Bahlis ${ }^{1}$, Philippe Moreau², Hareth $\mathrm{Nahi}^{3}$, Torben Plesner ${ }^{4}$, Hartmut Goldschmidt ${ }^{5}$, Kenshi Suzuki ${ }^{6}$, Robert Z. Orlowski ${ }^{7}$, Neil Rabin ${ }^{8}$, Merav Leiba ${ }^{9}$, Albert Oriol ${ }^{10}$, Ajai Chari $^{11}$, Jesus San-Miguel ${ }^{12}$, Paul G. Richardson ${ }^{13}$, Saad Z. Usmani ${ }^{14}$, Lisa M. O'Rourke ${ }^{15}$, Kaida $W_{u^{15}}$, Tineke Casneuf $^{16}$, Christopher Chiu ${ }^{15}$, Xiang Qin ${ }^{15}$, Meletios A. Dimopoulos ${ }^{17}$

${ }^{1}$ Tom Baker Cancer Centre, University of Calgary, Calgary, Alberta, Canada; ${ }^{2}$ Hematology, University Hospital Hôtel-Dieu, Nantes, France; ${ }^{3}$ Karolinska Institute and the Department of Medicine, Division of Hematology, Karolinska University Hospital at Huddinge, Stockholm, Sweden; ${ }^{4}$ Vejle Hospital and University of Southern Denmark, Vejle, Denmark; ${ }^{5}$ University Hospital Heidelberg and German Cancer Research Center, Heidelberg, Germany; ' Japanese Red Cross Medical Center, Department of Hematology, Tokyo, Japan; ${ }^{7}$ Department of Lymphoma/Myeloma, The University of Texas MD Anderson Cancer Center, Houston, TX, USA; ${ }^{8}$ Department of Haematology, University College London Hospitals NHS Trust, London, UK; ${ }^{9}$ Sheba Medical Center, Tel Hashomer, Ramat Gan, Israel; ${ }^{10}$ Institut Català d'Oncologia, HGTiP, Barcelona, Spain; ${ }^{11}$ Tisch Cancer Institute, Mount Sinai School of Medicine, New York, NY, USA; ${ }^{12}$ Clinica Universidad de Navarra-CIMA, IDISNA, Pamplona, Spain; ${ }^{13}$ Dana-Farber Cancer Institute, Harvard Medical School, Boston, MA, USA; ${ }^{14}$ Levine Cancer Institute/ Carolinas HealthCare System, Charlotte, NC, USA; ${ }^{15}$ Janssen Research \& Development, LLC, Spring House, PA, USA; ${ }^{16}$ Janssen Research \& Development, Beerse, Belgium; ${ }^{17}$ National and Kapodistrian University of Athens School of Medicine, Athens, Greece

Email ID for Correspondence: dbhatt19@its.jnj.com; Dipanjan Bhattacharjee (Presenting Authors)

Janssen India

Introduction: Daratumumab (DARA), a human CD38-targeting antibody, demonstrated a significant benefit to progression-free survival (PFS) when combined with standard of care in patients (pts) with RRMM. Objectives: To update data from a phase 3 study of DRd vs Rd in RRMM (POLLUX [NCT02076009]). Material and Methods: Eligible pts had received $\geq 1$ prior line of therapy (LOT) and were not refractory to lenalidomide. Rd ( $25 \mathrm{mg}$ PO lenalidomide on Days 1-21; $40 \mathrm{mg}$ dexamethasone weekly) \pm DARA $(16 \mathrm{mg} / \mathrm{kg}$ IV qw for Cycles 1 and 2, q2w for Cycles 3-6, then q4w until disease progression) was given in 28-day cycles. Pts were assessed for minimal residual disease (MRD) at sensitivities of $10^{-4}, 10^{-5}$, and $10^{-6}$ via next-generation sequencing at time of suspected complete response (CR) and at 3 and 6 months after suspected CR. Results: Pts received a median (range) of 1 (1-11) prior LOT and 55\% of pts had received prior treatment with IMiDs (18\% lenalidomide). At a median follow-up of 25.4 months, DRd significantly prolonged PFS (median: not reached vs 17.5 months; HR, 0.41; 95\% CI, 0.31-0.53; $P<0.0001)$. Overall response rate (ORR) was significantly higher with DRd vs Rd (ORR; $93 \%$ vs $76 \%, P<0.0001)$. The rates of very good partial response or better (79\% vs $48 \%)$ and CR or better $(51 \%$ vs $21 \%)$ were also higher with $\mathrm{DRd}$ vs $\operatorname{Rd}(P<0.0001)$. MRD negativity was $>3$-fold higher across all sensitivity thresholds with DRd vs Rd
(26\% vs $6 \%$ at the $10^{-5}$ threshold; $\left.P<0.000001\right)$. PFS was prolonged in MRD-negative pts compared with MRD-positive pts. With follow up ongoing, $63(22 \%)$ and 79 (28\%) deaths had occurred in the DRd and Rd arms, respectively. Consistent with earlier analyses, no new safety signals were identified. Conclusions: At 25 months of follow up, a significant benefit to PFS, ORR, and MRD negativity was observed with DRd vs Rd. The favorable safety profile of DRd was maintained with longer follow up. These data support the use of DRd in pts with RRMM who have received $\geq 1$ prior LOT.

\section{5}

First Report of Molecular Characterisation of Red Cell Membrane Protein Defect by Next-Generation Sequencing in Hereditary Spherocytosis in India

Prabhakar S Kedar", Prashant Warang, Manisha Madkaikar

Email ID for Correspondence: kedarps2002@yahoo.com National Institute of Immunohematology (ICMR) $13^{\text {th }}$ Floor, KEM Hospital; Campus, Parel, Mumbai-400012

Introduction: Hereditary spherocytosis (HS) is an inherited heterogeneous hemolytic anemia, characterized by the presence of spherical-shaped erythrocytes on the peripheral blood smear, and the clinical manifestation ranges from asymptomatic to severely anemic, and transfusion-dependent patients which requires treatment with transfusions and splenectomy. Main signs and symptoms are normocytic hemolytic anemia, icterus and splenomegaly. Gallstones are frequently seen. The molecular cause is a defect of erythrocyte membrane proteins, which play a key role in the stabilization and organization of the plasma membrane. Mutations in at least five genes (ANK1, EPB42, SLC4A1, SPTA1, and SPTB) have been identified so far, and mutations of SPTB gene are responsible for the majority of all HS cases. Objectives: To identify the cause of hereditary spherocytosis with reference to red cell membrane protein defect using targeted gene next generation sequencing (NGS). Material and Methods: Typically genomic DNA from HS patients is subjected to target exon capture. All coding exon and their flanking intronic sequences from the genes in the spherocytosis gene panel are analyzed by next generation sequencing (NGS) and confirmed by Sanger sequencing. Analysis of the resulting sequencing data against various genetic databases as well as comparison with detailed knowledge of erythrocyte biology and physiology is used to determine the etiology of a patient's defective red cells. Results: In this study, targeted next generation sequencing (NGS) was applied to identify mutation in 10 Indian patients of HS which were diagnosed clinically with only $10-30 \%$ spherical-shaped erythrocytes in the peripheral blood and low MCF on EMA test by flowcytometer. Mutations in at least five genes (ANK1, EPB42, SLC4A1, SPTA1, SPTB, PIEZO1) have been identified so far, and mutations of SPTB gene are responsible for the majority of all HS cases. In this study, targeted next generation sequencing (NGS) was identify a novel de novo ANK1 c.1669G > T (Het) (p.RGlu557Ter*) nonsense mutation in one cases, two SLC4A1 gene mutation c.2002G $>$ A (homo) p.Ala668Thr, and c.1454A > G (het) p.Glu485Gly in two HS cases, SPTA1 gene c.4453C $>$ T (het) p.Leu1485Phe and c71T $>\mathrm{C}$ (het) p.Ile24Thr in two HS patient and SPTB gene mutation c.4121A > G (Het) p.His1374Arg: c1886G > A (het) p.Arg629Gln: c.5589G > C (het), p.Gln1863His and spice site c. $1182+5 \mathrm{C}>\mathrm{T}$ (het) in a $3 \mathrm{HS}$ cases. Sanger sequencing further confirmed that one of the parents carried heterozygous mutation, 
while one is having homozygous mutation and history of consanguinity shown both parents are heterozygous for same mutation. Moreover, consistent with the genetic findings, the anaemia was ameliorated after splenectomy in one HS case. Our results also demonstrate that targeted NGS may provide a powerful approach for rapid genetic test of HS. Systematic Functional studies were undertaken in Indian HS patients to look for association of genetic defects on the clinical outcome of the patients. It will also be helpful for genetic counselling and better management of the patients as well as offering prenatal diagnosis to severely affected patients. Conclusion: Our results also demonstrate that targeted NGS may provide a powerful approach for rapid genetic test of HS.

\section{6}

\section{Light Chain Myeloma: A Single Centre Experience}

Neha Singh", Narendra Agrawal, Anurag Mehta, Radhika Sekhri, Dushyant Kumar, Narender Tejwani, Rayaz Ahmed, Dinesh Bhurani

Email ID for Correspondence: drnehasingh123@gmail.com Rajiv Gandhi Cancer Institute and Research Centre, New Delhi

Introduction: Light chain myeloma (LCM) has a reported worldwide incidence of approximately $15 \%$ among all multiple myeloma patients and is more commonly of the lambda subtype. Few western studies have shown strong correlation of LCM with anemia, higher ISS scores, proclivity to renal failure, elevated LDH levels, raised SFLC ratio, higher frequency of extramedullary plasmacytomas and poorer overall survival, attributable probably to lack of differentiation and skeletal destruction. Objectives: The primary aim of this study was to define the clinical and laboratory characteristics as well as prognostic outcome of Indian LCM patients in comparison to the IgG and IgA subtypes as defined on serum immunofixation. Material and Methods: This retrospective observational study involved the newly diagnosed multiple myeloma patients who presented to the Hematology OPD during the period from September 2013 to August 2016. All patients were accorded with International Myeloma Working Group diagnostic criteria 2016 and staged as per the International Staging System (ISS). Most patients received Bortezomib-based regimens followed by autologous stem cell transplantation and were evaluated for response to chemotherapy and progression-free survival (PFS). Results: Out of one-hundred and four patients of newly diagnosed multiple myeloma in which results of serum immunofixation were available, sixty-five were of $\operatorname{IgG}$ type $(62.5 \%)$, fifteen had $\operatorname{IgA}(14.4 \%)$ and twenty-four had light chain myelomas (LCM) $(23.1 \%)$. Among the LCM patients, thirteen $(13 / 24=54.2 \%)$ and eleven $(11 / 24=45.8 \%)$ were of lambda and kappa subtypes respectively. On univariate analysis, LCM patients showed statistically significant correlation with younger age group, lesser degree of anaemia and higher SFLC ratios in comparison to the $\mathrm{IgG}$ and $\mathrm{IgA}$ subtypes. IgA subtype was found to be significantly associated with higher bone marrow plasma cell percentage. However, no difference was found in terms of serum LDH, renal impairment, presence of bony or extramedullary lesions, ISS-scores, pre-transplant chemosensitivity and PFS. Furthermore, within the LCM subgroups, the lambda subtype was associated with higher median beta2-microglobulin levels and bone marrow plasma cell percentage than the kappa subtype. But there was no significant difference between the two subgroups with respect to anemia, serum LDH, hypercalcemia, bone marrow plasma cell percentage, proclivity to renal failure, response to chemotherapy and PFS (1 year). Conclusions: It may be emphasized that the Indian LCM patients have distinct clinico-hematologic profile when compared to other published western studies. Also, their prognostic outcomes do not differ significantly in comparison with the other serum protein subtypes, probably due to standardized treatment regimens applied.

\section{7}

High frequency of high risk CNAs in our cohort of pediatric B-ALL is associated with poor MRD and EFS: Data from a tertiary care centre of India

Dr. Prateek Bhatia ${ }^{1}$, Dr. Minu Singh ${ }^{1}$, Prof. Amita Trehan ${ }^{1}$, Prof. Neelam Verma ${ }^{2}$, Dr. Manupdesh Singh Sachdeva ${ }^{2}$, Prof. Deepak Bansal ${ }^{1}$, Dr. Richa Jain ${ }^{1}$

${ }^{1}$ Pediatric Hematology-Oncology unit, ${ }^{2}$ Department of Hematology -Post Graduate Institute of Medical Education and Research, Chandigarh, India

Context: Studies on genomic and sub-genomic alterations in ALL have shown promising results in correlating poor prognosis and survival in leukemia patients. Objective: Present study analyzes copy number variations (CNA) and fusion transcripts in paediatric B-ALL cases and correlates with standard risk factors and treatment outcome. Design: The chimeric fusion transcripts were detected by conventional RT-PCR method, while CNAs for following genes; IKZF1, PAX5, EBF1, BTG1, RB1, CDKN2A/B, PAR1, CSF2RA, IL3RA and CRLF2 were identified by multiplex ligation dependent probe amplification assay. Setting: The study was performed at a tertiary care centre of Northern India. Patients: Newly diagnosed B-ALL pediatric patients $(0-12 y)$ with blast count $\geq 60 \%$ were enrolled $(n=67)$ in the study. Ten healthy controls were included for normalization of MLPA results. Result: The median age of patients was 5.5 years with a M:F ratio of $2.3: 1$. The incidence of fusion transcripts of AML1-TEL, E2A-PBX, BCR-ABL and MLL-AF4 was 17\%, 14\%, $13 \%$ and $1.5 \%$, respectively. The CNAs were detected in $70 \%$ of all cases analyzed, with most common deletions of CDKNA/B (36\%), PAX5 (22\%) and IKZF1 (19\%). Out of 47 cases positive for CNA, $87 \%$ (41) of cases showed variation in $\geq 3$ genes, while only $3 \%$ (2) and $5 \%$ (4) of cases showed variation in 2 and 1 gene, respectively. The cases with high risk BCR-ABL and low risk AML1-TEL transcripts, showed CNAs in $78 \%$ and $40 \%$ of cases, respectively. The CNAs classified in our cohort as per the report by Moorman et al. (Blood, 2014) were good risk- 37 (55\%) and poor risk- 30 (45\%). On correlation, a statistically significant association of CNA groups was noted with high WBC count ( $p-0.001)$, NCI group $(p-0.02)$ and MRD at Day35 $(p-<0.001)$. The EFS and OS at 22 months for high and low risk CNA was $83 \%$ \& $65 \%$ ( $p$ value 0.05 ) and $84 \% \& 76 \%$ ( $p$ value 0.18 ) respectively. Conclusion: Therefore, the identification of CNAs has significant potential to be used in better risk stratification at early stage of treatment as highlighted by high number of high risk CNAs associated with poor MRD status and relatively poor EFS at 22 months. Keywords copy number alteration, fusion transcripts, B-ALL, minimal residual disease

Acknowledgement: Funding source- CSIR, New Delhi, India [grant no. 27 (312)/15, 2015-2018]. 


\section{8}

KIR receptor-HLA ligand match/mismatch in graft loss post transplant cyclophosphamide based haploidentical transplant

Selma Zenia D'Silva", Pankaj Diwedi, Sachin Punatar, Anant Gokarn, Avinash Bonda, Manisha Tambe, S.B. Rajadhyaksha, Navin Khattry and Meenakshi Singh

Email ID for Correspondence: selmadsilva@gmail.com Department of Transfusion Medicine, Tata Memorial Hospital, Mumbai-400012

Introduction: Hematopoietic stem cell transplantation (HSCT) is the treatment option for leukemic patients. However, not every patient finds a full HLA matched donor and hence, has to opt for haploidentical hematopoietic stem cell transplantation (HHSCT). Recently, Killer Immunoglobulin like receptor (KIR) gene, present on Natural Killer (NK) cells, which modulate NK alloreactivity has been linked with predicting transplant outcomes. Objectives: We present two HHSCT cases, wherein after engraftment there was graft loss and auto recovery of patient cells. However, there was no disease relapse. The objective of the research was to find out the probable reason for the graft loss and absence of disease relapse. Material and Methods: We hypothesized that this phenomenon could be due to KIR ligand matches/mismatches. HLA typing for patient and donor was done using PCR SSP (Bag Diagnostics, Germany). KIR genotyping for patients and donors was done using PCR-SSP (Innotrain, Germany). HLA C and Bw4 ligands for patients were assigned using the KIR HLA ligand software http://www.ebi.ac.uk/ipd/kir/). We examined the KIR ligand matches/mismatches in both the $\mathrm{GvH}$ and $\mathrm{HvG}$ direction. Results: We observed that for both cases, there was KIR activating receptor match and stronger iKIR mismatch in GvH direction. This resulted in removal of leukemic cells, since there was no inhibition of $\mathrm{NK}$ cell activity. Moreover in the $\mathrm{HvG}$ direction, the stronger aKIR match (C1 HLA C ligand is stronger activator of NK cell activity) led graft rejection. Conclusions: These novel findings highlight the importance of knowing both aKIR and iKIR mismatches in the $\mathrm{GvH}$ and $\mathrm{HvG}$ direction before selecting a HHSCT donor in order to have good GvL effect and avoid graft rejection.

\section{9}

Benefit of Early Treatment with Room Temperature Stable Recombinant Activated Factor VII (rFVIIA) in Patients with Hemophilia A OR B with Inhibitors: Subgroup Analysis from the Prospective, Post-Authorization, Non-Interventional Smart- ${ }^{\mathrm{TM}}$ STUDY

Shankar, $\mathrm{S}^{\#}$; Benson, G; Chambost, H; Demartis, F; Benchikh El Fegoun, S; Cepo, K; Sommer, L; Kavakli, K

Email ID for Correspondence: ssqq@novonordisk.com Novo Nordisk India

Introduction: SMART- ${ }^{\mathrm{TM}}$ (NCT01220141) was a prospective, postauthorization, single-arm, multinational, multi-center, non-interventional study investigating the safety and effectiveness of room temperature stable rFVIIa (NovoSeven ${ }^{\circledR}$ ) in patients with hemophilia A or B with inhibitors in a real-world setting. Objectives: Here, we present a subgroup analysis of the hemostatic response of bleeding episodes to treatment with rFVIIa. Material and Methods: Study medication was not provided; use was at the discretion of the treating physician in accordance with the local label. Bleeding history was collected at the initiation visit. Information on bleeding episodes, including home treatment, was recorded in patient diaries during the study. Patients evaluated the status of bleeding episodes after treatment as 'bleed stopped', 'bleed slowed', or 'no change/worsened'. Overall bleed outcome was defined as the last given patient evaluation of the bleed. Patients were observed until they had achieved $\geq 25$ exposure days, defined as any exposure to rFVIIa during one $24 \mathrm{~h}$ period. Results: From November 2010 to March 2015, 51 patients were enrolled at 24 sites in 14 countries; 31 (60.8\%) patients completed the study and 20 discontinued (11 of these due to global study discontinuation, a decision endorsed by the European Medicines Agency). Patients were aged 1.6-69.5 years (median 22.0 years) with an historical median bleeding rate of 1.0 episode per month. During the study, 48 patients experienced a total of 618 bleeding episodes: median number of bleeding episodes per patient was 10.5 ; median study duration per patient was 13.9 months. $63.4 \%$ of bleeding episodes occurred spontaneously and $31.2 \%$ were categorized as traumatic. Effectiveness evaluation at end of treatment was available for 609 bleeding episodes: 569 (93.4\%) resolved, 35 (5.7\%) slowed and 5 $(0.8 \%)$ were unchanged/worsened. Nine bleeds had no reported outcome. A total of 538 bleeds were treated with rFVIIa monotherapy: $507(94.2 \%)$ resolved, $27(5.0 \%)$ slowed and $4(0.7 \%)$ were unchanged/worsened. In a post-hoc analysis in which the data were divided by time to first treatment into 3 groups, the best hemostatic response $(96.5 \%)$ was observed when rFVIIa treatment was initiated $\leq 1 \mathrm{~h}$ after onset of bleed; effectiveness was also high $(93.1 \%)$ for bleeds treated $>1-\leq 4 \mathrm{~h}$ of onset, decreasing for those treated $>4 \mathrm{~h}$ after onset $(87.3 \%$; representing $13.1 \%$ of bleeds). Early treatment $(\leq 1 \mathrm{~h})$ with rFVIIa monotherapy was effective for both joint and muscle bleeds (96.2\% and $97.3 \%$, respectively). The initial rFVIIa dose administered was comparable for bleeds treated $\leq 1 \mathrm{~h}$ and those treated $>1-\leq 4 \mathrm{~h}$ or $>4 \mathrm{~h}$ after onset. The median number of rFVIIa doses was higher for bleeds treated $>4 \mathrm{~h}$ after onset than for those treated within $4 \mathrm{~h}$, however these data should be interpreted with caution due to the low number of bleeds represented (13.1\% of total). Conclusions: A subanalysis of the SMART- $7^{\mathrm{TM}}$ study demonstrates higher effectiveness of early treatment with rFVIIa, with $96.5 \%$ of bleeds resolved when treatment was initiated $\leq 1 \mathrm{~h}$ after bleed onset, and remaining high $(93.1 \%)$ for bleeding treatment $>1-\leq 4 \mathrm{~h}$. Total effectiveness was $>87 \%$ in bleeds treated $>4$ hours after bleed onset.

\section{0}

Multicolor Flowcytometry-Based Post-Induction MRD Status is a Powerful Indicator of Event-Free-Survival in Childhood T-ALL

Dilshad Dhaliwal $^{1 \#}$, Gaurav Chatterjee ${ }^{1}$, M Sanyal ${ }^{1}$, P Devre ${ }^{1}$, S Ghogale ${ }^{1}$, Y Badrinath ${ }^{1}$, N Patkar $^{1}$, N Deshpande ${ }^{1}$, B Arora ${ }^{2}$,

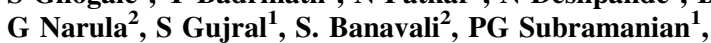
Prashant Tembhare*

Email ID for Correspondence: dlshddhaliwal@gmail.com Tata Memorial Center, Mumbai

Introduction: Minimal residual disease (MRD) is a powerful predictor of event-free survival in acute leukemia including T-cell acute lymphoblastic leukemia (T-ALL). Due to lower incidence of T-ALL, MRD studies are limited and restricted to a small cohort of 
patients. Moreover, flow-cytometry based MRD (FC-MRD) studies in T-ALL are very few. AIEOP-BFM group showed that late (Day78) MRD response determines overall risk-of-relapse and eventfree-survival (EFS) using RQ-PCR. However, a larger study by COG (Brent Wood et al. ASH, 2014) showed that post-induction (Day-29) FC-MRD was more relevant in the prediction of EFS. This indicates that the best time for MRD evaluation for the risk stratification in T-ALL is still not clear and need more studies. We investigated the value of post-induction FC-MRD response in an assessment of EFS in childhood T-ALL. It is a first T-ALL MRD study from India. Material and Methods: We studied the postinduction (Day-35) MRD (PI-MRD), and post-consolidation (Day78) MRD (PC-MRD) in bone marrow samples from 100 patients of T-ALL treated under modified MCP-841 protocol during January 2014 to October 2016. MRD was performed using 10-color FCMRD assay on Navios flow-cytometer and MRD analysis was performed with Kaluza software v-1.3 (Beckman Coulter). Any detectable level of MRD (Cluster of at least 20 events) was taken as MRD positive. Results: Median age of T-ALL patients studied was 11.5 years (range $2-16$ years; $\mathrm{M}: \mathrm{F}-4.6$ ). Based on the immunophenotypic criteria, 13 patients were diagnosed as earlythymic-precursor (ETP) type and remaining 87 as not-ETPALL type. PI-MRD was positive in $58 \%$ with median level of $0.23 \%$ (range, $0.002 \%-6 \%$ ). PC-MRD was not performed in $71.4 \%(30 / 42)$ of PI-MRD-negative and $1.2 \%$ (6/58) PI-MRD-positive patients. Thus, PC-MRD was available in only 64 patients and was positive in $28 \%(18 / 64)$ (median, $0.2 \%$ \&range, $0.009 \%-4 \%$ ). Frequency of PI-MRD positivity was seen in $12 / 13$ (93\%) patients in ETPALL using chi-square test (Chi-squared value 5.7, $p=0.01$ ). Median follow-up of all patients was 13.2 months (range, 3-38 months). Patients were considered MRD standard-risk (MRD-SR) if PI-MRD was negative and MRD high-risk (MRD-HR) if PI-MRD was positive with any level. On this MRD-based risk stratification,(42/ $100) 42 \%$ of them were MRD-SR, (58/100) 58\% MRD-HR. Twenty patients relapsed ( 2 were ETPALL \& 18 non-ETPALL; 3 MRDSR \& 17 MRD-HR) within 26 months (median, 12.3 months \& range, 6.1-26 months). Median EFS of MRD-HR patients was significantly inferior as compared to MRD-SR (26 months vs. did not reach; \& $70.67 \%$ vs. $92.86 \% ; p=0$.0017) (Kaplan-Mayer curve shown in Figure 1). Interestingly, there was no difference in EFS for PIMRD $<0.01 \%$ vs. $>0.01 \%$, suggesting any level of PI-MRD positivity indicates inferior EFS. Conclusions: We concluded that 10-color FC-based post-induction MRD response is a powerful indicator of EFS in childhood T-ALL. The frequency of PI-MRD positivity was significantly high in ETPALL indicating a lower

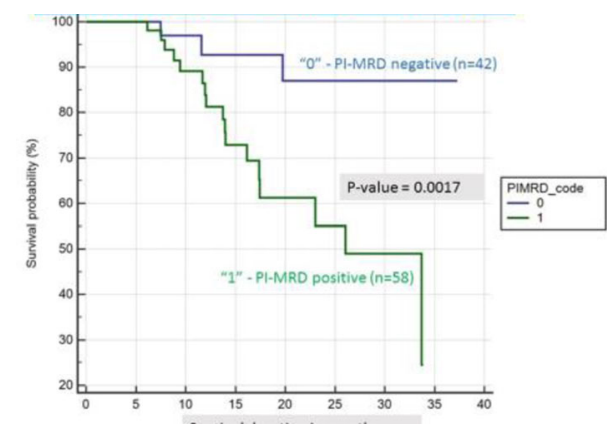

Figure 1 Kaplan Mayer Curve showing Event Free Survival (EFS) against PI-MRD status $(\mathrm{n}=100)$ tumor clearance rate. There was no difference in the EFS based on the level of PI-MRD-positivity indicating even a low level $(<0.01 \%$ MRD $)$ is important in risk-stratification of childhoodTALL.

\section{1}

A combination of Novel Markers "CD148, CD180 \& CD200" is Highly Useful in the Differential Diagnosis of B-Cell NonHodgkin's Lymphoma Involving Peripheral Blood and Bone Marrow

Neha J, Dilshad D, Gaurav C, Sitaram G, Y Badrinath, Ashok K, P G Subramanian, N Patkar, S Gujral, P Tembhare

Email ID for Correspondence: nileshdd1@ rediffmail.com; Nilesh Deshpande (Presenting Authors)

Tata Memorial Centre

Introduction: Flow cytometry is widely used in the diagnosis and classification of B-cell non-Hodgkin's lymphoma (B-NHL). B-NHL like classical chronic lymphocytic leukemia (CLL), and hairy cell leukemia (HCL) are well-defined by flow cytometry; however, due to lack of specific markers it is challenging to classify other B-NHL like marginal zone lymphoma (MZL), atypical CLL (aCLL), mantle cell lymphoma (MCL), and lymphoplasmacytic lymphoma (LPL) especially in the absence of tissue diagnosis. Objectives: To evaluate the utility of the combination of CD148, CD180 \& CD200 in the differential diagnosis of B-NHL involving peripheral blood $(\mathrm{PB})$ and bone marrow (BM). Material and Methods: We studied the expression of CD148 (clone, REA204), CD180 (clone, MHR73.11) \& CD200 (clone, B-ly6) in mature B-NHL using 10-color immunophenotyping on Cytoflex flow-cytometer. Mean florescent intensity (MFI) of these markers was determined as geometric mean and data-analysis were performed using Kaluza-v1.3 software. This study was conducted in Tata Memorial Centre and approved by institutional ethical committee. Results: 103 samples (40 PB \& 65 BM) from 105 B-NHL patients (age, 24-85 years; M:F-2.24) and 24 $\mathrm{BM}$ control samples (uninvolved staging BM; age 17-68 years) were studied. 79 B-NHL included 27-CLL, 6-aCLL, 15-diffuse large B-cell lymphoma (DLBCL), 19-follicular cell lymphoma (FCL), 20-MCL, 11-MZL, 2-Burkitt lymphoma (BL), 2-hairy cell leukemia (HCL) and 3-LPL. Median (range) of MFI of CD148/CD180/CD200 is mentioned in the table 1. Expression of CD148 was highest in MCL $(p<0.02)$, CD180 was highest in normal B-cells \& MZL $(p<0.01)$, and CD200 was highest in CLL $(p<0.01)$. Combination of bright CD148 with heterogenous/dim/negative CD180/CD200 expression diagnosed MCL correctly in 70\% (sensitivity-70\%/specificity$94.12 \%, p<0.001)$ and addition of CD5(+) \& CD10(-) expression improved the sensitivity to $80 \%$ \& specificity to $100 \%$. Similarly, combination of bright CD180 with heterogenous/dim/negative CD148/CD200 diagnosed MZL in $81 \%$ (6/9) (sensitivity-81.8\%/ specificity-89.4\%, $p=0.002$ ) and addition of CD5(-) \& CD10(-) expression improved sensitivity to $87.5 \%$ and specificity to $97 \%$. Combination of bright CD200 with heterogenous/dim/negative CD148/CD180 in $76.2 \%(16 / 21)$ (sensitivity-91\%, specificity-94\%, $p<0.001)$. In contrast to CLL, aCLL showed over-expression of CD148 and/or CD180 and under-expression of CD200 $(p<0.01)$. Conclusions: Strong expression of CD148 was significantly associated with MCL and strong expression of CD180 with MZL. The combination of these 3-novel markers- CD148, CD180 and CD200 along-with standard markers-CD5/CD10 is highly useful in the differential diagnosis of B-NHL involving peripheral blood and bone marrow. Thus, these novel markers improved the flow cytometric diagnosis and classification of B-NHL. 
Table 1 .

\begin{tabular}{|c|c|c|c|c|c|c|c|}
\hline & \multirow[t]{2}{*}{ NO. OF CASES } & \multicolumn{2}{|l|}{ CD148 } & \multicolumn{2}{|l|}{ CD180 } & \multicolumn{2}{|l|}{ CD200 } \\
\hline & & Median MFI & MFI RANGE & Median MFI & MFI RANGE & Median MFI & MFI RANGE \\
\hline NORMAL & 24 & 3.86 & $0.38-63.67$ & 91.6 & $12.19-190$ & 10.9 & $1.04-111.5$ \\
\hline CLL & 27 & 3.05 & $0.12-10.88$ & 7.43 & $0.13-46.17$ & 35.3 & $10.55-117$ \\
\hline ATY CLL & 6 & 7.89 & $1.81-10.86$ & 35.72 & $5.57-63.4$ & 14 & $4.97-43.08$ \\
\hline DLBCL & 15 & 4.32 & $1.27-16.2$ & 26.08 & $4.64-98.86$ & 4.7 & $0.41-29.9$ \\
\hline FCL & 19 & 4.6 & $1.41-26.62$ & 20.6 & $5.2-78.0$ & 2.65 & $0.59-33.8$ \\
\hline MCL & 20 & 17.6 & $1.66-165.3$ & 13.4 & $0.47-86.6$ & 1.2 & $0.17-16.63$ \\
\hline MZL & 11 & 4.38 & $1.33-59.63$ & 64.3 & $18.57-138.7$ & 8.02 & $1.03-16.21$ \\
\hline $\mathrm{BL}$ & 2 & 7.1 & $2.79-11.42$ & 77.45 & $13.57-141.3$ & 1.63 & $0.7-2.57$ \\
\hline HCL & 2 & 1.08 & - & 46.8 & - & 41.6 & - \\
\hline LPL & 3 & 19.8 & - & 12.8 & - & 21.95 & - \\
\hline
\end{tabular}

\section{2}

Bendamustine-Rituximab (BR) as Front-Line Therapy in Chronic Lymphocytic Leukemia (CLL) Patients: A Retrospective Study from Single Center in Western India

Dr D Patel ${ }^{\#}$, Dr R Roshan, Dr H Balar, Dr B.C.Mahadeva, Dr A Joshi, Dr R Phatale, Dr S Melinkeri, Dr S Kannan, Dr S Apte

Email ID for Correspondence: docdiiv@yahoo.com Sahyadri Speciality Hospital, Pune

Introduction: Although Fludarabine-Cyclophosphamide-Rituximab (FCR) forms the standard of care for patients with CLL, high incidence of adverse events especially in elderly patients precludes its use in most of the patients with CLL. BR chemotherapy in CLL has been evaluated in three prospective studies and has been shown to be safe and effective. Objectives: We retrospectively analysed the data of CLL patients treated with BR as front-line therapy. The aim was to analyse safety and efficacy of BR as upfront therapy in CLL. Material and Methods: Retrospective analysis of 26 patients who were diagnosed as CLL and were treated upfront with BR chemotherapy was done. The diagnosis was based on immunophenotyping of peripheral blood. Patients were staged as per Rai and Binet staging system. Additional risk factors including cytogenetic analysis were recorded for the available cases. The details of chemotherapy given, dose, number of cycles and adverse events were documented. Results: The study included 26 patients of CLL who received at least 1 cycle of BR as first-line treatment during June 2010 to Aug 2017. Median age was 67.5 years (47- 82 years); male: female ratio was 1.6:1. Rai staging results included Stage 0 in $30.76 \%$ while $15.38 \%$ were stage I, $11.53 \%$ stage II, stage III- $15.38 \%$ and stage IV-26.92\%. Binet staging results were Stage A- 53.8\%, Stage B- $7.7 \%$ and Stage C- $38.4 \%$. At diagnosis, Haemogram parameters included Median $\mathrm{Hb}$ $12.4 \mathrm{gm} / \mathrm{dl}(5.2-16.1 \mathrm{gm} / \mathrm{dl})$, median TLC $36900 \mathrm{cells} / \mathrm{cmm}$ (11600423600 cells $/ \mathrm{cmm})$, median platelet count 171500 cells $/ \mathrm{cmm}(26000-$ 369000 cells $/ \mathrm{cmm}$ ), median absolute lymphocyte count 30912 cells/ $\mathrm{cmm}(8816-347352$ cells $/ \mathrm{cmm})$. FISH analysis was available for 5 cases (1 had del11q, 1 had 14q and 2 had del13q). Treatment indication criteria included anaemia, thrombocytopenia, symptoms and lymphocyte doubling time $<6$ months in $61.5 \%, 50 \%, 23 \%$ and $38.4 \%$ patients respectively. Mean duration between diagnosis and treatment was 23.6 months. All patients received BR chemotherapy. 21 patients $(80.7 \%)$ completed 6 cycles of chemotherapy. Average duration of chemotherapy was 4.65 months. Only 6 patients $(23 \%)$ developed adverse reactions to chemotherapy out of which only 1 patient $(3.8 \%)$ died due to septicaemia and fungal pneumonia after 5 cycles. Median follow up was 31.5 months (range 4 to 122 months). $57.7 \%$ patients were in $\mathrm{CR}$ at last follow up while $15.3 \%$ patients had relapse. Conclusions: Chemo immunotherapy with BR was effective and well tolerated in our cohort of patients. BR can be used as first line chemo immunotherapy in CLL patients, even in elderly patients.

\section{3}

Stepwise FISH Strategy to Identify High Risk Multiple Myeloma (MM) Patients Using CD138 Positively Selected Plasma Cells: TMC Experience

Sumanta K Patel, Sipra Rani Patel, Arun SR, Manish K Singh, Neeraj Arora, Deepak K Mishra, Jeevan Kumar, Saurabh J Bhave, Vivek S Radhakrishnan, Reena Nair, Mammen Chandy, Mayur Parihar

Introduction: Pre-treatment cytogenetic risk stratification is an integral part of modern treatment protocols in Multiple Myeloma (MM). The minimal FISH panel as recommended by the Mayo clinic includes identification of high risk MM that includes deletion of short arm of chromosome $17, \mathrm{t}(4 ; 14)$ and $\mathrm{t}(14 ; 16)$. We report a stepwise FISH approach to identify high risk MM patients that can be applied where resources are limited. Objective: To identify high risk MM patients using FISH strategy on CD138 positively selected cells from bone marrow aspirate of MM patients. Materials \& methods: 108 MM patients were diagnosed and worked up at Tata Medical Center, Kolkata between February 2016- August 2017. Plasma cells were enriched by positively selecting CD138 positive cells using immunomagnetic bead method (EasySep ${ }^{\mathrm{TM}}$, STEMCELL ${ }^{\mathrm{TM}}$ Technologies) from the bone marrow aspirate sample using standard protocols. The FISH analysis on these selected cells included TP53 deletion probe (Zytovision Bremerhaven, Germany) and IgH break apart probe (Zytovision Bremerhaven, Germany) in the first step. In case of $\mathrm{IgH}$ gene rearrangements, the partners were identified using $\mathrm{t}(4 ; 14) / F G F R 3-I g H$ dual color dual fusion (Vysis; Abbott, Illinois), $\mathrm{t}(14 ; 16) /$ MAF-IgH dual color dual fusion (Vysis; Abbott, Illinois), $\mathrm{t}(11 ; 14) /$ CCND1-IgH dual color dual fusion (Zytovision Bremerhaven, Germany) in the subsequent FISH analysis. Results: We 
analyzed the data of 108 patients $(\mathrm{M} / \mathrm{F}=63 / 45$, median age $=62.5$ yrs, age range $=33-81$ ). TP53 deletion was seen in $17 / 108$ patients $(15.7 \%)$ and $\operatorname{IgH}$ rearrangement was noted in 23/104 patients $(22.1 \%)$. Five patients showed $t(11 ; 14)$, nine patients showed $t(4 ; 14)$ and none of the patients showed $\mathrm{t}(14 ; 16)$. In nine of these $\mathrm{IgH}$ rearranged patients, the partner chromosome were not identified. In seven patients, there was presence of both the deletion of TP53 gene and $\mathrm{I} g \mathrm{H}$ gene rearrangement. In one patient, there was the presence of rearrangement of both copies of $I g H$ gene with different partners. Conclusion: There are only a few published Indian studies, which had used sorted plasma cells to study the cytogenetic abnormalities for risk stratification in MM. We report a relatively high proportion of MM patients with TP53 deletion and low proportion of $\mathrm{IgH}$ rearranged patients in comparison to the western literature. Using a stepwise FISH analysis saves costs that can be essentially used in enriching plasma cells in patients of MM.

\section{4}

A Retrospective Study to Assess the Safety and Efficacy of Addition of Eltrombopag to Standard Immunosuppressive Therapy in Patients with Aplastic Anaemia

\section{Dr. Shruti Mantri", Dr. Chandrakala S., Dr. Farah Jijina., Dr. Rajesh P}

Email ID for Correspondence: shruti.mantri22@gmail.com Dr. J C Patel Department of Hematology, KEM Hospital, Mumbai

Introduction: Acquired Aplastic Anaemia (AA) is defined as pancytopenia with hypocellular bone marrow in the absence of an abnormal infiltrate with no increase in reticulin. Hematopoietic stem cell transplant and immunosuppressive therapy (IST)are the primary modalities of treatment for AA. An effective IST regimen is equine anti-thymocyte globulin (eATG) in combination with cyclosporine which produces hematologic responses in $60-75 \%$ of cases, among which the relapse rate is $33.3 \%$. Hematopoietic stem cell transplant offers response rate of $70-80 \%$, but has long term morbidity and almost $70 \%$ patients lack a matched sibling donor. Eltrombopag, a TPO agonist has activity in refractory SAA producing hematologic responses, the majority multilineage, in about $40 \%$ of patients when administered as a single agent (1), (2). In an phase II, single-center trial to test the efficacy of adding Eltrombopag $(150 \mathrm{mg})$ to h-ATG/ CsA (clinicaltrials.gov, NCT01623167), showed that addition of Eltrombopag to IST markedly increases overall and complete hematologic response rates in treatment-naive SAA. The 3-month overall response rates ranged from $77 \%$ to $92 \%$ across the three cohorts, and 6-month response rates ranged from $80 \%$ to $95 \%$ (3). Objectives: To assess safety and efficacy of combining low dose eltrombopag $(50 \mathrm{mg})$ with standard immunosuppressive therapy in patients with aplastic anaemia and to compare outcomes in patients only receiving standard immunosuppressive regimen. The primary outcome was hematologic response at 3 and 6 months. Secondary end points included overall response, survival. Material and Methods: Retrospective data collection done from August 2015 to August 2016, among patients with aplastic anaemia patients. Sample size was 46 patients. Patients were analysed in 2 groups, group 1 (received eltrombopag $50 \mathrm{mg}$ on day 1 of IST), group 2 (received only IST). Non-responders at 3 months in both the groups received danazol $200 \mathrm{mg}$. Demographic details, treatment details, response rates entered in MS excel 2010, analysis done using SPSS (Statistical package for social sciences). Results: Among both the groups, at 3 months and 6 months overall response was 54.3\% (25), complete response was $6.5 \%$ (3), $13 \%$ (6) respectively. According to the severity classification response rates 3 months and 6 months $89.5 \%$ and $89.5 \%, 43.8 \%$ and $50 \%, 0.0 \%$ and $0.0 \%$ in non-severe, severe and very severe groups respectively. 20 (43.5\%) received eltrombopag in addition to standard immunosuppressive regimen (GROUP 1), 26 $(56.5 \%)$ received only standard immunosuppressive regimen (GROUP 2). At 3 months, overall response in group 1 was $55 \%$, group $250 \%$ (P value 0.736). At 6 months, overall response in group 1 was $60 \%$, group 2 was $50 \%$ (P value 0.5 ). At 3 months, in group 1 , response rates were $90 \%$ in non-severe class, $40 \%$ in severe, none in very severe. Whereas in group 2 response rates were $88.9 \%$ in nonsevere, $45.5 \%$ in severe, none in very severe group ( $\mathrm{p}$ value for nonsevere, severe classes:0.93, 0.85) At 6 months, in group 1 response rates were $90 \%$ in non-severe class, $60 \%$ in severe class, none in very severe. Whereas in group 2 response rates $88.9 \%$ in non-severe class, $45.5 \%$ in severe class and none in very severe class ( $\mathrm{p}$ value for nonsevere, severe classes:0.93, 0.58). 3 patients succumbed to sepsis post IST, resulting survival rate of $93.4 \%$. Most common event post treatment in both groups was febrile neutropenia of more than grade 2 requiring admission and iv antibiotics. In group 1, 25\% patients had transient elevation of transaminases leading to temporary discontinuation of eltrombopag. 12 months was the maximum duration of continuation of eltrombopag $(n=1)$, rest $95.6 \%$ could continue for 3 months due to monetary constraints. Conclusions: Response rates with addition of eltrombopag $50 \mathrm{mg}$ are higher than with only IST, but statistically non-significant. Also we did not encounter major adverse events with the combination, although long term effects were not studied. Also there in no response in the very severe class with IST with or without eltrombopag, which raises a question whether they should be offered IST. Further prospective studies with more sample size are needed to confirm this finding.

\section{References:}

1. Olnes MJ, Scheinberg P, Calvo KR, Desmond R, Tang Y, Dumitriu $\mathrm{B}$, et al. Eltrombopag and improved hematopoiesis in refractory aplastic anemia. N Engl J Med. 2012 Jul 5;367(1):11-9.

2. Desmond R, Townsley DM, Dunbar C, Young NS. Eltrombopag in aplastic anaemia. Seminars in hematology. 2015;52(1).

3. Danielle M. Townsley, M.D., Phillip Scheinberg, M.D., Eltrombopag Added to Standard Immunosuppression for Aplastic Anemia. N Engl J Med 2017;376:1540-50.

\section{5}

Validation of Inhibitor Development Prediction Scoring System for Hemophilia A

Ravi Ranjan", Amit Sharma, Kamal Kishor, Hareram Pandey, Kanwaljeet Singh, Vineet Kumar Kamal, Tulika Seth, M Mahapatra, Renu Saxena

Email ID for Correspondence: raviranjanaiims@gmail.com All India Institute of Medical Sciences, New Delhi

Introduction: A 7 point scoring system was developed by the CANAL study group to stratify patients with severe haemophilia A according to their risk of developing inhibitory antibodies. As per the score two points were assigned to a positive family history and presence of a high-risk gene mutation each, three points to the presence of an intensive treatment moment at initial treatment with FVIII. There was a need to validate the above scoring system in Indian severe Hemophilia A patients so that it can be used by clinicians to tailor clotting factor treatment regimens according to the individual risk profiles for inhibitor development. Objectives: To validate the 7 point scoring system in severe Haemophilia A patients from India. Material and Methods: The study population consisted of 110 patients with severe haemophilia A, the score was based on risk 
factors available at the first treatment episode. All the relevant patient characteristics were recorded as per the requirement of the scoring system. Screening test for FVIII inhibitors was performed in all patients. Bethesda assay was done to obtain the inhibitor titre in patients who were positive for FVIII inhibitors. Inversion 22 and 1 was done in all the patients and CSGE followed by sequencing was done in Inversion negative cases to find out the causative mutation. The patients were grouped into different risk category based on the total scores thus patients with a score of 0,2 and $\geq 3$ were put in the low, medium and high risk category, respectively. The positive and negative predictive values (PPV, NPV) for each risk category were calculated to find out the probability of developing FVIII inhibitors. Area under the ROC curve for the scoring system was calculated to find out the ability of the score to discriminate who did and who did not develop FVIII inhibitors. Results: A total of 26 patients $(23.6 \%)$ developed FVIII inhibitory antibodies. According to the risk scoring system 33 patients were in the low risk category, 52 in medium risk category and 25 in High risk category. The observed inhibitor incidences were $2 / 33(\mathrm{PPV}=0.06, \mathrm{NPV}=0.62)$ in low risk category, $16 / 52(\mathrm{PPV}=0.31, \mathrm{NPV}=0.74)$ in medium risk category and $13 / 25$ $(\mathrm{PPV}=0.52, \mathrm{NPV}=0.80)$ in high risk category respectively. The low risk category had PPV of 0.06 i.e. a probability of $6 \%$ to develop an inhibitor and a negative predictive value of 0.62 i.e. a probability of $62 \%$ that a patient (outside the low category) in the medium or high risk category will not develop an inhibitor. The predicted inhibitor incidences as per score were $3 / 33,15 / 52,13 / 25$ in the low medium and high risk respectively, which were statistically indifferent fro actual incidence of FVIII inhibitors. The discriminative ability of the score was as good as actually tested (area under the receiver operating curve 0.78). Conclusions: These findings suggest that the development of FVIII inhibitor in patients with severe haemophilia A can be effectively predicted with the present risk stratification score. This scoring system provides a tool for clinicians to tailor clotting factor treatment regimens according to the individual risk profiles for inhibitor development of their patients with severe haemophilia A.

\section{6}

Identification of cancer stem cells in acute $\mathbf{T}$ cell lymphoblastic lymphoma/leukemia

Gayatri Gogoi", Anupam Sarma ${ }^{3,4}$, Rashmi Bhuyan ${ }^{1,2,3}$, Joyeeta Talkudar ${ }^{3,5}$, Hong $\mathbf{L i}^{1,2}$, BidishaPal ${ }^{2,3}$, Seema Bhuyan ${ }^{3,5}$, Sora Sandhya $^{3,5}$, Sukanya Gayan ${ }^{2,3}$, Debabrat Baishya ${ }^{3,5}$, Amal Chandra Kataki ${ }^{4}$, Dean W. Felsher ${ }^{1}$, Bikul Das ${ }^{3}$

Email ID for Correspondence: gayatrigogoi303@gmail.com KaviKrishna Laboratory, Guwahati Biotech Park, Indian Institute of Technology, Guwahati, India, ${ }^{6}$ Department of Pathology, Assam Medical College, Dibrugarh, India, ${ }^{4}$ Dr.B.Borooah Cancer Institute, Guwahati, India, ${ }^{5}$ Department of Bioengineering, Gauhati University Institute of Science and Technology, Guwahati, India, Division of Oncology, Departments of Medicine and Pathology, Stanford University School of Medicine, Stanford, California, ${ }^{2}$ Department of Immunology and Infectious Diseases, Forsyth Institute, Cambridge, MA, *address correspondence to bdas@forsyth.org and dfelsher@stanford.edu

Backgound: Acute T-cell lymphoblastic lymphoma/leukemia (TLBL) is a variant of acute lymphoblastic leukemia (ALL). The 5 year disease free survival rate is $80-90 \%$ in children and $50 \%$ in adult. Previous studies indicated that T-LBL might contain rare cancer stem cell population resistant to conventional chemotherapy. However, the distinct molecular identity of these CSCs are not clearly known. Characterization of the pathological phenotype of the T-LBL derived
CSCs would help in pathological grading of T-LBL. Materials and method: Transgenic mice, tumor isolation, flow cytometry sorting for Sca-1+ cells and injection to transgenic mice, Sca-1+ phenotype flow cytometry analysis, Intracellular GSH and ROS Measurement, Apoptosis and Proliferation Measurement, Senescence Associated Beta-galactosidase (SA-betagal) activity Measurement, Real time PCR (qPCR), Specific inhibition of genes, Chromatin Immunoprecipitation, Clonogenic assay, Human T cell leukemia cell line Molt-3 culture, Clinical Sample of leukemia, Suspension culture of T-LBL cells, Immunohistochemistry and Western Blot (WB). Statistical analysis: The statistical calculations were performed with Graph Pad Prism 4.0, using Student's t-test dilution analysis software, Statistical comparison of Kaplan-Meier curves was based on the log-rank test. Results: Using both mouse model of T-LBL as well as clinical samples of adult T-LBL, we have identified CSCs, and characterized their pathological phenotype. In a transgenic mouse model of MYC driven lymphoma, we identified a Sca-1+/ABCG2+ CSC population that showed high expression of Nanog, Sox2, and HIF-2 $\alpha$. Even brief inactivation of HIF-2 $\alpha$ led to increased reactive oxygen species (ROS) generation and $\mathrm{p} 53$, decreased glutathione (GSH) level, proliferative arrest, and the loss of self-renewal capacity. In human T cell lymphoblastic lymphoma/leukemia, we isolated an ABCG2+ CSC population that exhibited high expression of Nanog, Sox-2, HIF-2alpha and MYC. The ABCG2+ cells were resistant to chemotherapy-induced apoptosis. Specific knockdown of HIF-2alhpa resulted in the loss of self-renewal capacity of $\mathrm{ABCG} 2+$ cells. Discussion and Conclusion: Thus, we have identified $\mathrm{ABCG} 2+$ as the putative CSCs of adult T-LBL. There is also potential explanation as MYC maintains self renewal in cancer stem cells that may have general therapeutic implications. We also found that inhibition of HIF-2alpha, a hypoxia-induced transcription factor might target these CSCs.

Keywords T cell-LBL, CSC, MYC, HIF2 alpha, Self renewal, ABCG2

\section{7}

Incidence, Clinical Profile and Treatment Outcomes of Peripheral T Cell Lymphoma: A Single Centre Experience

Jyotsna Kapoor ${ }^{\#}$, Narendra Agrawal, Mukul Aggarwal, Pallavi Mehta, Priyanka Verma, Prashant Raj Verma, Rayaz Ahmed, Dinesh Bhurani

Email ID for Correspondence: jkapoor022@gmail.com Rajiv Gandhi Cancer Institute and Research Centre, New Delhi, India

Introduction: Peripheral T cell Lymphomas (PTCL) are heterogeneous group of non hodgkin's lymphoma (NHL) with a incidence of 4-21\% of all NHLs and have a dismal outcome. Objectives: To report the incidence, clinical profile, \& the variables predicting the treatment outcomes of PTCL from a single centre from northern India. Methods: Records of 417 NHL patients between January 2010 till March 2016 were reviewed and a total of 59 patients $(14.1 \%)$ were found to have PTCL according to WHO classification ( $3^{\text {rd }}$ edition). Follow up data of all 59 PTCL patients were collected till $31^{\text {st }}$ July 2017. Overall survival (OS) and event free survival (EFS) was calculated using Kaplan-Meier method with the help of SPSS software (IBM, version 21 for windows, Armonk, NY, USA). Univariate \& multivariate analyses were used to assess various variables predicting OS using Cox-regression analysis. Results: - The median age was 49 years (1274) with a male predominance of 2.1: 1 . Nodal type of PTCL was the most common $(\mathrm{n}=40,67.8 \%)$, followed by extranodal PTCL $(\mathrm{n}=14,(23.7 \%)$ and cutaneous $\mathrm{T}$ cell lymphomas $(\mathrm{n}=5,8.47 \%)$. The histological distribution was: PTCL-NOS 16 patients $(27 \%)$, anaplastic large cell ALK negative (ALCL ALK -) 9 patients 
(15.25\%), anaplastic large cell lymphoma ALK positive (ALCL $\mathrm{ALK}+) 8$ patients $(13.5 \%)$, angioimmunoblastic $\mathrm{T}$ cell lymphoma (AITL) 7 patients $(11.86 \%$ ), extranodal NK/T (NKTCL) 5 patients (8.47\%), Hepatosplenic T cell (HSTCL) 5 patients (8.47\%), subcutaneous panniculitis $\mathrm{T}$ cell Lymphoma (SPTCL) 4 patients $(6.67 \%)$ and 5 of cutaneous T cell Lymphoma (CTCL) $8.49 \%$. PTCL patients presented with B symptoms $(n=30,50.8 \%)$, raised $\mathrm{LDH}(\mathrm{n}=33$, $55.9 \%)$, extranodal involvement $(\mathrm{n}=31,64.4 \%)$, ann- arbor stage of III/IV ( $\mathrm{n}=38,64.4 \%)$, involved bone marrow $(\mathrm{n}=10,16.9 \%)$, IPI risk group of intermediate/high $(\mathrm{n}=31,52.54 \%)$ and PIT risk group of intermediate/high $(n=20,33.89 \%)$. Forty- nine patients $(83.05 \%)$ were treated with anthracycline based chemotherapy as the first line treatment. Complete Response (CR) and Overall response rate (ORR) was achieved in $38(64.4 \%)$ and $41(69.5 \%)$ patients post first line treatment. Sixteen $(27.1 \%)$ patients underwent hematopoietic stem cell transplant \{Auto-HSCT -12 (CR1-6, CR2-4, PR2-1, stable disease-1) \& Allo-HSCT - 4\}. At a median follow up of 16.2 months (2.9-186.9) median OS and EFS was found to be 30.1 months and 10.2 months respectively. Relapse rate of $25.4 \%$ (15/59). The estimated 3- year OS is found to be $14.3 \%$ for AITL, $60 \%$ for NKTCL, $42.7 \%$ for PTCL-NOS, $100 \%$ for SPTCL, $44.4 \%$ for ALCL ALK $100 \%$ for ALCL ALK+, while for HSTCL and CTCL could not be calculated because of lesser duration of follow up. On univariate analysis, age $>60$ years $(p=0.006)$ and failure to achieve CR $(\mathrm{p}<0.001)$ was found to be significantly associated with inferior survival, while greater than one extranodal involvement $(p=0.056)$, intermediate or high IPI risk group $(\mathrm{p}=0.06)$, no hematopoietic stem cell transplantation ( $\mathrm{p}=0.099)$ was found to have a non-significant trend. On multivariate analysis, age $>60(\mathrm{p}=0.023)$ and failure to achieve CR $(\mathrm{p}<0.001)$ maintained predictive value. Conclusions: PTCL constitutes $14.1 \%$ of all NHL's. PTCL-NOS was the most common type of PTCL with an estimated 3-year survival of $42.7 \%$. Failure to achieve $\mathrm{CR}$ and age $>60$ years are poor predictors of survival.

\section{8}

The Role of Cytogenetic Abnormalities as a Prognostic Marker: Pathogenesis and Clinical Course in Patients with B-Chronic Lymphocytic Leukemia

Yogita Rohil $^{1 \#}$, Dhanlaxmi Shetty ${ }^{1}$, Hemani Jain ${ }^{1}$, Manju Sengar ${ }^{2}$, Hasmukh Jain ${ }^{2}$, Bhausaheb Bagal', Navin Khattry $^{2}$ and PG Subramanian ${ }^{3}$

${ }^{1}$ Department of Cancer Cytogenetics, ACTREC, Tata Memorial Centre, Kharghar, Navi Mumbai; ${ }^{2}$ Department of Medical Oncology, Tata Memorial Hospital, Parel, Mumbai; ${ }^{3}$ Department of Hematopathology, ACTREC, Tata Memorial Centre, Kharghar, Navi Mumbai

Email ID: yogitarohila@gmail.com, shettydl@tmc.gov.in, dhanlaxmis22@gmail.com

Introduction: Chronic lymphocytic leukemia (CLL) is highly heterogeneous in terms of its clinical course and prognosis which impacts the clinical approaches, treatment strategies and survival times from diagnosis. Among prognostic markers reviewed, chromosomal aberrations have been validated and are currently used clinically to predict prognosis independent from conventional clinical markers for a risk-adapted management of CLL patients. Molecular cytogenetic methods such as fluorescence in situ hybridization (FISH) detects chromosome aberrations in approximately $80 \%$ of CLL cases. Objectives: To score the frequencies of the recurrent aberrations and to understand their role in prognostication and therapy selection. Material and Methods: Interphase fluorescence in situ hybridization (FISH) studies were performed on bone marrow aspirate and/or peripheral blood of 292 patients diagnosed as CLL from January 2016 till July 2017 using commercially available disease-specific probe set to score the frequency of $\operatorname{del}(13 \mathrm{q})$, trisomy 12 , del(11q), TP53 deletion, del(6q) and IgH translocation/rearrangement. In every specimen, 200 interphase cells were analysed for each probe and images were captured using the Genesis ASI system. Results: Among 292 CLL cases, $228(78 \%)$ had 1 or more Cytogenetic abnormalities; remaining 64 cases $(22 \%)$ had normal FISH results. Deletion/Monosomy of chromosome 13q/-13 was the most common cytogenetic abnormality detected in $48 \%(110 / 228)$ cases indicating del(13q) as a primary event followed by $\operatorname{IgH}$ rearrangement/ deletion in $21 \%(49 / 228)$, trisomy 12 in 19\% (44/228), 11q deletion in 17\% (40/228), TP53 deletion in $14 \%$ (34/228), 6q deletion in $6 \%(14 / 228)$ as secondary progressive events. The estimated median treatment-free interval was longest for patients with a deletion 13q14 as single aberration and shortest for patients with $17 \mathrm{p}$ deletions. Patients with a higher percentage of interphase nuclei that exhibit the cytogenetic defect were associated with poor risk factors such as hyperleukocytosis, extensive lymphadenopathy and splenomegaly. Time to treatment was same for patients with homozygous or heterozygous deletions. Conclusions: Interphase-FISH has proved to be a sensitive and efficient technique in detecting the prevalence of various recurrent cytogenetic abnormalities. The association of TP53 deletions, del(6q) and $\operatorname{IgH}$ translocations with high-risk clinico-laboratory features aids in the prognostication and implementation of targeted therapies. Continued investigation of the relationship between specific FISH defects and clinical outcome will lead to improved risk stratification and better disease management.

\section{9}

Clinical significance of Cytogenetic Findings in 630 Patients with B-Cell Precursor Lymphoblastic Leukemia (BCP-ALL)

Vrushali Mistri $^{1 \#}$, Dhanlaxmi Shetty ${ }^{1}$, Sharayu Kabre ${ }^{1}$, Hemani Jain ${ }^{1}$, Yogita Deshpande ${ }^{1}$, Manju Sengar ${ }^{2}$, S.D. Banavali ${ }^{2}$, Navin Khattry ${ }^{2}$, Gaurav Narula ${ }^{2}$, Bhausaheb Bagal $^{2}$, Hasmukh Jain ${ }^{2}$ and PG Subramanian ${ }^{3}$

Email ID for Correspondence: vrush.mistri@gmail.com

${ }^{1}$ Department of Cancer Cytogenetics, ACTREC, Tata Memorial Centre, Kharghar, Navi, Mumbai; ${ }^{2}$ Department of Medical Oncology, Tata Memorial Hospital, Parel, Mumbai; ${ }^{3}$ Department of Hematopathology, ACTREC, Tata Memorial Centre, Kharghar, Navi Mumbai

Introduction: Acute lymphoblastic leukemia (ALL) is a neoplastic disease characterized by clonal expansion of leukemic cells in the bone marrow (BM), lymph nodes, thymus, or spleen. B-precursor acute lymphoblastic leukemia is the most common childhood tumor and the leading cause of cancer-related death in childhood and young adults. Cytogenetics is the most important diagnostic parameters in the classification of acute lymphoblastic leukemia. Objectives: To understand the prevalence and clinical significance of cytogenetic markers in BCP-ALL cases. Material and Methods: The study was conducted retrospectively on BCP-ALL cases that were referred to Department of Cancer Cytogenetic, Tata Memorial Center, Mumbai, India. The present study includes 630 patients (Adults- 198 cases; Pediatric-432 cases) that were diagnosed with BCP-ALL between January, 2016 to December, 2016. Fluorescence In Situ Hybridization (FISH) was performed on interphase cells/ metaphase using the commercially available probes for BCR/ ABL1; MLL; ETV6/ 
RUNX1; TCF3/PBX1 rearrangements and centromeric probes for chromosomes 4, 10 and 17 for ploidy status. Ploidy analysis on metaphases was also conducted. Results: Our study revealed $68 \%$ cases of BCP-ALL (433/630) with cytogenetic abnormality, out of 198 adult cases, 66\% (132/198) showed cytogenetic aberrations, whereas $69 \%(301 / 432)$ of pediatric cases were found to have cytogenetic aberration. Cytogenetic profiling of BCP-ALL revealed prevalence of $\mathrm{t}(12 ; 21)[E T V / R U N X 1]$ in $15 \%$ of pediatric cases. The incidence of $\mathrm{t}(9 ; 22)$; BCR-ABL1 was high in adult cases $(53 \%)$ as compared to pediatric cases $(8 \%)$. Similarly, $\mathrm{t}(1 ; 19)$; TCF3-PBX1 was higher in adult $(4 \%)$ compared to pediatric cases $(3 \%)$. The MLL aberrations found were similar in frequency for both adult (3\%) and pediatric $(3 \%)$ cases. Hyperdiploidy (13\%) was observed more in pediatric cases than in adult. Trisomy $4,10,17$ were detected either as a sole or in combination with other abnormalities in 16\% (50/430) pediatric cases and 5\% (7/198) adult cases. Hypodiploidy was observedin pediatric cases $(2.5 \%)$ than in adult (1.6\%). Conclusions: The present study showed the heterogeneity of BCP-ALL that involved age and prevalence of distinct cytogenetic subgroups. The above study confirmed that various cytogenetic subtypes have significant impact on risk stratification and hence remain strong independent indicators of disease outcome.

\section{$\mathbf{O 2 0}$}

Ibrutinib vs Temsirolimus: Three-Year Follow-Up of Patients with Previously Treated Mantle Cell Lymphoma from the Phase 3, International, Randomized, Open-Label Ray Study

Simon Rule, Wojciech Jurczak, Mats Jerkeman, Rodrigo Santucci Silva, Chiara Rusconi, Marek Trneny, Fritz Offner, Dolores Caballero, Cristina Joao, Mathias Witzens-Harig, Georg Hess, Isabelle Bence-Bruckler, Seok-Goo Cho, Catherine Thieblemont, Wenjiong Zhou, Todd Henninger, Jenna Goldberg, Jessica Vermeulen, Manan Shah", Martin Dreyling

Email ID for Correspondence: mshah33@its.jnj.com Medical Affairs Janssen India, Johnson \& Johnson Pvt Ltd

Introduction: Ibrutinib (IBR), a first-in-class, once-daily, oral, covalent inhibitor of Bruton's tyrosine kinase, is highly active in relapsed/refractory (R/R) mantle cell lymphoma (MCL). The phase 3, randomized, open-label RAY study compared IBR with temsirolimus (TEM) in patients (pts) with $\mathrm{R} / \mathrm{R}$ MCL and $\geq 1$ prior rituximabcontaining therapy. At median 20.0-month follow-up, IBR was superior to TEM for independent review committee-assessed progression-free survival (PFS) (HR: 0.43; 95\% CI, 0.32-0.58; $p<0.0001$ ) (Dreyling et al. Lancet 2016). Objectives: To present 3-year follow-up results (end of study). Material and Methods: 280 pts were randomized $1: 1$ to oral IBR (560 mg once-daily; $\mathrm{n}=139)$ or intravenous TEM (175 mg: days 1, 8, 15 of cycle $1 ; 75 \mathrm{mg}$ : days 1,8 , 15 of subsequent cycles; $\mathrm{n}=141$ ) until disease progression/unacceptable toxicity. Long-term efficacy was investigator assessed. Results: At a median follow-up of 39 months for IBR and TEM, respectively, median PFS was 15.6 vs 6.2 months (HR: $0.45 ; 95 \%$ CI, $0.35-0.60 ; p<0.0001$ ) (Figure 1A); median PFS for pts with only 1 prior line of therapy (LOT) was 25.4 (IBR) vs 6.2 months (TEM) (HR: $0.40 ; 95 \%$ CI, 0.25-0.64) (Figure 1B). Overall response rate

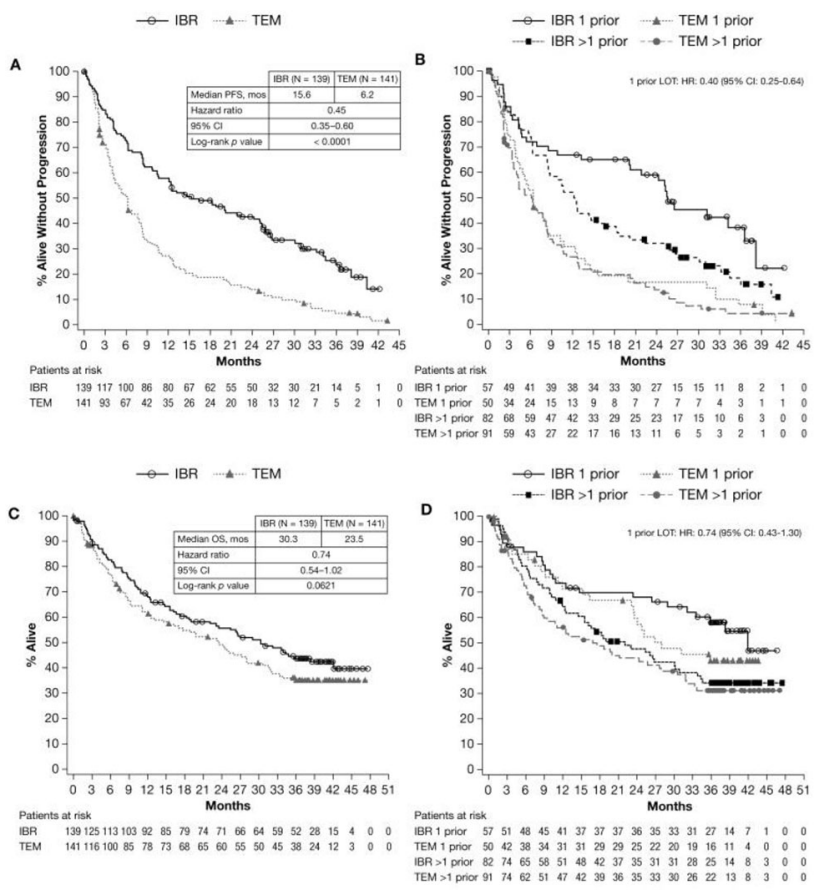

Figure 1 PFS (A) and PFS by line of therapy (B) and OS (C) and OS by line of therapy (D)

(ORR) was $77.0 \%$ (IBR) vs $46.8 \%$ (TEM); CR rate was $23.0 \%$ vs $2.8 \%(p<0.0001)$. ORR for pts with 1 prior LOT was $75.4 \%$ (IBR) vs $52.0 \%$ (TEM); complete response rate was $33.3 \%$ vs $4.0 \%$. Median duration of response was 23.1 (IBR) vs 6.3 months (TEM). Median time to next treatment was 31.8 (IBR) vs 11.6 months (TEM) (HR: 0.33 ; 95\% CI, 0.24-0.46; $p<0.0001$ ). PFS2 was 26.2 (IBR) vs 15.4 months (TEM) (HR: $0.67 ; 95 \%$ CI, $0.50-0.90 ; p<0.0079$ ). With $39 \%$ of pts randomized to TEM crossing over to IBR, median overall survival (OS) was 30.3 (IBR) vs 23.5 months (TEM) (HR: 0.74; 95\% CI, 0.54-1.02; $p=0.0621$ ) (Figure $1 \mathrm{C}$ ); median OS for pts with 1 prior LOT was 42.0 (IBR) vs 27.0 months (TEM) (HR: 0.74; 95\% CI, 0.43-1.30) (Figure 1D). Median treatment duration was 14.4 (IBR) vs 3.0 months (TEM), with $24 \%$ of IBR pts and 0 TEM pts on treatment at study end. Despite differences in exposure, overall frequency of adverse events (AEs) was lower with IBR vs TEM. AEs leading to treatment discontinuation: $17.3 \%$ (IBR) vs $31.7 \%$ (TEM). Most common treatment-emergent AEs: diarrhea, fatigue, and cough (IBR) and thrombocytopenia, anemia, and diarrhea (TEM). Grade $\geq 3$ AEs: $74.8 \%$ (IBR) and $87.1 \%$ (TEM). Serious AEs: $56.8 \%$ (IBR) and $59.7 \%$ (TEM). Conclusions: The RAY study 3-year follow-up results are consistent with primary analysis, showing clinically meaningful, statistically significant improvement of PFS for IBR vs TEM, with a strong trend in OS favoring IBR, despite nearly $40 \%$ crossover. Pts who had received IBR after only 1 prior LOT had the most durable and best PFS and OS outcomes, supporting earlier use of IBR in R/R MCL. Significantly longer PFS2 for IBR suggests treatment benefit is maintained after next LOT. No new safety signals were observed. Despite longer exposure, IBR pts experienced fewer grade 3/4 AEs and treatment discontinuations due to AEs. 


\section{1}

Single-Agent Ibrutinib vs Standard of Care for Patients with Treatment-Naïve (TN) Chronic Lymphocytic Leukemia (CLL): An Adjusted Comparison of Resonate-2 ${ }^{\mathrm{TM}}$ with the CLLEAR and French Lyon-Sud Databases

Michael Doubek, Emmanuel Bachy, Martin Spacek, Lucile Baseggio, Renata Urbanová, Hervé Besson, Joris Diels, Jamie Garside, Nollaig Healy, Wafae Iraqi, Evelyne Callet-Bauchu, Lukas Smolej, Deepak Chinchapattanam ${ }^{\#}$, Gilles Salles

Email ID for Correspondence: dchincha@its.jnj.com Medical Affairs Janssen India, Johnson \& Johnson Pvt Ltd

Introduction: The phase 3 RESONATE- $2^{\mathrm{TM}}$ study demonstrated significant improvement of progression-free survival (PFS) and overall survival (OS) with ibrutinib vs chlorambucil in TN (aged $\geq 65$ years) CLL patients. In the absence of direct comparison of single-agent ibrutinib with other frequently used treatments in this patient population, additional comparative evidence against standard of care as observed in clinical practice can provide useful insights on the relative efficacy of ibrutinib. Objectives: To investigate the relative treatment effect on PFS and OS for ibrutinib vs real-world (RW) treatment in daily clinical practice in TN CLL patients by adjusted comparison of patient-level data from RESONATE- $2^{\mathrm{TM}}$ vs RW data from the CLLEAR (Chronic Lymphocytic Leukemia Registry) and Lyon-Sud databases. Material and Methods: CLLEAR holds medical records for CLL patients from seven academic centers across the Czech Republic. The Lyon-Sud database holds medical records for CLL patients from the academic French hospital Lyon-Sud. Patients initiated on CLL frontline therapy were selected from CLLEAR and Lyon-Sud using the same inclusion-exclusion criteria as for RESONATE- ${ }^{\mathrm{TM}}$ (excluding patients aged $<65$ years and with del17p positive status). PFS and OS were compared between ibrutinib and RW treatment using patient-level data from RESONATE$2^{\mathrm{TM}}(\mathrm{n}=136)$ and pooled patient-level data from the two cohorts. To adjust for differences in patient characteristics between the trial population and both cohorts, a multivariate Cox proportional hazards model was fitted on patient-level data to estimate the hazard ratio (HR) for ibrutinib vs RW treatment, with age, sex, disease stage (based on Rai/Binet), and del11q presence/absence included as covariates. Results: Median age at treatment initiation for CLLEAR $(\mathrm{n}=418)$ and Lyon-Sud $(\mathrm{n}=102)$ was 71 and 72 years, respectively, vs 73 years for ibrutinib patients from RESONATE- $2^{\mathrm{TM}}$. The proportion of male patients was $63 \%$ in CLLEAR and $60 \%$ in LyonSud vs $65 \%$ in RESONATE- ${ }^{\mathrm{TM}}$. The median follow-up was 16.8 months in CLLEAR and 35.7 months for Lyon-Sud vs 28.1 months for RESONATE- ${ }^{\mathrm{TM}}$. Adjusted HR for ibrutinib vs physician choice in the pooled cohort (CLLEAR and Lyon-Sud; $\mathrm{n}=520)$ were $0.27(95 \% \mathrm{CI}, 0.16-0.45)$ for PFS and 0.40 (95\% CI, 0.19-0.82) for OS. Fludarabine + cyclophosphamide + rituximab (FCR; $\mathrm{n}=117)$, bendamustine $+\mathrm{R}(\mathrm{BR} ; \mathrm{n}=90)$, chlorambucil alone $(n=43)$, chlorambucil $+R(n=46)$, and other R-containing regimens $(n=157)$ were the most commonly used treatment regimens across both RW cohorts. Older age, male gender, advanced disease stage, and del11q positive status were independent risk factors for PFS and OS. The adjusted HRs (pooled estimates) for IBR vs the two most commonly used regimens were $0.33(0.19-0.60$, FCR $)$ and $0.38(0.17-0.82, \mathrm{BR})$ for PFS, and $0.49(0.22-1.12, \mathrm{FCR})$ and 0.38 (0.14-1.05, BR) for OS (Figure 1). Estimates of HR vs regimens in the cohorts were consistent across both databases. Conclusions: This adjusted comparison of patient-level data from RESONATE- $2^{\mathrm{TM}}$ with RW data from CLLEAR and Lyon-Sud demonstrates ibrutinib to be more effective compared with RW treatment, with a 3.7-fold

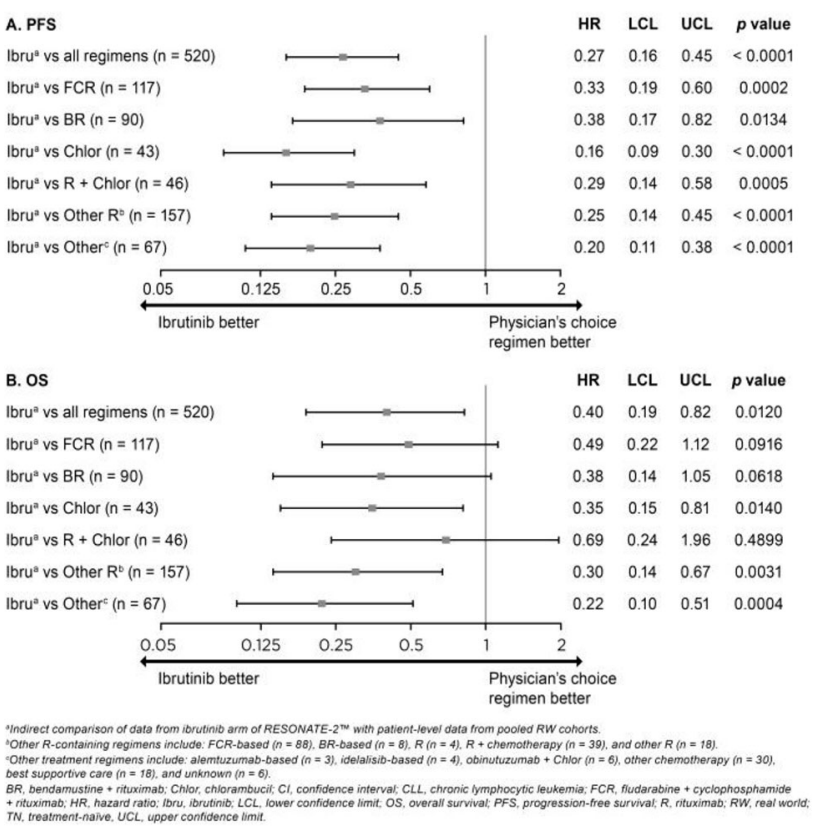

Figure 1 Adjusted Hrs (95\% CI) for PFS (A) and OS (B) for TN Patients With CLL ( $\geq 65$ Years Without Del17p) Treated With Ibrutinib Versus Most Frequently Used Psysician's Choice Regimen in the Pooled RW Cohorts

improvement in PFS and a 2.5 -fold improvement in OS. When comparing ibrutinib with the most commonly used RW treatments, statistically significant benefits for ibrutinib were consistently observed vs all treatment regimens on PFS and for some comparisons on OS. These results further support the existing evidence that ibrutinib significantly improves PFS and OS vs commonly used regimens in TN CLL settings, and has important implications for clinical practice.

\section{2}

Next generation sequencing based molecular diagnosis in rare red blood cell disorders presenting as hemolytic anemia

${ }^{1}$ Reena Das, ${ }^{1}$ Manu Jamwal, ${ }^{1}$ Anu Aggarwal, ${ }^{2}$ Arindam Maitra, ${ }^{1}$ Prashant Sharma, ${ }^{3}$ Deepak Bansal, ${ }^{3}$ Amita Trehan, ${ }^{4}$ Pankaj Malhotra

${ }^{1}$ Department of Hematology, Post Graduate Institute of Medical Education and Research, Chandigarh 160012, India; ${ }^{2}$ National Institute of Biomedical Genomics, Kalyani, West Bengal 741251, India; ${ }^{3}$ Hematology-Oncology unit, Department of Pediatrics, Advanced Pediatrics Centre, Post Graduate Institute of Medical Education and Research, Chandigarh 160012, India;

${ }^{4}$ Department of Internal Medicine (Clinical Hematology

Division), Post Graduate Institute of Medical Education and Research, Chandigarh 160012, India

Introduction: Diagnosing congenital anemias diagnosis requires a step-wise diagnostic algorithm to be followed. The clinical manifestations are jaundice, cholelithiasis, splenomegaly, with usually hemolytic anemia. Phenotypes vary from having fully compensated hemolysis (without anemia) to severe transfusion dependent hemolytic anemia. Causal genes implicated in congenital hemolytic anemia are numerous, making a gene-by-gene approach time consuming, 
expensive and labour intensive. Use of targeted resequencing can expedite the molecular diagnosis where the cause for hemolysis remains unexplained after routine laboratory tests. Methods: Sixteen patients with clinical and laboratory evidence suggestive of hemolytic anemia were enrolled. Various biochemical and molecular tests were used to exclude Glucose-6-phosphate dehydrogenase (G6PD) deficiency, thalassemias, hemoglobinopathies, autoimmune hemolytic anemia, hereditary spherocytosis and pyruvate kinase (PK) deficiency. Common $G 6 P D$ and $P K$ variants were excluded by molecular tests. Genomic DNA was extracted by the QIAamp DNA Blood Midi Kit and quantified on NanoDrop 2000 spectrophotometer and Qubit Fluorometer. TruSight One Sequencing Panel was used to prepare DNA libraries and sequenced on a MiSeq Sequencing System. MiSeq Reporter and VariantStudio were used for analysis, classification, and reporting of genomic variants reporting genomic variants. Results: Out of 16 patients sequenced, five patients with pyruvate kinase $(P K L R)$ deficiency, two patients with G6PD deficiency, two patients with Glucose-6-phosphate isomerase $(G P I)$ deficiency were found. One patient was found with each with probably causative mutations in RHAG, ABCG5, MTRR gene were diagnosed. Out of 16 cases we were unable to find the causative mutations in four of them and we plan to subject these samples for exome sequencing. Unexpected pyruvate kinase defects were found on targeted re-sequencing for four patients. Pyruvate kinase (PK) enzyme activity assay were within normal limits in all these cases. All the mutations were predicted deleterious by PolyPhen/ SIFT/ Provean/ mutpred and Mutationtaster. Mutations were validated in the parents/siblings (where available) to prove the mode of inheritance. Conclusions: PK deficiency may be missed by conventional testing approaches. We describe rare phenotypes for the first time from India. Our data demonstrates the clinical utility of next generation sequencing for molecular diagnosis. Timely detection of the cause in our patient is likely to be helpful not just in genetic counselling and future antenatal diagnosis, if required, but therapeutically as well.

\section{$\mathbf{0 2 3}$}

\section{Phenotypic Characteristics of Alpha-Globin Gene Triplication} in West Bengal Population

\section{Sujana Biswas", Rudra Ray, Kaushik Roy, Maitreyee Bhattacharyya}

Email ID for Correspondence: sujana.sujana@gmail.com Institute of Haematology and Transfusion Medicine, Medical College, Kolkata

Introduction: The human alpha globin gene cluster is located on chromosome 16 and spans about $30 \mathrm{~kb}$ including seven loci. Alphaglobin gene triplication (alpha alpha alpha anti 3.7) is the reciprocal recombinant chromosome of -alpha 3.7 deletion, and it occurs mostly in the tropical and subtropical regions of the world. These individuals carry a chromosome that contains three $\alpha$-globin loci as a result of unequal crossing-over. The frequency of the $\alpha \alpha \alpha$ anti 3.7 triplication have not been clearly determined. Objectives: There are few studies investigating alpha globin gene triplications in Indian population and its effect on phenotype. Hence it was the primary aim of this study. Material and Methods: 75 normal subjects (voluntary blood donors) were screened for triplication of alpha gene (alpha alpha alpha anti 3.7). Their clinical and laboratory parameters were recorded. Hematological analysis was performed by automated cell counter. HbA0, $\mathrm{HbA} 2$ and $\mathrm{HbF}$ were measured by HPLC. Triplication was done by Gap-PCR by optimizing the method as described by Wen et al (2003). Results: Among the 75 subjects, 16 showed alpha-globin gene triplication ( $\alpha \alpha \alpha$ anti 3.7). It is present in $21.33 \%$ within the population. 4 were male and 12 female. These 16 subjects showed no clinical symptoms nor were they associated with abnormal haematological parameters. Their mean $\mathrm{Hb}$ was $13.5 \pm 1.54$ for males and $12.36 \pm 0.46$ for females. The mean $\mathrm{MCV}$ was $90.69 \mathrm{fL} \pm 7.27$, $\mathrm{MCH}$ was $29.33 \mathrm{pg} / \mathrm{cell} \pm 2.24$ and $\mathrm{MCHC}$ was $32.19 \mathrm{~g} / \mathrm{dL} \pm 1.43$. All these results were well within the normal range. Also the mean $\mathrm{HbA} 0 \quad(87.68 \% \pm 0.79), \quad \mathrm{HbA} 2 \quad(2.93 \% \pm 0.25)$ and $\mathrm{HbF}$ $(0.39 \% \pm 0.22)$ did not show any significant deviation from the normal range. Conclusions: The phenotypic characteristics of the subjects having alpha-globin gene triplication were the same as that of normal healthy adults. So the only way to detect alpha gene triplication is by the molecular study. However, larger sample size is warranted for confirmation of these findings.

\section{4}

Differential Expression of Markers in Mesenchymal Stem Cell with Different Leukemia and Their Subsequent Differention Abilities

Trishna Anand", Dr. Bithiah Grace Jaganathan, Dr. Jina Bhattacharyya, Dr. Sewali Deka Talukdar, Dr. Damodar Das

Email ID for Correspondence: a.trishna@iitg.ernet.in Indian Institute of Technology, Guwahati

Introduction: Mesenchymal stem cells (MSCs) have the ability to differentiate into adipocytes, osteocytes, chondrocytes and other lineages of cells. Because of all these differentiating and immunomodulatory properties of MSCs, they have been used in various therapies in the field of regenerative medicines. These cells also form an important part of bone marrow microenvironment which is responsible for the regulation of hematopoiesis under normal physiological condition. MSCs are identified by expression of cell surface markers such as CD44, CD90, CD105, CD73 and CD49E. Objectives: Studies were done for the expression of specific cell surface marker expression using flow cytometry in order to verify their stemness. They were also compared for their differentiation ability into adipocytes and osteocytes. Material and Methods: MSCs of patients diagnosed with Acute Lymphoid Leukemia, Acute Myeloid Leukemia, Acute Leukemia, Chronic Myeloid Leukemia, Multiple Myeloma, Pancytopenia and Hodgkin's Lymphoma etc. of varying age groups were subjected to various experimental setups. Results: The MSC samples collected from patients showed positive expression of common MSC specific cell surface markers such as CD90, CD73 and CD49E etc. MSCs with acute leukemia showed highest expression of CD90 with a MFI value of 40, with lowest expressions having MFI value of 2 and 6 . In case of from AML, varying expression of CD90 was found with MFI ranging from 6 to 31 .

In case of CD73 expression on MSCs, the highest and the lowest was in case acute leukemia with a MFI value 12 and 1.3, while in AML, diverse expression of CD73 was reported with MFI value 8.5 and 5.8. The highest expression of CD49E was in AML with MFI 33. The patients suffering from acute leukemia showed erratic expression of CD49E with MFI values from 2.9 to 28 . The capacity of these stem cells to differentiate into adipocytes and osteocytes varied with respect to their origin (leukemia), age and passage number of MSCs. The MSCs samples from pancytopenia and acute leukemia showed higher level of adipogenic differentiation as compared to other leukemia. In case of osteogenic differentiation pancytopenia showed higher level of differentiation capacity than other leukemia. Conclusions: MSCs showed diverse surface marker expression as well as differentiation competency dependent on the patient's age, passage number as well as on the malignancy present. 


\section{$\mathbf{0 2 5}$}

Understanding Underlying Mechanisms of Chemoresistance in AML

\section{Amit Sharma\#, Sewali Deka Talukdar, Damodar Das,} Jina Bhattacharyya, Bithiah Grace Jaganathan

Email ID for Correspondence: amit.2015@iitg.ernet.in Indian Institute of Technology, Guwahati

Introduction: Acute myeloid leukemia (AML) is the most common adult leukemia with 30-35\% five-year survival rate in age group $<60$ and less than $10 \%$ long-term survival for $>60$ years age group yielding an overall survival rate of $26 \%$. Despite as high as $65 \%$ initial remission rate post chemotherapy, AML shows high relapse rates with the emergence of chemo-resistant leukemic cells. Objectives: This study aims at understanding the underlying mechanisms involved in the acquisition of chemoresistance by developing chemoresistant leukemic cells in-vitro and studying the aberrations in their gene expression profile. Material and Methods: THP-1 cells (human monocytic cell line derived from an acute monocytic leukemia patient) were repeatedly treated with different doses of daunorubicin and cytosine arabinoside (ara-C) which are prominent chemo-drugs used for treating AML. After 10-11 cycles of 72 hrs drug treatment, the expression of various cell surface markers was quantified using flow cytometry and compared to normal THP1 cells. Results: Among all the markers studied (CD13, CD34, CD44, CD45, CD49a, CD49b, CD49d, CD49e, CD49f, CD61, CD73, CD90, CD105, and HLA1) control THP1 showed expression of CD34, CD44, CD45, CD49d, CD49f, CD105, and HLA1. In comparison, a dose-dependent decrease in expression was observed for CD44, CD45, and CD105 in both daunorubicin and ara-C treated cells with complete loss of CD34 and HLA1. Although a 3.1-fold increase in expression of CD49d (integrin $\alpha$-subunit) was observed for low concentration $(1 \mathrm{nM})$ of daunorubicin, the expression was similar or lower as compared to control THP1 for higher concentrations (10 nM, $50 \mathrm{nM})$. Morphological changes could also be observed in drug treated cells with increased cell adhesion at higher concentrations of daunorubicin as well as ara-C. Conclusions: A dose-dependent decrease in expression of various cell surface markers was observed in drug treated cells in general, except for CD49d, wherein an increase was observed at lower doses of drugs. CD49d is involved in adhesion of cells and exposure to higher drug concentrations might have resulted in even higher expression of CD49d leading to complete cell adhesion of "resistant" cells. But since only the suspension cells were taken for phenotyping, the biasness in sampling cell population might have resulted in observation of lower expression levels of CD49d. The primary ligands for CD49d are VCAM-1, MAdCAM-1, and fibronectin and it plays critical role in adhesion. CD49d has been touted as the strongest flow cytometry-based predictor of overall survival in chronic lymphocytic leukemia (CLL) and correlates with poor prognosis. CD49d has also been implied in providing pro-survival signals and signals protecting CLL cells from drug-induced damages and might be playing a similar role in these drug treated AML cells.

\section{6}

Analysis of T Cell Large Granular Lymphocytic Leukemia: A Report of 62 Cases from a Single Tertiary Care Center in Southern India

Kotteeswari Kathirvel $^{\#}$, Nisham P N, Ansu Abu Alex, Yasir Jeelani Samoon, Uday Kulkarni, Anup J Devasia, Anu Korula, Aby Abraham, Alok Srivastava, Biju George, Vikram Mathews

Email ID for Correspondence: kotteevasu@gmail.com
Department of Haematology, Christian Medical College, Vellore, India

Christian Medical College, Vellore, India

Introduction: Large granular lymphocyte (LGL) leukemia is characterized by clonal expansion of cytotoxic $\mathrm{T}$ cells (CTL). They usually present with cytopenias and it usually associated with autoimmune conditions like rheumatoid arthritis. The World Health Organization (WHO) classification has recognized LGL leukemia as a specific entity among mature peripheral Tcell neoplasms, including CD3+ T-cell LGL (T-LGL) and CD3- natural killer (NK)-LGL leukemia subtypes. Objectives: Our study describes analysis of T-LGL cases with its baseline clinical, haematological characteristics, immunophenotypic findings and treatment outcomes. Material and Methods: We undertook a retrospective study of 62 patients diagnosed as T-LGL at our center from August 2009 to May 2017. The diagnosis of T-LGL was made based on the immunophenotypic analysis of chronic lymphoproliferative disorder (CLPD) panel. The samples were stained for T-CLPD panel comprises of markers such as CD3, CD7, CD5, CD2, CD4, CD8, CD57, CD56, CD16, TCR $\alpha \beta$ and $\mathrm{TCR} \gamma \delta$ followed by RBC lysis, wash and acquired in BD FACS Calibur/ BD FACS Canto II flow cytometer. Results: The median age of the cohort was 40.5 years (range: 5 -82) with male predominance (64.5\% males). The median WBC count was $7.1 \times 109 / \mathrm{L}$ (range: $0.7-$ 25.1), ANC was 1.42 x109/L (range: 0.04-11.9), Hb was $9.8 \mathrm{~g} / \mathrm{dl}$ (4.4$16.2)$ and platelet was $141.5 \times 109 / \mathrm{L}(7.0-770) .74 .1 \%$ of the patients were symptomatic and the common symptoms include fatigue (22patients, $47.8 \%$ ), fever ( 21 patients, $45.6 \%$ ) and $21 \%$ presented with infections. 21 patients had splenomegaly (33.8\%), 20 patients had hepatomegaly (32.2\%) and 13 had lymphadenopathy (21\%). $24.1 \%$ patients presented with B-symptoms. 34 cases (54.8\%) with T- LGL were found to be associated with different autoimmune, neoplastic disorders, stem cell and solid organ transplant. Immunophenotyping was done for all the cases which includes 42 bone marrow, 19 peripheral blood and 1 CSF sample. All cases were showing CD7 dim and CD5 was negative in 2 patients $(n=61)$. CD4/CD8 reversal is seen in all the patients. 25 out of 62 patients underwent treatment with different first line treatment regimens such as Cyclosporine \pm Prednisolone/Dexamethasone $(\mathrm{n}=9), \quad$ Methotrexate \pm Prednisolone/ Dexamethasone $(n=9)$, Cyclophosphamide \pm Prednisolone/Dexamethasone $(\mathrm{n}=5)$, G-CSF $(\mathrm{n}=1)$ and multiple drugs $(\mathrm{n}=1)$. Among the treated patients only $12 \%$ achieved complete remission and 28\% achieved partial remission. Conclusion: LGL Leukemia can have a varied presentation ranging from no symptoms to cytopenias and B symptoms. It can be associated with conditions like autoimmune and neoplastic disorders. A Subset is also seen post stem cell transplant and solid organ transplant. Flow cytometry can aid in the diagnosis of this condition. Symptomatic patients are treated with immunosuppressive medications and Cytotoxic drugs with or without steroids.

\section{O27}

Fluorescent Multiplex PCR Can Complement Multiplex Ligation Dependent Probe Amplification (MLPA) Screening for Detection of IKZF1 Deletions in B-ALL

Dr Sanjeev Kumar Gupta\#, Dr Sameer Bakhshi*, Dr Lalit Kumar*, Dr Ritu Gupta

Email ID for Correspondence: drskgupta1@gmail.com Laboratory Oncology Unit and *Department of Medical Oncology, All India Institute of Medical Sciences (AIIMS), New Delhi

All India Institute of Medical Sciences (AIIMS), New Delhi 
Introduction: IKZF1 (IKAROS) deletions independently affect the outcome in B-ALL. We investigated the prevalence of IKZFI deletions in B-ALL using multiplex ligation dependent probe amplification (MLPA) technique. Objective: To assess the value of breakpoint-specific fluorescent multiplex PCR in cases detected to be positive for $I K Z F 1$ deletions on MLPA. Material and Methods: The genomic DNA from newly-diagnosed, untreated B-ALL cases was prospectively analyzed for $I K Z F 1$ deletions over 26 months using MLPA (SALSA MLPA P335 ALL-IKZF1 and P202 IKZF1 kits (MRC-Holland)). Fragment analysis was done on Genome Lab GeXP genetic analysis system and the data analyzed using Coffalyser software. The breakpoint-specific fluorescent multiplex PCR followed by capillary electrophoresis was performed in cases having IKZFI deletions using primers described by Caye et al. The outcome of induction chemotherapy was noted. Results: The median age of 229 cases was 8.5 years ( 2 months-67 years) with 179 pediatric patients (upto $18 \mathrm{yrs})$. The $I K Z F 1$ gene deletions were seen in $56(24.5 \%)$ cases. Out of these, $34(19.0 \%)$ IKZFI deletions were seen in pediatric patients and $22(44 \%)$ in adults. Overall, 61 deletions were seen in 56 cases including complete deletions involving all the eight $I K Z F 1$ exons $(\mathrm{n}=22)$, dominant negative partial deletions involving exons 4-7 ( $\Delta 4-7)(\mathrm{n}=19)$ and other partial deletions- $\Delta 2-7(9), \Delta 2-3(3)$, $\Delta 4-8$ (3), $\Delta 2-8$ (2), and $\Delta 1$ (3). The deletions which could be confirmed on breakpoint-specific fluorescent multiplex PCR were $\Delta 4-7$, $\Delta 2-7$, and $\Delta 4-8$. The fluorescent multiplex PCR helped in resolving the extent or number of deletions in three cases. The cases with $I K Z F 1$ deletions had higher induction failure compared to other cases. Conclusions: Overall, MLPA could detect $I K Z F I$ gene deletions in $56(24.5 \%)$ cases of B-ALL. Fluorescent multiplex PCR was helpful in confirmation of most of the intragenic IKZFI deletions besides clarifying the extent or number of deletions in some cases. So, fluorescent multiplex PCR may act as complimentary technique to MLPA for better molecular detection of IKZF1 deletions in B-ALL.

\section{$\mathbf{O 2 8}$}

\section{Advantages of Short Tandem Repeat (STR) Based Chimerism Analysis Over VNTR Based Assays in Quantitative Estimation of Post-transplant Donor Engraftment}

\section{Mr. Biswajoy Pal ${ }^{\#}$, Dr. Deepak Mishra, Dr. Neeraj Arora. Dr. Mayur Parihar, Mr. Sourav Sarma Chowdhury, Dr. Saurabh Jayant Bhave, Dr. Vivek Radhakrishnan, Dr. Reena Nair, Dr. Sekhar Krishnan, Dr. Mammen Chandy}

Email ID for Correspondence: biswajoy.pal@tmckolkata.com Tata Medical Center, Kolkata

Introduction: Variable number tandem repeats (VNTRs) were among the first genetic markers used to quantitate bone marrow transplant engraftment. The limitations of PCR based VNTR markers in distinguishing some donor/recipient pairs has shown the need for additional genetic markers to analyze engraftment. Short tandem repeats (STRs) provide an excellent tool for this purpose because of their high degree of polymorphism and relatively short length. Objectives: The purpose of this retrospective study was to validate efficiency of STRs in BMT engraftment analysis by comparing VNTR and STR results for the same sets of post-transplant samples from patients who had undergone allogeneic BMT. Material and Methods: We compared STR analysis results with previous VNTR results for 15 post-transplant samples from as many allogeneic bone marrow transplant patients having single informative VNTR marker. The PowerPlex 16 System (Promega) used for these assays included 15 STR loci (Penta E, D18S51, D21S11, THO1, D3S1358, FGA, TPOX, D8S1179, vWA, Penta D, CSF1PO, D16S539, D7S820,
D13S317and D5S818) and the sex marker Amelogenin. All 16 loci were amplified simultaneously in a single tube (multiplex PCR). The amplified fragments from STR were analyzed in a single capillary injection using the 3500 Genetic Analyzer (Applied Biosystems). An internal lane standard 600 (ILS) (Promega) was injected with each sample for accurate sizing of STRs. Results: VNTR and STR analysis yielded comparable qualitative results in the majority of cases. The incidence of mixed chimerism (MC) by STR analysis was $13 \%$ compared to $0 \%$ in cases evaluated by VNTR analysis. STR markers were more informative; an average of five informative loci could be identified in all patients barring one donor-recipient pair of identical twins where no informative STR markers were observed. In two cases of matched sibling donor transplant four to five informative STR markers were identified. Conclusions: Compared to VNTR, STR analysis is more sensitive and therefore does not need as much DNA. The yield of informative alleles is higher with STR loci, and the assay offered the advantages of more rapid and accurate quantitative assessment of post-transplant chimerism.

\section{Haematocon 2017 Abstract}

\section{9}

Study of Platelet Aggregometry Comparison Between Chronolog Light Transmission Aggregometer and Automated Coagulation Analyser Sysmex CS-2000i

Nair SC, Mammen J J, Abraham A, Geevar T, Singh SS, Reena L Bapat Kalyani (Presenting Authors)

Department of Immunohematology and Transfusion Medicine, Christian Medical College, Vellore

Introduction: Light transmission aggregometry currently is the gold standard method for assessing platelet function in a bleeding patient suspected to have a platelet function disorder. However this method is labor intensive, technically challenging, time consuming along with the requirement of substantial blood sample volumes. As against this, automated coagulation analyzers have the advantages of need of smaller sample volumes, shorter turn around time, walk away technology and thus is technical feasible. Aims and Objectives of the study: To observe the agreement between results of aggregometry obtained on automated coagulation analyzer Sysmex CS-2000i with the Chrono-Log Light transmission aggregometer (LTA). Patients and Methods: The study is being conducted in Department of Transfusion Medicine and Immunohematology. All patients with history of bleeding symptoms or suspected to have bleeding disorder referred to the department for complete coagulation workup are subjected to evaluation. Patients should be in fasting state and should not be on any antiplatelet medications. History and other details are entered at the time of sample collection. Samples are collected in $3.2 \%$ of sodium citrate tubes and are processed within 4 hours of collection. Platelet rich plasma (PRP) and Platelet poor plasma (PPP) are prepared by centrifuging at appropriate speeds. Based on the results of primary screening tests, if platelet function abnormality is suspected, then samples are processed further for aggregometry. These samples are run simultaneously on Chronolog LTA and Sysmex CS-2000i. Aggregation response is assessed for five agonists namely Ristocetin (Ris), arachidonic acid (AA), collagen (Coll), ADP and epinephrine (Epi). Controls are run in parallel. The aggregation response obtained on Chrono-Log and Sysmex CS-2000i arethen compared for each agonist based on the percentage of maximal aggregation (\%MA). The agreement between results are obtained by using statistical method of Intra Class Correlation Coefficient (ICC). Results: 530patients were referred for coagulation workup over a period of 8 months (January 2017 till August 2017). Out of which 57 patients were suspected to have platelet 
dysfunction, and for whom aggregometry was performed. The age of patients ranged from an infant aged 10 months to an elderly aged 67 years (mean $=15.5 \mathrm{yrs}$ ). Out of 57 patients 31 were males and 26 were females $(\mathrm{M}: \mathrm{F}=1.1: 1)$. Amongst the 57 patients, 17 were diagnosed with Glanzmann thromboasthenia (GT), 1 with GT-Variant, 8 with Bernard Soulier Syndrome (BSS), 1-BSS-Variant, 5 with Acquired platelet dysfunction with eosinophilia (APDE), 8- Other platelet function defects, 1- diagnosed with Hemophilia -A, 1- Dysfibrogenemia, 2 -Thrombocytopenia. 13/57 cases were found to have normal platelet function. The aggregation response obtained on Chrono-log and Sysmex CS-2000i was compared by Intra Class Correlation Coefficient (ICC) for five agonists and which depicted strong agreement between results of both machines: AA $=0.90$ (95\% CI: 0.82-0.94), $\mathrm{ADP}=0.92$ (95\% CI:0.87-0.95), Coll $=0.89$ (95\% CI: $0.82-0.93$ ), Epi $=0.95$ (95\% CI:0.91-0.97), Ris $=0.85$ (95\% CI: 0.760.91). Conclusion: The results of this preliminary study show that automated platelet aggregometry in Sysmex CS-2000i offers a reproducible and robust alternative to manual aggregometry. Thus platelet aggregation on the routine coagulation analyser Sysmex CS-2000i is an easily accessible, handy and reliable, rapid tool to assess platelet function and allows to overcome the limitations of a stand alone light transmission aggregometer.

\section{O30}

\section{Spectrum of Elane Mutation in Congenital and Cyclical Neutropenia Patients}

Senthamizhselvi Anandan", Arun Kumar A, Uday Kulkarni, Anup Devasia, Anu Korula, Nisham P.N, Aby Abraham, Biju George, Alok Srivastava, Vikram Mathews, Poonkuzhali Balasubramanian

Email ID for Correspondence: selvi_lali2@rediffmail.com Christian Medical College, Vellore

Introduction: Congenital and Cyclical Neutropenia are rare inherited diseases, which become manifest in the hematopoietic system as a deficiency of neutrophils. Severe Congenital Neutropenia (Kostmann syndrome) is characterized by an absolute neutrophil counts (ANC) below $0.5 \times 10^{9} / \mathrm{L}$ leading to recurrent bacterial infections. Cyclical neutropenia patients show an oscillation of ANC with 3 weekly periods of severe neutropenia and is usually caused by inherited or de novo heterozygous ELANE mutations. Congenital neutropenia is more heterogeneous with mutations (heterozygous and homozygous) reported in ELANE, HAX-1, G6PC3, GFI1 and few other genes. Objectives: To identify the spectrum of ELANE mutations in patients with severe neutropenia from our population. Material and Methods: A total of 48 patients suspected with inherited neutropenia were included in our study. Diagnosis was based on ANC $<0.5 \times 10^{9} / \mathrm{L}$, myeloid maturation arrest and recurrent severe bacterial infections Mutations in ELANE gene were assessed by Sanger sequencing using specific primers. Results: Mutation(s) in ELANE gene were identified in 9 of the 48 patients and included missense, splice site and frameshift mutations. One patient had homozygous c. $597+1 \mathrm{G}>$ A mutation in the splice region of intron 4 while all the other patients had heterozygous mutations. The various mutations identified in our study include c.164G $>$ A (p.Cys55Tyr), c.169G > A (p.Ala57Thr), c.179T >C (p.Ile60Thr), c.302T > A (p.Val101Glu), c.468G > T (p.Try156Cys), c.596delT (Phe199Ser fs*13), the two splice site mutations c.367-8C $>$ A and c.597 + 1G $>$ A. Among these c.302T $>$ A, c. $468 \mathrm{G}>\mathrm{T}$ and c.596delT were novel mutations and have not been reported earlier. A common 5' upstream polymorphism, c-225C $>\mathrm{A}$ was also identified in four patients. The mean ANC in patients having ELANE mutations was lower $\left(0.398 \times 10^{9} / \mathrm{L}\right)$ when compared to the patients without the mutations $\left(0.418 \times 10^{9} / \mathrm{L}\right)$. Although mRNA expression was not analysed, data from other studies have shown both wild type and mutant mRNAs in different ratios, consistent with the autosomal dominant inheritance. Conclusions: Patients with ELANE mutations suffer from recurrent life threatening bacterial infections and are also at a high risk of developing myelodysplasia or acute myeloid leukemia. Hence it is important to screen for these mutations in patients presenting with neutropenia early in life. Results from our study show the widespread distribution of mutations and the need to screen all exons and exon-intron junctions when mutation analysis is requested in the ELANE gene.

\section{1}

Comparsion of Direct Sequencing and Allele Specific PCR Assay for Detection of MPL Mutations in Essential Thrombocythemia and Primary Myelofibrosis Patients

Hemamalini Thangavelu\#, Arun Kumar A, Uday Kulkarni, Anup Devasia, Anu Korula, Nisham P.N, Aby Abraham, Biju George, Alok Srivastava, Vikram Mathews, Poonkuzhali Balasubramanian

Email ID for Correspondence: hemaflowers10@gmail.com Christian Medical College, Vellore

Introduction: The myeloproliferative neoplasms (MPNs) include Polycythemia Vera (PV), Essential Thrombocythemia (ET), primary myelofibrosis (PMF) and other less well-characterized chronic myeloproliferative disorders. Majority of the patients have mutations in $J A K 2$ gene (predominantly $J A K 2 \mathrm{~V} 617 \mathrm{~F}$ and rarely exon 12). Recently mutations mapping to the juxtamemebrane region of the thrombopoietin receptor $M P L$ (exon 10) were described in the 5-10\% of the patients with PMF and $1 \%$ of the patients with ET who were negative for $J A K 2$ and $C A L R$ mutations. These $M P L$ mutations are gain of function mutations leading to receptor activation in the absence of thrombopoietin binding with constitutional activation of the JAK-STAT signaling. Four recurrent (W515L, W515 K, W515A and S505 N) and two rare (W515A and S505 N) mutations have been reported in ET and PMF patients. While Sanger sequencing is the commonly employed method for screening, mutations occurring at allelic levels below 15\% may not be detected. Objectives: To compare the sensitivities of allele specific PCR and Sanger sequencing for detection of $M P L$ mutations in essential thrombocythemia and primary myelofibrosis patients. Material and Methods: Retrospectively, we identified 80 patients suspected with ET or PMF between 2012 and 2016 (who were negative for JAK2 V617F, CALR and JAK2 exon 12 mutations. DNA from peripheral blood leukocytes was extracted using a commercial kit (Gentra Puregene Blood Kit, Qiagen, Hilden, Germany). Sanger sequencing and allele specific PCR (targeting W515L, W515 K, W515A and S505 N) were done according to established protocols. Results: $M P L$ mutation was identified in 12 out of 80 patients $(15 \%)$ using either of the two methods. Sanger sequencing and allele specific PCR identified the mutations in 10 and 12 patients respectively. Seven patients had W515L (c.1544G > T) and 5 patients W515 K (c.1543_1544delinsAA). We did not identify S505 N (c.1514G > A), W515A (c.1543_1544delinsGC) or other rare mutations in our study. Direct sequencing failed to detect the nucleotide change in 2 patients with W515L mutation. Conclusions: Although direct sequencing has an advantage of screening all possible mutations in exon 10 of MPL it is limited by its low sensitivity which is a major concern when dealing with somatic mutations. In our study sequencing did not yield any additional mutation other than the four common mutations tested by allele specific PCR while the high sensitivity of allele specific PCR enabled us to pick up two additional positive cases. 


\section{$\mathbf{O 3 2}$}

Spectrum of $I D H 1$ and $I D H 2$ Mutations in Patients with Cytogenetically Normal Acute Myeloid Leukemia

Shareen Stella Backia Royan", Arun Kumar A, Sathya M, Nancy Beryl Janet, Uday Kulkarni, Anup Devasia, Anu Korula, Nisham P.N, Aby Abraham, Biju George, Alok Srivastava, Vikram Mathews, Poonkuzhali Balasubramanian

Email ID for Correspondence: stella.shareen@gmail.com Christian Medical College, Vellore

Introduction: Mutations in the genes involved in epigenetic regulation namely DNA methyltransferase 3A (DNMT3A), Isocitrate Dehydrogenase 1 and 2 (IDH1 and $I D H 2)$ result in alterations in DNA methylation. Mutations in IDH1/2 are among the most commonly occurring mutations found in AML, especially in the intermediate- or normal cytogenetic risk category. IDH1 and IDH2 are key metabolic enzymes that converts isocitrate to alpha ketoglutarate. Studies on the prognostic impact of IDH1/2 mutations in AML patients have yielded conflicting results. Objectives: To study the frequency and significance of IDH1 and IDH2 mutations in AML patients with normal karyotype. Material and Methods: A total of 97 newly diagnosed AML patients with no cytogenetic abnormalities were included in this study. DNA from peripheral blood leukocytes was extracted using a commercial kit. Mutation analysis targeting exon 4 of both IDH1 and IDH2 was done by Sanger Sequencing. Results: IDH1 and IDH2 mutations were identified in $19(19.5 \%)$ and $20(20.6 \%)$ patients respectively in our study. The results were consistent with the frequencies reported in the Cancer Genome Atlas Research Network. Among the IDH1 mutations, c.395 $\mathrm{G}>\mathrm{A}$ was the most common mutation identified $(\mathrm{n}=8 ; 42 \%)$. The other mutations identified include c.394 C > T $(n=5)$, c.394 C > G $(n=4)$ and c.394 C > A $(n=2)$. All the base substitutions resulted in a missense mutation in codon 132 . On the other hand, IDH2 mutation analysis yielded two different hotspots namely codon 140 (c.419G > A, n = 13) and codon 172 (c.515G > A, n = 7). Majority of the patients with IDH1/2 mutations also had NPM1 or FLT3 mutations [59.5\% of IDH1/2 mutated patients also had NPM1 mutations in contrast to IDH1/2 wild type patients $(35.8 \%) ; \mathrm{p}=0.024]$. Also patients with IDH1/2 mutations presented with significantly higher blast counts compared to those who were wild type for IDH1/2 $(p=0.007)$. Conclusions: This study shows that IDH1/2 mutations are most commonly seen in normal karyotype AML patients. They are associated with high blast counts and by further assessing the prognostic impact of these mutations, it is possible to foresee a refinement of the intermediate cytogenetic risk group by stratifying patients into unique risk categories that can aid in determining treatment plans and use of newer strategies like hypomethylating agents.

\section{$\mathbf{O 3 3}$}

Minimal Residual Disease in Acute Myeloid Leukemia Using a Difference from Normal Approach is Predictive of Outcome

Chinmayee Kakirde", Rohan Kodgule, Goutham Raval, Sanjay Talole, Shraddha Kadechkar, Nikhil Rabade, Y Badrinath, Sitaram Ghogale, Nilesh Deshpande, Shruti Chaudhary, Swapnali Joshi, Russel Mascarenhas, Dhanlaxmi Shetty, HariMenon, Manju Sengar, Navin Khattry, Hasmukh Jain, Bhausaheb Bagal, Anant Gokarn, Prashant Tembhare, Sumeet Gujral, PG Subramanian \& Nikhil Patkar

Email ID for Correspondence: radhika.kakirde@gmail.com ACTREC, Tata Memorial Centre
Introduction: One of the mainstays of chemotherapy in acute myeloid leukemia (AML), other than acute promyelocytic leukemia, is induction with a goal to achieve morphological complete remission (CR) as evident by less than $5 \%$ blasts. Post remission strategies then focus on either consolidation chemotherapy or allogeneic bone marrow transplantation (aBMT). However, not all patients by this remission criterion achieve long term remission and a subset of patients relapse. Detection of minimal residual disease (MRD) following chemotherapy, is highly predictive of outcome and offers a window of opportunity to intensify treatment and prevent relapse. Here, we describe assessment of immunophenotypic MRD using a 10-colour two tube assay and a "difference from normal" approach. Objectives: To check the prognostic significance of post induction and post consolidation marrow analysis by flow cytometry for minimal residual disease detection. Material and Methods: We accrued 280 consecutive patients of adult ( $>18$ years) AML, other than with $\mathrm{t}(15 ; 17)$, over a 30 month period. MRD testing was done post induction and if feasible in post $1^{\text {st }}$ Cycle HiDac using a two tube 10 colour assay. (CD15, CD13, CD19, CD34, CD56, CD7, CD45, CD11b, HLA-DR, CD117, CD14, CD123, CD64, CD33, CD36 \& CD38). A minimum of 1.6 million events were acquired per tube on a Navios flow cytometer. Analysis of MRD was done using Kaluza 1.3 by a difference from normal approach that focused on the development of progenitors to monocytes. Conventional karyotyping and FISH was done as per standard recommendations. The presence of FLT3-ITD, NPM1 and CEBPA mutations was detected by a fragment length analysis based assay. Results of the MRD assays, cytogenetic and molecular risk groups were analyzed for their impact on OS (Overall Survival) and RFS (Relapse free Survival) using Log Rank test of Kaplan-Meier survival analysis. Results: A total of 280 AML patients were treated and followed up in a 30 months period. Based on cytogenetics, $28.6 \%$ were classified as favorable risk whereas $61.4 \%$ and $10 \%$ were intermediate and poor risk respectively. FLT3-ITD, NPM1 and CEBPA mutations were harbored by $20.7 \%, 26.4 \%$ and $10.3 \%$ of patients respectively. The median follow up was of 6 months (Ranging from 17 days to 30 months). Of these, 35 had induction death and 59 had refractory disease. Median OS was 6.5 months and RFS was 8.5 months. Post induction MRD was assessed in 235 patients of which $108(45.9 \%)$ had detectable residual disease (range 0.01-66\%, median: $1.38 \%$ ). Post consolidation MRD was assessed in 98 patients of which $22(22.4 \%)$ were MRD positive (range $0.002-7.7 \%$, median: $0.05 \%$ ). Favorable risk cytogenetics in cases excluding induction deaths was predictive of better OS than Intermediate $(p=0.031)$ and Poor risk $(\mathrm{p}=0.003$ )but not RFS. Patients harboring MRD at the end of induction were associated with worse OS $(p=0.004)$ but not RFS, whereas post consolidation positive MRD status did not show significant change in OS and RFS. Conclusions: Our data is in agreement with other studies that determination of immunophenotypic MRD is extremely important in predicting outcome. AML MRD is a very useful guide for guiding post remission strategies in AML and should be incorporated into routine treatment algorithms.

\section{4}

Bone Marrow Infiltration in Lymphoma: Can 18 F-FDG PET/CT Replace bone Marrow Aspiration Biopsy?

G. Smeeta , Manas Kumar Sahoo, Ritesh Sachdev, Ritu Chadha, Bhawana Jha, Salini Goel, Tushar Sahni, Nitin Sood, Ashok Sen, Ashok Vaid, Manorama Bhargav

\section{Medanta -The Medicity, Gurugram, Haryana-122001}

Introduction: Evaluation of bone marrow infiltration (BMI) plays a crucial role in the staging as well as prognosis of lymphoma. Bone marrow aspiration/biopsy (BMAB) is considered to be the gold 
standard modality for evaluation of BMI. BMAB is also helpful in detecting unsuspected lymphoma; however, it has few limitations such as inadequate sampling or focal marrow involvement. ${ }^{18} \mathrm{~F}-\mathrm{flu}$ oro-2-deoxy-D-glucose positron emission tomography/computed tomography $\left({ }^{18} \mathrm{~F}-\mathrm{FDG} \mathrm{PET} / \mathrm{CT}\right)$ has been proved to be an excellent non -invasive diagnostic tool for whole body assessment including bone marrow infiltration in lymphoma staging work up. However, in the era of $\mathrm{PET} / \mathrm{CT}, \mathrm{BMB}$ is an alternative or complementary or superior to PET/CT is still a topic of debate and is still under investigation. Objectives:

- To compare the utility of BMAB and ${ }^{18} \mathrm{~F}-\mathrm{FDG}$ PET/CT in detection of BMI in patients with Non-Hodgkin (NHL) and Hodgkin lymphoma (HL) and to highlight the limitations as well as advantages of both the modalities.

- To find if any group of patients with reference to initial diagnosis or other haematological parameters where one of these two modalities is superior to the other and BMAB can be avoided in those groups for evaluation of BMI.

Material and Methods: This was a retrospective study of patients with histologically confirmed lymphoma (NHL and HL) between January 2013 to December 2016 at Medanta-The Medicity, Gurugram, who underwent pre-treatment FDG PET/CT and BMB for staging which was performed simultaneously (time interval in both the procedure $<30$ days). All information was collected from the Hospital Information System (HIS) where all information pertaining to each patient is meticulously available since the time of registration. Results: Total 447 (age range: 02-86 yrs) patients of histology proven lymphoma who underwent bone marrow biopsy and PET/CT for staging were evaluated, out of which $362(81 \%)$ were of NHL and 85 (19\%) were of HL. Male: female ratio was 2.2:1 $(\mathrm{M}=309 ; \mathrm{F}=138)$. Among the NHL patients, 318 were B-cell and 44 cases were T cell NHL. B-NHL group included diffuse large B cell lymphoma (211), marginal zone lymphoma (25), follicular lymphoma (24), mantle cell lymphoma (20), $\mathrm{T}$ cell rich B cell lymphoma (17), Burkitt's lymphoma (12), lymphoblastic lymphoma (4), small cell lymphoma (4) and MALT lymphoma (1) patients. Out of $85 \mathrm{HL}$ patients, $26(30 \%)$ patients showed BMI either by PET/CT or BMAB, 23 by PET/CT, 11 by BMAB and 9 cases by both the modalities. Out of 318 B cell NHL patients, 137 (43.6\%) patients showed BMI either by PET/CT or BMAB, 109 by PET/CT, 110 cases. Conclusions: $18 \mathrm{~F}-\mathrm{PET} / \mathrm{CT}$ is a valuable diagnostic tool for detection of focal BMI in lymphoma patients. In HL, PET/CT should be the preferred modality for staging where BMAB may be avoided for routine bone marrow assessment. In case of High Grade NHL, BMAB may be avoided in PET/CT negative and focal/multifocal positive cases and should be performed in case of diffuse/focal low borderline marrow uptake in PET/CT. However, in Low Grade-NHL, BMAB should be mandatory as PET/CT shows lower accuracy in detecting BMI. In lymphoma patients associated severe anaemia PET/ $\mathrm{CT}$ poses an evaluation challenge due to high marrow uptake and BMAB should always be performed. Additionally, in synchronous malignancy involving bone marrow, $\mathrm{BMAB}$ can detect marrow involvement which PET/CT cannot differentiate.

\section{5}

Rituximab with Dose Adjusted Epoch in Non Hodgkin Lymphoma - A Single Center Experience

Ketan Modak, Mipsang Lama, Jeevan Kumar, Saurabh Bhave, Vivek Radhakrishnan, Reena Nair, Mammen Chandy

Objectives: To assess the benefit of Rituximab with dose-adjusted Etoposide, Cyclophosphamide, Doxorubicin, Vincristine and
Prednisolone (R-da-EPOCH) regimen as a first-line treatment in high grade Non Hodgkin Lymphoma (NHL). Methods: We retrospectively analyzed 57 newly diagnosed Non Hodgkin Lymphoma patients in Tata Medical Center who were treated with frontline R-da-EPOCH between 2013 to 2016. Data of all the patients was reviewed from electronic medical records. We determined the overall response rate (ORR), complete remission (CR), partial remission (PR), overall survival (OS) and relapse free survival (RFS) in all patients. Results: All 57 patients included in the analysis received minimum two or more cycles of therapy. The ORR, CR, and PR rates were $89.2 \%$, $80.3 \%$ and $8.9 \%$ respectively. 6 out of 57 patients could not be evaluated due to discontinuation of treatment. The OS at 3 years was $71.9 \%$ and RFS at 3 years was $60.1 \%$. The effect of dose intensity on patient outcome would be analyzed subsequently. Conclusion: This is one of the largest retrospective analyses of R-da-EPOCH as frontline chemotherapy in Non Hodgkin Lymphoma in eastern India. $\mathrm{R}$-da-EPOCH is a very effective first line treatment option in with high grade NHL irrespective of patient age.

\section{6}

Molecular Spectrum of Hemochromatosis in India

Madhavi. $\mathbf{M}^{\#}$, Neelagandan. K, Rekha. A, Eapen. C. E*, Anup Devasia, Anu Korula, Eunice S Edison

Email ID for Correspondence: dr.madhavimaddali@gmail.com Christian Medical College, Vellore

Introduction: Hereditary hemochromatosis $(\mathrm{HH})$ is a genetically heterogeneous disease caused by mutations in one or more different genes involved in iron homeostasis. It is characterized by decreased hepcidin levels and excessive iron absorption leading to iron overload in multiple organs. $\mathrm{C} 282 \mathrm{Y}$ mutation in HFE gene causes the commonest form of hemochromatosis and is found in a high frequency (80-100\%) of Caucasian population with HH. However, in India and other Asian countries, where iron deficiency and secondary iron overload following transfusion in thalassaemia are common, primary hemochromatosis is less documented and its molecular basis is not well established. Objectives: The aim of our study is to characterize the molecular spectrum of hemochromatosis. Material and Methods: Patients with suspected primary hemochromatosis based on clinical features and fulfilling AASLD criteria sent to the department of haematology for mutation analysis were studied from 2009 to 2017. Mutation analysis was done by direct Sanger sequencing in genes involved in hepcidin regulation (HFE, HFE2, HAMP, TFR2 \& SLC40A1). Results: This study group comprised of forty-seven cases. Majority of the patients were from Kerala (55\%) with a median age of 43 years (13-71), male preponderance $(\mathrm{M}: \mathrm{F}=5: 1)$. Clinical presentation was variable, with diabetes in $(37 \%)$, skin hyperpigmentation in $(23 \%)$ and altered liver function tests with a median AST of 62 (18-472) and ALT of 43 (13-329). This cohort had a mean hemoglobin $(\mathrm{g} / \mathrm{dl})$ of $11.0 \pm 2.5, \mathrm{MCV}$ (fl) of $93 \pm 11.7$, median serum ferritin (ng/ml) $1564(118-7380)$ and mean percentage transferrin saturation of $83.7 \pm 18.3$. Genetic variants associated with classic $\mathrm{HH}(H F E)$ were seen in $66 \%(\mathrm{n}=8)$, consisting of homozygous $\mathrm{C} 282 \mathrm{Y}$ in a family, homozygous for H63D $(\mathrm{n}=1), \mathrm{H} 63 \mathrm{D} / \mathrm{S} 65 \mathrm{C}$ $(\mathrm{n}=1)$, heterozygous E277 K $(\mathrm{n}=1)$ and heterozygous for H63D $(\mathrm{n}=4)$. HFE2 (type II a) variants were identified in three cases which consisted of A134Pfs112, E3Gfs18 and G336. All HFE2 variants identified in this study were novel. Type IIb (HAMP) was identified in 1 case in heterozygous state (R56*). However, no disease-causing variants were identified in TFR2 (type III) and SLC40A1 (type IV). Conclusions: We identified both HFE and non HFE causes of primary hemochromatosis in our study and next generation 
sequencing approaches may identify a distinct molecular basis in India.

\section{$\mathbf{0 3 7}$}

Comparison Between Pattern of TdT Expression in Bone Marrow Biopsy and Multicolour Flow Cytometry for Minimal Residual Disease in B Cell Precursor Acute Lymphoblastic Lymphoma

\author{
Surbhi Lahoti ${ }^{\#}$, Prashant Tembhare, Sumeet Gujral, \\ P G Subramanian
}

Email ID for Correspondence: drsurbhilahoti@gmail.com Department of Hematopathology, Tata Memorial Hospital, Mumbai

Introdcution: Post-induction monitoring of the treatment response and minimal residual disease status is a standard practice in the management of B-cell precursor acute lymphoblastic leukemia (BCPALL). It is done by using multicolor flow cytometry (MFC) determining the residual leukemic blast percentages in the bone marrow (BM). In many patients, simultaneous bone marrow biopsies are also performed which can provide additional information like cellularity or patchy involvement. Thus, BM biopsy can complement the residual disease assessment, especially in the hemodiluted BM aspirates. In the BM biopsy, the BCPALL blasts are identified by Terminal deoxynucleotidyl transferase (TdT) positivity on immunohistochemistry (IHC). However, TdT is also positive in the normal B-cell precursors which usually out-number residual leukemic blasts in post-induction (PI) marrow. To our knowledge, there is no data published which can help to differentiate normal B-precursors from the residual disease on the pattern of TdT positivity in BM biopsy. Hence, we studied the patterns of TdT positivity in the PI BM biopsy specimen and correlation with MFC-MRD results was performed. Objectives: To study the patterns of TdT positivity in the PI BM biopsy specimen and to correlate them with MFC-MRD results. Material and Methods: We studied the PI-BM biopsy specimen of total 17 cases of BCPALL. BM biopsies were processed as per TMHprotocol and evaluated with $\mathrm{H} \& \mathrm{E}$ stained sections. TdT was studied using standard TMH IHC protocol. The pattern of TdT positivity by blasts was studied. We categorized the TdT positivity in three categories: category- 1 defined as singly scattered positivity, category- 2 defined as clusters of 5 to $10 \mathrm{TdT}$ positive cells with diffuse involvement. Category-1 was reported as negative for the residual disease, and category-2 was reported as positive for the residual disease. MFC based MRD was performed using 10-color assay on Navios flow cytometer, and analysis was performed using Kaluza software. Result: Out of the total 17 cases, 12 cases showed category1 TdT positivity, and five showed category-2 TdT positivity. On MFC MRD assessment, MRD was positive in 13 samples with median MRD 2.77 (range 0.14 to 34.4). MRD was positive by flow cytometry in 12 samples which included all five cases with category-2 TdT positivity and eight samples with category-1 positivity. We determined the sensitivity and specificity of the pattern of TdT positivity and found that category-2 is highly specific for MRD positivity (specificity $100 \%$ ) but with low sensitivity of $33 \%$. Thus, a total of eight cases showed positive MRD detection by flow cytometry but were negative on bone marrow biopsy. Conclusion: Both flow cytometry and bone marrow biopsy (with Tdt) can be used to evaluate the residual disease in BCPALL. Clusters of 5 to $10 \mathrm{TdT}$ positive cells with diffuse involvement can be used to determine the MRD response status, but it has very low sensitivity and cannot detect MRD in twothird of cases. Flow cytometry is highly sensitive and remains the method of choice for MRD assessment. BM biopsy can help in the assessment of cellularity and quality of response regarding regenerating hematopoiesis but should not be used for MRD assessment.

\section{8}

Evaluation of SNPs Affecting Fetal Hemoglobin in Thalassemia Intermedia Patients from North India

Objectives: The study was planned to evaluate prevalence and effect of various single nucleotide polymorphisms (SNPs) at quantitative trait loci (QTLs) affecting fetal hemoglobin levels in thalassemia intermedia patients from North India. Methods: Polymerase chain reaction- restriction fragment length polymorphism was used for SNP analysis at three QTLs- Xmn $1{ }^{G} \gamma$ polymorphism (rs7482144), $B C L 11 A$ (rs11886868) and HBSIL-MYB intergenic-region (rs4895441). We compared the prevalence of SNPs among $52 \beta$ thalassemia intermedia patients, $42 \beta$-thalassemia major patients and 50 normal children from North India. We also attempted to correlate the genotypes at the SNPs with HbF levels in thalassemia intermedia patients. Results: The Mutant allele frequency (MAF) at rs7482144 $\left(X m n 1-{ }^{G} \gamma\right.$ polymorphism) was significantly higher in thalassemia intermedia patients compared to normal controls $(p<0.001)$ and thalassemia major $(p<0.001)$ patients. No significant difference was found in MAF at the other two sites among the three groups studied. Among the SNPs, only Xmn1- ${ }^{G} \gamma$ polymorphism showed a significant correlation with higher $\mathrm{HbF} \%\left(p=0.001, \mathrm{r}^{2}=0.26\right)$ and $\mathrm{HbF} \mathrm{g} / \mathrm{L}$ $\left(p=0.005, \mathrm{r}^{2}=0.21\right)$. We also noted a significant difference in $\mathrm{HbF}$ $\%$ and $\mathrm{HbF} \mathrm{g} / \mathrm{L}$ in the three genotypes at $\mathrm{Xmn} 1$ site. Conclusions: In a genetically heterogeneous group of thalassemia intermedia patients in the present study, rs7482144 $\left(\mathrm{Xmn} 1 \mathrm{-}^{G} \gamma\right.$ polymorphism) is the biggest player among the three SNPs studied in disease amelioration by increasing the $\mathrm{HbF}$ levels. A larger cohort study is needed to evaluate the effect of SNPs in other two regions in North Indian population. Keywords Fetal hemoglobin, Xmn $1{ }^{G} \gamma, H B S 1 L-M Y B, B C L 11 A$, Thalassemia intermedia, North India

\section{9}

Mutational Spectrum in Juvenile Myelomonocytic Leukemia (JMML)

Pratiksha Chandorkar", Nikhil Rabade, Rohan Kodgule, Goutham Raval, Prasanna Bansode, Shruti Chaudhary, Swapnali Joshi, Russel Mascarhenas, Prashant Tembhare, Sumeet Gujral, Gaurav Narula, Shripad Banavalli, Pratibha Amare, PG Subramanian, Nikhil Patkar

\section{Corresponding author: Dr Nikhil Patkar}

Email ID for Correspondence: nvpatkar@gmail.com

Tata Memorial Centre, ACTREC, Kharghar

Introduction: JMML is a rare disease affecting young children, accounting for $\sim 3 \%$ of all childhood haematological malignancies and is classified as myelodysplastic/myeloproliferative neoplasm by WHO. Mutations in the RAS signalling pathway leading to hyperactive RAS signalling are the hallmark of this disease. Although described in the literature, there is no data from India describing the baseline frequencies of these mutations because of its rarity. Objectives: To study the mutational spectrum of KRAS, NRAS, PTPN11 and $C B L$ gene mutations in Indian patients. Material and Methods: Thirteen cases diagnosed as per WHO 2008 criteria. Ten males, three 
females with median age 3.5 years (range 1-9 years). Clinical and cytogenetics details were obtained from patient records. DNA for mutational screening was extracted from fresh blood/bone marrow samples using Qiagen DNA mini kit. Exons 2, 3 of KRAS, NRAS; exons 3,13 of PTPN11 and exons 8, 9 of $C B L$ were amplified by PCR, diluted and subjected to Sanger sequencing for mutation detection. Results: Monosomy 7 was detected in $23.1 \%$ of the cases (3 of 13). Missense mutations within NRAS gene were detected in $50 \%$ (6 of 12) patients with five affecting codon 12 and two affecting codon 61. Mutations at both codons were detected in one case. None of the 11 cases tested harbored mutations in KRAS. Mutations affecting codons 61 (p.D61Y) and 76 (p.E76 K) were detected within exon 3 of PTPN11 whereas no mutations were detected within exon 13. No mutations were detected affecting $C B L$ gene. Conclusions: The mutational spectrum of JMML we report here varies from that reported in the west. To the best of our knowledge this is a first of its kind study from India. However studying a larger cohort with long term follow up is required to evaluate difference in mutational spectrum of Indian JMML as compared to the western literature and its prognostic impact.

\section{O40}

Characterization of Molecular Variants of G6PD DeficiencyA Single Center Study

\section{Sumithra $S^{\#}$, Eswari S, Aby Abraham, Shaji RV, Eunice S Edison}

Email ID for Correspondence: sumithra61@gmail.com

Christian Medical College, Vellore

Introduction: $G 6 P D$ deficiency is a monogenic, X-linked genetic defect with a worldwide prevalence of around 400 million people and an overall prevalence of $8.5 \%$ in India. Variable biochemical and molecular heterogeneity characterizes this disease. Although G6PD deficiency was reported in India more than 50 years ago, studies on molecular characterization and phenotypic correlation are few. Objectives: To correlate the phenotypic expression with molecular genotypes of G6PD deficiency. Material and Methods: All patients with haemolytic anaemia with a possible aetiology of G6PD deficiency based on the clinical, haematological and biochemical parameters referred to the department of haematology were studied. Molecular analysis of G6PD gene was done by direct Sanger sequencing. Results: A total of twenty-six $(n=26)$ haemolytic anaemia patients with a possible G6PD deficiency referred to the department of haematology for mutation analysis were studied from 2005-2017. Patients were categorized into two groups based on the presence or absence of mutations and their phenotype was compared. Phenotypic comparison between the two groups showed, lower mean G6PD level (U/gHb) (2.1 vs 8.7), mean haemoglobin (g/dl) (7.1 vs $8.7)$, higher mean retic count (\%) (10.2 vs 6.5), higher mean total bilirubin (mg/dl) (3.4 vs 3.0) and mean LDH (U/L) (2499 vs 1654) in the group with mutation suggesting a severe haemolytic picture. Molecular analysis of G6PD in twelve cases $(n=12,46 \%)$ revealed two novel missense mutations (p. P396A and p. L488F) and the rest were reported mutations which included, G6PD Orissa $(n=4)$, one each of G6PD Mediterranean, Kerala-Kalyan, Mahidol and Bangkok. No disease causing genetic variants were identified in the other cases $(54 \%, \mathrm{n}=14)$. Conclusions: Although G6PD values are variable and depend on the time of testing and testing method there is a correlation between G6PD levels and the presence of mutation as evident from our study. Hence molecular characterization to confirm the diagnosis is warranted in cases with low G6PD.

\section{1}

FANCD2 Ubiquitination Analysis by Western Blot as a Diagnostic Test for Fanconi Anaemia

Gaurav Joshi", Nancy Beryl Janet, Thulaj D Meharwade, Anu Korula, Vikram Mathews, Vivi M Srivastava, Alok Srivastava, Biju George, Shaji R Velayudhan

Email ID for Correspondence: gaurav.joshi@cmcvellore.ac.in Department of Haematology, Christian Medical College, Vellore

Introduction: Fanconi anaemia (FA) is a genetic disease characterized by bone marrow failure and increased risk of malignancies. The FA pathway proteins functions primarily in DNA interstrand crosslink (ICL) repair. The proteins in the FA pathway monoubiquitinate FANCD2 and FANCI proteins which form a complex at the site of DNA damage, thereby recruiting proteins that supress nonhomologous end joining (NHEJ) and promote error free homology directed repair (HDR). Mutations in 21 distinct Fanconi anaemia (FANC) genes have been shown to interfere with the DNA-replication-dependent repair of lesions involving cross-linked DNA. Although mutations in any of the FA pathway genes can cause the disease, the mutations in FANC A $(60 \%), \mathrm{C}(15 \%)$ and $\mathrm{G}(10 \%)$ genes are more prevalent. FANCD2 ubiquitination is the critical event in ICL repair and it is defective in $~ 95 \%$ of FA cases. Objective: Evaluation of FANCD2 western blot as a test for the diagnosis of FA. Materials and Methods: Patients with cytopenia in at least one cell lineage with and without the physical abnormalities suggestive of FA were recruited in this study. Peripheral blood (PB) and skin biopsy were collected from the patients after informed consent. Peripheral blood mononuclear cells (PBMNCs) obtained from the patients were cultured for 72-96hrs in RPMI medium containing $10 \%$ FBS and Phytohaemagglutinin (PHA) before the cell lysates were prepared for western blotting. The skin biopsy sample was digested with collagenase I and cultured in DMEM $+10 \%$ FBS for a week till fibroblasts were generated. Fibroblasts were then treated with mitomycin (MMC), a DNA crosslinker, for 16 hours to activate the DNA repair pathway after which the cells were harvested for the preparation of whole cell lysates. Western blot was performed with anti-FANCD2 antibody to see whether FANCD2 ubiquitination was defective (FANCD2 S+ L) or normal (FANCD2 S+ L+). Chromosome breakage analysis was performed with peripheral blood cells cultured for 48 hours in the presence of PHA, followed by treatment with Mitomycin C (50 ng/ $\mathrm{ml}$ and $100 \mathrm{ng} / \mathrm{ml}$ ) for $48 \mathrm{hrs}$. Before CBA analysis was performed the cells were treated with Colcemid for 1 hour and triradials, quadriradials and ring chromosomes were scored. CBA score greater than 40 was considered as positive for Fanconi anaemia. Results: Out of 101 patients with physical features suggestive of FA, 75 patients had high or borderline CBA scores. In this group, 73 were FANCD2S+ L- while 2 patients were FANCD2S + L+, who might have mutations in the genes involved in the downstream of FANCD2 ubiquitination. There were 26 patients with low CBA scores $(<30)$. In this group, 13 patients were analyzed in their peripheral blood and 7 were FANCD2S+ L-, and 17 were analyzed in their fibroblasts and 15 were FANCD2S+ L-. There were 6 patients in this group, who were FANCD2S+ L- in the fibroblasts but were FANCD2S+ L+, suggesting mosaicism in these cases. We also screened 58 patients with acquired aplastic anaemia without any physical features of FA. All the patients in this group had normal CBA scores and were FANCD2S+ L+. 


\begin{tabular}{|c|c|c|c|c|c|}
\hline \multirow{2}{*}{$\begin{array}{l}\text { CBA Scores } \\
\text { (Peripheral } \\
\text { Blood) }\end{array}$} & \multirow{2}{*}{$\begin{array}{l}\text { No of } \\
\text { Patients }\end{array}$} & \multicolumn{2}{|c|}{ Peripheral Blood } & \multicolumn{2}{|l|}{ Fibroblasts } \\
\hline & & $\begin{array}{l}\text { FANC } \\
\text { D2S+ L- }\end{array}$ & $\begin{array}{l}\text { FANC } \\
\text { D2S+ L+ }\end{array}$ & $\begin{array}{l}\text { FANC } \\
\text { D2S+ L- }\end{array}$ & $\begin{array}{l}\text { FANC } \\
\text { D2S+ L+ }\end{array}$ \\
\hline $\begin{array}{l}>40 \\
(\text { Mean }=66.09 ; \\
\quad \text { range }=40.8 \\
\text { to } 152)\end{array}$ & 64 & $\begin{array}{l}23 / 25 \\
(92.3 \%)\end{array}$ & $\begin{array}{l}2 / 25 \\
(8 \%)\end{array}$ & $\begin{array}{l}39 / 39 \\
(100 \%)\end{array}$ & $0(0 \%)$ \\
\hline $\begin{array}{l}30-40 \\
\quad(\text { Borderline }) \\
\text { (Mean = 33.37; } \\
\text { range }=30.3 \text { to } \\
\quad 36)\end{array}$ & 11 & $\begin{array}{l}10 / 10 \\
(100 \%)\end{array}$ & $\begin{array}{l}0 / 10 \\
(0 \%)\end{array}$ & $\begin{array}{l}1 / 1 \\
(100 \%)\end{array}$ & $0(0 \%)$ \\
\hline $\begin{array}{l}<30 \\
(\text { Mean }=8.16 ; \\
\text { range }=0 \text { to } \\
28.5)\end{array}$ & 26 & $\begin{array}{l}7 / 13 \\
(53.8 \%)\end{array}$ & $\begin{array}{l}6 / 13 \\
(46.2 \%)\end{array}$ & $\begin{array}{l}15^{*} / 17 \\
(88.24 \%)\end{array}$ & $\begin{array}{l}2 / 17 \\
(11.76 \%)\end{array}$ \\
\hline
\end{tabular}

* In 6 cases peripheral blood showed FANCD2 S+ L+ and fibroblasts showed FANCD2 S+ L-, suggestive of mosaicism in these cases.

Conclusions: The results of FANCD2 western blot correlated with CBA in $97 \%$ of the patients with high CBA scores. In case of borderline CBA scores, FANCD2 western blot was diagnostic in $100 \%$ of the patients. Fibroblast western blot is helpful in confirmation of mosaicism and diagnostic confirmation of Fanconi anaemia in patients with low CBA scores. This study suggests that FANCD2 ubiquitination analysis is a highly sensitive test for the diagnosis of Fanconi Anaemia.

\section{2}

\section{Results of Allogeneic Stem Cell Transplantation in Refractory Acute Myeloid Leukemia with Active Disease from a Tertiary Care Center in Western India}

\section{Sameer Melinkeri, I Anusree Prabhakaran, Urmi Sheth}

\section{Deenanath Mangeshkar Hospital, Pune}

Objectives: To study the outcome of allogeneic stem cell transplantation in refractory acute myeloid leukemia (AML) with active disease at the time of transplantation from a tertiary care centre in western India. Materials and methods: Medical details of all patients who underwent stem cell transplants from January 2012 to January 2017 in our centre were collected through computerised medical record system. Out of these 147 patients, 17 patients were diagnosed cases of refractory AML who had undergone allogeneic stem cell transplantation. All the clinical and laboratory parameters of these patients were collected and analysed with the help of SPSS version 20. Results: 17 patients underwent allogenic stem cell transplants for refractory acute myeloid leukemia. There were 10 males and 7 females. Mean age of the study population was 25 years. Disease was refractory in all 17 patients at the time of transplant. 4 out of 17 patients had FLT3/ITD positive status and one patient had NPM1 mutation. 3 patients had $\mathrm{t}(8 ; 21)$. Out of the total 17 patients, 9 underwent matched sibling allogeniec stem cell transplantation, 3 underwent matched unrelated donor transplantation and 5 underwent haplo-identical transplantation. Conditioning regimens used were Fludarabine-Busulfan $(n=8)$, Fludarabine-Melphalan $(n=4)$, Fludarabine-Cyclophosphamide $(\mathrm{n}=1)$, FLAG-Melphalan $(\mathrm{n}=1)$, Busulfan-Cyclophosphamide $(\mathrm{n}=2)$ and Cylophosphamide-Total Body Irradiation $(\mathrm{n}=1)$. GVHD prophylaxis was with Cyclosporine or Tacrolimus and short course Methotrexate in 12 patients and remaining 5 received post transplant Cyclophosphamide with Tacrolimus and Mycophenolate Mofetil. Stem cell source was peripheral blood in all patients. All 17 patients had active disease at the time of transplant. Mean time for neutrophil engraftment was 10.52 days and platelet engraftment was 7 days. 4 patients died before engraftment. 1 out of 17 patients had graft rejection. Acute graft versus host disease was documented in 13 out of 17 patients (76.5\%). Chronic GVHD was documented in 16 out of 17 patients (94.12\%). Day 30 mortality occurred in 7 out of 17 patients $(41.18 \%)$. Day 100 mortality occurred in 10 out of 17 patients (58.8\%). Day 180 mortality was 13 out of 17 patients $(76.5 \%)$. Between day 30 to day 180 , mortality due to GVHD was seen in 5 patients and disease progression was observed in 1 patient. At a median follow up of 843 days, 4 patients are alive and disease free. Progression free survival (PFS) and overall survival (OS) at the time of analysis was $23.5 \%$ for the cohort. Conclusion: Allogeneic stem cell transplant is a curative treatment modality for refractory acute myeloid leukemia in carefully selected patients. Infection and GVHD remain the major cause of mortality.

\section{3}

Results of Haploidentical Stem Cell Transplants for Relapsed Refractory Hematological Malignancies from a Tertiary Care Centre

Objectives: To study the outcome of haploidentical stem cell transplantation in relapsed or refractory hematological malignancies from a tertiary care centre in western India. Materials and methods: Medical details of all patients who underwent stem cell transplants from January 2014 to July 2017 in our centre were collected through computerised medical record system. Out of these 120 patients, 22 patients were diagnosed cases of relapsed/ refractory hematological malignancies who had undergone haploidentical stem cell transplants. All the clinical and laboratory parameters of these patients were collected and analysed with the help of SPSS version 20. Results: 22 patients underwent haploidentical stem cell transplants for various hematological disorders including acute myeloid leukemia $(\mathrm{n}=10)$, acute lymphoblastic leukemia $(\mathrm{n}=7)$, myelodysplastic syndrome $(\mathrm{n}=3)$, chronic myeloid leukemia $(\mathrm{n}=1)$ and non hodgkin's lymphoma $(\mathrm{n}=1)$. There were 16 males and 6 females. Mean age of the study population was 27.25 years. Disease was in remission in ... patients and refractory at the time of transplant in....patients. Conditioning regimens used were Fludarabine - Busulfan $(n=9)$, Fludarabine- Melphalan $(n=7)$, Fludarabine- Total body Irradiation $(\mathrm{n}=3)$, Cyclophosphamide-TBI $(\mathrm{n}=2)$, Fludarabine- CytarabineMelphalan $(\mathrm{n}=1)$. GVHD prophylaxis was post transplant Cyclophosphamide with Tacrolimus and Mycophenolate Mofetil. Stem cell source was peripheral blood in all patients. Mean time for neutrophil engraftment was 17.3 days. Only 1 patient had graft rejection. Acute Graft versus host disease was documented in 6 patients $(27.27 \%)$. Chronic GVHD was documented in 8 patients (61.54\%). Day 30 mortality occurred in 7 patients (31.82\%) Day 100 
transplant related mortality occurred in 9 patients $(40.9 \%)$. Progression free survival of the cohort was $36.3 \%$ ( 8 out of 22 patients). The overall survival observed was $45.45 \%$ (10 out of 22 patients). Conclusion: Haploidentical stem cell transplant is a curative treatment modality for relapsed refractory acute leukemias. The best results were noted in acute lymphoblastic leukemia.

\section{4}

Molecular Characteristics of AML in India: A Study from a Tertiary Care Center of Eastern India

\section{Akshatha Nayak U ${ }^{\#}$, Sreeparna Podder, Rudra Ray,} Arnab Chattopadhyay, Maitreyee Bhattacharyya

Email ID for Correspondence: anayakbejai@gmail.com Institute of Haematology and Transfusion Medicine (IHTM), Medical College and Hospital, Kolkata

Introduction: Acute myeloid leukemia (AML) is traditionally characterized based on immunophenotypic, and cytogenetic analysis. For further categorization into prognostic subgroups such as favourable, intermediate and unfavourable risk, molecular markers namely FLT3, NPM1, CEBPA are being routinely assessed. But inspite of these about $30 \%$ cases no mutation could be identified. Additional somatic mutations are also being assessed for further prognostication. In this study we have attempted to identify the regular molecular markers namely FLT3 (ITD \& D835) and NPM1 along with one somatic mutation IDH (IDH1\% IDH2) and tried to correlate with various clinicopathological characteristics. Objectives: 1) To explore prevalence of FLT3, NPM1 \& IDH mutations. 2) To correlate with various clinicopathological characteristics. Material and Methods: Sixty nine newly diagnosed AML patients attending IHTM, during the time period January 2017 to July 2017 were analysed for the presence ofFLT3 (FLT3 ITD \& FLT3D835), NPM1 and IDH (IDH1 \& IDH2) mutations in peripheral blood and bone marrow aspirate samples. DNA was isolated using Automated machine from QiaCube Qiagen. NPM1 was tested in only 31 patients and IDH1 was sequenced by sanger sequencing in only 33 randomly selected samples. FLT3, IDH2 was assessed in all the samples initially by amplification by PCR followed by analysis on agarose gel. Results: Out of the total 69 patients $41(59.4 \%)$ were males and $28(40.6 \%)$ were females. Median age at presentation is 42 years (Range 8-91 years) with $9(13 \%)$ patients in the $<18$ age group, $52(75.4 \%)$ patients in the 18-65 age group and $8(11.6 \%)$ patients in the $>65$ age group. Median WBC count at presentation was 19,000/cu.mm (700-205,000), blast percentage of $65 \%$ and platelet count of 23,000 respectively. Out of the 69 cases, cytogenetics were normal in $20(29 \%)$ patients, cytogenetics other than normal karyotype was seen in $18(26.1 \%)$ patients and no mitotic divisions in $21(30.4 \%)$ patients. Cytogenetics was not available for $10(14.5 \%)$ patients. Flow cytometry showed $10(14.5 \%)$ cases with Monocytic differentiation, 20 (29\%) cases with aberrant expression of CD7, $14(20.3 \%)$ cases with aberrant expression of CD56 and 6 (8.7\%) with CD19 expression. Molecular markers FLT3 was positive in 20 (29\%) cases with FLT3 ITD seen in $14(20.3 \%)$ cases and FLT3 D835 in $6(8.7 \%)$ cases, NPM1 was seen in $5 / 31$ $(16.7 \%)$ case and IDH was positive in $12(17.3 \%)$ cases with IDH1 positive in $3 / 33(9 \%)$ cases and IDH2 positive in $9 / 69(13 \%)$ cases. None of the patients with each of these mutations were older than 65 years. FLT3 mutations were more commonly seen in females $39.3 \%$ than in males $22 \%$; NPM1 mutations were more commonly seen in males $23.5 \%$ than in females $7.1 \%$ and IDH mutations were seen more in females $21.4 \%$ than in males $12.2 \%$. Both FLT3 and NPM1 positive patients had a higher mean WBC count at presentation. Aberrant CD7 expression in IPT was seen in 50\% of FLT3 positive cases more commonly in FLT3 ITD $57.1 \%$ which was significant. 35\% of cases with normal cytogenetics were FLT3 positive, $27.3 \%$ were NPM1 positive cases and $15 \%$ were IDH mainly IDH2 positive. Out of the 31 cases where NPM1 was tested, FLT3 was positive in 3 out 5 NPM1 positive cases (FLT3 ITD-2 \& FLT3 D8351 case. Coexisting FLT3 mutations specifically FLT3 ITD were seen in 3 of the IDH mutated patients mainly IDH2 whereas coexisting NPM1 mutation was seen in 1 patient with IDH2 mutation and none of the IDH1 mutated cases. Conclusions: Prevalence of most of the mutations in this study confers with those of other studies with few exceptions which included low prevalence of NPM1 mutations in normal cytogenetics cases, higher frequency of FLT3 positive cases showing CD7 aberrancy which was significant, none of the three molecular mutations positive cases were older than 65 years and IDH 2 mutations had more of coexisting FLT3 mutations than NPM1 mutations.

\section{5}

\section{OEPA/OPPA \& COPDAC/COPP Protocol in Paediatric Hodgkin Lymphoma-Experience of a Tertiary Care Hospital}

\section{Patra PC ${ }^{\#}$, Mandal PK, Dolai TK, De R, Baul S, Chakrabarti P}

Email ID for Correspondence: drpritishpatra@gmail.com NRS Medical College, Kolkata

Introduction: Hodgkin lymphoma (HL) constitutes approximately $40 \%$ of all lymphomas that present during childhood and is the most common malignancy in adolescents and young adults. The cure rate for children and adolescents has steadily improved over the years at the price of serious long-term toxicities. Therefore, the therapeutic paradigm has shifted towards reducing treatment-associated toxicities while maintaining high cure rates. Objectives: To assess the response rate of OPPA/OEPA \& COPP/COPDAC protocol in case of paediatric HL (upto 18 years of age) both as upfront as well as salvage therapy and also to compare the response rate with that of ABVD protocol. Material and Methods: All consecutive cases of paediatric HL patients (upto 18 years of age) attending NRS Medical College, were taken from October 2014 to September 2017 and were given chemotherapy either as per OPPA/OEPA \& COPP/COPDAC or ABVD protocol. Results: Among the total 70 cases of paediatric HL, initial 52 cases were given ABVD and subsequently 18 were treated with OPPA/OEPA \& COPP/COPDAC protocol. Median age was 9.5yrs (range: $3-18 \mathrm{yrs}$ ) and 8yrs (range: $4-16 \mathrm{yrs}$ ) respectively. In the former group, male:female ratio was $44: 8$ while in the later group it was 12:6. Cervical lymphadenopathy was the most common clinical finding during initial presentation $(89 \%$ and $94 \%$ in respective groups) either alone or with other lymph node regions. Thirty one cases $(60 \%)$ belonged to advanced stages in the former and $16(89 \%)$ in the later group, while B symptom accompanied the advanced stages in both (61\% and $87.5 \%$ respectively). Among those who received $\mathrm{ABVD}$ as upfront, overall response was seen in $39(87 \%)$ cases, among which 34 (76\%) had CR and $5(11 \%)$ had PR and one patient relapsed after 2 yrs of achieving CR. In the later group, overall response was seen in $11(84.6 \%)$ cases, among whom $10(77 \%)$ had CR; one (7.6\%) had PR. Out of those who went to CR, $2(15.3 \%)$ relapsed later. When the later was given as $2^{\text {nd }}$ line chemotherapy, CR and progressive disease was seen in $50 \%$ of cases each. Conclusions: The OPPA/OEPA \& COPP/COPDAC protocol when given as upfront chemotherapy, the response rates are not inferior when compared to ABVD. So it seems to be the preferred one as compared to ABVD as the long term toxicities is expected to be less. However larger studies and long term follow up may be needed for more detailed and better assessment. 


\section{6}

\section{Flowcytometry in Plasma Cell Neoplasms (PCN) - a cost effective} working protocol

Dr. Kiran Ghodke, Dr. Pankhi Dutta, Asha Priyadarshni, Shamika Murdas, Bhavini Chouhan, Swapnil More, Dr. Sameer Tulpule

Email ID for Correspondence: drkiranghodke@gmail.com; Anshita Dubey (Presenting Authors)

Koiklaben Dhirubhai Ambani Hospital and Research Institute

Introduction: Plasma cell neoplasm includes wide spectrum ranging from MGUS to Multiple myeloma. The role of flow cytometry is evolving and is used in diagnosis, prognostication, minimal residual disease detection and it also determines the risk of progression from MGUS to Multiple myeloma. Objectives: Standardisation of assay and to set up a cost effective working protocol (cytoplasmic staining) for immunophenotyping of plasma cell neoplasms by flow cytometry. Material and Methods: Consecutive thirty two suspected patients ofplasma cell neoplasm were accrued in this study from September 2016 to August 2017 (1 year). Patient's clinical and laboratory data was collected along with bone marrow (BM) aspiration, trephine biopsy and flowcytometric immunophenotypic (FCIPT) findings. BM specimen was further processed for FCI using a stain-lyse-wash technique and a six-color antibody-panel (2 tubes) for PCN on BD FACS CANTO II and analyzed using BD FACS Diva software. High number of events (5- 10 lakhs) were acquired. In FCIPT analysis, for cytoplasmic staining (clonality assay), we have used two methods method $1^{\text {st }}$ was the laboratory formulated while the method $2^{\text {nd }}$ was kit based. Results: Different approaches were used to calculate exact percentages of plasma cells. The results of both the methods were analysed and compared, the plasma cell percentages ranging from $0.1 \%$ to $57 \%$ in method 1 and $0.1 \%$ to $60.8 \%$ in method 2 . The details of all the cases will be presentedat the meeting. Conclusions: We have validated a standardised and cost effective working protocol for FC IPT of plasma cell neoplasm which gives an acceptable plasma cell counts (\%) and clear separation of plasma cells clonality. Both the methods are effective in providing clear plasma cell separation and with comparable plasma cell percentages however, the yield of second method is slightly high in few cases and hence suited well in samples with lower plasma cell percentage but the routine laboratory method is more cost effective.

\section{7}

Significance of Mosaicism in Molecular diagnosis of Haemophilia

Sankari Devi. G, Aby Abraham, Biju George, Mathews. V, Srivastava. A, Eunice S. Edison

Email ID for Correspondence: rajkumar.sve@gmail.com; S.V. Rajkumar (Presenting Authors) Christian Medical College, Vellore

Introduction: Haemophilia A (HA) and haemophilia B (HB) are inherited X-linked bleeding disorders caused by deficiency of coagulation FVIII and FIX respectively. Molecular characterization of haemophilia is important, especially for prenatal diagnosis. Although haemophilia is inherited in an X-linked manner, de novo mutations can also occur. De novo mutations may represent either germ line and or somatic mosaicism. We analyzed the frequency of mosaicism in haemophilia cases in our center. Objectives: To estimate significance of mosaicism in molecular diagnosis of Haemophilia. Material and Methods: We retrospectively analyzed the molecular data of haemophilia patients done in our center between 2008 and 2017. The algorithm for molecular diagnosis of haemophiliaA isinversion PCR followed by confirmation sensitive gel electrophoresis (CSGE) and DNA sequencing. InhaemophiliaB, mutations were screened by direct DNA sequencing. Gross deletions involving F8 and F9 genes were screened by gene dosage analysis. VNTR analysis was carried out using capillary electrophoresis. Results: A total of 511 cases opted for molecular diagnosis of haemophilia (HA-431; HB-80) between 2008-2017. A disease causing genetic variant could be identified in 479 cases $[\mathrm{HA}=399 / 431,92.57 \%$; $\mathrm{HB}=80 / 80,100 \%$ ]. Of these 479 cases, mothers' samples were available only in 69\% HA $(\mathrm{n}=275)$ and $71.2 \% \mathrm{HB}(\mathrm{n}=57)$ cases. Analysis of the disease causing variant in the mothers' sample revealed that 3.6\%[10/399] and $5.26 \%[3 / 80]$ of the cases were attributable to de novo mutations due to mosaicism. Twelve different mutations were identified. Inversions accounted for $30 \%(3 / 10)$ and the rest were point mutations $(7 / 10$, $70 \%)$ in HA. A gross deletion $(1 / 3,33 \%)$ andpoint mutations $(2 / 3$, $66 \%$ ) constituted these cases in HB. VNTR analyses ruled out parental issues. Conclusions: It is important to look for de novo mutations in haemophilia patients' presenting without a significant family history. Understanding mosaicism is important since the carriers go undetected during screening. Carrier screening and prenatal diagnosis should be performed with caution in families with de novo mutations due to mosaicism.

\section{8}

Evaluation of a Single Tube Flow Cytometric Method Using Five Antibodies Including CD157 in the Detection of PNH Clone

Roopam Deka", Hara Prasad Pati, Seema Tyagi, Renu Saxena

Email ID for Correspondence: roopamdeka1983sd@gmail.com All India Institute of Medical Sciences, New Delhi

Introduction: CD157 is a glycosyl-phosphatidylinositol (GPI)linked molecule which is expressed on both granulocytes and monocytes. It can be used in a single tube fluorescein-labelled proaerolysin (FLAER) based flow cytometric assay to detect paroxysmal nocturnal hemoglobinuria (PNH) clones on both these types of leucocytes, replacing CD24 (expressed on granulocytes) as well as CD14 (expressed on monocytes). Objectives: The aim of the study was to evaluate the ability of CD157 in a new "test" method to replace both CD14-CD24 used in the standard flow cytometric assay for detecting PNH clone. Material and Methods: Peripheral blood samples from 52 patients newly detected to have PNH clone by the standard flow cytometric assay (employing six antibodies in one tube, viz., CD45-CD15-CD64-CD14-CD24-FLAER) were subjected to the test method (using CD157 instead of CD14-CD24, with rest of the antibodies remaining same as in the standard assay). Patients were divided into two categories for analysis: i) PNH disease $(n=10)$ and ii) Aplastic anemia/Myelodysplastic syndrome (AA/MDS) with PNH clone $(n=42)$. Results: Receiver operating characteristic curve (in the test method) calculated cut-off for PNH clone on granulocytes to be $>0.4 \%$ and $>0.9 \%$ for monocytes (with sensitivities and specificities of $\geq 95 \%$ for both of these leucocyte subtypes). There was significant correlation between the clone sizes measured by both 
standard and test methods on granulocytes $(r=0.976, p<0.001$ in PNH disease; $r=0.980, p<0.001$ in AA/MDS with PNH clone) as well as on monocytes $(\mathrm{r}=0.806, \mathrm{p}=0.005$ in $\mathrm{PNH}$ disease; $\mathrm{r}=0.915, \mathrm{p}<0.001$ in AA/MDS with PNH clone). Bland-Altman analysis demonstrated performance agreement between the methods. Cost of the assay to the patient was reduced by about $15 \%$ in the "test" method as compared to the standard method. Conclusions: PNH clone sizes of $\leq 1 \%$ could be detected by the CD157 based single tube assay in PNH disease as well as in AA/MDS. This 5-colour method was found to be equivalent to the 6-colour standard assay, with reduction of the cost to the patient and sample preparation time, providing better use of technical resources.

\section{0}

Comparative Analysis of Role of ISO Electric Focussing \& High Performance Liquid Chromatography in Newborn Screening with Special Reference to DNA Analysis

Dr. Kavita Jain (PGT Pathology) ${ }^{\#}$, Dr. Mousumi kar (MD Pathology), Dr. Nikhil Kumar (MD Pathology), Dr. Moumita Sengupta (Asst.prof), Dr. Chhanda Datta (prof \& HOD), Dr. Uttara Chatterjee (Prof, Dept of pathology)

Email ID for Correspondence: dockavitajain@gmail.com Institute of Post Graduate Medical Education \& Research, Kolkata

Introduction: Hemoglobin disorder which includes thalassaemia and hemoglobin variants are the most frequent clinically significant single gene disorders affecting $7 \%$ of world population. To avoid the large financial burden to provide optimal treatment for patients and the innumerable fatalities from untreated, screening is the best cure for hemoglobin disorders. Prenatal screening, prospective antenatal screening and Community carrier screening was introduced according to WHO guideline since 1970. Recommended interventions are genetic counseling, disease education and antibiotic prophylaxis depending on diagnosis. $\mathrm{Hb}$ Variants are typically identified from their electrophoretic mobility, the quantity of the abnormal hemoglobin and the ethnic background of the individual. High performance liquid chromatography (HPLC) is now considered to be a sensitive, specific, and reproducible alternative to electrophoresis. Capillary electrophoresis is also a versatile analytical electrophoresis technique that uses numerous separation principles, including capillary isoelectric focusing (CIEF). Objectives: 1 . To evaluate the prevalence of occurrence of different haemoglobinopathies in study population. 2. To develop and compare, for both result and quantification capabilities of two methods suitable for different analysis of different HbsCIEF assay and a HPLC assay. 3. To report few of the predominant mutation of thalassaemia patients included in our study. Material and Methods: Study Design - This prospective and observational study was conducted in department of pathology in collaboration with department of Neonatology during the period of January, 2016 to July, 2017. Isoelectric focusing was carried out as primary screening programme using dried blood sample by heel puncture on filter papers followed by high performance liquid chromatography (HPLC). Study Population - 4200 neonates within 3-7 days of age. Inclusion criteria were 1) neonates- 28 days of age or below 2) informed consent from parents 3) participants who had no history of blood transfusion at the time of sample collection. Method: Complete haemoglobin parameter were noted using automated $\mathrm{Hb}$ analyser \& peripheral blood examination. IEF was employed to detect $\mathrm{Hb}$ variants. Hemoglobin was reported in decreasing order of concentration. FA, FE, FC, FD and FAV reporting tables were made and analyse accordingly. HPLC was employed to validate and tally the results of IEF to find out the positive predictive values between the two exclusive. Data were assembled and a chromatogram was prepared. Finally, molecular analysis at DNA level was done to cross verify the results of the both the IEF and HPLC in all beta thalassaemia variants and alpha thalassaemia. Mutational analysis were taken as the 'gold standard' in discordant cases and the reports of IEF and HPLC were statistically analyzed using Chi Square test, Mathews correlation test, Sensitivity, Specificity, Positive predictive value, Negative predictive value and Diagnostic accuracy etc. Results: Total 4200 neonates were included aged between $0-28$ days, with mean age 14.3 days, with a male preponderance. In this study we identified total 213 cases of hemoglobinopathies consisting of eleven Hbvariants named as $\mathrm{HbE}, \beta$-Thal Carrier $(\beta+), \beta$-Thal intermediate, HbD Punjab, HbS, $\beta$-Thal Major $(\beta 0), \mathrm{HbE} / \beta, \mathrm{HbD} / \beta$ (Hetero), Alpha, Sickle beta $\& \mathrm{HbEE}$ disease etc. We observed that $\mathrm{HbE}$ trait is quite prominent in this region as $3.54 \%$ within our targeted population and $69.95 \%$ among the total $\mathrm{Hb}$ variants. Comparison between IEF and CE - In diagnosis of affected child: Complete correlation between isoelectric focussing and HPLC was obtained in 25 cases out of 28 cases of major haemoglobinopathies. Most common major neonatal haemoglobinopathies in our study is sickle cell disease (10 cases, $4.69 \%)$ followed by thalassaemia major (6 cases, $2.81 \%$ ) and $\mathrm{Hb}$ D- Punjab (5 cases, $2.34 \%$ ). An abnormality was detected in HPLC chromatogram in all cases of $\mathrm{Hb} \mathrm{E}$ homozygous (2 cases, 0.93\%) detected by IEF revealing significant correlation (100\%). A significant drop in the diagnostic accuracy of HPLC was observed in alpha -thalassaemia. It was identified in 5/269 and 2/269 of the samples tested by IEF and HPLC, respectively. Early eluting peak was detected in HPLC chromatogram in 2 cases of alpha thalassaemia. Three cases which were positive in IEF remain undetectable in HPLC probably due to low concentration of $\mathrm{HbH}$.

- In diagnosis of healthy carrier: The common phenotype in present study was $\mathrm{HbE}$ trait (149 cases, 69.9\%) followed by betathal trait (24 cases, $11.26 \%$ ). All cases of $\mathrm{HbE}$ trait were confirmed to have hemoglobin abnormalities by both HPLC and IEF during neonatal screening. During IEF testing 26 cases neonates were suspected to have beta thalassaemia trait depending on the low intensity band of HbA compared to normal newborns. But by quantitative assay in HPLC only 24 cases were diagnosed and confirmed by further genetic study. There were 7 cases of $\mathrm{Hb}$ E-Beta detected by IEF and subsequently confirmed by HPLC. HPLC was able to detect 1 out of 3 double heterozygous conditions observed by IEF.

- Evaluation of IEF as a first-line screening test: Eleven $\mathrm{Hb}$ variants were identified by both techniques, with no discrepancy in the detection of variants. The results of IEF were comparable with HPLC with a statistically significant measure of agreement of $\kappa=0.928$ between the two.

- Mutation analysis: DNA analysis of $37 \beta$-Thal variants for 3 common mutations found in Asian Indian population was conducted, e.g. $\operatorname{cd} 26(\mathrm{G}>\mathrm{A})$, IVS1-5 $(\mathrm{G}>\mathrm{C})$ and $\operatorname{cd} 15(\mathrm{G}>\mathrm{A})$. The occurrence of these 3 mutations were seen in total 36 cases $(87.8 \%)$. IVS $1-5(\mathrm{G}>\mathrm{C})$ was found in $68.3 \%$ cases, cd15(TGG $>$ TAG) was found in $21.95 \%$ cases \& $\operatorname{cd} 26(\mathrm{G}>\mathrm{A})$ in $19.5 \%$ cases.

Conclusion: Beta-Thalassaemia trait and $\mathrm{HbE}$ trait are most prevalent abnormalities in this region. IEF \& CE-HPLC are both labor saving as objective screening tool for early detection of haemoglobinopathies. The order of frequency of mutational analysis were IVS1-5 $(\mathrm{G}>\mathrm{C})$ then $\mathrm{cd} 15$ (TGG $>$ TAG) followed by $\operatorname{cd} 26(\mathrm{G}>\mathrm{A})$. 


\section{P-AL 01}

\section{Leukemic Infiltration of Kidney in a Case of T-Cell all Dr. RonicaBaruah MD", Dr Naushad Shah MD, Dr Manjuri Sharma MD DM, Dr Manzoor Ahmad Parry MD DM scholar}

Email ID for Correspondence: ronicanagpal@yahoo.com Ekopath Metropolis Lab Services PvtLtd, G.S.Road, Christian Basti, Guwahati-781005, Assam

Introduction: AKI is an important complication in patients with acute leukemia, caused by the disease or treatment. Most common complications are sepsis, hypoperfusion injury and tumor lysis syndrome (TLS). ALL presenting with AKI and infiltration in kidney is rare and uncommon. Objectives: To present an uncommon case of renal involvement by acute leukemic presenting as acute kidney injury (AKI). Material and Methods: A 19 year old male presented with 3-month history of weakness, anorexia and weight loss (8\%). His past medical history was unremarkable. Physical examination revealed pallor with normal blood pressure, pulse rate, respiratory rate and body temperature. Results: Lab findings at presentation showed haemoglobin of $7.5 \mathrm{~g} / \mathrm{dL}$, leucocyte count of $10.35 \times 10^{3} / \mu \mathrm{L}$ (Differential count showed presence of few blast), creatinine $2.66 \mathrm{mg} / \mathrm{dl}$, urea $98 \mathrm{mg} / \mathrm{dl}$ and LDH $825 \mathrm{U} / \mathrm{L}$. Bone marrow biopsy was performed which showed features of acute leukaemia. Flowcytometry confirmed it as a case of T cell acute lymphoblastic leukaemia. The renal biopsy was done to determine the cause of renal dysfunction. The renal biopsy showed sheet like interstitial infiltration by tumour cells with atrophy and destruction of tubules. IHC showed positivity for CD 7 and TdT and CD 20 negative tumour cells, confirming the diagnosis of T cell ALL. Conclusions: It must be kept in mind that T cell ALL may rarely present as AKI with normal WBC counts in blood. Renal biopsy is required for the diagnosis at earliest. Treatment with chemotherapy can result in complete recovery of renal function.

\section{Poster Presentation}

\section{P-AL 02}

Blastic Plasmacytoid Dendritic Cell Neoplasm: A Case Report

\section{Vikranth Varma, Anil Aribandi}

Department of Haemato-Oncology and Bone marrow Transplantation, American Oncology Institute, Hyderabad, India

Background: Blastic plasmacytoid dendritic cell neoplasm (BPDCN) is a rare highly aggressive hematologic neoplasm characterised by the clonal proliferation of immature precursors of plasmacytoid dendritic cells, included among acute myeloid leukemia (AML) and related precursor disorders in the 2008 World Health Organization (WHO) classification of hematological diseases and then classified as a distinct entity among myeloid neoplasms in the 2016 revision. It accounts for about $0.7 \%$ of all skin and primary malignant lymphomas. The clinical features of BPDCN are infiltration of the skin and subsequent involvement of the hematopoietic system, including bone marrow and lymph nodes. Most of the patients are elderly with median age at diagnosis of 61-67 years. We report a case of BPDCN which had undergone a CHOP chemotherapy and recovered well. Case Report: A 72 years old male was admitted to our hospital for an evaluation of skin rashes. One month before presentation, the patient had noted an erythematous patches on his forehead, chest and abdomen that were increasing in size. Physical examination revealed conjunctival bleeding, reddish brown patches and bruise-like lesions on the scalp, face, upper limbs and trunk. The majority of the patches ranged from 2 to $5 \mathrm{~cm}$ in diameter. Bone marrow aspiration and skin biopsy were done showed similar morphology. Based on clinical and immunophenotyping studies, the patient was diagnosed with BPDCN with extensive cutaneous and bone marrow involvement. He had undergone CHOP chemotherapy and doing well. Conclusion: In conclusion, based on clinical and immunophenotyping studies, we treated a case of BPDCN, a rarely aggressive hemato-dermic neoplasm which has very poor prognosis with CHOP chemotherapy and he is on follow-up now.

\section{P-AL 03}

The Unexpected Challenge in Acute Lymphoblastic Leukemia Management- Invasive Aspergillus Infection

Dr. Rohith Chitrapur, Dr. Ramya $\mathbf{R}^{\#}$, Dr. Murali subramian, Dr. Berly ${ }^{\#}$

Email ID for Correspondence: ramya6991@gmail.com, berlydan02@gmail.com

Bangalore Baptist Hospital, Bangalore

Introduction: Childhood Acute Lymphoblastic Leukemia (ALL) was the first disseminated cancer shown to be curable. There has been a remarkable progress in the treatment of ALL improving overall 5 year survival rate upto $90 \%$. But invasive fungal infections are steadily on the rise causing significant mortality and morbidity in these patients. Invasive aspergillosis is one of the common serious fungal infections in patients with hematological malignancies affecting the outcome of the disease. Objectives: Management and difficulties encountered in diagnosing and treating Fungal Infection in children treated for ALL in a tertiary care hospital. Material and Methods: We are reporting two cases of fungal infection in children treated for ALL in our chemotherapy unit.10 year old boy following induction chemotherapy presented with seizures and found to have cerebral SOL, Stereotactic Biopsy done, Biopsy proven Aspergillus Fumigatus. 17 year old boy on induction chemotherapy for ALL present with nasal pain and found to have Aspergillus Fumigatus sinusitis. Results: Fungal infections are uncommon finding in ALL as compared to AML. We report two cases of documented Aspergillus infection and their management. Conclusions: Invasive Aspergillosis though uncommon in ALL, are being encountered these days, complicating ALL management. Hence it should be considered as a differential, diagnosed and treated adequately as it could have fatal outcome. Though various drugs can be used to treat fungal infections, however a difficult situation arises in case of patients with cerebral space occupying lesions since most of the drugs used do not cross the blood brain barrier.

\section{P-AL 04}

A rare case of hypoplastic Acute Myeloid Leukemia

Sonia Chhabra\#, Monika Gupta, Renuka Verma, Sunita Singh, Rajeev Sen

Email ID for Correspondence: dr.soniachhabra@gmail.com Pt BDS, Post Graduate Institute of Medical Sciences, UHS, Rohtak (Haryana)

Introduction: Hypocellular acute myeloid leukemia (AML) is an infrequent entity. Its frequency ranges between $5-12 \%$ of all cases of 
AML. Hypocellular AML is currently defined as AML with a bone marrow cellularity less than $20 \%$, although in some earlier reports, cellularity less than $40 \%$ or $50 \%$ was considered to be hypocellular. We report a rare case of hypocellular AML-M5 occurring in a 75-year-old man. Case report/ Material and methods: A 75-yearold male presented with complaints of fever, which is mild grade and intermittent in nature since last 20 days and dyspnea on exertion gradually progressive since last 15 days. There is history of black colour stools, decreased appetite and weight loss. Patient had past H/O pulmonary Koch's and had taken anti tubercular drug (Partially treated). On examination, Pallor was present but there was no lymphadenopathy or organomegaly. On investigation, $\mathrm{Hb}-5.5 \mathrm{gm} \%$, TLC -3050 cells/cumm, DLC: $\mathrm{P}_{06} \mathrm{~L}_{11} \mathrm{E}_{01}$ Monocytoid cells 82, Platelet count 41,000 cells/cumm. Peripheral smear showed macrocytic picture with moderate anisocytosis and presence of monocytoid cells. All biochemical investigations were within normal limit. Bone marrow aspiration and trephine biopsy both showed hypoplastic marrow with markedly suppressed erythropoiesis, myelopoiesis showed increase in blast population $(30 \%)$ revealing monocytic appearance with folded nuclei and abundant cytoplasm. Megakaryocytes were markedly reduced. Cytochemically, these blasts were nonspecific esterase positive and myeloperoxidase negative. Flowcytometry on peripheral blood showed CD13, CD33, CD64, HLA-DR, CD117 and CD4 positivity. Based on all these features, diagnosis of hypocellular AML-M5 was made. Discussion: Hypoplastic AML can be challenging for both pathologist and treating physician. The pathogenesis of the hypocellularity remains speculative. It is unclear whether the leukemia is secondary to the hypocellularity or if it is the primary event. It has been suggested that leukemia cell populations inhibit myelopoiesis through a humoral mechanism or an increased susceptibility of myeloid precursors to the inhibitor in older patients might play a role in the genesis of hypoplasia. Conclusion: Hypocellular acute monocytic leukemia is a rare entity, and more frequently seen in older patients, and patients tend to have more profound presenting pancytopenias. The limited published information about the clinical and prognostic parameters of this entity makes specific clinical recommendations difficult.

\section{P-AL 05}

\section{Altered Sensorium in T Cell Acute Lymphoblastic Leukemia - ?} Bleed ? Central Nervous System Leukostasis

\section{Dr. Rohith G Chitrapur, Dr. Lakshmi K S,} Dr Murali Subramanian Dr. P. Mary Dayana ${ }^{\#}$

Email ID for Correspondence: marydayana.siraj@gmail.com Bangalore Baptist Hospital, Hebbal, Bangalore

Introduction: Leukostasis is a poorly understood, life threatening complication of leukemia. It is a clinico pathological entity characterised by vascular occlusion by leukemic blast cells. Early diagnosis and treatment is essential as mortality of untreated leukostasis is around $40 \%$ in the first week. Objectives: We report a case of T cell ALL, an acute hyperleukocytic leukaemia with bilateral cerebral intraparenchymal haemorrhages secondary to CNS leukostasis in a $14 \mathrm{yr}$ old boy. Material and Methods: $14 \mathrm{yr}$ old male child with normal growth and development presented to the emergency room with complaints of 4 days history of fever, headache, epistaxis, drowsiness and vomiting. At the time of admission he was drowsy with GCS of 14/15, generalised lymphadenopathy, hepatosplenomegaly and no focal neurological deficit. Investigations showed WBC count of $4,42,000 / \mathrm{mm} 3$ with $85 \%$ blast cells with platelet count 50,000. Flow cytometry reports confirmed T cell ALL. He was treated with allopurinol, dexamethasone, Inj Vincristine and hyper hydration. CT scan done in view of depressed consciousness showed multiple intraparenchymal hemorrhages in bilateral cerebral hemispheres with surrounding oedema and mass effect. On day 2 of admission child's consciousness gradually worsened. He had a cardiac arrest and was revived with 2 cycles of CPR and started on mechanical ventilation. His sensorium further deteriorated and after 2 hours had absent brain stem reflexes with bilateral fixed dilated pupils. Repeat WBC counts showed drastic improvement and were $3,73,000 / \mathrm{mm}^{3}$ on day 2 which dropped to $37,000,7,000$ and $2,900 /$ $\mathrm{mm}^{3}$ over the next 4 days. Symptomatic treatment was continued for the next 72 hrs. Child however showed no improvement in general condition. Brain stem reflexes continued to be absent and cold calorie test done was suggestive of brain death. Results: T cell Acute lymphoblastic leukaemia presenting as CNS leucostasis is a dreaded complication, very difficult to manage with high mortality. Conclusions: Central Nervous system leucostasis is a complicated situation with poor outcomes in spite of timely intervention, cytoreduction and supportive treatement.

\section{P-AL 06}

CD86 is a Highly Sensitive Marker for Detecting AML with Monocytic Differentiation and is a Strong Indicator of KMT2A Mutation in Acute Monocytic Leukemia (AML-M5)

Karishma Girase $^{1 \#}$, Pratiksha Devre ${ }^{1}$, Gaurav Chatterjee ${ }^{1}$, PG Subramanian ${ }^{1}$, Pratibha Amare ${ }^{2}$, Nikhil Patkar ${ }^{1}$, Sitaram Ghogale ${ }^{1}$, Y Badrinath ${ }^{1}$, Nilesh Deshpande ${ }^{1}$, Sumeet Gujral ${ }^{1}$, *Prashant Tembhare ${ }^{1}$ (*corresponding author)

Email ID for Correspondence: karigirase@gmail.com

Tata Memorial Centre, Mumbai

Introduction: Acute myeloid leukemia (AML) is a group of heterogeneous disease with poor clinical outcome ${ }^{1}$. CD86 is a member of "B7" co-stimulatory molecule expressed on antigen presenting cells such as activated B-cells, monocytes, and dendritic cells $^{2}$. Expression of CD86 shown in B-cell acute lymphoblastic leukemia (B-ALL) and its role in B-ALL-MRD detection has been reported $^{3}$. We studied the expression-pattern of CD86 in AML, its subtypes and its relation with underlying cytogenetics. Objectives: To study CD86 Expression in AML (non-APML) cases and its association with KTM2A mutation in AML. Material and Methods: Expression-pattern of CD86 (PE, clone: 2331/FUN-1) was studied in AML (non-APML) as per WHO criteria, Morphology, cytochemistry, flow cytometric immunophenotyping (FCI) and cytogenetics were determined. FCI was performed using 10-color on Navios-flow-cytometer and Kaluza-software-V1.3. FISH was performed using Abott Mol LSI dual-fusion translocation and break-apart rearrangement probes. Results: Expression-pattern of CD86 studied in 107 cases of (non-APML) AML cases (age, 1-72 years; M:F, 64:43) diagnosed as per WHO criteria. Of 107 cases, CD86 positive-52.3\% cases. Morphological subtypes were M0-15.0\%, M1-14.0\%, M2-43.9\%, M4$12.1 \%$, M5-11.2\%, M7-0.9\%, and AML-MD-2.8\%. Cytogenetics were available in 95 cases, of them $39 \%$ showed recurrent cytogenetic abnormality which includes KMT2A-(11.6\%), inv(16)/t(16;16)(5.3\%), RUNX1-RUNX1T1 (15.8\%), and del(5q) (6.3\%). Association of CD86 expression with morphologic subtype and recurrent-genetic abnormality was studied using Fisher-Exact test. CD86 was positively associated with AML harbouring KMT2A mutation $(p=0.02)$ and negatively associated with AML harbouring RUNX1-RUNX1T1 $(p<0.001)$. Sensitivity-84\% and specificity-58\% of CD86 in detecting AML-monocytic differentiation (M4 \& M5) with $p$ value $<0.001$. The association of AML-M5 morphology with CD86 expression with underlying KMT2A mutation was found to be very 
high $(p<0.001)$ with sensitivity-90\% and specificity-99\%. Conclusions: CD86 is expressed in half of the (non-APML) AML cases and is a very sensitive marker for diagnosis of AML-monocytic differentiation. CD86 expression with acute monocytic leukemia (AMLM5) morphology is highly specific and sensitive indicator of KMT2A mutation and this combination can be considered as a surrogate marker for KMT2A mutation.

\section{P-AL 07}

\author{
Spectrum of Leukemia Cases and Its Significance \\ to Clinicopathological Parameters for a Period of 2 Years \\ in a Tertiary Care Hospital of Upper Assam

\section{Dr. Gayotri Gogoi, Dr. Mondita Borgohain, Dr. Nitumani} \\ Khakhlari, Dr. Dimpee Lahkar", Dr. S. M Baruah
}

Email ID for Correspondence: daina59@rediffmail.com Assam Medical College and Hospital, Dibrugarh

Introduction: Leukemias are classified into two broad groups, myeloid and lymphoid, based on the origin of the leukemic stem cell clone. In AML, the leukocyte count is elevated in more than one half of patients but is $>100,000$ cells $/ \mathrm{mm}^{3}$ in $<20 \%$. Blasts usually are identified on peripheral smear; Auer rods and Phi bodies are considered pathognomonic of AML. In ALL, hyperleukocytosis, arbitrarily defined as a blood blast count $>100,000 / \mathrm{mm}^{3}$. occurs in approximately $15 \%$ of patients and may exceed $200,000 \times 10^{6} / \mathrm{L}$. Approximately one third of patients have a platelet count $<25,000 \times 10^{6} / \mathrm{L}$. In CML, the median lymphocyte count at diagnosis is $30 \times 10^{9} / \mathrm{L}$. There is a marked granulocytic leukocytosis dominated by the entire spectrum of granulocytes including rare myeloblasts, basophilia, predominance of mature neutrophils and also myelocyte bulge. Objectives: To study: 1. The spectrum of leukaemia cases for a period of two years. 2. Clinicopathological correlation of each category to various parameters like TC, HB\%, Platelet, Age, Sex, blasts count, most common clinical presentation, remission rate at discharge, blast concentration and its role to therapy. Material and Methods: All newly diagnosed cases of leukaemia that presented to our hospital from 2015 january to 2017 january were included in a retrospective study. The data of clinical presentations, duration etc along with $\mathrm{Hb}$, TC, DLC, Platelet, PBS, Bone marrow were collected. The peripheral blood was tested for various parameters by using Leishman stain. Immunophenotyping was done wherever possible. Results: A total of 146 cases presented to our department from jan 2015 to jan 2017. Out of these, 86 cases were Acute Leukemia and 60 being Chronic. Among 86 cases, 60 belonged to AML, 23 belonged to ALL and 3 belonged to MPAL. Among chronic leukemia, 55 cases belonged to CML, 5 cases to CLL. The median age of onset in AML is 31.1 years, that of ALL is 7.4 years, CML is 40.7 years and in CLL it is 64.4 years. Male to female ratio in AML is 1.4:1, in ALL IS 2.7:1, in CML is 1.6:1 and in CLL it is 4:1. Total count in AML was mostly in the range of $80,000-2.5$ lacs/cumm and in ALL it was between 3000-5000/cumm. However in chronic leukemia, except for blast crisis, total count was within normal limits. Platelet count in AML was mostly in the range of 10,000-50,000/cumm and inALL it was between 7000-15,000/cumm. In chronic leukemia, most of the cases had platelet count more than 1 lacs/cumm. The mean $\mathrm{Hb}$ level in AML is found to be $7.5 \mathrm{~g} / \mathrm{dl}$, in ALL it is $5.2 \mathrm{~g} / \mathrm{dl}$, in CML IT IS $10.5 \mathrm{~g} / \mathrm{dl}$ and in CLL it is $10 \mathrm{~g} / \mathrm{dl}$. The blasts concentration in AML mostly belonged to $35-85 \%$, in ALL it was in the range of $30-50 \%$. Most common clinical presentation in AML and ALL is fatigue and for CML being splenomegaly. Conclusions: Acute leukemias are commoner than chronic leukemia. All leukemia incidence are more in males than females. ALL is a disease of childhood, whereas AML is a disease of young adulthood. Again among chronic CML median age is mid adulthood and CLL is of elderly. The leucocytosis of AML is very pronounced with average one lakh or more in contrast to leucocytosis of ALL is absent in most cases. So diagnosios of ALL requires strong clinical suspicion and accompaniment of low platelet count. Frequent repeat testing were observed in the study due to absent of leucocytosis or absence of classic blast features.

Keywords leukemia, immunophenotyping, clinicopathological parameters

\section{P-AL 08}

Detection of Acute promyleocytic leukemia (APML) variant $R A R a$ translocation using standard dual color/ dual fusion (DC/ DF) PML-RARA FISH probe: A tale of two cases

Sipra Rani Patel, Sumanta Patel, Arun SR, Manish K Singh, Neeraj Arora, Deepak K Mishra, Jeevan Kumar, Saurabh J Bhave, Vivek S Radhakrishnan, Reena Nair, Mammen Chandy, Mayur Parihar

Introduction: APML with variant $R A R a$ translocations comprises 1-2\% of APML cases. The diagnosis of these cases by employing routine techniques such as FISH and RT-PCR targeting the $P M L$ and $R A R A$ genes, is difficult. Here, we report two cases of variant $R A R A$ translocations diagnosed by FISH and conventional karyotyping, thus highlighting an alternative strategy and the importance of atypical FISH signal patterns in conjunction with karyotyping. Objective: To illustrate the importance of atypical FISH signal patterns using standard dual colour dual fusion PML-RARA probe in detection of variant $R A R A$ translocation. Materials and methods: Karyotyping and FISH was carried out on bone marrow aspirate samples cultured overnight using standard protocols. FISH analysis was performed using PML/RARa DC/DF probe (Vysis; Abbott, Illinois) and GTG banded karyotyping was reported as per the ISCN 2013. Results: Patient I: Outside diagnosed as APML \& All-trans Retinoic Acid (ATRA) was started on morphological suspicion and referred to our centre. Patient II: Diagnosed and treated as AML and referred to our centre post 3 cycles of HIDAC from Bangladesh. Both cases were negative for RT-PCR for $P M L-R A R A$ fusion transcript. FISH analysis using DC/DF PML-RARA probe showed an atypical signal pattern ( $2 \mathrm{R} 3 \mathrm{G})$, indicating gain of the $R A R a$ gene. Karyotyping with G-banded metaphases revealed a balanced translocation between the long arms of chromosomes 11 (11q23) and 17 involving 17q21 (RARa gene) leading to the ZBTB16-RARA fusion. Conclusion: An atypical signal pattern with an extra RARA on standard dual colour dual fusion FISH analysis indicates a variant $R A R A$ gene rearrangement that can be confirmed by using Karyotyping or a RARa breakapart probe. At least seven different translocation partners of RARa gene have been described in literature with clinical and pathological findings similar 
to APML. The identification of $\mathrm{t}(11 ; 17)(\mathrm{q} 23 ; \mathrm{q} 21) / Z B T B 16-R A R A$ fusion is important as the patients harboring this translocation do not respond to ATRA based therapy, traditionally used for APML.

\section{P-AL 09}

\section{A Retrospective Study of Antifungal Prophylaxis in Patients with Acute Myeloid Leukemia}

Pinki Mishra, Narendra Agrawal, Mukul Aggarwal, Jyotsna Kapoor ${ }^{\#}$, Pallavi Mehta, Priyanka Verma, Neha Vijay, Nidhi B. Agarwal, Rayaz Ahmed, Dinesh Bhurani

Email ID for Correspondence: jkapoor022@gmail.com Rajiv Gandhi Cancer Institute and Research Centre, New Delhi, India

Introduction: Invasive fungal infections (IFIs) are common in patients with acute myeloid leukemia (AML) undergoing intensive chemotherapy. Antifungal prophylaxis (AFP) is used commonly to prevent IFI in patients receiving intensive chemotherapies. Objectives: To study the incidence of IFI (possible, probable and proven) in patients with AML undergoing chemotherapy \& on AFP. Material and Methods: We retrospectively reviewed consecutive 46 patients (newly diagnosed 41, relapsed 5) with non-M3 AML, who received induction with intensive chemotherapy using $7+3$ regime $(n=40)$, $\operatorname{HAM}(\mathrm{n}=4)$ and $\operatorname{HiDAC}(\mathrm{n}=2)$ chemotherapy and systemic antifungal prophylaxis from August 2014 till December 2016. All of the patients recruited were free of IFI at the start of therapy. Patients were monitored for occurrence of IFI using HRCT of chest or PNS and test for Galactomannan antigen on serum or BAL and were followed up for 90 days after induction. Results: Forty-six patients with mean age of 39.5 years with male predominance to $1.5: 1$ were included. Majority of the patients had intermediate/high cytogenetic risk (low $=8$, intermediate $=18$, high $=18$ including 5 patients with relapsed disease, $\mathrm{NA}=2$ ). Out of the 46 patients on intensive chemotherapies, 41, 4 and 1 patients were started on posaconazole, amphotericin B and voriconazole prophylaxis respectively. Complete remission $(\mathrm{CR})$ rates were $58.7 \%$. The incidence of possible and probable IFIs was $34.8 \%(16 / 46)$ and $8.7 \%$ (4/46) respectively with a total IFI rate of $43.5 \%$ with onset at $13^{\text {th }}$ (4-24) day. Pulmonary infection was seen in 19 while 1 patient had PNS infection. The incidence of IFI was 19/41 (46.3\%), 1/4 (25\%) and 0/1 in patients on posaconazole, amphotericin-B and voriconazole prophylaxis respectively. Thirty patients received antifungal treatment $(20=$ IFI \& $10=$ empirical fungal infection) using monotherapy $(\mathrm{n}=22)$ and double antifungal therapy $(\mathrm{n}=8)$ for a median duration of $12.5(6-$ 84) days. Antifungal monotherapy consisted of amphotericin B $(n=14)$, voriconazole $(n=4)$ and caspofungin $(n=4)$. Fungal infection resolved in $12(60 \%)$ patients, while remain unresolved in four patients (20\%) and 4 succumbed to death because of refractory disease $(n=2)$ and bacterial infection $(n=2)$. Overall mortality in the study population was $11(23.9 \%)$ (induction mortality $=5$, refractory disease $=4 \&$ bacterial infection $=2$ ). Conclusions: IFI still remains a significant cause of morbidity in patients with AML despite universal use of antifungal prophylaxis. With effective pharmacotherapy, the mortality due to IFI is preventable. Appropriate antifungal prophylaxis strategy still need to be developed through larger and prospective studies.

\section{P-AL 10}

Treatment of Adolescent and Young Adults with Newly Diagnosed Non-philadelphia Positive Acute Lymphoblastic Leukemia with Pediatric Protocol: A Single Centre Experience

Narendra Agrawal, Priyanka Verma ${ }^{\#}$, Neha Yadav, Pallavi Mehta, Rayaz Ahmed, Dinesh Bhurani

Email ID for Correspondence: drpriyankaverma11@gmail.com Rajiv Gandhi Cancer Institute and Research Centre, New Delhi, India

Introduction: Adolescent and Young Adults (AYA) patients with Acute Lymphoblastic Leukemia (ALL) have shown superior outcome on treatment with pediatric protocols as compared to the adult ALL protocols. Objectives: To study outcome, toxicities and feasibility of treating AYA ALL and Lymphoblastic Lymphoma (LBL) patients with paediatric treatment protocol. Methods: Medical records of patients with ALL and LBL between age 10 to 35 years treated at our centre with COG0232 protocol between 1-Jan-2012 to 31-Dec-2016 were reviewed. Data regarding treatment protocol, survival outcome and toxicities were analyzed. Results: A total of 234 patients were diagnosed and treated with ALL and LBL at our centre during study period. Seventy nine patients fulfilled our study criteria and were included for analysis. Out of these 79 patients, 66 were males $(83.5 \%)$ and median age at diagnosis was 20 yrs (10-35 yrs). Total no. of patients with B-ALL, T-ALL and T-LBL were 49 (62\%), 25 (31.6\%) and $5(6.3 \%)$ respectively. Four (5\%) patients were CNS-3, $2(2.5 \%)$ had CNS-2 and one patient had testicular involvement at the time of diagnosis. Cytogenetics status was available for $\mathrm{n}=54$, out of which translocation $\mathrm{t}(1: 19)$ was positive in $\mathrm{n}=4$ while $\mathrm{t}(4 ; 11)$ and $\mathrm{t}(12 ; 21)$ was positive in 1 each, 8 had hyperdiploidy $(>53)$ out of which 4 were hi-hyperdiploidy ( $>56$ ). Five patients had hypodiploidy, 1 had complex cytogenetics, 3 had random abnormal cytogenetic abnormalities while 31 had normal cytogenetics. In patients with ALL $(\mathrm{n}=74)$ Day +14 bone marrow status was available for 69 patients, $52(75.3 \%)$ were in $\mathrm{M} 1$ while 8 patients $(11.5 \%)$ each were in $\mathrm{M} 2$ and M3. At the end of induction $57(77.0 \%)$ patients were in remission (Rapid early responder $=51 \&$ slow early responder $=6$ ). Sixteen patients $(21.6 \%)$ were induction failure. One patient died during induction due to fungal pneumonia. In patients with $\operatorname{LBL}(\mathrm{n}=5)$ at end of induction, $3(60 \%)$ were in remission and remaining $2(40 \%)$ were induction failure. Out of 60 patients who achieved remission, 38 $(63.3 \%)$ were in remission and are on regular follow-up and 14 $(23.3 \%)$ patients had relapsed $(n=10 \mathrm{BM} ; \mathrm{n}=2 \mathrm{CNS} ; \mathrm{n}=1$ $\mathrm{BM}+\mathrm{CNS}$ and $\mathrm{n}=1$ Extramedullary). 5 year Event free survival (EFS) of the cohort was $57.1 \%$ and overall survival (OS) was $68.6 \%$ at the median follow-up of 5 yrs. On comparing patients with age upto 19 yrs $(\mathrm{n}=33)$ with those with age between 20-35yrs $(\mathrm{n}=37)$, the EFS is $62.5 \%$ and $46.3 \%(\mathrm{p}=0.128)$ and OS was $69.3 \%$ and $52.5 \%$ $(\mathrm{p}=0.132)$ respectively. Two patients died due to treatment related toxicity 1 died due to sepsis while one developed secondary HLH, 9 (13.1\%) patients abandoned therapy. Conclusions: Treatment of ALL in AYA with pediatric protocols has shown improved survival and manageable toxicity in our centre. 


\section{P-AL 11}

\section{RBVD as Salvage for Refractory/Relapsed CD20+ VE Adult B-Cell Acute Lymphoblastic Leukemia (B-ALL)}

Objectives: To find a highly effective and less toxic salvage regimen. Adult B-ALL carries an inferior prognosis compared to pediatric and those who are CD20 positive and failed with standard chemotherapy, remains as nightmare for the clinicians. We tried to overcome this dismal situation making a salvage regimen avoiding toxic chemo agents by using immunological agents. Materials and Methods: Design of the protocol was RBVD (Rituximab $375 \mathrm{mg} / \mathrm{m} 2$ day1, Bortezomib $2 \mathrm{mg}$ IV or SC on day 1, 4, 8 and 11, Vincristine $1.4 \mathrm{mg} /$ $\mathrm{m} 2$ (maximum $2 \mathrm{mg}$ ) weekly and Dexamethasone $9 \mathrm{mg} / \mathrm{m} 2$ day 1-14). CASE -I: 26 years male without sibling, diagnosed as CD20+ VE B-ALL and treated with Hyper-CVAD, failed to show response, then given FLAG-Ida $\times$ two courses without any remission. Subsequently was put on palliative protocol. The boy had very poor PS (ECOG -III) as he had severe muscle wasting and remain bedridden. We rechecked and confirmed his diagnosis and started RBVD as per protocol. He achieved complete remission (CR) after $1^{\text {st }}$ cycle. CASE-II: 25 years female, a medical student after diagnosis, given standard chemotherapy and subsequently underwent allogeneic stem cell transplant from her full matched sibling with $\mathrm{Bu} / \mathrm{Cy}$ conditioning but relapsed after three months of transplant. She was given maintenance like chemotherapy with rituximab but did not achieve remission. She developed platelet refractoriness, became cushingoid with clear evidence of disease as shown circulating blasts. We offered her RBVD and after $1^{\text {st }}$ cycle, she also went to CR with initial two occasions of platelet transfusion. Result: Both patients underwent CR after the $1^{\text {st }}$ cycle of RBVD. Conclusion: Both these cases are very difficult and practically was not fit for further chemotherapy. Surprisingly both of them tolerated this salvage regimen and achieved CR without toxicities. This result needs to be validated by randomized multicenter trials.

\section{P-AL 12}

\section{Acute Panmyelosis with Myelofibrosis Versus Acute Megakaryoblastic Leukemia -Diagnostic Challenges}

\section{Narasimhapriyan K ${ }^{\#}$, Prasath S, Sree Rekha J, Jacob SE, Kar R, Basu D}

Email ID for Correspondence: knarasimhapriyan@gmail.com Jawaharlal Institute of Postgraduate Medical Education and Research, Pondicherry

Introduction: Acute panmyelosis with myelofibrosis (APMF) and Acute megakaryoblastic leukemia (AMKL) are classified under Acute Myeloid Leukemia-NOS in the current WHO classification. Although the criteria for diagnosing APMF and AMKL are somewhat distinct, there are clinical and morphological overlaps between the two. A careful assessment of bone marrow histomorphology aided by immunohistochemistry (IHC) is required for the diagnosis. Objectives: To compare the clinico-pathological and immunohistochemical profile of APMF and AMKL and to differentiate these two entities. Material and Methods: Cases diagnosed as APMF and AMKL in our institute in the last five years were retrieved from the archives. Clinical and haematological features along with bone marrow aspiration and biopsy findings were studied. IHC done in the bone marrow biopsies included CD34, CD61, CD 117, MPO, TdT, e- Cadherin, CD3 and CD20. Flow cytometric (FCM) correlation was done, wherever available. Results: There were seven cases of APMF and nine of AMKL with a median age of 31 and 32 years respectively. Splenomegaly was observed in 4 patients of AMKL while none in APMF. Pancytopenia was more often seen in APMF than AMKL (90\% vs $45 \%$ ). Normal platelet count with dysmorphic platelets was seen in 3 patients of AMKL. Median peripheral blast percentage was $<5 \%$ in both. In APMF, bone marrow aspirates were unsatisfactory in all. Biopsies were fibrotic with panmyelosis, foci of immature cells of erythroid, myeloid and megakaryocytes were seen. Prominent megakaryocytic dysplasia was noted. The blasts showed variable positivity for E-cadherin, CD34, MPO and CD61. Flow cytometry were available for two patients and were reported as AMLM1 and differential of AML and MDS with excess blasts in another owing to underrepresentation of blasts due to hemodilution. In AMKL the marrow was fibrotic in all the patients with prominent dysmorphic megakaryocytes and clusters of blasts. The blasts were predominantly positive for CD 61. FCM, done in 3 patients, was inadequate, discordant and AML- M1 respectively. Conclusions: Although APMF and AMKL have few overlapping features like marrow fibrosis, APMF has a more heterogenous cellular composition with a good representation of erythroid and myeloid precursors along with dysplastic megakaryocytes in contrast to AMKL which shows dyspoiesis and blasts only of megakaryocytic lineage. As FCM is often nonrepresentative, detailed marrow histomorphology coupled with IHC is essential to clinch the diagnoses of these two rare types of AML.

\section{P-AL 13}

Treatment Outcome of Acute Promyelocytic Leukemia Experience from a Tertiary Care Cent

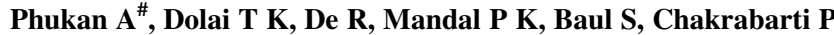

Email ID for Correspondence: abhijitphukan4747@gmail.com Nil Ratan Sircar Medical College \& Hospital

Introduction: Acute promyelocytic leukemia (APL) is one of the potentially curable human malignancies with unique clinical, cytogenetic, and molecular features. The reported data on APL is very limited from developing countries like India. From our registry data, the treatment outcome of newly diagnosed and relapsed APL patients from our centre is presented. Objectives: To study the response rate and treatment outcome APL in various risk groups. To study the relapse rate and their treatment outcome. Material and Methods: It is retrospective observational study which was conducted over a period of 5 years from Sept 2012 to Aug 2017. A total of 45 patients were included in our study. Bone marrow aspiration (BMA), Immunophenotyping (IPT), Cytogenetics and Fluorescent in situ hybridization (FISH) for PML RARA by RT PCR and was done in each and every cases and patients were treated according to the risk group. Relapsed APL cases were treated with ATRA + ATO protocol. Results: Mean age of this cohort was 26.8 years (range $=7$ to 65 years) with male female ratio was $1.7: 1$. No of cases in the low, intermediate and high risk group was $3(6.7 \%), 27(60.0 \%)$ and 19 $(42.3 \%)$ respectively. Out of total 45 patients, $36(80.0 \%)$ patients achieved first remission. Mortality during induction $17.7 \%(\mathrm{n}=8), 3$ in intermediate and 5 in high risk group respectively. In this cohort 25 (5 5.5\%) patients has successfully completed their therapy and 11 $(24.4 \%)$ patients are still on therapy. One patient $(2.2 \%)$ was lost to follow up. In this cohort $6(13.3 \%)$ patients had relapsed (Intermediate-2, High risk-4) of which $3(50 \%)$ patient went into $2^{\text {nd }}$ remission with ATRA + ATO protocol, 1 expired during induction and 2 patients expired before initiation of treatment. Conclusions: Although APL is a highly curable malignancy, mortality during induction is still high. The results very much comparable to those 
published in various literatures. The outcome could be further improved with early diagnosis and more efficient supportive care system.

\section{P-AL 14}

Immunophenotypic and Cytogenetic Profile in Paediatric Acute Leukemia: Experience from a Tertiary Care Hospital of South India

Pramitha M, Sridevi HB, Harsha Prasada L, Pooja K Suresh, Saraswathy Sreeram, Beshwanth Chowdhary

Introduction: Advanced diagnostic modalities in acute leukaemia (AL), have not only contributed in understanding of the complex association between morphology, immunophenotyping (IPT), and specific cytogenetic abnormalities but also has led to the development of personalised treatment modalities based upon specific genetic defects. IPT and cytogenetics also aid in monitoring responses to treatment such as detection of minimal residual disease; and for establishing a baseline against which future studies can be judged to assess disease progression. We intended to study the clinicopathological, immunophenotypic and cytogenetic profile of paediatric ALs diagnosed in our setup.

\section{Objectives:}

1. To correlate the morphological features associated with various immunophenotypic expression in pediatric acute leukemia.

2. To study the cytogenetic abnormalities in various types of pediatric acute leukemias and to correlate with the morphological and immunophenotypic expression.

3. To study the various prognostic markers in pediatric leukemias.

Material \& methods: Paediatric ALs (0 days-18 years) were analysed from January2016 to August2017 in the Haematopathology department of a tertiary care centre of South India. Clinical details along with complete blood count, peripheral smear, bone marrow, flow cytometry and cytogenetic findings were collected and pathological variables were correlated. Results: During the study period, paediatric AL constituted for 63 cases. Mean-age of presentation was 7.6 years (Range: 5 months-18 years) with $\mathrm{M}: \mathrm{F}$ ratio of 1.4:1. Infantile leukemia constituted for $9.5 \%(6 / 63$; M:F $=2: 1)$. Previous history of Down syndrome (4 cases) and Myelodysplastic syndrome ( 2 cases) was also noted. There were B-Acute Lymphoblastic Leukemia (ALL) (36 cases), T-ALL (7 cases), Acute Myeloid Leukemia (AML) with recurrent genetic abnormality (9 cases) and AML-NOS (11 cases). Cytogenetic evaluation done in 46/63 cases revealed normal karyotype in 13 cases. Hyperploidy was consistently seen only in B-ALL ( 7 cases); somatic trisomy-21 in 4 cases (AML-M7-3 cases; B-ALL-1 case); RARA translocation in 4 cases of PML [t(15;17)-3 cases; $\mathrm{t}(1 ; 17)-1$ case]; $\mathrm{t}(8,21)$ in 2 cases (AML-M2); monosomy 7 in 2 cases (AML-NOS and MDS-AML); $\mathrm{t}(9,22)$ in a case of B-ALL; inversion-16 in a case of AML-M4Eo; MLL gene abnormality in 4 cases. The study revealed specific morphological features associated with various cytogenetic abnormalities. Mortality rate was $12.9 \%$ [PML(1), B-ALL(3), T-ALL(1), AML-M4(3)]. Conclusion: Our study showed that specific cytogenetic abnormalities are associated with various subtypes of AL in children and some of these have unique morphology and IPT expression. Identification of such characteristics has direct impact on the risk stratification, choice of treatment modality, prognosis and survival rate.

\section{P-AL 15}

Clinicopathological Spectrum of T-Cell Acute Lymphoblastic Leukemia with Immunophenotypic Correlation - An Experience from a Tertiary Care Center

Dr. Arunima Deb ${ }^{\#}$, Dr. Ranjitha Rao, Mohammad Muiz, Dr. Sharada Rai, Dr. Prashantha B, Dr. Sridevi HB, Dr. Deepa Adiga

Email ID for Correspondence: arunimadeb91@gmail.com Kasturba Medical College, Mangalore

Introduction: $\mathrm{T}$ cell acute lymphoblastic leukemia (T-ALL) comprises of about $15 \%$ of childhood and $25 \%$ of adult ALL. Because of its rarity as compared to B-ALL extensive studies on this disease are seldom reported in the literature especially in the southern part of the India. Aims: To study the clinical, haematopathological and immunophenotypic features of T-ALL.

Objectives:

1. To correlate the clinical features and complete blood count in cases of T-ALL.

2. To correlate the morphological and immunophenotypic features in T-ALL.

3. To study the cytogenetic abnormalities associated with T-ALL.

4. To correlate the treatment response and prognostic factors in T-ALL.

Materials and methods: A retrospective study of all the T-ALL diagnosed between August 2014 and August 2017 in a tertiary care center of south India was conducted. The clinical features, complete blood count, morphological findings, immunophenotypic expression and cytogenetic abnormalities along with treatment response were analyzed. Results: T-ALL constituted for $10.8 \%$ (17 cases) of acute leukemia during the study period. Equal distribution was seen both in childhood and in adults and there was a male preponderance (M:F::4.3:1). Most patients (60\%) presented with symptoms related to infection such as ear infection (41.2\%) and pneumonia (35.3\%) and unusual presentations like neutropenic enterocolitis (5.9\%). Bleeding was uncommon $(11.8 \%)$ but lymphadenopathy (33\%), splenomegaly $(26.6 \%)$ and hepatomegaly $(13.3 \%)$ were commonly noted. The immunophenotypic analysis showed a consistent bright expression of CD7 in 100\% cases and bright to moderate expression of cytoplasmic CD3 in $92.3 \%$ cases. Surface CD3 expression was variable with $23.1 \%$ cases showing no expression. T-ALL were further divided into Pre-T-ALL (30.8\%), cortical T-ALL (15.4\%), and medullary T-ALL (46.2\%) immunophenotypically. There was no case of Pro-T-ALL (Early Precursor T-ALL-EPTL). Aberrant expression were noted with CD10 (23.1\%) being the commonest and others such as CD13 (15.9\%), CD33 (15.9\%), CD79a (7.6\%) and CD11b (7.6\%). Cytogenetic evaluation done in $7 / 17$ cases revealed normal karyotype in all. Mortality rate was $35.3 \%$ cases with sepsis being the cause of death during the early course of therapy. Conclusion: T-ALL is a rare hematologic malignancy with varied clinical presentation that depends on the severity of cytopenias. Diagnosis by immunophenotypic analysis is necessary which also aids in identifying the various stages of T-ALL as EPTL has worse prognosis. Early diagnosis and 
effective chemotherapeutic regimens with good supportive care can improve the outcome of these patients.

\section{P-AL 16}

\section{Acute Myeloid Leukemia (AML) with Mastocytosis: A Case Series of a Rare Entity from a Tertiary Care Hospital}

\section{Sabbavarapu Padmasree\#, T Roshni Paul, Shantveer G Uppin, Megha S Uppin, G Sadashivudu}

Email ID for Correspondence: spaddu2012@gmail.com Nizam's Institute of Medical Sciences (NIMS), Hyderabad, India

Introduction: Systemic mastocytosis with an associated hematological neoplasm (SM-AHN) is a variant of Systemic mastocytosis and is a rare entity. The hematological disorders may vary from myeloid neoplasms to non-Hodgkin's Lymphoma. A thorough bone marrow evaluation is required to identify mastocytosis as it may be masked at initial diagnosis. In such cases post-chemotherapy bone marrow shows variable blast percentage with persistence of mast cells. Cases associated with AML $t(8 ; 21)$ present with frequent relapses and show poor prognosis. Our case series is being presented because of its rarity, and to bring awareness that these patients do not do well with conventional chemotherapy. Objectives: The aim was to study the clinicopathological profile of AML cases associated with marrow mastocytosis. Material and Methods: It is a retrospective study from January 2005 to August 2017. Patients diagnosed as AML with marrow mastocytosis either at initial diagnosis or at relapse were included in the study. The clinical details were obtained from the patient case files. Giemsa stained peripheral blood smears and bone marrow aspirate smears were reviewed. Blast and mast cell counts were done on marrow aspirates for 500 nucleated cells. Cytochemistry with SBB and PAS were reviewed. Selected samples were submitted for immunophenotyping and cytogenetics. Results: During the study period, 9 such cases were diagnosed. The ages ranged from 7-65 years with a median age of 28 years and a M: F ratio of 1:1.25. These included 1 child ( 7 years) and 2 adolescents ( 16 and 18 years). Out of the 9 cases, there were 4 cases which were diagnosed as AML with mastocytosis at initial presentation and subsequent aspirates showed remission/relapse with persistent mastocytosis. The remaining cases were initially diagnosed at other hospitals and had their follow-up procedures at our hospital. The fresh cases presented with fever, signs and symptoms of anemia (4/4) and one of them had bleeding tendency and splenomegaly. The cases were diagnosed as AML-M2 by morphology and cytochemistry. Immunophenotyping was done in 1 case and was confirmed as AML. In 2 cases, cytogenetic analysis revealed $t(8: 21)$. The $\mathrm{BM}$ aspirates showed $>20 \%$ blasts along with mastocytosis in freshly diagnosed cases. All the follow-up cases showed varying number of blasts with persistent mastocytosis. Two cases showed mast cells admixed with plasma cells and eosinophils. One case had nodules of spindle-shaped mast cells. With cycles of chemotherapy, the blast percentages reduced but the prominence of mast cells persisted. Most of these cases had repeated relapses. Conclusions: SM associated with t $(8 ; 21)$ AML is extremely rare and carries a bad prognosis. Care should be taken to look for coexisting mastocytosis at the time of initial diagnosis and follow up, since the mast cell infiltrate may be subtle and masked. Diagnosis of AML with mastocytosis helps in assessing the treatment strategy and there should be an early consideration of Haematopoetic Stem Cell Transplantation (HSCT) in cases of SM associated with $\mathrm{t}(8 ; 21)$ AML.

\section{P-AL 17}

Arthritis and Bilateral Nephromegaly: A Rare Presentation of Acute Lymphoblastic Leukaemia

\section{Sudarshan Pandit ${ }^{\#}$, Vrinda Kulkarni, Amit Bhandare,} Mayur Mundhe

Email ID for Correspondence: sudarshanpandit21@gmail.com Department of Haematology, Topiwala National Medical College and b.y.1. Nair Charitable Hospital, Mumbai

Introduction: Acute lymphoblastic leukaemia is the most common malignancy in children. It account for $30 \%$ of all cancer diagnosed in children younger than 15 years. Leukemic infiltration in acute lymphoblastic leukaemia is most frequently seen in bone marrow, spleen, lymph nodes, and liver. Extramedullary involvement of the kidneys is uncommon finding at diagnosis. There are only a few reports of children with joint arthritis and palpable renal enlargement at initial presentation. We report a rare case of young girl who presented with unexplained bilateral renal enlargement and with large joint arthritis as an initial manifestation of acute lymphoblastic leukaemia. Case Report: A 13-year-old female was Symptomatic with Fever since 30 days on and off; abdominal pain; epistaxis; Pain and swelling in large joints of both hands and feet since 15 days. Pallor was present. There was a hepatomegaly with Splenomegaly and Kidneys are ballotable bilaterally. There was also swelling over bilateral (elbow, knee, ankle) joints. Routine blood picture showing pancytopenia. Peripheral Blood Smear Findings suggestive of pancytopenia with no abnormal cells or blast. USG local part (Elbow, Ankle, Knee joints bilateral) Suggestive of Small non tapable effusions in multiple joints. USG Abdomen and Pelvis suggestive of bilateral bulky kidney disease with hepatosplenomegaly. CT Abdomen and Thorax findings consistent with USG findings. Patient evaluated for arthritis but all her reports came negative. Finally Bone marrow Aspiration and biopsy and flow cytometry report came suggestive of Pre B cell ALL. Pt. was started as per BFM-90 modified protocol for chemotherapy. Discussion: The onset of the disease acute lymphoblastic leukaemia can be lengthy with a prodromal stage lasting for weeks, or even months. Clinically palpable renal enlargement occurs only in $2 \%-5 \%$ of cases. Renal enlargement in a case of leukaemia may be due to leukemic infiltration or simple hypertrophy or hyperplasia of cells. Joint involvement as an initial presentation of acute leukaemia in children has been described and about $15 \%$ to $30 \%$ of ALL patients manifest with osteoarthritic symptoms, some of which may mimic juvenile idiopathic arthritis. Conclusions: Bilateral enlargement of kidneys and arthritis should raise the suspicion of acute leukaemia and the patient should be subjected to bone marrow examination before ordering for other invasive tests. 


\section{P-AL 18}

Incidence and Prognostic Impact Of DNMT3A Mutations in Acute Myeloid Leukemia

Arun Kumar $A^{\#}$, Sathya M, Nancy Beryl Janet, Kavitha M. Lakshmi, Uday Kulkarni, Anup Devasia, Anu Korula, Nisham P.N, Aby Abraham, Biju George, Alok Srivastava, Vikram Mathews, Poonkuzhali Balasubramanian

Email ID for Correspondence: arun000_kumar@yahoo.com Christian Medical College, Vellore

Introduction: Acute myeloid leukemia (AML) is a complex multifactorial disease caused by mutations, deregulated gene expression and epigenetic modifications of genes leading to an increased proliferation and decreased differentiation of progenitor cells. Advent of whole-genome sequencing has led to the identification of a new class (class III/epigenetic regulators) of mutations in AML especially in AML patients with normal karyotyping (AML-NK). DNMT3A, an epigenetic regulator, is a de-novo methyl transferase that is involved in methylation of cytosine bases of transcription factors like GATA1, RUNX1. Studies on DNMT3A mutations have yielded conflicting results and there is no clear data on the incidence of these mutations in Indian population. Objectives: To study the frequency and impact of DNMT3A mutations in AML patients. Material and Methods: Samples from 284 AML patients with no favourable structural chromosomal abnormalities on karyotyping/FISH analysis were analysed retrospectively. Mutation analysis targeting the hotspot exon 23 (codon 882) of DNMT3A (60-70\% of DNMT3A mutations) was done by Sanger sequencing. FLT3-ITD \& NPM1, IDH1 \& IDH 2 mutations were assessed by multiplex fluorescent gene scan analysis and Sanger sequencing. Clinical details such as patient's age, blast count, AML morphological subclass, and relapse/survival status were collected from the hospital records. Statistical analysis was done with SPSS software. Results: DNMT3A mutations were seen in $8.8 \%$ and $12 \%$ of AML and AML-NK patients respectively. Mutations were more common in older individuals [median age for DNMT3 $\mathrm{A}^{\mathrm{MUT}}$ vs. $\mathrm{DNMT} \mathrm{A}^{\mathrm{WILD}}=43.5$ vs. 38 years; $\left.\mathrm{p}=0.02\right]$ and were associated with higher blast and lower CD34 counts. DNMT3A mutations were more common in patients with NPM1 $(\mathrm{p}<0.0001)$ and/or FLT3-ITD $(\mathrm{p}=0.0003)$ mutations and was morphologically associated with monocyte differentiation (FAB-AML M4/M5; $\mathrm{p}<0.0001$ ). DNMT3A mutations were associated with a difference in overall survival in AML-NK [DNMT3A ${ }^{\text {MUT }}$ vs. DNMT3A ${ }^{\text {WILD }}=37.6 \%$ vs. $50 \%$ ] and the difference was more pronounced in NPM1 ${ }^{\text {MUT }}$ AMLNK patients especially the good molecular risk (NPM1 ${ }^{\text {MUT }}$ FLT3 ${ }^{\text {WILD }}$ ) AML-NK patients $[\mathrm{p}=0.049(\mathrm{OS})$ and EFS $\mathrm{p}=0.043$ (EFS)]. Cox regression analysis showed high hazard ratio (HR) for both OS (1.603) and EFS (1.302) and DNMT3A mutations were second only to age indicating the poor prognosis associated with this mutation. Conclusions: This study shows that DNMT3A mutations are common in normal karyotype AML patients and are associated with a poor prognosis. Screening for these mutations helps to identify a subset of intermediate risk AML patients who have a poor prognosis and hence look for alternate/intensified treatment plans and newer strategies like hypomethylating agents.

\section{P-AL 19}

Therapy-related AML or AML as Second Primary Neoplasm: A Dilemma in a Treated Case of Retinoblastoma

\section{Dr. Abhirup Sarkar", Dr. Aparna Ningombam,}

Dr. Raghavendra Lingaiah

Email ID for Correspondence: abhirupsa@gmail.com All India Institute of Medical Sciences, New Delhi

Introduction: Retinoblastomas carry a significant risk of second nonocular malignancies. Third, fourth and fifth non-ocular malignancies have been described. Soft tissue and osteosarcomas occur at significantly higher rates whilst second primary leukemias are quite rare. The management of retinoblastoma necessitates the use of chemotherapeutic agents including Topoisomerase inhibitors and Alkylating agents, which increases the risk of therapy-related AML. Case Report: We report a case of a 5-year-old female child, who at the age of 3 years was diagnosed with retinoblastoma of the left eye. The child had no family history of retinoblastoma. Enucleation showed extensive choroidal and extra-scleral invasion (stage pT3). Following surgery, patient received 12 cycles of chemotherapy comprising intravenous vincristine, carboplatin and etoposide at 4-week intervals. The cumulative dose of etoposide was around $3000 \mathrm{mg} / \mathrm{m}^{2}$. Following 1 year 8 months after completion of chemotherapy, she was admitted with complaints of fever and bleeding from the surgical site. A routine peripheral blood smear revealed leukocytosis with $90 \%$ blasts showing increased nucleocytoplasmic ratio, fine chromatin, prominent nucleoli and numerous dysplastic features like nuclear blebbing, detached nuclear fragments and pseudo-Chédiak-Higashi granules. Immunophenotyping showed CD 45+, CD33+, CD117dim+, CD64+, CD4dim+, CD9+, MPO-, CD34-, HLA-DR-, CD13-, CD65-, CD25-, CD15-, CD11b-, CD7-, CD14-, CD19-, CD56-. NSE was positive. The AML was typed as M5 monocytic. The child developed tumor lysis syndrome and succumbed to the illness within two weeks of diagnosis. The child most probably had therapy-related AML. Cytogenetic study, which is confirmatory, could not be done due to economic constraints. Conclusion: AML as a second primary neoplasm in retinoblastoma is very rare. Chemotherapy-treated retinoblastoma patients are at increased risk of AML and usually they have a poor prognosis.

\section{P-AL 20}

Lineage Infidelity in Acute Undifferentiated Leukemia Difficulties In Diagnosis

Geeta Yadav ${ }^{1}$, Rashmi Kushwaha ${ }^{1}$, Mili Jain ${ }^{1}$, Ashutosh Kumar ${ }^{1}$, Madhumati Goel $^{1}$, Shailendra Prasad Verma ${ }^{2}$

${ }^{1}$ Department of Pathology, King George's Medical University, Lucknow-226003, U.P. India; ${ }^{2}$ Department of Hematology, King George's Medical University, Lucknow

Objective: Acute undifferentiated leukemia (AUL) is a rare subtype of acute leukemia (AL) which does not show specific morphology or lineage specific markers on immunophenotyping. AUL is difficult to 
diagnose and has poor treatment outcome. The aim of study was to analyze specific features of AUL. Materials \& methods: We retrospectively evaluated clinical details, hematological parameters, bone marrow aspirates, flow cytometry data, molecular analysis and treatment details of 6 cases of AUL from January 2016 to September 2017. Results: 6 cases of AUL were identified among 647 cases of AL diagnosed during this period. $\mathrm{M}: \mathrm{F}=4: 2$, Median age $=19$ years (range $=12-25$ years). Morphologically 4 cases showed large sized blasts with round nuclei, opened up chromatin, 1-2 prominent nucleoli and moderate agranular cytoplasm. 2 cases showed small to medium size blasts with clumped chromatin, inconspicuous nucleoli and scant cytoplasm. All cases were negative for cytochemical MPO and PAS. All 6 cases showed expression of at least one myeloid marker (CD13/CD33) and one early T cell marker (CD7/CD5/CD2) along with CD34 \& HLADR expression. None of them showed expression of lineage specific marker for myeloid origin (cytoplasmic myeloperoxidase (cMPO)) or $\mathrm{T}$ cell origin (cCD3). 2 patients were treated on ALL (BFM95) protocol and are in remission. 1 patient treated with AML protocol $(3+7$ regimen $)$ expired during induction. Conclusion: AUL represents a distinct stem cell leukemia. It is necessary to perform immunophenotyping with a comprehensive panel of monoclonal antibodies and molecular analysis of lineage markers before assigning a diagnosis of AUL.

\section{P-AL 21}

\section{Study of CD123 \& CD34 Expression in Acute Leukemias}

\section{Kumar A\#, Dr. Dawson L, Dr. Aggarwal M, Dr. Dhawan I}

Email ID for Correspondence: aman177ks@gmail.com Vardhman Mahavir Medical College \& Safdarjung Hospital, New Delhi

Introduction: Acute leukemia is a clonal expansion of tumour cells in blood or bone marrow. Antigenic expression on leukemic cells helps not only to diagnose \& prognosticate, but are useful predictive markers and potential therapeutic targets. CD123 is an IL3 receptor, which regulate the function \& production of hematopoietic cells \& its expression is also observed in repopulating stem cells. Antibodies to CD123 have been shown to have promising antitumor activity. CD34 is the commonest marker whose expression is sought on blasts as stem cell marker \& prognostic indicator. Objectives: To study the expression of aforesaid markers in acute leukemias \& to see if there is any correlation in their expression. Material and Methods: This study was conducted at the Department of Pathology, Vardhman Mahavir Medical College \& Safdarjung Hospital, New Delhi. This was a prospective observational study conducted over a period of one year. Thirty five newly diagnosed cases of acute leukemias were included in this study. Immunophenotyping was done on flow cytometer (CYTOMICS FC 500). In addition to specific diagnostic panels, CD34 \& CD123 were put up in all the cases. Results: The study group of 35 cases comprises of 9 cases of TALL, 11 cases of BALL \& 15 cases of AML. CD123 expression was seen in 6 cases of TALL $(66 \%), 8$ cases of BALL $(72 \%) \&$ in all 15 cases of AML $(100 \%)$. CD34 expression was seen in 6 cases of TALL $(66 \%), 11$ cases of BALL (100\%) \& 7 cases of AML (47\%). The range of percentage positivity for CD123 \& CD34, in TALL varies from $1 \%$ $70 \%$ \& 3\%-66\% respectively; in BALL varies from $11 \%-90 \%$ \& 5\%$82 \%$ respectively; in AML varies from 60\%-99\% \& 9\%-60\% respectively. Conclusions: The percentage expression of CD123 is very high in AML compared with other subtypes (T \&BALL) \& its detection in AML appears to be inevitable. However, there is no precise correlation with CD34 expression.

\section{P-AL 22}

Predominance of Hypogranular Variant of Acute Promyelocytic Leukemia in the Himalayan Region: An Unusual Observation

\section{Dr. Richa Sharma\#, Dr. Vibha Gupta, Dr. Kunal Das,} Dr Vikas Shrivastava, Dr. Mansi Kala

Email ID for Correspondence: ria.291401@gmail.com Himalayan Institute of Medical Sciences

Introduction: Acute promyelocytic leukemia (APL) is characterized by, $t(15 ; 17)$, life-threatening coagulopathy and unique sensitivity to all-trans-retinoic acid (ATRA) and arsenicals. Limited data is reported about APL from developing countries. Objectives: To observe the incidence of APL in the Himalayan region and patterns of presentations and treatment response in all the cases of APL. Material and Methods: In this retrospective study, we reviewed APL cases at our centre during the time period from June 2015 to March 2017. Physical examination and basic investigation as $\mathrm{CBC}$, coagulation profile, general blood picture with bone marrow examination and flow cytometry was done wherever possible. The diagnosis was confirmed by cytogenetics, RT-PCR analysis and flow cytometry. Treatment protocol was pathema protocol. Results: Out of 13 patients diagnosed, 6 underwent treatment and 4 patients expired before the initiation of treatment. Majority of the patients were between $20-$ 50 years. Male female ratio was 1:4.5. We observed hypogranular variants $(69.2 \%)$ were more compared to classical Acute Promyelocytic Leukemia (30.8\%). There was no significant difference between the clinical and laboratory profiles of both the groups. Patients who took treatment ( 4 with pathema protocol, 1 with modified pathema protocol and 1 with Arsenic Trioxide) achieved remission. Conclusions: The incidence of APL in Sub Himalayan area is high with hypogranular form of APL more as compared to classical form. Mean age of presentation was 36 years with $100 \%$ pediatric cases as hypogranular variant. Most common presentation was fever in both types of APL. Amongst 9 cases of the hypogranular variant 6 received treatment of which 5 survived and 1 expired. 3 patients expired before getting any treatment. Amongst the 4 patients with classical form, 3 patient expired without getting any treatment and one survived. The incidence of APL is high in the himalayan region and it's prompt diagnosis and treatment is important as it a leukemia with excellent prognosis.

\section{P-AL 23}

Clinicohematological profile of patients with subleukemic leukemia, a 5 year study

\section{Dr. Kirti Balhara\# ${ }^{\#}$ Dr. Richa Gupta}

Email ID for Correspondence: kirtibalhara21@gmail.com Maulana Azad Medical College, Department of Pathology

Introduction: Subleukemic leukemia is characterised by presence of more than $20 \%$ blasts in peripheral blood or bone marrow in the absence of an elevated total leucocyte count. Some of these cases may offer a diagnostic challenge especially if the blasts are very few on an initial PBS examination. The diagnosis is further supported by bone marrow examination and flow cytometry which should be carried out in all such cases. Aim and objective: The aim of the present study is to study the clinicopathological parameters, haematological and flow cytometry characteristics of such cases. Material and methods: Retrospective hematology and flow cytometry data was collected and evaluated over a period of 5 years (2013-2017) in a tertiary care 
centre. A total of 170 cases were analysed. Result and conclusion: The incidence of subleukemic leukemia in the present study was found to be $9.41 \%$. The mean age of the presentation was 23 years. Majority of patients came with vague complaints like generalised weakness (75\% cases), followed by fever, abdominal lump, bleeding gums, paraparaesis, and intractable hypotension. On physical examination, all these patients had pallor and around half of them had hepatosplenomegaly $(56.25 \%)$. Lymphadenopathy was found in $31.25 \%$ cases. Peripheral blood smear examination revealed Pancytopenia in $62.5 \%$ cases. The percentage of the blast varied from $0-79 \%$, with a TLC ranging from $360-3900$ cells $/ \mathrm{mm}^{3}$. $69 \%$ cases had blasts less than $20 \%$ in peripheral blood and $37 \%$ cases had very occasional blasts $(<5 \%)$. The bone marrow aspirate was hypercellular in all the cases thus ruling out hypoplastic Acute leukemia. Maximum cases were B cell ALL (68.75\%), followed by AML $(31.25 \%)$ No case of T cell ALL was found to be subleukemic. The present study highlights the importance of cautious screening of peripheral smears in cases presenting with vague complaints and showing pancytopenia or leucopenia. In the event of finding a few atypical cells, a prompt bone marrow examination and flow cytometric analysis should be carried out in all such cases.

\section{P-AL 24}

Patterns of Presentation in Childhood Acute Leukaemia Experience from a Single Tertiary Care Hospital

\section{Sen A $A^{\#}$, Baul SN, Dutta B, De R, Dutta S, Mandal PK, Dolai TK,} Chakrabarti $\mathbf{P}$

Email ID for Correspondence: dr.ankitasen@yahoo.in NRS Medical College

Introduction: Worldwide, acute leukaemia, chiefly Acute Lymphoblastic Leukaemia (ALL) is the commonest cancer in childhood. However, there have been no significant studies in the eastern part of India studying the presentation of childhood acute leukaemia. Exploring incidence patterns of different subtypes of leukaemia according to sex and age may generate new etiologic hypotheses and identify risk groups for further study, helping towards prevention of the disease.

\section{Objectives:}

- To study the pattern of childhood acute leukaemia among patients attending our tertiary care centre

- To study the dropout rate among children diagnosed with acute leukaemia prior to subtyping

Material and Methods: Retrospective analysis from an ongoing registry data of childhood acute leukaemia patients conducted at NRS Medical College, Kolkata, for 31/2 years from 2014- August, 2017. The cut off age was upto 18 years. All patients were assessed in detail by standard clinical methods, complete blood count, peripheral blood film, bone marrow aspiration \& biopsy, karyotyping and immunophenotyping (IPT). Results: Out of the total number of children who attended OPD, 672 were diagnosed as acute leukaemia, the majority, i.e. 427 , being male (M) $(63.5 \%)$. 28 children $(4.2 \%)$ belonged to the $\leq 1$ year group, with $17 \mathrm{M}(60.7 \%)$ and $11 \mathrm{~F}(39.3 \%)$. 326 children were $1-10$ year olds $(48.5 \%)$, with $209 \mathrm{M}(64.1 \%)$ and $117 \mathrm{~F}(35.8 \%)$, while 318 were $>10-18$ years of age $(47.3 \%)$, out of whom 201 were $\mathrm{M}(63.2 \%)$ and $117 \mathrm{~F}(36.8 \%)$. The most common presenting complaints were fever:in $449(66.8 \%)$ children, pallor:in $264(39.2 \%)$, bleeding manifestations:in $79(11.7 \%)$, bone pain:in 96 (14.3\%), neck swellings:in $97(14.4 \%)$ and less common presentations, such as testicular swelling and Superior Vena Cava Syndrome, were detected occasionally in 3 children each. The number of dropouts prior to acute leukaemia sub-classification were 227 (33.8\%), and those diagnosed by IPT were $445(66.2 \%)$. Out of 9 children in the $\leq 1$ year age group, who were sub-classified, 6 had B-ALL $(66.7 \%)$, followed by 2 with Mixed Phenotypic Leukaemia (MPAL) and 1 with T-ALL. There were no Acute Myeoid Leukemia (AML) patients in this cohort. In 205 patients of $>1-10$ years, the most common was ALL:175 patients (85.3\%), followed by AML:20 (9.8\%) and MPAL:8 (3.9\%). Among ALL, Precursor B-ALL:157 (76.6\%) is the commonest, followed by T-ALL:14 (6.8\%) and Pro B-ALL:4 (1.9\%). Out of 20 patients of AML, 6 (30\%) were Acute Promyelocytic leukemia (APML). 240 patients were $>10-18$ years of age, and again the majority had ALL:180 (75\%), followed by AML:49 (20.4\%) and MPAL:8 (3.3\%), with Pre B-ALL being the commonest subtype:142 patients (59.1\%). This was followed by T-ALL:35 (14.6\%) and Pro B-ALL:6 (2.5\%). APML was detected in 21AML patients $(42.9 \%)$. Conclusions: The analysis of our registry data concludes that childhood leukaemia is most common in the age group of 1-10 years and male sex is predominant across all age groups. The most common presentation is fever. Among all age groups, ALL is most common with Pre B-ALL being the commonest subtype and the incidence of AML increases with age.

\section{P-AL 25}

Immunophenotypic Characterization and Analysis of Outcomes of Early T Cell Precursor (ETP) Acute Lymphoblastic Leukemia

Madhavi Maddali", Nisham P N, Ansu Abu Alex,

Nancy Beryl Janet, Uday Kulkarni, Anup J Devasia, Anu Korula, Aby Abraham, Biju George, Alok Srivastava, Poonkuzhali Balasubramanian, Vikram Mathews

Email ID for Correspondence: dr.madhavimaddali@gmail.com Christian Medical College, Vellore

Introduction: $\mathrm{T}$ cell-acute lymphoblastic leukaemia is characterized by clonal expansion of malignant T-cells. It accounts for $10-15 \%$ of childhood and $25 \%$ of adult ALL. In 2009, early T cell precursor ALL (ETP) subset was identified based on characteristic gene expression profiling and immuno-phenotype. This subset is associated with worse prognosis when compared to the other T-ALL subtypes. Objectives: The aim of our study is to compare the baseline characteristics of ETP with non-ETP subtypes of T-ALL and their outcomes. Material and Methods: Newly diagnosed T-ALL patients who presented to the department of Clinical Haematology, CMC Vellore between 2012 and 2017 were included in this analysis retrospectively. Clinical details were obtained from patient records. The diagnosis and categorization of various subtypes of T-ALL was done by BD FACS CANTO II/BD FACS CALIBUR flow cytometer using the acute leukaemia panel (CD3, SCD3, CD7, CD 2, CD4, CD5, CD1a, CD8, CD19, CD20, CD 10, CD 13, CD 33, CD11b, CD11c, CD 56, CD117, HLA DR, MPO, CD 79a and CD 45). Identification of ETP ALL was based on absence of CD1a and CD8 with a weak/absent CD5 along with one or more stem cell/ myeloid specific markers. Cytogenetic analysis of both the groups was done by karyotyping. Of the 198 newly diagnosed patients 142 who took treatment at our institution were followed up. The overall survival and event free survival were analysed by Kaplan Meier method. Results: The prevalence of ETP in our study was $23 \%$ $(n=46)$. The other types of ALL $(n=152)$ were grouped as nonETP ALL. ETP ALL had a higher median age (years) at presentation $\left(35^{\text {ETP }}\right.$ versus $20^{\text {NON ETP }}$ ). Presence of CNS involvement and testicular mass at presentation were similar to that of non-ETP cases whereas the incidence of mediastinal mass was significantly lesser in 
ETP ALL group ( $7 \%^{\text {ETP }}$ vs $34 \%^{\text {NON ETP }}$ ). The baseline hematologic parameters such as Hemoglobin, WBC count and LDH were significantly lower in ETP ALL. Data from 23 of the 46 ETP cases (50\%) and 119 of 152 of non ETP ALL cases $(78 \%)$ were analyzed. There was a comparable response to steroid and induction therapy in both the groups. Allo-SCT in CR1 was done in $10 \mathrm{~T}$ ALL cases of which 4 were ETP ALL and the rest non ETP. ETP ALL had a lower OS $\left(62.6 \pm 16.2^{\text {ETP }}\right.$ vs $\left.75.7 \pm 5.0^{\text {NON ETP }}\right)$ and EFS $\left(48.9 \pm 17.9^{\text {ETP }}\right.$

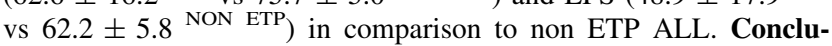
sions: ETP-ALL accounted for $23 \%$ of all T ALL cases and had a poor outcome in comparison to other subsets of T-ALL. Due to the characteristic immuno-phenotypic features, it is crucial to identify this subtype and analyse its impact in larger studies to define new treatment strategies.

\section{P-AL 26}

CD34 Expression in ALL and Its Correlation with Aberrant Antigen Expression and Other Prognostic Markers

Dr Neelakshi Goyal\#, Dr Richa Gupta, Dr Priyanka Saxena, Dr Prerna Arora, Dr Sarika Singh

\section{Department of Pathology, Maulana Azad Medical College, New Delhi}

\section{Objectives}

- To study the expression of CD34 positivity in cases of ALL

- To correlate CD34 expression with CD10(CALLA) positivity (BALL) and other prognostic markers (Total leucocyte count, age group, aberrant antigen expression)in cases of B-ALL and T-ALL

Introduction: ALL is the most common childhood malignancy, $75 \%$ of all leukemias are under the age of 20 years. Few western studies have reported that $70 \%$ of CALLA positive ALLs express CD34 positivity but no definite data is available in the Indian population. Absence of CD34 expression correlates with a better prognosis. Aberrant expression of antigens and CD34 positivity are independent prognostic markers but their correlation to each other is unknown. Methodology: A total of 43 cases were collected retrospectively from the Department of Pathology, Maulana Azad Medical College, New Delhi. Diagnosis of acute leukemia was based on the examination of peripheral smears, bone marrow morphology, cytochemistry and flow cytometric analysis. A total of 35 cases were B ALLs and 8 cases were T ALLs. Results: $57 \%$ of B-ALLs were CD34 positive (20 out of 35 cases) and $25 \%$ cases of T-ALLs were CD34 positive ( 2 out of 8 cases) 4 out of 35 cases of B-ALLs showed aberrant antigen expression ( 3 expressed aberrant CD33 and 1 expressed CD13). 3 out of 8 cases of T-ALLs showed aberrant antigen expression (CD10, $\mathrm{CD} 13, \mathrm{CD} 33$ respectively) $\mathrm{CD} 34$ positivity in B-ALL correlates with aberrancy (3 out of 4 cases, $75 \%$ ) whereas no correlation was found in cases of T-ALL CD34 positivity correlates with CALLA positivity (CD10) (18 out of 35 cases, 54.5\%) in B-ALL CD34 positivity correlates with TLC $<10,000 / \mathrm{mm} 3$ in cases of T-ALL and no correlation was found in cases of B-ALL CD34 positivity showed no correlation with age group in both B-ALLs and T-ALLS. Conclusion:
CD34 expression in B-ALLs correlates with aberrant antigen expression and CALLA positivity whereas no correlation was found with TLC and age while CD34 positivity correlates with low TLC in cases of T-ALL.

\section{P-AL 27}

\section{Prognostic impact of c-KIT in Core Binding Factor AMLs}

Dr. Sundar Shewale ${ }^{\#}$ Dr Mayur Parihar, Dr. Neeraj Arora, Dr. Nishit Gupta, Dr. Asish Rath, Dr. Saurabh Bhave, Dr. Mammen Chandy, Mr Kallol Saha,

Mr. Sourav Sharmachowdhary, Dr. Sushant Vinarkar, Dr. Deepak K. Mishra

Email ID for Correspondence: sundarshewale1988@gmail.com Tata Medical Centre, Kolkata

Introduction: Core-Binding Factor (CBF) - Acute Myeloid Leukemias (AML) includes $\mathrm{t}(8 ; 21)(\mathrm{q} 22 ; \mathrm{q} 22)$ and inv(16)(p13q22)/ $\mathrm{t}(16 ; 16)(\mathrm{p} 13 ; \mathrm{q} 22)$ and are generally considered as having favourable risk. However, approximately $50 \%$ of patients with CBF-AMLs remain incurable and c-KIT mutation status is one of the prognostic markers in the risk stratification of these patients. Material and Methods: This is a retrospective analysis of 21 cases of CBF-AMLs [AML with $\mathrm{t}(8 ; 21)-19$ cases and AML with inv(16) - 2 cases] over a period of 24 months (from August 2015 to August 2017) in which c-KIT mutation status was available. DNA was extracted from Peripheral Blood (PB)/Bone marrow (BM) samples, c-KIT exon 8 and 17 were analysed by Sanger Sequencing. Results: c-KIT mutations were detected in $8 / 21(38 \%)$ patients, all of which were $t(8 ; 21)$ positive. Six cases had exon 17 missense mutation whereas 2 cases had exon 8 deletion mutation. Exon $17 \mathrm{D} 816 \mathrm{~V}$ and D822 K missense mutations were seen in 2 cases each while D820G and D820Y missense mutations were seen in one case each. Exon 8 D419del was present in 2 cases. Within the c-KIT positive AMLs, mean WBC count, average blast \% in peripheral blood and bone marrow was $8.68 \times 10^{9} / \mathrm{L}, 31.4 \%$ and $51.2 \%$ respectively. Aberrant CD56 expression on flow-cytometry was seen in $12.5 \%$ (1/8) cases while aberrant CD19 expression on flowcytometry was noted in $75 \%$ (6/ 8)cases. The mean WBC count in wild type c-KIT CBF-AMLs was $30.14 \times 10^{9} / \mathrm{L}$ with $48 \%$ and $60 \%$ blasts in peripheral blood and bone marrow respectively. CD56 expression was seen in $15.3 \%(2 / 13)$ cases and $61.5 \%(8 / 13)$ cases revealed CD19 expression in the blasts. Among c-KIT positive CBF AML patients 4/8 (50\%) patients relapsed within 1 year of starting treatment while only 2 patients $(2 /$ $16,15 \%$ ) relapsed of the 16 patients with a wild type c-KIT at the end of one year. Conclusions: c-KIT positive CBF AMLs have less average WBCs count, peripheral and bone marrow blasts percentage as compared to negative group. We did not find comparative association between CD56 and c-KIT mutation status which may be due to the small number of cases. D816 V and D822 K are most common missense mutations noted in exon 17 while D419del was most common deletion in exon 8 . Similar to published literature we found a higher relapse rate in c-KIT positive CBF-AML. 


\section{P-AL 28}

\section{ACUTE MYELOID LEUKEMIA -Real World Data in a Tertiary Care Center in Bangalore}

Shilpa Prabhu", Karuna Kumar, Bharat Ram, Mukesh Chawla, Varun Bafna, Sunil Bhat, Shobha Badiger, Nataraj K.S, Sharat Damodar

Email ID for Correspondence: shilpasgp@gmail.com Mazumdar Shaw Medical Center, Bangalore

Introduction: ACUTE MYELOGENOUS LEUKEMIA (AML) is a heterogenous disease characterised by accumulation of genetic changes and somatic mutations in clonal hematopoeitic stem cells. Despite advances in treatment the prognosis remains dismal, especially in elderly population. Objectives: 1) To study the demographic profile of Acute myeloid leukaemia and to compare two different chemotherapy regimes with respect to remission on day 28 and overall survival. Material and Methods: The study involved a cohort of 84 cases of de novo acute myeloid leukemia patients of all age groups and both genders who presented to department of Haematology, Mazumdar Shaw Medical center, Bangalore over a period of two years.(January 2014-December 2015). The cases were confirmed by peripheral blood, bone marrow aspiration morphology and flow cytometric immunophenotyping. Based on the patients performance status and general conditions the patients were catagorised into three groups as" $3+7$ induction chemotherapy" arm, " $2+5$ chemotherapy" arm and hypomethylating agents (Decitabine/Azacytidine). These patients were followed up for two years. Results: Total of 84 cases presented to our department, out of these 78 patients opted to receive standard treatment. The age range was from 4 years 74 years. Of them, $48(56.4 \%)$ were males and $36(42.3 \%)$ were females. $14(16.47 \%)$ patients were above 60 years of age. $12(14.1 \%)$ patients belonged to paediatric age group. Median age of patients was 38 years. $67(78.8 \%)$ patients received induction chemotherapy. 32 patients received $3+7$ induction and 35 received $2+5$ chemotherapy. 11 patients were treated with hypomethylating agents as they were elderly and had preexisting comorbid conditionsInduction mortality was $16(18.8 \%)$. Induction deaths were primarily due to neutropenic sepsis. Gram negative sepsis was seen in 30 (35\%) of patients, gram positive $10 \%$. Induction mortality was higher with $3+7$ than with $2+5$ regime. Remission rate of $3+7$ chemotherapy was 21 of $32(65.2 \%)$ and that with $2+5$ regime was 16 of 35 $(45.7 \%)$. Two chemotherapy regimes were compared with respect to remission and overall survival, the difference was not statistically significant. However, $3+7$ regime was associated with slightly better overall survival than $2+5$ regime. Conclusions: In a tertiary care center, more than $90 \%$ of patients receive treatment for AML. Survival rates are marginally better in $3+7$ chemotherapy arm than $2+5 \mathrm{arm}$, however the difference was not statistically significant ( $\mathrm{p}$ $0.55)$.

\section{P-AL 29}

\section{GM-CSF Therapy Masking AML in a Case of Pancytopenia}

Paheli Maru\#, Harresh Kumar, Priyanka Gogoi, Mrinalini Kotru, Satendra Sharma, Preeti Diwaker

Email ID for Correspondence: ashjenny88@gmail.com University College of Medical Sciences
Introduction: GM-CSF therapy is known to accelerate hematologic recovery in cases of pancytopenia. Apart from the various known causes, pancytopenia can also be attributed to the clinical sequelae of a leukemia. Case Report: We present a case of 48 years old male who presented with 1 week history of fever, malaise, cough with expectoration, loose stools and generalised weakness. GPE showed pallor and systemic examination was normal. In view of these signs and symptom a hemogram was done which showed pancytopenia. The clinician initiated GM-CSF therapy for the same and a post therapy BM examination was performed. The peripheral smear examination showed $35 \%$ blasts whereas the BM showed $27 \%$ blasts which created a diagnostic dilemma for AML. Hence the clinician was suggested to stop GM-CSF therapy and keep the patient under close follow up. After withholding the therapy for 1 week, a repeat peripheral smear examination revealed a shoot up in blast count to $75 \%$ along with increase in TLC. Flowcytometry aided further diagnosis and a final report of AML-M2 was signed out. Conclusion: Cases of pancytopenia should be thoroughly evaluated before instituting therapy.

\section{P-AL 30}

Prevalence of IDH Mutations in Acute Myeloid Leukemia and Its Clinicopathological Characteristics

\section{Akshatha Nayak U\#, Sreeparna Podder, Rudra Ray,} Arnab Chattopadhyay, Maitreyee Bhattacharyya

Email ID for Correspondence: anayakbejai@gmail.com Institute of Haematology and Transfusion Medicine (IHTM), Medical College and Hospital, Kolkata

Introduction: Acute myeloid leukemia is routinely characterized using cytogenetic and various gene mutations. Gene mutations in AML are useful marker for diagnosis, prognostication, target for therapy and also for monitoring response to therapy. Cytogenetically normal cases are assessed for mutations of NPM1, FLT3, and CEBPA. Recently, mutations in the metabolic enzymes isocitrate dehydrogenase 1 and 2 genes (IDH 1/ IDH 2) have been reported in AML. Limited information is available on the clinicomorphological features of AML patients with IDH mutations worldwide and so far only one study reported from India. Objectives: 1) To explore prevalence of IDH mutations. 2) To correlate with various clinicopathological characteristics. Material and Methods: Sixty nine newly diagnosed AML patients attending IHTM during the time period January 2017 to July 2017 were analysed for the presence of IDH1 and IDH2 mutation in peripheral blood and bone marrow aspirate samples. DNA was isolated using automated machine from QiaCube Qiagen and IDH1 was sequenced by Sanger sequencing in only 33 randomly selected samples. IDH2 was assessed in all the samples initially by amplification by PCR followed by analysis on agarose gel. Results: The prevalence of IDH mutations was $17.3 \%$ (12/69) with prevalence of IDH 1 among the 33 cases sequenced was $9 \%(3 / 33)$ and that of IDH2 was $13 \%$ (9/69). Prevalence of IDH mutations among males was $12.2 \%(5 / 41)$ and females was $21.4 \%(6 /$ 28). Median age of presentation among IDH mutated patients was 44 years (Range:24-59 years) and among IDH unmutated patients was 42 years (Range:8-91 years). Median age of presentation in IDH1 mutation was 51 years and in IDH2 mutation was 44 years. Median WBC count \& blast percentage at presentation in IDH mutated patients was 20,450/cu.mm and 60\% (IDH $1-20,450 \& 75 \%$ and IDH2-46,000 \& 60\%) and in unmutated patients it was $18,700 /$ cu.mm and $65 \%$. Median platelet count at presentation in IDH 
mutated patients was 37,000/cu.mm (IDH1- 37,000 and IDH235,000 ) and in unmutated patients was $22,000 / \mathrm{cu} . \mathrm{mm}$. Out of the 12 IDH mutated patients only $3(27.3 \%)$ had normal karyotype, 5 had no mitotic divisions, 2 had Trisomy 8,1 had del17p and 1 patient's cytogenetics was not available. Flow cytometry analysis showed 2 out of the 12 IDH mutated patients showing CD56 aberrancy and 2 showed CD7 aberrancy and 1 patient showed AML with monocytic differentiation. Coexisting FLT3 mutations specifically FLT3 ITD were seen in 3 of the IDH mutated patients mainly IDH2 whereas coexisting NPM1 mutation was seen in 1 patient with IDH2 mutation. One patient showed the presence of both IDH1 and IDH2 mutations. Conclusion: Though literature reports IDH mutations in CN AML cases, in this study only $27 \%$ cases had normal cytogenetics. Both NPM and FLT3 mutations were found to be associated with IDH 2. Morphology and immunophenotypic characteristics are nondistinctive.

\section{P-AL 31}

Pattern of infections during intensive phase of chemotherapy for acute lymphoblastic leukaemia therapy in children and young adolescents

\section{Wanve Balasaheb, Nisham PN, Anup Devasia, Anu Korula, Uday P Kulkarni, Yasir Geelani Kavitha NL Aby Abraham, Alok Srivastava, Vikram Mathews, Biju George}

Department of Haematology, Christian Medical College, Vellore, Tamilnadu, India

Introduction: Chemotherapy for acute lymphoblastic leukaemia (ALL) includes an intensive phase for 6 months followed by a maintenance phase lasting 18-24 month. There may be repeated episodes of neutropenia due to marrow ablative chemotherapy and profound immunosuppression due to prolonged corticosteroid use. Both lead to an increased risk of bacterial, viral and fungal infections. This analysis was done to study the incidence of infection during first six months and their impact on overall survival. Methods: This is a retrospective chart based review of all patients $<21$ years of age who were treated for ALL in the department of Hematology CMC Vellore between 2006 and 2016. Data was collected from the electronic medical records and department databases. Results: Between January 2006 to December 2016834 patients underwent chemotherapy for ALL including 247 females (29.6\%) and 587 males (70.4\%). They were risk stratified into 3 groups, 125 (15\%) formed standard risk, 675 $(80.9 \%)$ formed intermediate risk and $34(4.1 \%)$ formed high risk group. Five hundred and five patients $(60.6 \%)$ had an absolute neutrophil count at $<500$ at presentation. Fever was presenting feature of acute leukemia in $471(56.5 \%)$ cases. One hundred and ninety eight patients $(23.7 \%)$ developed a documented infection during induction regimen. Out of 198 proven infections $80(40.4 \%)$ had proven bacterial infections. Out of bacterial infections $50(62.2 \%)$ were caused by gram negative organisms, $27(33.3 \%)$ were caused by gram positive organism and $3(3.75 \%)$ were caused by mycobacterial organism. Thirteen $(6.5 \%)$ were caused by common viruses (7 $(53.8 \%)$ were herpes simplex infections, 2 were varicella zoster $(15.3 \%)$ one was hepatitis B viral infection and $2(15.3 \%)$ were caused by influenza). Forty-four (22.2\%) were caused by fungal agents (36 had possible fungal pneumonia, 8 had proven disseminated fungal infection. $61(30.8 \%)$ infections were other infections likes line site infection, soft tissue infection etc, 21 had line site infection including tunnel track infection, 15 acute gastro-enteritis, 8 had pneumonia, 7 urinary tract infection, 3 had acute suppurative otitis media, 3 had axially hydra-adenitis, 1 had right popleteal fossa abscess, 1 had septic arthritis. During follow up, 62 patients (7.5\%) out of 834 had died; mortality was statistically significant among the patients with proven infections (18\%) as compare to mortality among patient without infections $(4.8 \%)[\mathrm{p}=0.0001]$. Out of 62 deaths $3.5 \%$ were in complete remission (out of 601 in complete remission), $41.7 \%$ were in partial remission (out of 12 in partial remission), and $16.2 \%$ had persistent or relapsed disease (out of 218 with persistent or relapsed disease. Conclusions: Proven infections were documented in $23 \%$ of patients during induction. There was significantly higher mortality in patients with proven infection during chemotherapy.

\section{P-AL 32}

Hypercalcemia: An Unusual Manifestation of Acute
Lymphoblastic Leukemia in Children

Naveen Gupta\#, Nivedita Dhingra, Meenakshi BA, Tulika Seth, Manoranjan Mahapatra, HP Pati, Seema Tyagi, Renu Saxena

Email ID for Correspondence: drnaveengupta1988@gmail.com All India Institute of Medical Sciences, New Delhi

Introduction: Malignancy associated hypercalcemia is rarely observed in children and adolescents, and is even more unlikely in leukemias, when compared to solid malignancies. It can be associated with devastating complications like pancreatitis, encephalopathy or arrhythmias and demands prompt intervention. We hereby describe presentation and successful management of 4 cases of pediatric acute lymphoblastic leukemia (ALL) who developed hypercalcemia. Objectives: The objective of this case series is to highlight an unusual manifestation of ALL. Material and Methods: All cases of ALL (age $<18$ years) receiving therapy at our institute from June 2011 to June 2017 were reviewed for presence of hypercalcemia at initial presentation. Clinical details, treatment records and follow up were noted. Results: 129 children underwent treatment for ALL in the study period. Hypercalcemia was noted in 4 patients (3.1\%) including 3 children with B-ALL ( 2 boys aged 3 and 15 years and a 9 year old girl) and a 13 year old boy with T-ALL. All patients had normal cytogenetics. Mean calcium level was $13 \mathrm{mg} / \mathrm{dL}$ (range 11.6 $13.6 \mathrm{mg} / \mathrm{dL}$ ). Two of the patients were symptomatic with abdominal pain, distention and altered mental status, and hypercalcemia was incidentally detected in two of them. None of them had renal dysfunction or radiographic evidence of bone lesions. Hypercalcemia responded to aggressive hydration, furosemide and initiation of chemotherapy and calcium levels normalised by 72 hours in all cases. Three patients developed laboratory tumor lysis syndrome during induction chemotherapy, of which two had evidence of bulky disease. Subsequent course of therapy was uneventful. Conclusions: Hypercalcemia at presentation is a rare manifestation of ALL. Clinical signs may be subtle or even absent in early stages. Prompt management yields favourable outcomes.

\section{Abstract for oral presentation}

\section{P-AL 33}

Role of flowcytometric analysis in distinguishing acute myeloid leukemia with monocytic differentiation from other subtypes of acute myeloid leukemia: A 5-year study in a tertiary care center

Nayanjyoti Barman", Manjari Kishore, Vijay Kumar, Sadhna Marwah, A.S. Nigam

Email ID for Correspondence: nayanjyoti71@gmail.com 


\section{PGIMER, Dr. RML Hospital, New Delhi}

Introduction: Flow cytometric immunophenotyping is a useful ancillary tool in the diagnosis and subclassification of acute myeloid leukemias (AMLs). It has been noted that CD64 is sensitive and specific for distinguishing AMLs with a monocytic component (ie, AML M4 and AML M5) from other AML subtypes. We present a series of cases of AML with monocytic differentiation highlighting the importance of flowcytometric immunophenotyping. Aims \& Objective: To evaluate the usefulness of CD14 and CD64 by flow cytometric immunophenotyping in distinguishing AMLs with monocytic differentiation from other AML subtypes. Materials \& Methods: A total of 60 cases of acute myeloid leukemia (AML) with flowcytometric analysis were included in the study. Among these, cases of AML with monocytic differentiation were selected and immunophenotypic analysis was done. Result: A total of sixteen cases of AMLs with monocytic differentiation was found, sub classified based on the French-American-British and World Health Organization classifications. Among sixteen patients, nine were male and seven were female. The age of the patients ranged from 7 to 80 years with median age of diagnosis at 33.5 years. A standard panel of markers, including CD2, CD3, CD5, CD7, CD10, CD13, CD14, CD19, CD20, CD33, CD34, CD45, CD56, CD64, CD117, and HLA$\mathrm{DR}$, were analyzed by flow cytometric immunophenotyping in all AMLs. Strong CD64 expression distinguishes AML M5 from other subtypes of AML. Conclusion: Strong CD64 expression distinguishes AML M5; however, heterogeneous, dim, or moderate expression in itself does not distinguish other sub-types of AML (M0-M4) from M5 with dim to moderate CD64 expression.

\section{P-AL 34}

\section{An Unusual Presentation of Childhood B-Cell ALL with Hyperleucocytosois and Priapism}

\section{Dr. Shilpi More\#, Dr. Mrinalini Kotru, Dr. Pooja Dewan, Dr. Meera Sikka}

Email ID for Correspondence: shilpimore@gmail.com University College of Medical Sciences \& GTB Hospital

Report of a case: Hyperleukocytosis (White blood cell count $\left.>100 \times 10^{9} / 1\right)$ is an uncommon presentation in acute leukemia. Factors related to the development of hyperleukocytosis in childhood acute lymphoblastic leukemia (ALL) are age $<1$ year, male gender, T-cell phenotype, mediastinal mass, massive hepatosplenomegaly and certain cytogenetic abnormalities. It can have a variety of manifestations owing to leucostasis and can range from non - specific symptoms like headache to fatal metabolic complications. Hyperleukocytosis presenting as priapism is very rare and reported in only $1-5 \%$ of adult patients with leukemia. The incidence is further down in pediatric age group. We report a case of 12 years old child who presented with priapism as the initial complaint. He had only mild hepatosplenomegaly and was found to have a TLC count of $>500 \times 10^{9} / 1$. On further work up the child was diagnosed as B cell ALL on flow cytometry. However the child succumbed to his disease before the completion of induction phase. The present case emphasizes on identifying the cause of priapism which is a rare event in childhood ALL.

\section{P-AL 35}

\section{Therapy Interruption During Induction Chemotherapy in Children with Acute Lymphoblastic Leukemia and the Risk of Relapse}

Nivedita Dhingra, Naveen Gupta, Tulika Seth, Manoranjan Mahapatra, HP Pati, Seema Tyagi, Renu Saxena

Email ID for Correspondence: nivedita_dhingra@yahoo.co.in All India Institute of Medical Sciences, New Delhi

Introduction: Multi-agent protocol based chemotherapy regimens with a risk adapted approach are the standard of care in pediatric ALL. Long-term survival rates exceeding $80 \%$ are reported by most western centres. In the Indian context the overall survival rates of $45-80 \%$ have been reported. Factors responsible for inferior outcomes are treatment abandonment, infections and disease relapse. We identified therapy interruptions during induction chemotherapy in ALL to be a major impediment to long-term relapse free survival. Objectives: To highlight the impact of interruption of protocolspecified induction chemotherapy on long term relapse free survival in children with ALL. Material and Methods: Medical records of children ( $<18$ years) with ALL who underwent treatment at our center between 2011-2017 were retrospectively reviewed and details of induction therapy were noted. All children were planned for a uniform 4-drug induction followed by risk adapted post remission therapy. Therapy interruption was defined as any deviation from the protocol other than pre-specified hepatic or renal dose modifications. Details of follow-up were recorded till last visit. Results: 129 children with a median age at presentation of 13 years (range 0.518 years) and male:female ratio of 3.6:1 underwent ALL induction during the study period. \%). The median duration of follow-up for the entire cohort of 113 cases that went into remission was 19 months (range 1-142 months). Twenty-six cases $(20.1 \%)$ had therapy interruption during induction on account of severe infections in 16 cases, jaundice in 5 cases, 1-asparaginase induced pancreatitis in 2 cases, seizures in 2 and severe hypersensitivity reaction in 1 patient. Amongst the infectious causes, fungal pneumonia was observed in the vast majority accounting for 11 of the 17 cases (64.7\%). At end of induction morphologic remission was attained in 25 of 26 cases with therapy interruptions (96.3\%). 12 out of these cases (46.2\%) had relapse (medullary or extramedullary or combined) as compared to $20.61 \%$ relapse rate in the group without therapy interruption $(\mathrm{p}<0.01)$. Kaplan Meier survival estimates at 24 months for relapse free survival in patients with therapy interruptions was $49.9 \%(95 \%$ CI $26.1-69.7 \%$ ) vs $73 \%$ (95\%CI $60.1-82.3 \%$ ) for patients with no treatment interruptions $(p=0.02)$. Conclusions: Infectious complications were found to be the most common cause for interruption of treatment. Despite achievement of morphological remission at end of induction patients with therapy interruptions were found to have adverse outcomes with inferior relapse free survival. 


\section{P-AL 36}

Presentation of Behcet's Disease with Acute Myeloid Leukemia in a 35 Year Old Male: A Rare Case Report

Amrita Anand", Dr Smita Singh, Dr Sunita Sharma, Dr Shruti, Dr Vivek Arya, Dr Ashish Sharma

Email ID for Correspondence: anandamrita@yahoo.in Department of Pathology, Lady hardinge Medical College

Introduction: Behcet's disease (BD) is an inflammatory disorder which affects multiple parts of the body causing oral and genital ulcers, uveitis, skin lesions, vascular lesions and arthritis.. Association of behcets with hematological disorders like chronic myelomonocytic leukemia (CMML) has been characterised but the association with acute myeloid leukemia (AML) has never been reported. We are presenting a case of 35 years old male with this rare presentation of AML-M5. Case Report: A 35 years old male presented with recurrent painless oral and genital ulcers since $1 \frac{1}{2}$ years, low grade fever and left lower limb swelling with induration and nodular thrombophlebitis. Perabdominal examination showed hepatosplenomegaly. UGI Endoscopy showed antral erosion. Hematological parameters showed anemia with leucopenia. Provisional clinical diagnoses of Behcets disease with bicytopenia with secondary MDS, EBV associated with genital ulcers with bone marrow suppression and thirdly, hematological malignancy with secondary DVT with febrile neutropenia. Bone marrow aspirate showed $72 \%$ blasts and promonocytes. Cytochemistry for Non Specific Esterase was positive, Myeloperoxidase positivity was seen in very few cells. On immunophenotyping, these blasts showed the expression of CD33, CD117, CD11b, CD11c, CD34. So, the diagnosis AML-M5 was confirmed. Also, the patient fulfilled the criteria for behcet's disease and Pathergy test was positive. Discussion: An association between BD or BD-like illness and CMML with a trisomy of chromosome 8 has been well established but very rarely with AML. Conclusion: This case is unique because of the rare coexistence of Behcets disease with acute monocytic leukemia (AML-M5).

\section{P-AL 37}

Outcome of Acute Promyelocytic Leukemia (APL): Retrospective Study from a Tertiary Cancer Center

\section{Mipsang Lama\#, Prakash Shekhawat, Ketan Modak, Jeevan Kumar, Saurabh Bhave, Vivek Radhakrishnan, Reena Nair, Mammen Chandy}

Objectives: To study the outcome of APL patients treated at Tata Medical Center. Methodology: A retrospective analysis of 53 APL patients treated at Tata Medical Center from Mar 2011 to Feb 2017 was performed. The electronic medical records of the patients were reviewed and analyzed. All the patients received induction for minimum 45 days and depending on day $45 \mathrm{BM}$ examination, induction was extended upto 60 days in those who were not in remission. It was followed by consolidation and then maintainance as per protocol. Molecular response by RQ-PCR for PML:RARA was also assessed post induction. Result: Eighty patients were diagnosed with APL at
Tata Medical Center from Mar 2011 to Feb 2017, out of which 53 patients continued treatment at our center. Median age of the patients was 33 years (range, 9-67 years). Out of them, 28 (53\%) were female and $25(47 \%)$ were male. The median range of hemoglobin, TLC and platelet count was $8.75 \mathrm{~g} / \mathrm{dl}, 19500 / \mathrm{mm} 3$ and $14500 / \mathrm{mm} 3$ respectively. Out of 53 patients, $8(15.1 \%)$ patients had fatal outcome. Molecular response post induction could be assessed only in 42 patients of which $27(64.2 \%)$ were negative and 15 (35.8\%) were positive. Five $(9.4 \%)$ patients relapsed during the study period, out of which 3 were salvaged in $1^{\text {st }}$ relapse and 1 in $2^{\text {nd }}$ relapse. RFS at 5 years was found to be $72.1 \%$. RFS was further calculated as per risk groups and treatment groups. OS at 5 years was $84.8 \%$. Conclusion: Relapse Free Survival (RFS) was found to be better in low risk group and ATO, ATRA and Anthracycline treatment group, but it was not statistically significant. A large prospective study is required to consolidate our results.

\section{Abstract for oral presentation}

\section{P-AL 38}

Role of Flowcytometric Analysis in Diagnosis of T-Acute Lymphoblastic Leukemia: A 5-Year Study in a Tertiary Care Center

Sivagama Sundari", Manjari Kishore, Vijay Kumar, Sadhna Marwah, A.S. Nigam

Email ID for Correspondence: vpgsiva25@gmail.com

PGIMER, Dr. RML Hospital, New Delhi

Introduction: Flow cytometric immunophenotyping is a useful ancillary tool in the diagnosis of acute leukemia. It helps in correctly distinguishing T- Acute lymphoblastic leukemia (ALL) from other subtypes of ALL. We present a series of cases of T-ALL highlighting the importance of flowcytometric immunophenotyping in their correct diagnosis. Aims \& Objective: To evaluate the usefulness of flow cytometric immunophenotyping in distinguishing T-ALL from other subtypes along with demographic correlation. Materials \& Methods: A total of 165 cases of acute lymphoblastic leukemia (ALL) presenting from 2012 (July) to 2017 (June) with flowcytometric analysis were included in the study. Among these, cases of T-ALL were selected and immunophenotypic analysis was done. Result: A total of 21 cases T-ALL was found, subclassified based on the FrenchAmerican-British and World Health Organization classifications. A standard panel of markers, including CD2, CD3, CD5, CD7, CD10, CD13, CD14, CD19, CD20, CD22, CD33, CD34, CD45, CD64, CD117, and HLA-DR, were analyzed by flow cytometric immunophenotyping in all cases of ALL. Among 21 cases of T-ALL, 17 were male and 4 were female. The age group ranged from 4 to 30 years with median age of presentation being 14.57 years. A detailed flowcytometric analysis was done and results were tabulated. Conclusion: Though the hallmark for the diagnosis of acute leukemia until recent past has been the morphology and cytochemistry, the role of flowcytometric immunophenotyping has emerged as a powerful tool in investigation of normal and neoplastic cells and their classification at each level. Hence, early and correct diagnosis of T-ALL help in initiation of therapy as early as possible. 


\section{P-AL 39}

Surface CD22 is as Reliable as CD79a and Easier-to-test Marker in the Diagnosis of B-ALL

\author{
Ravi Kiran Pawar, Manisha Daruwalla, Kainaz Sidhwa \\ and Amar Das Gupta
}

\section{SRL Limited, Mumbai, India}

Some earlier reports suggested that surface (s) CD22 expression by blast cells in B-acute lymphoblastic leukemia (ALL) was lower compared to $\mathrm{cCD} 22$ expression, thus rendering the former as a less reliable marker of B-lymphoid lineage than the latter. This might have prompted WHO to recommend testing of cytoplasmic (c) CD22 and cCD79a as the most reliable markers of B-lymphoid lineage of blast cells along with CD19. However, considering the complexity of the technique for testing of cytoplasmic markers, the variability of results and interpretation thereof, laboratories started using SCD20 in addition to or in place of sCD22 as a marker of B-lymphoid lineage in their immunophenotyping reagent panel. It was soon realized that CD20 is negative in a sizeable percentage of B-ALL cases necessitating the use of cCD22 and/or cCD79a also thereby increasing the cost of testing. So we wanted to check the expression of sCD22 by blast cells in B-ALL with the objective of using it as a reliable marker of B-lymphoid lineage thereby obviating the need to test for cCD22 and/cCD79a. Seventy five of 172 cases of B-ALL (CD19+, CD10+, $\mathrm{CD} 20+/-$ and $\mathrm{CD} 79 \mathrm{a}+$ ) were examined for sCD22 expression. While all the 75 cases $(100 \%)$ were positive for sCD22, only $95 / 172$ cases (55\%) were positive for CD20. All 23 cases in which cCD79a was tested as required for diagnosis, were positive for this marker. There was a $100 \%$ correlation in the expression of cCD22 and cCD79a in cases in which these two markers were tested. Our data show that sCD22 is a highly specific and sensitive marker for B-lymphoid lineage and can replace $\mathrm{cCD} 22$ and $\mathrm{cCD} 79 \mathrm{a}$ in an immunophenotyping reagent panel for the diagnosis of B-ALL.

\section{P-BMA 01}

Utility of E-Cadherin as a Marker of Erythroid Lineage in Bone Marrow Trephine Biopsy

Debdatta Basu\#, T. Mathumithra, A. Pavithra, J. Sree Rekha, Sajini E Jacob, Rakhee Kar

Email ID for Correspondence: ddbasu@gmail.com JIPMER, Pondicherry

Introduction: In bone marrow biopsies often the early erythroid precursors are difficult to distinguish from early myeloid precursors, especially blasts. This assumes importance in various clinical situations like cases of acute leukemia on chemotherapy for assessing remission status, differentiating megaloblasts from myeloblasts and in assessing the proportion and distribution of various cell lineages in Myelodysplastic syndromes and leukaemia. E-cadherin, epithelial calcium-dependent cell adhesion protein, has been identified as a marker of immature erythroid precursors in recent years However, the specificity of E-cadherin in bone marrow specimens for erythroblasts versus myeloblasts or other early hematopoietic precursors has not been fully elucidated. Objectives: To determine the usefulness of E-cadherin in delineating erythroid precursors in bone marrow in various hematological disorders. Material and Methods: We analysed 53 bone marrow biopsies including 30 cases of Acute leukemia on chemotherapy for assessing remission status and 23 miscellaneous conditions like Acute Myeloid Leukaemia ( 7 cases including one case of Erythroleukemia), MDS (4 cases), Megaloblastic anemia (4 cases),
Aplastic Anemia. (2 cases), Primary Myelofibrosis (2 cases), PRCA (1 case) and staging marrows (7 cases). Along with E-cadherin, the following markers were also used, as and when indicated, - CD 34, CD 117, MPO, CD 61, Glycophorin C and TdT. The distribution and the pattern of positivity of E-Cadherin in the spectrum of erythroid cells were studied. Results: In all the 30 cases of marrows done for assessing remission status in acute leukemia, E-Cadherin helped delineate the erythroid precursors from other blasts, both myeloid and lymphoid, and helped differentiate marrows in remission from those without. In the remaining cases, E-cadherin highlighted the early erythroid precursors and aided in diagnosis. E-Cadherin was expressed strongly in early precursors and weakly or not expressed in more mature erythroid precursors including RBCs. Conclusions: Our results demonstrate the usefulness of E-cadherin, as a marker specifically of erythroid differentiation, in bone marrow biopsies.

\section{P-BMA 02}

An audit of Bonemarrow Aspirations in a Tertiary Care Hospital Dr Kruti Bhut ${ }^{1 \#}$, Dr Amit H. Agravat ${ }^{2}$, Dr Gauravi Dhruva ${ }^{3}$

Email ID for Correspondence: drkruti_bhut@outlook.com Department of Pathology, P.D.U. Government Medical College and Hospital, Rajkot, Gujarat

Objective: To study the frequency of hematological and non-hematological disorders found on bone marrow examination in a tertiary care hospital of Rajkot, India. Methodology: Cross sectional descriptive study was conducted at the Pathology Department of P.D.U. Government Hospital, Rajkot from April 2016 to April 2017. A total of 66 patients were included in study. Bonemarrow aspiration was done, stained and examined. Results: Out of 66 patients, 4 patients $(6.0 \%)$ had Leukemia, 17 (25.7\%) had Megaloblastic anemia, Idiopathic Thrombocytopenic Purpura (ITP) was present in 4 patients (6.0\%), 10 patients (15.1\%) showed normal bone marrow, Aplastic Anemia in 2 patients $(3.0 \%)$ and 2 patients $(3.0 \%)$ showed Multiple Myeloma. Conclusion: Anemia was the commonest disorder amongst non-malignant disorders and acute leukemia was the commonest malignant disorder.

Keywords Bonemarrow aspiration, Leukemia, Megaloblastic Anemia, Aplastic Anemia

\section{P-BMA 03}

Phenytoin Induced Lymphoid Aggregates in Bone Marrow Biopsy: An Incidental Finding

Dr. Sant Prakash Kataria\#, Dr. Rajeev Sen, Dr. Padam Parmar, Dr. Gajender Singh, Dr. Sanjay Kumar, Dr. Ritika

Department of Pathology, Pt. B. D. Sharma Post Graduate Institute of Medical Sciences, Rohtak - 124001, Haryana, India

Introduction: Lymphadenopathy associated with the use of hydantoin derivatives such as phenytoin was first described in 1940. Phenytoin (diphenylhydantoin or Dilantin) is anticonvulsant agent used in the treatment epilepsy. The side effects of phenytoin create significant morbidity. Two most important drug reaction of phenytoin are anticonvulsant hypersensitivity syndrome and lymphadenopathy. Here we present a case of phenytoin induced pseudolymphoma. Case Report: A 24 years old female presented with complaint of pallor, easy fatigability and history of fever on and off. She had seizure disorders and was on phenytoin (Diphenyl hydantoin) therapy for the 
last 18 months. Physical examination revealed multiple cervical lymphadenopathy. Complete hemogram revealed pancytopenia with relative lymphocytosis. Bone marrow aspirations (done twice) were inadequate for evaluation. Bone marrow trephine biopsy showed normocellular to hypercellular marrow with equal myeloid and erythroid ratio revealing erythroid hyperplasia with normoblastic to megaloblastic erythropoiesis. Myelopoiesis and megakaryopoiesis were normal. There are two nodular aggregates comprising of lymphoid cells interspersed in between the trilineage hematopoietic cells. On immunohistochemical examination, these lymphoid cells were TdT negative, CD3 and 5 positive, CD20 positive and CD23 negative. Possibility of Non- Hodgkin lymphoma could not be ruled out on the basis. Conclusion: Phenytoin is a highly effective anticonvulsant agent. The side effects of phenytoin continue to create significant morbidity. Patients receiving phenytoin may develop benign lymphoid hyperplasia, pseudolymphoma, pseudopseudolymphoma, or, rarely, malignant lymphoma. Obtaining a history of phenytoin use is crucial in the diagnosis of such patients.

Keywords Phenytoin, side effect, pseudolymphoma

\section{P-BMA 04}

\section{Bone Marrow Metastasis In Non-Hematological Solid Malignancies}

Sandeep Abhijit Pattnaik ${ }^{\#}$, Somanath Padhi, Susama Patra, Mukund Namdev Sable, Pritinanda Mishra,

\section{Samanyoya Gochhayat, Sonali Mahapatra ${ }^{1}$, Asutosh Panigrahi ${ }^{1}$,} S.K. Dasmajumdar ${ }^{2}$

Email ID for Correspondence: sandeep.abhijit89@gmail.com Departments of Pathology \& Lab Medicine, ${ }^{1}$ Medical Oncology/ Hematology, and ${ }^{2}$ Radiotherapy, All India Institute of Medical Sciences, Bhubaneswar

Introduction: Bone marrow (BM) aspiration and trephine biopsy are useful tools in the staging of non haematological solid malignancies (NHSM); especially in pediatric age group. Objective: To study the pattern of marrow changes in NHSM and correlate with laboratory parameters. Materials/Methodology: Bone marrow changes in seventeen cases of NHSM undergoing BM study were analyzed retrospectively. Results:

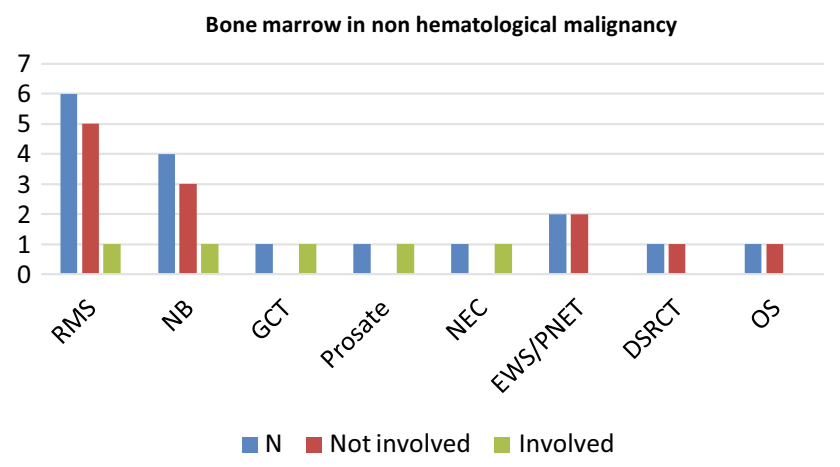

Abbreviations: RMS-rhabdomyosarcoma, NB-neuroblastoma,

MMGCT-malignant mixed germ cell tumor, NEC-neuroendocrine carcinoma, EWS/PNET-Ewing sarcoma/primitive neuroectodermal tumour, DSRCT-desmoplastic small round cell tumor, OSosteosarcoma.
Out of all the cases studied, one case each from MMGCT (27/M), NEC (68/M), NB (3/MCh), RMS (17/M) and carcinoma prostate (67/ $\mathrm{M})$ had metastasised to bone marrow. Involvement was focal in three (MMGCT, RMS, NEC) and diffuse in two (Ca prostate and neuroblastoma). Leukoerythroblastic blood picture was noted in three. BMA in with prostatic carcinoma revealed dry try; and the trephine core had a stony hard consistency. Trephine imprints were positive for malignant cells in three cases (neuroblastoma, RMS, and prostatic carcinoma) whereas neuropil like material with rosette formation; and focal gelatinous transformation were noted in neuroblastoma and MMGCT cases, respectively. On immunohistochemistry, MMGCT cases showed strong positivity for SALL-4, CD 30, PLAP, and Pan CK; whereas NEC showed positivity for Synaptophysin, Chromogranin, and Pan CK. Conclusion: Bone marrow metastasis from germ cell tumours and neuroendocrine carcinomas are extremely rare and carry a poor prognostic outcome.

\section{Abstract for Oral Presentation}

\section{P-BMA 05}

Metastasis of Solid Tumors Into Bone Marrow: A 5-Year Study in a Single Center

Shashikant Singh", Manjari Kishore, Vijay Kumar, Sadhna Marwah, A. S. Nigam, Minakshi Bhardwaj.

Email ID for Correspondence: shashisingh134@gmail.com PGIMER, Dr. RML Hospital, New Delhi

Introduction: Metastasis to bone marrow by non-haematological malignancies is a rare event and is often associated with a worsened prognosis. A reduction in overall survival, impaired response to therapy and multiple other manifestation resulting from decreased hematopoiesis are noted. Carcinoma prostate and carcinoma breast are the most common malignancies to metastasize in males and females respectively whereas small round cell tumor predominates in childhood. The patients with bone marrow involvement may or may not have any presenting complaint and a keen clinical observation is imperative in such cases. Here, we present a series of cases of bone marrow metastasis by solid organ malignancies over a period of 5 years. Objectives: The aim of this study was to investigate the clinical and hematological characteristics of patients with BM metastases of solid tumors diagnosed by bone marrow (BM) aspiration. Materials \& methods: In this study, we reviewed 3520 bone marrow aspirates in our hospital from May 2012 to June 2017. Patients diagnosed with solid malignancies metastasizing to bone marrow were selected. The patients' clinical information, hematological and histological findings (wherever available) were obtained from the medical records department and correlation was done. Results: We found a total of 26 cases of solid malignancies with metastasis to bone marrow. The proper clinical, radiological, hematological and histopathological correlation was done before giving a final diagnosis in these cases. The age of the patients ranged from 2-88 years (mean age of 46 years). Most of the patients were adults $(19 / 26)$, of which $77.7 \%$ were males. Seven of the 26 patients were in the pediatric age group, with an age range of 2-11 years. Various malignancies metastasizing to marrow were evaluated and clinical and hematological correlation was done. The common presenting sign \& symptoms were fever, anorexia and bodyache and organomegaly. Anemia was the most common hematological finding at the time of admission. Carcinoma prostate was the most common metastatic tumor in adults and small round cell tumor in children. Conclusion: $\mathrm{BM}$ is one of the important and infrequent sites of metastasis of solid tumors. Detection of bone marrow metastasis has significant clinical 
implication with impact on therapeutic decisions and is associated with poor prognosis. Aspiration of the BM provides an easy and quick way of detecting involvement, however, biopsy with immunohistochemical and/or molecular techniques may be needed in case of microscopical tumor burden.

\section{P-BMA 06}

Secondary Amyloidosis Involving the Bone Marrow, A Rare Case Report

Devi Anu. K* , Shilpi Agarwal, Amritha Anand

Email ID for Correspondence: k.devianu@gmail.com Department of Pathology, Lady Hardinge Medical College, New Delhi

Introduction: Amyloidosis encompasses a heterogenous group of disease caused by extracellular deposition of fibrillary protein. Amyloid deposits in AA amyloidosis are composed mainly of serum amyloid A protein, an apolipoprotein of HDL that serves as an acute phase reactant. Amyloid is usually not seen or is very sparse of present in aspirate or biopsy. Secondary amyloidosis involving bone marrow was reported only in $78.6 \%$ of patients. Case Report: A 54-year-old male patient, known case of chronic liver disease being treated for abdominal koch's for last 4 months presented with complaint of abdominal distension for 2 months. On examination, patient had icterus, gross ascites and hepatomegaly. Contrast enhanced CT scan revealed hepatomegaly with acute on chronic liver disease with findings suggestive of portal hypertension. Liver function tests and kidney function tests were deranged. Complete hemogram revealed leucocytosis and thrombocytopenia. Bone marrow aspirate smears were cellular with extensive deposition of pink amorphous extracellular material which was positive with methyl violet (suggestive of Amyloid). Bone marrow biopsy showed mildly hypocellular marrow with presence of abundant extracellular eosinophilic amorphous material (? amyloid) in the vessel wall and interstitium which on special stain was positive for methyl violet. Immunohistochemistry for Serum Amyloid A protein was positive. Serum protein electrophoresis with immunofixation revealed a polyclonal increase in the kappa light chain to $79.20 \mathrm{mg} / \mathrm{dl}$ and lambda light chain to $93.60 \mathrm{mg} /$ dl. The beta 2 microglobulin levels were $9687 \mathrm{ng} / \mathrm{ml}$. Myeloma protein panel showed an albumin level of $1.73 \mathrm{~g} / \mathrm{dl}$. No myeloma band was detected. Conclusion: Much of our information regarding amyloid involving bone marrow is based on primary amyloidosis. Secondary amyloidosis involving bone marrow is very unusual and is rarely reported.

\section{P-BMA 07}

Spectrum of Bone Marrow Findings in Extreme Elderly Patients A Single Tertiary Care Center Analysis

Dheeraj Chinnam", Praveen Sharma, Sreejesh Sreedharanunni, Narender Kumar, Prashant Sharma, Shano Naseem, Man Updesh Singh Sachdeva, Jasmina Ahluwalia, Reena Das, Neelam Varma, Pankaj Malhotra ${ }^{1}$

Email ID for Correspondence: dr.s.sreejesh@gmail.com Department of Hematology and ${ }^{1}$ Internal Medicine, Post Graduate Institute of Medical Education and Research, Chandigarh, India

Objectives: Bone marrow (BM) aspiration and trephine biopsy is one of the most valuable procedures in the evaluation of hematologic disorders. With increasing life expectancy, BM aspirates and trephine biopsies are increasingly performed even in extreme elderly patients ( $\geq 80$ years or older). There is a shortage of published literature regarding the indications, procedure, and spectrum of findings in the bone marrow of extreme elderly patients. The aim of the present study is to analyze the common indications of performing BM examination, the spectrum of findings and the utility of the procedure in this population. Materials and methods: A retrospective analysis of BM examinations performed in extreme elderly patients over a period of 5 years, between 2012 and 2016 was done. Results: A total 11698 BM examination procedures were performed in during the same period. Out of that, 84 patients aged 80 or above $(0.72 \%)$. The age of the patients ranged from 80 to 98 years (Median age -82 years). The male female ratio was 2.1:1. The most common indications for performing a bone marrow examination were suspicion of hematological malignancy $(60.7 \%)$, staging of lymphomas $(23.8 \%)$, evaluation of cytopenias $(12.5 \%)$ and others $(3.75 \%)$. Overall, $94 \%$ of the samples had adequate bone marrow for offering a diagnosis. The most common diagnosis based on bone marrow examination was infiltration by a lymphoma $(\mathrm{n}=11 ; 0.13 \%)$ followed by a myeloproliferative neoplasm $(\mathrm{n}=5)$. A definite diagnosis could not be offered in cases $(n=4)$. Conclusion: The most common hematological disorder diagnosed in extreme elderly patients based on bone marrow examination is infiltration by a lymphoma. BM examination not only helps to make specific diagnoses but it is an extremely valuable, quick and economically viable procedure to exclude major hematological disorders including certain forms of hematologic malignancy in appropriate clinical setting.

\section{P-CBCT 01}

The Grey Zone of Thrombocytopenia: Accuracy of Automated analyser vs Manual method

\section{Gayatri Gogoi ${ }^{1}$, Ashim Manta ${ }^{2}$, Shreya Kar $^{3 \#}$}

Email ID for Correspondence: kar.shreya1991@gmail.com Assam Medical College and Hospital, Dibrugarh; ${ }^{1}$ Assistant Professor, Department of pathology, AMCH; ${ }^{2}$ Demonstrator, Department of pathology, AMCH; ${ }^{3} \mathrm{PGT}$, Department of pathology, $\mathrm{AMCH}$

Introduction: Reliability of platelet estimation is essential to make clinical decision specially when platelet transfusion is considered. Most common cause of platelet not being accurately counted by automated analyser is the presence of giant platelet, satellitism and platelet clumping. Another cause in north-east region is Harris platelet syndrome most common cause of inherited thrombocytopenia characterised by low platelet count, high Mean Platelet Volume and absence of bleeding. Their low platelet count causes concern while administering chemotherapy or undertaking surgical procedure. Objective: To compare platelet count results of automated analyser with manual Peripheral blood smear examination and Neubauer slide counting. Materials and Methods: This study was conducted in department of pathology, AMCH, Dibrugarh from $1^{\text {st }}$ May, 2017 to $31^{\text {st }}$ May, 2017. All the blood samples in EDTA vial having low platelet count by automated analyser is evaluated by PBS examination and Neubauer chamber counting. Result: A total of 3803 patients were investigated for haematological parameters; 564 are males and 233 are females. On automation, 797 cases were thrombocytopenic (445 mild;257 moderate; 95 severe thrombocytopenia) whereas by manual method only 423 cases were thrombocytopenic ( 238 mild; 125 moderate;60 severe thrombocytopenia). 451 out of 797 cases have high MPV. Most of the transition occurred in mild and moderate thrombocytopenic group which converted to normal range of platelet. 
Discussion and Conclusion: Various reasons of false low platelet count by automated analyser was found such as large platelet size, micro clot formation during pre analytical period. So, manual examination by peripheral blood smear should always be considered whose platelet count is low by automation. This shows that the large numbers of cases labelled as thrombocytopenia by automated hematology analyzer are likely to cause confusion in clinical diagnosis and management. So both clinicians and laboratory personnel should be aware of this fact and should give due emphasis on manual method as well to establish accuracy and search for causes when possible.

\section{P-CBCT 02}

Study on the Performance of an Artificial Intelligence System for Image Based Analysis of Peripheral Blood Smears

Renu Ethirajan", Dheeraj Mundhra, Jaiprasad Rampure, Shreepad Potadar, Sukrit Mukherjee, Bharath Cheluvaraju

Email ID for Correspondence: renu@sigtuple.com SigTuple Technologies Pvt Ltd

Introduction: In this study, we evaluate SHONIT, a cloud based artificial intelligence (AI) system for automated analysis of images captured from peripheral blood smears using an automated digital microscope. We assess SHONIT's performance in categorization and classification of white blood cells (WBCs) by comparing SHONIT's results with haematology analysers and manual microscopy for manually stained smears. Objectives: To clinically validate the efficacy and accuracy of WBC differentials produced by SHONIT by comparison with existing state-of-the-art haematology analyser and manual microscopy. Material and Methods: The study was carried out over 100 samples. The cases included both normal and abnormal samples, wherein the abnormal cases were from patients with one or more quantitative or qualitative flagging. All the smears were created using Hemaprep auto-smearer and stained using May Grunwald Geimsa stain. They were scanned and analysed by SHONIT for WBC differentials under $400 \times$ magnification. WBC morphological classification by SHONIT was verified by an experienced haematopathologist. Quantitative parameters were analysed by computing the mean absolute error of the WBC differential values between SHONIT and Sysmex XN3000 and between SHONIT and manual microscopy. Results: The mean absolute error between WBC differential values of manual microscopy and SHONIT were $7.67 \%, 5.93 \%, 4.58 \%, 2.69 \%$, $0.44 \%$ for neutrophil, lymphocyte, monocyte, eosinophil, and basophil respectively. The mean absolute error between WBC differential values of Sysmex XN3000 and SHONIT were 8.73\%, 5.55\%, 3.63\%, $2.12 \%, 0.45 \%$ for neutrophil, lymphocyte, monocyte, eosinophil, and basophil respectively. Conclusions: SHONIT has proven to be effective in locating and examining WBCs. It saves time, accelerates the turnaround-time, and increases productivity of pathologists. It has helped to overcome the time-consuming effort associated with traditional microscopy.

\section{P-CBCT 03}

Interference of High Leucocytes Count in Estimation of Cellular Haemoglobin

Kritika Pandey ${ }^{\#}$, Sona Duseja, Dr. Ganesh Kumar, Vilas Lad, Shilpa Kushte, Shashikant Mahadik, Dr. Sumeet Gujral, Dr. P.G. Subramanian, Dr. prashant tembhare

Email ID for Correspondence: speedgallop@gmail.com Department of Haematopathology, Tata Memorial Centre, Mumbai, India

Introduction: Haemoglobin $(\mathrm{Hb})$ value is the main parameter that assesses the degree of anaemia in an individual patient. It is a principle parameter of complete blood count (CBC) and is estimated using automated analyzers based on colorimetric methods. This method involves lysing the RBCs, stabilizing the $\mathrm{Hb}$ with cyanide (or noncyanide reagents) and measuring the haemoglobin colorimetrically. Colorimetric measurement of $\mathrm{Hb}$ principally depends on light absorption which can be affected by other factors like Hyperbilirubinemia, Hyperlipidemia, etc. Similarly, high leucocyte count can also interfere with light absorption in a colorimetric method for $\mathrm{Hb}$ values which is seen in cases of Acute Leukemia, Chronic Myeloid Leukemia, etc. In addition to the colorimetric method, Siemens Advia 2120 gives a unique parameter CHCM in which the cellular $\mathrm{Hb}$ in each RBC is determined by flowcytometry (laserlight scattering characteristics) which has no interference in $\mathrm{Hb}$ estimation. In this study, we evaluated the interference of high leucocytes count in $\mathrm{Hb}$ estimation by a colorimetric method using cellular $\mathrm{Hb}$ method as a reference method. Objectives: To evaluate the interference of high leucocytes in the estimation of haemoglobin by colorimetric methods. Material and Methods: This prospective study was performed using 220 fresh blood sample anticoagulated with K2-EDTA. Included 200 samples with very high leucocyte count $(>80 \times 103 / \mathrm{ul})$ and 20 normal samples from healthy donors. Blood samples were analyzed using automated analyzer Siemens ADVIA-2120i that estimates

Table 1 .

\begin{tabular}{|c|c|c|c|c|c|}
\hline & \multicolumn{2}{|c|}{$\begin{array}{l}\text { COLORIMETRIC } \\
\text { Hb (Test method) }\end{array}$} & \multicolumn{2}{|c|}{$\begin{array}{l}\text { CELLULAR Hb } \\
\text { (Ref Method) }\end{array}$} & \multirow{2}{*}{$\begin{array}{l}\text { Mean (S.D) } \\
\text { of \% } \\
\text { Difference } \\
\text { of Hb } \\
\text { between two } \\
\text { methods }\end{array}$} \\
\hline & MEDIAN & RANGE & MEDIAN & RANGE & \\
\hline $\begin{array}{l}\text { HIGH } \\
\text { WBC }\end{array}$ & 8.4 & $\begin{array}{l}2.921- \\
13.87\end{array}$ & 7.5 & $\begin{array}{l}1.76- \\
13.23\end{array}$ & $29.4-48.03$ \\
\hline $\begin{array}{l}\text { NORMAL } \\
\text { WBC }\end{array}$ & 14.3 & $\begin{array}{r}11.65- \\
16.94\end{array}$ & 14.3 & $\begin{array}{r}11.77- \\
16.82\end{array}$ & $0.21-6.07$ \\
\hline
\end{tabular}


Comparison between $\%$ difference of $H b$ in samples with high WBC count vs. normal WBC count

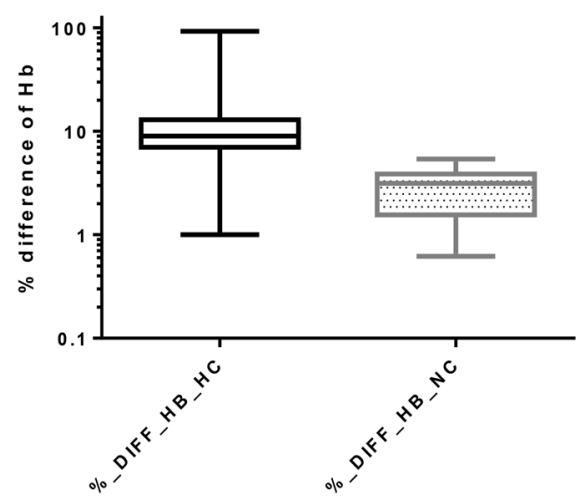

Figure 1 .

haemoglobin using two techniques: 1)colorimetric haemoglobin $(\mathrm{Hb})$ and 2) cellular haemoglobin $(\mathrm{Hb}$-cell $)=\mathrm{RBC} \times \mathrm{MCV} \times \mathrm{CHCM}$. Results- In samples with high leucocyte count, the median and range of WBC per microliterare mentioned in Table-1. In 15 samples with leucocyte count higher than 4,00,000, a dilution of 1:8 was performed with sheath fluid. Between calorimetric- $\mathrm{Hb}$ and cellular- $\mathrm{Hb}$ using Mann Whitney-U test. The percentage-differences between calorimetric- $\mathrm{Hb}$ and cellular- $\mathrm{Hb}$ was significantly high in samples with high WBC count with p-value. Conclusions: From above study we can conclude that $\mathrm{Hb}$ value measurement by colorimeter method are falsely high in sample with high leucocyte count. Hence it is suggested to use laser based cellular haemoglobin estimation in sample with high leucocyte count. To determine the level of interference of high leukocyte count as compared to normal counts, we compared the percentage-differences between calorimetric-Hb and cellular-Hb using Mann Whitney-U test. The percentage-differences between calorimetric- $\mathrm{Hb}$ and cellular-Hb was significantly high in samples with high WBC count with $p$-value $<0.001$ (refer to figure 1). Interestingly, there was no co-relation between WBC count and percentage-difference in $\mathrm{Hb}$ value (Pearson correlation, $r=0.0865$ ). Conclusions: We concluded that the $\mathrm{Hb}$ value estimated using colorimeter method gives falsely high $\mathrm{Hb}$ levels in samples with high leucocyte count. Hence, we suggest hemoglobin estimation using laser-based cellular-Hb as a method of choice in samples with high leucocyte count.

\section{P-CBCT 04}

\section{Determining the Stability of CBC Parameters in Blood Sample from Cancer Patients Pancytopenia}

Shilpa Jadhav", Nisha Adhikari, Shilpa Kushte, Shashikant Mahadik, Dr. Sumeet Gujral, Dr. P.G. Subramanian, Dr. Prashant Tembhare

Email ID for Correspondence: shilpaj0091@gmail.com Department of Haematopathology, Tata Memorial Centre, Mumbai, India
Table 1 (a): CV\% of three parameters of $\mathrm{CBC}$ in blood samples from normal individuals $(\mathrm{n}=20)$

\begin{tabular}{lll}
\hline Calculated fresh sample CV\% & & \\
\hline Haemoglobin $(\mathrm{CV} \%=3.0)$ & MEAN & 1 \\
& SD & 0.89 \\
Platelet counts $(\mathrm{CV} \%=5.5)$ & MEAN & 4.36 \\
Neutrophil percentages $(\mathrm{CV} \%=3.3)$ & SD & 3.82 \\
& MEAN & 1.66 \\
& SD & 1.6 \\
\hline
\end{tabular}

Introduction: Peripheral blood sample stability is defined as the capability of sample to retain original $\mathrm{CBC}$ parameter for defined period with specific storage criteria. Accurate measurement of hematologic analytes and blood cells is essential for correct diagnosis, treatment and follow up of patients with haematological disorders. It has been shown that some of the parameters of CBC are stable from 24-72 hours with proper collection, transport and storage. Pancytopenia is commonly seen in cancer patients, especially while taking chemotherapy. In these patients, CBC parameters like absolute neutrophil count, haemoglobin value and platelet counts are critical in the management of the patients. However, there is no data on the stability of CBC parameters in cancer patients with or without treatment with pancytopenia. Therefore, we evaluated the stability of these parameters in cancer patient samples with pancytopenia. Objectives: Determining the stability of CBC parameters in the cancer patients with pancytopenia at different time intervals. Material and Methods: : We studied CBC parameters in peripheral blood specimen of 100 cancer patients with pancytopenia on ADVIA2120I analyser.CBC was performed at four time-intervals that include 1-2 hours, 24 hours, 48 hours and 72 hours after collection of the sample in K2EDTA vacutainers in Tata Memorial Hospital, Mumbai. We calculated the percentage-difference between the values of various CBC parameters at $24 \mathrm{hrs}, 48 \mathrm{hrs} \& 72 \mathrm{hrs}$ and 1-2 hrs at room temperature and at $2-8^{\circ} \mathrm{C}$. We compared these results with laboratory CVs (from 20 normal samples) for all parameters. The percentage differences above the $\mathrm{CV}$ of individual parameter is considered as time-interval related differences. Results: We studied all the stability of all $\mathrm{CBC}$ parameters at different time-intervals in 100 peripheral blood samples from cancer patients (Age Range: 1-74; Male:Female-51:49). Due to limitation of words for the abstract, we mentioned the results of three important parameters i.e. absolute neutrophil counts, Hb levels and platelet counts. The values and percentage-differences of these three parameters at different time-intervals is summarized in Table 1(a), 1 (b), 1 (c) and 1 (d). Conclusion: Our study concluded that CBC parameters are not stable and shows significant deviation from the original results if tested time intervals greater than 24 hours in cancer patients with pancytopenia at room temperature i.e. $18-24{ }^{\circ} \mathrm{C}$ but not at $2-8{ }^{\circ} \mathrm{C}$. Hence, $\mathrm{CBC}$ must be performed with 24 hours of sample collection in cancer patients with pancytopenia and should be stored at room temperature. 
Table 1 (b) Number of samples within and out of CV\% limits with respect to three CBC parameters in pancytopenia samples (n $=100)$

\begin{tabular}{|c|c|c|c|c|c|c|c|c|c|c|c|c|c|c|c|c|c|c|}
\hline \multirow{3}{*}{$\begin{array}{l}\mathrm{CV} \% \\
\text { Time-intervals (in hours) }\end{array}$} & \multicolumn{6}{|c|}{ Haemoglobin CV\% $=3.0 \%$} & \multicolumn{6}{|c|}{ Platelet CV\% $=5.5 \%$} & \multicolumn{6}{|c|}{$\%$ Neutrophil CV\% $=3.3 \%$} \\
\hline & \multicolumn{3}{|c|}{ RT } & \multicolumn{3}{|c|}{$2-8^{\circ} \mathrm{C}$} & \multicolumn{3}{|l|}{ RT } & \multicolumn{3}{|c|}{$2-8^{\circ} \mathrm{C}$} & \multicolumn{3}{|l|}{ RT } & \multicolumn{3}{|c|}{$2-8^{\circ} \mathrm{C}$} \\
\hline & 24 & 48 & 72 & 24 & 48 & 72 & 24 & 48 & 72 & 24 & 48 & 72 & 24 & 48 & 72 & 24 & 48 & 72 \\
\hline Within acceptable lab CV\% & 83 & 83 & 80 & 51 & 55 & 35 & 24 & 21 & 21 & 29 & 26 & 20 & 27 & 22 & 22 & 28 & 31 & 21 \\
\hline Greater than acceptable lab CV\% & 17 & 17 & 20 & 49 & 45 & 65 & 76 & 79 & 79 & 71 & 74 & 80 & 73 & 78 & 78 & 72 & 69 & 76 \\
\hline
\end{tabular}

Table 1 (c) Mean and SD of out of acceptable lab CV\% (percentagedifference) of different parameters at RT

\begin{tabular}{|c|c|c|c|c|c|c|c|c|c|}
\hline & \multicolumn{3}{|c|}{$\begin{array}{l}\text { Haemoglobin } \\
\mathrm{n}=100\end{array}$} & \multicolumn{3}{|c|}{ Platelet $n=100$} & \multicolumn{3}{|c|}{$\begin{array}{l}\% \text { Neutrophil } \\
\mathrm{n}=100\end{array}$} \\
\hline & $\begin{array}{l}\mathrm{RT} \\
24\end{array}$ & RT48 & RT72 & $\begin{array}{l}\text { RT } \\
24\end{array}$ & RT48 & RT72 & $\begin{array}{l}\text { RT } \\
24\end{array}$ & RT48 & RT72 \\
\hline Mean & 3.03 & 3.03 & 3.45 & 12.21 & 14.19 & 14.19 & 17.61 & 19.54 & 22.79 \\
\hline SD & 8.06 & 7.54 & 9.23 & 9.35 & 9.16 & 9.16 & 22.82 & 24.97 & 33.32 \\
\hline
\end{tabular}

Table 1 (d) Mean and SD of out of acceptable lab CV\% (percentagedifference) of different parameters at $2-8^{\circ} \mathrm{C}$.

\begin{tabular}{|c|c|c|c|c|c|c|c|c|c|}
\hline & \multicolumn{3}{|c|}{$\begin{array}{l}\text { Haemoglobin } \\
\mathrm{n}=100\end{array}$} & \multicolumn{3}{|c|}{ Platelet $\mathrm{n}=100$} & \multicolumn{3}{|c|}{$\begin{array}{l}\% \text { Neutrophil } \\
\mathrm{n}=100\end{array}$} \\
\hline & 24 & 48 & 72 & 24 & 48 & 72 & 24 & 48 & 72 \\
\hline Mean & 6.25 & 5.59 & 7.75 & 11.12 & 13.82 & 19.87 & 15.34 & 13.52 & 21.85 \\
\hline SD & 10.77 & 9.92 & 11.31 & 10.41 & 12.63 & 18.14 & 19.82 & 18.91 & 56.99 \\
\hline
\end{tabular}

\section{P-CBCT 05}

\section{Mentzer Index for Differential Diagnosis of Iron Deficiency Anaemia and Beta Thalassemia Trait}

\section{Dr. Gayatri Gogoi ${ }^{1}$, Dr. Reshma Imran ${ }^{2 \#}$}

Email ID for Correspondence: reshmaimran27@gmail.com Assistant Professor, 2. PGT, second year; Department of pathology, Assam Medical College Dibrugarh, Assam

Introduction: Thalassemia trait is commonly seen in certain communities of India as well as North East. Individuals with thalassemia trait usually have an asymptomatic course with mild microcytic hypochromic anaemia. In India as well as in Assam, the most common cause of microcytic hypochromic anaemia is iron deficiency. Hence it is important to differentiate between the two. Although the definitive diagnosis of thalassemia trait can only be done by haemoglobin electrophoresis, there are certain indices which can differentiate between the two. Mentzer index is one such index. Objectives: The aim of our study is to evaluate the reliability of Mentzer Index in differentiating iron deficiency anaemia and thalassemia trait. Material and Methods: In our study we have taken a total of 60 patients, 30 patients of iron deficiency anaemia and another 30 of thalassemia trait who have been diagnosed by iron studies and haemoglobin electrophoresis respectively. Patients receiving blood transfusion in the last 3 months have been excluded. Results: Mentzer Index of $<13$ (indicating thalassemia trait) and $>13$ (indicating iron deficiency anaemia) has been found to be a reliable screening criteria to differentiate between the two. Conclusions: Since both iron deficiency anaemia and thalassemia trait are widely prevalent in our place, and being a resource poor state, Mentzer Index can be used as an important screening tool. The doubtful cases will however have to be confirmed by haemoglobin electrophoresis.

\section{P-CBCT 06}

Volume-Conductivity-Scatter (VCS) Parameter SD-AL2-LY of CBC Analyzer Beckman Coulter DXH800 is Highly Sensitive and Specific for Automated Detection of Malaria

Komal Bhanushali ${ }^{\#}$, Rashida Ansari, Arti Shinde, Shilpa Kushte, Shashikant Mahadik, DR. Ganesh Kumar, DR. Sumeet Gujaral, DR. P. G. Subramanian, DR. Prashant Tembhare

Email ID for Correspondence: komalbhanushali92@gmail.com Department of Hematopathology, Tata Memorial Centre, Mumbai, India

Introduction: Automated cell counters can be an adjunct to light microscopy in the diagnosis of malaria. Beckman Coulter DxH800 identifies malarial parasites in nucleated $\mathrm{RBC}$ region using the low angle and axial light scatter properties in nucleated red blood cell (nRBC) plot. This region is not specific to malarial parasites as debris and rarely smaller nuclear material can lie in this region. This study aims at identifying VCS parameters to detect malarial parasites.

Objectives:

1. To study the utility of volume, conductivity and scatter (VCS) parameters of leukocytes and nRBCs in the detection of malaria

2. To differentiate smear positive cases of malaria from smear negative controls using these parameters.

Material and Methods: A total of 678 peripheral blood samples were included in the study. Complete blood counts were done in a Beckman Coulter DxH800 automated counter. The samples included 20 normal controls (healthy volunteers). Peripheral blood film stained with Wright's stain was used for malaria detection. Peripheral smear examination by light microscopy was considered the gold standard (true positive). Volume, conductivity and light scatter properties of neutrophils, lymphocytes, monocytes, eosinophils, nucleated red blood cell (nRBCs) and Non-nucleated red blood cells (N-nRBCs) were studied. The parameters which could differentiate between malaria cases and the controls were identified. The cut off values for VCS parameters of CBC were defined using ROC analysis on MedCalc software. Results: Of the 678 samples, malarial parasite was detected in blood smears from $37(5.45 \%)$ samples by light microscopy. Haemoglobin did not show significant difference among the 
Table 1 .

\begin{tabular}{llll}
\hline Parameters & True positive & False positive & True negative \\
\hline SD-V-NE & High & WNL & WNL \\
SD-C-NE & WNL & High & WNL \\
SD-V-LY & High & WNL & WNL \\
SD-C-LY & High & WNL & WNL \\
MN-LMALS-LY & Low & WNL & WNL \\
SD-LALS-LY & High & WNL & WNL \\
MN-AL2-MO & High & WNL & WNL \\
MN-AL2-EO & High & WNL & WNL \\
MN-C-NNRBC & High & WNL & WNL \\
MN-LMALS-NNRBC & High & WNL & WNL \\
\hline
\end{tabular}

Table 2 .

\begin{tabular}{|c|c|c|c|c|c|}
\hline Parameters & AUC & $\mathrm{p}$ Value & $\begin{array}{l}\text { Sensitivity } \\
(\%)\end{array}$ & $\begin{array}{l}\text { Specificity } \\
(\%)\end{array}$ & Cut off \\
\hline SD-V-LY & 0.839 & $<0.0001$ & 77.78 & 85.51 & $>19.97$ \\
\hline SD-C-LY & 0.766 & $<0.0001$ & 83.33 & 64.57 & $>10.52$ \\
\hline $\begin{array}{l}\text { SD-LMALS- } \\
\text { LY }\end{array}$ & 0.774 & $<0.0001$ & 69.44 & 80.84 & $>22.51$ \\
\hline SD-LALS-LY & 0.795 & $<0.0001$ & 83.33 & 66.18 & $>13.48$ \\
\hline SD-AL2-LY & 0.872 & $<0.0001$ & 75 & 87.12 & $>13.34$ \\
\hline MN-V-MO & 0.696 & $<0.001$ & 75 & 62 & $>182$ \\
\hline SD-V-MO & 0.721 & $<0.0001$ & 86.11 & 56.36 & $>24.05$ \\
\hline $\begin{array}{l}\text { MN-UMALS- } \\
\text { MO }\end{array}$ & 0.666 & $<0.0001$ & 88.9 & 44.12 & $\leq 104$ \\
\hline $\begin{array}{l}\text { SD-LMALS- } \\
\text { MO }\end{array}$ & 0.598 & 0.0435 & 69.44 & 48.79 & $>15.02$ \\
\hline SD-AL2-MO & 0.639 & 0.0044 & 55.56 & 67.63 & $>15.37$ \\
\hline
\end{tabular}

groups. Leukopenia and thrombocytopenia were statistically significant in the group with malaria. The shift in the VCS parameters of $\mathrm{CBC}$ in malaria positive versus negative samples were studied and parameters with statistically significant $(\mathrm{p}<0.05$, Mann-Whitney) shift are outlined in Table 1 . The sensitivity and specificity using the ROC analysis defined cut off values of VCS parameters of CBC for the detection of malaria in the blood samples are shown in Table 2. Among these parameters "SD-AL2-LY" is found to be the most useful with sensitivity and specificity of $75 \% \& 87.12 \%$ respectively. Conclusions: VCS Parameter "SD-AL2-LY" of CBC analyzer Beckman Coulter DxH800 is highly sensitive and specific for automated detection of malaria. Thus, VCS parameters in Beckman Coulter DxH800 can be helpful in the initial screening of malaria and raise an alarm for microscopic examination. Better utilisation of VCS parameters help in development of algorithms like Malaria factor for malaria detection in automated cell counters.

\section{P-CBCT 07}

Assessment of Automated Red Cell and Reticulocyte ParametersUtility Anemia Categorisation

Brijendra bahadur singh", Mili jain, Rashmi Kushwaha, Wahid Ali, A.K.Tripathi, K.K.Sawlani, Ashutosh kumar

Email ID for Correspondence: brijendra72u@gmail.com; Loveena Rastogi (Presenting Authors)

King George's Medical University, Lucknow

Introduction: Differentiation of absolute and functional iron deficiency is essential for appropriate treatment. The automated red cell and reticulocyte parameters may show variable degree of change in different subtypes of anemia. Objectives: Assessment of automated red cell (MCV, MCHC, RDW, \%Hypo, \%Micro) and reticulocyte parameters (Reticulocyte hemoglobin content-CHr) in different subtypes of anemia and their utility in categorisation of anemia. Material and Methods: 112 cases of anemia (52 Iron deficiency anemia- IDA, 31Anemia of chronic disease -ACD, 13 ACD with IDA- ACD combi, 16 Thalassemia trait -TT) were enrolled after evaluation of hemoglobin, Iron profile, CRP and HPLC analysis. All samples were analysed on ADVIA 2120 (Siemens diagnostics). Statistical analysis was done using SPSS software. Results: \%Hypo cells were significant increased $(\mathrm{p}<0.01)$ in IDA as compared to ACD \& ACD combi. \% Micro cells were higher in IDA but not significant. CHr was significantly $(\mathrm{p}<0.001)$ lower in IDA as compared to ACD, however did not differ in ACD and ACD combi. \% Micro cells were significantly higher in TT and \%hypo cells were significantly higher in IDA. Ratio of \%micro and \%hypo cells was significantly higher in TT as compared to IDA. Conclusions: Automated parameters \% hypo and $\mathrm{CHr}$ are helpful in differentiating IDA from ACD and ACD combi. Conventional red cell parameters (MCV and RBC count) differ significantly between TT and IDA. Parameters measuring on individual cell basis \%micro, \%hypo, ratio of \%micro and \% hypo are useful in differentiating IDA and TT.

\section{P-CBCT 08}

A Comparative Study of Complete Haemogram and Iron Profile From Cord Blood of New Born with Respect to Gestation Age

Dr. Ashish Kumar Jha, Dr. Madhumita Mondal, Dr. Swapan kumar Sinha, Dr. Utpal Chaudhuri, Dr. Debdas Bose

Background: Infants and toddlers are vulnerable to the effects of anaemia due to rapid growth and development of brain and rest of the body from birth to age three. Preterm and/or low birth weight infants are more vulnerable. Iron-deficiency anaemia affects neurological development due to reduced myelination of the spinal cord, as well as a change in myelin composition. Objectives: To correlate complete hemogram, serum iron, TIBC and serum ferritin of new born of $\leq 32$ weeks (wks), 33-36 weeks, 37-40 weeks and $>40$ weeks gestation age from umbilical cord blood for early detection of anaemia and perinatal management of complications.

Material and Methods: Ninety-six new born cord blood was collected of gestation age $\leq 32$ weeks (10), 33-36 weeks (12), $37-40$ weeks (60), > 40 weeks (14). Comparison of complete blood count parameters with respect to gestation age was done in a tertiary 
care hospital, Kolkata by ANOVA (one-way analysis of variance) test with Graphpad Instat 3 software. Results: We found significant correlation coefficient for haematocrit $(\mathrm{p}=0.024)$, mean corpuscular volume $(\mathrm{p}=.0049)$, platelet count $(\mathrm{p}=0.0097)$, red cell distribution width $(\mathrm{p}=0.033)$, serum iron $(\mathrm{p}=0.0034)$, serum TIBC $(p=0.0482)$. Conclusion: Currently, there is no clear recommendation of iron supplementation to the new born with decreased iron content other than exclusive breast feeding. As cord blood detection of blood parameters is an early and non-invasive procedure of estimation of foetal iron deficiency, it may be recommended in health care programmes for early detection and treatment of iron deficiency before the development of actual anaemia.

Keywords Hemogram, cord blood, Iron profile

\section{P-CBCT 09}

Utility of Automated High Fluorescence Body Fluid (HF-BF\%) Parameter as a Predictor of Malignancy in Body Fluids

\section{Jasmita Dass, Vandana Arya, Jyoti Kotwal}

\section{Sir Ganga Ram Hospital, New Delhi}

Introduction: There has been a significant advancement in the Hematology analyzer technology for analysis of body fluids (BF) in recent years. Traditionally manual Neubauer chamber counts were used to determine total cell counts in BF. However, this carries a high degree of imprecision. BF analysis is also particularly important to diagnose malignant serous effusions and requires skilled personnel. Sysmex XN-1000 utilizes semiconductor laser flow cytometry and nucleic acid fluorescence techniques to study body fluids. Objective: The primary objective of this study was to look at the high fluorescence-body fluid (HF-BF) parameter and study its correlation with the presence of malignant cells in BF. Correlations between manual and automated differential counts were also evaluated to assess the performance of the Sysmex XN-1000. Methods: A total of 851 samples including 360 ascitic (AF), 264 pleural (PF) and 227 cerebrospinal fluid (CSF) specimens were analyzed by BF mode on Sysmex XN1000 cell counter. The samples were run within 2 hours of receipt in the lab. Two level BF controls was run each day for quality control. Giemsa stained deposit smears were examined for differential leucocyte counts. A corresponding cytopathology specimen was received for $254 \mathrm{AF}, 217 \mathrm{PF}$ and $96 \mathrm{CSF}$ samples. Of these, 20, 13 and 2 samples were positive for malignancy respectively. Receiver-operator-characteristic-curve analysis was performed to identify a cut-off for $\mathrm{HF}-\mathrm{BF} \% / 100 \mathrm{WBC}$ to predict malignant BF using SPSS v20.0 software. Correlation between the percentages of polymorphonuclear cells (PMNs\%) and mononuclear cells (MNs\%) between manual and automated methods were calculated using Microsoft Excel. Results: There was an excellent correlation between PMN\% and MNs\% using manual and automated BF analysis when only AF and PF samples were analysed $(\mathrm{R}=0.9$ and 0.91 respectively). For CSF samples however, there was a poor correlation between manual and automated $\mathrm{BF}$ analysis for the same parameters $(\mathrm{R}=0.18$ and 0.19 respectively). HF-BF\%/100WBCs was significantly different between all malignant and benign BF samples analysed (37.2 vs $4.2, \mathrm{p}=0.0001)$. There was a statistically significant difference in the HF-BF\%/ $100 \mathrm{WBCs}$ of the malignant and benign $\mathrm{AF}$ and $\mathrm{PF}$ samples analysed (38.8 vs $4.6, p=0.0001$ ). Using ROC analysis, a cut-off of $2.45 \%$ / $100 \mathrm{WBC}$ for HF-BF could differentiate all malignant $\mathrm{BF}$ from benign at a sensitivity and specificity of $60 \%$ and $59.6 \%$ respectively [AUC $=0.688 ; 95 \%$ CI-0.598-0.778]. If the same analysis was restricted to $\mathrm{AF}$ and $\mathrm{PF}$, a cut-off of $4.15 \% / 100 \mathrm{WBC}$ for $\mathrm{HF}-\mathrm{BF}$ could differentiate malignant $\mathrm{AF} \& \mathrm{PF}$ from benign at a sensitivity and specificity of $60 \%$ and $65.9 \%$ respectively [AUC $=0.727 ; 95 \%$
CI-0.647-0.807]. Conclusion: The Sysmex XN-1000 gives an excellent correlation between automated and manual differential counts for $\mathrm{AF}$ and PF. Although the HF-BF\%/100 WBC parameter is statistically different between malignant and benign BF, the cut-offs generated have a sub-optimal sensitivity and specificity to be able to reduce the requirement of manual examination of $\mathrm{BF}$.

\section{P-CBCT 10}

Evaluation of Platelet Parameters in Patients of Ischemic Heart Disease and Ischemic Stroke

Dr Imtiaz Ahmed ${ }^{\#}$, Krishna S Nair, Nishant Raman, Lt Col S Venkatesan

Email ID for Correspondence: imtiazamc@gmail.com Armed Forces Medical College, Pune

Introduction: Ischemic heart disease and ischemic stroke are among the most common causes of morbidity and mortality worldwide, which is by and large a lifestyle disease. One of the underlying pathology is atherosclerosis which may progress to a thrombus formation and subsequent lead to a thrombotic event, especially in an unstable atherosclerotic plaque, which is the result of a cascade of platelet reaction. Certain characteristics of the platelet may predispose an individual to risks of acute coronary syndrome (ACS) and ischemic stroke. It is known that large platelets are denser, aggregate more rapidly with sub endothelial collagen, produce more thromboxane A2 and express more glycoprotein Ib and glycoprotein IIb/IIIa receptors. These characteristics may lead to increased thrombosis, and possibly thrombotic events. Objectives: The objective of this study is to measure the various platelet parameters which include mean platelet volume (MPV), platelet distribution width (PDW), plateletcrit (PCT), Platelet large cell ratio (P-LCR) in patients with thrombotic events, and to evaluate their role, if any, as a marker of an impending thrombotic event. Material and Methods: Our study includes 200 healthy controls and 200 cases who were admitted to our hospital for Acute coronary syndrome and ischemic stroke during the period of April 2017 to October 2017. Total platelet count, MPV, PDW, PCT and P-LCR of all these patients were evaluated using a five part Hematology Analyzer (Model- Sysmex XT2000i). The platelet parameters were obtained and compared with normal healthy controls. Results: Among the parameters obtained, the risk of a thrombotic event in the P-LCR group was 1.89 times higher compared to the controls. Similarly those in the MPV and PDW group, the risk was 1.27 times and 1.11 times higher than the controls. While there was a negligible risk in cases those showed increased PCT and no risk in patients with high platelet counts. Conclusions: This study demonstrates that high P-LCR and MPV and PDW are associated with occurrence of thrombotic events. Evaluation of this parameters in high risk patients can predict the possibility of an impending thrombotic event.

\section{P-CBCT 11}

\section{More Specificity than Sensitivity increases Laboratory productivity}

\section{Ms. Hetali Shah", Mrs. Bhavini Chauhan, Dr. Pankhi Dutta}

Email ID for Correspondence: hetalishah2794@gmail.com Kokilaben Dhirubhai Ambani Hospital 
Introduction: In the present study, we have evaluated the 5-part WBC differential count and WBC flagging obtained by ABBOTT CELL-DYN Ruby which works on the principle of optical countingMAPSS (multi-angled polarised scatter separation) technology $\left(0^{\circ}\right.$, $10^{\circ}, 90^{\circ}$ and $90^{\circ} \mathrm{D}$ sensors) with the 5-part WBC differential count and WBC flagging obtained by SYSMEX XN-1000 which works on the fluorescence flowcytometry principle, while using the 200-cell differential count and WBC abnormalities obtained by the gold standard: manual review as reference. Objectives: Performance evaluation of WBC flags and 5-part differential obtained by ABBOTT CELL DYN Ruby, as compared to results obtained on the more popular XN1000 (Sysmex, Japan) keeping the gold standard: Manual Smear Review and 200-cell differential counts as the reference. Material and Methods: 207 random EDTA whole blood samples (including 7 known leukemic samples) were evaluated on Abbott CELL-DYN Ruby, and Sysmex XN-1000 and peripheral smears (PS) were reviewed for the same. Rumke's comparison method of acceptable range for the differential counts and truth table analysis method was utilized to compare different parameters. Results: Out of the 207 sample results obtained from CELL-DYN Ruby 75 sample results showed flags which necessitated PS review, while for Sysmex XN 1000, 122 sample results showed flags. The 5-part WBC counts by both the instruments were in good agreement with the 200-cell differential count. The sensitivity and specificity for Blast/Atypical Lymphocyte/Variant Lymphocyte flags by CELL-DYN Ruby was $48.83 \%$ and $92.07 \%$ respectively, while for Sysmex XN-1000 it was $92.85 \%$ and $57.57 \%$ respectively. The sensitivity and specificity for Immature Granulocytes/Left Shift flags by CELL-DYN Ruby was $45.45 \%$ and $100 \%$ respectively, while for XN-1000 it was $64.17 \%$ and $82.85 \%$ respectively. One sample with atypical (reactive) lymphocytes was missed by CELL-DYN Ruby. However, no blasts were missed by either instrument. Conclusions: CELL-DYN Ruby may reduce the slide review without compromising patient's safety.

\section{P-CBCT 12}

\section{Comparison of WBC Flags IN Coulter LH- 780 with Manual Smears: Role in Improving Laboratory Productivity}

\section{Anika Jain\#, Sushma Belukar, Sufiyan Ibrahim}

Email ID for Correspondence: jainanika445@gmail.com Kasturba Medical College

Introduction: Leucocyte differential count helps to identify distributional and morphological abnormalities. Automated hematologic analysers can perform them cost effectively within seconds. These analysers raise suspect and definite flags in case of deviations from normality. False positive rates for such flags are high on the Beckman Coulter LH780 and as such require microscopic examination of the slides, thereby increasing turnaround time and reducing laboratory productivity. In this study, we assessed the correlation between manual and automated leucocyte counts on LH -780. We also evaluated the sensitivity and validity of these flags and whether it can be used to reduce blood smear review. Objectives: 1) To determine the sensitivity and validity of LH -780 in predicating WBC flags by comparing with with manual peripheral smear. 2) To modify the criteria for peripheral smear review in order to increase the turnaround time. Material and Method: Retrospective study was conducted at KASTURBA MEDICAL COLLEGE over a period of one month (August- September 2017). 750 blood samples with WBC flags in LH -780 were selected in KMC laboratory. Corresponding peripheral smears were carefully studied and subsequently compared. Statical analysis using SSPS software, version 22 and Microsoft excel 2010 were done.

Results:

\begin{tabular}{lrrllll}
\hline $\begin{array}{l}\text { WBC } \\
\text { Parameter }\end{array}$ & Total & $\begin{array}{l}\text { True } \\
\text { positive }\end{array}$ & $\begin{array}{l}\text { False } \\
\text { positive }\end{array}$ & $\begin{array}{l}\text { False } \\
\text { negative }\end{array}$ & $\begin{array}{l}\text { Sensi- } \\
\text { tivity }\end{array}$ & $\begin{array}{l}\text { Validity } \\
\text { of WBC } \\
\text { Flags } \\
\text { (PPV) }\end{array}$ \\
\hline Leucopenia & 88 & 58 & 2 & 28 & $65 \%$ & $96.6 \%$ \\
Neutrophilia & 425 & 357 & 24 & 44 & $89 \%$ & $93.7 \%$ \\
Immature & 264 & 186 & 59 & 19 & $94.8 \%$ & $75.9 \%$ \\
$\quad$ granulocyte & & & & & & \\
Neutropenia & 157 & 81 & 11 & 58 & $58 \%$ & $81.8 \%$ \\
Eosinophilia & 89 & 41 & 30 & 18 & $69.5 \%$ & $57.7 \%$ \\
Monocytosis & 159 & 83 & 65 & 11 & $88 \%$ & $56 \%$ \\
Lymphocytos & 86 & 46 & 40 & 0 & $100 \%$ & $53.4 \%$ \\
$\quad$ Is & & & & & & \\
NRBC & 167 & 71 & 78 & 18 & $79.77 \%$ & $47.9 \%$ \\
Basophilia & 17 & 4 & 13 & 1 & $80 \%$ & $23.5 \%$ \\
NE blast & 29 & 4 & 25 & 0 & $100 \%$ & $14 \%$ \\
MO blast & 44 & 0 & 42 & 2 & $0 \%$ & $0 \%$ \\
LY-blast & 37 & 5 & 31 & 1 & $83.3 \%$ & $13.8 \%$ \\
\hline
\end{tabular}

Conclusion: The validity for leucopoenia, neutrophilia and neutropenia was high and therefore do not require peripheral smear review except for few conditions, in contrast to monoblast, neutrophilic blast and lymphoblast which had low validity. Peripheral smear reveiw should be done whenever blast flags are suspected by machine. This is similar to study conducted by Parya etal. which showed $74.9 \%$ invalid blast flags. Peripheral smear should also done in case of eosinophilia, basophilia and monocytosis.

\section{P-CBCT 13}

Evaluation of Body Fluid Mode on XN-1000 (Sysmex, Japan) Vis a Vis Classical Manual Methods \& Flowcytometry

Bhavini Chauhan, Anshita Dubey ${ }^{\#}$, Tushar Govekar, Jahnavi Naik, Kiran Ghodke, Pankhi Dutta

Email ID for Correspondence: anshita.doc @ gmail.com Kokilaben Dhirubhai Ambani Institute and Research Centre

Introduction: It is challenging to give accurate \& precise counts when they are very low (as often seen in body fluids) by the classical haemocytometer method. A fully automated body fluid counting method offered by the body fluid mode (BFM) of an automated cell counter, XN 1000 (Sysmex, Japan) which can also detect malignant cells, can be a boon for the haematology laboratory. Objectives: To evaluate the BFM of XN-1000 for measurement of cell counts, differential counts \& detection of abnormal cells vis-a vis the classical manual method with microscopy as well as an automated flowcytometric assay. Material and Methods: Precision studies were 
performed using 2 levels of controls and replicate analysis of a CSF with a count of 2 cells/ul. 686 samples of body fluids were analyzed by the BFM on $\mathrm{XN}-1000$ and results compared with those of the Neubaur's chamber and cytospin smear method for both total WBC counts and differential counts. 20 samples were analyzed by the BD leucocount method on the flow cytometer, BD FACS CANTO II. The High fluorescence WBCs (HF-WBC) on $\mathrm{XN}-1000$ were compared with the presence of macrophages, mesothelial cells \& malignant cells microscopically. Results: The coefficient of variation (CV\%) for low and high controls (range-47-99/ul and 216 - 360/ul) were $4.23 \%$ intra assay runs and $3.68 \%$ for interassay runs. For a CSF with a count of 3 cells/ul the CV\% of 10 runs was $16.11 \%$. The linearity was verified from 0 to 6750 cell/ul. The WBCs ranged from 0 to 80950/cumm (median -147.5/ cumm), the two methods showed a coefficient of correlation of 0.994. 20 fluids were compared with flowcytometry with a coefficient of correlation of 0.9915. Comparision of polymorphonuclear \& mononuclear cell counts showed an ' $\mathrm{r}$ ' value of $0.923 \& 0.750$ respectively. 475/666 fluids showed HFWBC, giving a sensitivity of $98.38 \%$ and a specificity of $53.05 \%$. Conclusions: Sysmex XN-1000 Body fluid mode is fast, accurate and precise with acceptable limits of linearity. In addition, it can detect non-haematopoietic cells.

\section{P-CBCT 14}

Can malaria factor become an indicator for diagnosing malaria in endemic country like India?

Ratnaprabha Maji", Parul Arora, Mamta Setia, A.K. Mukhopadhyay

Email ID for Correspondence: ratnaprabha.maji@rediffmail.com Department of Laboratory Medicine, All India Institute of Medical Sciences, New Delhi

Introduction: Malaria is a febrile illness, both life threatening and endemic in India, being transmitted to people by the bite of infected female Anopheles mosquito. Malaria factor can be used as a tool for giving a flag in order to alert the laboratory personnel. Objectives: Complete blood count is one of the simplest investigations, so we calculated malaria factor index for detection of malaria positive patients. Material and Methods: We collected 2 months retrospective data of 33 malaria positive patients and 30 healthy age matched controls. $3 \mathrm{ml}$ of peripheral venous blood samples, collected in EDTA vials, ran in Beckman Coulter LH750. Giemsa stained peripheral smears were microscopically examined. Hemozoin pigment, produced by parasite is ingested by monocytes, causing their activation with increased size and number. These monocytes were analyzed in hematology analyzer using the VCS (volume, conductivity, and scatter) technology for malaria detection. Results: The VCS parameters, SD of Lymphocyte Volume cases: $19.01 \pm 2.77$, Control: $14.70 \pm 1.48$, SD of Monocyte Volume cases: $27.72 \pm 4.41$, Control: $19.23 \pm 2.07$. A calculated malaria factor: (SD volume of lymphocytes $\times$ SD volume of monocytes/100) was used. Receiver operating characteristic curve of the malaria factor where the cut-off value of 3.7 gave $93.9 \%$ Sensitivity; $96.7 \%$ Specificity and area under the curve: 0.993. Conclusion: The gold standard for malaria is microscopic examination of blood films, but sometimes it can be missed in low parasitemia cases. The results of the malaria factor can easily be calculated along with the differential count with no extra expenses. It can also be used as a flag for routine screening for malaria.

\section{P-CBCT 15}

A Study to Compare Haematopoietic Progenitor Cell (HPC) Determined on the SYSMEX XN-9000 with Flowcytometric CD34 Count in Peripheral Blood and in the Harvested Peripheral Blood Stem Cell Graft from Autologous and Allogenic Donors

Introduction and Background: Hematopoietic stem cell transplantation is the current standard of curative care for all hemato-lymphoid malignancies as well as of many non-malignant conditions like marrow failure syndromes, inborn errors of metabolism and immunedeficiency syndromes. A successful bone marrow transplant requires a minimum of $2-3 \times 10^{\wedge} 6$ cells $/ \mathrm{kg}$ patient body weight of $\mathrm{CD}$ $34+$ cells in the product being transfused. CD34+ cell count in the peripheral blood has the best predictive value for the stem cell yield in the apheresis bag. The major disadvantages of this are the turn-around time and the cost, besides requiring specialized flow cytometric analysis equipment and trained operators. HPC is a parameter which is measured by automated platforms of Sysmex XN series. Recent studies have found a favourable correlation of XN-HPC with CD34 analysis and they inferred that it can be used as a surrogate marker for CD34 analysis in predicting optimal timing for stem cell collection by apheresis. Aims and Objectives: This is a pilot study which aims to analyse the reliability of HPC determined on the Sysmex XN-9000 in comparison with flowcytometric CD34 count in peripheral blood and in the harvested peripheral blood stem cell graft from autologous and allogenic donors. Materials and Methods: All donors both autologous and allogeneic who were mobilised in our centre will enrolled in our study after due consent. 30 donors were taken up for this pilot study. Paediatric donors were also included after due consents from parents and assents. TLC, MNC, HPC \& CD34 assays were done in both the peripheral blood prior to apheresis and the harvest product post-apheresis. Sysmex-XN 9000 was used for TLC, MNC and HPC assay. Slides were stained by automated slider stainer and evaluated by the investigator. CD34 Assay was done in flowcytometer using Modified ISH-AGE single platform protocol. Statistical Analysis: EPI data was used for data entry and statistical analysis was done using SPSS v20.0. Descriptive statistics were used to report Frequency and percentage for categorical data. Continuous data were evaluated by using Mean $\pm \mathrm{SD} /$ Median (IQR) based on the normality assumption. Correlations between the variables were calculated by using Pearson's correlation. Results: There were 12 autologous donors and 18 allogenic. 5 donors were of paediatric age group and rest were adults. XN-HPC and CD34+ cell counts in peripheral blood showed a correlation of $\mathrm{r}=0.90$ with $95 \%$ CI. Apheresis product showed a correlation of $r=0.85$ with $95 \%$ CI. Conclusions: XN-HPC compares favourably with CD34 analysis and may be a surrogate for CD34 analysis to predict optimal timing of PBPC collections in the future.

\section{P-CD 01}

Study of Von Willebrand Profiles of the Different Abo Blood Group Among Malay Population

\section{Rohaida Binti Abdul Rahman}

Email ID for Correspondence: rohaidaabdulrahman@yahoo.com Universiti Sains Malaysia- Advanced Medical and Dental Institute (AMDI), Malaysia

Introduction: In the year 2010, $0.002 \%$ von Willebrand patients were reported among 28 million of Malaysia population; $63 \%$ of them from the Malay ethnicity. Objectives: The objective of this research is to obtain the VWF profiles data of the different ABO blood type among 
Malays and to observe the association of demographic characteristic and smoking habit with the profiles. Material and Methods: One hundred and forty (140) Malay blood donors were involved in the research administered in the NBCKL. FVIII, vWF antigen, RiCof and CBA levels were measured by coagulometric clot detection, latex agglutination and ELISA methods. Results: Majority of the donor $(59.3 \%)$ were aged between $30-49$ years, male $(81.43 \%)$, non-smoker (74.3\%) and, overweight/obese (71.4\%). The Malays vWF profiles were slightly higher compared to Caucasians, Indian, Thais and Chinese but average ratio of vWF activity: antigen was slightly lower compared to other populations. The prevalence of low $(<50 \mathrm{IU} / \mathrm{dl})$ vWF antigen and CBA were also not common among the Malays. It was found that the highest level of vWF was among those with $\mathrm{B}$ blood group, followed by A and O. It was observed that the CBA levels were significantly interrelated with the age group while other demographic characteristics found to have no significant association. Conclusion: O-grouped Malay subjects had lower vWF profiles compared to non-O. The higher levels of vWF profiles and lower vWF activity: vWF antigen in Malays than other populations may suggest the impact of ethnicity on the plasma vWF levels and ethnic variation in the interaction of the vWF protein with collagen and platelet. These findings warrant for larger scale population-based study among our population as the small subjects in the current study represent a limitation. Molecular study among Malay patients is also suggested to find out the existence of vWF gene variant, which may result in a different vWF levels and activities in Malays.

\section{P-CD 02}

Quercetin 3, 3', 4', 5, 7-O-pentasulfate Prolongs Plasma Clotting Times and Reduces Thrombus Formation

Neha Gupta\#\#, Qudsia Rashid\#, Mohammad Abid§, Mohammad Zahid Ashrafi, Mohamad Aman Jairajpuri\#*

\#Protein Conformation and Enzymology Lab, §Organic Synthesis Lab, Department of Biosciences, Jamia Millia Islamia, New Delhi, India, ¿Department of Biotechnology, Jamia Millia Islamia, New Delhi, India

Email ID for Correspondence: nehadipas@gmail.com Jamia Millia Islamia

Introduction: Venous thromboembolism based complications are most common causes of morbidity and mortality worldwide. Current antithrombotic treatment strategies predominantly revolves around the use of heparin and its derivatives, however its polyanionic nature and multispecificity poses several complications including excessive bleeding. The quest for design of new oral antithrombotic molecules with reduced side effects envisages chemical modification of already known scaffolds like natural flavonoid for imparting the in vitro anticoagulant activity. Objectives: To test the anticoagulant and antithrombotic potential of the polysulfated derivative of Quercetin, a natural flavonoid. Material and Methods: In the present study, the pentasulfated form of the widely known flavanoid Quercetin, i.e. Quercetin 3, 3', 4', 5, 7-O- pentasulfate (QPS), was chemically synthesized, characterized and evaluated for its in vitro anticoagulant action. Following chemical synthesis, QPS was purified with RPHPLC and its structure was validated using FTIR, NMR and mass spectrometry. For in vitro anticoagulant assays, plasma was isolated from human blood. Activated Partial thromboplastin time (APTT),
Prothrombin Time (PT) and Thrombin Time (TT) were measured using commercial kits in the plasma samples pre-incubated with different concentration of QPS (10uM, $1 \mathrm{mM}, 2 \mathrm{mM}, 4 \mathrm{mM}, 7 \mathrm{mM}$ ) and Quercetin. In vivo antithrombotic potential was tested using rat model for venous thrombosis. Rats were injected with purified QPS at a dosage of $(0.5 \mathrm{mg} / \mathrm{kg})$, prior to thrombus induction and pro thrombotic read outs (thrombus weight and length) were measured and compared to vehicle controls. Anti-platelet activity of test compound was determined by ex-vivo whole blood platelet aggregation assay. Results: The clotting times (in sec) for non-sulfated Quercetin (viz. $\quad$ APTT $=41.67 \pm 0.032 ; \quad \mathrm{PT}=12.4 \pm 0.13 ; \quad \mathrm{TT}=9.45 \pm$ $0.11)$ and control plasma (APTT $=43.84 \pm 0.032 ;$ PT $=12.4 \pm$ 0.26 ; $\mathrm{TT}=9.28 \pm 0.18$ ) were found to be similar, even at maximum concentration tested $(10 \mathrm{mM})$. However, the presence of QPS exhibited prolonged APTT and PT with minimal effect on TT. At higher concentration of $7 \mathrm{mM}$, QPS completely blocked the intrinsic and extrinsic pathways (no clot formation). Our in vivo observations demonstrated that intravenous administration of QPS, significantly reduced thrombus formation in rat model of venous thrombosis. In addition, QPS injected rats also showed prolonged APTT with profound effect on PT as compared to non-sulfated Quercetin injected rats. Platelets isolated from QPS injected rats also showed a significant reduction in aggregation as compared to those from vehicle controls. Conclusions: QPS has significant anticoagulant potential with dose dependent retardation of intrinsic and extrinsic coagulation pathways and in vivo antithrombotic and antiplatelet action.

\section{P-CD 03}

Effect of FV Polymorphisms on FV Levels, APC Ratio and Its Association with DVT: AIIMS Experience

Background: FV polymorphisms have been associated with increased the risk of thrombosis. However, association of Arg485Lys and Met2120Thr polymorphisms with FV levels and APC ratio is controversial and role these polymorphisms with DVT is not studied in India. Aims: To study the effect of FV polymorphisms on FV levels, APC ratio and its association with DVT in India. Methods: 90 DVT patients and 90 age and sex matched healthy controls were study subjects. FV polymorphisms were detected by PCR-RFLP or allele specific PCR. FV activity levels were measured by one stage clotting assay using FV deficient plasma kit. APC ratios were determined as ratio of clotting time in the presence and absence of APC. Results: FV Leiden was seen in $14.4 \%(\mathrm{n}=13)$ of patients and absent in controls. $24.4 \%(\mathrm{n}=22)$ and $9 \%(\mathrm{n}=9)$ of patients and $15 \%$ $(\mathrm{n}=14)$ and $8 \%(\mathrm{n}=8)$ of controls carried Arg485Lys and Met2120Thr polymorphism respectively (Ala485Lys: $\mathrm{p}=0.010$, $\chi 2=5.61$; Met2120Thr: $p=0.255, \chi 2=1.29$ ). FV levels and APC ratio were significantly reduced in $485 \mathrm{Lys}$ carriers than non-carriers (FV levels: $p=0.001$, APC ratio: $p=0.003$ ). APC ratio was further reduced in carriers of both FV Leiden and 485Lys than FV Leiden alone $(\mathrm{p}=0.002)$. FV levels and APC ratio were not statistically significant when compared the carriers of $2120 \mathrm{Thr}$ with noncarriers (FV levels: $p=0.124$, APC ratio: $p=0.192$ ). Conclusions: In conclusion we observed significantly higher prevalence of Arg485Lys polymorphism in DVT patients as compared to controls. This polymorphism reduced FV levels and APC ratio, thus suggestive of its association with DVT. Met2120Thr polymorphism didn't show association with FV levels and APC ratio. However, study on larger sample size is required to strengthen these findings. 


\section{P-CD 04}

Impact of 603A > G Tissue Factor promoter polymorphism in the development of DVT in young Indians

Kamal Kishor", Amit Sharma, Hareram Pandey, Kanwaljeet Singh, Ravi Ranjan, Manoranjan Mahapatra, Renu Saxena

Email ID for Correspondence: kamal_kaushal23@yahoo.co.in All India Institute of Medical Sciences, Hematology, New Delhi, India

Introduction: Deep Vein thrombosis (DVT) is one of the most common complications in general population and leading cause of morbidity and mortality worldwide. Various hereditary and acquired factors play crucial role in the progression of DVT. Tissue factor (TF) acts as a major physiologic initiator of blood coagulation system by activating factor VII. High levels of TF have been associated with cardiovascular disease; however its association with DVT is controversial. In addition, the role of TF and its polymorphisms in Indian DVT patients is yet to be studied. Objectives: To find out the distribution of TF promoter polymorphism 603A $>\mathrm{G}$, its impact on TF plasma level, and to find out the association between TF and risk of DVT in young Indians. Material and Methods: Total 90 Doppler proven idiopathic DVT patients (age $<45$ years) and 90 age and sex matched healthy controls were studied. Patients on anticoagulants, with history of malignancy, pregnancy or sepsis were excluded. TF plasma level and TF promoter polymorphism $603 \mathrm{~A}>\mathrm{G}$ were determined by ELISA and PCR-RFLP respectively. Results: Total 90 DVT subjects, median age 35 years (range 20-43 years) and M: F (59:31) were recruited. DVT was seen in lower limb in 78 patients and upper limb in 12 patients. Mean TF level $(\mathrm{pg} / \mathrm{ml})$ in DVT patients $(84.95 \pm 17.16)$ was significantly higher than controls $(70.55 \pm 15.87, \mathrm{p}=0.001)$. The distribution of $603 \mathrm{~A}>\mathrm{G}$ polymorphism was found in $64.4 \%$ of patients and $46.6 \%$ of controls $(p=0.001)$. TF plasma level was significantly higher in patients and controls with $G$ allele than $A$ allele of $603 \mathrm{~A}>\mathrm{G}$ polymorphism (patients; $\mathrm{AG}+\mathrm{GG}$ : $93.4 \pm 12.55$, AA: $69.22 \pm 13.03 \mathrm{p}=0.001$, controls $\mathrm{AG}+\mathrm{GG}$ : $76.42 \pm 17.16$, AA: $64.90 \pm 12.22 \mathrm{p}=0.001)$. Conclusions: High plasma level of TF was significantly associated with DVT in young patients. AG and GG genotype of TF $603 \mathrm{~A}>\mathrm{G}$ polymorphism was associated with its high plasma levels and risk of DVT. Thus TF promoter polymorphism $603 \mathrm{~A}>\mathrm{G}$ detection may help in identifying young Indians having high risk of DVT. A study with larger sample size is required to confirm these findings.

\section{P-CD 05}

Effect of Thromboelastography Defined Coagulopathy in Traumatic Brain Injury

Dr Dibyendu De ${ }^{\#}$, Dr Suvro Sankha Datta, Dr Satyajit Das, Dr Manas Banerjee

Email ID for Correspondence: de.dibyendu@gmail.com

The Mission Hospital, Durgapur, West Bengal

Introduction: The presence of coagulopathy is common after severe trauma. It is associated with increased morbidities, increased transfusion requirement and mortality. Objectives: The aim of this study was to identify presence of coagulopathy in isolated traumatic brain injury, and its association with outcome. Material and Methods: This is a prospective observational study, with a cohort of patients admitted to neuro ICU with traumatic brain injury were evaluated. Conventional coagulation tests and Thomboelastography were done within first 24 hour of admission to identify coagulation status. The cohort is divided in 3 groups of hypercoaguable, normal, or hypocoaguable according to TEG report and the groups are compared. The SAPS II score and mortality rates were compared between the groups. Results: Total 112 patients were included in the study during the period of December 2015 to November 2016. The overall mean age of the cohort was 58.65 years, and $80.4 \%$ were male. The overall mortality was $32.1 \%$. by using TEG, about $44.64 \%$ patients showed hypercoaguability whereas hypocoaguable status was found in $4.46 \%$ patients. The mean age, sex ratio and other baseline parameters were comparable. The sensitivity of diagnosis of coagulopathy by conventional tests were $80.7 \%$, but specificity was only $8.9 \%$. Hypercoagulation status could not be picked up by conventional coagulation tests. However, the accuracy of diagnosing hypocoagulation status was $88.39 \%$ by conventional coagulation tests. The SAPS II score and mortality rates were comparable between hypercoaguable and normal hemostasis group (SAPS II score 43.66 and 42.46 respectively, $p$ value $>0.05$, mortality rate were $32 \%$ and $28.1 \%$ respectively, $\mathrm{p}$ value $>0.05$ ) but hypocoaguable group showed significant higher SAPS II score and higher mortality compared to normal hemostasis (SAPS II score 60.0 and 42.46 respectively, $\mathrm{p}$ value 0.002 , mortality rate were $80 \%$ and $28.1 \%$ respectively, $p$ value 0.034 ). Conclusions: Traumatic brain injury is often associated with coagulopathy. Thromboelastography is more reliable for diagnosing coagulopathy in these settings. The hypocoaguability is associated with higher SAPS II score and higher mortality rates.

\section{P-CD 06}

Impact of Combined JAK2V617F Mutation and the Inherited Thrombophilic Factors on Thrombotic Risk in CMPNs: AIIMS Experience

Kanwaljeet Singh", Sudha Sazawal, Amit Sharma, Sunita Chikkara, Rekha Chaubey, Kamal Kishor, Manoranjan Mahapatra, Renu Saxena

Email ID for Correspondence: kanwaljeet2009@gmail.com Departments of Hematology, AIIMS New Delhi, India (Pin code110029)

Introduction: It is still a dilemma regarding associative role of JAK2V617F mutation and inherited thrombophilia in CMPN (chronic myeloproliferative neoplasms) patients with thrombosis. We aimed to clarify the contribution of combined role of JAK2V617F and inherited thrombophilia in hypercoagulative state in CMPN patients. Objectives: We aimed to clarify the impact of combined JAK2V617F mutation and the inherited thrombophilic factors on thrombotic risk in CMPNs. Material and Methods: A total of 131 CMPN patients (PV, PMF and ET) positive for JAK2V617F with thrombosis and 85 controls (JAK2V617F negative CMPN patients without thrombosis) were study subjects. The DNA samples were screened for inherited thrombophilia which included FV Leiden, MTHFR C677T polymorphism, PT 20210 mutation and deficiencies of Protein C, Protein S and AT III. Results: Of 131 patients, 74 were males and 57 were 
females (M: F:: 2.3:1) with median age 52 years (range 16-85 years). $37 / 131$ (28.2\%) patients were positive for combined JAK2V617F and inherited thrombophilic factors (predominantly FVLeiden) as compared to $7 / 85(8.2 \%)$ in controls. Among the inherited thrombophilic factors, FVLeiden was exclusively present in JAK2V617F positive patients and negative in controls $(\mathrm{p}<0.004)$. The frequency of MTHFR C677T polymorphism was higher in JAK2V617F positive patients as compared controls but the difference was not statistically significant $(\mathrm{p}<0.07)$. Conclusions: This study emphasizes that JAK2V617F mutation in combination with inherited thrombophilia may increase the risk of thrombosis in CMPN patients. This finding could further assist in risk stratification, setting up the investigation and treatment protocols in CMPN patients.

\section{P-CD 07}

Factor VII Deficiency; An ENIGMA- Clinico Hematological Profile in 12 Cases

Tripathi $\mathbf{P}^{\#}$, Udayakumar DS, Kishor K, Mahapatra M, Saxena $\mathbf{R}$

Email ID for Correspondence: contactdoctorpreeti@gmail.com All India Institute of Medical Sciences, New Delhi

Introduction: Factor VII deficiency is the "commonest" of the rare bleeding disorders. Current knowledge on clinical profile or treatment of such cases is limited. The clinical heterogeneity with which these patients present, poses a dilemma to clinician and diagnostician. Objectives: To study the clinico- hematological profile of Factor VII deficient patients. Material and Methods: It is an observational study covering eleven months. All cases of probable inherited FVII deficiency found during this period were included and their clinical profile, family history, investigation profile and treatment records were studied in detail. Results: The study group comprised of total 12 cases $(n=12$, females -7 males -5$)$ with mean age of onset of symptoms - 17.5 yrs. Presenting symptoms included; epistaxis $(\mathrm{n}=4)$ gum bleedings $(\mathrm{n}=1)$ easy bruisability $(\mathrm{n}=3)$ menorrahagia $(\mathrm{n}=5)$ joint bleeds $(\mathrm{n}=2)$ post operative bleeds $(\mathrm{n}=1)$ prolonged bleeding from wound $(\mathrm{n}=1)$ hemoperitoneum $(\mathrm{n}=1)$ and asymptomatic $(n=1)$. Patients were divided according to their bleeding severity: severe bleeding - 02, moderate bleeding- 03 , mild bleeding - 06. Mean PT of the study group was $35.4 \mathrm{sec}$ (Range $18-50 \mathrm{sec}$ ) and mean prolongation of PT from upper limit of normal was $19.4 \mathrm{sec}$ (Range $2-34 \mathrm{sec}$ ) Factor VII levels ranged from less than 1\% to $40 \%$ in these patients. Patients having similar Factor VII level belonged to different categories as per bleeding severity symptoms Five out 12 patients required treatment other than local measures. Conclusions: Factor VII deficiency is notoriously heterogeneous in presentation regarding age, site and severity of presentation. Clinical symptoms can be discordant with the factor levels. Exclude all possible acquired causes and repeat factor VII levels in patients before labeling them as hereditary F VII deficient. Genetic correlation can be helpful in such cases.

\section{P-CD 08}

Safety Of Turoctocog Alfa for Prevention and Treatment of Bleeds in Patients with Severe Haemophilia A: Final Results from the Guardian ${ }^{\text {TM }} 2$ Trial
Kolla, $\mathbf{R}^{\#}$; Lejniece, S; Martin-Salces, M; Matytsina, I; Korsholm, L; Santagostino, E

Email ID for Correspondence: roko@novonordisk.com Novo Nordisk India

Introduction: Turoctocog alfa (NovoEight $\left.{ }^{\circledR}\right)$ is a B-domain-truncated recombinant factor VIII (rFVIII) for the treatment and prophylaxis of bleeding in patients with haemophilia A. The safety and efficacy of turoctocog alfa were assessed in guardian ${ }^{\mathrm{TM}_{2}}$, an openlabel, non-controlled, international extension to pivotal trials in previously treated adults, adolescents and children. Patients from pharmacokinetics trials could also enter guardian ${ }^{\mathrm{TM}} 2$. Interim analyses of guardian ${ }^{\mathrm{TM}} 2$ data showed turoctocog alfa to have a favourable safety profile. Objectives: Here, we report the final safety data, which, also includes 14 patients enrolled into an on-demand sub-trial. Material and Methods: Male patients with severe haemophilia A without FVIII inhibitors were enrolled from 19 countries. Patients received turoctocog alfa prophylactically (20-50 $\mathrm{IU} \mathrm{kg}^{-1}$ every second day or 20-60 IU kg ${ }^{-1}$ three times weekly) or on-demand (20-200 $\mathrm{IU} \mathrm{kg}^{-1}$ day $^{-1}$ ). Turoctocog alfa was used to treat any bleeds. The primary endpoint was inhibitor development $\left(>0.6 \mathrm{BU} \mathrm{mL}^{-1}\right)$; all adverse events (AEs) were also reported. Results: A total of 213 patients were dosed; mean (SD) patient age was 23.6 (14.6) years and most patients $(83.1 \%)$ were white. Total exposure to turoctocog alfa was 752.9 years, and individual patient participation in the study was up to 6 years, 7 months. No patients developed FVIII inhibitors $(>0.6$ BU). In total, 1278 AEs occurred in $184(86.4 \%)$ patients (1.7 AEs per patient year of exposure). There were 49 serious AEs in $41(19.2 \%)$ patients, including two fatalities (subdural haemorrhage and hepatic cancer); all but one were judged unlikely related to turoctocog alfa (both fatal cases were unlikely related to turoctocog alfa). One serious $\mathrm{AE}$ of acute myocardial infarction was judged probably related to turoctocog alfa; the patient recovered with sequelae. Seven other patients $(3.3 \%)$ experienced eleven AEs that were possibly or probably related to turoctocog alfa, but all were mild or moderate in severity. There were no hypersensitivity or allergic reactions to turoctocog alfa, and no other safety concerns. Conclusions: Final safety data from guardian ${ }^{\mathrm{TM}} 2$ support the long-term use ( $>6$ years for the patient with longest participation) of turoctocog alfa in previously treated patients with severe haemophilia A. No inhibitors or other safety concerns were observed.

\section{P-CD 09}

Severity of Injury and Coagulopathy in Patients with Isolated Head Trauma

\author{
Meera Sikka ${ }^{\#}$, Ruchika Sodhi, Mrinalini Kotru, \\ Gurubachan Singh
}

Email ID for Correspondence: meerasikka55@gmail.com Department of Pathology, UCMS \& GTB Hospital, Delhi

Introduction: The outcome of patients with Traumatic brain injury (TBI) is affected by hemostatic derangements which contribute to mortality and morbidity. As coagulation abnormalities are evident soon after trauma, their identification and subsequent treatment will help improve prognosis in these patients. This study aimed to assess the prevalence of coagulopathy in patients with varied severity of TBI. Methods Hundred patients admitted within $12 \mathrm{~h}$ of head injury were enrolled in the study. Based on the GCS, head injury was classified as mild (GCS13-15), moderate (GCS 9-12) and severe (GCS 3-8). The following investigations were done on all patients: complete blood counts including platelet count, Prothrombin time 
(PT) and Activated partial thromboplasin time (APTT). Based on the value of PT $(11 \mathrm{sec})$ and APTT $(28 \mathrm{sec})$ of laboratory controls, coagulopathy was defined as prolonged PT $(>14 \mathrm{sec})$ and/or APTT (>34sec). Results The prevalence of coagulopathy was 39\%. In 30/ $39(76.9 \%)$ patients associated thrombocytopenia was present, while 18 patients had isolated thrombocytopenia. The prevalence of coagulopathy and thrombocytopenia increased with increasing severity of injury. Both were present in patients with mild injury also. A highly significant $(p<0.001)$ association was seen between GCS and coagulopathy as also thrombocytopenia. Conclusions Coagulopathy is frequent in patients with TBI and was seen even in mild TBI. Hemostatic parameters must be measured in all patients at admission irrespective of severity of TBI to help identify patients who will benefit with additional therapy thus improving prognosis.

\section{P-CD 10}

\section{Can Clinicall Laboratory Coagulometer Help in Antiplatelet Drug Monitoring?}

Dr. D. Febe Renjitha Suman ${ }^{\#}$, Dr. Jesu Magdalene, Mrs. Uma Lakshmi, Mr. Ananda Kumar

Email ID for Correspondence: febemd@gmail.com Sri Ramachandra Medical College\&Research Institute, Chennai

Introduction: Platelets are critical in haemostasis and development of thrombosis. Damaged endothelium activates platelets which respond by adhesion and aggregation. Release of thromboxane $\mathrm{A}_{2}$ and adenosine diphosphate (ADP)amplifies the process. Aspirin irreversibly blocks the formation of thromboxane $A_{2}$, Clopidogrel a thienopyridine class of ADP receptor $\mathrm{P}_{2} \mathrm{Y}_{12}$ inhibitor is a prodrug which requires $\mathrm{CYP}_{2} \mathrm{C}_{19}$ for its action. The action of Prasugrel is similar but rapid potent, consistent and independent of $\mathrm{CYP}_{2} \mathrm{C}_{19}$. After administration of these drugs its highly essential to monitor their effects. Objectives: 1 . To study the aggregation of platelets in patients on antiplatelet drugs, 2. To assess the aggregation in drug change 3 . To assess the end users' satisfaction with platelet aggregometry using coagulation analyser. Material and Methods: This prospective study was done for a period of 8 months on patients whose samples were sent for adenosine diphosphate (ADP) induced platelet aggregometry. The demography, clinical details, drug intake and platelet counts were collected. Light transmission aggregometry was done with $2 \mu \mathrm{mol} / 1$ of ADP using automated coagulation analyser Sysmex CA 2400. The waves and the percentage of aggregation was analysed statistically. Survey was done with lab personel and clinician for satisfaction. Results: The study includes 50 patients whose age ranged between 12-76yrs. Male:female ratio was 1.3:1. Clinical conditions include arterial stenosis, aneurysm and thrombosis. The number of patients on prasugrel, clopidogrel, aspirin and dual therapy with prasugrel and aspirin, aspirin and clopidogrel were 20 (40\%), 10 $(20 \%), 8(16 \%), 6(12 \%), 6(12 \%)$ respectively. The median percentage of platelet aggregation for prasugrel, clopidogrel, aspirin and dual therapy with prasugrel and aspirin, aspirin and clopidogrel were $10.5,17.3,24.3,21.4$, and 14.15 respectively. Though median platelet aggregation was less with prasugrel, than others, the difference was statistically not significant $(\mathrm{P}=0.773) .6$ patients showed low platelet aggregation after changing drugs. 2 patients were noncompliant and counseled and showed response later. The clinicians were very happy about the turn around time and the results. As some of them needed the result in pictorial form to explain the results to patients, software modifications were done. Conclusion: Platelet aggregation on the routine coagulation analyzer is an easily accessible, handy, reliable, standardized, and rapid tool to assess platelet function. This is a very useful investigation tool to monitor and change drug usage.

\section{P-CD 11}

Coagulopathy at Admission Predicts Poor Outcome in Traumatic Brain Injury

Dr. Garima Dwivedi, Dr. Ruchika Sodhi, Dr. Meera Sikka, Dr. Mrinalini Kotru, Dr. Gurbachan Singh

Introduction: Coagulopathy is frequently observed in patients with traumatic brain injury and associated with an adverse outcome. This study aimed to evaluate the association between coagulopathy detected within the first 12 hrs of Traumatic brain injury and outcome in Indian patients with isolated head trauma. Material and Methods: One hundred patients admitted within $12 \mathrm{~h}$ of head injury were enrolled in the study. Based on the GCS, head injury was classified as mild (GCS13-15), moderate (GCS 9-12) and severe (GCS 3-8). Complete blood count including platelet counts, Prothrombin time (PT) and Activated partial thromboplastin time (APTT)were done in all patients. Based on the value of PT (11sec) and APTT (28sec) of laboratory controls, coagulopathy was defined as prolonged PT $(>14 \mathrm{sec})$ and/or APTT ( $>34 \mathrm{sec}$ ). Patients were followed up till the time of discharge/death. Results: The prevalence of coagulopathy was $39 \%$. Thirty of $39(76 \%)$ patients also had associated thrombocytopenia while 18 patients had only thrombocytopenia. The mortality was $26 \%$. Platelet count was significantly $(\mathrm{p}<0.001)$ lower and PT and APTT significantly $(\mathrm{p}<0.001)$ higher in non survivors as compared to survivors. The mortality was significantly $(p<0.001)$ higher in patients with thrombocytopenia and coagulopathy as compared to patients in whom these parameters were normal. Conclusions: Screening tests of hemostasis done at admission in patients with traumatic brain injury may be used to identify patients with worse outcome. Their identification and early management will reduce morbidity and mortality.

\section{P-CD 12}

Plasma Fibrinogen and D-Dimer in Children with Sepsis

Abhimanyu Sharma, Indu sharma\#, Meera Sikka, Sunil Gomber, Satendra Sharma

Email ID for Correspondence: dr.indu89@gmail.com Department of Pathology, UCMS \& GTB Hospital, Delhi

Introduction: In sepsis, enhanced fibrin formation, impaired fibrin degradation and intravascular fibrin deposition lead to a prothrombotic state. Objective: to measure various coagulation parameters so as to predict an early marker for DIC. Methods: A prospective study was conducted on50 children 1-10 years of age with clinically suspected sepsis and 50 age matched controls. patients were evaluated according to criteria for infection (i.e. symptoms less than seven days) which was confirmed in all patients in the laboratory. Patients who had received antibiotics $24-48$ hours preceding admission were excluded from study. Prothrombin time, activated partial thromboplastin time, plasma fibrinogen and D-dimer were measured at the time of admission. Results: D-dimer was positive in $36(72 \%)$ patients and negative in all controls, the difference being statistically significant $(\mathrm{p}<0.01)$. plasma fibrinogen was significantly $(\mathrm{p}<0.01)$ higher in patients as compared to controls. PT and APTT were significantly $(\mathrm{p}<0.01)$ higher in patients as compared to controls. Conclusion: Though none of the patients developed overt DIC, the high positivity of D-dimer as observed in the study suggests that it should be measured in children with sepsis for early identification of DIC and it's management with appropriate therapy. 


\section{P-CD 13}

\section{Level of Endogenous Anticoagulants in Patients with End Stage} Renal Disease

\section{Dr Mrinalini Kotru\#, Dr Kiran Saini, Dr Meera Sikka,} Dr Anil Yadav, Dr Satendra Sharma

Email ID for Correspondence: mrinalini.kotru@gmail.com UCMS and GTB Hospital

Introduction: Chronic renal insufficiency influences the hemostatic system to a large extent. Patients undergoing dialysis exhibit numerous abnormalities of coagulation, fibrinolysis and vascular thrombosis. End stage renal disease (ESRD) is also known to influence the level of endogenous anticoagulants in these patients. Objectives: To determine the difference in the level of Endogenous anticoagulants (Protein S, Protein C and Antithrombin III) in patients with ESRD and controls. Material and Methods: Thirty-five patients with ESRD on whom dialysis was to be started or those who had not undergone dialysis for last 1 month were included in the study. Ten age matched healthy controls were also included. Venous blood was collected from all subjects and the level of following Endogenous anticoagulants: Protein C (PC), total Protein S (Total PS) and Antithrombin (ATIII) were evaluated in patients and controls. Results: The levels of PC and total PS were significantly $(\mathrm{p}<0.05)$ lower in patients as opposed to controls, while there was no difference in the level of ATIII. PC was decreased in 24 (68.6\%) patients and total PS was decreased in $25(71.4 \%)$ patients. Conclusions: Patients with ESRD showed a decrease in endogenous anticoagulants such as PC and total PS which can contribute to a hypercoagulable state. These abnormalities may be involved in the pathogenesis of vascular thrombosis and episodes of bleeding seen in this population. So, their measurement may help in early identification of patients who are at increased risk of thrombosis and help in their management.

\section{P-CD 14}

\section{Spectrum of Protein $\mathrm{C}$ and Protein $\mathrm{S}$ Deficiency in a Tertiaryy Care Centre}

Dr. D. Febe Renjitha Suman\#, Dr. Tejaswita Bisht, Dr. B. Archana, Dr. Rithika Rajendran, Mrs. UmaLakshmi, Mr. Ananda Kumar

Email ID for Correspondence: febemd@gmail.com Sri Ramachandra Medical College and Research Institute

Introduction: Protein $\mathrm{C}$ and Protein $\mathrm{S}$ are Vitamin $\mathrm{K}$ dependent coagulation inhibitors, synthesized in liver. Activated protein $\mathrm{C}$ complexed with protein $\mathrm{S}$ as cofactor degrades factor VIIIa and factor $\mathrm{Va}$ to limit thrombin formation. Congenital or acquired deficiencies predispose to thrombosis. Objectives: (i)To analyze the clinico-hematological aspects of Protein $\mathrm{C}$ and Protein S deficiency (ii) To analyse the importance of protein $\mathrm{C}$ and protein $\mathrm{S}$ testing in the clinical laboratory. Material and Methods: This prospective study was done between 2015- 2017. The demography and clinical details were collected. The functionally active Protein $\mathrm{C}$ and protein $\mathrm{S}$ were quantitatively determined by chromogenic method using automated coagulation analyzer Sysmex CS 2400. The patients who are deficient in either one or both the proteins are taken for study. The other thrombophilic markers were also taken into consideration. Statistical analysis done. Results: Among the protein $\mathrm{C} \&$ protein $\mathrm{S}$ estimations done 84patients were found to have deficiciency in either one or both.
The age of the patients ranges from 1 day- 65 yrs. 12 patients were children of which 3 were neonates. Male:Female ratio is 1.7: 1 . Protein $\mathrm{C}$, protein $\mathrm{S}$ and combined deficiency were present in $64 \%$, $37 \%$ and $23 \%$ respectively. Statistically significant increase in thromboembolic events were noted with Protein $\mathrm{C}$ deficiency $(\mathrm{p}$ value $<0.001$ ). Pregnancy loss was seen in combined deficiency and none of them were lupus anticoagulant positive. Two cases of neonatal purpura fulminans, one being hereditary and the other sepsis induced were diagnosed. Acquired deficiencies were noted in end stage hepatic and renal disease and also in pulmonary tuberculosis and acute lymphoblastic leukemia. Conclusions: Protein $\mathrm{C}$ and protein $\mathrm{S}$ estimations indicate that it is essential to screen the patients for deficiency not only in thromboembolism and neonatal purpura fulminans but also in pregnancy loss, liver and kidney disorder. The deficiency may alter the therapeutic modality. Certain drug therapies may also be ordered with caution.

\section{P-CD 15}

\section{Decreased Protein C Function Predicts Mortality in Patients with Cirrhosis}

\section{Anupama G Patil", Chhagan Bihari, Hemant D Shewade,} Neha Nigam, Shiv K Sarin

Email ID for Correspondence: itsagp@gmail.com Institute of Liver \& Biliary Sciences, New Delhi, India

Introduction: Cirrhosis is characterized by a pro-coagulant imbalance. Protein $\mathrm{C}(\mathrm{PrC})$ is a physiological anticoagulant, the activated form of which is involved in regulating inflammation and cell death. It has long been noted to have predictive and therapeutic roles in sepsis. Accumulating evidences suggest plasma hypercoagulability results in progression of fibrosis and formation of micro-clots causing end-organ dysfunction. Objectives: We investigated a possible association between natural anticoagulants- PrC, protein S (PrS), antithrombin III (AT3) and clinical outcomes in cirrhotics. Material and Methods: The levels of PrC, PrS and AT3 functional activity were analysed in the plasma of 515 patients with cirrhosis and compared with 229 controls (non-cirrhotic cases). Child-Pugh (CP) and MELD scores were used to assess the severity of cirrhosis. Among those with cirrhosis, we conducted multivariable predictive model using Poisson regression analysis on 3-month survival to assess the prognostic ability of anticoagulants. Results: The $\operatorname{PrC}(\mathrm{p}<0.001)$, PrS $(\mathrm{p}<0.001)$ and AT3 $(\mathrm{p}<0.001)$ levels were lower in patients with cirrhosis compared with controls. In addition, patients with CP-C had significantly lower $(\mathrm{p}<0.05)$ functional $\operatorname{PrC}, \operatorname{PrS}$ and AT3 levels than CP-B, CP-A, and non-cirrhotic patients. Low functional $\mathrm{PrC}$ levels correlated with markers of liver dysfunction and inflammation: INR $(r=-0.72, p<0.001)$, serum bilirubin $(r=-0.620, p<0.001)$, albumin $(\mathrm{r}=0.539, \mathrm{p}<0.001)$, creatinine $(\mathrm{r}=-0.417, \mathrm{p}<0.001)$, ferritin $(\mathrm{r}=-0.68, \mathrm{p}=0.035)$, procalcitonin $(\mathrm{r}=-0.79, \mathrm{p}=0.01)$ raised ESR $(r=0.56, p<0.001)$, in addition to quantitative fibrosis $(r=-0.840, p<0.001)$. Also, the rate of occurrence of spontaneous bacterial peritonitis (SBP) was higher in patients $(n=108)$ with lower PrC levels $(p=0.034)$. However, PrS and AT3 deficiency failed to correlate with any of these markers. Patients who died $(n=160)$ at the end of three months had significantly lower functional PrC levels (median $=23.8 \%$, IQR: 16.3-33.0) compared with those who remained alive $($ median $=74.9 \%, \mathrm{IQR}=59.7-92.5$ ); $\mathrm{p}<0.001$. In a multivariable predictive model using $\operatorname{PrC}, \operatorname{PrS}$, AT3, haemoglobin, total leucocyte count, platelet count, MELD score, CP score and liver enzymes; we found a significant impact of low $\operatorname{PrC}$ 
levels on survival $(\mathrm{p}<0.001, \mathrm{IRR}=0.96,95 \% \mathrm{CI}=0.95-0.97)$. Receiver operating characteristic (ROC) curve analysis revealed that functional PrC levels $<52 \%$ were associated with increased mortality $(p<0.001)$. Conclusions: Low functional protein $\mathrm{C}$ level correlates with markers of liver dysfunction, inflammation and sepsis. It negatively correlates with survival in cirrhotics, especially if functional PrC levels are $<52 \%$.

\section{P-CD 17}

Early Detection of JAK2 Mutation in Young Patients Presenting as Acute Abdomen Due to Thrombosis in a Tertiary Care Hospital

\section{DR. Guntupalli Nadeswari (PGY2) ${ }^{\#}$, DR. Naval Kishore Bajaj} (Assoc. Prof), DR. Ezhil Arasi. N (Professor \& HOD)

Email ID for Correspondence: nadeswari.guntupalli@gmail.com Osmania Medical College

Introduction: Venous thrombosis is the third most common cause leading to death after ischemic heart disease and stroke. The pathogenesis of venous thrombosis is multifactorial. Portal or mesenteric venous thrombosis may be an early or presenting complication of Myeloproliferative disease (MPD). JAK2 V617F mutation is seen in some of these patients. Objectives: Early detection of JAK2 mutation in young patients who suffered with thrombotic episodes for better clinical and therapeutical outcome. Material and Methods: Prospective study from June 2016 to August 2017. Young and middle aged patients who presented with acute abdomen to surgery department, were clinically examined and were evaluated with CT scan and other radiological investigations. Cases with evidence of thrombosis in splanchnic circulation were selected and were investigated for Antiphospholipid antibody, Protein C, S, Anti thrombin, Lupus anticoagulant, Factor V leiden mutation and lastly JAK2. Results: 20 cases were studied, Out of which 08 cases were investigated for JAK2 mutation and 05 cases found to be significantly positive. Rest of 12 cases had varied etiology. Conclusions: Early detection of JAK2 V617F mutation in young patients who suffered with thrombotic events is useful in unearthing MPD, which can be followed up medically with anticoagulant therapy, thus avoiding unnecessary surgery.

\section{P-CD 18}

Study of Effectiveness of Low Dose Secondary Prophylaxis in Children with Severe Hemophilia A - A Tertiary Care Centre Experience

\section{Gulshan S ${ }^{\#}$, Mandal PK, Phukan A, Dolai TK, De R, Chakrabarti P}

Email ID for Correspondence: shzgulshan@gmail.com Nil Ratan Sircar Medical College, Kolkata

Introduction: Hemophilia A is an X-linked inherited disease. Patients usually present with joint and muscle bleed which may cripple the child. Once joint damage has occurred, it progresses over lifetime. Primary prophylaxis is the gold standard of treatment. However, secondary prophylaxis $(\geq 2$ joint bleed and before onset of joint disease) is a feasible option in developing countries. Objectives: To study the effectiveness of low dose secondary prophylaxis in children in terms of lowering bleeding episode \& improving quality of life. Material and Methods: A prospective interventional study was conducted from January 2016 to December 2016 in Haematology Department of NRS medical College, Kolkata. Twenty-nine children with severe Hemophilia A were given low dose long acting recombinant factor VIII prophylaxis $20 \mathrm{IU} / \mathrm{kg}$ twice weekly for 6 months. All the patients were previously receiving on-demand f VIII or FFP. Patients were evaluated every week for 6 months and data recorded. Outcome was measured in terms of median annual bleeding rate (MABR), number of days of school absenteeism per month (SA), school activity participation score (SAPS)\&daily activity score (DAS) as per published Beijing Children Hospital score compared to their 'on demand' therapy. Results: In this study, MABR on "on demand" $\&$ on prophylaxis is $14.5 \& 2$ respectively $(\mathrm{p}<0.00001)$. The mean SA while on "on demand' \& on prophylaxis is is $17.38 \& 2.42$. On prophylaxis the SAPS shows mild, moderate improvement in $57.14 \%$ \& $38.09 \%$ respectively. Moderate improvement was seen in $43.33 \%$ in DAS. Conclusions: Low dose secondary prophylaxis reduces joint bleeds with improvement in school attendance, school activity participation \& daily activities. In resource limited countries like India low dose secondary prophylaxis is an acceptable alternative in children with severe Hemophilia A.

\section{P-CD 19}

Retrospective Study of Clinical and Haematological Parameters in Patients with Haemophilia

Dr. Omkar Ramakrishna Puvvala\#, Dr. Hanagavadi Suresh, Dr. Rajashekar S Kurvatti, Dr. Chatura K.R, Dr. Parismrita Borah

Email ID for Correspondence: doctorpuvvala@gmail.com JJM Medical College, Davangere, Karnataka

Introduction: Haemophilia is an inherited bleeding disorder characterized by deficiency of certain clotting factors, most common being factor VIII (Haemophilia A/Classic haemophilia) followed by factor IX (Haemophilia B) and rarely factor XI (Haemophilia C). It is an X-linked recessive hereditary bleeding disorder usually affecting males and females being carrier. Affected individuals develop a variable phenotype of hemorrhage into joints and muscles, and prolonged bleeding from wounds. Management includes replacement of deficient factors and if not treated early may lead to crippling arthropathy. Knowledge of the pattern of clinical presentation helps in early diagnosis and management, thereby preventing complications. Development of inhibitors is a major challenge in the management of these patients.

Objectives:

1. To study the pattern of clinical presentation in 200 patients with haemophilia.

2. To correlate the various clinical symptoms with factor level.

Material and Methods: Retrospective study of 200 patients with Haemophilia who were referred to the Department of Pathology, JJM Medical College, Davangere from Kanataka Haemophilia Society Davangere. Detailed clinical history and coagulation profile was evaluated from the record sheets. Results: Out of a total of 200 cases (199 males and 1 female), $80.5 \%$ belonged to Haemophilia A while $19.5 \%$ belonged to Haemophilia B. $65.5 \%$ had severe, while $28.5 \%$ had moderate and $6 \%$ had mild form of the disorder. Haemarthrosis is the most common clinical presentation $(91.5 \%)$ with knee joint being most commonly involved ( $74 \%$ of the cases). 131 cases were evaluated for presence of inhibitors of which 24 were found positive (23 in Haemophilia A and 1 in Haemophilia B). Conclusions: Haemophilia A caused by deficiency of Factor VIII is the most common type, majority of the cases belonged to severe category. Musculoskeletal bleeding episodes are the most common clinical manifestation. The clinical pattern depends upon the level of factor deficiency. 


\section{P-CD 20}

Prothrombotic conditions in isolation or in combination is the dominant cause of cerebral venous sinus thrombosis

\section{J Kalita, BK Singh, UK Misra}

\section{Sanjay Gandhi PGIMS, Lucknow}

Background: Cerebral venous sinus thrombosis (CVST) is an important cause of young stroke in South East Asia. Prothrombotic conditions and hyper viscosity are important hematological conditions attributed to CVST. Early recognition and management of these conditions are important for better outcome and prevention of recurrence. Purpose: To evaluate the risk factors in CVST and its relationship with the clinical severity, extent of MRI and MRV abnormality, and outcome. Methods: 128 consecutive patients with CVST aged 3-76 (median 29) years were prospectively included. Their demographic, presenting symptoms and neurological findings were noted. The patients were evaluated for underlying female specific causes, prothrombotic state, hematological abnormality, vasculitis and infective causes. The outcomes were assessed using modified Rankin Scale (mRS) into death, poor, partial, and complete recovery. Results: Eighty-two patients had prothrombotic state (hyperhomocysteinemia 41, protein S deficiency 44, protein C deficiency 17, APLA syndrome 19, antithrombin III deficiency 4 , factor $\mathrm{V}$ Leiden mutation in 3), female specific in 22 , infection in 20, other hematological disorders in 4 (paroxysmal nocturnal hemoglobinuria, post renal transplant polycythemia, multiple myeloma, hyperlipidemia in 1 each) and systemic and cancer related in 3 patients. In 26 patients ( $20.3 \%$ ) no risk factor could be identified. The patients with factor V Leiden mutation, APLA syndrome and PNH had recurrent venous thrombosis and had worse outcome. Sixteen out of 22 (72.7\%) patients with female specific risk factors also had other prothrombotic conditions. On MR venography, more than two sinuses were involved in $35(27.3 \%)$ patients and $94(73.4 \%)$ patients had parenchymal brain lesions on MRI. 31 (24.2\%) had poor outcome $(\mathrm{mRS}>3)$ at discharge and $25(19.5 \%)$ at 3 months. On multivariate analysis, age (OR 1.06, CI 1.01-1.12, $\mathrm{P}=0.01)$ and mechanical ventilation (OR $0.003,95 \% \mathrm{CI} 0.00-0.08, \mathrm{P}=0.001$ ) predicted outcome at discharge. Conclusion: The commonest cause of CVST is prothrombotic state and multiplicity of risk factors usually present in majority of the patients. Patients with PNH, factor V Leiden mutation and APLA syndrome need more close monitoring due recurrence of venous thrombosis.

\section{P-CD 21}

Factor $\times$ deficieny presenting with massive cephalhematoma an uncommon factor deficiency with an uncommon presentation

\section{Mishra Sanjay**, Kumar A*, Tripathi AK**, MIshra KL*}

* Department of Pathology, King George's Medical University, Lucknow; ** Department of Clinical Hematology, King George's Medical UnIversity, Lucknow

Introduction: Congenital factor $\times$ defi ciency is considered very rare in Western literature. About 50 cases have yet been described. This deficiency affects one individual per 500000 to 1000000 population worldwide. It is common in areas where consanguinity is frequent like Pakistan and India. Case report: We present a case of factor deficiency who presented with massive cephalhematoma, first time at 7 years of age. The patient attendant gave history of prolonged umbilical cord bleed. Since birth patient did not have any significant bleed. She did have mucosal and subcutaneous bleed after minor trauma. On examination she did not have pallor. She have massive hematoma, proved after suction through the syringe. There were pressure symptoms of the cephalhematoma and she was restless. Her both eyes showed marked congestion and proptosis. His haemoglobin was $6.4 \mathrm{~g} \%$. Platelet count was $250000 / \mathrm{mm}$. Bleeding time was normal. Hess test was negative. Prothrombin time (PT), activated thromboplastin time (APTT) was prolonged and thrombin time (TT) was normal. The PT and APTT corrected with aged plasma and serum and did not correct with adsorbed plasma. Coagulation factor assays showed marked factor $\times$ deficiency $(<1 \%$ of normal activity). Factor $\times$ deficiency can present at any age and with variable manifestations. Common presentations are umbilical cord stump bleeding, nose bleed, easy bruising, bleeding into soft tissues and muscles, GIT bleeding, menorrhagia, haematuria, intracranial bleed, hemarthrosis, recurrent epistaxis and bleeding after trauma or surgery. Discussion: The case is worth reporting as the case has typical rare presentation and was interesting to report. The patient was treated with fresh frozen plasma and operated for cephalhematoma. She improved after surgical intervention. She is now on regular follow up and doing well.

\section{P-CD 22}

Study of Circulating Microparticles (Platelet, Endothelium Derived \& Tissue Factor Bearing) in Traumatic Brain Injury and Its Corelation with TBI Associated Coagulopathy and Mortality

Venencia Albert ${ }^{\#}$, Arulselvi Subramaniam, Haraprasad Pati*, Deepak Agrawal\#, A K Mukhopadhayay

Email ID for Correspondence: albertvenencia@gmail.com Department of Lab Medicine, Hematology* and Neuro surgery\#, Jai Prakash Narayan Apex Trauma Center, All India Institute of Medical Science, New Delhi

Introduction: Incidence of early coagulopathy in brain injury patients is reported to be $32.7 \%$, in absence of severe bleeding and restricted fluid administration, suggesting that TBI associated coagulopathy (TBI-AC) follows a distinct pathway that differs from multisystem trauma. Cellular microparticles (MPs) generated by injured cells serve as a platform to promote systemic coagulation and thrombosis, potentially contributing to the development of TBI-AC. Objectives: We designed a study to assess the systemic impacts of cellular microparticles [Tissue factor bearing (TFMP), Platelet (PDMP) and endothelium (EDMP) derived microparticles] released following brain injury with specific emphasis on TBI-AC and mortality. Material and Methods: 120 isolated severe TBI patients admitted $\leq 12 \mathrm{hrs}$ of injury to trauma center were enrolled. Clinical and laboratory data was recorded and Flow cytometric MP analysis was performed on sample drawn prior to any blood transfusion. Flow cytometry analysis of TFMP, PDMP \& EDMP was done using triple gating based on (i) particle size (meagamix beads) (ii) expression of cell surface markers (CD142, CD 42a \& CD62E) and (iii) phosphatidylserine (Anx V). Tissue factor and tissue factor pathway inhibitor (TFPI) levels were assessed by ELISA. Results: 120 patients met the inclusion criteria, aged $35.7 \pm 12.12$ years, $96 \%$ males, and mean Glasgow coma score of $6.5 \pm 1.7$. Brain injury resulted in a significant depletion of tissue factor levels [1.7 (1.0-3.6) vs. 3.9 (3.04.7) $\mathrm{p}$ 0.0002] and a concordant surge of microparticle release [TFMP 5.8 (3.9-9.45) vs. 3.83 (3.0-5.0) p 0.007; PDMP 4.21 (2.8-6.5) vs. 2.45 (2.1-3.2) p 0.002; EDMP 2.83 (1.9-4.0) vs. 1.46 (1.25-2.7)] compared to control. Spearman's correlation was positively moderate between TFMP, PDMP and EDMP respectively. Strong positive correlation was seen between PDMP and EDMP [rho 0.62(P $<0.0001)]$. TBI coagulopathy was identified in $58(48.3 \%)$. Patients with TBI coagulopathy had low MP count regardless of cellular origin compared to 
normocoagulable patients [TFMP 5.34 (4.03-8.3) vs. 6.45 (3.4-9.97); PDMP 3.8 (2.6-6.5) vs. 5.2 (2.9-6.6); EDMP 2.7 (2.0-3.9) vs. 2.9 (1.84.6)] which was not statistically significant. Significant depletion in tissue factor and TFPI were observed between patients with and without TBI associated coagulopathy. Early TBI associated coagulopathy was an independent predictor of mortality [61.1\%, OR: 4.73 (CI 1.68-13.3)]. Compared to survivors, non-survivor TBI patients had a significant fall in the tissue factor, PDMP and EDMP levels, interestingly the levels of TFMP and anticoagulant TFPI levels were high in non-survivors vs. survivors. Conclusions: Enhanced activation and increased procoagulant MP generation is predominant following isolated TBI. Low counts of TFMP, PDMP, and EDMP in coagulopathic TBI compared to non-coagulopathic, suggest their involvement in the development of TBI induced coagulopathy and possibly result in early mortality.

\section{P-CD 23}

\section{Study of Hyperfibrinolysis in Acute Traumatic Brain Injury (TBI) Patients Using Thromboelastography (TEG) and It's Correlation with Coagulation Markers (Activation, Inhibition, Thrombin Generation and Fibrinolysis)}

\section{Arulselvi Subramaniam", Venencia Albert, Haraprasad Pati*, Deepak Agrawal\#, A K Mukhopadhayay}

Email ID for Correspondence: arulselvi.jpnatc@gmail.com Departments of Lab Medicine, Hematology* and Neurosurgery\#; Jai Prakash Narayan Apex Trauma Center, All India Institute of Medical Science, New Delhi

Introduction: Hyperfibrinolysis is a key mediator of coagulation abnormalities in brain injury. Maladaptive over activation of hyperfibrinolysis identified as [ $\geq 3 \%$ lysis at 30 mins (LY30)] on thromboelastograph (TEG) and large utilization of blood products is associated with higher mortality rates exceeding $70 \%$. Objectives: We designed a prospective study to compare TEG and pathwayspecific coagulation biomarkers at time of admission among isolated severe TBI patients (GCS $\leq 8)$ with/without early hyperfibrinolysis. Material and Methods: Prospective observational study of 57 severe TBI patients admitted $\leq 12$ hrs of injury at a Level I trauma center was done. Clinical and laboratory data was recorded and TEG was performed before any blood transfusion support, ELISA analysis of tissue factor (TF), tissue factor pathway inhibitor (TFPI), protein C (PC), free protein $\mathrm{S}$ (PS), thrombin antithrombin complex (TAT), soluble fibrin monomer $(\mathrm{sFM})$, tissue plasminogen activator (TPA) and plasminogen activator inhibitor (PAI-1) were done. Results: Fifty-seven patients were included (average, $35.7 \pm 12.1$ years; 97\% male). On basis of clot strength as measured by TEG in TBI patients was as follows: normal (MA 51-69 $\mathrm{mm}(\mathrm{n}=15)$, hypercoagulable $(\mathrm{MA}>69 \mathrm{~mm})(\mathrm{n}=16)$ and hypocoagulable $(\mathrm{MA}<51)(\mathrm{n}=26)$. Distribution of fibrinolysis as measured by TEG was as follows: physiologic/normal (LY30 $\leq 2.9) 74 \%(\mathrm{n}=42)$; and hyperfibrinolysis, $26 \%(\mathrm{n}=15)$. TEG parameters for clot initiation ( $\mathrm{r}$-time 7 (5$8.5)$ vs. 3.8 (2.3-7.2) p 0.03), and cross linking ( $\kappa$-time 3.7 (2.0-6.7) vs. $1.8(1.0-4.0)$ p 0.03$)$ were significantly elevated with hyperfibrinolysis. Coagulo-fibrinolytic assays did not vary among these patients. Early hyperfibrinolysis was significantly correlated with increased thrombin generation [TAT 72.8 (34.4-146.7) vs. 72.8 (34.4146.7) p0.0002], significant TF [1.6 (1.0-2.6) vs. 3.8 (1.4-5.1) p 0.03] and anticoagulants: TFPI [51.7 (34.6-118.0) vs. 105.1 (93.4-132.0), p 0.03] \& PAI [76.9 \pm 27.1 vs. $95.2 \pm 35.0$ p0.03]. We also observed a severe depletion of PC levels in patients with hyperfibrinolysis [56 (22-103) vs. 76 (47-94) p 0.51] compared to patients without Hyperfibrinolysis. 3 (20\%) patients in the Hyperfibrinolysis group had overt DIC. Mortality rate was $40 \%$ in the HF group, compared to $26.1 \%$ (11) in the physiologic fibrinolysis group. Conclusions: Hyperfibrinolysis was observed in $26 \%(\mathrm{n}=15)$ of TBI patients using TEG, which was not identified by routine conventional assays. Hyperfibrinolysis correlated with low TF enhanced coagulation activation, exacerbation of thrombin generation (high TAT) and lastly supressed anticoagulation as depicted by elevated PAI levels adjunct to depleted PC. However, it was not associated with higher mortality.

\section{P-C 01}

\section{Haematological Indices and Bone Marrow Morphology} in Pancytopenia/Bicytopenia

Introduction: Peripheral cytopenia is defined as reduction in the cellular elements of blood i.e. red cells, white cells, platelets. Bicytopenia is reduction in any two cell lines and pancytopenia is reduction in all the three. There is considerable overlap between the causes and diagnostic approach of bicytopenia and pancytopenia. It's not a disease in itself but a triad of findings that may arise from a number of disease processes. The aetiology of bicytopenia and pancytopenia ranges from transient marrow suppression to marrow infiltration by malignancy. These may also be caused iatrogenically, secondary to certain drugs, chemotherapy or radiotherapy for malignancies. The presenting symtioms are usually attributable to anemia, thrombocytopenia or leucopenia. Underlying pathology determines the management and prognosis of the patients. Some experts suggest that marrow examination is essential to the diagnosis, but it has not been established whether the procedure is necessary in all pancytopenic patients.

\section{Objectives:}

1. To evaluate clinic-haematologic profile and bone marrow morphology of patients with pancytopenia/ bicytopenia.

2. To determine common causes of pancytopenia and bicytopenia in this geographical area in different sex and age groups.

3. To determine protocol for work up in such patients

Material and Methods: The study includes patients of all ages, both sexes, coming to the hospital with complaints of weakness, giddiness, fever, breathlessness, bleeding tendency, or body pain. Patients were clinically examined and further haematological workup was done. Patients showing all three or any two of the following parameters on $\mathrm{CBC}$ examination were included in the study.

1. haemoglobin $<10 \mathrm{~g} / \mathrm{dl}$

2. Total leucocyte count $<400 / \mathrm{cu} \mathrm{mm}$

Platelet count $<1.5 \mathrm{lakh} / \mathrm{cu} \mathrm{mm}$

Patients fulfilling the above criteria were subjected to bone marrow aspiration and trephine biopsy with imprint cytology with due informed consent.

Materials used were

1. $\mathrm{CBC}$ (Beckman coulter LH 750 analyser)

2. Salah's Bone marrow aspiration needle (11G- adults, 13Gchildren)

3. Jamshedi bone marrow biopsy needle (11G- adults, 13Gchildren)

Results: I studied total 75 patients of pancytopenia/ bicytopenia in the department of pathology in our institute. Among the total 75 patients studied 35 patients were males and 40 patients were females with M:F ratio of 0.87:1. Haematological data of 75 cases was collected and studied under four headings: haemoglobin in $\mathrm{g} \%$, total leucocyte count in cells/cu mm, platelet count in cells/cu mm. Megaloblastic anaemia is observed as the commonest cause of pancytopenia/ bicytopenia in all age groups except 61-80 years in which hypoplastic 
anaemia, AML and MDS are commonest causes with $22.22 \%$ each. Hypoplastic anaemia is reported as the $2^{\text {nd }}$ commonest cause in 1-20 and 41-60 year age group while nutritional anaemia is $2^{\text {nd }}$ common cause in $21-40$ year age group. In the total 75 cases studied pancytopenia was observed in 60 cases and bicytopenia in 15 cases. Among the 15 cases of bicytopenia, anaemia and leucopenia was present in 3 cases while 12 cases presented with anaemia and thrombocytopenia. Pallor was noted in $100 \%$ cases, spleenomegaly was seen in $20 \%$ cases while hepatospleenomegaly was mainly seen in patients with hypersplenism and in some cases of leukemia. Lymphadenopathy was observed in $10 \%$ cases. Conclusion: haematological indices and bone marrow morphology is a simple, economical and safe diagnostic tool in the evaluation of pancytopenia/ bicytopenia and help in arriving at a diagnosis in most cases. However hematologic malignancies and MDS have to be supplemented with immunohistochemistry and cytogenetic studies. In megaoblastic anaemia if no clinical response to treatment is achieved then bone marrow examination and further work up becomes essential to find out the specific cause of pancytopenia/ bicytopenia. In aplastic anaemia, bone marrow examination is gold standard diagnostic tool for the diagnosis of the condition except rare exceptions. In aleukemic/ subleukemic leukemias bone marrow examination is very helpful to institute the appropriate treatment at the earliest because mnay a times it is a clinical emergency. In this series itself, three cases of AML M3 died of the disease before the appropriate treatment could be started. In hypersplenism the clinical finding of spleenomegaly with typical haematological indices, bone marrow examination is essential for the diagnosis of the condition. In other causes of pancytopenia/ bicytopenia the bone marrow morphology plays important role in diagnosis along with supplementary investigations.

\section{P-C 02}

\section{An Unusual Clinical Presentation of a Case of Paroxysmal Nocturnal Hemoglobinuria (PNH) Diagnosed on Flowcytometry}

\section{Monika Gupta\#, Manpreet Tanwar, Sonia Chhabra, Rajeev Sen}

Email ID for Correspondence: monikashashwat@hotmail.com Pt BDS, Post Graduate Institute of Medical Sciences, UHS, Rohtak (Haryana)

Introduction: Paroxysmal nocturnal hemoglobinuria $(\mathrm{PNH})$ is an acquired clonal stem cell disorder resulting from a somatic mutation in the hematopoietic stem cell. It is characterized by intravascular hemolysis, cytopenias, frequent infections, bone marrow hypoplasia, and a high incidence of life threatening venous thrombosis. An absent glycosylphosphatidylinositol (GPI)-anchored receptor prevents several proteins from binding to the erythrocyte membrane. We present a 28-year-old male with combination of symptoms and signs that made us to diagnose this rare disorder. Patient/ Material and methods: A 28-year-old man presented with a history of recurrent jaundice over the past 10 years usually following fever episodes and requiring blood transfusions during the episodes. Examination revealed moderate pallor with mild icterus and moderate splenomegaly. His blood picture showed bicytopenia with elevated reticulocytes and indirect hyperbilirubinaemia. Urine examination was within normal limits. Coombs test, HPLC, bone marrow aspiration and lactate dehydrogenase was done. Flow cytometry was performed using 6 colour 2-laser flow cytometey (BDBiosciences, FACS CANTO II). Immunophenotypic analysis was performed using gating antibodies CD45, CD15, CD64 and the GPI-linked antibodies CD59, CD14,
CD24, as well as fluorescent Aerolysin (FLAER) showed evidence of PNH clone on RBCs, granulocytes and monocytes. Discussion: We studied the clinico-hematological profile of the case as it presented a unique diagnostic challenge and hence remained undiagnosed for a period of 10 years. The presence of splenomegaly with an elevated reticulocyte count pointed towards a haemolytic anaemia. Confirmatory diagnosis of PNH was done on the basis of flowcytometry. The delay in the diagnosis of this disease was due to its rarity and nonspecific clinical features. This was possible because diagnostic testing has evolved significantly due to the better understanding of the molecular basis of the disease and eventually the pathogenesis of hemolysis in PNH. Prompt and accurate diagnosis is important as effective therapies have become available. Conclusion: Flow cytometry is now widely accepted as the method of choice for detecting GPI-anchor protein-deficient clones in clinical, subclinical $\mathrm{PNH}$ and related bone marrow disorders. Clinical and laboratory findings together should raise the suspicion for $\mathrm{PNH}$, and to proceed for confirmatory diagnosis by flow cytometry.

\section{P-C 03}

\section{Effectiveness of Low Dose Generic Antithymocyte Globulin and cyclosporine In Idiopathic Aplastic Anemia}

Background: In transplant ineligible idiopathic aplastic anemia (IAA) patients, immunosuppressant therapy (IST) with equine Antithymocyte globulin (eATG) and cyclosporine is a standard treatment option. In resource constraint settings, where cost is an indisputable determinant of care, haematologists perennially face challenges to come forward with economical yet effective treatment strategies to ensure wider affordability. To explore cost-effective therapeutic options for this under addressed patient population, we studied the efficacy of a lower dose of generic eATG. Methods: Transplant ineligible AA patients (age $>12$ years) treated with generic eATG (Thymogam) and cyclosporine from January 2010 to December 2016 were retrospectively analysed. Efficacy of low dose generic eATG $(25 \mathrm{mg} / \mathrm{kg} / \mathrm{d}$ for 4 days) was analysed and compared with standard dose of generic eATG ( $40 \mathrm{mg} / \mathrm{kg} / \mathrm{d}$ for 4 days). The choice of eATG dose was based on affordability of the patient. The patients who had completed eATG therapy and followed up at 3 months and 6 months were included in efficacy analysis. Overall response rate (ORR) [including complete response (CR) and partial response (PR)] at 3 months and 6 months were analysed. Results: Thirty-four AA patients treated with generic eATG and cyclosporine were analysed. Mean age was 23.74 years (range 13-45 years); 22 $(64.7 \%)$ were males. PNH clone was present in 13 (38.2\%) patients. Median duration of follow up was 21.1 months (range 3-42 months). Out of 34 patients included in the study, one patient died on day 15 of therapy due to febrile neutropenia, 32 followed up at 3 months and 30 completed 6 months follow up. ORR in the study population was $48.8 \%$ at 3 months and $60.6 \%$ at 6 months. Difference between ORR at 3 months with low dose (42.9\%, CR 0, PR 6) and standard dose eATG $(47.3 \%$, CR 0, PR 9) was not significantly significant $(\mathrm{p}=0.8)$. Similarly, difference in ORR at 6 months was not statistically significant between low dose $(53.8 \%, \mathrm{CR} 0, \mathrm{PR} 7)$ and standard dose $(61.1 \%$, CR 1, PR 10) eATG $(\mathrm{p}=0.537)$. Serious adverse events were observed in three patients in low dose group [non-fatal febrile neutropenia 1; seizure 1; acute kidney injury (AKI) 1] and 7 patients in standard dose group [death due to febrile neutropenia 1; non-fatal febrile neutropenia 2; intracranial bleed 1; AKI 2; flash pulmonary edema 1]. On multivariate analysis for ORR at 6 months, 
none of the expected variables (age, absolute neutrophil count, absolute lymphocyte count, interval between onset of symptoms to initiation of therapy) could reach statistical significance. Two patients relapsed (after 30 months and 36 months, respectively) after receiving low dose ATG and three patients relapsed after receiving standard dose ATG (after 18 months, 26 months and 38 months, respectively). Conclusion: We found that efficacy of low dose generic eATG was similar to the standard dose generic eATG. We conclude that IST with low dose generic eATG and cyclosporine is an efficacious and economical approach that can potentially enhance treatment affordability in IAA patients. However, prospective studies with larger number of patients are needed to further validate these results.

\section{P-C 04}

\section{Pregnancy as a Cause of Pure Red Cell Aplasia}

\section{Chandan Baranwal, Uday Prabhakar, Sushil Yadav, Sukdev} Manna

Presenting author: Saumyaleen Roy

Institute - Banaras Hindu University, Varanasi

Introduction: Pure red cell aplasia is a syndrome characterized by severe normocytic anemia, reticulocytopenia and absence of red cell precursor in bone marrow. Pathophysiologically it can be immunologically mediated. It is associated with pregnancy and others with viral infection, lymphoma, collagen vascular disease. Pregnancy associated Pure red cell aplasia is a rare entity. Here we mention a 26-year-old female with history of abortion admitted with severe anemia. Objective: Pregnancy as a cause of pure red cell aplasia. Materials and Methodology: 26-year-old female with history of single abortion admitted with generalized weakness and easy fatigability. She had no history of concurrent fever, jaundice previously, joint pain, photosensitive rash, oral ulceration, any history of similar admission in past for low hemoglobin. She takes mixed diet and drug history is nothing suggestive. On admission she had severe pallor with pedal edema. No lymphadenopathy or organomegaly detected. CNS, CVS, CHEST, ABDOMEN examinations were within normal limits. Her routine blood investigation, bone marrow biopsy, collagen profile, PCR for virus detection in blood, CECT chest done to look for all associations. Results: Her routine investigation showed HB-4.8 g/dl, TRBC-1.6 millions, TLC-5370, DLC-N48L42, Platelet-adequate, MCV -91, LFT and RFT are within normal limits. General blood picture showed decreased red cell lines while other cell lines showed cells of normal morphology and number, Corrected reticulocyte count (CRC) were .4\%. Her iron/tibc level 233/249.vit b12 and folic acid stores were normal. Bone marrow biopsy showed markedly depressed normoblastic erythroid series, with M: E ratio -40:1, other cell lines within normal limits. A picture of pure red cell aplasia is being made from bone marrow studies. Viral markers were negative, collagen profile negative, test for parvovirus, cytomegalovirus and Epstein bar virus PCR from blood came to be negative, USG -neck done along with CECT chest showed no evidence of thymoma. Patient was transfused 2 units of PRBC and is then discharged with HB -9.8, WBC-5600 and platelet adequate. Patient was evaluated after 3 months, her $\mathrm{HB}$ was $12 \mathrm{~g} / \mathrm{dl}$, total RBC count 3.5 million, R.C $1.5 \%$, with other parameters normal. Bone marrow examination showed normoblastic erythropoiesis with erythrocytes in different stages of maturation.

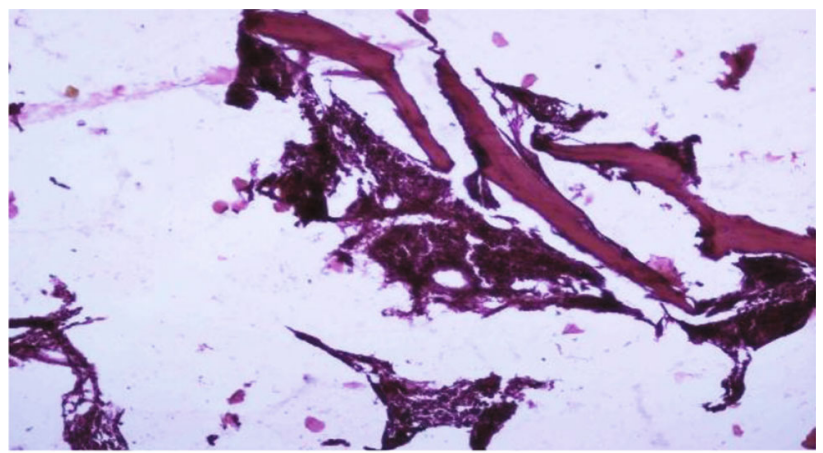

\section{slide showing pure red cell aplasia.}

Conclusion: Pure red cell aplasia is a hematological disorder where the precursor of red blood cells are absent in bone marrow with other cell lines intact Pregnancy associated PRCA is very rare. It is said that igG immunoglobulin is formed against the erythroid precursors during pregnancy with pure red cell aplasia. Pregnancy associated pure red cell aplasia has got good prognostic factor. In our case diagnosis of pure red cell aplasia was made on the ground of bone marrow study, peripheral blood study. Other association causing pure red cell aplasia have been ruled out and only association was pregnancy.

\section{P-C 05}

Study of Clinico-hematological Evaluation of Pancytopenia in Adult at tertiary Care Hospital in Eastern Part of India

\section{Chandan kumar, JR III General Medicine IMS BHU Varanasi (U.P.)}

Introduction: Pancytopenia is quite common in day to day clinical practice with variable clinical presentation from anemia, mild petechial spots and increased susceptibility to infections to severe anemia, life threatening bleeding and gaining of overwhelming infections. It is not the disease, rather it is outcome of various disease and disease processes. The underlying pathology determines the prognosis of disease and the condition of pancytopenia.

\section{Objective:}

a) To analyze the etiology and its distribution in causing pancytopenia.

b) To evaluate the clinical profile \& its correlation in patients with pancytopenia.

Material and methods: A prospective study was carried out among 141 cases of pancytopenia, evaluated clinically along with hematological parameter and bone marrow examination in department of General Medicine in collaboration with department of Pathology, IMS BHU during the period of July, 2015 to June, 2017. Results: Among all 141 cases studied, of age group $>15$ years were included in study. Most of the patients were in younger age group i.e. 
15-25 year age group with nearly equal occurrence in both sexes. The commonest cause among all etiology was found to be megaloblastic anemia $(27 \%)$ followed by hypoplastic and visceral leishmaniasis, presented with predominantly generalized weakness and fever and exertional breathlessness. In all patients pallor was almost universal finding. In most of the patients Hemoglobin found to be ranged between 4.2-7.5 g/dl with mean MCV 98.67, Total leucocyte count ranged between $1700-3255 / \mathrm{mm}^{3}$ and Platelet count ranged between $20000-70500 / \mathrm{mm}^{3}$. Conclusion: Being megaloblastic anemia the most common cause of pancytopenia and it is more common in female can be explained by their dietary habits, which is reversible cause with adequate management and the result will help in effective reinforcement of fortification of food and effective counselling regarding dietary habits as most coming from low socio economic groups. Hypoplastic anemia and visceral leishmaniasis are the $2^{\text {nd }} \&$ $3^{\text {rd }}$ most common cause. This suggest the need of detail work up along with bone marrow examination is extermination must be done in evaluation, to see prognosis and management of pancytopenia.

\section{P-C 06}

A Clinicohematological Evaluation of Pancytopenia in Southern Odisha-A 1 and 1/2 Years Institutional Study

Dr. Krusnasis Dash*\#, Dr. M.K. Patro**, Dr. Jayanti Nayak***, Prof. Dr. Debiprasad Mishra****

Email ID for Correspondence: krisnadoc@gmail.com M.K.C.G.Medical College, Brahmapur, Odisha

Introduction: Pancytopenia is not a disease rather a relatively common haematological entity encountered in clinical practice. It is characterised by decrease in all the three formed elements of blood. It is a laboratory finding in a wide range of diseases that range from transient marrow suppression due to viral illness to dreaded malignant neoplasms both hematopoietic and metastatic. Objectives: 1. To identify the causes of pancytopenia in this region of the state by studying the clinical, peripheral blood and bone marrow findings. 2 . To observe the prevalence of various causes of pancytopenia. Material and Methods: A prospective institutional based study conducted in the Department of Pathology, MKCG MCH Brahmapur over a1 $1 / 2$ year period. After careful recording of the detailed clinical history first a routine peripheral blood examination that includes CBC and PBS evaluation was done and findings are recorded. Finally a Bone marrow aspiration and Trephine biopsy was performed. Ancillary studies as and when required for a diagnosis were performed. Results: Pancytopenia was encountered in all age groups. Most common cause of pancytopenia was found to be Megaloblastic anemia $(40 \%)$ followed in decreasing orders of frequency by Hypoplastic anemia (25\%), Transient marrow suppression due to drugs (20\%), Malaria (10\%), Hypersplenism (2\%) and Hypoplastic MDS, AML \& Tuberculosis of marrow $1 \%$ each. Conclusions: The present study concludes that detailed primary haematological investigations also with bone marrow study in cytopenic patients are helpful for understanding disease process, to rule out possible causes, planning for further investigations and management.

\section{P-C 07}

"Serum Thrombopoietin Levels in Aplastic Anemia and Myelodysplastic Syndrome- A Comparative Study"
Anu Singh", Wahid Ali, U.S.Singh, Rashmi Kushwaha, Mili Jain, Dr. S.P.Verma, Ashutosh Kumar

Email ID for Correspondence: dr.anusingh08@gmail.com King George Medical University, Lucknow

Introduction: Thrombopoietin (TPO) regulates platelet production by controlling megakaryocyte growth and differentiation. Thrombocytopenia in aplastic anemia (AA) and myelodysplastic syndrome (MDS) has different underlying pathophysiology. In Aplastic Anemia there is decrease overall production while in MDS there is abnormal production of megakaryocytes and precursors. In both the conditions there is decrease in platelet counts while there increase in thrombopoetin levels are not proportionate to platelet counts in both the conditions. Objectives: To measure circulating serum TPO in AA and MDS \& to compare serum TPO levels between the two categories and with controls. Material and Methods: 64 cases comprising of AA (43) and MDS (21)) and 21 healthy individuals were enrolled in the study. TPO was measured by using Human TPO ELISA kit (Catalog No: E-EL-H1588). Statistical analysis was done using SPSS (Statistical Package for Social Sciences) Version 15.0 statistical Analysis Software. Association between attributes was analysed using chi square test and $\mathrm{p}$ value $<0.01$ was considered significant. Results: AA was most common in younger age group, while MDS patients were $>40 \mathrm{yrs}$. Mean TPO $(\mathrm{pg} / \mathrm{ml})$ in AA $(1469.23 \pm 475.25)$ were statistically significantly higher as compared to MDS $(324.14 \pm 82.13)$ and control group $(186.90 \pm 94.27)$. TPO levels in AA categories were highest among severe AA followed by very severe AA. TPO values in subcategories of MDS were not significant. TPO levels are inversely proportional to platelet count. Most common symptom among AA \&MDS patients was pallor. Conclusions: TPO levels are significantly raised $(\mathrm{p}<0.001)$ in AA \& MDS in comparison to healthy individuals. The levels are significantly higher in AA as compared to MDS Platelet counts were comparable in both the groups but rise in TPO level was not proportionate to it, Hence platelet count is not the only factor that regulates TPO levels.

\section{P-C 08}

Androgens are associated with reasonable responses in patients with aplastic anaemia especially with non severe disease

Nisham PN, Yasir Jeelani Samoon, Kavitha M L, Anup J Devasia, Anu Korula, Uday P Kulkarni, Aby Abraham, Alok Srivastava, Vikram Mathews, Biju George

Department of Haematology, Christian Medical College, Vellore, Tamilnadu, India

Introduction: Haematopoietic stem cell transplant (HSCT) or Immunosuppressive therapy (IST) with antithymocyte globulin and Cyclosporine remains the standard of care in patients with aplastic anaemia (AA). Androgenic steroids are used where both are not feasible due to financial constraints. We studied the outcomes of newly diagnosed patients with acquired AA who received first line therapy with androgenic steroids (Stanazolol and Danazol) in the department of Haematology, CMC Vellore between 2004 and 2014. Materials and Methods: We reviewed data on 107 patients who received treatment with androgenic steroids for AA. The diagnosis was based on blood counts and bone marrow analysis and was classified as per Camitta criteria. In general males received stanazolol [adults $40 \mathrm{mg} /$ day; children $1 \mathrm{mg} / \mathrm{kg} /$ day) while females received danazol [adults $200-600 \mathrm{mg} /$ day in 2-3 divided doses; children $1 \mathrm{mg} / \mathrm{kg} / \mathrm{day}]$. Only patients who had atleast 1 follow up after 3 months were included in this analysis. Responses were classified as 
complete or partial. Results: 107 patients with a median age of 30 years (range- 2-79)and a male: female ratio of 1.3: 1 were studied with $21(19.6 \%)$ being less than 15 years of age. 9.3\% of patients were classified as very severe (VSAA), $40.2 \%$ as severe (SAA) while $50.5 \%$ had nonsevere AA (NSAA). Stanazolol was given to 63 patients $(58.9 \%)$ while $44(40.1 \%)$ received Danazol. The median time from onset of symptoms to initiation of treatment was 75 days (range: 2-730). An overall response (ORR) was seen in 49 patients (45.8\%) patients of which $19(17.4 \%)$ had a complete response and 30 $(28.1 \%)$ had a partial response. The median duration of follow up for the entire cohort was 22 months (range: 2-134). ORR was significantly higher in patients with NSAA [57.4\%; CR26\% PR31.4\%] compared to SAA [37.2\%; CR9.3\% PR 27.9\%) and VSAA [20\%; CR10\% PR10\%] $(\mathrm{p}=0.019)$. There was a trend towards better ORR with Danazol [56.8\%] compared to Stanazolol [38.1\%; $\mathrm{p}=0.07$ ]. Age, Sex distribution, time to initiation of therapy, clinical symptoms, prior history of jaundice did not affect response. None of patients lost response to androgenic steroids or underwent clonal evolution during the two year follow up period. However 4 patients lost response on subsequent follow up. 3 patients out of 107 patients transformed to MDS during subsequent follow up (6-7 years) while 1 patient developed a PNH clone (after 28 months). Among the non responders 11 patients received ATG, 11 patients received Cyclosporine and 6 patients underwent stem cell transplant. The median $5 \mathrm{yr}$ overall survival and event free survival of these patients were $83.8 \pm 5 \%$ and $41.2 \pm 5 \%$ respectively. The median $5 \mathrm{yr}$ overall survival for the nonsevere group was compared with Severe and very severe group and was found to clinically significant $(89.5 \pm 6.1 \%$ Vs $78.5 \pm 7.4 \%, \mathrm{p}=0.021)$. The median $5 \mathrm{yr}$ EFS for the nonsevere group was compared with severe and very severe group and was found to be clinically significant $(51.2 \pm 7.3 \%$ vs $31.6 \pm 6.5 \%$, $\mathrm{p}=0.017$ ). Conclusion: Androgens are associated with reasonable response and survival in patients with acquired aplastic anaemia.

\section{P-C 10}

\section{Outcome of Immunosuppressive Therapy in Patients with Acquired Aplastic Anaemia}

\section{Dutta B ${ }^{\#}$, Dolai TK, Mandal PK, Baul S, De R, Chakrabarti P}

Email ID for Correspondence: bijitadutta123@gmail.com Department of Haematology, NRS Medical College and Hospital

Introduction: Aplastic anaemia (AA) is an immune-mediated bone marrow failure disorder. Majority of AA patients could not undergone HSCT due to non-availability of matched donor, co-morbidity and financial reasons. Objectives: To evaluate response after immunosuppressive therapy (IST) consisting of horse anti-thymocyte globulin (ATG) and cyclosporine (CSA) in patients with acquired AA. Material and Methods: This study was conducted from year March'2011 to September'2017. AA was diagnosed as per established criteria. Horse ATG was administered at the dose of $40 \mathrm{mg} / \mathrm{kg} /$ day for 4 days and cyclosporine at $5 \mathrm{mg} / \mathrm{kg} /$ day in two divided doses from day 14 of ATG therapy. Response assessment was done at three months and six months. Published response criteria was followed for assessing response. Results: A total of 57 AA patients was included in the study. Male and Female ratio was 2:1. Median age at presentation was 35 years (range 6 to 65yrs). Majority of patients presented with anaemia and bleeding manifestation. At presentation mean $\mathrm{Hb}$ was $7.19 \mathrm{~g} / \mathrm{dl}$ (range 4.4 to 13.8), mean ANC was $838 / \mu$ l (range 200 to 2000 ) and mean platelet count was $18355 / \mu 1$ (range 1000 to 91000 ). Forty (83.3\%) patients had severe AA, 2 (4.16\%) patients had very severe AA and $6(12.5 \%)$ patients had non-severe AA.
Median interval from diagnosis to ATG therapy was 90 days (range 10 days to 5 years). ATGAM was received 29 patients and 28 patients received THYMOGAM. Four patients received ATG one month back and four patients died before 3 months of post ATG. At the end of three months $18(33.96 \%)$ patients showed partial response (PR), $35(66.03 \%)$ patients showed no response (NR). At six months 16 (30.18\%) showed complete response (CR), 12 (22.64\%) were in PR and $25(47.16 \%)$ patients showed NR. As side effects two patients (4.167\%) developed serum sickness and one (2.08\%) developed progressive mulifocal encephalopathy. Conclusions: Overall response to ATG at the end of 3 months and 6 months was $33.96 \%$ and $52.82 \%$ respectively.

\section{P-C 11}

Clinical, Hematological and Pathological Profile of Patients with Pancytopenia and Prolonged Fever in a Tertiary Care Hospital in Northern INDIA

Introduction: Pancytopenia, a frequently encountered clinico-hematological condition can be attributed to a myriad number of causes, most common of them being, aplastic anemia, megaloblastosis, hematological malignancies, hypersplenism, bone marrow infiltration disorders and infectious conditions apart from cytotoxic agents and radiotherapy. This common clinical condition, its varied etiologies, different clinical presentations and response to treatment have been investigated and reported by only a few studies in literature. Aims and Objectives: To study the clinical, hematological and pathological profile of patients with pancytopenia admitted in Department of Medicine and Hematology at All India Institute of Medical Sciences, Delhi. Methodology: Prospective observational study for duration of 1 year from January 2016 to December 2016 of all patients presenting to the Medicine and Hematology Out-patient Department or admitted in the wards with age $\geq 14$ years, history of prolonged fever (more than 3 weeks duration) and constitutional symptoms, pancytopenia in peripheral blood smear examination and those who gave consent were evaluated. Patients with pancytopenia due to diagnosed infections (e.g., dengue, malaria, septicaemia) or iatrogenic cause (e.g., radiation, cancer chemotherapy) and those who refused consent for bone marrow examination were excluded. Results: A total of 32 patients satisfied the eligibility criteria, out of which $59 \%$ were females and $41 \%$ males with $68 \%$ of them aged less than 40 years. The etiological distribution was as follows - Hematological malignancies constituted $68 \%$ of which most common was aplastic anemia, Infectious causes including disseminated tuberculosis was $16 \%$ followed by hypersplenism in $7 \%$, autoimmune in $3 \%$ and miscellaneous causes in $6 \%$ of patients. The most common symptom was fatiguability $(68 \%)$ followed by pallor (46\%), loss of weight $(43 \%)$ and shortness of breath $(40 \%)$. Ninety three percent had clinical pallor while significant hepatomegaly, splenomegaly and lymphadenopathy was present in $28 \%, 34 \%$ and $7 \%$ respectively. The mean hemoglobin level was $7.06 \pm 2.47(2-10.6)$ with $50 \%$ having moderate (8-10.9) and $50 \%$ having severe $(<8)$ anemia. The mean TLC and platelet count were $2466 \pm 1058 \quad(400-3750)$ and $40,093 \pm 25,909 \quad(7000-94000)$ respectively. The corrected retic count varied between $<1 \%(56 \%)$, $1-2 \%(35 \%)$ and $>3 \%(9 \%)$. Bone marrow examination revelaed cellular marrow in $50 \%$, hypocellularity in $37 \%$, blasts in $15 \%$, hemophagocytosis in $9 \%$ and granulomas in $11 \%$ patients. The follow up data showed mortality in $28 \%$, complete recovery in $12.5 \%$ and persistent of pancytopenia in the remaining. The results of this study show the varied etiologies of pancytopenia when they present with prolonged fever with infectious conditions being reversible causes. 


\section{P-HL 01}

Rare case of HLH (Hemophagocytic Lymphohistiocytosis) secondary to chronic malaria

\section{R P Singh}

\section{Dr. Ankita Katara (Presenting Author)}

Institute - Forrd Hospital, Lucknow

Haemophagocytic lymphohistiocytosis (HLH), is a disorder characterized by proliferation of histiocytes in the bone marrow, liver and spleen. Histiocytes demonstrate phagocytosis of hemopoietic cells. HLH (Haemophagocytic lymphohistiocytosis)can be primary (Familial) and secondary (acquired) forms. Secondary HLH mainly occurs in various infections, malignancy (associated with lymphomas), autoimmune diseases (SLE) and drugs (Phenobarbitone and cotrimoxazole). Primary HLH mainly occurs in childhood whereas the secondary forms can occur at any age. HLH secondary to infections can occur with viral (Mainly Epstein-Barr virus (EBV)), bacterial, fungal or parasitic infections (Malaria, Leishmaniasis etc.). Literature on malaria-associated HLH is very less. Here, We present a case of malaria-associated secondary HLH in a $19 \mathrm{yr}$. old boy admitted in medicine department of Forrd Hospital, Lucknow, UttarPradesh, who presented with fever, anorexia and weight loss since 1 year. On P/S examination Pancytopenia was detected. Blood tests for Malaria, typhoid and Filaria were negative. Bone marrow aspiration was done, gametocytes of Plasmodium Vivax was found and a diagnosis of HLH secondary to malaria was made.(Rapid Card Test based on immunochromatographic method are unable to detect malaria in case of only gametocytes) Patient was treated empirically for malaria and his blood counts and his condition was improved.

\section{P-HL 02}

Hemophagocytic Lymphohistiocytosis in Traumatic Brain Injury Patient Infected with Epstein-Barr Virus: A Case Report

\section{Dr Damodar Das, Dr P C Bhattacharyya, Dr Anuj Sarma, Dr} Sumitav Barua

Email ID for Correspondence: dr.lalitmohan7@gmail.com; Presenting Authors: Dr Lalit Mohan Bhardwaj Down Town Hospital, Kamrup, Guwahati, Assam

Hemophagocytic lymphohistiocytosis (HLH) is an uncommon, lifethreatening hyperinflammatory syndrome caused by severe hypercytokinemia with excessive activation of lymphocytes and macrophages due to a highly stimulated but ineffective immune process. HLH is primarily a paediatric disorder and epidemiological data for adults are sparse. Epstein-Barr virus (EBV) is the most common infective cause of acquired HLH. EBV associated HLH is associated with high mortality if not treated early. A high index of suspicion, early diagnosis, and prompt treatment is very crucial in saving patient's life. Here we report a case of Traumatic Brain Injury in 52 years old Bhutan resident complicated by EBV induced HLH.

\section{P-HL 03}

Hemophagocytic Lymphohistiocytosis in Disseminated Mycobacteriaum Tuberculosis
Somanath Padhi ${ }^{\#}$, Manoj Kumar Panigrahi ${ }^{1}$, Yara Dhanurdhar ${ }^{1}$, Susama Patra

Email ID for Correspondence: somanath.padhi@gmail.com Departments of Pathology and Laboratory Medicine, and ${ }^{1}$ Pulmonary Medicine, All India Institute of Medical Sciences, Bhubaneswar

Introduction: Hemophagocytic lymphohistiocytosis (HLH) is a potentially fatal hyperinflammatory syndrome which can rarely complicate the clinical course of tuberculosis (TB). Nearly 80 cases of TB associated HLH (TB-HLH) have been reported in the world literature. Objective: To describe a case of TB-HLH in a middle aged female patient with correlation of clinicolaboratory parameters and brief literature review. Case report: A 38-year-old female presented with prolonged fever, cough, fatigue, and worsening pancytopenia, hyperbilirubinemia, markedly elevated liver transaminases, prolonged prothrombin time and activated partial thromboplastin time, and hyponatremia. Chest $\times$ ray and CECT-thorax revealed mediastinal lymph nodes encasing left pulmonary trunk; suspicious of a lymphoma or TB. Bone marrow aspiration and trephine biopsy showed marked hypocellularity, numerous caseating necrotizing granulomas with numerous Acid Fast Bacilli and prominent histiocytic hemophagocytosis. Marked hyperferritinemia $(>31000 \mathrm{ng} / \mathrm{ml})$, hypertriglyceridemia $(>500 \mathrm{mg} / \mathrm{dl})$, and hypofibrinogenemia $(<1 \mathrm{~g} /$ L) confirmed TB-HLH (HScore $=279$ ). The patient is closely being followed up with antitubercular drugs and immunosuppressive therapy with a favourable outcome. Conclusion: TB-HLH is rare with unpredictable biological behaviour and outcome. Literature review have found nearly 80 such cases already reported in the world literature with an overall survival of nearly $50 \%$. Age $>30$ years, associated comorbidities, delayed diagnosis and/or initiation of ATT, marked hyperferritinemia, prominent hemophagocytosis in the marrow were reported to be associated with inferior survival in such patients. Usage of immunosuppressive and immunomodulator drugs did not alter the outcome in such patient. HLH may coexist; follow; or precede the diagnosis of Tuberculosis which can pose great challenge both for the diagnostic and treating physician. Hepatic injury in HLH makes the usage of hepatotoxic ATT and/or immunomodulator such as Etoposide quite intriguing.

\section{P-HL 04}

Hemophagocytic Lymphohistiocytosis-A Rare Series of 5 Cases *Dr. Roopa Urs AN, Dr. Renu Kumari, Dr. Sushma BJ

Introduction: Hemophagocytic lymphohistiocytosis (HLHL) is a rare, serious and potentially life threatening histiocytic disorder affecting children and adults. It is characterized by dysfunction of cytotoxic $\mathrm{T}$ cells leading to activation of cytototoxic $\mathrm{T}$ lymphocytes and macrophages resulting in cytokine storm and hence hemophagocytosis. Objective: To describe the clinico- hematological profile and chemical dearrangements in cases of HLH. Materials and methods: A retrospective record based study over a period of 2 years showed five cases of HLH. Results: The patients had varied clinical presentations ranging from abdominal tuberculosis, pulmonary tuberculosis, pneumonia, chronic diarrhoea to unprovoked bleeding etc. All five patients had fever for more than one week and spleenomegaly. The spleenic enlargement was mild in one patient and two each had moderate to massive enlargement. Hepatomegaly was present in one patient. All five patients had hyperferritinemia, two had hypofibrinogenemia and four had hypertriglyceridemia. All patients had thrombocytopenia and anaemia. One had leucopenia. Bone marrow aspirate in all cases revealed varying degrees of hemophagocytosis of erythroid, granulocyte and platelet elements. 
Hence the diagnosis was infection related HLH in all five patients. On follow up 3 patients died and 2 were lost to follow up. Conclusion: $\mathrm{HLH}$, though rare is a fatal disease arising commonly secondary to infection. Early diagnosis and appropriate treatment is essential in preventing mortality of such patients.

\section{P-HL 05}

Clinicohematological Profile of Hemophagocytic Lymphohistiocytosis (Hlh) - A Case Series

Nadeem Kocheri, Dr. Renu Saxena, Dr. Aastha Gupta

\section{All India Institute of Medical Sciences, New Delhi}

Introduction: Hemophagocytic Lymphohistiocytosis (HLH) is a life threatening hyper inflammatory syndrome defined by clinical and laboratory criteria. Diagnosis and treatment is based on the HLH 2004 criteria which include 8 parameters of which 5 are required for the diagnosis. Here we try to present a case series of HLH came across in our setting in AIIMS, New Delhi. Objectives: To compare the clinical and pathological findings in the rare disease hemophagocytic lymphohistiocytosis. Materials and Methods: We have selected the cases which showed hemophagocytosis in bone marrow from July 2015 to December 2016. Case files were taken out and analysed for clinical details (HLH criteria 2004). Data was analysed. Results: Of the total 13 cases which showed evidence of hemophagocytosis in bone marrow, 5 were females and 8 were males. Median age at the time of presentation was 18 years. On applying the HLH 2004 criteria to these patients, of the 14 cases, only 8 cases fulfilled the criteria for HLH. Of these 8 cases, 3 patients received HLH 2004 protocol based treatment and rest of them received steroids only. On follow up, only 2 patients survived after treatment which included one patient who received HLH 2004 protocol. Conclusion: Being a rare and life threatening disease, timely diagnosis and treatment is of utmost important in HLH. The HLH 2004 criteria for diagnosis is the only way for a successful diagnosis. Therefore it is important to investigate for the parameters included in the HLH criteria.

\section{P-HL 06}

\section{A Case Series of $\mathbf{H L H}$ Syndrome in Adults at a Tertiary Care Hospital}

\section{Kautuk lohia", Muralimohan B V, Ravichandra MRK,} Manisha Daware

Email ID for Correspondence:kautuklohia@gmail.com

Narayana health city, Bangalore

Introduction: Hemophagocytic lymphohistiocytosis (HLH) is a lifethreatening syndrome of excessive immune activation. It most frequently affects infants but also observed in adults of all ages. HLH can occur as a familial or sporadic disorder, triggered by an event. Infection being common trigger in both. Here we are reporting case series of HLH in adults. Diagnosis is based on combined clinical, laboratory, genetic, and morphological criteria. According to the Histiocyte Society in the absence of family history or of specific genetic tests, five out of eight criteria must be present in order to establish a diagnosis and initiate treatment. Objectives: To describe the clinical and laboratory presentation of hemophagocytic lymphohistiocytosis in adults treated at a referral institution. Material and Methods: A prospective descriptive study was carried out, three adults were diagnosed with hemophagocytic lymphohistiocytosis between 2016 and 2017. The HLH 2004 TRIAL criteria were used for diagnosis. Results: The patient's ages at diagnosis ranged from 24 to 68 years. All patients had splenomegaly, fever, cytopenias, hyperferritinemia and hypertriglyceridemia \&/or hypofibrinogenemia. Bone marrow hemophagocytosis was not detected in one patient. In another one case, no trigger was found. Two cases received only steroids, while one case receive steroids with cyclosporine. All patients expired within 48 hours of diagnosis of HLH. Conclusions: Hemophagocytic lymphohistiocytosis can have vivid presentation with nonspecific signs and symptoms. Inspite of initiation of immunosuppressive treatment the mortality remains high. Early diagnosis of cases and prompt initiation of treatment with immunosuppression may help.

\section{P-I 01}

A Uncommon Cause of Isolated Thromocytopenia in South India

Rohith G Chitrapur ${ }^{\#}$, Dr Murali Subramanian, Dr Preethi S chari

Email ID for Correspondence: rohithchitrapur@gmail.com Bangalore Baptist Hospital, Bangalore, Karnataka

Introduction: Isolated Thrombocytopenia in a elderly patient can be a diagnostic dilemma. Timely diagnosis is required for proper management of these patients. Objectives: Thrombocytopenia can be a presentation of many of the conditions varying from Malaria, Dengue fever, Immune Thrombocytopenia and Marrow infiltration disease Leukaemia, Lymphoma, Metastatic diseases. Presenting a uncommon cause of isolated thrombocytopenia. Material and Methods: 60 year old female patient with no comorbid conditions, with $\mathrm{p} / \mathrm{h} / \mathrm{o}$ travel to Badrinath and Hrishikesh presented with 1 week history of fatigue and low grade Fever. On examination patient had petechiae over the limbs, abdomen, chest and few patches in the mouth. Evaluation showed Platelet count of 10,000 with normal Haemoglobin, Normal WBC count and differential count. Peripheral smear showed isolated thrombocytopenia. Viral markers, malaria and dengue serology was negative. Bone marrow aspiration and biopsy was done reported as Megakaryocytic hyperplasia. She was started on High dose Dexamethasone for 4 days followed by oral prednisolone $1 \mathrm{mg} / \mathrm{kg} /$ day . There was no response till day 10 . Bone marrow was reviewed again, showed LD bodies and patient was started on Liposomal Amphotericin. Day 3 of Liposomal Amphotericin platelet count rose to 70,000. Results: Patient platelet count normalised by $5^{\text {th }}$ day of Liposomal Amphotericin. To repeat a Marrow for disappearance of LD bodies. Conclusion: Kalaazar presenting as a isolated thrombocytopenia can be a very tricky diagnostic dilemma in south India.

\section{P-I 02}

\section{Spectrum of Haematological Changes in HIV Infection}

Vinu K V*, Venkatesan S, Sampath K S, Bansal V, Bharadwaj R

Email ID for Correspondence: vbalraam@gmail.com Armed Forces Medical College

Introduction: Haematological abnormalities are frequently observed in HIV infected individuals. Bone marrow examination is usually performed in these patients to evaluate peripheral cytopenias or when systemic infections or malignancies are suspected. A spectrum of bone marrow changes is usually seen over and above the usually 
encountered peripheral cytopenias in the individuals infected with HIV. Objectives: The main objective of this study was to elucidate the spectrum of haematological changes encountered in HIV infected individuals with special emphasis on bone marrow examination. Material and Methods: A cross sectional study was conducted in the department of pathology on a sample size of 110 between Jan 2015-Aug 2017. Relevant clinical history, complete blood count and bone marrow findings were recorded. Results: Our studied included 24 females and 86 males. The commonest haematological abnormality we found was anaemia. 103 of the 110 patients (94\%) suffered from anaemia. Of these 103 patients, 62 patients suffered from anaemia of chronic disease $(60 \%), 24$ suffered from microcytic hypochromic anaemia (23\%), 16 had a macrocytic peripheral blood picture $(16 \%)$ and 01 had dimorphic anaemia (1\%). 44\% of them had leukopenia and $25 \%$ cases had neutropenia. As much as $36 \%$ patients had left shift in the neutrophil series. $31 \%$ cases had low platelet count. The commonest bone marrow abnormality was reactive plasmacytosis $(52 \%)$, which was in the range from as low as $05 \%$ to as high as $25 \%$ of marrow nucleated cells.Other abnormalities included megaloblastoid changes (35\%) and erythroid hyperplasia (28\%). Granulomatous inflammation was seen in $23 \%$ of cases. 05 cases showed haemophagocytosis. Lymphoma depositswere seen in 04 cases. One of the marrow studied showed involvement by plasma cell neoplasm. Conclusions: Bone marrow study is an important investigation in HIV infected patients with peripheral haematological abnormalities.

\section{P-I 03}

\section{Idiopathic CD4+ T- Lymphocytopenia with Diffused} Cryptococcosis

Sushil Yadav", Jagveer Singh, Saumyaleen Roy, Prof K K Gupta, Dr Nilesh Kumar, Dr pankaj kannauje

Email ID for Correspondence: sushil001rocks@gmail.com Institute of Medical Sciences, Banaras Hindu University, Varanasi

Introduction: Decrease in CD4+ T cell counts has varied detrimental effects on immune system of body. Idiopathic CD4+ T cell lymphocytopenia (ICL) is a rare syndrome in which persistent CD4+ T cell lymphopenia occurs in absence of infection with HIV, or any other cause of immunodeficiency. (ICL) is characterised by opportunistic infections, malignancies, autoimmune disorders. Lymphocytopenia may exists long before opportunistic infections are detected. Objectives: patient- A 55 years old female, presented with fever, headache, maculopapular rash for 2 months, no history of seizures, hemoptysis, chronic drug intake, ATT intake in past. On examination patient GCS- 7 (E2V1M4), febrile- (101.f) with neck rigidity, kernigs sign present. Maculopapular rash present over upper and lower limb present. Other systemic examination were within normal limits. Material and Methods: On investigations, TLC- 20350 N-95\%, NCCT head, Digital CXR, USG abdomen within normal limits, CSFprotein 195 , total cells $500, \mathrm{~N} 90$, india ink- capsulated yeast cells seen, CSF c/s- cryptococcus growh present, Urine c/s- cryptococci growh present, HIV negative, Absolute lymphocyte count- 180 cells/ microliter, CD4+ cells - 19.2\% - total 34 cells, CD3+ cells $-80.8 \%$ total 146 cells. Results: Counts were repeated after 6 weeks Absolute lymphocyte count- 171 cells/microliter, CD4+ cells $-18.7 \%$ - total 32 cells, CD3+ cells $-81.3 \%$ total 139 cells. HIV negative. No other cause of lymphocytopenia identified, suggestive of Idiopathic CD4+ lymphocytopenia with diffused cryptococcosis. Conclusions: Idiopathic CD4+ lymphocytopenia is a rare syndrome with very few cases repoted till now. It is possible cause of opportunistic infections in an individual who is HIV negative, until patient is screened for
CD4+ cells. Further study of genetic basis and immunopathogenesis of this heterogenous condition is required so that targeted treatment for Idiopathic CD4+ lymphocytopenia patients at risk can be safely established.

\section{P-I 04}

A Rare Case of Leucoerythroblastosis in a Preterm Very Low Birth Weight Baby Due to Parvo Virus B19 Infection

\section{Dr. Prashant Arora}

Presenting Author: Ankita Katara

Forrd Hospital, Lucknow

Leucoerythroblastosis is a rare finding characterized by presence of immature erythroid and myeloid precursors in peripheral blood. A female baby weighing around 700 grams, was born prematurely at the 28th week of gestation by Cesarean section. Baby was immediately shifted to NICU (Neonatal Intensive Care Unit) of Forrd Hospital, Lucknow, Uttarpradesh due to prematurity and respiratory distress. Physical examination revealed tachypnea and hepatosplenomegaly. Routine laboratory measurements showed significant leucocytosis (35,000cells/cumm) and anemia (Hb: $11.8 \mathrm{~g} / \mathrm{dL}$ and Hct: 33.6\%). The platelet count was initially normal, but later on decreased to 50,000 . The peripheral blood smear suggested leucoerythroblastosis with the presence of normoblasts, myeloid precursors, and $4 \%$ blasts. Serum IgM and IgG levels for Human Parvovirus B19 were done and results turned out to be POSITIVE. The causative factors for leucoerythroblastosis occurring during neonatal and early childhood are viral infections, JMML (Juvenile Myelomonocytic Leukemia) and osteopetrosis. According to various literatures, very few cases of leucoerythroblastosis has been reported in premature and very lowbirth weight neonates. Intrauterine parvovirus B19 infection was considered to be the cause of premature delivery and leucoerythroblastosis. Here we present the case of a prematurely born newborn who was admitted in intensive care and was on ventilator due to prematurity and respiratory distress and was diagnosed with leucoerythroblastosis.

\section{P-I 05}

\section{Microparticles as Prognostic Biomarkers in Dengue Virus Infection}

Rucha Patil ${ }^{1}$ Bajpai $5^{2}$, and Shrimati Shetty ${ }^{1}$

Email ID for Correspondence: rucha colours@hotmail.com National Institute of lmmunohaematology (ICMR), 13th floor, KEM Hospital, Parel, Mumbai 400 012, India

Introduction: Dengue virus infection (DVI) manifests as a spectrum of illnesses ranging from mild febrile illness to the severe forms, i.e. dengue haemorrhagic fever and dengue shock syndrome (DSS). According to the recent WHO guidelines, cases are now classified as dengue without warning signs (DWOS), dengue with warning signs (DWS) and severe dengue (WHO, 2009). There are no vaccines or specific therapeutics currently available. Rapid diagnosis along with appropriate immediate fluid replacement is what is essential to achieve success in these cases. Promising biomarkers which may help predict the risk of developing severe DVI are lacking and will be helpful. Objectives: To analyze the role of cell-derived microparticles (M P) in DVI. Material and Methods: 60 patients with DVI (18: 
DWS; 1: DSS and 41: DWOS); along with 15 controls (other febrile illness) were included in the study. The following MPs were assessed: annexinV, platelet (CD41a), red blood cell (RBC)(CD235a) and activated endotheliai (CD62e) MPs. MP assessment by flow cytometry was standardized by participating in the International Society of Thrombosi s and Haemosta sis workshop. M P levels of the different groups were compared with those of controls using the 2 tailed Student's t-test. Statistical significance was assumed at $p<0.05,95 \%$ confidence interval (CI). Results: Platelet MP (PM P) levels in majority of our patients were found to be lower (536 $\pm 111 \mathrm{PM}$ Ps/ 111) than that observed in healthy subjects (731.5 \pm 377.3 PMPs/1-1-1; cut off: $>1486$ PMPs/l11). Patients with profound thrombocytopenia without bleeding (platelet count $<20000$, range: $4000-20000$ platelets/Ill) had statistically elevated PMPs ( $\mathrm{p}<0.001,95 \% \mathrm{Cl}$ : 922.532909.12 ) i.e. mean $\pm \mathrm{SD}=2270.6 \pm 990.1 \mathrm{PMPs} / 111$ when compared to those of the symptomatic patients with profound thrombocytopenia (mean $\pm \mathrm{SD}=354.8 \pm 88.3 \mathrm{PMPs} / 111)$. RBC MPs were found to be significantly elevated in the 2 nd phase in patient with DWS which wa s seen earliest on Day 4 of infection with a cut off of $2200 \mathrm{M}$ Ps/ Ill when compared to patients with DWOS ( $<1100 \mathrm{MPs} /$ 11l) $(\mathrm{p}<0.0001,95 \% \mathrm{Cl}: 2946-4706)$.

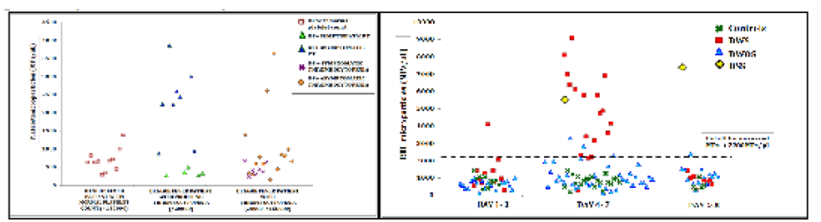

Conclusions: If procoagulant PM Ps; carrying all the surface antigens of platelets and thus functioning like platelets; are elev ated; their hae mostat ic f unction $w$ ill rema in sta ble. Thus such patients wit $h$ a low platelet count but elevated PM P levels will be protected a nd not suffer from hemorrhagic manifestations a nd thus will not require platelet tra nsf usion. PMP lev els may prove to be a promising nov el biomarker $\mathrm{w}$ hich helps discriminate patients in need of prophylactic platelet tra nsf usion. RBC MPs, on the ot her hand could be potentia 1 biomarkers capa ble of identifying potentially sev ere patients who req uire immediate ca re.Thus, $M$ Ps seem to be a promising importa nt biomarker in many aspects of DVI.

\section{P-I 06}

\section{Myelofibrosis in HIV Infected Patient: A Rare Case Report \\ Dr. Gajender Singh ${ }^{\#}$, Dr. Sanjay Kumar, Dr. Sant Prakash Kataria, Dr. Rajeev Sen, Dr. Sucheta}

Email ID for Correspondence: drgajender@rediffmail.com Pt. B. D. Sharma, PGIMS, Rohtak

Introduction: We are presenting a case of HIV infected patient who was diagnosed with myelofibrosis on bone marrow examination which is a rare feature and very few such cases have been reported in literature till now. Case Report: Patient was a 35 years HIV positive female and was on Anti-Retroviral Therapy (ART) since four months. Now she presented with persistent pancytopenia and hepatomegaly. Bone marrow aspiration slides of the patient were markedly hemodiluted and revealed few atypical cells with low to high N/C ratio with abundant basophilic cytoplasm, fine to coarse nuclear chromatin. Bone marrow biopsy revealed normoblastic erythropoiesis with normal myeloid maturation but reduced in number with increased number of megakaryocytes (micromegakaryocytes to large cells). Blast cells were less than 5\%. Diagnosis of Chronic myeloid neoplasm: Idiopathic myelofibrosis (Fibrosis Grade II) was offered. Discussion: As mentioned in the literature the changes in the bone marrow of HIV patients include myelodysplasia, myelofibrosis and hypocellularity. Myelofibrosis and hypocellularity are usually seen in advanced cases. Direct effect of HIV on progenitor cells, effect of drugs and other infective diseases can lead to such changes in bone marrow. For treatment of these cytopenias, optimal treatment of HIV infection is essential. Treatment of opportunistic infections, stopping drugs leading to cytopenias are essential. Supportive care of anemia includes use of erythropoietin in addition to red blood cell transfusion. Therapy for neutropenia includes use of myeloid growth factors G-CSF and GM-CSF. Thrombocytopenia associated with bleeding due to decreased production will require platelet transfusions. ART in these patients may have a variable response. Conclusion: Hence examination of bone marrow and proper diagnosis is important in such patients to modify the treatment accordingly.

\section{P-I 07}

\section{A Rare Case of Leishmaniasis in Bone Marrow Without Organomegaly}

Ankita Katara (Presenting Author)

\section{Forrd Hospital, Lucknow}

Leishmaniasis or kala-azar is a protozoan disease that occurs through tropical areas of the world. In India, it is usually localized in areas of Bihar, Jharkhand, West Bengal, and parts of Eastern Uttar Pradesh. The causative agent is a protozoan Leishmania Donovani and is transmitted by the bite of sand fly -Phlebotomus. Visceral leishmaniasis (VL) involves the visceral organs, mainly the liver, the spleen and bone marrow. It can present in cutaneous, mucocutaneous, visceral, and disseminated forms. VL is characterized by prolonged fever, malaise and moderate to massive splenomegaly. In peripheral blood present as Pancytopenia. Here we present a case of 10 year old boy who presented with fever and weakness for 6 months at Forrd Hospital, Lucknow, Uttar pradesh. On examination no organomegaly found. USG findings showed mild degree of hepatomegaly and splenomegaly only. On Peripheral smear, Pancytopenia was present. Blood tests for malaria, typhoid and filaraia were negative. Bone marrow aspiration was done and LD bodies were detected. Boy was treated for leishmaniasis, his fever subsided and blood counts became normal.

\section{P-I 08}

Visceral Leishmaniasis Presenting as Autoimmune Hemolytic Anemia: A Rare Phenomenon

Meena Rachana", Sharma Sunita, Singh Smita, Chauhan Shruti, Parakh Nupur*, Singh Varinder*

\section{Email ID for Correspondence: drrachana89@gmail.com}

Department of Pathology \& Paediatrics*, Lady Hardinge Medical College, New Delhi

Introduction: One of the major presenting clinical feature of Visceral leishmaniasis is anemia. Although various mechanisms of anemia have been described, anemia due to immune mediated hemolysis is rarely seen in these patients. Case Summary: We present a case 
report of 11 year old female child, resident of Nepal, presented with complaints of fever and abdominal distension for past 2 months. Physical examination showed pallor and hepatosplenomegaly. Laboratory Investigations revealed raised liver enzymes, serum lactate dehydrogenase and hemoglobinuria. Complete hemogram showed pancytopenia with reticulocytosis. Direct coomb test was positive (4+) for IgG type. Autoimmune workup and viral markers were negative. Bone marrow aspiration showed normoblastic erythroid hyperplasia with presence of few intracellular amastigote forms of Leishmania donovani and evidence of hemophagocytosis. Hence, the patient was diagnosed as kala azar associated with $\operatorname{IgG}$ mediated coombs positive haemolytic anemia. Severity of hemolysis was reduced (DCT 2+) after starting liposomal amphotericin B at a dose of $0.5 \mathrm{gm} / \mathrm{kg}$. However the patient died during the course of treatment. Conclusion: Visceral leishmaniasis can present with clinical and laboratory manifestations suggestive of autoimmune disease and can cause misdiagnosis. This case is being presented because of its rarity.

\section{P-I 09}

Study of hematological manifestations in HIV patients and correlation with CD4 level

Gaurav Kumar", Rashmi Kushwaha, Geeta Yadav, S.P.Verma, D. Himanshu, Wahid Ali, Ashutosh Kumar

Email ID for Correspondence: dr.gauravgupta08@gmail.com King Georges Medical University, Lucknow

Introduction: Hematological abnormalities are a common complication of HIV infection. CD4 level is a direct indicator of viral load. HIV affects the micronutrients levels in the body. Objectives: To characterize the bone marrow changes in the HIV and to correlate with CD4 level, iron profile, Vitamin B12 and folate levels. Material and Methods: 50 patients were taken in the study. These patients were divided into two groups, group I (CD4 count $<200 \mu$ l) and group II (CD4 count $>200 / \mu 1)$. Blood samples were taken and CBC, CD4 levels, Iron profile, Vitamin B12 \& Folate were measured. Bone marrow examinations was done. Discriptive statistics were applied. Association between these two attributes was calculated by chi-square test and $\mathrm{p}<0.05$ was considered statistically significant. Results: Anemia was seen in $33(66 \%)$ cases. The most common type of anemia in both groups was normocytic normochromic anemia. Bone marrow was normocellular in $34(64.67 \%)$ of cases. Dysplasia was seen in 18 $(36 \%)$ of cases. Bone marrow was normocellular $(p=0.884)$ and in both the groups. Erythroid maturation was megaloblastic in $40 \%$ of cases in group I as compared to $8.11 \%$ in group II $(p=0.025)$. Erythroid dysplasia was most commonly seen in both the groups with significantly increased in group I $(\mathrm{p}=0.029)$. Serum iron and folate was significantly decreased in group I with $p=0.042$ and $p=0.006$ respectively. Leukemia was seen in 3 cases while cryptococcus, histoplasma and L.D. bodies were seen in 1 case. Conclusion: Anemia, serum iron and folate levels can be a good predictor for immune status in these patients. These patients should be evaluated and treated for the hematological abnormalities to decrease the morbidity.

\section{Abstract Submission Form for scientific oral paper}

\section{P-I 10}

\begin{abstract}
A Study to Discriminate Malarial from Nonmalarial Acute Febrile Illness by Determining Changes in Hematological Parameters
\end{abstract}

\section{Dr Sheetal G. Gole ${ }^{\#}$, Associate Professor Pathology}

Email ID for Correspondence: drsheetalgole@ rediffmail.com

World College of Medical Sciences and Research \& Hospital, Gurawar, Jhajjar, Haryana

Introduction: In tropics malaria is main differential diagnosis of acute febrile illness. Hematological parameter changes involving major cell types such as erythrocytes, leucocytes and platelets are observed in malaria patients. Objectives: To ascertain if certain hematological parameters increase the probability of malaria in patients with acute febrile illnesses. Material and Methods: Present study is a two year hospital based prospective cohort study and was carried out at a tertiary care rural hospital, Telangana. Patients with fever of less than seven days in duration were included in the study. Patients where localizing cause for fever could be determined were excluded. Hemoglobin, Red cell distribution width, leukocyte count, and platelet counts were determined by using automated cell counter. Peripheral smear examination for malaria parasite was taken as gold standard for diagnosis of malaria. Diagnostic accuracy was measured by computing sensitivity, specificity, predictive values and likelihood ratios. Precision of these estimates was evaluated using 95\% confidence intervals. Results: Study included 368 patients and those with positive peripheral smear for malaria parasite were $140(38.04 \%)$. Thrombocytopenia alone (platelet count $<150,000 / \mathrm{cu} \mathrm{mm}$ ) was a predictor for malaria (Sensitivity $60 \%$, Specificity $88 \%$, Likelihood ratio +5.04$)$ and in combination with anemia $(\mathrm{Hb}<10 \mathrm{~g} / \mathrm{dl})$ it was next best parameter (Sensitivity 69\%, Specificity 74\%, Likelihood ratio +2.77$)$. Conclusions: Thrombocytopenia is an important predictor of malaria infection. When used in combination with other clinical and gold standard microscopy methods, hematological parameters could improve malaria diagnosis and treatment.

Keywords Plasmodium falciparum, Plasmodium vivax, Thrombocytopenia, Anemia

\section{P-I 11}

Evaluation of Cd64 on Neutrophils and HLA-DR on Monocytes: A Combined Parameter as Marker of Neonatal Sepsis

Dr. Suman Tomer", Dr. Gajender Singh, Dr. Geeta Gathwala, Dr. Monika Gupta, Dr. Harish punia, Dr. Rajeev Sen

Email ID for Correspondence: dr. suman117@gmail.com Pt. B.D.Sharma P.G.I.M.S. Rohtak

Introduction: Neonatal sepsis remains a diagnostic burden globally responsible for about $30-50 \%$ of the total neonatal deaths each year in developing countries. It is estimated that up to $20 \%$ of neonates develop sepsis and approximately $1 \%$ die of sepsis related causes. Objectives: This study evaluated the potential of the neutrophilic CD64 (nCD64) and monocytic HLA-DR (mHLA-DR) median fluorescence index as marker of neonatal sepsis. Material and Methods: This was a prospective study carried out in Department of Pathology in collaboration with Neonatal services division, Department of Paediatrics, Pt.B.D.Sharma Post Graduate Institute of Medical Sciences, Rohtak. The peripheral venous blood sample was collected for flow cytometry, blood culture and sepsis screening in Seventy full term, appropriate for gestational age neonates with clinical suspicion of sepsis and warranting antibiotic therapy provided they had not received antibiotics in the preceding 72 hours after an informed parental consent. The sepsis screen included C-reactive protein (CRP), total leukocyte count (TLC), absolute neutrophil count 
(ANC), immature to total neutrophil ratio (I:T) and micro erythrocyte sedimentation rate (mESR). Sepsis screen was taken as positive if two parameters out of 5 were positive. We evaluated a parameter by division of MFI (mean fluorescence intensity) values of nCD64 and their respective mHLA-DR and multiplying the ratio by 100 , and termed as sepsis index (Sepsis Index $=$ nCD64/mHLA-DR $\times 100$ ). The cut-off value of positivity for parameters (n CD64 and Sepsis index) which were upregulated was derived by Mean +2 SD (standard deviation) after running thirty samples of healthy neonates and the value below $10^{\text {th }}$ percentile of the healthy controls was taken as cut off value for the parameters which was downregulated (mHLA DR). Results: The cases were categorized into "Sepsis group" and "No Sepsis group". The sepsis group was further sub-grouped in to "Definite Sepsis" (Blood culture positive) and "Probable Sepsis" (Symptomatic baby with sepsis screen positive but sterile blood culture). The cases that failed to meet the criteria of sepsis group were categorized as No Sepsis group (Sepsis screen negative and blood culture sterile). All cases of definite sepsis had a positive nCD64. In probable sepsis group nCD64 positivity was seen in $88.89 \%$ cases. However $12.50 \%$ cases of no sepsis group also had a positive nCD64. In definite sepsis group, mHLA DR was positive in $63.16 \%$ cases, $85.19 \%$ in probable sepsis and $54.17 \%$ of no sepsis group. Out of 19 cases of definite sepsis $94.74 \%$ cases had positive sepsis index while one case had sepsis index less than cutoff value. Conclusions: Flow cytometric assessment of neutrophils CD64 may be considered as a rapid and reliable marker for the diagnosis of bacterial neonatal sepsis and can be used independently as a diagnostic marker for neonatal sepsis. It is also useful to identify a separate group among culture negative sick neonates and may be useful to guide antibiotic administration especially in these neonates. mHLA-DR may be beneficial for monitoring of patients at later point in time for identification of delayed immunosupression in neonatal sepsis. Sepsis index also showed a significant difference between various groups that is definite sepsis, probable sepsis, no sepsis group and in healthy controls.

\section{P-I 12}

\section{Evaluation of the Significance of IL-6 and Procalcitonin as a Biomarker for Infection and Correlation} with Haematological Parameters for Early Detection of Sepsis

\section{Dr. Dipsha Kriplani ${ }^{\#}$, Dr. F. Udwadia, Dr. V.P.Antia,} Ena Laurenco

Email ID for Correspondence: dipkrip@gmail.com Breach Candy Hospital

Introduction: Sepsis is a common and serious complication in intensive care hospitalized unit patients which can be caused by bacterial, fungal and viral infections. Many biomarkers have been proposed and assessed clinically, but none alone is specific enough to definitively determine diagnosis. Procalcitonin is the most accurate biomarker of bacterial infection which rises within 2-4 hours of infection and peaks at 6-8 hours. However, it is positive only in bacterial infections. IL-6 is a pleiotropic cytokine which is rapidly induced in the course of acute inflammatory reactions associated with bacterial, viral and fungal infections. It is an early indicator of inflammation. Dysfunction of the haematological organ system is an early manifestation of sepsis and is seen in virtually all patients with this disease. Recognizing these changes induced by sepsis and understanding the underlying pathophysiology is the key to improving outcomes in these patients. Thus, studying the haematological parameters and morphology along with the biomarkers can enable in early detection and treatment of sepsis. Objectives: 1. To identify alterations in haematological parameters like immature granulocytes, eosinopenia, immature platelet fraction, changes in morphology. 2 . To correlate the haematological parameters with clinical findings and biomarkers of sepsis like IL-6 and procalcitonin .3. To establish haematological parameters as reliable initial markers of infectionsbacterial and viral. Material and Methods: Study design: prospective, cross sectional analytical study. Study duration: 3 months. Study venue: Breach Candy Hospital Trust. Sample size: 100 patients. Inclusion criteria: $>18$ years. Exclusion criteria: $<18$ years, pregnant women. Results: There was significant correlation $(p<0.005)$ of the haematological parameters of immature granulocytes, eosinopenia, immature platelet fraction, changes in morphology with the biomarkers procalcitonin and IL-6. IL-6 was seen to be of value as it was an early marker for detection of sepsis. Procalcitonin is a more specific marker for sepsis. Conclusions: correlation of haematological parameters with biomarkers is an early indicator of sepsis.

\section{P-I 13}

Human Parvo B19 and Epstein-Barr Virus Infection in Anemia with Special Reference to Aplastic Anemia and Cytopenias of Different Cell Lines

\section{Dr. Shekhar Jyoti Gogoi*\#, Dr. Avinash Saikia**, Dr. Alal Uddin*, Dr. B.C Kalita***}

Email ID for Correspondence: shekhargogoi23@gmail.com *PGT Department of Medicine AMCH; ** Scientist 2, RMRC (ICMR) Dibrugarh; ***Associate Professor, Department of Medicine AMCH, Dibrugarh

\section{Assam Medical College and Hospital, Dibrugarh}

Introduction: Aplastic anemia is characterized by pancytopenia with hypoplastic bone marrow. The various etiologies that have been implicated to aplastic anemia include drugs, irradiation and preceding viral infections. Human parvovirus B19 and Epstein-Barr virus have been known to be associated with red-cell aplasia, leukopenia, agranulocytosis, thrombocytopenia, transient aplastic crisis and severe aplastic anemia. The present study was carried out to determine the association of parvovirus B19 and Epstein-Barr virus in aplastic anemia and patients with cytopenia of different cell lines. Objectives: To study the prevalence and association of Parvo B19 and EpsteinBarr virus infection in patients with aplastic anemia and cytopenias. Material and Methods: We had undertaken a hospital-based casecontrol study of 30 patients of anemia including aplastic anemia and cytopenia of different cell lines and 30 age and gender-matched controls from the same geographical area. Aplastic anemia was diagnosed with the help of Complete Blood Count, peripheral blood smear and Bone Marrow study. Presence of Parvo B19 infection was diagnosed by Parvovirus B19 IgM antibody by ELISA. Parvo B19 DNA was detected by PCR designed to amplify a 204-bp fragment of constant region of parvovirus B19. Epstein-Barr Infection was detected by EBV VCA IgM by ELISA. Results: Of the30 patients with anemia group, Parvo B19 IgM, ParvoB 19 DNA and EBV IgM could be detected in $14(46.67 \%), 2(6.67 \%)$ patients, respectively. None of the 30 control subjects showed the presence of Parvo B19viral DNA and EBV IgM, whereas B19 IgM antibodies could be detected in $2(6.67 \%) .4$ patients in the anaemia group showed equivocal respose to Parvo B19 IgM. The occurrence of IgM antibodies in anemia group was significantly higher than in normal controls ( $p<0.01$; OR 12.25; 95\% CI 2.46 to 60.91$)$ ). Conclusions: The present study shows significant association of parvovirus infection with aplastic anemia and Cytopenia of different cell lines. However, more studies involving larger numbers of subjects need to 
be conducted to determine the exact role of parvovirusB19 in this regards.

\section{P-I 14}

Common Hemoparasite with An Uncommon Effect of Pancytopenia

Aparna Ningombam ${ }^{1}$, Raghavendra Lingaiah ${ }^{2}$, Amit Kumar $^{3}$, Rita Sood ${ }^{4}$

1. Department of Laboratory Medicine, AIIMS, New Delhi; 2. Department of Laboratory Medicine, AIIMS, New Delhi; 3. Department of Laboratory Medicine, AIIMS, New Delhi; 4. Department of Internal Medicine, AIIMS, New Delhi

Background: Pancytopenia is associated with many diseases including infectious etiologies. Malaria manifesting initially as pancytopenia is very rare and it is more common with Plasmodium falciparum when compared to other species of malaria. Here we present a case of pancytopenia due to plasmodium vivax which was detected incidentally by a screening pathologist over an unusual histogram as reported by the hematology counter. Case report: An 18 year old male presented to emergency medicine with 14 to 15 episodes of non bilious projectile vomiting since 12 hours. He also had high grade fever with chills, head ache along with generalised myalgia for the past 5 days. On presentation, he was dehydrated, febrile, conscious and oriented. Clinical examination showed mild hepatosplenomegaly. Initial lab investigation revealed pancytopenia (TLC- $2.5^{*} 10^{3} / \mu \mathrm{L}, \mathrm{Hb}-$ $11.2 \mathrm{~g} / \mathrm{dL}$, platelet count $-52^{*} 10^{3} / \mu \mathrm{L}$ ). Bedside optimal test for malaria was equivocal. Patient was admitted for evaluation of fever and pancytopenia. The screening pathologist in the laboratory noticed the unusual histogram in the counter machine along with a corrected TLC of $0.2 * 10^{3} / \mu \mathrm{L}$ (Uncorrected TLC of $2.3 * 10^{3} / \mu \mathrm{L}$ ) to account for cellular interference and nucleated RBCs. More over Briggs' Malaria factor was more than 3.7 (4.8). All these findings were suggestive of malarial infection. The peripheral smear confirmed the malarial infection as there were Schizonts and Gametocyte of Plasmodium vivax in the smear. The patient responded to treatment with IV Artesunate and the blood parameters improved in 3 days. Conclusion: Malaria can present with pancytopenia. Clinicians cannot rely only on bedside testing procedures such as optimal test to rule out malaria.

\section{P-I 15}

Parvovirus B19 Related Red Cell Hypoplasia in a Post Renal Transplant Patient-A Rare Case Report

Dr. Mahender Nishad, $1^{\text {st }}$ year Post Graduate, Dr. Zaheda Kausar-Assistant Professor, Dr. Anunayi.J-Associate Professor, Dr. Ezhil Arasi.N-Professor and HOD

Upgraded Department of Pathology, Osmania Medical College, Osmania General Hospital, Hyderabad, Telangana State, India

Objectives: To access the role of Parvi virus B19 in post renal transplant patients. Materials and Methods: We present a case of 27 year old male, post renal transplant on immunosuppression presenting with severe persistent anemia. Bone marrow study and special stains were done. Results: Parvo virus B19 was diagnosed on on bone marrow examination. Qualitative PCR which detected B19 DNA confirmed the diagnosis. The patient was treated with $0.4 \mathrm{G} \mathrm{IV} / \mathrm{kg} / \mathrm{day}$ for 5 days and packed red cells. Hb levels and reticulocyte count improved on treatment. Case is under follow up. Conclusion: When post renal transplant patients on immunosuppressive present with isolated and persistent anemia, reticulocytopenia and no other evidence of of anemia, Parvo virus induced pure cell aplasia should be excluded.

\section{P-LD 01}

Primary Central Nervous system Lymphoma - Is R-BEAM a optimum conditioning prior to Autologous transplant?

Rohith G Chitrapur ${ }^{\#}$, Dr Murali Subramanian, Dr Srinivas Prasad

Email ID for Correspondence:rohithchitrapur@gmail.com Bangalore Baptist Hospital, Bangalore, Karnataka

Introduction: Autologous Transplant in Primary CNS lymphoma is still debatable. Conditioning regimen prior to stem cell rescue is still in the nascent phase in India with published data from other countries using Busulphan/Thiotepa/Cyclophosphamide as Conditioning regimen. Objectives: Is R-BEAM a adequate conditioning regimen for Primary CNS lymphoma considering the cost of conditioning for BU/ TT/CY in India. Material and Methods: 43 year old male, with no comorbid conditions evaluated for Blurring of Vision, gait disturbance of 6 months duration. Diagnosed to have a Primary CNS lymphoma with Ocular Involvement. He was treated with 5 cycles of High dose Methotrexate, Cytosine Arabinoside with Intrathecal Methotrexate. Post 5 cycles patient persisted to have blurred vision and Gait disturbance. Patient was planned for Autologous transplant in view of Progressive Disease on Imaging and clinically. Stem cell Mobilisation was done with G-CSF and Plerixafor. He was conditioned with R -BEAM (Rituximab, Carmustine, Etoposide, Cytosine Arabinoside and Melphalan). Patients neutrophil engraftment happened on $\mathrm{D}+10$ and Platelet engraftment on $\mathrm{D}+12$. Patient at the time of discharge his vision was normal with no gait disturbance. (Evaulation with Imaging will be done in the Month of September 2017). Result: Patient at the time of discharge had no gait disturbance and vision disturbance. Will be imaged after 6 weeks to look at the response. Conclusion: R- BEAM is probably a adequate and a feasible conditioning regimen in Primary Central Nervous system Lymphoma.

\section{P-LD 02}

LCA-Negative Diffuse Large B Cell Lymphoma: A Potential Diagnostic Pitfall

Sunil Pasricha, Neha Singh ${ }^{\#}$, Gurudutt Gupta, Anila Sharma, Anurag Mehta

Rajiv Gandhi Cancer Institute and Research Centre, New Delhi Email ID for Correspondence: drnehasingh123@gmail.com

Introduction: Loss of LCA expression is frequently observed in immature B-cell neoplasms, plasma cell dyscrasias and anaplastic T-cell lymphomas. However, it is uncommon in mature B-cell lymphoproliferative disorders. We present a case of LCA negative primary diffuse large-B cell lymphoma (DLBCL) of the urinary bladder, which in itself is a rare presentation. Case Report: A 83-year -old male presented to the OPD with complaints of hematuria and acuteonset pelvic pain. He had recurrent episodes of urinary tract infections for last one year. Hemogram and biochemical tests were within normal limits. Computed Tomography (CT) scan abdomen revealed 
right moderate hydroureteronephrosis, eccentric thickening of the right lateral wall of urinary bladder with encasement of the distal ureter and conglomerate iliac lymph nodal mass. Histopathological examination revealed diffuse infiltration of the muscularis propria by large, round to polygonal neoplastic cells with high mitotic activity, and unremarkable urothelial lining. Primary panel of antibodies comprising of cytokeratin, LCA, EMA, desmin, GATA-3, p40, synaptophysin, S-100, HMB-45 etc was applied but all of them came out to be negative, favouring a diagnosis of undifferentiated carcinoma. However, on reassessing the histopathological findings, round cell tumor morphology, diffuse pattern of infiltration and unremarakable urothelial lining with involvement of iliac lymph nodes behoved us to rule out the possibility of lymphoma in spite of LCA negativity in tumor cells (with positive intrinsic and internal controls). The subsequent IHC panel revealed diffuse positivity for CD20, Bcl-2 and MUM-1and negative expression of CD10, CD138, ALK-1 and bcl-6; Ki -67 index was $\sim 90 \%$. Bone marrow was uninvolved at presentation. Hence, a final diagnosis of LCA negative primary DLBCL-non germinal centre like, of urinary bladder was established. The patient was started on R-CHOP based protocol, following which partial response was achieved at four months. Discussion: Monoclonal antibodies directed against LCA have been routinely used as a tool to differentiate between lymphomas and other poorly-differentiated tumors. Loss of LCA expression on lymphoma cells usually suggest that neoplastic transformation has occurred during the differentiation pathway to plasma cells just before its CD20 expression. The few LCA negative large B-cell lymphomas reported in literature have an extranodal presentation and have been re-classified as plasmablastic lymphoma or ALK-positive Large B-cell Lymphoma. Rare cases of LCA negative DLBCL have also been reported in thyroid gland and cerebellum. But LCA negative primary urinary bladder DLBCL has not been reported earlier. Conclusions: It can be concluded that the pathologist must apply judicious IHC panel before labelling a case as poorly differentiated carcinoma and overlooking a plausible possibility of lymphoma thereby subjecting the patient to unwarranted radical surgery.

\section{P-LD 03}

\section{Primary Splenic Diffuse Large B cell Lymphoma: Report of a Rare Entity}

\section{Dr. Adity Sharma, Dr. Alankrita Deka ${ }^{\#}$}

Email ID for Correspondence: alankritadeka@gmail.com Department of Pathology, Assam Medical College, Dibrugarh

Introduction: Primary splenic Lymphoma (PSL) is a rare entity and constitutes less than $1 \%$ of all the Non-Hodgkin's lymphoma. The spleen is usually involved by lymphoma as part of the systemic illness. However, rarely it could be a primary site of lymphoma. DLBCL is the most common lymphoma in the spleen accounting for 22-33\% of the cases. Objectives: Presentation of an incidental detection of a rare case. Materials and Methods: A 30 year old female patient presented with abdominal pain, vague abdominal mass, weakness and weight loss for 6 months. Physical examination revealed massive splenomegaly. Peripheral smear showed normocytic normochromic anemia. Bone marrow aspirate showed normoblastic reaction. Imaging study (CECT) showed large heterogenous hypoechoic splenic mass without any nodal involvement. Patient was planned for splenectomy. Results: Histopathology examination showed replacement of splenic white pulp by dispersed population of malignant cells in sheets. On IHC, the tumor cells were positive for CD20, CD79a while they were immunonegative for CD3, CD5, CD10, CD23 and Cyclin-D1. Ki-67 labeling index was more than
$70 \%$. Post -surgery, patient is on remission for last 2 years on follow up. Conclusion: Primary Splenic DLBCL is rare and splenectomy is often required for diagnostic and curative modality. Prognosis is favourable if the disease is confined to the spleen. Post-operative adjuvant therapy is necessary in most of the cases.

\section{P-LD 04}

Clinical characteristics and outcome of lymphoplasmacytic lymphoma in resource constraints settings

Introduction: Lymphoplasmacytic lymphoma is an uncommon and indolent B-cell chronic lymphoproliferative disorder. All patients don't require therapy and those who require have multiple options, yet Rituximab (R) containing regimes are preferred. However, financial burden of R-containing regimens limit their use in resource constraints settings. Method: We retrospectively analyzed the data from central registry department, PGIMER Chandigarh from year 2011 to 2016. Patients' clinical characteristics and outcomes were analyzed using SPSS software. Results: A total of $25(\mathrm{n}=25)$ patients who were included in the study. Mean age was 59.5 years (37-78 yrs, SD 10.3 years). Fourteen patients $(56 \%)$ had 'B' symptoms at presentation. Twenty-two patients $(88 \%)$ had advanced disease (stage IV). Clinically, 14 (56\%) had hepatomegaly, $10(40 \%)$ had splenomegaly and $9(36 \%)$ had lymphadenopathy. None of them presented with features of hyper viscosity. Mean duration of symptoms was 11.5 months (1-72 months). Eleven (44\%) patients were treated with rituximab containing regimens while $14(56 \%)$ patients were treated with non-rituximab containing regimens. The overall response rate of ' $R$ ' based regimen was $91 \%$ and non- $R$ based regimen was $43 \%$ $(\mathrm{p}=.013)$. The overall survival of $\mathrm{R}$ based and non- $\mathrm{R}$ based regimens had a mean of 43.8 and 28.6 months $(p=0.07)$. Conclusion: Rituximab containing regimens have significantly better overall response rate than non-rituximab containing regimens. However, overall survival in not significantly improved with rituximab containing regimens. The overall survival is low in resource constraints settings in comparison to developed world.

\section{P-LD 05}

Clinicopathological Study of Pattern of Malignant Lymphomas Diagnosed in a Tertiary Care Centre Over a Period of One Year

Dr. Mondita Borgohain ${ }^{1}$, Dr. Gayatri Gogoi ${ }^{2}$, Dr. Swagata Dowrah ${ }^{3}$, Dr. Panchalika Borgohain ${ }^{4 \#}$

Email ID for Correspondence: panchalika.xyz@gmail.com 1. Professor, 2. Assistant Professor, 3. Assistant Professor 4. PGT, second year; 1,2,4: Department of pathology, Assam Medical College \& Hospital Dibrugarh, Assam; 3: Department of Pathology, Silchar Medical College \& Hospital, Assam

Assam Medical College, Dibrugarh

Introduction: Malignant lymphomas are one of the most common haematological malignancies in the world with a remarkable increase in incidence in the recent years. The distribution of various subtypes of lymphomas in India is different from the rest of the world. Lymphomas are divided as Hodgkin lymphoma (HL) \& Non Hodgkin lymphoma (NHL). Objectives: To subtype the different malignant lymphomas \& to correlate with clinicopathological parameters. Material and Methods: A total of 25 cases were studied from March 2016 to March 2017 in the department of Pathology, AMCH. Patient's important informations including personal details, 
presenting complaints \& previous investigations were noted in a predesigned proforma. The cases were diagnosed provisionally using Histopathological features as lymphoproliferative disorders \& were taken for immunophenotyping with IHC studies. The cases were subtyped according to WHO classification. Results: Out of the 25 cases studied histologically, NHL was confirmed in 18 cases $(72 \%)$ \& subtyped only in 15 cases; 3 cases were diagnosed as HL (12\%) \& subtyped in 2 cases. 3 NHL \& 1 HL case couldn't be subtyped due to inadequate tissue samples. Out of the other 4 cases, 3 were diagnosed as metastatic lymph nodes (12\%) \& 1 case as myeloid sarcoma (4\%). These 4 cases were excluded from our study. Median age of involvement of lymphoma patients was 45 years with a male preponderance of $61.9 \%$. most common symptom was nodal enlargement (76.2\%). Diffuse large B cell lymphoma (DLBCL) was the most common subtype followed by Mantle cell lymphoma (MCL) among NHL. Both cases of HL were of Nodular lymphocyte predominant HL type. Discussion \& Conclusions: Lymphomas are potentially curable malignancies with proper subtyping based on morphology \& immunohistochemical diagnosis. But there are various challenges \& overlapping of not only morphological features but also immunohistochemical features. DLBCL is an aggressive high grade disease with variable outcome so further subclassification \& prognostication is important.

\section{P-LD 06}

HHV8 Unrelated Sinusoidal PEL Like B Cell Lymphoma in HIV Positive Patients

\section{Sumit Gupta\#, Tanuja Shet}

Email ID for Correspondence: agent.sumit88@gmail.com Department of Pathology and Adult hematolymphoid unit, Tata Memorial hospital, Parel, Mumbai

Background: Aggressive B cell lymphomas are common malignancies in HIV positive patients. We describe 4 cases of an unusual variant of large B cell lymphoma in HIV positive that has a sinusoidal pattern and is the HHV8 unrelated counterpart of primary effusion lymphoma. Only few reports exist of this tumor. Material and Methods: A total of 4 cases were studied with ages ranging from 35-60 years. A wide Immunohistochemistry and in situ hybridization panelfor DLBCL viz: - CD3, CD20, PAX-5, CD10, BCL-6, MUM-1, EBV-LMP1, EBERISH, HHV-8 was done. Results: All patients were aged 35 - 60 years. Sections from lymph nodes showed features of high grade non Hodgkin lymphoma immunoblastic type with sinusoidal involvement of nodes. The immunoprofile was CD20 weak or strong positive/CD30negative/ MUM1+ or CD1138+. All four cases were positive for EBV-LMP1 and were negative for HHV-8. Three cases were positive for MUM-1 and one positive for CD138 One case was CD20 positive. One case showed a background of Castleman's disease. Of the four patients, only one with Castleman's disease is alive while two others are dead. Conclusion: HHV8 unrelated PEL like sinusoidal B cell lymphoma in HIV positive patients seems to be an unique entity and may show association with Castleman's disease in HIV positive patients. As HHV8 is not common in India this may be a disease similar to PEL but caused due to EBV infection.

\section{P-LD 07}

Non hodgkin's lymphoma presenting as a solitary liver mass

\section{Dr. Bhanita Baro ${ }^{\#}$, Dr. Vikas Kavishwar, Dr. Shilpa Lad}

Department of Pathology, Topiwala National Medical College and B.Y.L Nair Hospital, Mumbai

Email ID for Correspondence: bhanita102@gmail.com

Aims \$ objectives: We wish to discuss the unusual presentation of non Hodgkin's lymphoma as a solitary liver mass. Materials \$ methods: A 45 year old male chronic alcoholic who was treated for liver disease since 1 year. C.T scan performed showed large illdefined mass $25 \times 20 \mathrm{~cm}$ with mesenteric adenopathy and presence of ascites. Provisional diagnosis of hepatocellular carcinoma was made. Patient expired before final diagnosis and a complete autopsy was performed. Results: At autopsy liver was enlarge and showed a solitary ill- defined solid homogenous mass involving $80 \%$ of liver parenchyma. Similar masses were seen in mesentery and omentum. Histology revealed diffuse proliferation of atypical cells with nuclear enlargement. Possibility of lymphoma was considered and immunohistochemistry confirms the diagnosis of non hodgkin's lymphoma. Conclusion: This is an autopsy study of an unusual case of non hodgkin's lymphoma presenting with solitary liver mass.

Keywords Solitary liver mass, Non hodgkin's lymphoma, Histopathology

\section{P-LD 08}

Flow Cytometry as Diagnostic Tool for Diagnosis of Non Hodgkin's Lymphoma on FNAC

Dr Sanjay Kumar", Dr Meena, Dr Monika Gupta, Dr Sant Prakash Kataria, Dr Gajender Singh, Dr Rajeev Sen

Email ID for Correspondence: drsanjayk@yahoo.co.uk Department of Pathology, Post Graduate Institute of Medical Sciences, Rohtak-124001 (Haryana)

Introduction: Flow cytometry is an objective and quantitative diagnostic tool for quick multiparametric analysis of large number of cells. It is still evolving fast and needs expertise for accurate information on FNA samples for diagnosis and subtyping of lymphoma. Objectives: To establish the utility of flow cytometry for screening and diagnosis non -Hodgkin's lymphoma fine-needle aspiration samples. Material and Methods: FNAC was performed by using $23 \mathrm{G}$ needle. Two pricks were given to each patient. From one prick smears were prepared and stained with Leishman stain for cytological diagnosis. Second prick material was aspirated in Eppendrof tube for flow cytometric immunophenotyping. Sample was processed using fluoresceinated antibodies: CD3, CD4/CD8, CD2/CD7/CD3, CD5/ CD10/ CD19, CD19/ $\kappa / \lambda$, FMC7/CD23/CD19, CD38/CD56/CD19. Both cytologic findings and FCI data were interpreted together to diagnose and sub-classify NHL according to WHO classification. Data was acquired on FACS Canto II Flow cytometer and analyzed by FACS Diva software. FC/FNAC diagnoses of reactive lymph node and NHL were confirmed either histologically or clinically. Results: Total of 20 cases were included comprising 10 cases each of NHL and reactive lymph node. All 10 cases of reactive lymph node were polyclonal on FCM. Light chain restriction was demonstrated in 8 out of 10 cases of B-NHL. Four cases of NHL showed $\kappa$ chain restriction and 4 showed $\lambda$ light chain restriction while 2 cases were negative for both $\kappa$ and $\lambda$. With the help of combined FCI and FNAC, it was possible to sub-classify 4 cases as small lymphocytic lymphoma and rest were classified as B cell NHL. Conclusions: FNAC combined with FCM was successful in differentiating between reactive lymph node, B-NHL, T-NHL. Further sub-classification is also possible in most of the cases. 


\section{P-LD 09}

\author{
A Unique Case of Hairy Cell Leukemia Variant (HCL-V) \\ Following Transitional Cell Carcinoma Renal Pelvis \\ Dr Rajni Kaushik, Dr Anantika Thakur", \\ Dr Sudarshan Kumar Sharma, Dr Anchana Gulati
}

Email ID for Correspondence: nants_53@yahoo.com Indira Gandhi Medical college, Shimla (H.P.)

Introduction: Hairy cell leukemia variant (HCL-v) is a B-cell disorder, which accounts for $0.4 \%$ of the chronic lymphoid malignancies and $10 \%$ of HCL cases. It is included in the World Health Organisation (WHO) classification as a provisional entity among mature B cell neoplasms and affects elderly or middle-aged males. The main presenting features are splenomegaly, leukocytosis, lymphocytosis, anemia and thrombocytopenia without monocytopenia. As compared to the classical HCL, the hairy cell leukemia variant (HCL-v) is a more aggressive disease. Here we report a case of HCL-v in a patient of Transitional cell carcinoma (TCC) renal pelvis. Objectives: The aim of the article is to present a rare case of Hairy cell leukemia variant ( $\mathrm{HCl}-\mathrm{v})$ which is a distinct clinicopathological entity with intermediate features between classical $\mathrm{HCl}(\mathrm{HCl}-\mathrm{C})$ and $\mathrm{B}$-cell prolymphocytic leukaemia. Material and Methods: A 78 years old patient was admitted with shortness of breath for the past 2 weeks. He had low grade transitional cell carcinoma renal pelvis diagnosed 3 years back for which nephrectomy was done. On physical examination, the patient had massive splenomegaly. Complete haemogram and peripheral blood smear examination was done. Bone marrow aspiration revealed a dry tap. Bone marrow biopsy was stained with H\&E and reticulin stain. Results: Complete haemogram revealed high TLC (140 thou/microl). Peripheral blood smear revealed numerous circulating mononuclear cells with high $\mathrm{N}: \mathrm{C}$ ratio, round nuclei, prominent nucleoli and moderate basophilic cytoplasm with fine circumferential projections. Flow cytometry analysis showed cells expressing CD19, CD20, CD22, CD11c, CD25 and CD103. The bone marrow biopsy revealed diffuse infiltration with hairy cells, dislocation of normal hematopoietic cells with isolated megakaryocytes. Based on the clinical details, immunophenotyping and morphological features diagnosis of hairy cell leukemia variant was given. Conclusions: Hairy cell leukemia variant is a rare type of lymphoproliferative disorder with a characteristic immunophenotype. Its association with TCC renal pelvis is uncommon, hence being reported.

\section{P-LD 10}

CD38 in CLL; A good surrogate marker for adverse cytogenetics in CLL

Subhajit Brahma\#, Dr. Neeraj Arora, Sambhunath Banerjee, Dr. Nishit Gupta, Dr. Asish Rath, Dr. Saurabh Bhave, Dr. Anupam Chakrapani, Dr. Reena Nair, Dr. Mammen Chandy, Dr Mayur Parihar, Dr. Deepak K. Mishra

Email ID for Correspondence:

brahma2710@gmail.com/subhajit.brahma@tmckolkata.com TATA Medical Center, kolkata

Introduction: CLL is a heterogeneous disease. Accurate staging and prognostic studies are necessary to guide therapy and determine clinical outcome. By flowcytometry, CD38 is a useful and reliable prognostic marker because it typically remains stable over time and correlates with detection of del (17p13.1) [TP53 gene], del (11q23) [deletion ATM gene], trisomy 12 by FISH. Objectives: In the present study we tried to observe the relationship between CD38 positivity by flowcytometry and FISH abnormalities. Material and Methods: We retrospectively analyzed immunophenotypic data from consecutive cases $(\mathrm{n}=91)$ of CLL referred to our flowcytometry laboratory from August 2014 to August 2017. These cases were diagnosed based on immunophenotyping, morphology and genetics as per WHO 2008 guideline. FISH for del (17p13) [TP53], del (11q23) [ATM], trisomy 12 were done using standard protocols. Results were compared with FISH data wherever available. Results: The median age at diagnosis was 63 years; the male to female ratio was $4: 1$. Of the total 91 CLL cases, $27(30 \%)$ cases were CD38 positive of which FISH data was available in $\mathrm{n}=14$ patients. TP53 gene deletion was seen in $6 / 14$ (42\%) patients of which concomitant deletion of ATM gene was seen in two patients (14\%), one (7\%) of which also had trisomy 12 . ATM deletion was seen in three $(21 \%)$ patients. Isolated trisomy 12 was seen in one patient (7\%). $\mathrm{n}=6 / 14$ cases $(42 \%)$ were FISH negative. Out of total $64(70 \%)$ CD38 negative cases, FISH data was available in $\mathrm{n}=38$ patients. TP53 gene deletion was seen in $5 / 38$ patients (13\%), 1 case (3\%) showed deletion of ATM Gene, 3 cases $(8 \%)$ showed presence of Trisomy 12.29 cases (45\%) were negative for all. Overall out of total CD38 positive cases where FISH data was also available, TP53 deletion and ATM deletion were seen in $42 \%$ and $21 \%$ respectively. Conclusions: Adverse risk cytogenetic features are more common in CD38 positive CLL patients. Further studies are required to establish the same as the data sets analysed in the study are small in number.

\section{P-LD 11}

Role of Flowytometric Immunophenotyping in Diagnosis of CLPD- Three Year Data with Discussion of Interesting Cases

Kushwaha $\mathbf{R}^{\#}$, Jain M, Yadav G, Verma SP, Singh US, Tripathi AK, Goel MM, Kumar A

Email ID for Correspondence: docrashmi27@yahoo.co.in King George's Medical University, Lucknow

Introduction: Multiparametric flowcytometric immunophenotyping is a powerful tool for diagnosis of various CLPD's by virtue of its high throughput and low turn around time. It can be used to differentiate various chronic lymphoproliferative disorders. Objectives: To collect, analyse and correlate the morphological and immunophenotypic data from the cases of chronic lymphoproliferative disorders which were diagnosed at King George's Medical University. Material and Methods: After detailed history patients were subjected to PBS examination, bone marrow aspiration and trephine biopsy. Flowcytometric immunphenotyping was done in samples diagnosed morphologically as CLPD. BD FACS Calibur, 4 color flowcytometer was used with a panel consisting of CD5, CD10, CD19, CD20, CD79a, CD22, FMC7, CD23, CD7, CD4, CD8, CD3, kappa and Lambda. CD25, CD 103, CD38, CD138 were used as second line markers. Cyclin D1 (IHC) was used when required. Three year data was analyzed from September 2014 to September 2017. Results: 82 cases of CLPD were analyzed over period of 3 years. $73.75 \%$ cases were males. Average age of presentation was $6^{\text {th }}$ decade. $96 \%$ cases were B-CLPD, $4 \%$ T-CLPD. 41 cases were of CLL, one case each of HCL and HCL-v, 4 cases of Prolymphocytic leukemia, 3 cases of Mantle cell lymphoma, 32 cases of B-CLPD unclassifiable. Among CLL Cases one case had aberrant CD 8 expression. Conclusions: Flowcytometric immunophenotyping helps in early diagnosis and subclassification of CLPD cases. In a case of CLPD, a screening panel comprising CD19, CD5, CD23, FMC7, CD10, CD20, CD3, kappa and lambda would be successful in the diagnosis of most of CLPDs. 
This can be followed by a secondary panel as required. CD5, CD23, and FMC7 were identified as most sensitive markers in differentiating CLL from other CLPDs.

Keywords CLPD, Immunophenotyping, CLL

\section{P-LD 12}

Hodgkin Lymphoma Hiding in Bone Marrow - A Series of Eight Clinically Unsuspected Cases Diagnosed Primarily on Bone Marrow Biopsy

\section{Mathumithra T ${ }^{\#}$, Basu D, Sree Rekha J, Jacob SE, Kar R}

Email ID for Correspondence: madhumithra59@yahoo.com JIPMER, Pondicherry

Introduction: Marrow involvement in Hodgkin Lymphoma (HL) is seen in $10-16 \%$ of cases. Most patients of HL present with asymptomatic lymphadenopathy and diagnosis is often straightforward. However, at times the diagnosis can be challenging especially for patients who present with marrow involvement at diagnosis, due to varied clinical manifestations. Here we present a series of eight cases, with no clinical suspicion of HL, in whom bone marrow biopsy was the primary site of detection of the disease. Objectives: To study the clinicopathological features and discuss the diagnostic issues in patients in whom bone marrow biopsy was the primary site of detection of HL. Material and Methods: A retrospective record based study over the last ten years revealed eight cases of HL who presented with bone marrow biopsy being the primary site of diagnosis paving way for subsequent investigations and confirmation of the disease. The clinical, hematological and bone marrow histopathological details along with follow up and management were recorded. Results: The initial clinical diagnoses were varied and included abdominal tuberculosis, sub acute intestinal obstruction, Pyrexia of unknown origin, aplastic anemia, Non Hodgkin lymphoma with autoimmune hemolytic anemia and disseminated tuberculosis. Three patients had peripheral lymphadenopathy and four had intraabdominal lymph nodes. One patient had no identifiable lymph nodes. Two patients had anemia, five had pancytopenia and one had a leucoerythroblastic blood picture. Aspirates were diluted in five patients and cellular in the rest. One of the paucicellular diluted marrow showed a few atypical large cells, suspicious of a lymphoma. The bone marrow trephine biopsy revealed features suggestive of HL infiltration in all patients in the form of scattered Reed Sternberg/ Hodgkin cells in typical reactive inflammatory milieu and secondary myelofibrosis in four patients. Immunohistochemistry was done in all cases. The large cells showed CD 30 positivity in all cases with weak positive staining for PAX5 in two patients, while none showed CD 15 expression. Subsequently, lymph node biopsy proved the diagnosis of HL in five cases with a FNA diagnosis in one more. Three patients were successfully treated for HL, two succumbed, and the rest were lost to follow up. Conclusion: Marrow involvement by HL, at diagnosis, poses considerable diagnostic challenges owing to varied clinical spectrum, peripheral pancytopenia and dilution of marrow, secondary to myelofibrosis. A thorough examination of bone marrow trephine biopsy and a high index of suspicion are needed to clinch the diagnosis.

\section{P-LD 13}

Hepatosplenic T Cell Lymphoma: A Rare Case Report
Chowdhury Z\#, Khonglah Y, Raphael V, Mishra J, Gogoi BB

Email ID for Correspondence: chowdhury.zachariah@gmail.com North Eastern Indira Gandhi Regional Institute of Health and Medical Sciences (Neigrihms)

Introduction: Hepatosplenic $\mathrm{T}$ cell lymphoma is a rare peripheral $\mathrm{T}$ cell lymphoma that comprises less than $1 \%$ of Non-Hodgkin lymphomas (NHL). It is derived from cytotoxic T-cells, usually of $\gamma \delta \mathrm{T}$ cell receptor type, and is characterized by primary extranodal disease with typical sinusoidal infiltration of the liver, spleen and bone marrow by medium-sized lymphoid cells. Objectives: Here we report a case of a 20 year old male presenting with fever, pallor, weight loss, pancytopenia, hepatomegaly, massive splenomegaly and lymphadenopathy. Material and Methods: Bone marrow aspirate and biopsy was done, which showed infiltration by abnormal mediumsized lymphoid cells. Results: These cells on four-color flow cytometric evaluation showed positivity for both Surface and Cytoplasmic CD 3, CD 8, CD 7 and CD 56 and negativity for CD 4, CD 5, CD 34, CD 19, CD 20, CD 10, CD 33, TdT, MPO \& HLA DR, suggesting the a $T$ cell NHL infiltrating the marrow, with the possibility of hepatosplenic $\mathrm{T}$ cell lymphoma, taking into consideration the clinical context. Subsequently, fine needle aspiration cytology from the spleen and biopsy from the liver showed infiltration by morphologically similar atypical lymphoid cells, thus confirming the diagnosis of hepatosplenic T cell lymphoma. Conclusions: Diagnosis was difficult to establish without the help of immunophenotyping. We report this case for its rarity and to highlight the importance of considering hepatosplenic $\mathrm{T}$ cell lymphoma as differential diagnosis in the clinical setting of pancytopenia with hepatosplenomegaly to allow timely diagnosis of these aggressive neoplasms.

\section{P-LD 14}

Spectrum of Extra Nodal Lymphomas in a Tertiary Care Center Dr. N.Priyathersini", Dr. S.SriGayathri, Dr. M.Arthi, Dr. J. Thanka

Email ID for Correspondence: Nagarajan.priyathersini@gmail.com Sri Ramachandra Medical College and Research Institute, Chennai

Introduction: Extra nodal lymphomas are lymphomas arising in nonlymphoid organs. The incidence of extra nodal lymphomas is rising all over the world. The accurate diagnosis of them is very essential since the treatment modalities are different and unnecessary surgeries can be avoided. Objectives: This retrospective study is aimed at analysing the incidence of extra nodal lymphomas, their distribution and their immunohistochemical profile. Material and Methods: The cases diagnosed as lymphomas in extra nodal sites in our department of pathology were collected. The slides were retrieved from the archival collections. The histopathological and the immunohistochemical slides were reviewed for morphology and immunophenotype. The data collected were analyzed. The basic immunohistochemically panel included CD45, CD20 and CD3. Other markers like CD 10, CD 5, Cyclin D1, CK7, CK20, and EMA were included when needed. Results: Extra nodal lymphomas accounted for 82 cases. It was found in patients from 3 years to 84 years. The most common site being GIT with 23 cases followed by 17 cases in brain, 11 cases in skin, 8 cases in sino nasal region. The other sites included bone, soft tissues, testis, thyroid, head and neck, urinary bladder, breast, lungs, liver and kidney. Diffuse large B cell lymphoma was the most common type. Conclusions: Extra nodal lymphomas are a heterogeneous disease with varied presentations. 
Gastro intestinal tract is the most common site and diffuse large B cell lymphoma is the most common type.

\section{P-LD 15}

Extra Nodal Marzinal Zone Lymphoma Involving Bilateral Ocular Adnexa

\author{
Dr Naushad Shah", Dr Ronica Baruah, Dr Nazima Nashrin, \\ Dr Dipali C. Deka
}

Email ID for Correspondence: drnaushadshah@gmail.com Ekopath Metropolis Lab Services Private Ltd.

Introduction: Intraocular and ocular adnexal non-Hodgkin lymphoma are a heterogeneous group of malignancies, accounting for approximately $1 \%$ to $2 \%$ of non-Hodgkin lymphomas (NHLs) and 5 to $15 \%$ of all extranodal lymphomas. Majority of them are low-grade tumours of the MALT-type and usually carry a good prognosis. The diagnosis of ocular adnexal lymphoma (OAL)is made by the clinical and imaging studies followed by the combination of histopathologic, immunophenotypic and molecular genetic studies. Management of OAL is a multidisciplinary approach first requiring a comprehensive staging evaluation. Objectives: To present an uncommon case of bilateral OAL, describe its clinical, histological and immunohistochemical features. Material and Methods: 64 years male presented with tiny nodules on bilateral upper eye lids since 2 years duration.The lesions gradually increased and involved conjunctival tissues, orbital soft tissue, muscle, lacrimal gland, entire eyeball and adnexa. He presented to ophthalmology department with bilateral proptosis, eyelid mass, conjunctival mass and ocular motility restriction. Results: Histology shows diffuse infiltration by small to medium lymphoid cells having irregular nuclei.Immunohistochemistry examination shows positivity for CD 20, Bcl-2 and Mum 1 with Lambda/kappa cocktail favouring monoclonality and Ki 67 index of 10 to $15 \%$. Based on histoimmunoprofile diagnosis of Extra Nodal Marzinal Zone B- cell Non-Hodgkin Lymphoma was made. Conclusions: Primary OAL are uncommon, extranodal lymphomas. Usually unilateral and slow growing, these may mimic benign tumours and hence the awareness and understanding of this lymphoma helps in timely diagnosis and its treatment.

\section{P-LD 16}

\section{Profile of Paediatric Lymphoma in a Tertiary Care Centre-A Bird's Eye View \\ Gulshan S ${ }^{\#}$, Mandal PK, Patra PC, Dolai TK, De R, Chakrabarti P}

Email ID for Correspondence: shzgulshan@gmail.com Nil Ratan Sircar Medical College, Kolkata

Introduction: Lymphomas are the $3^{\text {rd }}$ most common childhood malignancy. Hodgkin lymphoma (HL) is one of the most curable pediatric cancers. In children Non-Hodgkin Lymphoma (NHL) is somewhat less common than HL. However incidence of NHL appears to be on the rise. HL has long-term survival rates now exceeding $90 \%$ after treatment with chemotherapy alone or combined with radiotherapy. Objectives: To determine the spectrum of HL \& NHL patients from 0-18 years attending NRS Medical College \& Hospital, Kolkata. Material and Methods: A retrospective study was conducted from May 2014 to September 2017 of patients diagnosed as lymphoma between 0-18 years attending NRS Medical College. Data of medical records from an ongoing cancer registry was reviewed and analysed. Results: Median age at presentation is $11 \mathrm{yrs}$ (range 4-18yrs) with a Male:Female ratioof 3.6:1. Lymphadenopathy is the most common presentation $(97.8 \%)$. B symptoms are present in 65 (58\%) case. There are $84(75.3 \%)$ HL\&28 (24.7\%) NHL patients. Classical HL (cHL) comprises 95\% (80) of HL patients. Of the NHL patients, $8(28.5 \%)$ were $\mathrm{T}$ lymphoblastic lymphoma, $5(17.8 \%)$ DLBCL, 6 (21.4\%) Burkitt's lymphoma, 3 (10.7\%) ALCL, 1 (3.5\%) Mycosis fungoides, 1 (3.5\%) Grey zone lymphoma, 2 (7.1\%) unclassified T-cell lymphoma \&2 (7.1\%) B-cell lymphomas. Seventy seven cases $(68.7 \%)$ presented in advanced stage (III\&IV). ABVD and OEPA/COPDAC was received by 52 and 18 patients respectively. Conclusions: In this study majority of paediatric patients were HL;cHLbeing the commonest histologic subtype. Among NHL patients majority were T-lymphoblastic lymphoma followed by Burkitt's lymphoma.

\section{P-LD 17}

An Unusual Presentation of Adult T - Cell Leukemia/Lymphoma Dr. Shabnam Roohi, Dr. Pooja Sarda

Email ID for Correspondence: pooja_diya24@yahoo.co.in Sakra World Hospital

Introduction: Adult T-cell leukemia/lymphoma (ATLL) is an aggressive malignancy of activated mature $\mathrm{T}$ lymphocytes associated with Human T-cell lymphotropic virus type 1 (HTLV-1). Clinical presentations and morbidity is determined by leukemic infiltration of the ATLL cells into different organs. The disease is resistant to multiple chemotherapy agents and has a poor survival. Objectives: The objective was to study a case of ATLL which was unique in its features that it primarily presented like a Chronic Lymphoproliferative disorder (CLPD) associated with a solid organ involvement in the form of abdominal discomfort and vomiting, lymphoadenopathy, hepatosplenomegaly, marked leucocytosis, hypercalcemia and high serum LDH levels along with atypical lymphocytes, finally progressing to an explosive cutaneous involvement and early death of the patient. Material and Methods: A 22 years old male patient presented with on and off abdominal pain and vomiting. His peripheral smear was suggestive of a CLPD and bone marrow was suggestive of a Non-Hodgkin Lymphoma (NHL), which later was confirmed by flow cytometry to be possibly a Peripheral T cell Lymphoma. After the fourth cycle of chemotherapy, however, he developed explosive rapid skin infiltration along with fever and cough, to which he finally succumbed to death. Skin biopsy confirmed involvement by NHL of T cell type, high grade. Results: The acute type of ATLL is characterized by massive lymph nodes, hepatosplenomegaly, lytic bone lesions, and multiple visceral lesions with skin, GI tract, and lung infiltration. Other important findings associated with the acute type of ATLL are hypercalcemia, elevated LDH levels. Clinical subtypes, age, performance status, and serum calcium and LDH levels are the major prognostic factors for ATLL. The skin is the most common extralymphatic site of involvement in 50\% (43-72\%) patients with ATLL. Conclusions: In conclusion, it is important to differentiate between primary and secondary cutaneous ATLL and from other T cell malignancies like Sezary Syndrome and Mycosis Fungoides in order to better evaluate the prognosis. Clinicians should have a high suspicion when patients present with a CLPD like blood picture and subsequently should always screen for HTLV-1 infection when patients are finally diagnosed with peripheral T-cell lymphoma. 


\section{P-LD 18}

\section{Plasmablastic Lymphoma vs Its Morphologic Mimics: Report of Two Cases with Unusual Presentation and Associated Diagnostic Dilemma}

Introduction: Plasmablastic Lymphoma (PBL) is an aggressive growth of neoplastic cells that resemble B immunoblasts in morphology and are immunophenotypically indistinguishable from plasma cells and lack B cell markers. Although originally reported in oral cavity of HIV positive patients, PBL shows significant heterogeneity in terms of location and clinical presentation. The diagnosis is often challenging, as other hematolymphoid neoplasms with plasmablastic morphology like Plasmablastic myeloma, Plasmacytoma, Primary effusion lymphoma, ALK positive large B cell lymphoma etc. can show similar clinical, morphological and immunophenotypic pictures. PBL has an aggressive course with high rates of mortality. Objective: To describe the clinicopathological features of two cases diagnosed as Plasmablastic Lymphoma (PBL) with unusual presentations. Material and Methods: Relevant clinical history and examination, imaging, lab parameters, light microscopic and immunohistochemical findings were studied in both the cases. Results: The first patient was an elderly male with history of dyspepsia with episodes of hematemesis and melaena. A tumor mass was seen arising from the gastric fundus and body. The second patient was a young female with history of simple partial seizure. An SOL was seen arising from left posterior frontal region. Both patients were HIV negative. On light microscopy, both the tumors were composed of sheets of atypical lymphoid cells with plasmablastic morphology. Apoptotic bodies, tingible body macrophages and frequent mitotic figures were noted. On IHC, both the tumors were positive for CD138 with negative B \& T cell markers and Alk-1. Proliferative index were high (Ki67 > 60\%). EBV serology done in one case came negative. However EBER in-situ hybridization was not performed. Absence of $\mathrm{CRAB}$ features, myeloma defining events or bone marrow infiltration favoured the diagnosis of PBL. Conclusion: PBL can occur in HIV negative patients outside oral cavity. Combined clinical and laboratory approach is necessary to distinguish from its morphological mimics.

\section{Abstract Haematocon 2017}

\section{P-LD 19}

Cutaneous Infarcts and ANCA Positive Vasculitis in AITL: A Rare Presentation

\section{Sqn Ldr Sourya Sourabh Mohakuda (Resident, Internal Medicine), AFMC, Surg Lt Cdr Abhish Mohan (Resident, Internal Medicine), AFMC, MajSandeep Kumar (Asst Prof, Internal Medicine), AFMC}

Introduction: AITL is one of the rare subtype of peripheral T-cell lymphoma, comprising $2-5 \%$ of all non-Hodgkin lymphomas ${ }^{1}$. The lymph node architecture is often effaced, with retention of only a few benign follicles. The infiltrate characteristically extends beyond the lymph node capsule, with preserved and dilated subcapsular sinus. Often there is interfollicular proliferation of endothelial venules. The neoplastic $\mathrm{T}$ cells are positive for CD2, CD3, CD4, CD10, CXCL-13, PD1, and often BCL- $6 .^{2-4}$ AITL is associated with many systemic features like fever, rash or arthritis ${ }^{5}$. These systemic features may precede the appearance of other features of lymphoma. The disease may present with autoimmune phenomena, such as the presence of circulating immune complexes, cold agglutinins, hemolytic anemia, and rheumatoid factor and anti-smoothmuscle antibodies. In approximately half of the patients, polyclonal hypergammaglobulinemia is present. ${ }^{6}$ Vasculitis is not common, but comprises ANCA negative vasculitis. ${ }^{7}$ Case: 65 year old male a nonsmoker with no known comorbidities presented with complaints of short febrile illness associated with weight loss, dry cough, generalized weakness, pruritus and paresthesias of bilateral lower limb. He was admitted to Medical ward and after 12 hrspatient developed dyspnea and blackish discolouration of digits. Clinical examination revealed scattered polyphonic wheeze over bilateral chest. A working diagnosis of secondary vasculitis was made and was started on methylprednisolone pulsein view of impending digital gangrene. He responded to the treatment with no further progression of digital ischaemia. Evaluation revealed anemia $(\mathrm{Hb}-5-6 \mathrm{~g} / \mathrm{dl})$, thrombocytopenia (Platelet$5000 / \mathrm{mm} 3)$, raised ESR $(78 \mathrm{~mm} / \mathrm{hr}$ ) and eosinophilia (AEC-2910). Biochemistry panel revealed azotemia (Serum Urea/ Serum Creatinine:70/3.5 mg/dl). Autoimmune workup revealed pANCA strongly positive by ELISA, however ANA, DCT/ICT were negative. Tumour markers including AFP, CEA and CA-19-9 were negative. CECT Chest and abdomen showed hepatosplenomegaly with hilar and pretracheal lymphadenopathy. MR Angio Aorta \& Branches showed small scarredRt kidney with small calibre right renal artery with no evidence of aorto-arteritis. Nerve conduction studies was suggestive of mononeuritis multiplex with involvement of ulnar and peroneal nerves. Bone Marrow studies showed Cellular (80\%) reactive marrow with slight increase in plasma cells. Serum protein electrophoresis revealed monoclonal gammopathy, Ig G lamda restricted. A lymph node biopsy of left axilla was done and HPE was suggestive of Angioimmunoblastic $\mathrm{T}$ cell lymphoma. He was started on Chemotherapy CHOP and has shown response. Discussion Angioimmunoblastic T-cell lymphoma (AITL) is one of the uncommon but aggressive neoplasm which has a varied clinical presentation including constitutional symptoms, lymphadenopathy, organomegaly, autoimmune phenomena especially hemolytic anemia and thrombocytopenia and polyclonal hypergammaglobulinemia. ${ }^{8}$ The average age of presentation is 60-65 years with no sex predilection. The clinical spectrum include weight loss, fever, night sweats, pruritus, skin rash hepatosplenomegaly, pleural effusion, oedema and ascites ${ }^{9,10,11}$ Autoimmune phenomena is seen in a vast majority of patients including autoimmune hemolytic anemia, vasculitis, polyarthritis, autoimmune thyroiditis ${ }^{12}$. Laboratory finding include anaemia, eosinophilia, hypergammaglobulinemia, autoantibodies, elevated lactate dehydrogenase serum levels, elevated erythrocyte sedimentation rate, and bone marrow involvement. Generally paraneoplasticvasculitis associated with lymphoid malignancies are ANCA negative, however our Patient presented with vasculitis which was ANCA positive ( $\mathrm{p}$ ANCA). There are two reported cases of ANCA positive vasculitis one with AITL and other with chronic lymphocytic leukaemia both being c ANCA positive ${ }^{13}$. AITL is associated with hypergammaglobinemia but rarely monoclonal gammopathy has been reported. Our patient had monoclonal gammopathy with features simulating POEMS syndrome with neuropathy, organomegaly, skin changes and endocrinal dysfunction. Conclusion This case emphasizes the atypical presentation of a rather rare disease and the need for high index of suspicion and close followup for diagnosis of AITL. The early diagnosis will help in initiating chemotherapy well in time and to prolong median survival in this aggressive disease.

\section{Conflicts of Interest}

The authors have none to declare.

\section{References}

1. Rüdiger T, Weisenburger DD, Anderson JR, et al: Peripheral T-cell lymphoma (excluding anaplastic large cell lymphoma): Results from the Non-Hodgkin's Lymphoma Classification Project. Ann Oncol 13:140-149, 2002. 
2. Attygalle AD, Diss TC, Munson P, et al: CD10 expression in extranodal dissemination of angioim- munoblastic T-cell lymphoma. Am J SurgPathol 28:54-61, 2004

3. Dupuis J, Boye K, Martin N, et al: Expression of CXCL13 by neoplastic cells in angioimmunoblas- tic T-cell lymphoma (AITL): A new diagnostic marker providing evidence that AITL derives from follicular helper cells. Am J SurgPathol 30:490494, 2006

4. Dorfman DM, Brown JA, Shahsafaei A, et al: Programmed death-1 (PD-1) is a marker of germinal center-associated T cells and angioimmunoblastic T-cell lymphoma. Am J SurgPathol 30:802-810, 2006

5. Tsochatzis E, Vassilopoulos D, Deutsch M, Filiotou A, Tasidou A, Archimandritis AJ. Angioimmunoblastic T-cell lymphoma-associated arthritis: case report and literature review. J ClinRheumatol. 2005; $11: 326-8$

6. Frizzera G, Moran EM, Rappaport H: Angio- immunoblastic lymphadenopathy with dysproteinaemia. Lancet 1:1070-1073, 1974

7. M A Hamidou, D El Kouri, M Audrain, J-Y Grolleau: Systemic antineutrophil cytoplasmic antibody vasculitis associated with lymphoid neoplasia. Ann Rheum Dis 2001;60:293-295

8. Emilio Iannitto, Andrés J.M. Ferreri, VivianaMinardi, Claudio Tripodo, Hans H. Kreipe :Angioimmunoblastic T-cell lymphoma: Critical Reviews in Oncology/Hematology 68 (2008) 264-271

9. Patsouris E, Noel H, Lennert K. Angioimmunoblastic lymphadenopathy-type of T-cell lymphoma with a high con- tent of epithelioid cells. Histopathology and comparison with lymphoepithelioid cell lymphoma. Am J SurgPathol 1989;13:262-75.

10. Frizzera G, Moran EM, Rappaport H. Angio-immunoblasticlymphadenopathy with dysproteinaemia. Lancet 1974;1:1070-3.

11. Siegert W, Nerl C, Agthe A, et al. Angioimmunoblasticlymphadenopa- thy (AILD)-type T-cell lymphoma: prognostic impact of clinical observations and laboratory findings at presentation. The Kiel Lym- phoma Study Group. Ann Oncol 1995;6:659-64.

12. Dogan A, Attygalle AD, Kyriakou C. Angioimmunoblastic T-cell lym- phoma. Br J Haematol 2003;121:681-91.

13. M A Hamidou, D El Kouri, M Audrain, J-Y Grolleau: Systemic antineutrophil cytoplasmic antibody vasculitis associated with lymphoid neoplasia: Ann Rheum Dis 2001;60:293-295

\section{P-LD 20}

Anaplastic Lymphoma Kinase-Positive Large B Cell Lymphoma (ALK + LBCL): An Aggressive Yet Under-Recognized Entity

Shivani Sharma", M. Murthy, Vandana Sharma, Arjun Singh Rana, Shipra Garg, Samriti Arora, Lata Kini, Naveen Aggarwal

Email ID for Correspondence: shivani.sharma0701@gmail.com CORE Diagnostics

Introduction: ALK + LBCL was described first in 1997 by Delsol and colleagues, and was listed as a distinct entity in 2008 WHO classification of tumors of hematopoietic and lymphoid tissues. It is a lymphoma with plasmablastic or immunoblastic morphology with lack of CD30 expression and diffuse positivity for ALK. It is important to recognize this subtype, as this is an aggressive neoplasm with poor response to conventional therapies. Objectives: To review the histopathologic and immunohistochemical profile of ALK + LBCL and analyse the pitfalls in the diagnosis of this rare or rather under recognized neoplasm while reporting a case of 32 year male patient with cervical lymphadenopathy. Material and Methods: We received a paraffin block from cervical lymph node for review and immunohistochemistry. Formalin fixed paraffin embedded tissue underwent routine haematoxylin and eosin $(\mathrm{H}$ and $\mathrm{E})$ staining and microscopic observation. An extensive immunohistochemical panel was performed including PanCK, CD45, CD20, CD3, CD5, CD56, CD34, CD4, CD8, BCL6, BCL2, CD10, CD30, CD7, ALK-1, desmin, CD79a, Pax5, CD43, MPO, HMB45, CD138, and Ki67. For each antibody external and internal controls were examined. A review of literature of ALCL + LBCL was done. Results: H and E stained section showed solid sheets of monomorphic atypical intermediate to large sized lymphoid cells that had round to oval nuclei with dispersed chromatin and prominent nucleoli. The cells had moderate to scant amount of amphophilic cytoplasm. Mitosis was brisk. These neoplastic cells were positive for CD45 (strong and diffuse), CD79a and Pax 5 (weak and focal), ALK (strong and diffuse), CD138 (strong and diffuse), CD4 (weak and focal), BCL6 (weak and focal) with Ki67 proliferation index of $80 \%$. No loss of T cell markers was seen. Conclusions: The differential diagnoses that should be considered whenever a plasmablastic and immunoblastic morphology is seen on histomorphology are ALK + LBCL, ALK + Anaplastic large cell lymphoma, Diffuse large B cell lymphoma (NOS), and plasma cell dyscrasia including plasmacytoma. These neoplasms are usually CD20 negative, hence extensive panel of markers including CD79a, Pax5, and CD138 are essential. It is important to note that CD4 can be focally positive in these neoplasms. They comprises of $<1 \%$ of all Diffuse large B cell lymphoma and under recognition of these neoplasms could be one of the reasons for the same. The response to conventional therapies is poor and there is a possible targeted therapy hence, diagnosis of this neoplasm becomes all the more important.

\section{P-LD 21}

Concomitant Hodgkin Lymphoma (HL) and Langerhans Cell Histiocytosis (LCH) in a 24 Year Male Patient: Case Report with Review of Literature

\section{Shivani Sharma", Aditi Dewan, Gulruh Botiraliyeva, Aditi Aggarwal, Lata Kini}

Email ID for Correspondence: shivani.sharma0701@gmail.com CORE Diagnostics

Introduction: Hodgkin lymphoma and Langerhans cell histiocytosis are known to occur simultaneously and exhibit a complex relationship. Differentiation of these two diseases is very crucial because of their different treatment protocols. The prompting question remains whether $\mathrm{LCH}$ is a reactive or a neoplastic process. In cases where LCH follows HL, proliferation of Langerhans cells is thought to occur as a response to radiotherapy and chemotherapy. Objectives: We report a lung mass involving the mediastinum in a 24 year old male patient from Uzbekistan, wherein both these diseases are concordant. The patient had a significant history of receiving chemotherapy for an untypified lung tumor in the past. We review the clinical, histopathologic, and immunohistochemical profile of concurrent HL and LCH and analyse the diagnostic pitfalls. Material and Methods: A needle core biopsy and paraffin blocks from lung mass were receieved for review and immunohistochemistry. Formalin fixed paraffin embedded tissue underwent routine haematoxylin and eosin ( $\mathrm{H}$ and $\mathrm{E})$ staining and microscopic examination. An extensive immunohistochemical panel was performed including PanCK, CD45, CD30, CD15, BCL2, Pax5, CD3, CD20, CD4, CD8, CD1a, CD68, S100 and Ki67. For each antibody, external and internal controls were examined. A review of literature to understand the complex relationship between HL and LCH was done. Results: $\mathrm{H}$ and E stained 
sections showed areas of fibrosis infiltrated by few large binucleate cells that had prominent nucleoli and amphophilic cytoplasm (Reed Sternberg cells). A second population of histiocytic cells was seen that had acidophilic cytoplasm, lobulated indented nuclei with longitudinal clefts. Another significant finding on histomorphology was presence of sheets of eosinophills with interspersed lymphocytes and neutrophils. Immunohistochemistry panel results showed positivity for CD30, and CD15, weak positivity for Pax5, and negativity for CD45 in RS cells. The histiocytic cells (Langerhans cells) were positive for CD1a, S100 and CD68. Conclusions: A careful histomorphologic analysis is important in identifying the Langerhans cells in the background of Reed Sternberg cells. An extensive immunohistochemical panel is indispensible to confirm the two population of neoplastic cells. It has been concluded that lymphomas including Hodgkin lymphoma are capable of inducing the proliferation of abnormal Langerhans cells that leads to $\mathrm{LCH}$. The dendritic cells can be present in the microenvironment of HL but these are usually CD1a negative and langerin positive as described in the literature. In the index case large population of cells showed copositivity for S100, CD68, CD1a and Ki67, hence this population was favoured to be clonal.

\section{P-LD 22}

\section{Primary Lymphoma of the Breast: Report of Three Cases}

\section{Rajesh Kashyap, Pradeep Kumar, Hira Lal, Ram Nawal Rao}

\section{Sanjay Gandhi Postgraduate Institute of Medical Sciences,} Lucknow

Introduction: Primary breast lymphoma (PBL) is a rare form of extra nodal non-Hodgkin's lymphoma (NHL). It accounts for less than $0.5 \%$ of all breast malignancies and $2 \%$ extra nodal NHL. Diffuse large B-cell lymphoma (DLBCL) is the most common histological subtype. We report 3 cases of PBL diagnosed and treated at our center. Material \& Methods: The clinical records of 3 female patients diagnosed with PBL from 2004 to 2015 were reviewed. Two patients had diffuse large B-cell lymphoma (DLBCL) and the third patient had anaplastic large cell (ALCL) NHL. The mean age at presentation was 56 years. One patient with DLBCL was treated with CHOP chemotherapy and the second patient with rituximab plus CHOP chemotherapy. Both the patients showed complete response and have had a disease free survival of 84 and 96 months respectively. The third patient with ALCL refused further treatment after confirmation of diagnosis. Conclusion: PBL-DLBCL can be successful treated with chemotherapy and long-term survival is similar to nodal NHL. Running Head; Primary Breast Lymphoma

\section{P-LD 23}

Epstein Barr Virus IN Classical Hodgkin's Lymphoma: A Study at Tertiary Care Centre

Preeti Rani $^{1 \#}$, Mili Jain ${ }^{2}$, Nishant Verma ${ }^{3}$, Amita Jain ${ }^{4}$, Archana Kumar $^{5}$, A.K. Tripathi' ${ }^{6}$, Ashutosh Kumar ${ }^{7}$

1-Junior resident 2- Assistant Pofessor 7- Professor, Department of Pathology, 3- Assistent Professor 5- Professor, Department of Pediatrics, 4-Professor, Department of Microbiology, 6-Professor, Department of Clinical Hematology, King George's Medical University, Lucknow U.P.

Email ID for Correspondence: thakkarpreeti23@gmail.com
King George's Medical University

Introduction: Classical Hodgkin's lymphoma (HL) is characterised by scattered Reed Sternberg cells (RS) in a milieu of inflammatory cells. Pathogenetic link of HL and Epstein Barr Virus (EBV) has been postulated. EBV status in HL has limited reports from India. Objectives: To evaluate the prevalence of immunohistochemical and molecular positivity for EBV in classical HL and its correlation with clinicopathological parameters and treatment response. Material and Methods: 88 histopathological and immunohistochemistry (CD15, CD30) proven cases of classical HL were enrolled. Histomorphologic evaluation (Capsule, nodal architecture, residual follicle, nodule formation, sclerosis, granuloma, necrosis, RS cell atypia, RS cell clusters, RS cell sheets, type and percentage of inflammatory infiltrate) Immunohistochemistry (IHC) for EBV Latent Membrane Protein 1 (LMP1) and polymerase chain reaction (PCR) analysis was done. Treatment response was evaluated according to InPOG protocol. Statistical analysis was done by using SPSS software. Results: Majority cases were of nodular sclerosis (NS) (79.55\%) followed by mixed cellularity (MC) (11.45\%). EBV positivity was seen in $87.14 \%$ and $100 \%$ in patients of NS and MC respectively on either IHC, PCR or both. In comparison of IHC, sensitivity of $83.3 \%$, specificity $43.8 \%$, and diagnostic accuracy of $75.6 \%$ for PCR was evaluated. A higher proportion of EBV negative cases presented as an earlier clinical stage but the difference was not significant. No significant difference was seen for various clinicopathologic parameters and treatment response with respect to EBV status. Conclusions: No significant difference was seen for various clinicopathologic parameters and treatment response with respect to EBV status. A high proportion of EBV positivity indicates pathogenic role in HL. However, no significant correlation of EBV status was seen with clinical parameters, histomorphologic parameters and treatment response.

\section{Poster Presentation}

\section{P-LD 24}

Primary Burkitt's Lymphoma of Endometrium and Bilateral Ovaries in a 6-Year-Old Female: Report of a Rare Entity

\section{Kalpana Deshpande, Jai Vijay Juvekar ${ }^{\#}$, Purva Patil, Madhumita Kurdukar, Wasif Ali Z. A. Khan}

Email ID for Correspondence: nezazzuri@gmail.com Grant Medical College and Sir J. J. Group of Hospitals, Mumbai

Background: Lymphomas are a group of malignant tumors involving cells of the lymphoreticular or immune system such as B-lymphocytes, T-lymphocytes, and monocytes. Burkitt's lymphoma is the eponym given to a malignant tumor of the hematopoietic system, characterized by undifferentiated lymphocytes. Case Summary: A 6 year old girl was hospitalized for pain and lump in abdomen since one month. On per abdomen examination, a large bosselated lump was palpable. CT Scan of Abdomen showed three well defined heterogenously enahancing large masses in lower abdomen, Uterus and either ovaries could not be identified separately from mass. Panhysterectomy was performed. Pathological Findings: The received specimen of uterus showed bilateral ovarian masses. On section, all the masses were homogenous grayish-white. Microscopically the tumor showed a diffuse monotonous population of intermediate-sized cells with scattered tingible body macrophages imparting a 'starry-sky' pattern. Abundant apoptosis and frequent mitosis were present. Immunohistochemical analysis revealed the cells expressing positivity for CD10, CD 20, ki67 > 95\% and negative for Tdt and bcl 2 confirming the diagnosis of Burkitt's lymphoma. Discussion: Burkitt's Lymphoma is a highly aggressive but 
potentially curable lymphoma. This tumor specifically involves jaw bones in African children, however, in non-endemic areas, extranodal sites like the intestinal tract, mesentery, kidney, liver, skin, central nervous system and gonads are commonly involved. Conclusion: Although rare, Burkitt's lymphoma should be one of the differential diagnoses in a case of young girl presenting with endometrial and bilateral ovarian masses.

\section{References:}

1. WHO Classification of Tumours of Haematopoietic and Lymphoid Tissues Jaffe ES, Harris NL, Stein H 2008

2. A sarcoma involving the jaws in African children Burkitt $\mathrm{D} \mathrm{Br} \mathrm{J}$ Surg, 1958

3. Comparative immunohistochemical analysis of pediatric Burkitt's lymphoma and diffuse large B-cell lymphoma Frost M, Newell J, Lones MA Am J Clin Pathol, 2004

\section{P-LD 25}

Bone Marrow Changes in Hodgkin Lmphoma: A Retrospective Analysis of 12 Cases from a Tertiary Care Center

Aditi Kundoo", Somanath Padhi, Pritinanda Mishra, Susama Patra, Mukund N Sable, Suvendu Purkait, Samanyoya Gochhayat, R R Das ${ }^{1}$, A Satpathy ${ }^{1}$, Samarendra Mahapatro ${ }^{1}$, Sonali Mohapatra ${ }^{2}$, Asutosh Panigrahi $^{2}$

Departments of Pathology and Laboratory Medicine, ${ }^{1}$ Pediatrics, and ${ }^{2}$ Medical Oncology/Hematology, All India Institute of Medical Sciences, Bhubaneswar

Email ID for Correspondence: aditi.kundoo@gmail.com

Introduction: Bone marrow (BM) involvement in Hodgkin lymphoma (HL) is uncommon and is reported in 5-15\% cases. Objectives: To study the spectrum of marrow changes in newly diagnosed cases of HL undergoing staging procedure. Materials and methods: BM changes in twelve newly diagnosed patients with HL (stage IIB and above) (males $=8$, females $=4$; mean age $=42 \pm 1.7$ ) were analysed retrospectively and correlated with the routine laboratory parameters. Results: BM involvement was noted in four out of twelve cases (33\%), at the time of diagnosis. The involvement was focal in three; and one patient showed diffuse involvement with myelonecrosis and fibrosis. Classical Reed Sternberg (RS) giant cells in appropriate milieu were seen in three cases. One of the four patients with marrow involvement had HIV associated secondary myelodysplasia related changes. Marrow hyper cellularity, lymphoplasmacytosis and focal granulomatous response were common in both involved and uninvolved cases $(\mathrm{N}=8 / 12$ ). Immunohistochemistry (IHC) using CD30, PAX5, and ALK-1 were useful in delineating the RS cells and ruling out close differential (ALCL). Anemia, B-symptoms, hepatosplenomegaly, raised transaminases, deranged coagulation parameters and hyponatremia were more commonly observed in the involved group. Conclusion: In an era of PET-scan, adequate bone marrow trephine biopsy still remains gold standard in the staging of Hodgkin lymphoma; and should be evaluated routinely in resource poor setting.

\section{P-LD 26}

Expression of Epigenetic Regulator (EZH2) in Primary Diffuse Large B Cell Lymphoma and Correlation with P16, P53 Expression
Rimlee Dutta", Moien Rasheed Lone, Tryambak Srivastava, Aruna Nambarajan, Prashant Ramteke, Chandan K Das ${ }^{2}$, Ajay Gogia ${ }^{2}$, Mehar Chand Sharma, Saumyaranjan Mallick

Email ID for Correspondence: rimdutt369@gmail.com All India Institute of Medical Sciences, Department of Pathology, Department of Medical Oncology ${ }^{2}$, Dr B. R. Ambedkar Institute Rotary Cancer Hospital, New Delhi

Introduction: Diffuse large B cell lymphoma (DLBCL) is the most common lymphoma worldwide, accounting for $30 \%$ of all nonHodgkin lymphomas. It exhibits a marked heterogeneity in its morphological and biological features. Despite numerous advances, the overall survival rate of DLBCL is only $50 \%$ with currently available chemotherapeutic regimens. Recent studies have highlighted the role of epigenetics in prognostication and treatment of many solid tumors. In this study, we have tried to compare the cell cycle regulators with epigenetic regulator EZH2 and H3k27 expression in DLBCL. Objectives: To analyse epigenetic regulator EZH2 and H3k27 expression in primary diffuse large B-cell lymphoma and correlation with cell cycle regulators p16, p53 expression. Material and Methods: This was a retrospective study. A total of 35 cases of histopathologically confirmed primary diffuse large B-cell lymphoma diagnosed in the department of Pathology and treated at All India Institute of Medical Sciences (AIIMS) and Institute Rotary Cancer Hospital (IRCH) in 2014 were included. Detailed histomorphological examination was carried out on hematoxylin \& eosin (H\&E) stained sections. Immunohistochemistry for CD3, CD20, CD10, BCL6, MUM1, EZH2, H3K27, P53, Rb, p16 were performed using streptavidinbiotin peroxidase method. Results: The age of the patients ranged from 23 to 78 with an average of 49 years. Male to female ratio was 2:1. The most common clinical presentation was lymphadenopathy. According to Hans algorithm, germinal center phenotype comprised $42 \%$ of cases $(n=15)$ while post germinal center comprised $58 \%$ cases $(n=20)$. There was positive correlation between EZh2 and p53 immunohistochemical expression $(\mathrm{p}=0.003)$, similar observation was also made between p16 and EZh2 $(\mathrm{p}=0.04)$, However, no correlation was found between other markers. There was no correlation between EZH2 expression and subtypes of DLBCL $(p=0.6)$. Conclusions: Our study demonstrates that there is positive correlation between p16 and EZH2 expression which indicates that the loss of p16 in DLBCL may be epigenetically regulated. The gene expression profile have shown that EZH2 mostly alter in germinal center phenotype of DLBCL however, we did not find any correlation between EZH2 and DLBCL subtypes. The limitation of our study was that we could not confirm our result by gene expression studies.

Acknowledgement: The study was funded by AIIMS, New Delhi.

\section{P-LD 27}

Rare Epstein Barr Virus Related Lymphoproliferative Lesion of Skin: A Case Report

Rimlee Dutta ${ }^{\#}$, Vineet Govinda Gupta*, Ajay Gogia*, Prashant Ramteke, Maher C. Sharma, Saumyaranjan Mallick

Email ID for Correspondence: rimdutt369@gmail.com Departments of Pathology and Medical Oncology*, All India Institute of Medical Sciences, New Delhi

Introduction: Lymphomatoid granulomatosis is one of the rare B-cell lymphoproliferative disorders amongst the spectrum of 
malignancies associated with Epstein Barr virus (EBV). The median age is fifth to sixth decade with a male preponderance. Most common organs involved include the lungs, followed by skin and the CNS. The histological triad comprises of mixed atypical lymphoid infiltrate along with vascular invasion and coagulative necrosis. Consistent association with EBV is seen. Material and Methods (Case details): A 39 year old male presented with waxing and waning lesions over his back for the past 8 years, with sudden development of rapidly increasing mass over a period of 4 to 5 months. CECT imaging revealed nodular soft tissue masses measuring $10 \times 10 \mathrm{~cm}$. The lesion was confined to skin, the underlying muscle being free of tumor. Axillary lymphadenopathy was also seen. Skin biopsy showed unremarkable epidermis, dermis showed polymormphous infiltrate, comprising of small lymphocytes, histiocytes, eosinophils and interspersed large atypical cells. Large areas of necrosis was also seen. On immunohistochemistry, the large cells were immunopositive for CD20, CD30, and EBV- LMP1 while negative for EMA, CD3, CD4, ALK, CD8 and CD15. EBV- LMP1 staining revealed 10-15 positive cells per high power field. A diagnosis of lymphomatoid granulomatosis stage IV (Lugano) was rendered. Post six cycles of dose adjusted EPOCH chemotherapy, the patient achieved complete remission and has been disease free for 1.5 years, since end of therapy. Results \& Conclusion: Lymphomatoid granulomatosis is a rare EBV related B-cell Non Hodgkin lymphoma with variable treatment options, spanning from only observation in minimal stable disease to chemotherapy along the lines of NHL in high grade cases. Hence, a careful combined approach integrating both morphological and immunohistochemical findings is required for proper diagnosis.

\section{P-LD 28}

\section{Primary Ovarian non Hodgkins Lymphoma : A Case Report}

Dr B Sreekanth", Dr T Kannan, Dr Bhargavi, Dr Ravi Shankar, Dr Ram prahlad, Dr Praveena, Dr Shilpa

Email ID for Correspondence: sreekanthboga@gmail.com Sri Venkateswara Institute Of Medical Sciences, Tirupati, Andhra Pradesh

Introduction: The ovarian lymphoma is rare entity. Ovarian involvement by NHL is usually secondary, as a part of systemic disease. Accounts for $0.5 \%$ of non hodgkins lymphomas and $1.5 \%$ of all ovarian neoplasms with diffuse large B cell lymphoma being the commonest type. We present a case of ovarian non hodgkin's lymphoma, manifesting like an advanced ovarian cancer, which was managed by chemotherapy only. After six cycles of BR regimens patient achieved complete metabolic and morphological remission. Case Report : A 58 years old post menopausal hypertensive female presented with the complaints of right side lower abdominal pain and fullness.On examination found to have large vague mass palpable in right iliac fossa and hypogastrium. Initial CECT abdomen shows mass of $16 \times 11.5 \times 10.2 \mathrm{cms}$ arising from right adnexa, no ascites. Serum CEA was $82 \mathrm{U} / \mathrm{ml}$. Biopsy suggestive of lymphoproliferative neoplasm, Immunohistocyto chemistry positive for CD45, CD20, BCL2, BCL6, CMYC, MUM1 and CD5.Ki 67 index 80\%.CD10 and CD 23 negative.Pathological diagnosis as Diffuse large B cell lymphoma-Activated B cell type. Retrospectively we evaluated staging work up for lymphoma.Serum LDH was 830 IU/L. Whole body PET/ $\mathrm{CT}$ shows extensive hypermetabolic mass lesion arising from right adnexal mass measuring $13.6 \times 15.9 \times 18 \mathrm{cms}$, fat planes lost between mass lesion with uterus and sigmoid colon. Metabolically mildly active aorto caval, para aortic, multiple mesenteric, bilateral inguinal lymphadenopathy. Then we came to final diagnosis as DIFFUSE LARGE B CELL LYMPHOMA -ACTIVATED B CELL
TYPE, STAGE II AE, HIGH INTERMEDIATE RISK (IPI Score 3).We treat with six cycles of Bendamustine and Rituximab with growth factor support.After chemotherapy patient achieved complete metabolic and morphologic response.Now patient was on regular followup.Till now patient disease free survival was six months. Conclusions: Primary Ovarian lymphoma is a rare disease. Its a challenge for Oncology team for proper diagnosis, staging \& management. Chemotherapy \pm Immunotherapy is the cornerstone of treatment. Clinicians should be aware of this rare presentation to avoid radical surgical resection.

\section{P-LD 29}

Retrospective Study to Evaluate the Outcomes of Patients with Primary Mediastinal B-Cell Lymphoma (PMBCL) Treated with Dose Adjusted R-Epoch Regimen and Utility of an Interim18-FDG PET-CT

Background: PMBCL is a biologically distinct subset of diffuse large B- cell lymphoma. The optimal management with regards to chemotherapy regimen, role of radiation (RT) and use of 18-FDG PET- CT is not clearly defined. A phase II study showed superior outcomes with DA R-EPOCH regimen alone obviating the need for RT in majority of the patients. As majority of our patients present with advanced stage, poor performance status and with a higher incidence of extranodal site involvement; the efficacy of DAREPOCH and interim PET-CT in this setting needs to be addressed. Materials and methods: This is a retrospective observational study conducted on a treatment-naive PMBCL cohort treated with DA-REPOCH from January 2012 till January 2016 at Tata memorial centre, Mumbai. The histology was reviewed by expert hematopathologists. PMBCL was diagnosed based on clinico-pathological features. On histology, patients with intermediate to large cell morphology, with expression of CD30, CD20 and CD 23 were included. In doubtful cases, Rel and PU.1 were tested. Data for patients $>15$ years was retrieved from case files and electronic medical records. Staging details, lactate dehydrogenase levels, treatment details, toxicities, response assessment, last follow- up and outcomes were recorded. 18-FDG PET-CT scan was done at the end of 4 cycles, and repeated at the end of 6 cycles in those who had residual disease. RT was given to those who had single site residual disease. Patients who received atleast 1 cycle were included. Baseline clinical, epidemiological data, treatment, toxicities and response were summarised using descriptive statistics. Survival analysis was done using Kaplan-Meier method. Overall survival was calculated from registration till death or last follow up. Patients who were on palliative care and lost to follow up were censored as an event. Event-free survival was calculated from the registration date till progression, relapse or death due to any cause or last follow-up. Positive and negative predictive value of interim PET CT scan in confirmed PMBCL was calculated. Results: 30 patients were analysed out of which 24 patients had a clinicopathologically confirmed diagnosis of PMBCL, in the remaining 6 patients complete pathological classification was not possible. Baseline features are summarised in Table 1 . Notable is that $19(64 \%)$ patients had extranodal disease. All but one patient received 6 cycles of $\mathrm{R}$ EPOCH regimen. Dose escalation was possible in 21 (70) patients, 19 (63\%) patients could reach atleast till level 3 dosing. CNS prophylaxis in the form of intrathecal methotrexate was administered in 9 patients. Grade $3 / 4$ toxicities were seen in 24 (80\%) patients, febrile neutropenia was the most common with 30 episodes (In 179 cycles administered), peripheral neuropathy in 5, Left ventricular dysfunction in 1 , mucositis in 4 , Ileus in 1 , anemia in 7 patients. 12 (40\%) patients required hospitalisation, median duration was 4 days (2-19 days) and only 2 patients had interruptions in therapy. 
Table 1 Baseline characteristics

\begin{tabular}{ll}
\hline Baseline characteristics & Total no. - 30 \\
\hline Age in years, median (range) & $30(15-50)$ \\
Female, n (\%) & $16(53)$ \\
Duration of symptoms, in months. Median (range) & $2(1-12)$ \\
B symptoms, $\mathrm{n}(\%)$ & $12(40)$ \\
PS (ECOG scale) n (\%) & \\
0 & $3(10)$ \\
1 & $21(70)$ \\
2 & $2(7)$ \\
3,4 & $4(13)$ \\
Bulky disease $>7 \mathrm{~cm}, \mathrm{n}(\%)$ & $29(96)$ \\
Stage III/IV, $\mathrm{n}(\%)$ & $17(57)$ \\
Extranodal sites, $\mathrm{n}(\%)$. & \\
0 & $11(36)$ \\
1 & $5(17)$ \\
2 & $8(27)$ \\
$\geq 3$ & $6(20)$ \\
Pleural effusion, $\mathrm{n}(\%)$ & $6(20)$ \\
Pericardial effusion, $\mathrm{n}(\%)$ & $3(10)$ \\
IPI score $3 / 4, \mathrm{n}(\%)$ & $14(46)$ \\
Serum albumin $<4 \mathrm{~g} / \mathrm{dL}, \mathrm{n}(\%)$ & $18(60)$ \\
\hline
\end{tabular}

Radiation was administered to $16(54 \%)$ patients. In the overall cohort, there was a complete response in 25 (83\%) and partial response in $5(17 \%)$. In confirmed PMBCL, Deauville score $\leq 3$ were seen in 21 patients on interim PET CT scan with $\geq 3$ in 3 patients. The positive predictive value of an interim PET scan was $33 \%$ and negative predictive value was $76 \%$. There were 8 relapses, and 7 deaths. All the deaths were due to disease progression. With a median follow up of 28 months, the 2-year overall survival is $75 \%$ and 2-year event free survival is $72 \%$. Conclusions: $\mathrm{R}$ EPOCH regimen is an effective regimen in PMBCL patients even with adverse features and can reduce the need for consolidation radiotherapy. Interim PET CT scan is not of much help in these patients.

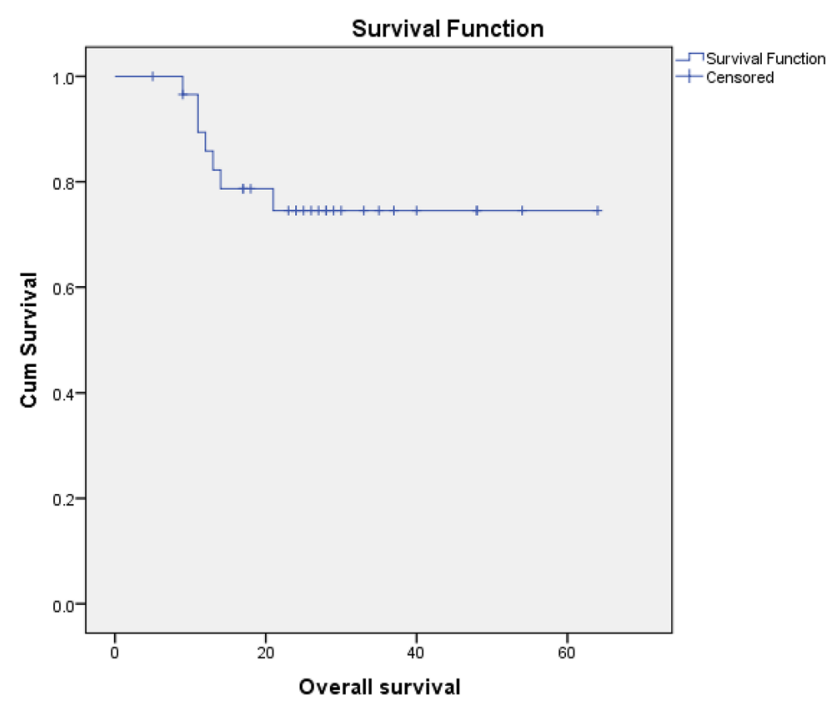

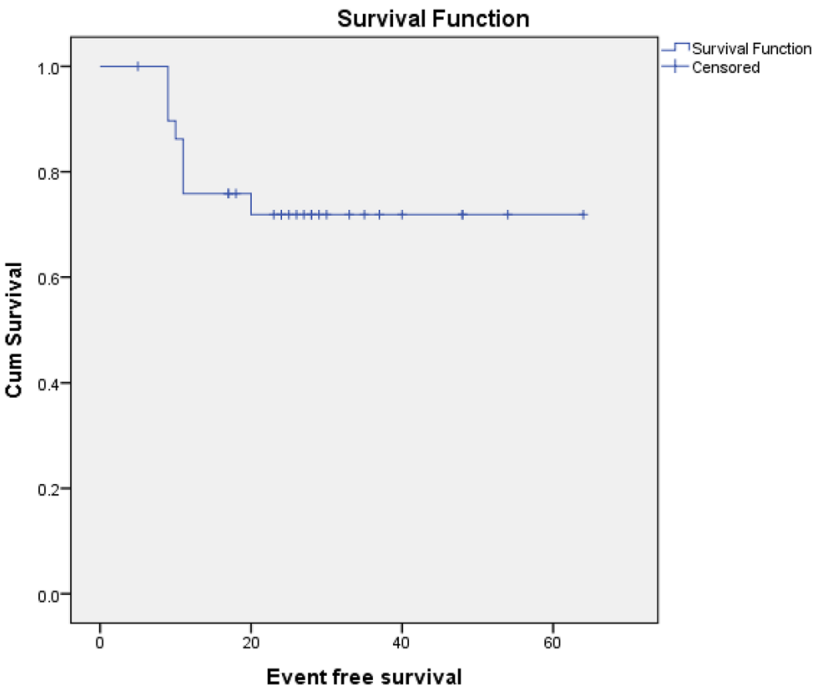

P-LD 30

Role of IgM Flow Marker in CLPD in the Era of New Markers Aishwarya Sharma, Dr Prashant Tembhare

Tata Memorial Hospital, Mumbai

Introduction: CLPD is a heterogeneous group of disorders characterised by monoclonal proliferation of lymphocytes in peripheral blood, spleen or bone marrow. Flow cytometry is used for the diagnosis and classification of CLPD. By flow cytometry large number of antigens can be assessed in a single measurement which allows for more precise identification and characterisation and also predicts the prognosis. With the availability of so many new flow markers it is not fair to ignore the age old markers of CLPD.

\section{Objectives:}

1. To study the relevance of IgM antibody in different CLPDs

2. Role of new flow markers like CD180, CD148, CD49d in the diagnosis of CLPD

3. To check for the need of revising the CLPD antibody panel Materials and Methods

Flow cytometry was performed on the peripheral blood, bone marrow aspirate and body fluids of 285 newly diagnosed CLPD cases by using Navios 10 coloured flow cytometer. The flow cytometry findings were also correlated with the lymph node biopsy and cytogenetics findings wherever available.

Results: We studied IgM expression in 285 cases of CLPD. 103 out of 155 cases of CLL $(66.4 \%), 21$ out of 25 cases of mantle cell lymphoma (84\%), 7 out of 12 cases of BCLL/SLL (58.3\%), 6 out of 8 cases of Burkitt's lymphoma (75\%), 10 out of 13 cases of hairy cell leukaemia (76.9\%), 9 out of 14 cases of DLBCL (64.2\%), 14 out of 17 cases of marginal zone lymphoma (82.3\%), 12 out of 13 cases of atypical CLL (92\%), all 11 cases of lymphoplasmacytic lymphoma and all 3 cases of CD5/CD10 negative lymphoma showed IgM expression. Conclusion: IgM expression was seen in the majority of cases of lymphoplasmacytic lymphoma, atypical CLL, mantle cell lymphoma, CD5/CD10 negative lymphoma, Burkitts lymphoma, hairy cell leukaemia and CLL. IgM can serve as a useful marker in 
characterizing CLPDs. The role of CD180, CD148 and CD49d will be discussed after further analysis.

\section{P-LD 31}

Pediatric-type Follicular Lymphoma (Case Report of a 6 Year Old Male Child with Nodal and Extra-Nodal Involvement)

\section{Dr. Maliha Afsar", Dr. Sai Mallikarjun, Dr. Triveni Bhopal, Dr. N.Ezhil Arasi}

Email ID for Correspondence: dr.malihaafsar@gmail.com Osmania Medical College (Hyderabad, Telangana)

Introduction: Pediatric-type Follicular Lymphomas (PFLs) are indolent Non-Hodgkins Lymphomas with clonal follicular proliferation resulting in lymphadenopathy or extra-nodal lesions. They are distinct from the usual adult Follicular Lymphomas in being extremely rare, have lower clinical stage and excellent prognosis inspite of having a higher histological grade with high proliferation index, have blastoid follicular centre cells rather than classic centroblasts and do not recur after excision even without systemic treatment. Pediatrictype Follicular Lymphomas are positive for germinal centre markers CD10 and BCL6, and should lack BCL2 gene rearrangements, though BCL2 protein expression (IHC) may be present in some. PFLs at different anatomic sites behave differently. Further, MUM1 expression dichotomizes Follicular Lymphoma into Low grade FL (CD10+, BCL6+, MUM1-, Ki-67 low) and High grade FL (CD10+/-, BCL6+/ weak, MUM1+, Ki-67 high). Case Report: 6 year old male child presented with right cervical lymphadenopathy of 30 days duration. On examination discrete cervical nodes measuring 0.5 to $1 \mathrm{~cm}$ were present on right side. FNAC was suggestive of a Lympho-Proliferative Disorder. Further examination revealed an enlarged Right tonsil. PET CT showed multiple bilateral subcentimetric level II to V cervical nodes with enlarged metabolically active tonsils. Peripheral smear had no abnormal findings and HIV status was non-reactive. Bone marrow was within normal limits (Ann-Arbour Stage II). Cervical nodes were excised and sent for histopathological examination which revealed partially effaced nodal architecture with a starry sky pattern. Expansile follicles with attenuated mantle zones, with prominent blastoid follicular centre cells were seen.IHC profile highlighted follicles with blastoid cells being CD10+ CD20+ PAX5+ MUM1 strong+ BCL2+ and Ki-67 80\%. CD3 highlighted the T cells, CD30- TdT-. Paraffin blocks have been sent for BCL2 rearrangements and reports of cytogenetics awaited.

Keywords Pediatric, follicular, lymphoma, stage, grade, blastoid, BCL2, MUM-1, prognosis

\section{P-LD 32}

\section{Primary Bone Lymphoma: A Rare Case Report}

Pansi Gupta\#, Sunita Singh, Rajnish Kalra, Nisha Marwah, Gauri Munjal, Rajeev Sen

Email ID for Correspondence: pgdoc1703@gmail.com PT BD Sharma, PGIMS, Rohtak

Introduction: Primary bone lymphoma ia an extremely rare entity with only retrospective studies mentioned in literature. Case report: Here we mention a case of 10 year old girl presented with lytic lesion in right femur distal end. MRI showed involvement of soft tisuue as well. FNAC and Histopathological examination confirmed it to be Bcell lymphoma. Discussion: Mostly patients are $>45 y$ rs age and with male preponderance. The cause is not well known but viral infection, immunodeficiency, organ transplant, inherited diseases are suggested in various studies. Chronic osteomyelitis, primary bone tumors, metastases have to be ruled out. Conclusions: The present study reports a case of primary bone lymphoma and a review of literature.

\section{P-LD 33}

How CD34 \& CD105 Can Help in Therapy and Prognosis of NonHodgkin's Lymphoma?

Sucheta ${ }^{\#}$, Sant Prakash Kataria, Rajeev Sen

Email ID for Correspondence: suchetamalik19@yahoo.com Pt. B. D. Sharma PGIMS Rohtak

Introduction: Prognosis in Non Hodgkin Lymphoma depends on many factors including extent of angiogenesis measured as microvessel density (MVD). Several studies have shown that increased MVD is predictive of poor prognosis and associated with high tumor grade or high grade transformation in NHLs. Other studies have failed to confirm these results.

Objectives:

1. Evaluation of microvessel density by using immunohistochemical methods and computer assisted quantitative image analysis in nodal Non-Hodgkin Lymphoma patients.

2. Comparison of CD34 and CD105 expression in lymph nodes of Non-Hodgkin Lymphoma patients.

Material and Methods: The present study was conducted on 60 lymph node biopsies that were divided into two groups - control group included 20 cases of reactive lymphadenitis \& test group included 40 cases of Non-Hodgkin Lymphoma. Representative paraffin embedded tissue sections of all the cases were stained with routine haematoxylin and eosin alongwith immunohistochemical stains with monoclonal antibodies to CD34 and CD105. "Hot spots", that is, areas of maximal microvessel density (MVD) were identified. In four such hot spots vessel number and MVD were analyzed at $400 \times$ using automated image analyzer by two investigators independently. The mean value of both investigators was considered as the MVD in each case. Sections of lymph node were used as positive controls for anti CD34 and anti CD105 antibodies. Negative controls were prepared by replacing the antibody by PBS or nonimmune serum. The quantitative morphometric studies were done by image analysis. For comparison between immunohistochemical staining between CD34 and CD105, Chi square test was applied. To compare the number of microvessels and microvessel density between reactive lymphadenitis and various categories of Non-Hodgkin Lymphoma, T-test was applied.

Results:

- There was an increased angiogenesis in aggressive lymphomas (DLBCL, PTCL) as compared to indolent lymphomas (CLL/SLL) and this difference was statistically significant.

- When CD34 was used as an endothelial marker it highlighted the vessels in both benign lymph nodes as well as lymph nodes involved in lymphoma. Whereas CD105 highlighted the vessels more in cases of lymphomas as compared to benign lymph nodes.

- CD105 was observed to be a more specific marker for counting of tumor blood vessels in lymphomas.

Conclusions: The present study concluded that Non-Hodgkin Lymphomas exhibit potent angiogenic activity which increased significantly with increasing aggressiveness. Microvessel density determined by CD34 and CD105 antibody highly correlated with 
different categories of NHL. Higher microvessel density was observed in cases of aggressive NHL as compared to indolent NHL. These findings further suggested the potential significance of characteristics of microvessel density as potential prognostic marker as well as its application in improved selection of patients for antiangiogenic and other treatments. In the present era of newer antiangiogenic therapies, Non-Hodgkin Lymphomas' angiogenesis and alteration in vascular morphology definitely has prognostic significance and thus helps to improve the treatment modalities and patient care.

\section{P-M 01}

\section{Cytotoxic Effect of Adenanthera Pavonina Seed Extracts on Cancer and Normal Cell Lines}

Ayisha Basheer Vakkath, Kartik Sachdeva, Nair Ashish Dinesh", N.G.R Srikanth, Neha Mardia, Sreelakshmi Chandrashekharan, Devi Arikketh

Email ID for Correspondence: ashishnair28@gmail.com SRM University, Chennai

Introduction: Adenanthera Pavonina is a tropical tree which is of religious importance in Kerala and of medical importance in Ayurveda. It is colloquially called as "Manjadikuru" and several research papers state its blood pressure lowering effect and anti-diabetic effect. My team was interested to analyse the effect of crude seed extract on cancer cells. Objectives: To prepare extracts from boiled and unboiled seeds and to evaluate the cytotoxicity of seed extracts on cancer and normal cell lines. Material and Methods: Methanolic Extraction was performed on boiled and unboiled crude seed extracts; HepG2 (cancerous) cell line and HEK (normal) cell lines were cultured; MTT Assay was performed and subsequent IC50 values were analysed.

Results:

\begin{tabular}{|c|c|c|c|c|c|}
\hline $\begin{array}{l}\text { Sl. } \\
\text { No. }\end{array}$ & Objectives & Methods & Cell lines & $\begin{array}{l}\text { IC50 } \\
\text { (mg/ml) } \\
\text { (boiled; } \\
\text { unboiled) }\end{array}$ & Status \\
\hline 1. & $\begin{array}{l}\text { To prepare } \\
\text { extracts from } \\
\text { boiled and } \\
\text { unboiled seeds }\end{array}$ & $\begin{array}{l}\text { Methanolic } \\
\text { extraction of } \\
\text { A. pavonina } \\
\text { seeds }\end{array}$ & - & - & Completed \\
\hline \multirow[t]{3}{*}{2.} & $\begin{array}{l}\text { To evaluate the } \\
\text { cytotoxicity of } \\
\text { seed } \\
\text { extracts on } \\
\text { cancer } \\
\text { cell lines }\end{array}$ & $\begin{array}{l}\text { Procurement of } \\
\text { cell lines } \\
\text { MTT assay }\end{array}$ & $\begin{array}{r}\text { HepG2, } \\
\text { HEK }\end{array}$ & - & Completed \\
\hline & & & HepG2 & $\begin{array}{l}464.5849 ; \\
266.7395\end{array}$ & Completed \\
\hline & & & HEK & $337.15 ; 76.15$ & Completed \\
\hline
\end{tabular}

Conclusions: Cytotoxic activity is evident in cancer cell line (HepG2) and normal cell line (HEK). Boiled samples were safer than unboiled samples as per the IC50 values of cancer (HepG2) and normal (HEK) cell lines.

\section{P-M 02}

"In-Vitro evaluation of Betulinic Acid against Molt-3, Lymphoblastic Cell line and Immunostimulant Effect on Raw264.7 Cell line."

Soumodeep Karmakar ${ }^{1}$, Nilanjana Deb ${ }^{1}$, Santanu Basu ${ }^{2}$, Shila Elizabeth Besra ${ }^{1 *}$

E-mail: soumo92kol@gmail.com/shilabesra@iicb.res.in

${ }^{1}$ Cancer Biology \& Inflammatory Disorder Division, CSIR- Indian Institute of Chemical Biology, 4 Raja S.C. Mullick Road, Kolkata700032, West Bengal, India; ${ }^{2}$ Employees State Insurance Hospital, Sealdah, 301/3, A.P.C. Road, Kolkata-700009

Betulinic acid (3 $\beta$, hydroxy-lup-20 (29)-en-28-oic acid), is a pentacyclic triterpenoid. BA induces death in cancer cells however, previous studies have not reported the anti- cancer activity of BA in Molt -3 (relapsed patients) acute lymphoblastic leukemia cells. Therefore, we investigated the anti-leukemic activity of Betulinic acid against acute human lymphoblastic leukemia cell line i.e. Molt-3 where the $\mathrm{IC}_{50}$ value is calculated to be $37.90 \mu \mathrm{g} / \mathrm{ml}$. Morphologically cell shrinkage, membrane blebbing, chromatin condensation, nuclear fragmentation and formation of apoptotic bodies are characteristic features of apoptosis studied by fluorescence and confocal microscopy. Gel electrophoresis study showed fragmented DNA in the form of ladder and Flow cytometric analysis showed appreciable number of cells in early \& late apoptotic stages. The cell gets arrested in the $\mathrm{G}_{0} /$ $\mathrm{G}_{1}$ phases of cell cycle. Nitric oxide (NO) a short-lived gaseous radical, is a potent multifunctional reactive metabolite that is a major effector molecule of immune cells against tumor cells and pathogens. Nitric oxide (NO) production in non-primed and $\mathrm{rIFN}-\gamma$ primed (stimulated) was investigated in murine macrophage Raw264.7 cell line. Also, the effect of PDTC and NOS inhibitor $\mathrm{N}^{\mathrm{G}} \mathrm{MMA}$ on Betulinic acid induced NO production was determined to address its immunostimulant properties. Our results demonstrate that BA can accelerate the activation of Raw 264.7 cells by $\mathrm{rIFN}-\gamma$ via a process

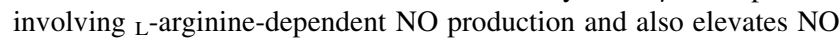
production via activation of $\mathrm{NF}-\kappa \mathrm{B}$ signaling pathway. So, in conclusion it can be said that the immunomodulatory activity of BA might explain its beneficial effect in the treatment of acute lymphoblastic leukemia/cancer.

Keywords Betulinic Acid, Leukemia, Lymphoid, Apoptosis, Nitric Oxide.

\section{P-M 03}

"Anti-neoplastic impact of triterpenoid, TUA on acute lymphoid leukemia cell growth and cell cycle arrest"

Nilanjana Deb ${ }^{1}$, Samrat Chakraborty ${ }^{1}$, Santanu Basu ${ }^{2}$, Snehasikta Swarnakar ${ }^{1}$, Tarun Jha ${ }^{3}$, Shila Elizabeth Besra ${ }^{1}$ *

${ }^{1}$ Cancer Biology \& Inflammatory Disorder Division, CSIR -

Indian Institute of Chemical Biology, 4 Raja S.C. Mullick Road, Kolkata-700032, West Bengal, India; ${ }^{2}$ Employees State Insurance Hospital, Sealdah, 301/3, A.P.C. Road, Kolkata-700009; ${ }^{3}$ Department of Pharmaceutical Technology, Jadavpur University

E-mail-neeludeb@yahoo.co.in, shilabesra@iiicb.res.in

Triterpenoids are ubiquitous in the plant kingdom. Anti-cancer potential of triterpenoids along with their anti-proliferative and pro- 
apoptotic effects are well established in in-vitro and in-vivo models. This encouraged us to investigate the anti-neoplastic effect of TUA in PBMNCs of ALL patients as well as MOLT-4 cell line. The $\mathrm{IC}_{50}$ value for ALL patient cells was calculated to be $38.25 \mu \mathrm{g} / \mathrm{ml}$ and $45.88 \mu \mathrm{g} / \mathrm{ml}$ for MOLT-4 cell line respectively. Cell cytotoxicity assay by MTT helped to evaluate the cytotoxic potential of TUA on both the metabolically active leukemic cells. The cell morphology was determined by Fluorescence and Confocal microscopy. DNA fragmentation was studied by gel electrophoresis. To examine whether cell death occurred via apoptosis or necrosis, double staining flow cytometry was performed. Cell cycle study helped to establish the phase distribution of the leukemic cells. TUA significantly inhibited cytotoxicity in a concentration dependent manner. Fluorescence microscopy study showed characteristic features of membrane blabbing, chromatin condensation the sign of early and late apoptotic changes in the cells after treatment. Propidium iodide staining showed presence of apoptotic bodies. Gel electrophoresis study showed fragmented DNA in the form of ladder. Flow cytometric analysis confirmed the presence of apoptotic cells. This study merits the consideration and further investigation of TUA as a potent therapeutic in acute lymphoid leukemia.

Keywords Triterpenoid, Leukemia, Lymphoid, Cell cycle, Apoptosis

\section{P-M 04}

\section{"Studies on the Anti-Proliferative Effect of Diospyros melanoxylon Bark on U937 CELL line"}

Shayantani Chowdhuri ${ }^{1}$, Nilanjana Deb ${ }^{1}$, Anita Hansda ${ }^{1}$, Twinkle Roy $^{1}$, Samrat Chakraborty ${ }^{1}$, Santanu Basu ${ }^{2}$, Snehasikta Swarnakar', Tarun $\mathrm{Jha}^{3}$, Shila Elizabeth Besra ${ }^{* 1}$

\section{E-mail: shayantanic@yahoo.com, shilabesra@iicb.res.in/} shilabesra@gmail.com

1 Cancer Biology \& Inflammatory Disorder Division, CSIR, Indian Institute of Chemical Biology, 4 Raja S.C. Mullick Road, Kolkata 700032, West Bengal, India. Tel: 91-33-2413-3223, Fax-91-33-24735197; 2 Employee State Insurance Hospital Sealdah, 301/3, A.P.C. Road. Kolkata-700009; 3. Department of Pharmaceutical

Technology, Jadavpur University, Kolkata-700032

Objective: Acute myeloid leukemia attributes to significant number of deaths as compared to any other form of Cancer. Elimination of leukemic cells through apoptosis is the main goal of specific therapy for killing the leukemic cells. Majority of chemotherapeutic drugs are highly expensive and cytotoxic to normal cells. The importance of developing totally new classes of chemopreventive agents is stressed. The present study investigates the cytotoxic potential of Diospyros melanoxylon bark extract (DMBE) and its active fractions (N-butanol and ethyl acetate) show anti-proliferative effect on human monocytic lymphoma cell line (U937). Materials and Methods: Cell viability by Trypan blue assay and Cytotoxicity study by MTT assay, apoptotic study by fluorescence microscopy and Agarose gel electrophoresis for DNA fragmentation were performed. Results: DMBE and its active fractions exhibited significant antiproliferative effect on U937 cells in a time and dose dependent manner. Fluorescence microscopic study demonstrated remarkable chromatin condensation, formation of apoptotic body and nuclei fragmentation in the treated cells as compared to control. DNA fragmentation study by Agarose gel electrophoresis also showed the presence of DNA in a ladder like form, which serves as the hallmark of programmed cell death. Conclusion: Furthermore, detailed study of DMBE has to be carried out for identifying its active constituents. We also aim to study the antiproliferative activity of DMBE on PBMNCs of leukemic patients in the near future.
Keywords Leukemia, Myeloid, Bark, Diospyros melanoxylon

\section{P-M 05}

Etiological Study of Anemia in Rheumatoid Arthritis

*Sk Sarafat Hossain, **Mrinal Gogoi, *** A. K. Sen

* Post graduate trainee Medicine, Assam Medical College and Hospital; ** Registrar, Department of Medicine, Assam Medical College and Hospital; *** Professor of Medicine, Assam Medical College and Hospital

Introduction: Since very few studies has been done on anemia in rheumatoid arthritis from north eastern India with this facts in view this present study has been undertaken with the following aims and objectives- To study the prevalence and cause of anemia in rheumatoid arthritis and relationship of anemia with the disease activity. Materials: Hospital based observational study for a duration of one year. 137 ( 32 males and 105 females) patients of Rheumatoid arthritis (RA) $\geq 13$ years of age, diagnosed by the 2010 ACR/ EULAR criteria for Rheumatoid Arthritis meeting inclusion and exclusion criteria were included in the study. After enrolling and through clinical examination following investigations was done in each patient- Complete blood counts, Peripheral blood smear, Erythrocyte sedimentation rate, Serum ferritin estimation, TIBC, $\mathrm{Hb}$ typing, Serum B12 estimation (if MCV > 100), Stool for occult blood, UGI endoscopy (whenever indicated). Observations: Among the 137 patients anemia was found in 91 [66.42\% (Anemia chronic disease in $42.86 \%$, Iron deficiency anemia in $36.26 \%$, hemoglobinopathies $17.58 \%$, megaloblastic anemia in $3.30 \%$ )]. Stool for occult blood is positive in 10 patients. Upper GI endoscopy shows antral erosions in 4 and chronic duodenal ulcer in 6 patients. mDAS28 was found higher in anemic patients compared to non-anemic $(\mathrm{p}<0.0001)$. Also there is significant association found between early morning stiffness, mean tender joint count and swollen joint count in anemic and non-anemic patients. Disease activity score in anemic patients has an inverse co-relation with hemoglobin $(\mathrm{r}=$ $0.4291, \mathrm{p}<0.0001)$. There is positive co-relation between tender joint count and disease activity in anemic patients $(r=0.8999$, $\mathrm{p}<0.0001)$. Conclusions: Anemic patients with RA have higher disease activity compared to non-anemic patients. Cause of anemia in RA is multifactorial and in this part of country it is affected by the higher prevalence-of hemoglobinopathies.

\section{P-M 06}

IgG4 Related Disease Masquerading as Lymphoma Sree Rekha\#, J, Debdatta Basu, Srinivas BH, Biswajit D*

Email ID for Correspondence: sree.path77@gmail.com JIPMER, Puducherry

Introduction: IgG4 Related disease (IgG4RD) is a systemic fibroinflammatory disorder of unknown origin. It involves multiple organs and produces tumefactive lesions. The disease shows characteristic features on histopathology like dense lymphoplasmacytic infiltrate rich in IgG4 plasma cells, storiform fibrosis and obliterative endarteritis. Objectives: To present and discuss two cases of IgG4RD clinically suspected as lymphoma. Material and Methods: The clinical scenarios along with histopathological findings of various biopsies like lymphnode, salivary gland, renal biopsy and biopsy from the ocular swelling received in these two cases has been discussed. 
Immunohistochemistry with CD3, CD20, CD15, CD30, CD38, IgG, IgG4 has been done. Results: Case 1: A 51 year old lady presented with multiple neck swellings and axillary swellings since one year. She was evaluated with a suspicion of lymphoma. FNAC from the cervical lymphnode showed features of Hodgkin lymphoma. Subsequently a submandibular lymph node biopsy was done and it showed salivary gland with features of chronic sclerosing sialadenitis. Histopathology of the Axillary lymphnode was diagnosed as probable IgG4RD associated lymphadenopathy due to increase in IgG4 plasma cells and immunoblastic proliferation. Subsequently she developed proteinuria for which a renal biopsy was done. The renal biopsy showed acute interstitial nephritis with lymphoplasmacytic infiltrate. Thus, this patient had multiple system involvement by IgG4RD. Case 2: An 11 yr old girl presented with complaints of right eye swelling resulting in proptosis. With a suspicion of lymphoma tissue from the right eye swelling was biopsied. The tissue showed dense lymphoplasmacytic infiltrate, storiform fibrosis and increased IgG4 plasma cells. Subsequently, serum IgG4 levels were done and were found to be elevated, confirming the diagnosis of IgG4RD. Conclusions: IgG4 $\mathrm{RD}$ masquerades as lymphoma clinically. Histopathology plays a major role in the diagnosis of this entity emphasising the need for awareness of this entity.

Keywords IgG4RD, masquerading, lymphoma

\section{P-M 07}

\section{Pattern of Hematological Disease in Patients Attending Out- Patient Department of a Tertiary Care Centre: Real World Experience}

\section{Jitani AK", Dolai TK, Chakrabarti P, Dutta S, De R, Mandal PK,} Baul SN

Email ID for Correspondence: ankitjitani@gmail.com

Nilratan Sircar Medical College and Hospital, Kolkata

Introduction: The epidemiology of hematological diseases in India is largely unknown due to paucity of data. The number of hematology centre in our country is also limited. Our centre caters to a major chunk of hematological patients in eastern India and is one of the largest centres in terms of patient attendance. Objectives: We planned to analyze our OPD data to decipher the disease pattern in our region. Material and Methods: We retrieved the data from July 2014 to June 2017 from our hospital management information system. We analyzed the number of new and follow-up patient attending our OPD for 3 consecutive years 2014-15, 2015-16, 2016-17 from the month of July to June. We also assessed the disease pattern for the mentioned years. Results: The total number of OPD consultations for the 3 years was 78809 , of which $21851(27.7 \%)$ were new cases and 56958 $(72.3 \%)$ were follow-ups. Disease characterization could be done for 15517 patients. Out of these cases, 73.3\% (11377/15517) were benign and $26.7 \%(4140 / 15517)$ were malignant hematological diseases. Common reasons for consultation in hematology OPD were anemia $59.1 \%$ (9169/15517), leukemia 16.4\% (2548/1517), bleeding disorder $12.3 \%$ (1899/15517), lymphoma $6.1 \%$ (941/15517) and myeloma $2.9 \%$ (450/15517). Among the benign diseases, the most common diagnosis was hemoglobinopathy $26 \%(2962 / 11377)$ followed by iron deficiency anemia 12\% (1367/11377), immune thrombocytopenia $6.3 \%$ (720/11377), aplastic anemia 4.7\% (535/11377), hemophilia $4.3 \%$ (487/11377) and thrombosis 0.7\%79/11377). Among the malignant cases, common causes were leukemia 61.5\% (2548/4140), lymphoma $22.7 \%$ (941/4140), and myeloma $10.9 \%$ (450/4140). Conclusions: Anemia remains the most common reason for OPD consultation even in a tertiary care hematology centre. The major burden of malignant hematological disease is leukemia.

\section{P-M 08}

Correlation of Serum Immunoglobulin $E$ Level and Absolute Eosinophil Count in Chronic Urticaria Patients

\section{K Lekshmi Priya, Chakrabarty Barun Kumar", Bajpai Mukul}

Email ID for Correspondence: bkcdc@yahoo.co.in Base Hospital

Introduction: Urticaria (hives) is relatively common clinical presentation with a point prevalence of about $0.5-1 \%$ in general population. Chronic urticaria is defined as urticaria that persists for at least 6 weeks. Chronic urticarial conditions can be subdivided into chronic spontaneous urticaria (CSU) and chronic inducible urticaria (CINDU). Increase in Immunoglobulin E (IgE) level and eosinophil counts are commonly associated with cutaneous inflammation and are thought to incite the occurrence of urticaria. However, the relationship of these factors with the disease process of chronic urticaria remains unclear. Objectives: The aim of this study is to measure the total serum IgE level and absolute eosinophil count (AEC) in chronic urticaria patients and to assess the correlation between these parameters and the severity of urticaria. Material and Methods: A total of 30 patients of chronic urticaria and 30 age and sex matched controls were enrolled in this study. The demographic and clinical features were recorded in all patients and routine investigations were performed. Autologous serum skin test (ASST) was performed as per the standard guidelines. Serum levels of total $\operatorname{IgE}$ and blood eosinophil count was compared with the clinical severity of urticaria. Established urticarial activity scoring system was used in assessing the severity of urticaria. Results: Descriptive statistical analysis has been carried out in the present study. Chi-square/Fischer exact test has been used to find the significance of study parameters on categorical scale between two or more groups. Student's $t$-test (2-tailed, independent) has been used to find the significance of study parameters on between the two groups, intergroup analysis. Conclusions: The pathophysiologic basis in all types of chronic urticaria is due to activation of dermal mast cells and basophils. However, the levels of eosinophils and serum IgE can be well correlated with the clinical presentation of chronic urticaria. Further large scale studies are required to authenticate this association.

\section{P-M 09}

An Uncommon Occurrence of Hypopharyngeal Malignancy in a Case of Fanconi Anemia

\section{Dr. Arunima Deb ${ }^{\#}$, Dr. Sridevi HB}

Email ID for Correspondence: arunimadeb91@gmail.com Kasturba Medical College, Mangalore

Introduction: Fanconi's anemia (FA) is a rare autosomal recessive disorder with an incidence of 1 in 350000 live-births. First described in 1927 by Guido-Fanconi, it is most commonly diagnosed between 5-15 years of age. The prognosis of this entity is poor with high mortality in the first 10 years of age and has high incidence of developing cancers in the later years of their life. It is characterized by aplastic-anemia (AA) and the most common malignancies that develop are acute leukemias (AL) followed by solid tumors (ST). We report a case of 20 year old student who came with history of dysphagia and course of his disease evaluation.

Case report: A 20 year old student presented with dysphagia of 1 month duration. Patient had pallor, hyperpigmentation of hands and knuckles with pigmented tongue. He was short statured (height- 
$142 \mathrm{~cm}$ ). There was no hepatosplenomegaly. In the past, patient had three admissions for repeated respiratory infections with routine investigations revealing pancytopenia. $\mathrm{He}$ is a non-smoker and nonalcoholic without any history of betel-nut chewing. On $70^{\circ}$ scopy, there was a post-cricoid mass and on CECT, this mass $(2.3 \mathrm{~cm} \times 1.4 \mathrm{~cm})$ was seen posterior to cricoid compressing the esophagus. Peripheral-smear showed pancytopenia with macrocytosis and neutropenia. The post-cricoid mass biopsy showed an infiltrating tumor with features of moderately-differentiated squamous cell carcinoma. Bone-marrow (BM) was hypocellular having increased fat cells and absence of megakaryocytes with extreme paucity of maturing myeloid cells. Occasional clusters of erythroid cells with a relative increase in lymphocytes, plasma cells and mast cells were seen. The BM-biopsy confirmed hypoplastic marrow.

Conclusion: FA is a rare hereditary disorder with increased risk of malignancy which increases the associated morbidity and mortality especially at older ages. This case illustrates the occurrence of ST in FA at a young age. BM-transplantation at early age may delay the occurrence of ST.

\section{P-M 10}

Pattern of Leukemia and Lymphoma Incident and Mortality In Tripura, 2010-2014

\section{Dr. Biswajit Deb Barma ${ }^{\#}$}

Email ID for Correspondence: drbiswajitonco77@gmail.com Radiation Oncology, Regional Cancer Centre Agartala, RCC Agartala Tripura India, Agartala, Tripura, India, 799006

Background/ Purpose: To know the First, Second, Third highest site of incidence in Male and female, Common Age Group, Stages of Detection and Mortality. Methods: Total No of patients 461 nos,(Male 278 Female 183) Age Group 0-75 yrs, Data from year 2010-2014. Results: Out of total 183 female patients 1st was Myeloid Leukemia with 120 nos, 2nd was Lymphoid Leukemia 29 nos, 3rd was Multiple Myeloma 28 nos cases diagnosed. While in Male out of total 278 patients 1st was Myeloid Leukemia 154 nos, 2nd was Lymphoid Leukemia 68 nos and 3rd was Multiple Myeloma 41 nos. case diagnosed. MI Ratio was 45.5 and overall MI ratio of the state was 42.5. Total Nos of death recorded was 210 in this period. Discussion \& Conclusion:Patients are in the middle age group, highest diagnosed was Myeloid Leukemia both in Male and Female. There was a lack of awareness among the society and spread lots of Myth for Hematological Malignancies too. More specialized setup and more specialized medical fraternity to treat Hematological Malignancies required in Tripura to cope up with the disease more efficiently and effectively.

\section{P-M 11}

Role of Interleukin-6 (IL-6) In Hematological Malignancies

Dr Devgan Kirti ${ }^{1 \#}$, Dr Gaur Dushyant Singh ${ }^{1}$, Dr Verma $\mathrm{SK}^{2}$, Dr Gupta Vibha ${ }^{2}$, Dr Kusum Anuradha ${ }^{2}$

Email ID for Correspondence: kirti.devgan@gmail.com HIHT University, Swami Rama Nagar Doiwala Dehradun-248140

Introduction: Haematological malignancies affect the blood, marrow and lymph nodes.IL-6 is a pleiotropic cytokine involved in immune responses, inflammation and hematopoiesis.IL-6 acts as cell growth modulator in haematological malignancies. Elevated levels of serum
IL-6 have been co related with adverse patient prognosis. Thus decrease in levels of IL-6 post treatment in various haematological malignancies can be considered as an important prognostic factor.

Objectives: 1.To compare various haematological parameters $(\mathrm{Hb}$, TLC, Plt count, MPV, MCV \& PCV) pre and post treatment. 2. To compare the levels of IL-6 pre treatment and post treatment. Material and Methods: Newly diagnosed 81 cases and 81 controls were included for the study of 12 months duration. Blood parameters were elicited by LH750 Beckman Coulter. Serum IL-6 was measured according to manufactures instruction. Nominal categorical data between the groups was compared using Chi Square test. Independent t-test was used between pre treatment cases and controls and post treatment cases and controls. Paired t-test was used between pre treatment cases and controls. $\mathrm{P}$ value $<0.05$ was considered significant. Results: Of 81 cases, 50 were male and 31 were females. The maximum cases were distributed in the age of 61-70 years.Multiple myeloma was the most common malignancy. All haematological parameters (Hb, TLC, Plt count, MPV, MCV \& PCV)all showed significant decrease post treatment $(p<0.001)$.Serum levels of IL-6 pre treatment cases was $0.0326 \mathrm{pg} / \mathrm{ml}$ and post treatment was $0.029 \mathrm{pg} / \mathrm{ml}$, showing significant decline $(\mathrm{p}<0.001)$. AML, CML, HL and PCM cases showed decrease in levels following treatment which when compared to their pre treatment levels was found highly statistically significant ( $\mathrm{p}$ value $<0.001$ ) except in CLL and NHL. Conclusions: Significant decrease in the levels of serum Interleukin 6 was seen in post-treatment cases. It was also found be a proven independent prognostic marker in most of the malignancies. Lower levels of IL-6 is related to increased survival, which when compared with higher levels, showed decrease in survival. However, no corelation between IL-6 levels and individual malignancy could be established owing to small number of cases in certain hematological malignancies Further studies on a larger population needs to be done to determine its clinical utility in correlating with the survival status and establishing the efficiency of the treatment.

\section{P-M 12}

\section{Langerhans Cell Histiocytosis in a Two and a Half Year Old} Female: A Rare and Unusual Case Report

\section{Dr. Medhavi Vimal ${ }^{\#}$, Dr. Sunita Sharma, Dr. Smita Singh,} Dr. Shruti Chauhan, Dr. Sharmila B Mukherjee*

Email ID for Correspondence: medhavivimal.15@gmail.com Department of Pathology and Department of Paediatrics*, Lady Hardinge Medical College, Delhi

Introduction: Langerhans cell histiocytosis $(\mathrm{LCH})$ is characterised by lytic bony lesions and presence of coffee bean/grooved nuclei along with eosinophils in bone marrow and tissue biopsies. Hence, absence of any of these commonly seen features pose a diagnostic challenge

Case report: A two and a half year old female presented with off and on fever, progressive pallor and reddish maculopapular lesions on body for one and a half year. There was hepatosplenomegaly and no lymphadenopathy. Skeletal survey was normal. Clinical provisional diagnosis of severe acute malnutrition, disseminated Kochs or langerhans cell histiocytosis was suggested. Complete blood examination showed normocytic anemia with thrombocytopenia. BMA smears were hypercellular and showed many histiocytes lying in clusters as well as individually showing characteristic coffee bean/grooved nuclei. There was no increase in eosinophils. Serum ferritin, plasma fibrinogen and serum triglycerides were within normal limits. 
Sections from skin biopsy showed infiltration of epidermis and dermis by sheets of histiocytic cells having cleaved, irregularly contoured coffee bean shaped nuclei. However, no eosinophils were noted. On IHC: CD1a and S100 positivity was seen confirming the diagnosis of Langerhans cell histiocytosis.

Conclusion: LCH is clinically divided into three categories on the basis of organ system involvement: unifocal, multifocal unisystem and multifocal multisystem category. This case comes under multifocal multisystem category. This was a rare and unusual presentation of LCH without any lytic bony lesions alongwith absence of eosinophils on BMA smears as well as on skin biopsy.

\section{Abstract for Oral Paper}

\section{P-M 13}

Immunophenotyping and Cytogenetics -Diagnostic Challenge for Leukaemia

Introduction \& Background: Leukaemias are neoplasms caused by mutation of the bone marrow pleuripotent stem cells and characterized by widespread proliferation of leucocytes and their precursors in the bone marrow and almost always in the peripheral blood. The various subtypes pose a great challenge in the diagnosis which is very vital for treatment. Thus combined evaluation by morphology, immunophenotyping and cytogenetics is very important for accurate diagnosis and planning the appropriate treatment.

Objectives:

1) To determine the type of leukaemia based on morphology and/or immunophenotyping findings as well as cytochemistry, in patients attending different departments in a span of one year.

2) To analyse the karyotyping profile of these patients based on conventional cytogenetic techniques.

Material and Methods: All cases with abnormal findings on routine examination suggestive of haematological malignancies were included in the study which spanned fpr a period of 12 months. Detailed history taking, clinical examination, peripheral blood and bone marrow samples were collected from these patients and analysed for morphology and karyotypic profiles along with antigen expression by neoplastic cells using flowcytometry for proper subtyping of leukaemias. Results: Out of total 70 cases of leukaemia diagnosed in a span of one year, CML was the commonest followed by AML, ALL, CLL and Acute leukaemia of Ambiguous Lineage (ALAL).Morphology alone could identify all cases of acute and chronic leukaemias but further subtyping and diagnosis were possible with the help of Flowcytometry and cytogenetics. Conclusions: Leukaemia is one of the dreadest cancer which mandates early and specific diagnosis for proper treatment.Thus the present study emphasises the indispensable role of flowcytometry and cytogenetics in supplementing the conventional morphology in accurately diagnosing and subtyping of leukaemias. Hence it can be concluded that immunophenotyping along with cytogenetics play a vital role in elucidating the lineage specificity and help in arriving at final diagnosis specially in cases with ambiguous morphology on peripheral blood smear and bone marrow examination.

\section{P-M 14}

Study of Neutrophil Lymphocyte Ratio in Type 2 Diabetes Mellitus and Its Correlation with Renal Function

\section{Dr Seema Rahar\#, Dr Sadhna Marwah, Dr Bindu Kulshreshta}

Email ID for Correspondence: chehdisambar@gmail.com PGIMER, Dr. RML Hospital, New Delhi

Introduction: Diabetes mellitus (DM) is a common metabolic disease with various vascular complications. Chronic inflammation plays an important role in development and acceleration of micro and macroangiopathy in these patients. Neutrophil lymphocyte ratio (NLR) is a novel potential inflammatory marker in many diseases including diabetes mellitus. Measuring NLR is simple and inexpensive than measuring other inflammatory cytokines like IL6, IL1, TNF alpha. Objectives: To study neutrophil lymphocyte ratio in type 2 diabetes mellitus and to correlate with renal function. Materials and Methods: One hundred patients with type 2 Diabetes Mellitus (50 patients with normal renal function and 50 with deranged renal function) and fifty controls were included in the study. Complete blood count including NLR, blood sugar, HbA1c, Kidney function tests, urine microalbumin and urine albumin creatinine ratio were assessed for these patients. Estimated Glomerular Filtration Rate (eGFR) was calculated with MDRD -4 variable formula. NLR was studied in diabetics and controls. Results: NLR was found to be higher in diabetic patients as compared to controls and was significantly higher in diabetic patients with deranged renal functions as compared to diabetics with normal renal function. TLC (total leucocyte count) was also significantly higher in diabetics with deranged renal function as compared to diabetics with normal renal function and controls. Anaemia was found to be more in diabetics with deranged renal function, $82 \%$ cases were found to have anaemia while among diabetics with normal renal function, $56 \%$ cases were found to be anaemic. Conclusions: NLR was significantly higher in diabetic patients as compared to controls and NLR was higher in diabetic patients with deranged renal function as compared to diabetic with normal renal function. Hence NLR may serve as a useful alternative marker in recognizing diabetic nephropathy earlier.

\section{P-M 15}

Haematological Abnormalities Among Alcoholic Liver Diseases and Its Correlation with Meld Score

\section{Prof Dr S. K Mahapatra \& Prof Dr L. K Dash}

Email ID for Correspondence: kumar.bismaya@gmail.com; Dr Kumar Bismaya (Presenting Author)

VSSIMSAR, Burla

Introduction: Alcoholism represents one of the most serious worldwide socioeconomic \& health related problem. Patients with alcoholic liver disease have various haematological abnormalities in various proportions, which are to a greater extend determine the mortality and morbidity among them.

\section{Objectives:}

1. To evaluate the spectrum of haematological abnormalities among alcoholic liver diseases.

2. To evaluate the correlation between haematological abnormalities with respect to MELD Score.

Material and Methods: This was a prospective observational study in which spectrum of various haematological indices of alcoholic liver disease were observed on clinical examination substantiated by histocytological evidence and imaging. The haematological indices were assessed in the subjects and were categorized under the different groups of MELD score. The relationship of these variables with MELD score was studied and statistical analysis was done. Results: 
We observed anaemia, leokocytosis as well as leukopenia and thrombocytopenia in various proportions in patients with ALD. There was progressive fall in haemoglobin levels with the increase in MELD score. Macrocytic anemia was mostly found in Cirrhotic patients in which MELD Score was closely related to the development of Macrocytic Anemia. Patients with group 1 MELD Score had normal leukocyte count. Leukocytosis predominated in MELD group 2 and 3 patients. In group 4, leukopenia was more prevalent. All the patients in group 5 had leukopenia. Group 1 and 2 patients did not have thrombocytopenia. Thrombocytopenia started occurring in MELD group 3 patients, while involving all the patients of group 4 and 5. Conclusions: Haematological parameters are progressively deteriorated with the progression of the disease. MELD Score could be an important tool in the assessment of these patients. These association strongly depicts that the clinicians could effectively apply the classification in predicting the haematological complications in these patients and take precautions early in preventing the further progression of the disease thus decreasing the mortality in these patients.

\section{P-M 16}

\section{Renal Osteodystrophy - A benign mimicker of multiple myeloma?}

Introduction \& Background: Renal osteodystrophy is a combination of disorder of mineral and bone metabolism associated with chronic renal insufficiency, or a long term complication of end-stage renal disease. It represents a spectrum of skeletal lesions. The most frequent cause of renal osteodystrophy is secondary hyperparathyroidism, which results in high-bone turnover and fractures. Case report: We report a case of 47 year old female, who presented with renal failure, multiple bony lytic lesions, fractures, and anemia. Bone marrow examination was performed on a high clinical suspicion of multiple myeloma. Results: Bone marrow examination revealed only $2 \%$ plasma cells. On examination of Bone marrow biopsy, histopathological changes of renal osteodystrophy were seen. Subsequently, her Parathyroid hormone levels were evaluated which were found to be markedly increased. Moreover, serum electrophoresis was normal and urine tested negative for Bence Jones proteins. Ultimately, a diagnosis of seconary hyperparathyroidim, leading to renal osteodystrophy was rendered. Conclusions: Overlapping clinical features of renal failure, anemia, hypercalcemia and bony lesions may provide a clinical diagnostic challenge. Bone marrow examination is a simple method which helps in clinching the diagnosis. Our case report intends to highlight the importance of identifying the subtle, and not so commonly encountered morphological changes associated with hyperparathyroidism, to not miss the diagnosis. This ultimately, has an impact on patient treatment and prognostic outcomes.

\section{P-M 17}

Knowing the Unknown - Primary Autoimmune Myelofibrosis

Dr. Sumeet P. Mirgh", Dr. Dinesh Chandra, Dr. Tulika Seth, Dr. M. Mahapatra, Dr. R. Saxena, Dr. H.P. Pati, Dr. S. Tyagi, Dr. P. Mishra, Dr. S. Sazawal, Dr. M. Aggarwal, Dr. R. Dhawan

Email ID for Correspondence: drsumeetmirgh@gmail.com AIIMS, New Delhi
Introduction: Bone marrow fibrosis is commonly seen in the setting of chronic myeloproliferative disorders, most commonly primary myelofibrosis (PMF). Autoimmune myelofibrosis (AIMF) is a recently described, lesser known entity, which, in contrast, to its counterpart PMF has favourable outcomes. They present with cytopenias, autoantibodies, reticulin fibrosis on bone marrow in absence of any clonal markers. AIMF is distinguished from primary myelofibrosis by the absence of splenomegaly/minimal splenomegaly, absence of eosinophilia/basophilia, and any abnormal myeloid, erythroid, or megakaryocytic morphology. Objectives: The objective of the study was to describe the clinical profile and treatment response of patients with AIMF. Material and Methods: We conducted a single tertiary centre retrospective review of patients diagnosed with AIMF inorder to review the clinical profile, therapies and outcomes with immunosuppressive therapy. Patients were excluded if they met diagnostic criteria for PMF or other neoplastic hematologic disorders associated with MF. Bone marrows were assessed as described by Pullarkat et al: presence of diffuse reticulin fibrosis, lack of clustered/ atypical megakaryocytes; lack of myeloid or erythroid dysplasia, eosinophilia, or basophilia; lymphocyte infiltration; lack of osteosclerosis; absent or mild splenomegaly and presence of autoantibodies. Results: 6 patients ( 5 males, 1 female, age range -1.8 - 53 years) were diagnosed with a mean follow up period of 13.3 months. Most common presentations were fatiguability and muco-cutaneous bleeding. All patients had features in bone marrow favouring AIMF and positive autoantibodies. However, none of them had a concomitant autoimmune disorder (no secondary AIMF). One patient achieved a CR (complete response) whereas four patients achieved PR (partial response) following immunosuppression with oral steroids. One patient did not achieve any response. Conclusions: Primary AIMF is a rare, clinicopathologic entity. Physicians and hematologists should be cognizant of this lesser known entity - as it responds to steroids with a good clinical outcome.Knowledge of this will prevent irrational tests and prompt treatment with steroids. There is paucity of data to support a role of stem cell transplant in patients not responding to steroids.

\section{P-M 18}

\section{Langerhans Cell Histiocytosis \\ Dr Yadlapalli C Deepak", Dr Ankur Punia, Dr (Col) PG Chitalkar}

Email ID for Correspondence: cdeepakyadlapalli@gmail.com Sri Aurobindo Institute of Medical Sciences

Introduction: Langerhans cell histiocytosis ( $\mathrm{LCH}$ ) describes a spectrum of clinical presentations ranging from a single bone lesion or trivial skin rash to an explosive disseminated disease. $\mathrm{LCH}$ is composed of three distinct clinical syndromes that show indistinguishable histology. Characteristically, these lesions stain positively with histochemical stains, S-100 and CD1a. Evaluation of the pathologic dendritic cells in blood and bone marrow has proven that LCH is caused by myeloid dendritic cells with activation of the RAS pathway. Objectives: The annual incidence of LCH is reported to be 0.5 - 5.4 per million children per year. We present two boys with $\mathrm{LCH}$ with two different entities of clinical presentation, one with single system LCH and other with multisystem LCH. Material and Methods: Case 1: First child for non-consanguineous parents, 22 month boy, was born following an uncomplicated normal vaginal delivery, full-term pregnancy. He developed swellings over the scalp and left iliac bone which on evaluation diagnosed as LCH. Received induction phase with vinblastine and steroid followed by maintainance vinblastine, steroid and 6 MP. Case 2: Eight year old male 
child for non-consanguineous parents, developed nail changes and itching which on evaluation diagnosed as $\mathrm{LCH}$, treated with induction phase vinblastine and steroid. Developed bulging of right eyeball with vision loss, on further work up found to be having multisystem $\mathrm{LCH}$. Treated with 4 cyles of cladiribine and cytosar followed by maintainance vinblastine, 6-MP, steroid. Conclusions: LCH can be difficult to diagnose since it is an uncommon disease that can affect many organ systems. LCH must be distinguished histologically and immuno-phenotypically from other histiocytic and dendritic cell disorders, metastatic solid or hematopoietic neoplasms, and hemophagocytic lymphohistiocytic and macrophage activation syndromes.

\section{P-M 19}

Clinico - Haematological Correlation in Patients of Chronic Renal Failure, An Observational Study in a Tertiary Care Centre Over a Period of 3 Months

1) Dr. Afreen, $1 \mathrm{~s}^{\mathrm{t}}$ yr PG., 2) Dr. Kalyani (Assistant Professor), 3) Dr. Naval Kishore Bajaj (Associate Professor),

4) Dr. Anunayi. J (Associate Professor),

5) Dr. Ezhil Arasi. N (Professor and HOD)

Upgraded Department of Pathology, Osmania Medical College/ Osmania General Hospital, Hyderabad, Telangana

Introduction: Chronic Kidney Disease is worldwide disease of varied etiology. Chronic renal failure results due to structural and functional glomerular damage or when the GFR is less than $60 \mathrm{ml} /$ $\mathrm{min} / 1.73 \mathrm{~m}^{2}$ of body surface area for more than 3 months. Anaemia in CRF results due to impaired production of erythropoietin by diseased kidneys. Objective: To assess haematological changes in patients with chronic renal failure and its correlation with renal parameters and duration of disease wherever possible. Material and Methods: It is an observational study from June 2017 to September 2017 in 66 patients who were diagnosed to have CRF and admitted in Department of Nephrology, Osmania General Hospital, Hyderabad, Telangana. Patients with known haemotological disorders and haematological malignancies were excluded. Haematological parameters were analyzed by Automated Haematology Analyzer (SYSMEX- XN1000). Results: Present study included 66 patients with age group ranges from 5 to 75 years, with 35 males $(53 \%)$ and 31 females $(47 \%)$. Most of the cases were in the age group of 20 to 60 years. Majority of the renal failure were due to hypertension (37.8) followed by diabetes (22.72). As per WHO classification of severity of anaemia - the patients were graded in to three categories, mild anaemia (11 cases), moderate anaemia ( 28 cases), severe ( 25 cases) $.65 .1 \%$ cases presented with normocytic normochromic anaemia followed by microcytic hypochromic anaemia (18 Cases) of probable iron deficiency associated with CRF and dimorphic picture seen in 5 cases. The mean Haemoglobin value and the mean value of haematological indices were found to be low except for mean MCV. Mean haemoglobin $-7.45 \mathrm{gm} / \mathrm{dl}$, mean haematocrit $-24.12 \%$, the mean $\mathrm{MCH}$ $-23.3 \mathrm{pg}$, the observed mean MCHC $-31.6 \mathrm{gm} / \mathrm{dl}$ and mean MCV 83.92 fl. WBC counts were raised in most of the cases. 44 cases showed neutrophilia with 5 cases showin g leucocytopenia platelet counts were normal in most of the patients decreased in 16 cases. Conclusion: Anaemia is the commonest haematological abnormality in patients of chronic renal failure with commonest morphological subtype being normocytic normochromic picture. Chronic anaemia of normocytic normochromic type when present, an underlying renal disease should be investigated. Hypertension and Diabetes are the predominant causes of chronic renal failure, such patients should be investigated for chronic kidney disease.

\section{P-M 20}

Inflammation and Efficacy of Iron Supplementation in Anemia in End Stage Renal Disease

Dr Santosh Kumar Roy", Dr Sajini Elizabeth Jacob, Dr Sreejith Parameswaran, Dr Zachariah Bobby

Email ID for Correspondence: roysaintoshkumar@gmail.com JIPMER, Puducherry

Introduction: Anemia in CKD has many factors like deficiency of iron, decrease in production of erythropoietin, increase in inflammation evidenced by an increase in inflammatory mediator like, ferritin hepcidin \& hsCRP. Objectives: To assess the effectiveness of IV Iron treatment in anaemic CKD subjects who are on regular haemodialysis with respect to inflammatory markers like Hepcidin and hsCRP by study of haemoglobin, ferritin, serum iron, TIBC, TSAT. Material and Methods: Blood samples were taken twice from 17 patients who are going to receive IV iron; first sample before IV iron injection and second sample after 3 months of iv iron treatment $(2 \mathrm{ml}$ in EDTA container and $3 \mathrm{ml}$ in plane container).All the patients were already on erythropoietin. Haematological parameters (by Sysmax XT 2000i), Iron parameters (by Chemiluminescent ADVIA, Beckman CoulterAU680 analyser), Hepcidin and hsCRP (by ELISA kit)were measured for both pre and post treatment samples. Results: Pre-treatment $\mathrm{Hb}$ range-6.5-9.5 g/dl; post treatment $\mathrm{Hb}$ range-8-11.2 g/d. Pre-treatment ferritin range-25.1-379 ng/ml; post treatment range-1408-335 ng/d. Pre-treatment TSAT range 3-45\%; post treatment-25-10. Pre-treatment hsCRP range-0.031-0.142 mg/l; post-treatment $0.058-0.81 \mathrm{mg}$. Pre-treatment Hepcidin range-26.5-158 ng/ml; post-treatment460$>880 \mathrm{ng} / \mathrm{ml}$. Data was analysed by Wilcoxon rank test for of indices of iron homeostasis, and by Pearson/Spearman correlation analysis between iron homeostasis with Hepcidin \&hsCRP. Conclusion: Even after IV iron supplementation Anemia did not get corrected in 35\% of the subjects, they also showed higher level of hsCRP and very high Hepcidin level than those responded better. This lack of response to iv iron treatment can be due to high level of inflammation in them, which needs to be tackled along with iv iron supplementation.

\section{P-M 21}

Detection of Celiac Disease in Patients of Nutritional Anemia and There Complete Hematological

Amandeep Singh, Dr. Sudhir Mehta, Dr. Sahdev Patel, Dr Bhawani Shankar Sharma

\section{SMS Medical College}

Objectives: To find out the proportion of celiac disease among patients diagnosed with nutritional anemia and also to describe the complete hematological manifestation of celiac disease presenting with anemia. Material and methods: A hospital based prospective and observational, descriptive study involving 150 patients with nutritional anemia taken on first come first serve basis after applying inclusion and exclusion criteria. In all the subjects, other than routine investigations, IgA anti-tissue transglutaminase (IgA anti-tTG) antibody by ELISA was done, upper GI endoscopic guided duodenal biopsy was taken and assessed. Results: 16 out of 150 subjects had celiac disease. Among 16 cases, 9 had iron deficiency anemia, 2 had Vit-B12 deficiency, 2 had iron + B12 deficiency, 1 had iron + folate deficiency, 1had iron $+\mathrm{B} 12+$ folate deficiency, 1 had B12 + folate deficiency. Mean duration of Symptoms was higher in Celiac disease group (11.06 months) as compared to Non celiac group (5.52 months) which 
was significant. History of diarrhoea was present in only one case of celiac disease. $\mathrm{H} / \mathrm{O}$ blood transfusion was found in significantly more number of subjects in Celiac group (43.8\%) as compared to non celiac group $(8.2 \%) 8$ patients who had serum IgA anti tTG $>100 \mathrm{AU} / \mathrm{ml}$ had stage 3 of Marsh classification on histology. The mean of CBC parameters between celiac and non celiac group revealed no significant difference. Conclusion: Screening for celiac disease should be considered in patients having unexplained long-standing and non responsive nutritional anemia, especially iron deficiency anemia, even if they do not have any typical gastrointestinal symptoms. Patients with celiac disease and anemia have a younger age of presentation, higher frequency of blood transfusions and longer duration of symptoms.

\section{P-MS 01}

\section{5 q Deletion Myelodysplastic Syndrome Among Men, Commoner} Than Expected

Objective: The objective is to assess the 5 q deletion in Myelodysplastic Syndrome among men in a tertiary care hospital. Materials and Methods: Cases of $5 \mathrm{q}$ deletions among men diagnosed to have Myelodysplastic Syndrome in a span of 2 years. Introduction: Myelodysplastic Syndrome (MDS) is a hematopoietic stem cell disorder primarily affecting CD34+ cells, characterized by ineffective hematopoiesis, often transforming into acute Myelogenous Leukaemia (AML). Deletion of part of the long arm of chromosome 5 is the most common structural chromosome aberration. Most patients documented are women. Among cytogenetic abnormalities, partial/complete deletion of the long arm of chromosome 5 is the most commonly found in MDS, $10-15 \%$ of all de novo cases and up to $30 \%$ with abnormal karyotype. Isolated del $(5 q)$ is associated with good prognosis in primary MDS, proper recognition of this chromosomal abnormality is of fundamental importance. Results: In a single institutional study which included 10 diagnosed cases of 5 q deletion Myelodysplastic Syndrome, Out of which two men were found to have $5 \mathrm{q}$ deletion whereas only one woman was found positive for $5 \mathrm{q}$ deletion. Conclusions: The molecular basis for the $5 \mathrm{q}$ deletion Syndrome has been the subject of extensive investigation for decades. Patients with the typical $5 \mathrm{q}$ deletion syndrome have a relatively good prognosis with a low risk of Leukaemic evolution. As the incidence of MDS is on a constant rise, the presence of $5 \mathrm{q}$ deletion has to be essentially considered.

\section{P-MS 02}

Two Cases of Myelodysplastic Syndrome with Ring Sideroblasts (MDS-RS)

\section{Sampath KS ${ }^{\#}$, Vinu KV, Nabeel A, Venkatesan S, Sharma S}

Email ID for Correspondence: dr.sampathks@gmail.com Armed Forces Medical College

Introduction: MDS-RS and single lineage dysplasia (MDS-RS-SLD) and MDS-RS and multilineage dysplasia (MDS-RS-MLD) are two subtypes of MDS-RS. They have overlapping clinico-pathological profile. Morphologically, the only differentiating feature between these two subtypes is the number of hematopoietic cell lineages exhibiting dysplasia. MDS-RS-SLD shows dysplasia in one of the myeloid lineage while MDS-RS-MLD shows dysplasia in two or more lineages. Both are characterised by anemia or other cytopenias with bone marrow (BM) ringed sideroblasts count $\geq 15 \%$ and myeloblast count $<5 \%$, without any circulating blasts. The secondary causes of RS need to be excluded before making these diagnoses.
Objectives: To document the clinico-pathological profile of two cases of MDS-RS. Material and Methods: One case of MDS-RSSLD another case of MDS-RS-MLD studied to compare the clinicopathological profile. Results: (a) Case 1 (MDS-RS and SLD): 73 year lady, presented with anemia requiring transfusion. $\mathrm{Hb}: 7.3$ gm/dL, Peripheral smear: Dimorphic picture composed of macrocytes admixed with normocytic normochromic cells, Absolute neutrophil count (ANC): 2,805/cumm, Platelets: 4, 93,000/cumm. Bone marrow aspirates (BMA): Erythroid hyperplasia with dyserythropiesis, Normal maturation of other lineages. No increase in blasts seen. Perl's stain showed increased iron stores with $>15 \%$ ringed sideroblasts. Trephine biopsy showed hypercellular BM with erythroid hyperplasia and dyserythropoiesis and no increase in blasts and normal reticulin pattern. (b) Case 2 (MDS-RS-MLD): 76 year man, presented with anemia requiring transfusion. $\mathrm{Hb}$ : $7.1 \mathrm{gm} / \mathrm{dL}$, Peripheral smear: Dimorphic picture composed of macrocytes admixed with normocytic normochromic cells, ANC: 6,472/cumm, Platelets- 3, 15,000/cumm. BMA: Erythroid hyperplasia with dyserythropoiesis, myeloid series showed hypogranular forms. No increase in blasts seen. Perl's stain showed increased iron stores with $>15 \%$ ringed sideroblasts. Trephine biopsy showed erythroid hyperplasia with dyserythropiesis and megakaryocyte dysplasia and no increase in blasts and normal reticulin pattern. Conclusions: Morphological distinction between MDSRS-SLD and MDS-RS-MLD is critical as they have distinct disease progression and outcome profile.

\section{P-MS 03}

Bonemarrow in Myelodysplastic Syndrome - A Study of 40 Cases

Dr. Priyanka D. Dhende ${ }^{\#}$, Dr. Manoj Kumar, Dr. Meenakshi Balasubramanian, Dr. Vrinda Kulkarni, Dr. Vikas Kavishwar

Email ID for Correspondence: priyankadhende3071991@gmail.com Department of Pathology, Topiwala National Medical College and B.Y.L Nair Hospital, Mumbai

\section{Objectives:}

1. To study morphological changes in bone marrow in aspirates and trephine biopsy in the background of cytogenetics study.

2. To estimate those morphological features that could be present in the eve of a leukemic translocation.

Materials And Methods:

1. Five years Retrospective study.

2. Age, sex, chief complaints, investigative findings, features on aspirate, biopsy and cytogenetic findings were recorded.

Results: Myelodysplastic syndrome was found to be more common in elderly, in males and weakness and breathlessness were the main complaints. Anemia, presence of nRBCs, macrocytes, pseudo-pelgher huet anomaly was seen in peripheral blood smear. Hypercellularity is seen in trephine biopsy and aspirates. Megakaryocyte monolobation was a frequent finding. Hypogranularity was the prominent feature in myeloid series. Erythroid hyperplasia with dyserythropoisis was a common finding. Cytogenetic s was positive in $60 \%$ cases. 10 cases transformed into acute myeloid leukemia during the course of the study. Careful examination of peripheral smear for blasts and aspirate for blasts count more than $5 \%$ and dyserythropoisis were the parameters to pick up patients undergoing leukemic translocation. Conclusion: Careful examination of megakaryocyte monolobation, micromegakaryoctes, hypogranular myeloid cells with left shift, blast count, erythroid hyperplasia and dyserythroposis all were suggestive 
of myelodysplastic syndrome in cases that were negative on cytogenetics.

Keywords micromegakaryocytes, megakaryocyte monolobation, dyserythopoisis

\section{P-MS 04}

Utility of 5-Methylcytosine Immunohistochemical Staining to Assess Global DNA Methylation and Its Prognostic Impact in MDS Patients

Dinesh Chandra\#, Seema Tyagi, Jasdeep Singh, Roopam Deka, Pravas Mishra, Hara Prasad Pati, Renu Saxena

Email ID for Correspondence: dinesh3224@gmail.com

Department of Hematology, All India Institute of Medical Sciences, New Delhi, India, 110029

Introduction: Myelodysplastic syndrome (MDS) is a heterogeneous group of clonal hematopoietic stem cell (HSC) disorders. DNA methylation plays a vital role in pathogenesis of MDS, and is an independent prognostic factor affecting overall survival. Objectives: Our aim was to analyze the role of global DNA methylation using an anti5-methylcytocine (5-MC) antibody by immunohistochemistry (IHC) on bone marrow biopsy in MDS patients and its correlation with various clinical and biological prognostic factors in MDS. Material and Methods: A total of 59 MDS cases were evaluated over a period of 4 years. Clinical data were retrieved from the departmental case records. All these cases were classified as per World Health Organization (WHO) 2008 classification of MDS. Anti 5-MC expression was analyzed on formalin fixed paraffin embedded sections of bone marrow biopsy (BM Bx) of MDS patients and compared with normal BM Bx. Results: The median age at diagnosis was 52 years (15-85 years). Patients were categorized into low risk (59\%) and high risk (41\%) according to International Prognostic Scoring System (IPSS). The median follow-up time was 10 months (1 to 37 months). We compared methylation score (M-score) between the high-risk and low-risk MDS, and the difference between two groups was statistically significantly $(\mathrm{p}<0.001)$. The $\mathrm{M}$-score was significant on univariate analysis for overall survival $(\mathrm{OS})(\mathrm{p}=0.002)$ and progression free survival (PFS) $(\mathrm{p}=0.03)$ but not in multivariate analysis. Bone marrow blast percentage $(\mathrm{p}=0.009)$ and progression to AML were found to be significant independent prognostic factor for OS. Conclusions: Immunostaining with anti 5-MC antibody on bone marrow biopsy is a simple and cost effective technique to detect global methylation. The global methylation may be used as a powerful tool to predict overall survival in patients with MDS.

Keyword DNA methylation, 5-methylcytosine, Myelodysplastic syndrome

\section{P-MS 05}

\section{? Refractory Anaemia with Ringed Sideroblasts with Thrombocytosis (MDS/MPN-RS-T): A Rare Case Report}

\section{Dr. Meenu Angi" ${ }^{\#}$ Dr. Atoshi Basu, Dr. Soumya Bhattacharya}

Email ID for Correspondence: dr.meenuangi@gmail.com Apollo Gleneagles Hospital, Kolkata

Introduction: RARS-T was a provisional entity in WHO 2008. It has now been accepted as a full entity termed MDS/MPN with ring sideroblasts and thrombocytosis in the recommended 2016 revision of WHO. Diagnostic criteria include anemia associated with erythroid lineage dysplasia with or without multilineage dysplasia, $>15 \%$ ring sideroblasts (even if SF3B1 mutation is detected), $<1 \%$ blasts in $\mathrm{PB}$ and, $<5 \%$ blasts in the $\mathrm{BM}$ and Persistent thrombocytosis with platelet count $\geq 450 \times 10^{9} / \mathrm{L}$ (1). Objectives: We report a rare case of ? RARS-T with $10 \%$ ring sideroblasts but all other features of this rare entity. Material and Methods: A 71 yrs old hypertensive, non-diabetic male patient presented with the complaints of weakness, weight loss, anorexia and dizziness. Blood counts on admission was $\mathrm{Hb}-9.1 \mathrm{~g} / \mathrm{dl}$, TLC - 21360/cumm, Platelet count - 12.39 lacs/cumm. Serum uric Acid - 8.8 and other biochemical parameters were within normal limits. After initial evaluation of patient, bone marrow was done which was hypercellular with marked megakaryocyte hyperplasia and MDS/ MPN like features. Bone marrow showed excess of Iron stores, grade $6 / 6$ with $10 \%$ Ring sideroblasts. The samples were negative for JAK $2 \mathrm{~V} 617 \mathrm{~F}, \mathrm{BCR} / \mathrm{ABL} 1$ and failed culture on karyotype (done at outsourced laboratory). Results: Based on the bone marrow and CBC findings. a provisional diagnosis of ?RARS$\mathrm{T}$ was made. Patient was started on Hydroxyurea. Subsequently, patient started having melaena, had multiple superficial ileal ulcers on endoscopy and was given treatment for that. Platelet counts came down with treatment but patient required transfusion support off and on for low haemoglobin. After one year of treatment as CBC parameters continued to be deranged, $\mathrm{Hb}: 6.9 \mathrm{~g} / \mathrm{dl}$, TLC: 13280/ cumm, Plt: 16.45 lacs/cumm, Darbepopoetin and Ecopsrin $75 \mathrm{mg}$ were added to the regimen. As patient had compliance issues and platelet count again increased, Azacytidine and Inj PegInterferon Alpha were added. Post treatment CBC was $\mathrm{Hb}: 5.1 \mathrm{~g} /$ dl, TLC: $3400 /$ cumm, Plt: 3.24 lacs/ cumm but within a month of this treatment platelet count again increased to 25.5 lacs/ cumm. Patient was revaluated for molecular markers and was found to JAK2 V617F positive this time. Meanwhile patient developed pancytopenia and bone marrow aspiration showed marrow hypoplasia with necrosis and marrow fibrosis. Patient's counts did not improve and patient expired. Conclusions: We report a rare case of ? RARS-T with $10 \%$ ring sideroblasts, BCR/ABL1 negative, failed culture on karyotype and JAK2-V617F positive. SF3B1 mutation was not tested in this patient.

\section{References:}

Arber DA, Orazi A, Hasserjian R, et al. The 2016 revision to the World Health Organization classification of myeloid neoplasms and acute leukemia. Blood. 2016;127:2391-2405

\section{P-MS 06}

Mast Cell Immunophenotyping and KIT Gene Exon 17 Mutation Studies in Cases of Mast Cell Hyperplasia Associated with Hematological Neoplasms

Sweta Rajpal ${ }^{\#}$, Man Updesh Singh Sachdeva, Sreejesh Sreedharanunni, Praveen Bose, Anu Aggarwal, Reena Das, Pankaj Malhotra, Richa Jain, Neelam Varma

Email ID for Correspondence: swetarajpal12@gmail.com Postgraduate Institute of Medical Education and Research

Introduction: Systemic mastocytosis with an associated hematological neoplasm (SM-AHN) is rare disease diagnosed when the criteria for both systemic mastocytosis and the hematological neoplasm are met. The treatment of SM-AHN requires therapy for both components. The frequency and molecular profile of SM-AHN is unknown in India. Objectives: To screen cases of hematological neoplasms for presence of mast cell hyperplasia and subject such cases to 
immunophenotyping and KIT gene exon 17 sequencing. Material and Methods: This was prospective study from January-June 2017. Cases of hematological neoplasm having mast cell hyperplasia were subjected to immunophenotyping of mast cells (MC), sorting of MC (in two cases) and KIT gene exon 17 sequencing. Pre-titrated antibody cocktail comprising of CD2FITC, CD25PE, CD34PerCPCy5.5, CD117PECy7, CD33APC, and CD45APCH7 was used to characterize MC. Flow-assisted sorting of MC on basis of very bright CD117 positivity was performed in case 1 and 2 . Sorted MC (case $1 \& 2$ ) and bone marrow sample in case $3 \& 4$ were subjected to DNA extraction using QIAmp DNA Blood Mini kit (Qiagen). Extracted gDNA was used as template in PCR for amplification of Exon 17 of KIT by using primer pair 5'TGTATTCACAGAGACTTGGC3' (forward) and 5' GGATTTACATTATGAAAATCAC3' (reverse). Purified PCR product was sequenced on automated sequencer (ABI 3130 Genetic Analyzer; Applied Biosystems). Sequencing data was analyzed for mutation analysis using SEQUENCHER software. Immunohistochemical stain for tryptase on trephine biopsy section was also done. Results: There were four cases of MC hyperplasia associated with hematological neoplasms. The mean age was 31 years (range: 658 years). Three patients were females. The commonest clinical presentation was weakness $(n=3)$. Skin lesions were seen in two patients. Organomegaly was seen in two patients. The hematological neoplasm were AML $t(8 ; 21)$ in two cases, T-lineage ALL and chronic eosinophilic leukemia. The mean mast cell percentage in bone marrow aspirate was $12.5 \%$ (range- 3-30\%). Only two cases showed immunophenotypic aberrancy of mast cells (CD25 and/or CD2 positive). All four cases had no mutation in exon 17 of KIT gene. Conclusions: Two out of four cases showed CD25 and/or CD2 expression, while none had KIT gene-exon 17 mutation. It is possible that the mutation spectrum of cases of SM-AHN may be different in India. More number and whole gene sequencing is needed to characterize such cases.

\section{P-MN 01}

Platelet Aggregation Study in Eighty Cases of Chronic Myeloid Leukaemia. A Tertiary Care Centre Experience

Zaw Htet Min", Venkatesan S, Arijit S, Sharma S, Bharadwaj R

Email ID for Correspondence: hminzaw178@gmail.com Armed Forces Medical College, Pune

Introduction: Chronic myeloid leukemia (CML) is one of the chronic myeloproliferative disorders. It is a clonal stem cell disorder characterized by the attainment of an oncogenic BCR/ABL fusion protein, usually the result of a reciprocal translocation $(9 ; 22)$. There may be abnormal in platelet aggregation functions in CML patients. Objectives: To carry out platelet aggregation tests in cases of CML and to identify the presence of platelet function defects. Material and Methods: Clinical and laboratory and data of all cases of CML, which were diagnosed and managed at a tertiary centre, from June 2015 to May 2017 were analyzed. Patient's platelet rich plasma was subjected to platelet aggregation tests with Chronolog Dual Channel Platelet Aggregometer. Platelet aggregation study was carried out with Arachidonic acid, Epinephrine, ADP and Ristocetin. Results: Among total eighty cases of CML patients, maximum number of patients were in the age group of 31 to 40 year and mean age was 41 year, with 66 cases being males. $20 \%$ of cases presented with fatigue, $95 \%$ of cases presented with splenomegaly and one presented with bleeding manifestation in the form of epistaxis and petechial haemorrhages. Three cases showed aggregation defects with
Arachidonic acid, five cases showed aggregation defects with Epinephrine, two cases showed aggregation defects with ADP and one case showed aggregation defect with Ristocetin. Conclusions: Platelet aggregation abnormalities are not specific for MPD, however it is observed in cases of CML. Doing this investigation in these cases will add value to the comprehensive management of CML cases.

\section{P-MN 02}

Influence of JAK2V617F Allele Burden on Clinical Phenotype of Polycythemia Vera Patients: AIIMS Experience

Sudha Sazawal ${ }^{\#}$, Kanwaljeet Singh, Sunita Chikkara, Dinesh Kumar, Rekha Chaubey, Manoranjan Mahapatra, Renu Saxena

Email ID for Correspondence: sudha_sazawal@hotmail.com Departments of Hematology, AIIMS New Delhi, India (Pin code110029)

Introduction: Elevated JAK2V617F allele burden is associated with enhanced expression of downstream target genes in Philadelphia chromosome-negative chronic myeloproliferative neoplasms (CMPNs) which include PV, ET \&PMF. Worldwide, various studies have shown the impact of JAK2V617F allele burden on clinical phenotype of CMPNs. Studies from India including our previous study, have reported prevalence of JAK2 V617F mutation in CMPNs and correlated it with the clinico-hematologic characteristics. However, there is no data regarding the association between JAK2V617F allele burden and clinical phenotype in Indian polycythemia vera (PV) patients. Objectives: We aimed to investigate the effect of allele burden on clinical phenotype in a study group of JAK2V617F positive PV patients and its influence on disease related complications. Material and Methods: Blood samples were obtained from a total of 90 PV patients with JAK2V617F positivity. Allele burden was quantified by Real-time polymerase chain reaction (RQ-PCR). Results: Of the 90 patients, $74(82.22 \%)$ were males and $16(17.78 \%)$ were females (median45 years, range 35-78). Patients with age $>50$ years had significantly higher JAK2V617F allele burden (median $40.15 \%$, range $0.49-91.62 \%$ ) when compared to patients with age $\leq 50$ years (median $48.59 \%$, range $0.56-86.74 \%$; $\mathrm{p}<0.032$ ). Patients with splenomegaly had significantly higher JAK2V617F allele burden (mean $50.24 \%$, range $6.91-84.17 \%$ ) as compared to patients without splenomegaly (mean $33.82 \%$, range $0.49-71.83 \%$; $p<0.017$ ). Patients with higher allele burden (median 57.20, range 43.4-72.03\%) had significantly raised thrombotic events as compared to patients with lower allele burden (median 37.38, range 0.49-84.17\%; $\mathrm{p}<0.043$ ). Out of 90 patients, $49(54 \%)$ were homozygous and $41(46 \%)$ were heterozygous by ASO-PCR. 32/49 (66\%) homozygous patients had allele burden $>50 \%$ by RQ-PCR, however $17 / 49$ (34\%) patients had allele burden $<50 \%$ by RQ-PCR. Conclusions: In PV patients, higher JAK2V617F allele burden has been associated with increasing age, splenomegaly and thrombotic events. The inclusion of JAK2V617Fallele burden by RQ-PCR may be considered for prognostication and setting up the treatment protocol in these patients.

\section{P-MN 03}

Comparative Analysis of the Sokal, Hasford, and EUTOS Scores in Prognostication and Response to Imatinib Therapy in Chronic Phase Chronic Myeloid Leukemia Patients: AIIMS Experience 
Sunita Chhikara ${ }^{\#}$, Sudha Sazawal, Rekha Chaubey, Kanwaljeet Singh, Rahul Sharma Tulika Seth, Manoranjan Mahapatra, Renu Saxena

Email ID for Correspondence: sunita1409@gmail.com Departments of Hematology, AIIMS New Delhi, India (Pin code110029)

Introduction: Over the years, many prognostic models have been developed for risk stratification in CML. Sokal, Euro, and EUTOS scores were developed to predict the outcome of patients on TKIs. Objectives: To perform a comparative assessment of the Sokal, Euro and EUTOS scoring system in the Indian CML patients receiving front-line imatinib mesylate. Material and Methods: This was a retrospective study performed in $260 \mathrm{Ph}+\mathrm{CML}-\mathrm{CP}$ patients attending the Hematology Out Patient Department (OPD) of the AIIMS, New Delhi from July 2010 to July 2014. Results: Out of 260 patients, 166 were male and 94 female (M:F::1.6:1) with median age 35 years (range 20-70 years). The median follow-up period of the surviving patients was 38 months (range, 2-48 months).Using Sokal score, 92 (35.38\%), 83 (31.92\%) and $106(40.76 \%)$ patients were divided into low, intermediate and high risk score. As per the Euro score, 102 $(39.23 \%), 106(40.76 \%)$ and $52(20 \%)$ patients were discriminated into low, intermediate and high risk score. According to the EUTOS score, $210(80.7 \%)$ patients were categorized as low risk, while 50 $(19.2 \%)$ patients had high risk score group. According to Sokal score, CI of MMR was $90 \%, 86 \%$ and $73 \%$ for low, intermediate and highrisk score $(\mathrm{p}=0.016)$. As per Euro score, $\mathrm{CI}$ of MMR was $92 \%, 86 \%$ and $69 \%$ for low, intermediate and high-risk groups $(p=0.001)$. CI of MMR for low and high risk group as with EUTOS score was $80 \%$ and $81 \%(\mathrm{p}=0.764)$. Both EFS and OS was found to be significantly correlated with Sokal score $(\mathrm{p}=0.004, \mathrm{p}=0.007)$ and Euro score $(\mathrm{p}=0.009, \mathrm{p}=0.001)$, however no correlation was observed with EUTOS score $(\mathrm{p}=0.581, \mathrm{p}=0.927)$. Conclusion: Sokal and Euro scores have significant predictive efficacy in the Indian population with CML-CP, however the present study did not validate the effectiveness of the EUTOS score. Further research work in this field may play a pivotal role in identifying the better prognostic scores for the risk stratification in CML patients on TKIs. Both event-free survival (EFS) and median overall survival (OS) with imatinib therapy using Kaplan-Meier analysis was found to be significantly correlated with Sokal score $(p=0.004, p=0.007)$ and Euro score $(p=0.009$, $\mathrm{p}=0.001$ ), however no correlation was observed with Eutos score $(\mathrm{p}=0.581, \mathrm{p}=0.927)$.

\section{P-MN 04}

\section{Status of DNA Double Strand Breaks, Machinery for Their Repair and Chromosomal Aberrations in Imatinib Sensitive and Resistant Cells Representing CML-BC}

Rahul Mahendra Mojidra*1\#, Shiva Shankaran Chettiar ${ }^{2}$, Mythreyi Narasimhan', Aneri Parekh', Alpesh Patel', Devendrasinh Jhala ${ }^{2}$, Raghuram Gorantla ${ }^{1}$, Rukmini Govekar ${ }^{1}$ ( ${ }^{1}$ ACTREC, Navi Mumbai; ${ }^{2}$ Genexplore, Ahemedabad)

Email ID for Correspondence: rmojidra@actrec.gov.in; rahul.m2212@gmail.com

Advanced Centre for Treatment, Research and Education in Cancer (ACTREC)

Introduction: Chronic myelogenous leukemia (CML) is a haematological malignancy, marked by the presence of Philadelphia chromosome which is formed due to an unequal reciprocal translocation $t$ $(9 ; 22)(\mathrm{q} 34 ; \mathrm{q} 11)$. The resultant fusion gene BCR-ABL is a constitutively active tyrosine kinase which is causally associated with induction of Chronic Phase (CP) of CML in experimental systems. Inhibition of BCR-ABL with imatinib, a tyrosine kinase inhibitor (TKI) has been the most successful targeted therapy which brings about remission of disease in $90 \%$ patients in CP. However resistance to TKIs occurs during the course of treatment and patients with resistance to TKIs progress to the acute phase called Blast Crisis (BC) wherein the survival is restricted to 4-5 months. In BC, whether de novo or due to progression of TKI resistant $\mathrm{CP}$, pathways other than $\mathrm{BCR} / \mathrm{ABL}$ drive disease progression. Thus majority of patient in $\mathrm{BC}$ are unresponsive to TKIs which raises the need to identify alternate therapeutic target for treatment of TKI resistant CP and BC patients. Objectives: In majority of the leukemias including CML, the driver molecular alterations result from Chromosomal Aberrations (CAs). Thus generation of a profile of CAs in different phases of CML would assist in identification of therapeutic targets for TKI resistant $\mathrm{CP}$ and $\mathrm{BC}$ patients. Towards this objective, the present study generates the profile of CAs in Imatinib resistant and sensitive cell lines representing $\mathrm{BC}$ of $\mathrm{CML}$, and attempts to understand its relation to the proneness of cells to formation of Double Strand Breaks (DSB), and status of DSB repair machinery. Material and Methods: Array CGH was carried out on Agilent SurePrint G3 array, $8 \times 60 \mathrm{~K}$. ADM-2 algorithm of Agilent's cytogenomic software was used to identify the CAs such as deletion, duplication and copy number variations (CNVs) in different CML cell lines. Status of DNA damage was screened by comet assay and immuno-fluorescence staining of $\gamma$ H2AX, marker of DNA damage. Status of repair machinery was analysed by western blotting for different proteins involved NHEJ and HR pathways. Results: Increase in DSB generation has been observed in the imatinib-resistant cell lines upon treatment with mitoxentrone (topoisomerase II inhibitor) in comparison to sensitive cells. Also alterations in expression pattern and phosphorylation status of NHEJ and HR proteins indicate that the DNA repair activity is affected in resistant cell lines. These factors can contribute to accumulation of CAs during the course of disease progression. The profile of differential CAs across different CML cell lines has been generated through Array CGH. Further, CAs specifically present in resistant cell lines when compared to sensitive counterpart has been identified. Conclusions: Profile of CAs for different CML cell lines has been generated and differential CAs are identified in resistant cell lines which can be further investigated to assess their translational potential. This study has provided a background for undertaking profiling of immature cells from CML patient samples representing different clinical phases to identify CAs with therapeutic potential.

\section{P-MN 05}

\section{Clinico-Pathological Spectrum of Myeloneoproliferative Neoplasm}

\section{Anita Tahlan", Sapna Yadav, Anshu Palta}

Email ID for Correspondence: anitatahlan@gmail.com Government Medical College \& Hospital, Chandigarh

Introduction: Myeloproliferative neoplasms (MPNs) are clonal haematopoietic stem cell disorders that include Chronic Myelogenous Leukemia, Chronic Neutrophilic Leukemia, Polycythemia Vera, Primary myelofibrosis, Essential Thrombocythemia, Chronic Eosinophilic Leukemia, and MPN, unclassifiable. Objectives: To study the clinical profile, haematological parameters and bone marrow histomorphology in the cases diagnosed as Myeloproliferative neoplasms. Material and Methods: In the present study, 120 cases of myeloproliferative neoplasms diagnosed over a period of 12 years were reviewed. The clinical features including hepatosplenomegaly were 
noted. The haemogrm findings and bone marrow aspiration and trephine findings were analyzed. Results: Out of total 120 cases, 97 were diagnosed as Chronic Myeloid Leukemia (CML), 6 as Polycythemia Vera (PV), 5 as Essential Thrombocythemia (ET), 11 as Primary Myelofibrosis (PMF) and 1 as Chronic Neutrophilic Leukemia (CNL). $53.3 \%$ of the patients in the study were male and $46.7 \%$ were female. The overall male - female ratio was 1.1:1. CML was the most common type of MPN followed by PMF. Fever was the most common clinical presentation $(53.3 \%)$ followed by abdominal symptoms $(34.2 \%)$. Haematological parameters and bone marrow aspiration and trephine biopsy findings were analysed for the various MPNs and compared with previously published studies. Conclusions: MPNs share many biological, clinical and morphological features. The multidisciplinary approach to the diagnosis of MPNs includes the evaluation of a bone marrow biopsy along with cytogenetics and molecular studies. Histopathology is especially crucial to distinguish between MPNs in which the clinical and laboratory data overlap. This analysis can be beneficial to physicians and haematologists in developing countries who do not have easy access to the iagnostic techniques like cytogenetics, RT-PCR or FISH.

\section{P-MN 06}

Blast Crisis at Initial Presentation of Chronic Myelogenous Leukemia - Series of 11 Cases

\section{Praveena $\mathbf{E}^{\#}$, Jamir TS, Sree Rekha J, Jacob SE, Kar R, Basu D}

Email ID for Correspondence: praveenaedura19@gmail.com JIPMER, Pondicherry

Introduction: Chronic Myelogenous Leukemia (CML) generally presents in chronic phase of disease. Blast crisis at presentation is uncommon, accounting for $10 \%$ cases. The blast crisis is usually myeloid in lineage. Lymphoid and megakaryoblastic are also seen, although less frequently. Amongst the lymphoid crisis it is mainly B-lymphoblastic crisis, that has been reported. We report a series of 11 cases of CML in blast crisis at presentation. Objectives: To study the clinico-haematological profile with emphasis on the immunohistochemical faetures of CML in blast crisis at presentation. Material and Methods: It is a retrospective record based study of the last four years. In this period 160 cases of CML were diagnosed in our department. 11 of these cases presented in blast crisis at diagnosis of the disease. Details of clinical and haematological features, bone marrow findings, immunohistochemistry were studied. The panel of markers used were CD 34, CD 117, MPO, CD 14, CD 11c, CD 61, TdT, CD 10, CD 3, CD 20 as and when indicated. Ancillary investigations like flow cytometry, karyotyping and FISH/RT-PCR for BCL-ABR fusion were recorded wherever available. Results: CML presenting in blast crisis constituted $6.8 \%$ of all CML diagnosed in our department. The mean age of presentation was 38 years. The youngest was a 9 year old boy. Eight cases had splenomegaly and one patient presented with generalised lymphadenopathy. There were 9 males and 2 females. Hemoglobin ranged from $4.5 \mathrm{gm} / \mathrm{dl}$ to $11.7 \mathrm{gm} /$ dl with a mean of $7.8 \mathrm{gm} / \mathrm{dl}$. TLC ranged from $2 \times 10^{9} / 1$ to $500 \times 10^{9} / 1$ with a mean count of $54.7 \times 10^{9} / 1$. Platelet count was normal in five cases, two showed moderate thrombocytopenia and one showed thrombocytosis. Blasts in peripheral blood ranged from $7 \%$ to $46 \%$. In five cases, peripheral blood was suggestive of blast crisis in CML. Bone marrow aspirates were particulate in only six cases. Blasts were more than $20 \%$ in eight cases. Three cases where aspirate was paucicellular with low number of blasts showed clusters of blasts in bone marrow biopsy. Six cases showed Grade 2 or 3 fibrosis. Immunohistochemistry revealed that six of the crises were myeloid in lineage with blasts being positive for CD 34, CD 117 and MPO, two cases were megakaryoblastic, including the nine year old child. One case was myelomonocytic while another was mixed myeloid, megakaryocytic and erythroid. There was a single case of extramedullary blast crisis in lymph node and that was T-lymphoblastic. All patients had either 9; 22 translocation or BCR-ABL gene p210 fusion. Additional cytogenetic abnormality was not detected in the karyotyping done in four cases. Flow cytometry was available in three cases; it correlated in two cases and was inadequate in one. Conclusions: Blast crisis at presentation in CML is uncommon and was seen in $6.8 \%$ of our cases. In our series, two rare entities of a nodal $\mathrm{T}$-Lymphoblastic crisis and a paediatric megakaryoblastic crisis were encountered.

\section{P-MN 07}

\section{Standarization and Validation of Bcrabl Fusion Transcript by Droplet Digital PCR}

Neeraj arora, Pragya gupta, kallol saha, Saurav Sharma chowdhury, mammen chandy, Deepak mishra

Email ID for Correspondence: poonam.santra@tmckolkata.com; Poonam Santra (Presenting Authors)

Tata Medical Center

Introduction: CML is caused by the formation of the Philadelphia $(\mathrm{Ph})$ chromosome, a reciprocal translocation between chromosomes 9 and 22, resulting in the formation of the BCR-ABL1 fusion gene. Quantitation of BCR-ABL1 transcript levels using qRT-PCR has become the standard-of-care protocol in CML molecular monitoring. For the current challenges of developing a efficient method which facilitate fast, inexpensive and sensitive BCR ABL1 quantification, Droplet digital PCR is an highly sensitive technique which has the potential to monitor minimal residual disease in patients with BCR-ABL positive leukemias. Objectives: To validate the sensitivity and specificity of ddPCR and to find its concordance with qRT PCR results in detecting MRD in CML patients. Material and Methods: The study includes $51 \mathrm{CML}$ cases who were either at diagnosis or undergoing TKI therapy. The patients includes low $(<0.1)$, intermediate (0.1-10) and high $(>10)$ range groups level of their BCR ABL/ABL ratio after analyzing them on ABI 7500. All of these cases were further compared by Droplet Digital PCR platform (QX200). For detection of false positivity and generate the negative threshold in BCR ABL assay by Digital droplet PCR, 16 samples of PML-RARA were run in duplicates. Results: The median copy no of BCR-ABL and $\mathrm{ABL}$ was 1005.53 (range 0 -17220) and 22768.3 (range 739-139000) by dPCR and 9005.19 (range 0- 63021.91) and 56960 (range $12316.48-125554.3$ ) by qPCR, respectively. Out of 51 cases 29 patients were in lower range, 15 patients were, intermediate range and 7 patients were in higher range. Both methods detected similar numbers of BCR-ABL+ samples only there was a discordance found to be between the values of both the techniques in almost all cases in higher range group. In intermediate group values were concordant only one case was found in which more than one log difference were present in both the techniques. In low range group, the distribution in MR classes was different between the methods, with dPCR indicating decreased cumulative rates of MR4 and MR4.5. Significantly fewer pts achieved MR4 and MR4.5 when analyzed by dPCR in comparison to RQ PCR ( $n=8$ vs. 11$)(n=1$ vs $n=2)$ respectively. On examining 16 samples of PML RARA cases in duplicates, 11 wells $(n=32)$ showed presence of positive droplets ranging from 1 to 4 which determined the range of 4 droplets or less to be considered as false positive. Conclusions: RT-dPCR can perform absolute 
quantification by counting cDNA molecules and offers high precision and accuracy without the requirement of a standard curve which makes it a simpler method. However, to further improve assay sensitivity and measure MR4.0 and MR4.5 with confidence, changes to the upstream processing are required.

\section{P-MN 08}

\section{Common BCR-ABL Fusion Transcripts and Their Impact on Outcome in Imatinib Treated Adult CML Patients in India: AIIMS Experience}

\section{Sudha Sazawal ${ }^{\#}$, Sunita Chikkara, Kanwaljeet Singh, Rekha} Chaubey, Manoranjan Mahapatra, Tulika Seth, Renu Saxena

Email ID for Correspondence: sudha_sazawal@hotmail.com Departments of Hematology, AIIMS New Delhi, India (Pin code110029)

Introduction: Chronic myelogenous leukaemia (CML) is the commonest leukaemia in Asia and is characterised by the presence of $\mathrm{Ph}$ chromosome/t(9;22). The most common breakpoint cluster region in chronic myeloid leukemia (CML) is b2a2 and b3a2. The impact of the type of transcript on response to therapy is largely controversial. Data from India is sparse regarding the distribution of various BCRABL fusion transcripts and response to Tyrosine Kinase Inhibitor therapy (TKI) is sparse. Objectives: To look for the fusion transcripts in CML patients and to assess their impact on outcome in patients treated with TKIs. Material and Methods: CML patients diagnosed at the Department of Hematology, AIIMS, New Delhi, between 2014 to 2016 were the study subjects. Diagnosis was done by complete hemogram and peripheral blood smear. This was confirmed by RT-PCR for BCR-ABL fusion transcript followed by 3 monthly real time PCR for the molecular response. All the patients were started on Imatinib (dose $400-800 \mathrm{mg}$ /day). Results: Of the 200 patients, $146(73 \%)$ were males and $54(27 \%)$ were females (median 37, range 18-70 years). CML-CP was diagnosed in 190 (95\%), CML-AP in 10 (5\%) patients and progression from CP to AP was seen in 6 out of 190 CML-CP patients. The overall frequency of b3a2 transcripts was observed in $72 \%$ patients followed by $\mathrm{b} 2 \mathrm{a} 2$ in $25 \%$ and $\mathrm{b} 3 \mathrm{a} 2+\mathrm{b} 2 \mathrm{a} 2$ in $3 \%$ cases. When the clinical and hematological parameters were assessed in b3a2 and b2a2 transcripts, it was observed that major molecular response (MMR) was attained in $60 \%$ patients with b3a2 transcript as compared to $40 \%$ patients with b2a 2 and the difference was statistically significant $(\mathrm{p}<0.05)$. Other parameters such as age, gender, $\mathrm{Hb}$, TLC and platelet were not significantly different among the fusion transcripts. Patients who showed poor response to Imatinib were negative for Tyrosine Kinase domain mutations. Conclusion: The frequency of b3a2 transcript was more as compared to b2a2 transcript. MMR was significantly higher in patients with b3a2 transcript as compared to patients with $\mathrm{b} 2 \mathrm{a} 2$ transcript.

\section{P-MN 09}

Chronic Myeloid Leukemia in Known Case Of Carcinoma Breast Dr. Prasad Chaudhari, Dr. Deepak Ghosh, Dr. Mangal Pandure

Rural Medical College, Pravara Institute of Medical Sciences (DU), Loni, Maharashtra
Introduction: There is a $1-5 \%$ lifetime risk of developing therapyrelated myeloid neoplasms (t-MN) after breast cancer treatment. The most common types of t-MN in the World Health Organization classification include AML, MDS and Myelodysplastic/ Myeloproliferative neoplasms. Case Report: A 48 years old female, a known case of infiltrating ductal carcinoma treated with Modified radical mastectomy, Tamoxifen, 8 cycles of radiotherapy about 8 years back; presented with abdominal pain and massive splenomegaly. She had severe anemia (Hb- $2.3 \mathrm{~g} / \mathrm{dl})$ and a total count of 2 lacs WBC/ cumm. Bone marrow examination confirmed it to be a case of chronic myeloid leukemia. (BCR-ABL positive). Discussion: Leukemia is an unusual event in the natural history of breast cancer, though its association with breast cancer therapy has been reported. Several types of leukemia can be observed such as acute lymphocytic leukemia, acute myeloid leukemia (AML), chronic myeloid leukemia (CML), chronic myelomonocytic leukemia, myelodysplastic syndrome (MDS) and T cell prolymphocytic leukemia [4]. CML is a slow-growing tumour of white blood cells, characterized by an unregulated growth of the myeloid precursor cells and its accumulation in the bone marrow and the lymphoid organs. CML is more frequent in men between 25 and 60 years. These tumours occur as a carcinogenic effect of ionizing radiation and have shown to be occurring with increased frequency among atomic bomb survivors [3]. Conclusion: The presented case and related literature reveals that there is risk of developing secondary leukemia after receiving radiotherapy and/or chemotherapy for breast carcinoma. Secondary chronic myeloid leukemia is a rare presentation in post-therapy carcinoma breast cases. The interval between the adjuvant treatment of breast cancer and CML is 4.7 years and this risk persist over 25 years after breast cancer diagnosis. It is recommended that patient should undergo regular haematological check up after completion of radiotherapy and/or chemotherapy.

\section{References:}

1. Leone G, Fianchi L, Pagano L, Voso MT. Incidence and susceptibility to therapy-related myeloid neoplasms. Chem Biol Interact. 2010;184:39-45.

2. Vardiman JW, Arber DA, Brunning RD, Larson RA, Matutes E, Baumann I, et al. Therapy-related myeloid neoplasms. In: Swerdlow SH, Campo E, Harris NL, Jaffe ES, Pileri SA, Stein $\mathrm{H}$, et al., editors. WHO Classification of Tumours of Haematopoietic and Lymphoid Tissues. 4th ed. Lyon: IARC Press; 2008. pp. 127-129.

3. Preston DL, Kusumi S, Tomonaga M, et al. Cancer incidence in atomic bomb survivors. Part III: leukemia, lymphoma and multiple myeloma, 1950-1987. Radiat Res. 1994;37 suppl 2:S68-97.

4. Singhal M, Raina V, Gupta R, Das P. T cell-prolymphocytic leukemia detected in a patient of breast cancer at the time of recurrence: a case report. Cases J. 2010;7(12):683-92. doi:10.1186/1757-1626-3-4

5. Howard RA, Gilbert ES, Chen BE, Hall P, Storm H, Pukkala E, et al. Leukemia following breast cancer: An international population-based study of 376,825 women. Breast Cancer Res Treat. 2007;105(3):359-68.

\section{P-MN 10}

CML Masquerading As Acute Promyelocytic Leukemia

Vibha Gupta, Lalita Jyotsna P, Shivali Sehgal, Sangeeta Pahuja, Sunita Sharma

Himalayan Institute of Medical Sciences 
Introduction: Chronic myeloid leukemia (CML) is a clonal myeloproliferative disorder of the primitive hematopoietic stem cell and is characterized by the presence of unique translocation, i.e., BCR/ ABL1 known as Philadelphia chromosome. CML is one of the commonest adult leukemia in Indian population. The most common symptom was splenomegaly followed by hepatomegaly, fatigue, weakness, dragging pain, pallor or may be asymptomatic (30\% cases). Rare manifestations include bleeding, thrombosis, gouty arthritis, priapism, retinal hemorrhages, and upper gastrointestinal ulceration and bleeding. Objectives: To highlight an unsual case CML which presented with coagulopathy and imitated Acute Promelocytic Leukemia. Case report: A 45 year-old-male was admitted to the emergency unit with a history of high grade fever and ecchymotic patches all over his body. On examination, he was conscious and oriented, febrile, pale and had ecchymotic patches all over his body. Abdominal examination revealed mild splenomegaly. Investigations revealed hemoglobin $7 \mathrm{~g} / \mathrm{dl}$, TLC-1,63,000/cumm, DLC- showed lef shifed maturation with eosinophilia and basophilia, platelet count- 73,000/ cumm PT- 16.2 and APTT- 43 seconds, TT- 20 seconds and D-dimers were significant. Molecular studies were positive BCR-ABL fusion gene product and negative for PML-RARA and $t(11 ; 17)$ for variant APL. This patient was started on Imatinib therapy and following two weeks the patients TLC decreased and coagulopathy improved. The patient is on molecular remission and on imatinib. Conclusions: CML is known to cause DIC in blast crisis and on therapy. Coagulopathy is a rare presentation in CML, that too in chronic phase. Hyperleukocytosis (white blood cell count, $>100 \times 109 / 1$ ), an uncommon presentation of leukemia, is associated with an increased risk of early mortality. It may present with a variety of symptoms secondary to leukostasis. DIC is a coagulopathy induced by the formation of small clots consuming coagulation proteins and platelets, resulting in disruption of normal coagulation and a severe bleeding tendency. One of the leading theories to explain DIC in this setting is that high leukocyte counts and cell turnover exposed the circulation to very high levels of tissue factor which triggered the extrinsic pathway via Factor VII.

\section{P-MN 11}

Efficacy and Safety of Imatinib in Chronic Myeloid Leukaemia Patients - An Experience from a Tertiary Centre

Phukan A*, Dolai T K, De R, Mandal P K, Baul S, Chakrabarti P

\section{Nil Ratan Sircar Medical College \& Hospital}

Email ID for Correspondence: abhijitphukan47@gmail.com

Introduction: Tyrosine kinase inhibitors (TKIs) have dramatically improved the treatment and survival outcome of chronic myeloid leukemia (CML) patients. Response efficacy of the TKIs are measured in terms of haematological, cytogenetic and molecular response at specified time points. Objectives: To analyse the efficacy and safety profile of CML patients treated with Imatinib. Material and Methods: It was a prospective study conducted over a period of 1 year from $1^{\text {st }}$ Jan 2016 to $31^{\text {st }}$ Dec 2016. A total of 91 newly diagnosed CML-Chronic phase (CML-CP) patients were enrolled in the study and were treated with recommended dose of Imatinib. The response was assessed as per published European leukaemia net (ELN) recommendation 2013. Haematological response assessment was done at 3 months, cytogenetic response at 6 months and molecular response at 3, 6, 9 and 12 months. Dose modification and toxicity monitoring was done as per published recommendations in the literature. Results: The mean age of this cohort was 35.2 years (ranges 10 to 74 years). Male to female ratio was 3.1:1. Haematological response at 3 month was $97.8 \%$ (89/91). One patient
Table 1 Molecular response rate at 3,6 and 12 months of Imatinib therapy

\begin{tabular}{llll}
\hline & Optimal Response & Warning sign & Failure \\
\hline At 3 months & $68.1 \%(64 / 91)$ & $29.7 \%(27 / 91)$ & $2.2 \%(2 / 91)$ \\
At 6 months & $46.1 \%(42 / 91)$ & $37.5 \%(34 / 91)$ & $16.4 \%(15 / 91)$ \\
At 12 months & $51.2 \%(20 / 39)$ & $17.9 \%(7 / 39)$ & $30.8 \%(12 / 39)$ \\
\hline
\end{tabular}

progressed to blast crisis at 5 months of therapy. At 6 month, cytogenetic response assessment was done in 32 patients. Out of 32 patients, 9 patients did not showed any growth in metaphase. Among the rest 23 patients, $69.5 \%(n=16)$ showed complete cytogenetic response (CCyR). Molecular response rate at 3,6 and 12 months of Imatinib therapy are shown in the Table 1 . Most common adverse effect while on Imatinib were oedema (55.9\%), Myalgia (48.3\%), Nausea (41.7\%). Conclusions: Cytogenetic response (CCyR) achieved after 6 months of therapy was $69.5 \%$ and optimal molecular response achieved at 12 month was $51.2 \%$ in this cohort. In comparisons to IRIS trial, there is an inferior response rate of Imatinib in CML CP patients in this study.

\section{P-MN 12}

Megakaryocyte Morphology and Morphometry in Philadelphia Negative Myeloproliferative Neoplasms

\section{Dr. Tanya ${ }^{\#}$, Dr. Debdatta Basu, Dr. Rakhee Kar}

Email ID for Correspondence: gemtanya@gmail.com JIPMER, Puducherry-605006

Introduction: Myeloproliferative neoplasms (MPN) are a group of stem cells disorders characterized by a variable degree of myeloid, erythroid, megakaryocytic hyperplasia. It can be broadly classified into Philadelphia $(\mathrm{Ph})$ positive Chronic myelogenous leukemia and $\mathrm{Ph}$ negative entities which includes Essential thrombocytosis (ET), Polycythemia vera (PV), Primary myelofibrosis (PMF) and others. There is considerable overlap in the clinical, hematological as well as morphological features of the various subtypes of Ph negative MPN. The current WHO classification scheme stresses the importance of bone marrow trephine biopsy especially the morphology of megakaryocytes to distinguish the various subgroups of $\mathrm{Ph}$ negative MPN. Objectives: To assess and compare megakaryocytic morphology and morphometry in $\mathrm{Ph}$ negative MPN. Materials and Methods: All newly diagnosed (based on WHO 2008 criteria) cases of Ph negative MPNs $(\mathrm{n}=22)$ between January 2014 and June 2017 were included, out of which 7 cases were of ET, 7 were of PMF, 8 were of PV. The following parameters were studied in and the bone marrow trephine biopsy:

1. Morphology -Location, type, number of megakaryocyte $/ \mathrm{mm}^{2}$, megakaryocytes in clusters, attached to trabeculae, intrasinusoidal megakaryocytes, megakaryocytes with naked nuclei, and showing emperipolesis along with megakaryocyte atypia and degree of marrow fibrosis.

2. Morphometric analysis was done on a minimum of 10 megakaryocytes in each case by Olympus Microscope using Progress ${ }^{(\mathrm{R})}$ Capture Pro software at $40 \times$ and these parameters were assessed: (a) Cytoplasmic major diameter (CMD), (b) Nuclear major diameter (NMD), (c) Nuclear to cytoplasmic area ratio, (d) Nuclear circularity 
Results: Number of megakaryocytes $/ \mathrm{mm}^{2}$ was found to be significantly high in ET as compared to PMF (8.6 vs 4.13 ; p < 0.05 ). Megakaryocytes in both dense and loose clusters were seen more in ET as compared to PV and PMF. Paratrabecular location of megakaryocytes were more in PMF as compared to ET and PV. One of the case of PMF showed a very high number of intrasinusoidal megakaryocytes $\left(45.3 \mathrm{megs} / \mathrm{mm}^{2}\right.$ ). Megakaryocytes with naked nuclei and with emperipolesis were commonly seen in all the cases. Atypia was seen in 4 out of 7 cases of PMF and non in ET and PV. The degree of fibrosis (using WHO 2008 criteria) was significantly high for PMF ( 7 out of 8 cases showed grade 3 fibrosis). 6 out of 8 cases of PV and 5 out of 7 cases of ET showed grade 1 fibrosis. The mean CMD for PV was 13.02 which was more than both ET and PMF. Hence, the cytoplasmic area was more in PV as compared to ET and PMF. PV showed more of cloud- like nuclei and the NMD for PV was more as compared to ET and PMF. ET showed more of staghorn type of nuclei. The nuclear to cytoplasmic area was more in PV as compared to ET and PMF. Conclusions: Although there was an overlap between the 3 entities, the morphology and morphometry of megakaryocytes can aid in reaching the diagnosis in various $\mathrm{Ph}$ negative MPN. The number of megakaryocytes $/ \mathrm{mm}^{2}$ and clustering was more in ET, large size of megakaryocytes were seen in PV, atypia and fibrosis was more in PMF.

\section{P-MN 13}

A Clinico-Pathological Study on Chronic Myelogenous Leukemia in Neigrihms

Dr Pranoy Paul ${ }^{\#}$, Dr Vandana Raphael, Dr YookarinKhonglah, Dr Bhupen Barman, Dr L. Purnima Devi

Email ID for Correspondence: drpranoypaul @gmail.com North Eastern Indira Gandhi Regional Institute of Health and Medical Sciences

Introduction: Chronic myeloid leukaemia (CML) is the most common leukemia encountered in India. The median age of presentation in CML is around 50 years. It is consistently associated with BCRABL fusion gene (t 9:22) in the Philadelphia ( $\mathrm{Ph})$ chromosome. Since the last decade Tyrosine kinase inhibitors have been the main stay of treatment with complete cytogenetic response achieved in 70-90\% cases. Objectives: To review the clinical presentation, natural history, and pathobiology of CML by studying the cases presenting to NEIGRIHMS and to assess the hematological and cytogenetic response to treatment. Material and Methods: Peripheral blood, bone marrow aspiration and biopsy samples and clinical data collected from 34 consecutive patients of CML over past 2 years were included in this hospital based cross sectional study after obtaining approval from Institute's Ethics Committee. According to PBS and BMA they were categorized into chronic, accelerated and blast crisis cases. FISH was done to detect BCR-ABL gene and assess cytogenetic response. Results: Among the 34 cases, 20 were males and 14 females; 33 were in chronic phase with only one case presenting with blast crises. Mean age of presentation was 34.5 yrs with youngest and oldest patients being 3yrs and 60yrs respectively. Twenty four patients were followed up of which 10 showed CHR within 1 month. Five patients showed progression of disease within a mean period of 22.5 months from diagnosis. Response at the end of 1 month was indicative of progression of disease as only 1 patient who progressed showed CHR (20\%) as opposed to $45 \%$ CHR in patients maintaining at the same stage. Of the 5 patients who progressed, $4(80 \%)$ of them presented with fever compared to only 8 patients (27.5\%) among the rest. FISH was done on 26 patients and cytogenetic response assessed for 8 patients with only one patient showing CCyGR. Conclusions:
Average age of presentation is significantly lower in our study compared to western data. Other findings were consistent with similar studies carried out in India and abroad. After 1 month of therapy with TKI's, significant decrease in blast count, basophil count and TLC was seen. Fever was seen to be associated with worse prognosis. FISH was seen to be not only a good diagnostic tool but also in monitoring treatment.

\section{P-MN 14}

\section{Rare Case of JAK2 Negative Primary Polycythemia Vera}

Dr. Aradhana Deka, Dr. Shaheen Kanpurwala, Dr. Shweta Watane, Dr. Grace d'Costa, Dr. Tousif Mulla

\section{Department of Pathology Grant Govt Medical College and Hospital, Mumbai}

Objective: To review and emphasize importance of modern diagnostic criteria (British Committee for Standards in Hematology (BCSH)) and emerging molecular findings in Polycythemia vera. Method: Case Report

- A $50 \mathrm{yrs} / \mathrm{male}$ admitted with c/o of generalized weakness since two years and erythromalalgia since birth. No H/O hypertension/ smoking/alcohol.

- O/E: Average built with erythromelalgia on hands and cheeks. RS/ CVS/CNS examination- Normal. P/A - mild splenomegaly, confirmed on USG. Lab investigation: $\mathrm{Hb} \% 17.8 \mathrm{gm} \%$, hematocrit $0.53 \%$, TLC 14,740 cells/cumm with neutrophils $79 \%$, and a platelet count of 10.96 lakhs/cumm. Coagulation assays were normal, LFT, RFT and ABG- WNL.

- Bone marrow shows trilineage hyperplasia with erythoid series showing normoblastic maturation.

- Serum Erythropoitin level reduced - $1.53 \mathrm{mIU} / \mathrm{ml} .(\mathrm{N}-2.54$ to 3.54$)$

- Secondary causes of erythrocytosis were ruled out.

- Cytogenetic analysis for

1) JAK2V617F- NEGATIVE

2) JAK2 exon 12- NEGATIVE

3) CALR - NEGATIVE.

- The patient underwent phlebotomy and was put on hydroxyurea. The patient symptomatically responded well to the treatment.

Result: Previously diagnosis was made on WHO (2008) guidelines which included only Jak2 positive mutation cases. JAK2 negative mutation patients BCSH guidelines are used which includes both; hence a diagnosis of Primary Polycythemia vera was made. Treatment: JAK2 mutation in positive patients was more sensitive to hydroxyurea and not to anagrelide, than in those without JAK2 mutation. Conclusion: Thus we emphasize the need of separate modern molecular diagnostic techniques/ diagnostic criteria evaluation and treatment modalities of patients of Polycythemia Vera with JAK2V617F negative mutations (rare occurrence).

Keyword Polycythemia vera, JAK2 gene, BCSH guidelines

\section{P-MN 15}

Chronic Myeloid Leukemia in Juvenile and Adolescents: A Relatively Rare Entity 
Dr Neha Singh", Dr Monika Gupta, Dr Sunita Singh, Dr Nisha Marwah, Dr Rajnish Kalra, Dr Sant Prakash, Dr Sonia Chhabra, Dr Pansi Gupta, Dr Rajiv Sen

Email ID for Correspondence: drnehasingh25@gmail.com Pt B.D.Sharma, PGIMS, Rohtak, Haryana

Introduction: Chronic Myeloid Leukemia (CML) commonly known as a disease of the elderly rarely affects the children or adolescent and accounts for around 3\% of leukemia in this age group. Incidence of CML in this age group merits much attention as outcome and response to standard treatment often differs significantly from the adult group. Objective: The aim of the study was to evaluate the distribution and clinical profile of CML in the children and adolescent age group. Material and Method: It is a retrospective study carried out in the Department of Pathology, Pt B D Sharma, PGIMS, Rohtak over a period of five years between June, 2012 to July, 2017. The demographic data, clinical features, haematological parameters including the bone marrow analysis reports of all the patients were studied. All the patients were diagnosed to have CML based on the presence of BCR ABL transcript (either by Fluorescence Insitu Hybridisation or Reverse Transcriptase Polymerase chain Reaction test). Result: Out of total 95 cases of chronic myeloid leukemia, 14 cases $(14.8 \%)$ were children and adolescents, and 81 cases $(85.2 \%)$ were adults. Among the children and adolescents, 8 cases were males and 6 cases were females (M:F- 1.3:1) with age range between 5 month to 20 years and mean age of 8.24 years. The commonest symptoms were anemia, fever and hepatosplenomegaly. The mean hemoglobin levels, total leukocyte counts, platelet counts and marrow blast frequencies were $7.91 \mathrm{~g} / \mathrm{dl}, 1.19 \mathrm{lac} / \mu \mathrm{l}, 2.07 \mathrm{lac} / \mu \mathrm{l}$ and $8 \%$ respectively. All the patients were diagnosed to have CML chronic phase based on bone marrow aspiration and the diagnosis of CML was confirmed by the presence of BCR ABL transcript. Discussion: The data on the clinical and laboratory parameters of CML in the children and adolescent age group are scanty, due to the rarity of the disease in this age group. Fatigue and abdominal swelling were the predominant symptoms and splenomegaly was the predominant sign in our study which was similar to studies reported in literature. The majority of patients presented in the chronic phase. Conclusion: Our study demonstrated the incidence (14\%) and presenting features of CML in the children and adolescent age group. However; being a single centre study further studies should be undertaken to find out the true incidence of CML in pediatric and young adolescent age groups. As, the response to drugs used according to current protocols, may also differ, the clinicians should be cautious.

\section{P-MN 16}

To Study Morphological Changes and Angiogenesis in Bone Marrow of Patients of Chronic Myeloid Leukemia on Imatinib Therapy

Neetu Pandey", Geeta Yadav, Rashmi Kushwaha, U.S Singh, S.P Verma, Ashutosh Kumar

Email ID for Correspondence: neetu.npandey@gmail.com King George Medical University Lucknow

Introduction: Chronic myeloid leukemia (CML) is a clonal stem cell disorder of hemopoeitic stem cells, characterized by a significant change in the microenvironment of the bone marrow and imatinib is effective in normalizing this microenvironment. Objectives: To document the morphological changes produced by imatinib therapy in the bone marrow of CML patients and to assess and compare angiogenesis pre and post therapy. Material and Methods: 31 patients of CML on imatinib therapy were included in our study. Morphological changes in bone marrow aspirate and biopsy were evaluated at diagnosis and at follow up period of 3 months. Angiogenesis was assessed at diagnosis and at follow up by immunohistochemical staining of $\mathrm{CD} 34$. Hematological and cytogenetic evaluation was also done. Hematological, morphological and cytogenetic response was assessed and correlation between them was calculated by descriptive statistics. Chi-square test was used and $p$ value of $<0.05$ was considered statistically significant. Results: Significant reduction in various hematological parameters were observed post therapy including reduction in TLC ( $\mathrm{p}$ value $<0.001$ ), basophil ( $\mathrm{p}$ value $<0.001)$ and blast percentage $(\mathrm{p}=0.043)$. Complete hematological response was seen in $51.7 \%$ of patients. Bone marrow aspirates and biopsies showed reduction in cellularity and myeloid precursors with regeneration of erythroid precursors. A significant decrease in myelofibrosis (p-value $<0.001$ ) was observed. hypoplasia was noted in $10 \%$ of cases and lymphoid aggregates in $27.6 \%$ of cases. A significant correlation of morphological response with hematological response and cytogenetic response was found ( $\mathrm{p}<0.05$ ). MVD (mean vascular density) of CML biopsies at diagnosis was found to be higher than in controls. A significant reduction of MVD was observed post therapy $(p<0.001)$. Conclusions: Imatinib produces significant effects on bone marrow morphometry and angiogenesis in patients of CML and causes consistent decrease in bone marrow cellularity, reticulin fibrosis and microvessel density.

\section{P-MN 17}

Single Center Analysis of Chronic Myeloid Leukemia Treatment Jeevan Kumar", Reena nair, Vivek S Radhakrishnan, Saurabh Bhave, Sanket shah, Dibakar podder, Neelesh jain, Neeraj Arora, Mayur Parihar, Deepak Mishra, Mammen Chandy

Email ID for Correspondence: grgjvn@gmail.com Tata Medical Center, Kolkata, India

Introduction: Chronic myeloid leukemia (CML) is the most common form of adult leukemia in India. The introduction of Imatinib led to change in the parameters of documenting disease response in CML. Objectives: To analyze the consistency of CML response monitoring and our real world data of treatment response rates. Material and Methods: This is a retrospective data analysis of CML patients who were registered in Tata Medical center, Kolkata from June 2011 to Dec 2015. Diagnosis was confirmed by FISH and/or RT PCR for BCR $\mathrm{ABL}$ on peripheral blood. Response assessments were done by RQ PCR or FISH for BCR-ABL on peripheral blood. Responses were evaluated according to European Leukemia Net (ELN) guidelines. Results: From June 2011 to Dec 2015 a total of 480 CML patients were registered. Out of 480 patients, 272 patients were on regular follow-up and were analyzed. Out of 272 patients $244(89.7 \%), 16$ $(5.9 \%)$ and $12(4.4 \%)$ had CML-CP, CML-AP and CML-BT respectively. Median age of CML CP patients was 40.5 years (6 yrs$81 \mathrm{yrs}$ ) and male to female ratio was 1.83 . The most common presentation was splenomegaly $(82.3 \%)$. According to the Sokal score, $126(51.6 \%), 88(36.1 \%)$ and $30(12.3 \%)$ patients had low risk, intermediate risk and high risk respectively. Imatinib was started as first line treatment in $97.5 \%$ CML-CP patients and $91.8 \%$ of these patients achieved complete hematological response at median duration of 38 days. Early molecular response (BCR-ABL $\leq 10 \%$ at 3 months) was evaluated in $75(30.7 \%)$ patients, out of which $62 \%$ 
patients had achieved early molecular response. At 12 months molecular response was evaluated in $101(41.4 \%)$ patients, out of which $32.6 \%$ patients achieved major molecular response (MMR). MMR at any time (12 months or after) was evaluated in $138(56.5 \%)$ patients, out of which 55.8\% patients achieved MMR. Imatinib failure was evaluated in $185(75.8 \%)$ patients, out of which $38.9 \%$ patients had Imatinib failure. Disease was progressed to CML-AP and CML BT in $4.5 \%$ and $4.1 \%$ patients respectively. Most common adverse effects noted with Imatinib were hematological (anemia 15.5\%, neutropenia $15.9 \%$ and thrombocytopenia $17.2 \%$ ). Median duration of follow up was 30.93 months. The 5-year overall survival and progression free survival rates were $94.3 \%$ and $92.7 \%$, respectively. Conclusions: Imatinib was well tolerated in this retrospective analysis of Indian patients but Imatinib failure was seen $38.9 \%$ patients. Regular monitoring will identify patients who fail to reach response milestones and may help to identify the factors associated with or contributing to Imatinib resistance.

\section{P-MN 18}

\section{ASXL-1 Mutations in Myelofibrosis}

Russel Mascarenhas ${ }^{\#}$, Nikhil Rabade, Swapnali Joshi, Shruti Chaudhary, Prasanna Bhanshe, Prashant Tembhare, Sumeet Gujral, PG Subramanian, Nikhil Patkar*

Email ID for Correspondence: nvpatkar@gmail.com Hematopathology Laboratory, Tata Memorial Hospital, Mumbai

Introduction: $J A K 2 V 617 F$, CALR and MPL mutations are seen in $50-60 \%, 20-25 \%$ and $6-7 \%$ cases of myelofibrosis respectively. Additional sex comb like 1 (ASXL1) mutations are seen in approximately $15-25 \%$ cases of primary myelofibrosis. Presence of ASXL1 mutations is associated with poorer prognosis. A CALR and ASXL1 based molecular prognostication model has also been proposed. Objectives: To determine the frequency of ASXL1 mutations, their association with $C A L R$ mutations and correlation with DIPSS score. Material and Methods: 58 patients of MF with a median age 52.5 (range; 17-89 years, 68.9\% males). ASO PCR and fragment length analysis was performed on genomic DNA for detection of JAK2, MPL and CALR mutations respectively. Exon 12 of ASXL1 was amplified using 10 overlapping fluorescent primer pairs followed by fragment length analysis. All positive cases were validated by Sanger sequencing. Results: JAK2V617F, CALR and MPL mutations were present in $50 \%, 24.1 \%$ and $1.7 \%$ cases respectively. ASXL1 mutations were detected in $17.7 \%$ ( 8 of 45 ) cases. Of the five different frameshift mutations detected, $23 \mathrm{bp}$ deletion (p.R634fs* c.1900_1922del23) was the commonest. Frequency of ASXL1+/ CALR-, CALR+/ASXL1-, CALR-/ASXL- and CALR+/ASXL1+ cases was $6.9 \%, 17 \%, 50 \%$ and $6.8 \%$ respectively. Seven out of 8 ASXL1 mutated patients were in the intermediate-2 risk category and 1 was in high risk category according to the DIPSS scoring system.70\% CALR+/ASXL1- patients were in low to intermediate-1 risk group. Conclusions: We report frequency of ASXL1 mutations similar to western literature. ASXL1 mutated cases have higher risk categorization as compared to the wild type ones. To the best of our knowledge this is the first report of ASXL1 mutations from India. Long term follow up is required to ascertain their prognostic impact.

\section{P-MN 19}

Coexistence and Impact of Tuberculosis on Chronic Myeloid Leukaemia: A case report
Dr. Gauri Munjal", Dr. Sant Prakash Kataria, Dr. Rajeev Sen, Dr. Shivani Dua, Dr. Pansi Gupta

Email ID for Correspondence: drgaurimunjal11@gmail.com Pt B.D Sharma PGIMS

Introduction: $\mathrm{CML}$ is a hematologic malignancy driven by unregulated tyrosine kinase signalling. The disease develops from a single, pluripotent, hematopoietic stem cell acquiring the BCR-ABL fusion gene, which results in proliferation. TB is a devastating disease responsible for 1.7 million deaths worldwide per year. TB can develop from transmission by actively infected persons or reactivation of a quiescent infection. Suffering from impaired immunity, patients with CML may be at higher risk of developing TB. Case Report: A 40 year old male patient presented at PGIMS Rohtak with complains of generalised weakness, reduced appetite and cervical lymphadenopathy over a period of one month. The patient was thoroughly investigated including lab investigations comprising of complete hemogram, bone marrow aspiration and trephine biopsy and was diagnosed with Chronic Myeloid Leukaemia in blast crisis. The patient was also a known case of extrapulmonary TB on treatment since 6 months. Discussion: Chronic inflammatory conditions predispose to rapid cell turnover and risk of malignancies. Tubercular mycobacteria can cause DNA damage. It may increase BCL-2 synthesis leading to amplified antiapoptotic activity. Imatinib affects immune system and $\mathrm{T}$ cell response by influencing signal transduction, thus increasing risk of TB and advocated assessment of risk of TB before starting Imatinib. Conclusion: The risk of TB is increased in malignancies. This is due to decrease in immunity because of steroid treatment or dysfunction of immune system. The association of TB and malignancy can be sequential, concurrent or masquerading and therefore can pose a diagnostic challenge in some cases. Thus a multidisciplinary approach and concurrent treatment of both the pathologies are must for optimum outcome.

\section{P-MN 20}

Chronic Neutrophilic Leukemia - A Rare Malignant Disorder

Amandeep Singh, Dr. CL Nawal, Dr. R S Chejara, Dr PD Meena, Dr. Pradeep Bansal

\section{SMS Medical College}

Introduction: Chronic Neutrophilic Leukemia (CNL) is a rare myeloproliferative neoplasmcharacterized by sustained mature neutrophilic leukocytosis, hepatosplenomegaly and bone marrow granulocytic hyperplasia. Case Description: 33 year old Hindu male presented with complains of fatigue 3 month, myalgia from 8 days. He consulted nearby hospitals where he detected with raised white blood cells. On examination - Splenomegaly present. Systemic examination was normal. Investigations: Blood Hb-10.8 g\%, WBC $51.83 \times 109 / \mathrm{L}$, platelet count- $2.4 \times 109 / \mathrm{L}$ DLC: N94.6 L3.7 E.5 B.3. Morphology of neutrophils normal with segmented and band cells present. ESR- $120 \mathrm{~mm} / 1 \mathrm{st} \mathrm{hr}$. RFT and LFT Normal. Blood and urine culture sterile. Leucocyte Alkaline Phosphatase score normal. Bone marrow aspiration - Hypercellular marrow with marked neutrophilic proliferation. BCR - ABL, PDGFRA and PDGFRB genes were negative. Differential diagnosis - CNL, Atypical CML. Discussion: Applying WHO diagnostic criteria, diagnosis of CNL was made. Gene analysis CSF 3 receptor mutation was not done due to financial constraints of patient. Tab Hydroxyurea $500 \mathrm{mg}$ BD given. Patient improved symptomatically with TLC $(6.03 \times 109 / \mathrm{L})$ after 7 days. Conclusion: Rare chronic myeloproliferative neoplasms like 
CNL should be considered in patients of persistent neutrophilia and not merely treating it as sepsis.

\section{P-PCD 01}

Plasma Cell Myeloma with Unusual Bone Marrow Morphology: A Case Series

\section{Murari M, Sharma S, Verma R, Vallipriya $\mathbf{P}$}

\section{Department of Pathology, SGPGIMS, Lucknow}

Background: Morphological diagnosis of myeloma is based on characteristic peripheral blood and bone marrow findings. Variant morphology is rare and infrequently reported in myeloma. Material and methods: Diagnostic work-up including peripheral blood, bone marrow aspiration and biopsy findings was carried out in Department of Pathology, SGPGIMS, Lucknow. Morphological findings were correlated with biochemical profile, electrophoresis and radiological findings. Results: All patients were adults and included both males and females. The cases Included in the series are atypical lymphoid/lymphoplasmacytic proliferation that were suggestive of lymphoma, anaplastic plasma cell proliferation mimicking metastasis, myeloma associated with marked fibrosis that precluded diagnosis on aspiration. In addition, few cases had atypical morphology of plasma cells including crystalline inclusions, Clover leaf-like nuclei, numerous Mott cells and Russel bodies. Conclusion: Atypical clinical presentation and morphological variants in plasma cells neoplasms may cause diagnostic difficulty. Such cases are rare and often necessitate use of ancillary techniques. Identification of variant morphology in myeloma has diagnostic and prognostic significance.

\section{P-PCD 02}

Clinico-Pathological Profile of Waldenstrom Macroglobulinemia: A Tertiary Care Centre Experience of Six Cases

\section{Venkatesan $S^{\#}$, Sampath K, Min H, Sharma S, Bharadwaj R}

Email ID for Correspondence: 1tcolsvenkatesan@gmail.com Armed Forces Medical College

Introduction: Waldenstrom macroglobulinemia (WM) is a mature B cell Non Hodgkin lymphoma, characterized by a monoclonal Ig M in the serum and the presence of lymphoplasmacytic infiltration in the bone marrow. The common clinical manifestations of this disease are anemia, organomegaly, lymphadenopathy and hyperviscocity. The majority of the patients typically present with anemia and monoclonal IgM in the serum. Objectives: The aim of the study was to document the clinico-pathological profile of six cases of Waldenstrom macroglobulinemia, which were diagnosed and treated in a tertiary care center. Material and Methods: In this observational cross sectional study, clinico-pathological profile of six consecutive cases of Waldenstrom macroglobulinemia was documented. Results: Median age of patients was 65 years with a male preponderance. All patients presented with clinical features of moderate to severe anemia and some severe cases with features of cardiovascular decompensation. Pateints also had B symptoms, bleeding manifestations along with varying degrees of cytopenias. All cases had albumin:globulin ratio reversal. Peripheral smear in all cases showed background staining and rouleaux formation. Peripheral smear revealed plasmacytoid cells in two cases and such cases needs to be differentiated from plasma cell neoplasms. Bone marrow aspirates and biopsy showed features of lympho-plasmacytic infiltration with suppression of other lineages. Marrows in all cases were hypercellular and commonly showed a diffuse pattern of marrow infiltration by lymphoplasmacytic cells. All the bone marrow biopsies showed Grade 1 marrow fibrosis. Serum protein electrophoresis and immunofixation electrophoresis confirmed Ig M type of monoclonal gammopathy. Conclusions: WM is a rare haematological malignancy and needs to be recognised and differentiated from plasma cell neoplasms as the management and prognosis are different in these cases.

\section{P-PCD 03}

Clinical Value of Classification of Multiple Myeloma Samples Based on Their Gene Expression Profiles

Pratyush R Behere, Jina Bhattacharyya, Bithiah Grace Jaganathan

Multiple myeloma is a hematological cancer that is caused by malignant plasma cells. These abnormal plasma cells overproduce monoclonal antibodies and suppress the growth of other cells in the bone marrow, this can lead to suppression of immune system, anemia, bone lesions and impaired kidney function. There has been an improvement in the median survival of multiple myeloma in recent years nevertheless it remains a disease in which has an unpredictable clinical course. This can be attributed to the molecular heterogeneity associated with multiple myeloma. The objective of this study is to exploit this molecular heterogeneity to classify multiple myeloma samples according to their gene expression profiles and to see if the obtained classes have any clinical value. Materials and methods: Microarray data (gene expression profiles) for newly diagnosed multiple myeloma patients has been obtained from NCBI Gene Expression Omnibus (GEO) specifically the dataset GSE2658 has been used for analysis. All statistical analysis has been done using $\mathrm{R}$ software (version 3.4.1). The genes exhibiting highly variable gene expression were selected (standard deviation $>1.34$ ) for further analysis. Dimensionality reduction using tSNE (t-distributed stochastic neighbor embedding) was done followed by unsupervised clustering using DBSCAN (Density based spatial clustering of applications with noise) in order to identify the subclasses. Statistically significant genes across all classes were selected using SAM (Significance analysis of microarrays) following which a classifier was built and validated using PAM (Prediction analysis for microarrays). Survival curves for expression free survival and overall survival made for each class using Kaplan-Meier method. Also, survival curves comparing the effect of two therapy regimes named as TT2 and TT3 was made for each class. Log-rank tests used to check independence of the survival curves. Results: Using the abovementioned techniques six subclasses were identified for newly diagnosed multiple myeloma based on their gene expression profiles. A 644-gene classifier was built and validated for the aforementioned classes. Survival analysis for expression free survival (relapse) and overall survival (death) done for each of the obtained classes followed by log-rank tests to check independence of the survival curve. A clear overall survival advantage was seen in case of groups 1,2 and 4 while groups 2 and 5 had a favorable expression free survival. Survival analysis for expression free survival (relapse) and overall survival (death) was repeated for each class taking the two therapy regimes TT2 and TT3 into consideration. It's seen that TT3 confers a significant favorable overall survival in all classes except for groups 4 and 5. Also, TT3 shows significantly better expression free survival in all classes. Conclusion: Each class has an associated survival different from the other, this shows that each class has an associated prognostic value and is thus of clinical importance. Also by analyzing 
in the information that is seen in the survival curves relating to we can decide which therapy is better suited for each class.

\section{P-PCD 04}

\section{Clinical Profile of Multiple Myeloma in Atertiary Care Centre from North East India}

Introduction: Multiple Myeloma is a neoplastic disease of the plasma cells characterized by clonal proliferation of terminally differentiated B lymphoid cells, which accumulate in bone marrow and produce a monoclonal protein (Immunoglobulin), usually IgG or IgA, often referred to as $\mathrm{M}$ or myeloma protein. Aims and Objectives: Our study aims to describe the common clinical and laboratory features, stages and outcome of patients with Multiple Myeloma coming to a Tertiary Care Center in North East India. Material and Methods: Newly diagnosed and previously diagnosed untreated patients of MM coming to Assam Medical College over a period of one year from June 2013 to May 2014 were randomly included in this prospective observational study. Diagnosis was confirmed by the 2009 updated criteria of International Myeloma Working Group and clinical features, laboratory findings and outcome were studied. Results and Observations: Multiple myeloma mostly from 5th and 6th decade with the age varying from 38 to 82 and a mean age of 57.7 years (SD 10.29); 27 (61\%) patients were male and $17(39 \%)$ were females with a male-to-female ratio of $1.6: 1 ; 38(86 \%)$ presented with backache and other bone pains, $35(80 \%)$ had generalized weakness and easy fatigability, $13(30 \%)$ had symptoms of spinal cord compression, 14 (32\%) had fever. On examination 37 (84\%) had pallor, 34 (77\%) had bony tenderness, 14 (32\%) had paraplegia; 40 (91\%) cases revealed positive M Band by serum protein electrophoresis. In rest of the 4 cases, band was determined by immunofixation method. Urinary Bence-Jones protein was estimated in 28 out of 44 patients and of them 4 (14\%) were positive; $40(91 \%)$ had radiologically detectable abnormalities; $35(80 \%)$ had lytic lesions, 17 (39\%) had pathological fractures, 21 (48\%) had generalized osteoporosis and only $4(9 \%)$ had normal skeletal survey. Out of 35 cases of lytic lesions, most frequent area was spine in $25(71 \%) ;$ $(16 \%)$ cases were found to be in stage I, $20(45 \%)$ were found to be in stage II and $17(39 \%)$ were in stage III. Conclusion: We conclude that though most patients were in sixth decade, but we got significant involvement in younger age group. Bone pain, mostly low backache was the most common presenting symptom along with fatigue and weakness. Majority of patients were in higher stages (ISS II, III) at the time of diagnosis and severe anaemia, lytic lesions, pathological fractures and renal insufficiency were most observations in these patients in higher stages.

\section{P-PCD 05}

\section{Retrospective Fish Analysis in 95 Multiple Myeloma Cases and Their Co-relation with Clinico- Biochemical Parameters}

Hemani Jain ${ }^{1 \#}$, Dhanlaxmi Shetty ${ }^{1}$, Yogita Deshpande ${ }^{1}$, Nishigandha Masurkar', Manju Sengar ${ }^{2}$, Navin Khattry², Hasmukh Jain ${ }^{2}$, Bhausaheb Bagal ${ }^{2}$, Nitin Inamdar ${ }^{3}$, P. G. Subramanian ${ }^{4}$

Email ID for Correspondence: hemanijain7@gmail.com Department of Cancer Cytogenetics, ACTREC, Tata Memorial Centre, Kharghar, Navi Mumbai; 2 Department of Medical Oncology, Tata Memorial Hospital, Parel, Mumbai; 3 Biochemistry Department, Tata Memorial Hospital, Parel, Mumbai; 4 Department of
Hematopathology, ACTREC, Tata Memorial Centre, Kharghar, Navi Mumbai

Introduction: Multiple myeloma (MM) is a cytogenetically heterogenous plasma cell malignancy. The detection and interpretation of cytogenetic abnormalities in MM is of critical importance for prognosis and risk stratification. Objectives: To check the prevalence of various cytogenetic abnormalities, analyze their association with biochemical characteristics and therapy response in patients with multiple myeloma. Material and Methods: Retrospective cytogenetic analysis was carried out in 95 de novo multiple myeloma patients registered in Tata Memorial Hospital, Mumbai from August 2016 to December 2016. All the patients were studied for cytogenetic abnormalities on purified CD138 positive plasma cells and bone marrow aspirate cultured cells by fluorescent in situ hybridization (FISH) using commercially available DNA probes: LSI IgH-CCNDI, LSI $I g H-F G F R 3$, LSI $I g H-M A F$, LSI $I g H-M A F b$ dual fusion translocation probes, LSI IgH break apart probe, LSI D13S319 (13q), LSI TP53/CEN 17 deletion probes, LSI 1p36/q21, LSI 15q22, LSI 9q34, LSI 5q31/D5S235 (5p12.2) and centromeric probes for 3 and 7. Hyperdiploidy was defined as trisomy of $\geq 2$ chromosomes. Results: Cytogenetic abnormalities by FISH were detectable in $66 \%(63 / 95$ cases) of patients. Monosomy 13/del(13q) was seen in $40 \%$ patients followed by $\mathrm{IgH}$ translocations in $32 \%$, gain (1q21) in $26 \%$ and $T P 53$ deletion/monosomy 17 in $8 \%$ patients. Hyperdiploidy was found in $36 \%$ patients. Patients' median age was 55.5 years (range, 27 to 84 years) and 65 were males (M: F: 2.1: 1). IgH translocation group and TP53 deletion were identified as high-risk group due to correlation with advanced disease, stage III $(\mathrm{P}<0.042) \&(\mathrm{P}<0.052)$ respectively, whereas monosomy $13 / \mathrm{del}(13 \mathrm{q})$ was significantly associated with high plasma cells $(\mathrm{P}<0.043)$. Response to treatment was assessed at a median follow up of 3 months. Patients on cyclophosphamide based therapy showed an overall response rate [complete response (CR)+ very good partial response (VGPR)] of $86 \%$ whereas in case of lenalidomide based therapy the overall response rate was $61.5 \%$. Conclusions: Our patients presented lower median age at 55.5 years which is a decade younger than those from other countries. Low prevalence of chromosome 13 aberrations and $\mathrm{IgH}$ translocations was observed, as compared to Western population probably due to geographic heterogeneity. Association of $\mathrm{IgH}$ translocations, chromosome 13 aberrations and TP53 deletions with high-risk clinicolaboratory features such as advanced disease and high plasma cells revealed importance of genomic aberrations for prognosis. Cytogenetic abnormalities detected on standard FISH testing should be routinely used for risk stratification and making treatment decisions to aid in clinical practice and patient management.

\section{P-PCD 06}

Initial Experience with Daratumumab from a Tertiary Cancer Care Centre

Rayaz Ahmed, Pallavi Mehta, Neha Yadav", Priyanka Verma, Pragya Bhandari, Narendra Agrawal, Dinesh Bhurani

Email ID for Correspondence: nehayresearch@gmail.com Rajiv Gandhi Cancer Institute and Research Centre, New Delhi, India

Introduction: Patients with multiple myeloma (MM) typically have recurrent relapses and poor survival despite the availability of newer proteosome inhibitors (PIs) and immunomodulatory drugs (IMDs). Therefore, novel therapeutic strategies are needed for these high risk patients. Daratumumab, a human monoclonal antibody targeting CD38, has been approved as monotherapy or combination therapy in heavily treated patients. No clinical data is available till now on the 
efficacy and toxicity of this drug from developing countries. We are hereby presenting our experience with this novel drug in relapsed and refractory patients treated in Rajiv Gandhi Cancer Institute from year 2017. Objectives: To study the toxicity profile and efficacy of daratumumab. Methods: Patients with relapsed or refractory MM treated with daratumumab, were evaluated for clinical efficacy and safety profile. Daratumumab was administered in the dose of $16 \mathrm{mg} /$ $\mathrm{kg}$ /week either in combination with an IMID or a proteosome inhibitors. Patient's clinical and laboratory parameters were retrieved from hospital records. Response to treatment and disease progression was evaluated according to the IMWG response criteria. Results: Seven patients with relapsed or refractory MM during the year 2017 received daratumumab as combination therapy. IgG Kappa was the commonest (3/7) subtype among these patients. FISH analysis was available in 2 patients, with one having del13q14 and del17p. The median number of lines of chemotherapy prior to daratumumab was 2 (1-5). Four (4/7) patients were refractory to both PIs and IMDs; 3/7 patients were refractory to PIs. Three patients had relapse, 2 patients had progressive disease and 1 patient had minimal response while one patient had inadequate response to their last therapy. Median duration of daratumumab therapy was 5 weeks ( 1 dose per week, 1-8 doses). Median time to response was 1.5 months (1-2 months). Overall response rate (ORR) was $42.7 \%$ which included $14.2 \%(n=1)$ patients had VGPR, 28.5\% $(\mathrm{n}=2)$ patients had PR .14.2\% $(\mathrm{n}=1)$ patient had progressive disease. Two patients could not be assessed because of death. One patient is yet to be assessed. One patient underwent stem cell transplant post daratumumab therapy in PR. The most common significant toxicities with daratumumab were hematologic in nature with 5 patients had neutropenia out of which 2 had grade 3-4 febrile neutropenia, thrombocytopenia was seen in 3 patients out of which 2 patients had an episode of grade 3-4 thrombocytopenia and 2 patients had anemia. Infusion related reaction was seen in 1 patient which was managed with steroids and antihistaminics. Two deaths were observed due to secondary infections (sepsis with gram negative bacteria and HINI infection). Conclusions: Our study shown response rate above $40 \%$. There were significant toxicities such as neutropenia, sepsis and infection with HINI.

\section{P-PCD 07}

\section{An Unusual Presentation of Multiple Myeloma: A Case Report Trushali Dobariya $^{1 \#}$, Amit H.Agravat ${ }^{2}$ Gauravi A Dhruva ${ }^{3}$}

Email ID for Correspondence: trushali.dby@gmail.com ${ }^{1}$ Resident Doctor, Department of Pathology, P.D.U. Government Medical College, Rajkot; ${ }^{2}$ Associate Professor, Department of Pathology, P.D.U. Government Medical College, Rajkot; ${ }^{3}$ Professor \& Head, Department of Pathology, P.D.U. Government Medical College, Rajkot

Introduction: Multiple myeloma (MM) is a primary malignancy of bone marrow in adult characterized by clonal proliferation of plasma cells and production of monoclonal immunoglobulin. The clinical presentation varied and depends on the sites and extent of involvement. Most common clinical symptoms of multiple myeloma may mimic pain of musculoskeletal origin. Aim: To report unusual presentation of multiple myeloma and highlight how early detection of these unusual features will encourage early investigations, diagnosis and early management and consequently better prognosis of disease. Method: It was a case of 60 year old male who presented with recurrent nasal bleeding since 20 days and joint pain since 15 days. Clinical presentation, physical examination, bone marrow examination and other laboratory investigations confirmed the diagnosis of multiple myeloma. Result/Conclusion: A thorough complete blood count $(\mathrm{CBC})$ and peripheral blood smear examination will help to detect the disease like multiple myeloma.

Keywords Multiple myeloma, nasal bleeding, CBC

\section{Abstract for Oral Presentation}

\section{P-PCD 08}

Unusual presentation of multiple myeloma: A 5-year study in a tertiary care center

Manjari Kishore", Vijay Kumar, Sadhna Marwah, A.S. Nigam, Minakshi Bhardwaj

Email ID for Correspondence: drmanjarik@gmail.com PGIMER, Dr. RML Hospital, New Delhi

Introduction: Multiple myeloma is the most common bone malignancy characterized by malignant proliferation of plasma cells leading to production of a monoclonal paraprotein. Varied clinical presentations are noted in the patients like fever, fatigue, weight loss, paraesthesias, hypercalcemia, hyperviscosity, renal failure, bone pain or pathological fractures, cutaneous lesions, etc. We present a series of multiple myeloma cases with unusual presentation over a period of 5 years. Objectives: To study the unusual clinico-hematological and histopathological presentation in patients with multiple myeloma. Materials \& methods: In this study, we reviewed bone marrow aspirate \& biopsy slides in our hospital from May 2012 to June 2017. Patients diagnosed with multiple myeloma were selected. The patients's clinical information, hematological and histological findings were obtained from the medical records department and correlation was done. The rare and unusual clinical and hematological findings of the patients were studied and correlation was done. Results: We found a total of 14 cases of multiple myeloma with unusual and rare presentations. Proper clinical, radiological, hematological and histopathological correlation was done before giving a final diagnosis in these cases. Unusual cutaneous and gastrointestinal involvement of multiple myeloma along with a different case of anaplastic myeloma were noted, especially in younger individual, which further added to the rarity of our cases. Conclusion: Multiple myeloma is a debilitating malignancy. In the past few years, substantial progress has been noted in treatment of this entity. Early diagnosis of multiple myeloma along with appropriate management will prevent serious complications, especially in younger individuals. The present case series attempts to study the clinicopathological correlation in cases of multiple myeloma and associated rare and unusual presentations.

\section{P-PCD 09}

\section{Plasma Cell Myeloma: A Series of 6 Unusual Cases}

Background: Plasma cell myeloma is characterized by monoclonal proliferation of plasma cells in the bone marrow producing serum or urinary $\mathrm{M}$ protein, lytic bone lesions and various other clinical and laboratory abnormalities. We report six cases of plasma cell myeloma to highlight the unusual morphologic and electrophoretic abnormalities of this entity and the diagnostic dilemma these can pose. Objectives: To study the additional/ unusual clinicopathologic features of patients diagnosed with MM. Materials and method: This is a retrospective study of clinicopathologic features of 168 patients diagnosed with multiple myeloma in GMCH-32, Chandigarh over a period of 12 years (June 2005- June 2017). The detailed clinical data, 
radiological features, plates of agarose gel electrophoresis and slides of bone marrow aspirate and trephine biopsies were retrieved from archival material and reviewed. Results: Of the 168 patients diagnosed with multiple myeloma, $6(3.6 \%)$ patients had features diverging from the usual morphologic/ biochemicalcharacteristics of MM. All the patients were males in $6^{\text {th }}-7^{\text {th }}$ decade of life. All the patients presented with complaints of backache. On bone marrow examination, all cases had $>10 \%$ plasma cells (15-54\%). Two of the cases $(1.1 \%)$ demonstrated two monoclonal bands on serum electrophoresis (biclonal myeloma). Another two patients (1.1\%) showed amyloid deposition. Presence of fibrinoid necrosis was observed in the bone marrow of one patient $(0.6 \%)$ while one of the patients $(0.6 \%)$ was diagnosed with crystal storing histiocytosis along with presence of $15 \%$ plasma cells. Conclusion: This report highlights the additional and unusual electrophoresis patterns and morphologic features that could be encountered in cases of plasma cell myeloma. An acquaintance with these features is essential for establishment of a correct diagnosis.

R. A. Kyle, M. A. Gertz, T. E. Witzig et al., "Review of 1027 patients with newly diagnosed multiple myeloma," Mayo Clinic Proceedings, vol. 78 , no. 1 , pp. 21-33, 2003.

\section{P-PCD 10}

\section{Morphological Features of Bone Marrow Biopsies in Systemic} Amyloidosis and Their Follow Up

\section{Dr Kusha Sharma\#, Dr Jasdeep Singh, Prof Prabal Deb, Dr} Tarun Verma, Dr P Suresh

Email ID for Correspondence: drkushasharma@gmail.com Command Hospital (EC), Kolkata

Introduction: The pathognomic clinical findings of amyloidosis are found in less than one-fifth of patients and are not reliable for disease detection. Rare presentation of this disease in the form of Immunoglobulin light chain amyloidosis (AL) caused by misfolded $\mathrm{AL}$ protein is associated with an underlying plasma cell dyscrasia and in rare cases with multiple myeloma.

Objectives:

1. To study the varied morphological features of systemic amyloidosis on bone marrow biopsies .

2. To follow up these patients clinically and with follow up biopsies.

Material and Method: We analysed 200 bone marrow biopsies retrospectively from Jan 2015 till Sep 2017. 12 bone marrow aspirate and biopsies were done with suspicion of systemic amyloidosis. 08 cases were confirmed on Congo red special staining to have amyloid deposition. These cases were analysed for clinical features, baseline laboratory parameters, morphological features on bone marrow biopsy. The response to therapy was also assessed over a mean duration of 09 months. Results: The incidence of amyloidosis with bone marrow involvement was $4 \%$ in our study. The median age of presentation was 64 years with commonest complaint of generalised weakness $(75 \%)$. Anaemia with mean haemoglobin of $85 \mathrm{~g} / \mathrm{l}$ was the commonest haematological finding. Significant bleeding events occurred in $50 \%$ of the patients with factor $\times$ deficiency seen in $25 \%$ cases. Raised creatinine was the commonest biochemical finding. The most common pattern of bone marrow involvement was interstitial infiltration by $7 \%$ plasma cells $(5-18 \%)$ and perivascular amyloid deposition. Rare presentations like amyloidoma and associated myeloma were also seen. The first line treatment regimens were Melphalan based in 06 patients while patient with multiple myeloma was started on Bortezomib and the median follow up from diagnosis was 15 months (range 1-24 months). During this period 06 patients survived while 01 died of cardiac failure. Conclusion: The commonest presentation of systemic amyloidosis in our study was perivascular thickening and interstitial infiltration by plasma cells. However this study highlights varied morphological features on bone marrow biopsies with rare presentation like amyloidoma and associated multiple myeloma. The overall survival of patients is fair if therapy is instituted early.

\section{P-PCD 11}

Case Report of Monoclonal Gammopathy of Undetermined Significance Associated with Scleromyxedema

Jai Vijay Juvekar", Kalpana Anand Deshpande, Rachna Binayke, Akshay Kumar Tiwari, Grace D'costa

\section{Grant Medical College and Sir J. J. Group of Hospitals, Mumbai}

Introduction: MGUS denotes the presence of a monoclonal immunoglobin, also called M-protein in the serum or urine in persons without evidence of multiple myeloma, Waldenstroms macroglobulinemia, amyloidosis or other lymphoproliferative disorders. It is thought to be a precursor to multiple myeloma and other plasma cell dyscrasias and is seen in persons over 50 years of age. Herewith we present a case of monoclonal gammopathy of undetermined significance associated with scleromyxedema. Case Summary: A 71 year old diabetic, hypertensive male patient presented with chief complaints of convulsions, altered state with irrelevant talk. Also noted were skin lesions over hand and abdomen. Skin biopsy showed features consistent with scleromyxedema. Peripheral smear showed normocytic normochromic smear with neutrophilic leukcocytosis and eosinophilia. Bone marrow was mildly hypercellular with plasmacytosis $<10 \%$. Serum electrophoresis showed M- band (0.75 gms) in gamma region. A thorough workup of the patient revealed no end organ damage. Discussion: WHO has defined MGUS as the presence of M-protein in serum $<30 \mathrm{mg} / \mathrm{L}$, bone marrow clonal plasma cells $<10 \%$, no end organ damage and no evidence of B-cell lymphoma or other disease known to produce a n M-protein. Our patient has satisfied all WHO criteria and hence labelled as MGUS. Conclusion: Etiology of MGUS is not always associated with a plasma cell dyscrasias or B cell disorders and it has been shown by various studies to be an entity which may be associated with dermatological conditions like scleromyxedema as in our case.

\section{Abstract for Haematocon}

\section{P-PCD 12}

Plasma Cell Leukemia- A Coincidental Finding Presenting as Chest Pain

\section{Dr Sarika Singh ${ }^{1}$, Ashutosh Rath ${ }^{2}$, Surekha Yadav ${ }^{2}$ \\ Institutional Affiliation: Department of Pathology Maulana Azad Medical College, New Delhi \\ 1: Professor Pathology MAMC, 2: Senior resident Pathology}

Introduction: Plasma cell leukemia (PCL) is the most aggressive\& rare form among plasma cell dyscrasias. Although many therapeutic regimens have been suggested, no specific drug or regimen seems to be promising enough that would significantly enhance the survival period. Here we present a case of primary plasma cell leukemia to signify the symptomatology, biochemical and clinical course. Case Report: A 69 
male presented in cardiology with dyspnea and chest pain. On investigations his $\mathrm{Hb}$ wasfound to be $7.2 \mathrm{~g} / \mathrm{dL}$, TLC of $12000 / \mu \mathrm{L}$ and platelet count of $97000 / \mu \mathrm{L}$. ESR- $70 \mathrm{~mm} / \mathrm{hr}$. Peripheral smear showed marked rouleaux formation with normocytic normochromic picture. Plasma cells constituted $18 \%$ and atypical lymphocytes comprised of $15 \%$ of total leukocytes. Many of the plasma cells showed bipolar cytoplasm. Atypical lymphocytes exhibited central to eccentrically placed nuclei with moderate amount of cytoplasm showing fuzzy borders. A diagnosis of plasma cell leukemia was made and an urgent bone marrow examination was advised. Bone marrow aspiration revealed atypical plasma cells constituting $61 \%$ of marrow nucleated cells along with thesaurocytes and few binucleated plasma cells. Bone marrow biopsy showed hypercellular marrow spaces with near total replacement by plasma cells. The plasma cells were positive for CD38, focally positive for CD20, negative for CD19 \& p53, showed Kappa light chain restriction. Blood urea $-147 \mathrm{mg} / \mathrm{dL}$, serum creatinine- $3.3 \mathrm{mg} / \mathrm{dL}$, serum uric acid- $13.0 \mathrm{mg} / \mathrm{dL} \&$ serum calcium $(8.5 \mathrm{mg} / \mathrm{dL})$. His serum protein electrophoresis showed total proteins- $15.20 \mathrm{~g} / \mathrm{dL}$ (normal .40$8.10 \mathrm{~g} / \mathrm{dL}$ ), albumin- $3.83 \mathrm{~g} / \mathrm{dL}(3.50-5.64 \mathrm{~g} / \mathrm{dL}), \alpha 1$ globulin- $0.62 \mathrm{~g} /$ dL (0.17-0.41 g/dL), $\alpha 2$ globulin- $1.19 \mathrm{~g} / \mathrm{dL}(0.31-0.85 \mathrm{~g} / \mathrm{dL})$, beta globulin $0.59 \mathrm{~g} / \mathrm{dL}(0.49-1.32 \mathrm{~g} / \mathrm{dL})$, gamma globulin $8.97 \mathrm{~g} / \mathrm{dL}(0.62-$ $1.53 \mathrm{~g} / \mathrm{dL}$ ), A/G- 0.34 (normal -0.90-2.00) and $M$ spike in gamma globulin region. Serum IEF revealed the raised globulins to be IgD. Urine was negative for Bence Jones proteins. Sflc showed, kappa free light chains $204.00 \mathrm{mg} / \mathrm{L}$ (3.30-19.40 mg/L) and kappa/lambda ratio of 27.717 (0.26-1.65). Serum $\beta 2$-microglobulins was $15576 \mathrm{ng} / \mathrm{mL}$ $(609.00-2366.00 \mathrm{ng} / \mathrm{mL})$. With a final diagnosis of primary plasma cell leukemia, the patient was started on Bortezomib based chemotherapy. In the first cycle itself, the leukocyte count normalized to $8720 / \mu \mathrm{L}, \mathrm{Hb} \mathrm{d}$ to $10.6 \mathrm{~g} / \mathrm{dL}$, platelet count to $157 \times 10^{3} / \mu \mathrm{L}$. After six cycles of chemotherapy patient is doing fine, his renal functions reverted to normal and chest pain subsided. Conclusion: Sternal pain in an elderly patient with normal ECG \& echo findings a strong clinical suspicion of haematolymphoid malignancy is to be considered and patients to be worked up energetically to derive a final diagnosis.

\section{P-PCD 13}

\section{The Prognostic Impact of CD20 and Cyclin D1 Expression in Multiple Myeloma - A Pilot Study}

Nabhajit Mallik", Ram Nampoothiri ${ }^{1}$, Sreejesh Sreedharanunni, Narender Kumar, Prashant Sharma, Shano Naseem, Man Updesh Singh Sachdeva, Jasmina Ahluwalia, Reena Das, Neelam Varma, Pankaj Malhotra

\section{Email ID for Correspondence: nabs.mallik@gmail.com PGIMER, Chandigarh}

Introduction: The expression of CD 20 and cyclin D1 have been reported at varying frequencies in patients with multiple myeloma, and their clinical significance has remained unclear. This study aims to investigate the expression of CD20 and cyclin D1 in myeloma cells in the bone marrow, and any relationship with disease stage and progression. Objectives: (1) To study the frequency of expression of CD 20 and cyclin D1 by immunohistochemistry in multiple myeloma. (2) To correlate their expression with response to treatment and other known prognostic parameters. Material and Methods: A retrospective evaluation was performed on multiple myeloma patients on therapy. A total of 62 consecutive patients on therapy and with a clinical follow up were chosen for the study. The trephine biopsy performed at diagnosis was retrieved and evaluated for the expression of CD20 and cyclin D1 by immunohistochemistry. The expression of these markers was correlated with the treatment response. Results:
The age of the patients ranged from 36 to 82 years of age (median56 years), and showed a male to female ratio of 1.69:1. The bone marrow plasma cell count ranged from $7 \%$ to $94 \%$ (median - $41 \%$ ). $46 \%$ of the patients were in ISS stage III at diagnosis while $16 \%$ and $38 \%$ of the patients were in stage I or II respectively. Overall, $14.7 \%$ $(n=9)$ cases were positive for CD 20, whereas $21.6 \%(n=13)$ were positive for cyclin D1. The follow-up period ranged from 8 months to 108 months (median 18 months). All the patients with CD20 positivity and $91.7 \%$ of the patients with cyclin D1 positivity presented in ISS stage II (33.3\% and 50\%) or III (50\% and 41.7\%). However, $75 \%$ of patients with $\mathrm{CD} 20$ positivity or cyclin D1 positivity showed complete or a better response; while only $58.3 \%$ of the CD20 negative and $57.8 \%$ of the cyclin D1 negative myeloma cases showed complete or a better response. Conclusions: Only $14.7 \%$ and $21.6 \%$ of the cases in our study showed expression of CD 20 and cyclin D1 respectively, which is lower than what has been reported in the literature. Our pilot study indicates better response to therapy in multiple myeloma with CD20 or cyclin D1 expression despite presentation in higher ISS stages.

\section{P-PCD 14}

\section{Varied Presentations of Multiple Myeloma - A Report of Two Cases}

Dr Shubhashree $\mathbf{P}^{\#}$, Dr Suchitha S, Dr Manjunath S Shetty

Email ID for Correspondence: shubhashreep2@gmail.com JSS Medical College, JSS University, Mysore

Introduction: Multiple myeloma (MM) is a multifocal neoplasm of clonal plasma cells characterized by the presence of a monoclonal protein and end-organ damage. It is primarily a disease of the elderly with the median age at diagnosis approximately 70 years and less than $0.3 \%$ patients being younger than 30 years. We report two extremely rare cases of MM in young patients presenting with renal failure. Objectives: To study renal and bone marrow biopsy features in two young patients presenting with renal failure of unknown aetiology. Material and Methods: Two patients, one a 25 year-old male and other a 26 year-old female presented with nausea and anorexia, blurring of vision, pedal oedema, and facial puffiness since one month. Renal biopsy showed cast nephropathy in one and light chain deposition disease in the other. The patients were later investigated for multiple myeloma. Serum protein electrophoresis showed two distinct bands in the beta- gamma region in one and normal study in the other. Subsequent bone marrow examination showed plasmacytosis. No lytic lesions were seen on skeletal survey. Both were diagnosed as MM and started on bortezomib therapy. Results: MM in this age group may present with unusual features such as absence of bone pains or lytic lesions and may have an atypical course. During the course of disease, approximately $20 \%$ of the patients develop progressive renal failure. Our study delineates the importance of renal and bone marrow biopsy in early diagnosis of MM with renal involvement. Conclusions: Determination of optimal treatment regimens for younger subset of patients has been hampered by the rarity of MM in this age group. There seem to be differences in presentation and also a better response to treatment in younger patients. Clinically, it may present as progressive renal insufficiency or acute renal failure of unexplained aetiology. The diagnosis of light chain deposition disease or cast nephropathy indicates the presence of multiple myeloma; in other words, it is considered a "myeloma-defining event" and hence it 
should be considered as an important pre-biopsy differential diagnosis in renal failure of unknown etiology. It is imperative that all renal biopsies be analyzed with anti-light-chain antibodies to detect a possible underlying gammopathy.

\section{P-PCD 15}

\section{Walderstorm's Macroglobulinemia \\ Dr Jagveer singh", Dr Sushil yadav, Prof Kailash kumar, Dr Nilesh kumar, Dr Pankaj Kannauje}

Email ID for Correspondence: jagveer21@gmail.com Institute of Medical Sciences, BHU

Introduction: Plasma cell disorders are group of disease which having a variable nature of ' $\mathrm{M}$ ' component whether, a intact antibody molecule of any heavy chain or an altered antibody or fragment. Waldenstorm's macroglobulinemia, a relatively rare plasma cell disorder, originates from a post germinal centre B cell, has an characteristic IgM bearing memory cell which secretes IgM type of immunoglobulin in excess amounts causing the symptoms. Objectives: Patient- 70 years male presented with complaints of generalised body weakness and easy fatigability for 8 months. No history of fever, rashes, haemoptysis, headache, malena, per rectal bleeding, radiation exposure, chronic medication. On examination- pallor++, bilateral inguinal lymph node of size $2 \times 2 \mathrm{~cm}$, pulse 94/min, BP- 140/84 $\mathrm{mm} \mathrm{hg}$, respiratory rate 16/min, per abdomen- spleen $3 \mathrm{~cm}$ palpable. HIV- negative. Material and Methods: Patient was managed with 3 units of PRBC and 6 units RDP. In routine investigations $\mathrm{CBC}$ - pancyotpenia hb 3.1, tlc 3200(N74, L20), mcv 92. Corrected rc 1.2, crt/urea 1.3/43, ca++/ phosphate-9/4.8, tp/alb 10.5/3.1, sgot/sgpt-37/48, viral markers negative, BM aspiration- s/o hypoplastic anaemia, no malignant cells seen, $21 \%$ lymphocytes, $8 \%$ cells with plasmacytoid morphology. Serum electrophoresis- s/o mm peak in beta region s/o plasma cell dyscrasisa. Immunofixation showed a IgM peak with low level of IgG. patient was not assessed for MYD88 L256P mutation. Results: A diagnosis of Waldenstorm's macroglobulinemia proposed and patient planned for Bortezomib based chemotherapy. long term outcome planned to be monitored. Conclusions:. Waldenstorm's macroglobulinemia, a type of plasma cell disorder, differ very much in symptoms, pathogenesis and management from a well known form of multiple myeloma.

\section{P-PD 01}

Mean Platelet Volume in Polycystic Ovarian Syndrome and it's Correlation with Body Mass Index. A Potential Risk Factor for Thrombosis?

\section{Dr Pooja Suteri", Dr Sadhna Marwah, Dr Indu Chawla}

Email ID for Correspondence: drpoojaxyz@gmail.com PGIMER, Dr RML Hospital, Delhi

Introduction: Polycystic ovarian syndrome (PCOS) is an endocrine disorder. PCOS patients have insulin resistance, hyperinsulinemia, hyperandrogenism, altered adipose metabolism and obesity. PCOS is a prothrombotic state. According to androgen excess society insulin resistance and hyperandrogenemia are risk factors for cardiovascular events in patients with PCOS. Platelets play an important role in thrombogenesis, they are sites of insulin action and can be subject to variation in insulin sensitivity. Thus platelet dysfunction is a parameter which can have adverse cardiovascular effects and thus needs to be evaluated. This study included obese and non obese patients with PCOS and studied MPV levels in these patients.
Objectives: To study mean platelet volume in patients of polycystic ovarian syndrome and it's correlation with body mass index. Material and Methods: A total of 30 newly diagnosed cases of PCOS (using Rotterdam criteria) and 30 age matched healthy controls were included in this study. Weight and height were measured to calculate the BMI. Complete blood count including MPV, fasting sugar, insulin, HOMA2-IR, hormonal analysis including free and total testosterone, prolactin, 17 hydroxyprogesterone (17-OHP), TSH were done for cases as well as controls. Prolactin, 17-OHP and TSH were ascertained to detect chronic disease other than PCOS and to select suitable candidates. Cases and controls groups were stratified according to their BMI into obese and non obese sub groups respectively. MPV and hormonal parameters were compared between the PCOS group and controls as well as the sub groups. MPV was correlated with BMI, insulin resistance, HOMA2-IR, free and total testosterone Results: Mean values of MPV, Free Testosterone, fasting insulin, HOMA2-IR were significantly higher in the PCOS group as compared to controls. These parameters were higher in the obese PCOS subgroup as compared to control subgroup as well. In addition, MPV showed positive correlation with BMI and free testosterone levels. Conclusions: The elevated MPV might be considered to be a marker of increased cardiovascular disease risk in patients with PCOS.

\section{P-PD 02}

\section{Rifampicin Induced Thrombocytopenia}

Dr Jagveer singh", Dr Sushil yadav, Prof Kailash kumar, Dr Nilesh kumar, Dr Pankaj Kannauje

Email ID for Correspondence: jagveer21@gmail.com Institute of Medical Sciences, BHU

Introduction: Tuberculosis being very common disease in India. Most of the anti-tuberculosis drugs (ATT) has side effects. Rifampicin being essential component of ATT. Adverse effects being hepatotoxicity, skin rash, git symptoms, and very rarely potentially life threatning complication thrombocytopenia. Objectives: patient60 years male with history of pulmonary tuberculosis 3 years back for which he took DOTS regimen for 6 months now presented with cough with expectoration, fever, for 1 months. No history of hemoptysis, loss of consciousness, seizures, headache, malena, per rectal bleeding. On examination- pulse 98/min, BP- 116/78 mm hg, resp rate 18/min, chest-Crepitations in left upper zone apical region. Chest $\mathrm{x}$-ray and sputum for AFB s/o active pulmonary tuberculosis. HIV- negative, gene expert negative. Material and Methods: Patient was started with ATT (HRZES) on daily basis. On day 2 patient complained of oral mucosal bleed. CBC s/o low platelets $25000 / \mathrm{mi}$ crolitre. Possibility of drug induced thrombocytopenia was kept and ATT stopped. Patient was managed conservatively, on $4^{\text {th }}$ day platelets 60000, ATT again started and patient developed mucosal bleed, platelets 15000/microlitre. Again ATT stopped and methyl-prednisolone started at $1 \mathrm{mg} / \mathrm{kg}$ and platelets showed rising trend. Third time only rifampicin stopped and other ATT continued along with Moxifloxacin, after which he didn't developed low platelets count. Results: ATT was continued for 6 months. Rifampicin was not added to regimen and steroids were tapered and finally stopped within few weeks when hemogram was normal with adequate platelets count. Conclusions: Rifampicin induced thrombocytopenia is rare etiology of thrombocytopenia. It Is rare but can be life threatening. Early recognition and cessation of medication might prevent from grave outcome. 


\section{P-PD 03}

Immune Thrombocytopenia is the Commonest Diagnosis on Consultative Hematology - A Single Centre Experience

Ram V. Nampoothiri MD\#, Charanpreet Singh MBBS, Kundan Mishra MD, Aditya Jandial MD, Deepesh Lad DM, Gaurav Prakash DM, Alka Khadwal MD, Pankaj Malhotra MD, Neelam Varma MD, Subhash Varma MD

Email ID for Correspondence: ramvnampoothiri@gmail.com Post Graduate Institute of Medical Education \& Research, Chandigarh

Objectives: Thrombocytopenia is one of the commonest reasons for hematology consultation. We assessed prospectively prevalence, etiology \& clinical features of admitted patients for whom hematology consultation was taken for thrombocytopenia. Methods: All patients seen in hematology consultation for thrombocytopenia were included and baseline parameters, baseline investigations, department from where call was made and etiology of thrombocytopenia, transfusions received were analysed. Results: Out of 277 patients who had a hematology consultation over a period of two months, $88(31.77 \%)$ were for thrombocytopenia. The median age of these 88 patients was 30 years (22-53), 62.5\% were female, and median platelet of 40500/ $\mu \mathrm{L}$ (5500-65000). Mild, moderate \& severe thrombocytopenia was seen in $6.8 \%, 27.3 \% \& 65.9 \%$ patients respectively. $22.7 \%$ consults were in emergency ward, $25 \%, 23.9 \%$ \& $28.4 \%$ in medical, surgical $\&$ gynecology wards respectively. Fifty percent of patients had primary hematological diagnosis. ITP was the commonest diagnosis (38.6\%). DIC (11.4\%) was commonest non hematological cause for consultation. $9.1 \%$ of consultations each were for drug induced thrombocytopenia \& pseudo-thrombocytopenia. Etiology could not be ascertained in $4.5 \%$ of consultations. Bleeding manifestations were present in $48.9 \%$ patients with $20.5 \%$ having major bleed. Conclusions: One third of hematology consultations in a tertiary care hospital are for thrombocytopenia. Almost in half, the etiology of thrombocytopenia is related to a primary hematological disorder. This information should help in decision making of use of appropriate resources.

\section{P-PD 04}

\section{Management of Subdural Hematoma in Adults with Immune Thrombocytopenia}

\section{Prasad R. Koduri (Presenting Author)}

Mahavir Hospital \& Research Center, Mahavir Marg, Hyderabad

Purpose: Subdural hematoma is an extremely rare complication of immune thrombocytopenia in adults. Guidelines for the management of subdural hematoma complicating immune thrombocytopenia (ITP$\mathrm{SDH})$ do not exist. Reports of adult patients with ITP-SDH were reviewed to better understand the management of this complication. Methods: A search of the literature for reports of ITP-SDH was done using Pubmed database, Google Scholar and relevant cross-references using search terms subdural hematoma, intracranial hemorrhage and immune thrombocytopenia. The clinical and imaging data, treatment and outcome was recorded in each case. Also included are cases of four patients with ITP-SDH treated by the authors. Results: A total of 40 adults with ITP-SDH have been reported to date. Reports of three patients could not be accessed and are not included in the analysis. Including our four patients, 41 patients with ITP-SDH are analyzed. Since 1998 a majority ( 24 out of $30,80 \%$ ) of patients were managed with medical therapy. Intravenous immunoglobulin was used in a minority of patients (20\%) during these years. All patients had good outcome except for three. One was brought in decerebrate and died in 24 hours and the second patient died of lupus nephropathy and severe metabolic acidosis; residual neurologic deficit was noted in a single patient treated surgically. Conclusion: Chronic subdural hematoma is a circumscribed, self-perpetuating inflammatory process involving the dura mater that develops following minor trauma. The anti-inflammatory and anti-VEGF effects of steroids impair the formation and expansion of the dural neomembrane and aid in the resolution of the subdural hematoma. Available data support conservative therapy with steroids, platelet transfusions and intravenous immunoglobulin in ITP-SDH. Operative intervention is reserved for patients with progressive neurologic deficit or poor response to conservative therapy. The disproportionate number of patients with ITP-SDH reported from India (26 of 41 cases) lacks a clear explanation.

\section{P-PD 05}

\section{Dapsone; An Excellent Second -line Drug in ITP \\ Dr Meera. $\mathbf{V}^{\#}$}

Email ID for Correspondence: meerachakra@yahoo.com Department of Clinical Haematology, Bangalore Medical College and Research Institute, Bangalore

We retrospectively analysed 20 patients of ITP who were treated with dapsone. $14(70 \%)$ of them showed a response and $9(45 \%)$ showed a Complete Response. The median interval between diagnosis of ITP and dapsone therapy was 25.15 months. The mean pre-treatment platelet count was $15,250 \pm 6810.83 /$ cumm. The average time for response was 30.35 days (range 11-60 days) and the average duration of treatment with dapsone in responders was 9.92 months (range 4-30). The mean post-treatment platelet count in responders was $1,18,142.85 \pm 49726.19 /$ cumm. Side effects requiring discontinuation of therapy were observed in three $(15 \%)$ patients. Thus the study suggests that dapsone is a safe and effective second-line agent for steroid-dependent or refractory ITP patients.

\section{P-PD 06}

A Study on Haematological Manifestations in Pulmonary Tuberculosis Pre and Post Treatment (DOTS)

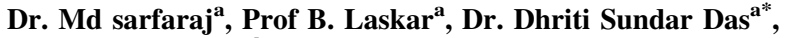
Prof R K Hazarika ${ }^{b}$, Dr B N Mahanta ${ }^{a}$

${ }^{\text {a }}$ Department of Medicine, Assam Medical College \& Hospital, Dibrugarh; ${ }^{b}$ Department of Pathology, Assam Medical College \& Hospital, Dibrugarh

Dhriti Sundar Das (Corresponding author)

Introduction: Tuberculosis is a major public health problem in India. Haematological changes associated with tuberculosis have been incompletely investigated. There is no comprehensive study assessing the haematological abnormalities in these patients from this part of the country. In the present study, peripheral blood and bone marrow findings in patients with pulmonary tuberculosis were analysed. An attempt has also been made to assess the effect of antituberculosis therapy on the haematological abnormalities.

Materials and methods: Fifty one patients with pulmonary tuberculosis were prospectively studied (from Sept'10 to Aug'11) to determine the various haematological manifestations in tuberculosis 
and the effect of antituberculosis therapy. All patients received standard antituberculosis treatment. They were subjected to a detailed hemogram including peripheral blood examination, which was repeated on completion of antituberculosis therapy. Bone marrow aspiration was also done in all patients before starting antituberculosis treatment.

Results \& Observation: Microcytic hypochromic anaemia was the most common abnormality observed (54.9\%) followed by normocytic hypochromic $(45.1 \%)$. Other haematological abnormalities of the white blood cells include leucopenia (9.8\%), neutropenia $(5.9 \%)$, lymphocytopenia $(19.6 \%)$, monocytopenia $(52.9 \%$; $\mathrm{p}<0.000)$, leucocytosis (15.7\%), neutrophilia (15.7\%) and lymphocytosis (3.9\%). Pancytopenia was observed in only 2 patients (2.4\%). Thrombocytopenia was rare. Bone marrow showed granulomas (1.96\%), AFB $(1.96 \%)$ and megaloblastosis. The hemogram improved with antituberculosis therapy in these patients. Conclusion: The present study suggest that tuberculosis can present with a spectrum of haematological manifestations and most common manifestation was the microcytic hypochromic anemia. Prominent haematological abnormalities associated with tuberculosis patients improved substantially with DOTS.

\section{P-PD 07}

Identification and Classification of Thrombotic Thrombocytopenic Purpura patients

\section{Ms. Sneha Yadav", Dr. Shrimati Shetty, Dr. Bipin Kulkarni}

Email ID for Correspondence: snehayadav69@yahoo.in National Institute of Immunohaematology- ICMR, Parel, Mumbai

Introduction: Thrombotic microangiopathy (TMA) shows presence of thrombocytopenia and hemolytic anemia leading to small blood vessel thrombosis, accompanied by varying degrees of organ dysfunction. Thrombotic Thrombocytopenic Purpura (TTP) is caused due to deficiency of von Willebrand factor cleaving protease ADAMTS13, which could be genetic, or acquired resulting from production of inhibitory autoimmune anti-ADAMTS13 antibodies. Objectives: To conduct detailed and comprehensive study of various inherited and acquired parameters involved in the pathogenesis of TTP. Material and Methods: Patients with clinical diagnosis of TTP were tested for plasma ADAMTS13 activity by immunofluorescence, plasma concentration of ADAMTS13 auto antibody was estimated by ELISA and genetic analysis of ADAMTS13 gene by direct DNA sequencing of the 29 exons and their flanking intronic regions by Sanger sequencing. Variants were analyzed using Polyphen, SIFT, Panther and Mutation Taster. Results: 45 TTP-HUS suspects were screened. 19 patients had low ADAMTS13 Activity, of which 16 patients had autoantibody against ADAMTS13 (Table 1).

- 3 patients had low ADAMTS13 activity and absence of inhibitor so ADAMTS13 gene for these patients was sequenced and mutations were found (Table 2).

- A237Gfs*153 and G231C are novel mutations (GenBank. Accession nos. KY924825 and MF630952).

Conclusions: In this study, 16 Acquired TTP and 3 Congenital TTPs were Characterized at clinical and molecular level. There is a need to establish comprehensive evaluation of TTP, which will help in early laboratory \& molecular characterization of the disorder, aiding in better prognosis as well as optimum and early treatment to the patients.
Table 1 Classification of cases of acquired TTP based on clinical details.

\begin{tabular}{lllll}
\hline $\begin{array}{l}\text { Sr. } \\
\text { No. }\end{array}$ & $\begin{array}{l}\text { Clinical } \\
\text { Manifestation }\end{array}$ & $\begin{array}{l}\text { No. of } \\
\text { cases }\end{array}$ & $\begin{array}{l}\text { Relapse } \\
\text { cases }\end{array}$ & $\begin{array}{l}\text { No. of patients } \\
\text { deaths }\end{array}$ \\
\hline 1 & $\begin{array}{l}\text { Pregnancy } \\
\text { associated TTP }\end{array}$ & 2 & 1 & 1 \\
2 & $\begin{array}{c}\text { Cancer associated } \\
\text { TTP }\end{array}$ & 3 & 1 & 0 \\
3 & $\begin{array}{c}\text { Dengue associated } \\
\text { TTP }\end{array}$ & 1 & 1 & 1 \\
$4 \quad \begin{array}{c}\text { Other Secondary } \\
\text { TTP }\end{array}$ & 10 & 4 & 1 \\
\hline
\end{tabular}

Table 2 ADAMTS13 Activity and Mutation Analysis in cases of congenital TTP

\begin{tabular}{|c|c|c|c|c|c|c|}
\hline $\begin{array}{l}\text { Sr. } \\
\text { No. }\end{array}$ & $\begin{array}{l}\text { ADAMTS13 } \\
\text { Activity } \\
\text { (Ref Range: } \\
50-150 \% \text { ) }\end{array}$ & $\begin{array}{l}\text { ADAMTS13 } \\
\text { auto-Ab (Ref } \\
\text { Range: }< \\
9.8 \mathrm{AU} / \mathrm{ml})\end{array}$ & Mutation & $\begin{array}{l}\text { Nucleotide } \\
\text { change \& } \\
\text { Exon }\end{array}$ & $\begin{array}{l}\text { Amino } \\
\text { acid } \\
\text { change }\end{array}$ & $\begin{array}{l}\text { SIFT/ } \\
\text { Provean } \\
\text { Prediction }\end{array}$ \\
\hline 1 & Reduced & 6.47 & $\begin{array}{l}\text { Insertion } \\
\quad \text { Mutation }\end{array}$ & $\begin{array}{l}\text { c.708_709 } \\
\text { insG } \\
\text { Exon } 7\end{array}$ & $\begin{array}{c}\text { A237Gfs } \\
* 153\end{array}$ & Deleterious \\
\hline 2 & 3.71 & 9.16 & $\begin{array}{l}\text { Missense } \\
\quad \text { Mutation }\end{array}$ & $\begin{array}{c}\text { c. } 1852 \mathrm{C} \\
>\mathrm{G} \\
\text { Exon } 16\end{array}$ & P618A & Deleterious \\
\hline 3 & 8.27 & - & $\begin{array}{l}\text { Missense } \\
\quad \text { Mutation }\end{array}$ & $\begin{array}{r}\text { c.691G } \\
>\mathrm{T} \\
\text { Exon } 7\end{array}$ & $\mathrm{G} 231 \mathrm{C}$ & Deleterious \\
\hline
\end{tabular}

\section{P-PD 08}

Study of Antiphospholipid Antibody in Patients with Acute Immune Thrombocytopenia

\section{Divya $M^{\#}$, Pahuja S, Sharma S, Chandra J, Goel A}

Email ID for Correspondence: its4div@yahoo.com Lady Hardinge Medical College

Introduction: Immune thrombocytopenia (ITP) is an autoimmune disease characterised by peripheral thrombocytopenia, with a normal or increased number of megakaryocytes in bone marrow, in the absence of splenomegaly. Thrombocytopenia is observed in around $30 \%$ cases of anti phospholipid syndrome. Antiphospholipid antibodies (Lupus Anticoagulant, Antibody to Anticardiolipin and Beta 2 glycoprotein) are also demonstrated among $23-73 \%$ of ITP patients. Objectives: To study lupus anticoagulant, anti cardiolipin antibody and beta 2 glycoprotein antibody among patients of acute immune thrombocytopenia. Material and Methods: Thirty one newly diagnosed Immune thrombocytopenia patients between the age group 2 years to 50 years of age were included in the study. All the cases at the time of diagnosis were subjected to $\mathrm{CBC}$ with platelet count, peripheral smear, PT and APTT tests. Antiphospholipid Antibody (APLA) were tested using lupus anticoagulant test (PTT-LA test or dRVVT) and ELISA to detect anticardiolipin antibody (IgG, IgM) and Beta 2 Glycoprotien I Antibody (IgG, IgM). Tests were repeated at third month to confirm APLA positivity. Results: The age group of the study population ranged from 3 years to 48 years with mean age 
of 14.78 years. The maximum number of cases, twelve out of thirty one $(38.71 \%)$ belonged to the age group 7-12 years. Male to female ratio was $1.38: 1$. Petechiae was the most common bleeding manifestation $(87.09 \%)$. Other bleeding manifestations were ecchymosis (84\%), epistaxis $(51.6 \%)$, melena $(19.4 \%)$, hematuria $(9.7 \%)$ and subconjunctival haemorrhage $(3.2 \%)$. Platelet count at initial presentation ranged from $3000 / \mu \mathrm{L}$ to $90,000 / \mu \mathrm{L}$ with a mean platelet count of $17,700 / \mu \mathrm{L}$. Seventeen cases out of $31(54.83 \%)$ showed initial platelet count below $10,000 / \mu \mathrm{L}$. Twenty nine out of 31 cases had platelet count below 50,000/ $\mu \mathrm{L}(93.54 \%)$. Fourteen out of 31 cases $(45.2 \%)$ showed persistence of thrombocytopenia. APLA positivity was observed in a total of $19.35 \%$ of cases. Lupus anticoagulant positivity was observed in $3 / 31$ (9.68\%). ACL antibodies and Anti- $\beta 2$ - glycoprotein antibodies were observed in $1 / 31(3.22 \%)$ and $3 / 31(9.68 \%)$ cases respectively. None of these cases retained APLA positivity after 12 weeks duration. There was no statistically significant difference in age, gender, bleeding symptoms, initial platelet count among APLA positive and negative groups. There was no significant association between APLA positivity and persistence of thrombocytopenia. ( $\mathrm{p}$ value $=0.517$ ). Conclusions:, The transient presence of APLA antibodies in few patients of ITP could be due to immunological perturbations in the natural course of ITP. None of the cases showed any evidence of thromboembolism during the follow up period. Due to shorter follow up, correlation of these antibodies with thromboembolic events could not be judged. More extensive studies with larger number of patients and longer follow up period are required to assess the clinical significance of APLA positivity in ITP patients.

\section{P-PD 09}

\section{Inflammatory Markers and Platelet Indices in Preeclampsia}

Taruna Bansal ${ }^{\#}$, Pallavi Sinha, Priyanka Gogoi, Bindiya Gupta, Preeti Diwaker

Email ID for Correspondence: taruna.pathology@gmail.com University College Of Medical Sciences

Introduction: Hypertensive disorders of pregnancy complicates $5-11 \%$ of all pregnancies, of which preeclampsia occurs in $3-5 \%$ of all gestations. ACOG defines preeclampsia as blood pressure elevation $>140 / 90 \mathrm{~mm} \mathrm{Hg}$ on two occasions 4 hours apart after 20 weeks of gestation with proteinuria or any other severe features of preeclampsia such as impaired liver function, thrombocytopenia, new development of renal insufficiency, pulmonary edema, cerebral or visual disturbances. Owing to hyper activation of inflammatory and immunologic responses in preeclampsia, there is marked increase in the neutrophil count. Preeclampsia is also known to cause platelet activation manifesting as low platelet count and high mean platelet volume (MPV). Objectives: To compare the values of inflammatory markers such as neutrophil lymphocyte ratio (NLR) and platelet lymphocyte ratio (PLR) in women with preeclampsia and healthy pregnant women to determine the association of platelet indices $\{$ MPV, Platelet distribution width (PDW) and plateletcrit (Pct) $\}$ and preeclampsia if any. Material and Methods: This prospective case control study was conducted on 65 pregnant women having preeclampsia and 65 age matched healthy pregnant females. Complete blood count were analysed on an automated hematology analyser (AB micros E360, Horibamedical). Differential counts were done on peripheral smear. Results: NLR was significantly higher in women with preeclampsia (Mean \pm SD $6.8 \pm 7.6$ ) compared to healthy pregnant females (Mean \pm SD $3.0 \pm 0.98, \mathrm{p}<0.001)$. There was a significant increase in PLR $(\mathrm{p}=0.01)$ and MPV $(\mathrm{p}=0.03)$ in patients with preeclampsia compared to healthy pregnant controls.
There was a significant decrease in platelet count in cases (Mean $\pm \mathrm{SD} 188 \pm 89.7$ ) as compared to controls (Mean $\pm \mathrm{SD}$ $200.1 \pm 62.36$ ). However, there was no significant difference in Pct and PDW. Conclusions: NLR, PLR and MPV are significantly increased in preeclampsiaand might serve as useful tool in screening pregnant females for same.

\section{P-PD 12}

Platelet Aggregation Study in Chronic Renal Failure Patients Pre And Post Hemodialysis

Gupta AK ${ }^{\#}$, Khonglah Y, Raphael V, Marbaniang E, Jamil Md*, Lyngdoh $M *$ (Department of Pathology and *Department of General Medicine)

Email ID for Correspondence: akguptamb090005@gmail.com NEIGRIHMS, Shillong, Meghalaya

Introduction: Chronic renal failure (CRF) typically corresponds to stage 3-5 CKD. Patients with end-stage renal disease (ESRD) develop hemostatic disorders mainly in the form of bleeding diatheses. Patients undergoing hemodialysis may show both thrombotic complications and bleeding abnormalities. Hemostatic changes in patients on hemodialysis may result from alterations in vessel wall integrity, platelet function, and reduced blood flow in the native arteriovenous fistula. The normal platelet response to vessel wall injury with platelet activation, recruitment, adhesion, and aggregation is defective in advanced renal failure. Hemodialysis may partially correct these defects, but cannot totally eliminate them. It is suggested that abnormal platelet function is a major contributor, since haemorrhage occurs despite a coagulation profile of normal or elevated levels of coagulation factors and normal platelet counts.

Objectives:

1. Primary objective: Platelet aggregation study in chronic renal failure (CRF) patients in both pre and post hemodialysis samples.

2. Secondary objective: To study the complete hematological profile and coagulation profile including PT, APTT, INR, Fibrinogen in CRF patients pre and post hemodialysis

Material and Methods: Total 27 subjects were selected for the study. Among them 20 were chronic renal failure patients on hemodialysis and 7 samples were from normal healthy subjects which served as controls.

1. Platelet aggregation study using Optical platelet aggregometer (BORN principle)

2. Complete Hemogram using fully automated BECKMAN COULTER LH 750 hematology analyser

3. Coagulation profile including (PT, APTT, INR, Fibrinogen) using fully automated STAGO coagulation analyser

\section{Results:}

1. Platelet aggregation study :- Using the paired sample $-t$ test the difference between the mean percentage of platelet aggregation in CRF patients pre and post hemodialysis for ADP, arachidonic acid, collagen and epinephrine showed statistical improvement in post hemodialysis samples. $(\mathrm{P}<0.05)$

2. Coagulation profile study - There was significant increase in APTT and fibrinogen post hemodialysis in CRF patients $(\mathrm{p}<0.05)$.

3. 3.Hematological profile - There was a slight improvement in hemoglobin levels in CRF patients post hemodialysis ( $\mathrm{p}<0.05$ ). Out of the 20 cases of CRF, 13 showed normocytic normochromic anemia, 4 showed dimorphic anemia and 3 showed microcytic hypochromic anemia. 
Conclusion: In this study, there was significant improvement in platelet aggregation in CRF patients in post hemodialysis samples. Few of the possible reasons for this finding have been put forward in this study, which needs to be further validated by future studies on the same.

\section{P.PD 13}

\section{Evaluation of Platelet Indices and It's Role in Pre-Eclampsia And Eclampsia}

Dr. Samatha Kurapati (PGY2) ${ }^{\#}$, Dr. Naga Kalyani (Assit.Prof), Dr. Ezhil Arasi.N (Professor\&HOD)

Email ID for Correspondence: samathakurapati@gmail.com Osmania Medical College

Introduction: Hypertension is one of the common medical complications of pregnancy and contributes significantly to maternal, perinatal morbidity and mortality. The dysfunction of blood coagulationfibrinolysis system is a salient characteristic feature of pre-eclampsia that varies in severity, require different treatments. Therefore, it is necessary to find suitable predictors for the onset and severity of preeclampsia. Objectives: To evaluate the relationship between platelet indices \& platelet counts with pre-eclampsia, eclampsia. Material and Methods: 22 cases of pre-eclampsia \& 18 cases of eclampsia diagnosed between June - September 2017. Blood samples were analysed by sysmex 1000 automated haematology analyser. Platelet count, MPV, PDW, PL-LCR compared. Results: The platelet counts are lower while mean platelet volume, platelet distribution width \& platelet large cell ratio were increased in pre-eclampsia \& eclampsia as compared to control groups. Conclusions: The present study observes the relationship between platelet indices \& severity of preeclampsia. Therefore estimation of platelet indices can be considered as an early, simple, \& rapid procedure in assessment of severity of pre-eclampsia and to prevent progression to HELLP syndrome \& DIC.

\section{P-PD 14}

\section{Thrombocytopenia in Children Admitted to Intensive Care Unit}

Dr. Ranjani Upadhyay ${ }^{\#}$, Dr. Suneel .C. Mundkur, Dr. ShriKiran Hebbar, Dr. Nalini Bhaskaranand, Dr. Pushpa G Kini

Email ID for Correspondence: ranjani.upadhyay@gmail.com Kasturba Medical College, Manipal

Introduction: Thrombocytopenia is a common complication among children admitted to Paediatrics intensive care unit and is a risk factor for major bleeding manifestation, prolonged length of ICU or hospital stay, and mortality. Prevalence of thrombocytopenia varies from $13 \%$ to $58 \%$ among different ICUs setting. The advantage of using platelet count as a predictor of ICU outcome, is the dynamic nature of daily platelet count which takes the disease progression into account in contrast to various mortality scores which use only the worst parameters within $24 \mathrm{hrs}$ after admission or at admission. Objectives: To study clinical profile and outcome of children admitted with thrombocytopenia in intensive care unit. To study platelet transfusion practices employed in intensive care unit. Material and Methods: It is a prospective and retrospective observational study done in 150 children admitted to paediatric intensive care unit with thrombocytopenia. Clinical profile and outcomes of children with thrombocytopenia were studied.Platelet count done on $1^{\text {st }}$ day and $4^{\text {th }}$ day of admission were analyzed between survivors and non survivors .Platelet transfusion practices followed in PICU were studied. Results: Among 150 children infection was most common cause of thrombocytopenia followed by sepsis. No statistical difference was noted in platelet count and platelet indices at admission among survivors and non survivors. Rise in platelet count among survivors and non survivors on $4^{\text {th }}$ day of hospitalization was $79.1 \%$ and $15.9 \%$ respectively. Platelet transfusion trigger among children with and without bleeding manifestation 18500 (IQR 31000, 14000) and 20500 (IQR 25750, 11000) respectively. This study illustrates that failure of rise in platelet count on $4^{\text {th }}$ day of admission was significantly associated with mortality. ( $\mathrm{p}$ value .001 ).

\section{P-PD 15}

\section{Thrombocytopenia in Iron Deficiency Anemia \\ D V Phaneendra\# ${ }^{\#}$ Chethan Manohar}

Email ID for Correspondence: drphaneendradatari@outlook.com Kasturba Medical College, Manipal

Introduction: Iron deficiency anemia is a regular encounter to a physician day in and day out, and is easily treatable with oral iron supplements. Iron deficiency anemia is frequently associated with reactive thrombocytosis of mild to moderate degree. Though less frequently, cases of thrombocytopenia associated with iron deficiency anemia are also being reported worldwide, the causes of which are thought to be the dual deficiency and immune thrombocytopenia. However, rapid correction of thrombocytopenia following iron replenishment have also been reported. Objectives: To evaluate the proportion of iron deficiency anemia cases associated with thrombocytopenia and explain the possible etiology. Material and Methods: We took 1000 cases of iron deficiency anemia with an inclusion criterion of low ferritin $(<11.1$ in females and $<23.9$ in males $)$ and evaluated the platelet parameters. Thrombocytopenia was seen in 45/1000 cases. Biochemistry values, RBC \& platelet indices, peripheral smear, Bone marrow findings and DAT when available, along with a follow-up CBC (1 week - 1 month), final diagnosis and the treatment course from the patient records were evaluated. Results: Among the cases with thrombocytopenia, the Chronic liver disease was seen in $18 / 45$ cases (40\%), the Dual deficiency was seen in $6 / 45$ cases (13.3\%), Immune thrombocytopenia was seen in $6 / 45$ cases (13.3\%). 10/45 cases $(22.2 \%)$ had no other findings except iron deficiency, similar to the studies of Morris et al., Cunha et al., Lopas \& Rabiner. Follow-up count in this group (available in $4 / 10$ cases) showed improvement in 3 cases with mere iron supplements similar to the above studies. Improvement in platelet count was seen in $18 / 45$ cases, of which 14 cases $(77.8 \%)$ received iron supplements along with disease-specific treatment. Conclusions: It is proposed that iron deficiency itself causes thrombocytopenia in a significant proportion of cases; in this study, it was seen more than in the dual deficiency. Iron affects thrombopoiesis in mechanisms similar to those described by Evastiev et al. and supplementation of iron has a beneficial effect on platelet count irrespective of the etiology.

\section{P-QCH 01}

Optimizing the Use Ofpatient Samples in Conjunction with Commercial Controls for Internal Quality Control and Inter Laboratory Quality Assessment of Hematology Analyzers 


\section{Dr Rakhee Kar}

G H Jhansi Priya (Presenting Author) JIPMER

Introduction: Quality control (QC) measures are essential in hematology analyzers to ensure desired result from every blood sample. It is not always feasible to use commercial controls as they are expensive and have a limited shelf life. An alternative is to use patient sample for quality measures especially in a resource limited setup. Materials and Methods: This study was performed in a tertiary care hospital in southern India. The participating laboratories were those performing complete blood counts by using cell counters and side labs performing Hemoglobin $(\mathrm{Hb})$ estimation by Sahli's or Drabkin's method. The QC tests performed using patient samples were:

a. Daily -Average of numbers (AON) which was compared with Levy-Jennings (LJ) charts using commercial controls

b. Weekly - Replicate Test (RT), Duplicate Test (DT), Correlation Check (CC) and Weekly AON

c. Monthly- Deviation Index (DI).

Results: In daily AON, the graphs of RBC indices were comparable with LJ charts of commercial controls. The co-efficient of variation (CV) in RT for low, normal and high levels were $1.06,0.69,0.58$ for $\mathrm{Hb}, 2.28,1.16,1.23$ for WBC count and 6.66, 2.22, 1.61 for platelet count. The precision was higher for $\mathrm{Hb}$ and least for low platelet counts. In DT, an occasional random error was detected. In CC, few flags like thrombocytopenia, NRBCs did not show $100 \%$ correlation with peripheral smear. DI using z-score showed satisfactory performance of the participating labs. Conclusion: Use of patients' blood sample was helpful for internal QC, inter- method/inter- instrument and inter-laboratory comparability.

\section{P-QCH 02}

An Easy Way of Implementing Quality Indicators in HematoOncology Laboratory in a Tertiary Care Centre M Tiwari", P Chavan

Email ID for Correspondence: manik_tiwari@yahoo.co.in ACTREC, Tata Memorial Hospital, Navi Mumbai - 410210

Introduction: Assessing the quality of laboratory using quality indicators $(\mathrm{QI})$ or performance evaluators $(\mathrm{PE})$ requires a transparent, regular and sincere effort in collecting and analysing data generated during pre- analytical, analytical and post-analytical stages. QI data is collected periodically to identify, correct, and continuously monitor problems and improve performance of the laboratory. Hence it is necessary to identify and evaluate QIs so as to achieve an effective and procedural intervention in achieving good laboratory practices (GLP). Objectives: To identify, establish and evaluate QIs at all stages in hemato-oncology laboratory and to achieve the required set threshold, so as to provide a useful tool for continuous quality improvement in laboratory services. Material and Methods: QIs or PEs were identified as shown in table below of hemato-oncology laboratory. Required thresholds for all the QIs were planned. Continuous monitoring and achievement of QIs were planned on monthly basis so as to achieve GLP. We started in the month of May 2017 and henceforth the monthly QIs data were gathered and analysed statistically using excel sheet and histogram charts. Thus every month based on this evaluation study we planned our scope of improvement where the pitfalls were observed. Results: The table shows the result of monthly monitoring various QIs and its implication in improving the quality of laboratory services. 


\begin{tabular}{|c|c|c|c|c|c|c|c|c|c|c|}
\hline \multicolumn{4}{|c|}{$\begin{array}{l}\text { QUALITY INDICATORS (QI) OR } \\
\text { PERFORMANCE EVALUATORS (PE) }\end{array}$} & $\begin{array}{l}\text { Required } \\
\text { Threshold }\end{array}$ & $\begin{array}{c}\text { May } \\
17\end{array}$ & $\begin{array}{c}\text { June } \\
17\end{array}$ & $\begin{array}{c}\text { July } \\
17\end{array}$ & $\begin{array}{c}\text { August } \\
17\end{array}$ & $\begin{array}{c}\text { Sept } \\
17\end{array}$ & $\begin{array}{c}\text { Observed } \\
\text { Improvement }\end{array}$ \\
\hline \multicolumn{11}{|l|}{$\mathbf{A}$} \\
\hline \multirow[b]{3}{*}{ 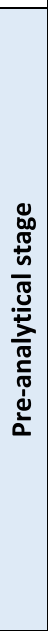 } & 1 & \multicolumn{2}{|c|}{ Incomplete requisitions } & 0 & 1 & 0 & 0 & 0 & 0 & 100 \\
\hline & 2 & \multicolumn{2}{|c|}{$\begin{array}{l}\text { Patient preparation before collection } \\
\text { - Resting posture } \\
\text { - Prevent prolonged tourniquet tying }\end{array}$} & Always & 0 & 0 & 0 & 0 & 0 & 100 \\
\hline & 3 & \multicolumn{2}{|c|}{$\begin{array}{l}\text { Sample rejections noted } \\
\text { - Clotted, labelling error, } \\
\text { - Quantity not sufficient, } \\
\text { - Anticoagulant: blood 1:9 ratio is not maintained, } \\
\text { - Heparin contamination noted post-analysis, } \\
\text { diluted sample noted post-analysis, } \\
\text { - Lipemic sample causing falsely elevated } \\
\text { haemoglobin results noted post-analysis, } \\
\text { - Hemolysed sample causing variation in RBC } \\
\text { indices, } \\
\text { - Brought beyond acceptance time, sample } \\
\text { - Expired vacutainer }\end{array}$} & $0 \%$ & 0.25 & 0.20 & 0.22 & 0.14 & 0.11 & $\begin{array}{l}\text { Gradual decrease in } \\
\text { sample rejection }\end{array}$ \\
\hline \multicolumn{11}{|l|}{ B } \\
\hline \multirow{15}{*}{ 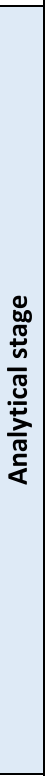 } & \multirow{12}{*}{1} & \multirow{6}{*}{$\begin{array}{l}\text { EQAS performance } \\
\text { (Z-score } \\
\text { evaluation) }\end{array}$} & (AlIMS) & \multirow{12}{*}{$\begin{array}{c}0 \text { outliers } \\
\text { (Z score } \\
\leq 2 / \leq 3 \& \text { no } \\
\text { trend) }\end{array}$} & 0 & - & - & - & 0 & \\
\hline & & & (Biorad) & & 0 & 0 & 0 & 1 & - & \\
\hline & & & PBS $\quad$ (AIIMS) & & 0 & - & - & - & 0 & \\
\hline & & & (AlIMS) & & - & - & - & - & 0 & \\
\hline & & & (CMC) & & - & - & 0 & - & - & \\
\hline & & & (Biorad) & & 0 & 0 & 0 & 0 & 0 & Overall satisfactory \\
\hline & & \multirow{6}{*}{$\begin{array}{l}\text { EQAS: AIIMS, CMC, } \\
\text { BIORAD }\end{array}$} & (CMC) & & - & - & 0 & - & - & Performance in EQAS \\
\hline & & & (Biorad) & & 0 & 0 & 0 & 0 & 0 & \\
\hline & & & APTT $\quad$ (CMC) & & - & - & 0 & - & - & \\
\hline & & & (Biorad) & & 0 & 0 & 0 & 0 & 0 & \\
\hline & & & Fibrinogen (CMC) & & - & - & 1 & - & - & \\
\hline & & & Fibrinogen (Biorad) & & 0 & 0 & 0 & 0 & 0 & \\
\hline & 2 & \multicolumn{2}{|c|}{$\begin{array}{l}\text { Internal Quality Control performance } \\
\text { (Improvement in \% Coefficient of Variation) }\end{array}$} & $\begin{array}{c}\text { outliers } \\
\text { (Decreasing } \\
\% \mathrm{CV})\end{array}$ & 0 & 0 & 0 & 0 & 0 & $\begin{array}{l}\text { Overall satisfactory } \\
\text { Performance in IQC }\end{array}$ \\
\hline & 3 & \multicolumn{2}{|c|}{$\begin{array}{l}\text { Interpretation of the flags displayed by cell counter } \\
\text { for presence of blasts cells, LUCs, pancytopenia, } \\
\text { giant platelets, platelet clumps by technical staff } \\
\text { for each CBC sample tested }\end{array}$} & \begin{tabular}{|c|}
0 wrong reports \\
transmitted \\
(Misinterpretation \\
may lead to wrong \\
reporting)
\end{tabular} & 0 & 0 & 0 & 0 & 0 & $\begin{array}{l}\text { Satisfactory } \\
\text { performance in } \\
\text { interpretation of flags }\end{array}$ \\
\hline & 4 & \multicolumn{2}{|c|}{$\begin{array}{l}\text { Calibration of cell counter done instantly as } \\
\text { required }\end{array}$} & $\begin{array}{c}\text { Done in } \\
\text { time }\end{array}$ & - & - & - & - & Done & $\begin{array}{c}\text { Calibration is done in } \\
\text { time }\end{array}$ \\
\hline \multicolumn{11}{|l|}{$\mathbf{C}$} \\
\hline \multirow{4}{*}{ 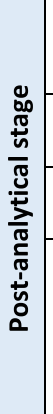 } & 1 & \multicolumn{2}{|c|}{ Number of wrong report released } & 0 & 0 & 0 & 0 & 0 & 0 & $\begin{array}{l}\text { No wrong reports } \\
\text { released }\end{array}$ \\
\hline & 2 & \multicolumn{2}{|c|}{ Percentage TAT achievement } & $100 \%$ & 81.3 & 87.2 & 93.1 & 94.9 & 96.1 & $\begin{array}{l}\text { TAT is achieved } \\
\text { effectively }\end{array}$ \\
\hline & 3 & \multicolumn{2}{|c|}{ Critical or alert values communicated instantly } & $100 \%$ & 95.2 & 97.2 & 99.5 & 100 & 100 & $\begin{array}{l}\text { Instant reporting } \\
\text { critical value }\end{array}$ \\
\hline & 4 & \multicolumn{2}{|l|}{ Delta check practice } & $\begin{array}{l}\text { Do delta } \\
\text { check for } \\
\text { all } \\
\text { abnormal } \\
\text { values }\end{array}$ & Done & Done & Done & Done & Done & $\begin{array}{l}\text { Doing delta check for } \\
\text { all abnormal values } \\
\text { prevents wrong } \\
\text { release of reports }\end{array}$ \\
\hline
\end{tabular}




\begin{tabular}{|c|c|c|c|c|c|c|c|c|c|c|}
\hline \multicolumn{4}{|r|}{$\begin{array}{l}\text { QUALITY INDICATORS (QI) OR } \\
\text { PERFORMANCE EVALUATORS (PE) }\end{array}$} & $\begin{array}{l}\text { Required } \\
\text { Threshold }\end{array}$ & $\begin{array}{c}\text { May } \\
17\end{array}$ & $\begin{array}{c}\text { June } \\
17\end{array}$ & $\begin{array}{c}\text { July } \\
17\end{array}$ & $\begin{array}{c}\text { August } \\
17\end{array}$ & $\begin{array}{c}\text { Sept } \\
17\end{array}$ & $\begin{array}{c}\text { Observed } \\
\text { Improvement }\end{array}$ \\
\hline \multicolumn{11}{|c|}{ Non conformities (NC) observed } \\
\hline & \multirow{3}{*}{1} & A & In routine work & Minimum & 0 & 0 & 0 & 0 & 0 & \multirow{3}{*}{$\begin{array}{l}\text { Minimum NCs } \\
\text { observed }\end{array}$} \\
\hline & & $\mathrm{B}$ & Internal audit & Minimum & - & - & 3 & - & - & \\
\hline & & C & NABL assessment & Minimum & 3 & - & - & - & - & \\
\hline \multirow{13}{*}{ 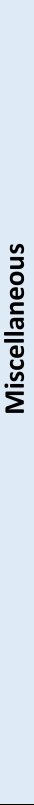 } & & & dback and suggestions by end users (Clinicians & nd patients) & & & & & & \\
\hline & & A & Number of good feedbacks & Many & 0 & 0 & 0 & 4 & 0 & Many good feedbacks \\
\hline & 2 & B & $\begin{array}{l}\text { Number of feedbacks where improvement is } \\
\text { required }\end{array}$ & Less & 0 & 0 & 0 & 1 & 0 & $\begin{array}{l}1 \text { feedback for } \\
\text { improvement }\end{array}$ \\
\hline & & C & $\begin{array}{l}\text { Percentage of feedbacks solved and } \\
\text { redressed }\end{array}$ & $100 \%$ & 0 & 0 & 0 & 5 & 0 & $100 \%$ \\
\hline & \multirow{6}{*}{3} & \multicolumn{9}{|c|}{ Staff performance } \\
\hline & & A & Percentage of planned internal CME achieved & $100 \%$ & 0 & 0 & 0 & 0 & 0 & 0 Internal CME seen \\
\hline & & B & $\begin{array}{l}\text { Number of staff attended National / } \\
\text { International CME }\end{array}$ & Many & 0 & 0 & 0 & 0 & 0 & $\begin{array}{l}0 \text { staff attending } \\
\text { outside } \mathrm{CME}\end{array}$ \\
\hline & & C & $\begin{array}{l}\text { Incident reported in form of } \\
\text { accident/exposure to biohazard or chemical } \\
\text { hazard }\end{array}$ & All & 0 & 0 & 0 & 0 & 0 & Good \\
\hline & & D & $\begin{array}{l}\text { Managements intervention for incident } \\
\text { reported in percentage }\end{array}$ & $100 \%$ & NA & NA & NA & NA & NA & NA \\
\hline & & $E$ & $\begin{array}{l}\text { Total number of staff suggestions received } \\
\text { and implemented }\end{array}$ & All & 0 & 0 & 0 & 0 & 0 & $\begin{array}{l}0 \text { staff suggestion } \\
\text { received \& } \\
\text { implemented } \\
\end{array}$ \\
\hline & \multirow{3}{*}{4} & \multicolumn{9}{|c|}{ Equipment performance } \\
\hline & & A & $\begin{array}{l}\text { Number of breakdowns of analytical } \\
\text { equipment }\end{array}$ & Minimum & 1 & 0 & 1 & 0 & 1 & $\begin{array}{l}\text { Minimum breakdown } \\
\text { of equipment }\end{array}$ \\
\hline & & $\mathrm{B}$ & Number of reagents rejected back to vendor & Minimum & 0 & 0 & 0 & 0 & 0 & 0 reagent rejection \\
\hline
\end{tabular}

After evaluating performance of hemato-oncology laboratory based on the above QIs, it was found that the laboratory is showing overall good performance in EQAS, IQC, TAT, Critical value reporting, sample rejections, equipment calibration and maintenance but few QIs needs attention like conducting regular internal CMEs and encouraging lab personnel for attending national or international CME to upgrade their knowledge. Also laboratory management must encourage staff suggestions and its implementation. Conclusions: Quality indicators or performance evaluators proved to be an effective tool for monitoring and establishing good laboratory practise.

\section{P-QCH 03}

Bull's Algorithm as an Effective Tool for Monitoring Systemic Errors in Hematology Cell Counters at Zero Cost

\section{Tiwari", T Mestry, P Chavan}

Email ID: manik_tiwari@yahoo.co.in ACTREC, Tata Memorial Center, Navi Mumbai

Introduction: Bull's algorithm or moving average analysis is an effective tool for monitoring quality control of haematology cell counters. In this method, by using patient's red cell indices (MCV, $\mathrm{MCH}$ and $\mathrm{MCHC}$ ) we can detect systemic errors associated with functioning of impedance channel, flow cytometer and spectrophotometer in any cell counter. The advantage of this algorithm is that, systemic error (SE) can be detected while processing the patient's samples instantly at zero cost. Objectives: To set an easy way for understanding the use of Bull's algorithm for detecting systemic errors in cell counters by using patients data at zero cost. Material and Methods: 400 samples of oncology patients analysed between 04/09/2017 to 08/09/2017) on Advia 2120i, a calibrated cell counter (calibrated on 02/09/2017) with IQC and EQAS results within acceptable limits were used to establish the target mean value and acceptable limits for red cell indices (MCV, $\mathrm{MCH}$ and $\mathrm{MCHC}$ ). The population of 400 samples constitute patients of Bone Marrow Transplant, solid tumour, haematological malignancies who are on chemotherapy or radiation therapy and show gross variations in CBC values. This population becomes a representative population for this analyser. The success of using this algorithm is based on assumption that same type of population will be analysed on this analyser in future also. Further by using excel sheet 20 batches were made and 20 mean values of red cell indices were obtained. These 20 mean values were correlated with each other for any gross outliers so that they can be excluded and few more patient data may be incorporated. In our study we didn't see any gross outliers in the 20 mean values. Further we calculated a target mean value by using the 20 mean values obtained earlier. Acceptable criteria of $3 \%$ were applied to the target value of MCV, MCH and MCHC. Finally we got a Levey-Jennings plot with target mean and acceptable lower-upper limits for MCV, $\mathrm{MCH}$ and $\mathrm{MCHC}$. These values were set in QC program of Advia2120i. After every 20 sample analysed on the analyser, a point is plotted on the Levey- Jennings chart. Monitoring of the plot was started. With every sample analysed, any outlier is instantly detected which may be a cause for systemic errors. This is why it is also known as moving average analysis. Results: After implementing Bull's algorithm for QC monitoring it was observed that $\mathrm{MCH}$ and $\mathrm{MCHC}$ plot showed outliers above the target mean on 22/09/2017 (Fig 1, 2). This may be a systemic error associated with the measurement of either haemoglobin or RBC count. So we tried to correlate this with our commercial control results of 22/09/2017, which also showed outlier for RBC count and MCH (Fig 3). Corrective action in terms of maintenance of $\mathrm{RBC}$ flow cell was done. This corrected the systemic error. On 26/09/2017 there was outlier observed in the Bull's algorithm which was again correlated with commercial control data which were within acceptable limits. This time we saw the data of patients 


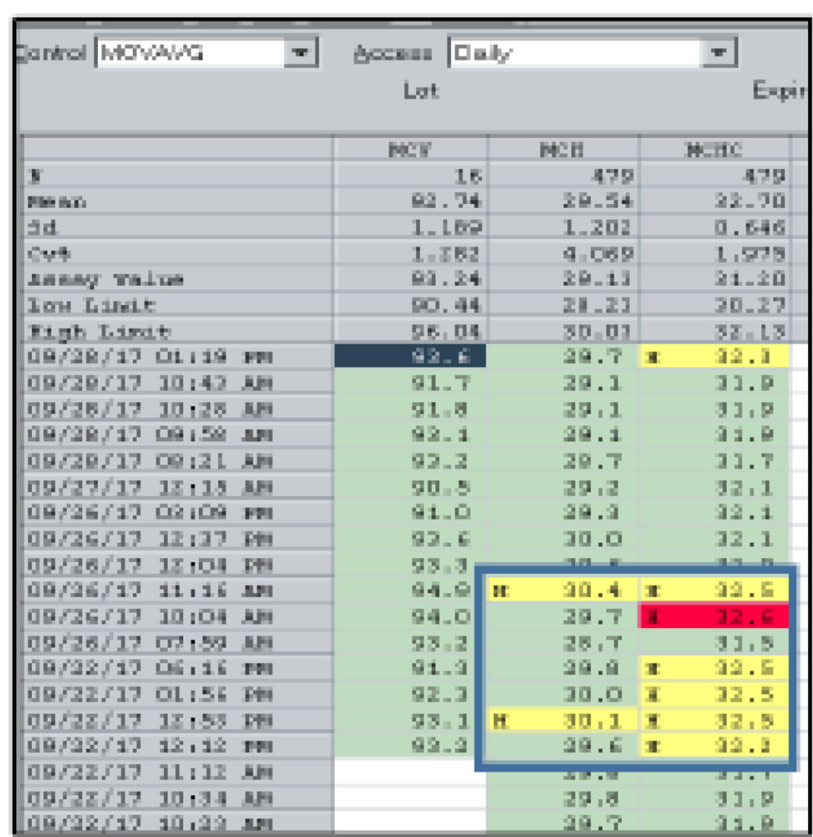

Figure 1 .

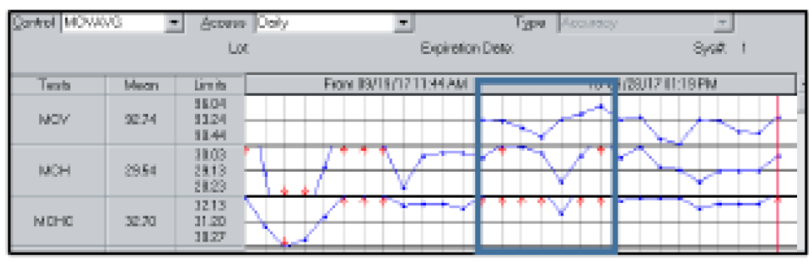

Figure 2 .

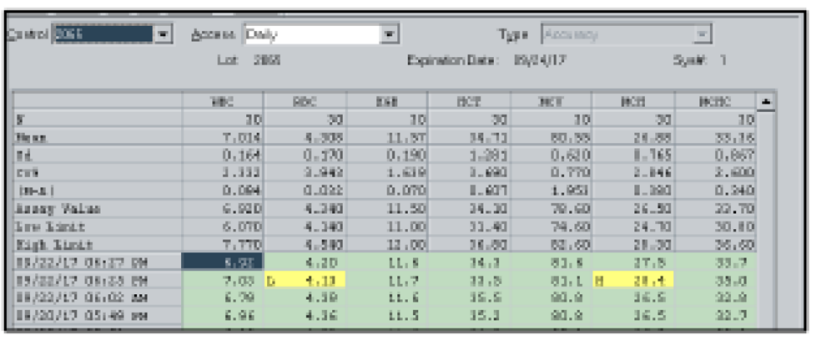

Figure 3 .

and found that the patient population was skewed which was the reason for the outlier observed. Conclusions: Bull's algorithm is an effective and instant tool for detection of systemic errors in haematology cell counters. However, some assumptions need to be considered, such as there should be consistent flow of same type of patient population; skewed patient population may lead to false outliers. So an outlier in Bull's algorithm must always be correlated with either commercial control results or the patient data must be reviewed before taking corrective action.

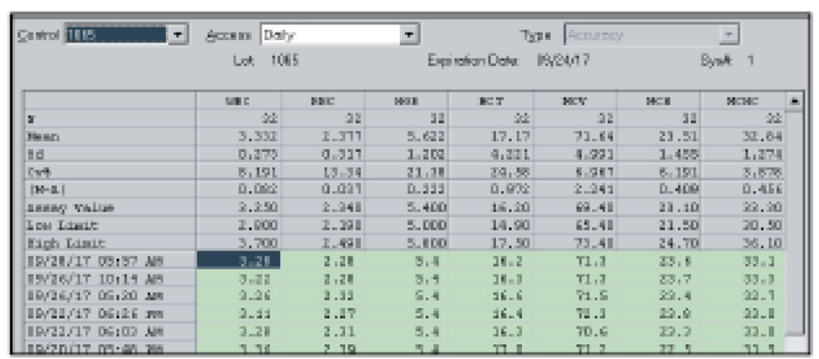

\section{P-RCD 01}

Effect of Maternal Iron Deficiency Anaemia on Blood Hemoglobin Levels and Iron Stores of Cord Blood

Dr M.V.Surekha\#, Dr P.Uday Kumar, Dr N.Balakrishna, M.Srinivas, Dr Tripurasundari and Dr Jijiya Bai

Email ID for Correspondence: surekha_mv@yahoo.com

National Institute of Nutrition (ICMR), Hyderabad, Telangana State

Introduction: Iron deficiency anaemia (IDA) is the most prevalent problem afflicting pregnant women and severe maternal iron deficiency has been shown to adversely affect neonatal iron status $[1,2]$. Although several studies have been done, some have reported negative impact of maternal IDA on iron stores of newborns while others could not find any relationship and the results are also inconsistent and inconclusive [3-8]. Hence, this study was planned to study the effect of maternal iron deficiency anaemia on cord blood iron status. Objectives:

1) To evaluate the severity of anaemia in maternal and cord blood.

2) To correlate mother's iron status to that of cord blood iron content.

Materials and methods:

Our study was carried out in 48 pregnant women, in their third trimester of pregnancy, reporting to the antenatal outpatient department (OPD) of Government Medical College and Hospital, Secunderabad, between the years 2013-2014 and included women of age 18 years and above, with singleton pregnancy and in third trimester of pregnancy. Women with history of hemolytic anaemia, hypertension, diabetes mellitus, Human immunodeficiency virus (HIV), Hepatitis C Virus (HCV), Hepatitis B surface antigen (HBsAg) positive and twin pregnancies were excluded from the study. Based on $\mathrm{Hb}$ levels and World health organization (WHO) standards, the pregnant women were categorized into mild, moderate and severe grades. Women with $\mathrm{Hb}>11.0 \mathrm{~g} / \mathrm{dl}$ were considered as normal. RBC indices as suggested by WHO [9] and Centers for Disease Control and prevention (CDC) for pregnant women were used [10]. Serum ferritin and serum transferrin receptors were estimated by enzyme-linked immunosorbent one-step process assay (ELISA). Statistical analysis was done by SPSS (Statistical package for Social Science) version 19. Descriptive statistics like mean, standard deviation (SD) and prevalence were calculated for all variables. Mean values for all variables were compared by unpaired 't' test across both normal and anemia groups. ANOVA and Post Hoc test of LSD were also performed. The level of significance was considered as 0.05 . Results: Among the 96 pregnant women recruited in our study, $40(41.7 \%)$ had normal $\mathrm{Hb}$ levels and the remaining $56(58.3 \%)$ had anaemia of varying grades with 20 women $(20.83 \%)$ presenting with mild anaemia, 18 women $(18.75 \%)$ with moderate anaemia and 18 women $(18.75 \%)$ with severe anaemia. Comparison of values of $\mathrm{Hb}$ and red cell indices between maternal and cord blood was done. In maternal blood most of the values were 
found to be significantly lower $(p<0.05)$ in anaemic mothers when compared with normal mothers. However, in contrast, cord blood from babies born of anaemic mothers had normal to slightly higher levels when compared to those of babies born to normal mothers. Significant correlations between maternal $\mathrm{Hb}, \mathrm{PCV}, \mathrm{MCV}, \mathrm{MCH}$, MCHC, RDW HDW and cord blood PCV, MCV, MCHC and ferritin were also observed. Conclusion: Our study showed that the foetus remains unaffected by maternal iron deficiency anaemia by maintaining adequate iron stores at the expense of maternal iron.

\section{References:}

1) Kalaivani K: Prevalence and consequences of anemia in pregnancy. Indian J Med Res 2009, 130:627-633.

2) Scholl TO. Iron status during pregnancy: setting the stage for mother and infant. Am J Clin Nutr.2005;81(5):1218S-1222S.

3) Fatin AS, Mamdooh G, Safaa Q, Nadiah B and Adel A. Prevalence of iron deficiency and iron deficiency anemia among females at university stage. J Medical Laboratory and Diagnosis.2;2011:5-11.

4) Shyamala KV, Ravichandra V, Subbalakshmi NK, Sheila PR, Raghuveera K. Iron Status Indicators of Neonates of Mild to Moderate Anaemic Mothers. Research J. Pharm. and Tech.2012; 5 (2): 2012.

5) Usha R, Nishi M, Neera A, Meera S, Sood SK. Effect of maternal iron deficiency anaemia on foetal outcome. Indian J. Pathol. Microbiol.1995; 38(3):273-79.

6) Lao TT, Loong EP, Chin RK, et al. Relationship between newborn and maternal iron status and hematological indices. Biol. Neonate .60(5);1991:303-307.

7) Adriana de A, PaivaI Patrícia H C, RondóII Regina A, PagliusiIII Maria R D O, Latorre IV, Maria A A Cardosol Sheila S R, GondimI. Relationship between the iron status of pregnant women and their newborns Rev Saúde Pública 2007; 41(3):321-7.

8) Emamghorashi F, Heidari T. Iron status of babies born to iron deficient anaemic mothers in an Iranian hospital. IJMS 10; 2004:808-14.

9) WHO/UNICEF/UNU (2001). Iron Deficiency Anaemia: Assessment, Prevention and Control: A Guide for Program Managers. Geneva: World Health Organization.

10) Centers for Disease control and prevention: Recommendations to prevent and control iron deficiency in the United States. MMWR Recomm Rep. 1998; 47(RR-3): 1-36.

\section{P-RCD 02}

Evaluation of One-Tube Osmotic Fragility as a Screening Test for Beta Thalassaemia Trait in Sri Lanka

Pilapitiya PHMAU ${ }^{1}$, Thayaparan $\mathbf{M}^{1}$, Warushahennadi $\mathbf{R}^{2}$, Nilam JM², Kulathilake HWCK ${ }^{1 \#}$

${ }^{1}$ Faculty of Medical Sciences, University of Sri Jayewardenepura, Sri Lanka; ${ }^{2}$ National Thalassaemia centre, Teaching Hospital Kurunegala, Sri Lanka

Email ID for Correspondence: chandimawarawita@yahoo.co.uk Faculty of Medical Sciences, University of Sri Jayewardenepura, Sri Lanka

Introduction: Thalassaemia is highly prevalent in Sri Lanka. The highest number of patients are seen in the North-Western province, especially in the Kurunegala district, in comparison to the other provinces in the country. Screening of children and adults to detect beta thalassaemia carriers using automated full blood count reports and HPLC are only limited to few centers in Sri Lanka. So, the diagnostic process is not easy in under resourced laboratories in a developing country like this. In this context, the value of one tube osmotic fragility test as a screening test is immense. Our study tried to find out the sensitivity and specificity of this test in beta thalassaemia carriers/trait (BTT) in Sri Lanka. Objectives: To evaluate the efficiency of $0.36 \%$ buffered one tube osmotic fragility test as a screening test for beta thalassaemia trait in Sri Lanka. Material and Methods: This was a comparative cross sectional study on randomly selected 700 subjects, carried out at National Thalassaemia Centre of Kurunegala Teaching Hospital. Subjects were categorized into four groups based on red cell indices, HPLC and serum ferritin assay as normal group, BTT group, IDA group and other haemoglobinopathies. (Hb E trait, E thalassaemia, 6 thalassaemia, Hb Strait) .OFT was performed in all cases before the other investigation results became available. Results: Out of 700 subjects 396 subjects (56.6\%) were female and $304(43.4 \%)$ were male. OFT gave definitely positive or equivocal results in 194 of 201 patients with BTT and $96.52 \%$ of sensitivity was observed. The test was false positive in $2(0.75 \%)$ of 268 normal subjects and $99.25 \%$ of specificity was observed. There were $2.56 \%$ of false negative results with $97.44 \%$ of NPV and the $98.97 \%$ of PPV. The efficiency of test was $98.08 \%$ indicating that only $1.92 \%$ of BTT cases were misclassified. We found only 2 confirmed IDA patients and 2 suspected alfa thai trait patients in which both categories gave OFT positivity of $1 / 2(50 \%)$.0ur test also found positive results as follows: $\mathrm{Hb} \mathrm{E}$ trait, $5 / 9(55.6 \%)$; E thalassaemia, 4/4 (100\%); Hb S trait, 1/1 (100\%); delta beta, 1/1 (100\%); beta thai major, 2/3 (66.7\%). Conclusions: OFT is a sensitive, cost effective, rapid and reliable primary screening test for the detection of BTT a nd other haemoglobinopathies in a population with financial restrictions. It can be easily performed in the field. Al so a negative OFT is very useful in ruling out BTT and OFT positive samples need further investigations to establish the diagnosis.

\section{P-RCD 03}

Hemoglobin E-Beta Thalassemia in Uttar Pradesh and Approach for Prenatal Diagnosis

Sarita Agarwal, Usha Prasad, Saroj Gautam

Objective: To evaluate the molecular makeup of hemoglobin E-beta thalassemia to facilitate diagnosis, genetic counseling and prenatal diagnosis in Uttar Pradesh. Method: Referred hemolytic anemia cases of Genetics OPD of Tertiary care centre were included in the present study from the year 2012-2016. All anemias related [9547] patient were evaluated at hematological, biochemical and molecular level. We found 113 subjects of Hemoglobin E variants of which 44 subjects as E-thalassemia [EB thalassemia]. Red Blood cell indices, osmotic fragility, hemoglobin electrophoresis/ quantitative evaluation of fetal hemoglobin, HbA2/E on Hemoglobin variant system, serum iron and total iron binding capacity estimation were carried out in all the blood samples as hematological and biochemical evaluation. However for molecular evaluation genomic DNA was extracted and subjected for sequencing analysis to confirm $\mathrm{HbE}$ mutation and beta thalassemia mutations. Results: The commonest, IVS I-5(G-C) mutation $(70 \%)$ was found along with $\mathrm{HbE}$ mutation. All 44 cases belonged to the group of common Beta-thalassemia mutations as described in literature. Conclusion: Establishment of antennal diagnostic services is necessary in the parts of India where both these mutations are commonly seen. 


\section{P-RCD 04}

\section{Determining Nt-proBNP Levels with Diastolic Dysfunction in Thalassemia Major Patients}

Introduction: Beta thalassemia is an autosomal, recessive disorder, characterized by ineffective erythropoiesis. Chronic transfusions and inability of body to eliminate iron lead to an iron overload, thereby causing damage to heart. Natriuretic peptides (NPs) are produced within the heart, which are then released into the circulation in response to ventricular wall stress. We, therefore, aimed to study the relation between ventricular dysfunction and N-terminal pro-B-type natriuretic peptides (NT-proBNPs). Material and Method: We enrolled 105 patients with increased serum ferritin levels and echocardiography was performed. We collected blood samples and NT-proBNP levels were measured in them. Result: Though, we found that the studied group had no significant difference in the mean serum NT-proBNP levels, in patients with or without hypertrophy $(\mathrm{n}=37, \mathrm{p}<0.992)$, The NT-proBNP levels were found to be significantly increased in patients with diastolic dysfunction. NTproBNP levels correlated well with the echocardiography indices like left ventricular end-systolic diameter (LVESD); ratio between early mitral in flow velocity and mitral annular early diastolic velocity (E/ E'ratio); and ratio of the early (E) to late (A) ventricular filling velocities (E/A ratio), and were found to have significant positive correlation with the serum ferritin levels. Conclusion: NT-proBNP levels correlated significantly with diastolic dysfunction; thus, serum ferritin levels could be useful for assessing the diastolic dysfunction in patients with Beta Thalassemia major.

\section{P-RCD 05}

\section{A rare Hb Variant Hemoglobin O Indonesia}

Ravindra Kumar", Purshottam Patel, Anil Gwal, Subash Godbole, Praveen Bharti, S Rajasubramaniam

Email ID for Correspondence: ravindrachhabra@gmail.com Department of Genetics, ICMR-National Institute for Research in Tribal Health, Jabalpur, India

Background: Hemoglobinopathies are the most common genetic disorder and around 1275 Hemoglobin variant have been identified across the world. Hemoglobin variants are formed due to missense mutation in either $\alpha$ or $\beta$ globin gene. Here we are reporting the $\mathrm{Hb} \mathrm{O}$ Indonesia (HBA1 c. 349 $(\mathrm{G}>\mathrm{A}$ ) which is a rare alpha globin variant. Case Characteristics: An 85 year old female patient was admitted to tertiary care center with a complaint of shortness of breath. Her general condition was poor, afebrile. She had a bilateral pedal edema for 3 months prior to admission and had no history of blood transfusion or jaundice. Clinical examination revealed vitals within normal limits. No organomegaly was observed. Complete blood count revealed hypochromic and microcytic RBCs. with $5.3 \mathrm{~g} / \mathrm{dL} \mathrm{Hb}$. Hemoglobin electrophoresis showed a faint slow moving band immediately behind $\mathrm{HbS}$. Cation Exchange High Performance Liquid Chromatography showed unknown peak with retention time of $4.87 \mathrm{~min}$. DNA Sequencing of alpha and beta globin gene revealed the presence of $\mathrm{HB}-\mathrm{O}$ Indonesia. Screening for $\mathrm{Hb}$ Variants in the family surprisingly revealed that woman's husband and their 3 offspring also carried HB-O Indonesia. Conclusion: HB O Indonesia is a rare variant and only 11 sporadic cases are currently reported across the world. This variant has heterogeneous phenotypic effect and hence clinical presentations need to be studied to correlate its effect.

\section{P-RCD 06}

Effect of Vitamin-D and Calcium Supplementation on Bone Mineral Content (Bmc) in Children with ThalassemiaProspective Longitudinal Study

Dr. Thiagarajan N.R.\#, Dr. C.G. Delhi Kumar, Dr. Jayapraksh Sahoo, Dr. Sriram Krishnamurthy

Email ID for Correspondence: thiagu07ace@gmail.com Jawaharlal Institute of Postgraduate Medical Education And Research (JIPMER)

Introduction: The frequency of osteoporosis is $13.6 \%-50 \%$ and osteopenia is $45 \%$ even in well-treated thalassemia patients. The mechanism of osteoporosis is multifactorial like progressive marrow expansion, chronic hypoxia, direct iron toxicity and iron chelators, endocrinopathies and nutritional deficiency. Objectives: To study the effect of calcium and vitamin D supplementation for osteoprotection and to assess the factors associated with BMC in pediatric thalassemia. Material and Methods: Twenty nine $(n=29)$ children aged 2- 12 years with thalassemia (major or minor) were studied. Dual energy X-ray absorptiometry (DXA) was done to study BMC and BMD, at baseline and at the end of study. 25- hydroxy cholecalciferol, iPTH, serum osteocalcin, urine pyrillinks D/creatinine ratio, calcium, phosphate and alkaline phosphatase were done at baseline, at 6 months and at 12 months and analysed. All patients were supplemented with oral vitamin D (1000 IU/day) and calcium ( $500 \mathrm{mg} /$ day) for 1 year. Patients with low vitamin D levels $(<20 \mathrm{ng} / \mathrm{ml})$ at baseline received vitamin D $2000 \mathrm{IU} /$ day for initial six weeks. Results: At the end of study, percentage of patients with low BMC and BMD decreased from $69 \%(\mathrm{n}=20)$ to $38 \%(\mathrm{n}=11)$ $(\mathrm{p}<0.01)$. BMC increased from baseline value of $8.37 \pm 2.77 \mathrm{~g}$ to $10.78 \pm 3.54 \mathrm{~g}$, at the end of the study $(\mathrm{p}<0.01)$. BMD increased from baseline value of $0.38 \pm 0.07 \mathrm{~g} / \mathrm{cm}^{2}$ to $0.43 \pm 0.08 \mathrm{~g} / \mathrm{cm}^{2}$ at the end of study $(\mathrm{p}<0.01)$. Percentage of patients with low vitamin D levels $(<20 \mathrm{ng} / \mathrm{ml})$ decreased from $72 \%(\mathrm{n}=21)$ at baseline, to $24 \%(\mathrm{n}=07)$ at the end of study $(\mathrm{p}<0.05)$. Mean vitamin D level increased from baseline value of $16.01 \pm 5.75 \mathrm{ng} / \mathrm{ml}$ to $23.40 \pm 6.58 \mathrm{ng} / \mathrm{mL}(\mathrm{p}<0.01)$ at the end of study. The change in serum osteocalcin and urine DPD at the end of study were not significant $(\mathrm{p}>0.05)$. Mean corrected serum calcium value increased from $8.90 \pm 0.80 \mathrm{mg} / \mathrm{dl}$ to $9.60 \pm 0.70 \mathrm{mg} / \mathrm{dl}(\mathrm{p}<0.01)$. Age, sex, anthropometry, duration of disease, number of previous transfusions, serum ferritin, vitamin D, iPTH and bone turn-over markers (serum osteocalcin and urine DPD) did not have any significant correlation with BMC. Conclusions: Our results showed that simple measures like vitamin D and calcium supplementation significantly increased BMC and BMD without having any adverse effects. As children still had low bone mass and vitamin D insufficiency at the end of study, further studies are needed to find out the exact dose of vitamin D and calcium supplementation.

\section{P-RCD 07}

\section{A Community Based Study on Prevalance of Anaemia} in Geriatric Population in an Urban Area of Dibrugarh Town

Introduction Anaemia should not be accepted as an inevitable consequence of ageing. Approximately $8-44 \%$ of the geriatric population, specially those in the age group above 85 years suffers from anaemia. A cause of anaemia is found in approximately $80 \%$ elderly population using WHO criteria for anaemia (Haemoglobin $<12 \mathrm{gm} / \mathrm{dl}$ for woman and $13 \mathrm{gm} / \mathrm{dl}$ for man). Human ageing is characterised by progressive constriction of homeostatic reserve of every organ 
system. This decline reffered to as hoemostenosis is evident by $3^{\text {rd }}$ decade and is gradually progressive. Objectives The present study was primarily aimed at studying the prevalence of anaemia among the geriatric population in Dibrugarh town and secondly to study the morphological patterns of anaemia in the same study group. Materials and Method The present cross sectional study was conducted in the geriatric population for a period of 1 year using stratified random sampling among a sample size of 430 . Each of the study subjects were tested for $\mathrm{Hb} \%$, RBC Count, Red cell morphology and Indices by appropriate laboratory methods. Results In this study prevalence of anaemia in geriatric in urban area was $36.28 \%$. Total number of anaemic geriatric was 156 out of total 430, out of which $68(30.63 \%)$ were male and $88(42.31 \%)$ were female. Out of total 156 geriatric people having anaemia number and percentage of microcytic hypochromic, normocytic normochromic and macrocytic anemias were 97 $(62.18 \%), 45(28.85 \%)$ and $14(8.97 \%)$ respectively. Among the geriatric people with microcytic hypochromic anaemia number of males and females were $41(42.27 \%)$ and $56(57.73 \%)$ respectively. Out of total 45 normocytic normochromic anemia 19 (42.22\%) and 26 $(57.78 \%)$ were males and females respectively, and out of total 14 with macrocytic anaemia number of males and females were 8 $(57.14 \%)$ and $6(42.86 \%)$ respectively. Conclusion Anaemia is prevalent among the geriatric population in this part of the country and prevalence increases as the age increases. Further well coordinated study is required to know the aactual scenario of anaemia in geriatric population in this part of the country. It ill be too early to conclude from a study which includes 430 geriatric people with one year duration only.

\section{P-RCD 09}

\section{Genotype-Phenotype Correlation of $\delta \beta$-Thalassemia and Hereditary Persistence of Fetal Hemoglobin Under Influence of Inherited Cofactors}

Hareram Pandey", Ravi Ranjan, Amit Sharma, Kamal Kishor, Kanwaljeet Singh, Manoranjan Mahapatra, Renu Saxena

Email ID for Correspondence: hareram.aiims@gmail.com All India Institutes of Medical Sciences, New Delhi

Introduction: $\delta \beta$-thalassemia and hereditary persistence of fetal hemoglobin (HPFH) are heterogeneous disorders characterized by the continuous production of fetal haemoglobin $\left(\mathrm{HbF}-\alpha_{2} \gamma_{2}\right)$ during adult life. These syndromes are frequently associated with variable-size deletions within the $\beta$-globin gene complex. Asian Indian inversion deletion $\mathrm{G} \gamma(\mathrm{A} \gamma \delta \beta) 0$ - thalassemia is one of the rare entities characterized by high $\mathrm{HbF}$. Due to its interaction with various inherited factors, patients with $\mathrm{G} \gamma(\mathrm{A} \gamma \delta \beta) 0$-thalassemia showed clinical variability. Worldwide, there is sparse data pertaining to correlation of genotype and phenotype in $\delta \beta$-thalassemia and HPFH under influence of inherited cofactors like $\alpha$ deletion and $\beta$ mutation. In view of these findings, we conducted this study. Objectives: To look for the molecular and hematological correlation of $\delta \beta$-thalassemia and HPFH under influence of inherited cofactors like $\alpha$ deletion and $\beta$ mutation in the patients with raised $\mathrm{Hb}$ F. Material and Methods: A total of 50 individuals with raised $\mathrm{Hb} F$ levels detected during routine screening in the last 4 years were the study subjects. Complete blood count was measured by automated cell analyser. Giemsa-stained peripheral blood smears were examined for red cell morphology. Quantification of Haemoglobin $\mathrm{HbA}, \mathrm{HbA}_{2}$ and $\mathrm{HbF}$ were done by high performance liquid chromatography (HPLC). Asian Indian Inversion Deletion break point type A (5'deletion) and type B (3'deletion), G $\gamma(\mathrm{A} \gamma \delta \beta) 0$ Thalassemia, alpha deletion \& HPFH-3 was done by GAP-PCR where as ARMS-PCR was used for beta mutation. All the patients with absence of high $\mathrm{HbF}$ were excluded. Results: Out of these 50 cases, 15 cases of $\delta \beta \mathrm{G} \gamma(\mathrm{A} \gamma \delta \beta)$ zero thalassemia and 28 cases of HPFH-3 were characterized. $9 / 15(60 \%)$ were heterozygous for $\mathrm{G} \gamma(\mathrm{A} \gamma \delta \beta) 0$ break point type A and 6/15 (40\%) were heterozygous for break point type B. $2 / 9$ patients with type A $\mathrm{G} \gamma(\mathrm{A} \gamma \delta \beta) 0$ inversion deletion showed heterozygous alpha $3.7 \mathrm{~kb}$ deletion and $3 / 9$ patients showed IVS $1-5(\mathrm{G}-\mathrm{C})$ mutation. $1 / 6$ patients with type $\mathrm{B}$ G $\gamma(\mathrm{A} \gamma \delta \beta) 0$ inversion deletion showed heterozygous alpha $3.7 \mathrm{~kb}$ deletions and $2 / 6$ patients showed IVS $1-5(\mathrm{G}-\mathrm{C})$ mutation. All the patients had elevated level of $\mathrm{HbF}$ with normal to reduced $\mathrm{Hb} \mathrm{A} 2$ levels. In the $\mathrm{G} \gamma(\mathrm{A} \gamma \delta \beta) 0$-thal deletion-inversion carriers the mean $\mathrm{Hb} \mathrm{F}$ levels were $16.0 \pm 3.20$. The highest $\mathrm{Hb} F$ levels were observed among HPFH-3 heterozygotes (mean, $27.0 \pm 7.22 \%$ ). The $\delta \beta$-thal showed reduced indices (MCV $68.6 \mathrm{fl} \pm 3.8, \mathrm{MCH} 24.50 \mathrm{pg} \pm 3.30$ ). The mean MCV (78.65 $\mathrm{fl} \pm 6.40)$ and $\mathrm{MCH}(27.1 \mathrm{pg} \pm 2.15)$ values in HPFH carriers were near normal levels (MCV $81.2 \mathrm{fl} \pm 4.72, \mathrm{MCH}$ $27.40 \mathrm{pg} \pm 2.34)$. Conclusions: Co-inheritance of $\alpha$-thal or $\beta$-thal with $\delta \beta$-thal may play a pivotal role in understanding the clinicopathological profile of these patients as the patients with co-inherited $\alpha$-thal may present clinically as thalassemia intermedia and co-expression with beta thalassemia mutation may behave as thalassemia major. HPFH-3 deletion showed RBC indices near-normal range and higher $\mathrm{Hb} \mathrm{F}$ levels as compared to $\delta \beta$-thal.

\section{P-RCD 10}

Vaccine-Associated Immune Hemolytic Anemia: A Case Report

Gurmeet Singh (Presenting Author)

Institute - Jawaharlal Nehru Hospital \& Research Centre, Bhilai

Background: Immune hemolytic anemia (AIHA) developing after vaccination is extremely rare. Only 55 confirmed cases of new-onset IHA from 1991 through 2000 out of 4.5 million children has been reported in a study. Case Reports: A 15 months old child presented with sudden onset of pallor. On admission his $\mathrm{Hb}$ was $5 \mathrm{~g} / \mathrm{dl}$. PS was suggestive of hemolytic anemia with clumps of RBC's. His direct coomb's test was positive. She gave a history of receiving MMR vaccine 2 days before the onset of symptoms at which time she was asymotomatic. Results: The diagnosis of Vaccine associated AIHA was made in accordance with established criteria (hemolysis, positive DAT, and lack of other reasons for the hemolysis. Conclusion: Vaccination-induced AIHA is an extremely rare entity and should be kept in mind if patient presents with sudden onset anemia.

\section{P-RCD 11}

\section{Pregnancy as a Cause of Pure Red Cell Aplasia}

\section{Chandan Baranwal, Uday Prabhakar, Sushil Yadav, Sukdev} Manna

Saumyaleen Roy (Presenting author)

Institute - Banaras Hindu University, Varanasi

Introduction: Pure red cell aplasia is a syndrome characterized by severe normocytic anemia, reticulocytopenia and absence of red cell precursor in bone marrow. Pathophysiologically it can be immunologically mediated. It is associated with pregnancy and others with viral infection, lymphoma, collagen vascular disease. Pregnancy associated Pure red cell aplasia is a rare entity. Here we mention a 26-year-old female with history of abortion admitted with severe anemia. Objective: Pregnancy as a cause of pure red cell aplasia. 
Materials and Methodology: 26-year-old female with history of single abortion admitted with generalized weakness and easy fatigability. She had no history of concurrent fever, jaundice previously, joint pain, photosensitive rash, oral ulceration, any history of similar admission in past for low hemoglobin. She takes mixed diet and drug history is nothing suggestive. On admission she had severe pallor with pedal edema. No lymphadenopathy or organomegaly detected. CNS, CVS, CHEST, ABDOMEN examinations were within normal limits. Her routine blood investigation, bone marrow biopsy, collagen profile, PCR for virus detection in blood, CECT chest done to look for all associations. Results: Her routine investigation showed HB-4.8 g/dl, TRBC-1.6 millions, TLC-5370, DLC-N48L42, Platelet-adequate, MCV -91, LFT and RFT are within normal limits. General blood picture showed decreased red cell lines while other cell lines showed cells of normal morphology and number, Corrected reticulocyte count (CRC) were $.4 \%$. Her iron/tibc level 233/249.vit b12 and folic acid stores were normal. Bone marrow biopsy showed markedly depressed normoblastic erythroid series, with M: E ratio $-40: 1$, other cell lines within normal limits. A picture of pure red cell aplasia is being made from bone marrow studies. Viral markers were negative, collagen profile negative, test for parvovirus, cytomegalovirus and Epstein bar virus PCR from blood came to be negative, USG -neck done along with CECT chest showed no evidence of thymoma. Patient was transfused 2 units of PRBC and is then discharged with HB -9.8, WBC-5600 and platelet adequate. Patient was evaluated after 3 months, her HB was $12 \mathrm{~g} / \mathrm{dl}$, total RBC count 3.5 million, R.C $1.5 \%$, with other parameters normal. Bone marrow examination showed normoblastic erythropoiesis with erythrocytes in different stages of maturation. Conclusion: Pure red cell aplasia is a hematological disorder where the precursor of red blood cells are absent in bone marrow with other cell lines intact Pregnancy associated PRCA is very rare. It is said that igG immunoglobulin is formed against the erythroid precursors during pregnancy with pure red cell aplasia. Pregnancy associated pure red cell aplasia has got good prognostic factor. In our case diagnosis of pure red cell aplasia was made on the ground of bone marrow study, peripheral blood study. Other association causing pure red cell aplasia have been ruled out and only association was pregnancy.

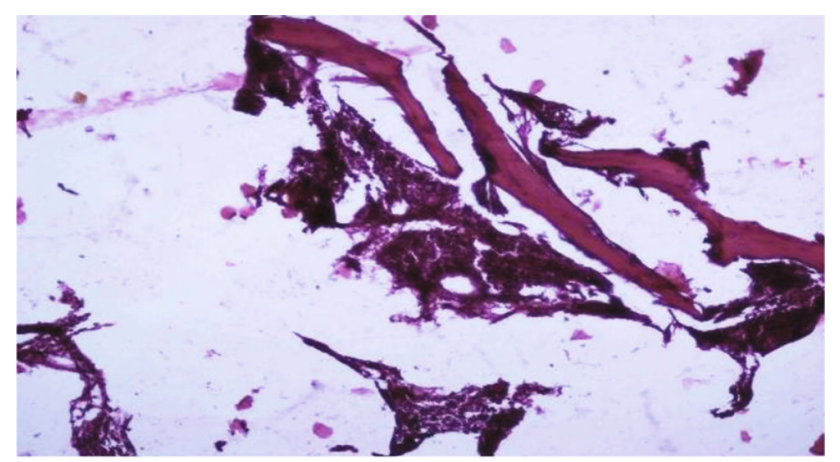

slide showing pure red cell aplasia.

\section{P-RCD 12}

Initial Experience with New Program of Autologous Stem Cell Transplantation in a Resource Constraint Settings - Single Centre Data from Eastern India
P P Gupta, R N Ghosh, Madhumita Patra, Shipla Roy

Introduction: Autologous stem cell transplantation is the standard consolidation therapy for newly diagnosed multiple myeloma patients who are eligible for the procedure. ASCT is also indicated for a variety of haematologic malignancies like relapsed NHL and Hodgkin lymphoma (HL). Method: Patients with various haematologic malignancies who underwent ASCT between Jan 2013 to August 2017 were analyzed. Result: Forty two patients [23 (55\%)males and 19 (45\%) females with a median age of 50 (range 15- 69) years received ASCT during this period. Disease specific distribution: newly diagnosed multiple myeloma (NDMM) -28, relapsed refractory multiple myeloma (RRMM) 5, relapsed NHL (DLBCL) 3, relapsed Hodgkin Lymphoma 3, plasma cell leukaemia (PCL) 1 and relapsed APL 1. Twenty six out of 34 MM patients received MEL 200 and the remaining 8 patients received MEL 140 as conditioning chemotherapy. BEAM was given to all the HL and NHL patients, APL patient received BuCy and MEL 200 was given to the PCL patient. The median stem cell dose $5.36 \times 10^{6}$ cels $/ \mathrm{kg}$ (range 2.34 to $\left.55.36 \times 10^{6}\right)$, Median to engraftment was day +11 (range $d+9$ to $\mathrm{d}+15$ ) and no patient died due to post transplant complications. $89 \%$ patients with NDMM are progression freeon their last follow up. Conclusion: Although preliminary, our ASCT data are encouraging and no transplant related mortality among 42 patients is exciting for a new program. More mature data is needed for comparison with standard centre of India and western literature.

\section{P-RCD 13}

Spectrum and Hematological Profile of Hereditary Anemia: Cross Sectional Study of 9468 Subjects

Priyanka Vishwakarma\#, Sarita Agarwal*

Email ID for Correspondence: priyankav375@gmail.com Sanjay Gandhi Postgraduate Institute of Medical Sciences (SGPGIMS), Raiebareli, Lucknow, U.P.

Introduction: Thalassemia and other hemoglobinopathies together with red cell enzymopathies are common cause of anemia which can be prevented by population screening and genetic counseling. This study was designed to screen the anemic patients for the thalassemia and other hemoglobinopathies and red cell enzymopathies. Objectives: A total of 9468 anemia cases were evaluated for thalassemia, hemoglobinopathies, glucose 6 phosphate dehydrogenase deficiency, Pyruvate Kinase deficiency and hereditary spherocytosis. Patients records were entered in Microsoft excel and spectrum of disorders was evaluated. Year wise spectrum was also analyzed to see the difference in incidence at different time period. Material and Methods: A total of 9468 anemia cases were evaluated for thalassemia, hemoglobinopathies, glucose 6 phosphate dehydrogenase deficiency, Pyruvate Kinase deficiency and hereditary spherocytosis. Patients records were entered in Microsoft excel and spectrum of disorders was evaluated. Year wise spectrum was also analyzed to see the difference in incidence at different time period. Results: Incidence of Beta thalassemia trait and thalassemia major was 9.0 and $2.9 \%$ respectively whereas hemoglobinopathies were observed in $3.2 \%$ of the cases. $0.2 \%$ of the cases were G6PD deficient and $0.5 \%$ had hereditary spherocytosis. No significant difference was observed in incidence of thalassemia and other hemoglobinopathies at different time point. Conclusions: In view of high incidence of thalassemia and other hemoglobinopathies a routine hematological screening at primary health center may be introduced as a prospective premarital screening under thalassemia control program. 


\section{P-RCD 14}

Is Nestrof Test - A Reliable Screening Tool for the Detection of B Thalassemia Trait in Paediatric Age Group

Dr Ankur Goyal ${ }^{\#}$, Dr Anjali Khare, Dr Jaskirat Singh, Dr Rani Bansal

Email ID for Correspondence: ankur.goya1299@gmail.com Subharti Medical College

Introduction: The Thalassemia syndromes, the most common heterogeneous group of Mandelian disorders, characterized by a lack of/or decreased synthesis of either the alpha-globin chains (alpha thalassemia) or the beta-globin chains (beta thalassemia) of haemoglobin. Objectives: To find NESTROF TEST as a suitable screening method for Thalassemia Trait in paediatric patients. Material and Methods: The study was conducted in Department of Pathology, Subharti Medical College and CSS Hospital, Meerut. 441 blood samples of paediatric cases (1to18 years of age) taken in EDTA vacutainer, NESTROF Test was performed. NESTROF Positive cases were subjected to HPLC (Bio Rad D10). Results: Out of 441 cases subjected to NESTROF test, HPLC was performed on 19 cases (4.3\%) which were NESTROF positive. 9/19 cases showed HbA2 values less than $3.5 \%$ (False positive NESTROF). Ten cases displayed abnormal haemoglobin fractions on HPLC .6/10 cases were diagnosed as Thalassemia trait ( $\mathrm{HbA} 2$ between $4.1 \%$ and $7.6 \%$ ). One of the cases showed elevated $\mathrm{HbF}(29.2 \%)$ suggesting beta Thalassemia major.3/10 cases showed raised $\mathrm{HbA} 2$ levels and were diagnosed as $\mathrm{HbE}$ disease. Sensitivity and specificity of NESTROF (calculated by $2 \times 2$ contingency table) was $100 \%$ and $97 \%$ respectively. Conclusions: NESTROF test is a highly sensitive and reasonable specific tool for detection of thalassemia but HPLC should be used as a gold standard.

\section{P-RCD 15}

\section{Unfolding Mystery of Paraspinal Mass}

Dr. Purwa Patil, Dr. Samruddhi Rajpurkar, Dr Grace F D'Costa, Dr. Sneha Chandwani ${ }^{\#}$

Email ID for Correspondence: snehachandvani2508@gmail.com Grant Govt Medical College and Sir J.J. Group of Hospitals, Mumbai

Introduction: Extramedullary hematopoiesis (EMH) refers to hematopoiesis that occurs outside the bone marrow, spinal cord being a very rare site. EMH generally occurs as a compensatory mechanism in cases of chronic anemia such as thalassemia, of which Thalassemia minor forms a very rare entity. Objectives: To make aware that Thalassemia minor patient can present with EMH as pseudotumor in the spine producing morbidity in the form of spinal cord compression. Material and Methods: 43 year old male presented with lower backache since 1 month, weakness bilateral lower limbs since 15 days. MRI spine revealed multiple lesions in paraspinal region. Patient was operated for the lesions and squash smears were prepared and studied. A provisional diagnosis of EMH was made. Specimen was subsequently received as two brownish black tissue bits with fatty areas in the periphery. H\&E stained revealed trilineage proliferation of hematopoietic cells along with mature fat tissue. The diagnosis was further confirmed on IHC, which showed positivity for glycophorin C, Myeloperoxidase and CD61. On examination patient had spleenomegaly. Hematological work up proved that the patient was having thalassemia minor. Conclusions: EMH is known to occur in varying etiologies and in varying locations. However, thalassemia minor comprises of a very rare situation causing EMH. More so, spinal cord forms a very rare site and still presenting itself as a highly morbid condition due to the space occupying nature of the lesion, in a region already compromised for space. We present the case for its rarity.

\section{P-RCD 17}

Utility of PET-CT and Bone Marrow Biopsy in Detecting Bone Marrow Infiltration in the Initial Evaluation of Lymphoma

Jitani AK ${ }^{\#}$, Chakrabarti P, Dutta S, Dolai TK, De R, Mandal PK, Baul SN

Email ID for Correspondence: ankitjitani@gmail.com Nilratan Sircar Medical College and Hospital, Kolkata

Introduction: Evaluation of bone marrow infiltration (BMI) in lymphoma is of prognostic and therapeutic importance. This is assessed by invasive bone marrow biopsy (BMB). PET-CT scan is now routinely done for staging of lymphoma. Objectives: This study was conducted to analyze the utility of PET-CT in BMI detection compared to BMB. Material and Methods: All newly diagnosed, treatment naive lymphoma cases presenting in our out-patient clinic between January 2017 to August 2017 were included in the study. All the included patients had undergone both PET-CT and BMB before initiation of treatment. Two analyses were conducted for the estimation of sensitivity/specificity of BMB and eventual role of PET-CT in detecting BMI. Results: A total of 27 cases were identified. The age range was 4 to 79 years (mean 34.5), with male to female ratio of 2.3:1. Nodal disease was seen in 59\% (16/ 27 ) cases whereas $41 \%(11 / 27)$ cases showed both nodal and extranodal involvement. Non hodgkins lymphoma (NHL: $52 \%$ ) was more common than hodgkins lymphoma (HL: 48\%), and diffuse large B-cell lymphoma was the most common type of NHL $(6 / 14 ; 43 \%)$. Considering $\mathrm{BMB}$ as the gold standard and the only true positive procedure for detecting BMI, PET showed sensitivity of $80 \%$ and specificity of $68.1 \%$. The negative predictive value (NPV) of this test was $93.8 \%$ and the positive predictive value (PPV) was $36.4 \%$. Considering BMI as true positive, whether detected by BMB or PET, sensitivity of PET-CT was $91.7 \%(11 / 12)$ vs. $41.7 \%$ (5/12) for the BMB. The specificity was $100 \%$ for both tests. Conclusions: PET-CT has an excellent sensitivity and NPV for detection of BMI in lymphoma. A negative PET-CT result may eliminate the need of BMB in evaluation of BMI. In cases with positive BMI on PET-CT, a targeted biopsy from metabolically active area may be performed.

\section{P-RCD 18}

Prevalence of Anemia Among Tribal Children in Rural Part of West Bengal

Suman Kumar Maji, Tuphan Kanti Dolai, Rajib De, Prakas Kumar Mandal, Shuvraneel Baul, Indrani Mandal, Prantar Chakrabarti

Email ID for Correspondence: ankitjitani@gmail.com; Ankit Kumar Jitani (Presenting Authors)

Haematology Department, NRS Medical College, Kolkata

Introduction: Prevalence of anemia in the tribal children of India is estimated to be $73-74 \%$, with iron and vitamin B12 deficiency being the most common cause. In West Bengal tribal community comprises $5.5 \%$ of the overall population and distributed among 40 different tribes. Objectives: This study was conducted to estimate the prevalence and causes of anemia in the tribal children. Material and 
Methods: A cross sectional study was conducted over a period of 1 year from June 2016 to July 2017. The target population was children between the age of 5 to 14 years in the tribal areas of 4 districts of West Bengal. EDTA blood and serum sample were collected to investigate the hemoglobin, red cell indices, serum iron, ferritin, TIBC, vitamin B12 and folate level in order to classify the type of anemia. Anemia was defined by WHO guidelines as hemoglobin of $11.5 \mathrm{gm} / \mathrm{dl}$ in 5 to 12 years and $12 \mathrm{gm} / \mathrm{dl}$ in $12-15$ years. Results: A total of 481 children were included in the study. The mean age was 10.3 years (range 5 to 14 years) with $44.5 \%$ (214/481) cases being male and $55.5 \%$ (267/481) being female. The mean hemoglobin was $11.6 \mathrm{gm} / \mathrm{dl}(5.8$ to $14.9 \mathrm{gm} / \mathrm{dl})$, hematocrit $37.66 \%$ (23.5 to 46.7\%), MCV $73.64 \mathrm{fl}(57.8$ to $95.2 \mathrm{fl}), \mathrm{MCH} 22.74 \mathrm{pg}$ (14.4 to $30.8 \mathrm{pg}$ ), and MCHC $30.8 \%$ (24.7 to $34.4 \%$ ). The overall prevalence of anemia was $48.23 \%$ (232/481). The prevalence of anemia was $44 \%$ $(137 / 309)$ and $55 \%(95 / 172)$ in the age group of 5 to $<12$ years and 12 to 14 years respectively. In the age group of 5 to $<12$ years, the common causes of anemia were folate deficiency (47\%), iron deficiency $(29 \%)$ and vitamin B12 deficiency (18\%). In the age group 12 to 14 years, folate deficiency was seen in $41 \%$ followed by iron deficiency (30\%) and vitamin B12 deficiency (22\%). Conclusions: In the tribal children, the overall prevalence of anemia was $48.23 \%$ and more prevalent in the age group of 12 to 14 years. Folate deficiency is the most common cause of anemia in all age group. Supplementary food enriched with micronutrients may reduce the morbidity in these children.

\section{P-RCD 19}

Prothrombotic Markers in Patients with End Stage Renal Disease and Its Change with Dialysis

Dr Kiran Saini", Dr Meera Sikka, Dr Anil Yadav, Dr Mrinalini Kotru, Dr Satendra Sharma

Email ID for Correspondence: kiran.saini4@gmail.com UCMS and GTB Hospital

Introduction: End stage renal disease (ESRD) is known to profoundly influence the hemostatic system. Coagulation disorders resulting in episodes of bleeding as well as recurrent thrombosis have been reported in these patients. Inflammatory and prothrombotic markers are reported to be elevated in patients with renal disease and hemodialysis is known to influence the level of these markers in these patients. Objectives: To determine change in the level of Prothrombotic (Factor VIII, Plasma Fibrinogen), Inflammatory (CRP) markers and screening tests of hemostasis (PT, APTT) in patients with ESRD following dialysis. Material and Methods: Thirty-five patients with ESRD on whom dialysis was to be started or those who had not undergone dialysis for last 1 month were included in the study. Venous blood was collected from all subjects and changes in the levels of following markers: Factor VIII (FVIII), Plasma Fibrinogen (Fib), C-Reactive Protein (CRP), Prothrombin Time (PT) and Activated partial thromboplastin time (APTT) were evaluated just before and 2 weeks after starting regular dialysis. Results: There was a significant $(\mathrm{p}<0.05)$ increase in the level of FVIII and CRP after dialysis. There was no significant change in the level of Fibrinogen. FVIII was increased in $9(25.7 \%)$ and $21(60 \%)$ patients in predialysis and postdialysis period. Increased CRP was seen in $100 \%$ patients postdialysis. Conclusions: ESRD patients show abnormalities of blood coagulation and high degree of inflammation both of which increased with dialysis. These abnormalities can increase the risk of cardiovascular disease. Measurement of these parameters will aid in management of patients with ESRD.

\section{P-RCD 20}

Spectrum of Hemoglobinopathies and Thalassemias Diagnosed on Hplc in A Tertiary Teaching Hospital of Northern India

\section{Jaskirat Singh (Presenting Author)}

\section{Subharti Medical College Meerut}

Introduction: Hemoglobinopathies constitute an increasing global health burden. They are the most common genetic disorders in the World. As per WHO, the incidence is highest in the Middle East and Indian subcontinent. Various hemoglobin variants including $\mathrm{HbF}$ and $\mathrm{HbA} 2$ can be screened using a single, highly excellent method with the use of high performance liquid chromatography (HPLC). Aim: The present study was carried out to diagnose hemoglobinopathies and thalassemias by the use of HPLC in a tertiary care teaching hospital of Northern India. Materials and Methods: A prospective study of 22 months duration was conducted including 100 patients screened for the presence of thalassaemia or any other hemoglobin structural variants. All cases of microcytic hypochromic anemia ( $\mathrm{MCV}<80 \mathrm{fl}$ and $\mathrm{MCH}<27 \mathrm{pg}$ ) not responding to conventional treatment and with clinical suspicion of hemoglobinopathy were included in the present stunventional treatment and with clinical suspicion of hemoglobinopathy were included in the present study. Patient with a history of recent blood transfusion of less than 3 months duration were excluded from the study. Results: A total of 100 cases (57 males and 43 females) were included in the present study. The age group of patients ranged from 2 months to 40 years. Complete blood count, Red blood cell indices and peripheral blood examination were done in all the cases. Out of the 100 cases, 51 $(51 \%)$ cases displayed abnormal hemoglobin fractions on HPLC of which. $42(42 \%)$ cases were diagnosed as thalassemia trait, 4 cases (4\%) as beta thalassemia major ( $\mathrm{HbF}$ more than $75 \%$ ), 2 cases as $\mathrm{HbE}$ (2\%) and, 3 cases as HPFH (3\%). 49 cases had a normal HPLC pattern. Conclusion: HPLC is a rapid, accurate and useful method for diagnosing hemoglobinopathies. It serves as an important tool in diagnosing beta thalassemia traits especially in developing countries like India, where, the resources for detection of hemoglobinopathies are limited. Early diagnosis may help in proper and specific management.

\section{P-RCD 21}

Prevalence and Genetic Characterization of Glucose-6-Phosphate Dehydrogenase Deficiency Among North Indian Population

\section{Deepika Delsa Dean, Sarita Agarwal}

Glucose-6-phosphate dehydrogenase deficiency is caused by one or more mutations in the G6PD gene on chromosome X. It affects approximately 400 million people worldwide. The present study was conducted on 1069 referred cases with suspected G6PD deficiency. All the samples were screen for G6PD deficiency by Dichloro phenol indophenols decolorizing test (DCIP Bernstein test), fluorescence spot test and the deficient samples were further process for mutation analysis by sequencing. This study revealed that the G6PD Mediterranean mutation $(563 \mathrm{C} \rightarrow \mathrm{T})$ is the most common variant among G6PD deficient individuals. Prevalence was found $8.8 \%$ in northern population. Our findings will helps in designing population screening approach and provide better management for G6PD deficiency patients. We concluded that, all symptomatic patients (anemic or jaundice) should be investigated for G6PD deficiency before giving them any oxidant drugs. Government should launch educational 
awareness program and investigating facility at community health centers (CHC) for better management of G6PD deficient patient.

\section{P-SCT 01}

Bone Marrow Transplantation as Permanent Curative Treatment in Red Cell Pyruvate Kinase Deficiency

\section{Bharath Ram S, Shobha Badiger, Sunil Bhat, Pooja P Mallya}

\section{Pediatric Hemato-Oncology, Mazumdar Shaw Cancer Centre, Narayana Heath, Bangalore}

Background: Pyruvate kinase (PK) deficiency is the most common RBC enzymopathy of glycolytic pathway leading to congenital nonspherocytic hemolytic anemia. Its prevalence being 51 cases per million white population transmitted as autosomal recessive trait. The degree of hemolysis is variable, ranging from mild or fully compensated forms to life-threatening neonatal anemia including documented hydrops fetalis. Treatment of this disease remains mainly symptomatic, including blood transfusion and splenectomy with only bone marrow transplantation having potential to cure the disease. Clinical Case: We report a case 7 year old boy who developed neonatal jaundice and later severe transfusion-dependent hemolytic anemia diagnosed at 4 years of age with pyruvate kinase deficiency. He later underwent BMT from an HLA-identical younger sibling (Normal PK activity) after conditioning with thiotepa, treosulfan, fludarabine and thymoglobulin and GVHD prophylaxis of methotrexate and cyclosporine. He underwent matched sibling peripheral blood stem cell transplantation (CD34 cell dose of $5.3 \times 10^{6}$ cells $/ \mathrm{kg}$ and $\mathrm{MNC}$ of $3.5 \times 10^{8}$ cells $\left./ \mathrm{kg}\right)$ in $2016 \mathrm{He}$ attained neutrophil ANC $>0.5 \times 10^{9}$ cells/ 1 and platelet engraftment $>20 \times 10^{9}$ cells $/ 1$ on day +16 . The post-transplant course was uneventful. Day 28 chimerism showed $98.47 \%$ donor chimerism. He maintained stable donor chimerism and is presently 1 year post transplantation followup with transfusion independance. Conclusion: PK deficiency is a rare disease where bone marrow transplantation can be successfully used as curative option for severe transfusion dependant disease.

\section{P-SCT 02}

Initial Experience with New Program of Autologous Stem Cell Transplantation in a Resource Constraint Settings - Single Centre Data from Eastern India

\section{P P Gupta, R N Ghosh, Madhumita Patra, Shipla Roy}

Introduction: Autologous stem cell transplantation is the standard consolidation therapy for newly diagnosed multiple myeloma patients who are eligible for the procedure. ASCT is also indicated for a variety of haematologic malignancies like relapsed NHL and Hodgkin lymphoma (HL). Method: Patientswith various haematologic malignancies who underwent ASCT between Jan 2013 to August 2017 were analyzed. Result: Forty two patients [23 (55\%)males and19 (45\%) females with a median age of 50 (range15- 69) years received ASCT during this period. Disease specific distribution: newly diagnosed multiple myeloma (NDMM) -28 , relapsed refractory multiple myeloma (RRMM) 5, relapsed NHL (DLBCL) 3, relapsed
Hodgkin Lymphoma 3, plasma cell leukaemia (PCL) 1 and relapsed APL 1. Twenty six out of 34 MM patients received MEL 200 and the remaining 8 patients received MEL 140 as conditioning chemotherapy. BEAM was given to all the HL and NHL patients, APL patient received $\mathrm{BuCy}$ andMEL 200 was given to the PCL patient. The median stem cell dose $5.36 \times 10^{6}$ cels $/ \mathrm{kg}$ (range 2.34 to $55.36 \times 10^{6}$ ), Median to engraftment was day +11 (range $d+9$ to $\mathrm{d}+15$ ) and no patient died due to post transplant complications. $89 \%$ patients with NDMM are progression freeon their last follow up. Conclusion: Although preliminary, our ASCT data are encouraging and no transplant related mortality among 42 patients is exciting for a new program. More mature data is needed for comparison with standard centre of India and western literature.

\section{P-SCT 04}

To Compare and Study, Blood Tacrolimus Drug Level of BMT Cancer Patients Analysed on Two Different Analysers Having Different Principles

UlkaGosavi", S Kumar, M.Tiwari, A Karmore, U A Gavhane, B.G.Pillai, S Yaykar, R. Mohite, Dr. Preeti Chavan Dr.Vivek Bhat, P. Poladia

Email ID for Correspondence: ulka.gosavi@yahoo.co.in ACTREC, Tata Memorial Centre, Kharghar, Navi-mumbai

Introduction: Tacrolimus, an immunosuppressive drug is used to decrease the risk of organ rejection in Bone Marrow Transplant (BMT) patients. The therapeutic monitoring of Tacrolimus is necessary since low doses may cause graft rejection while over dosage is linked to nephrotoxicity, neurotoxicity, and many other adverse effects. Tacrolimus drug levels may be estimated on different biochemistry analysers. In our laboratory, ARCHITECTi1000 which works on chemiluminescent microparticle enzyme immunoassay (CMIA) and EXL with LM which works on immunoassay technique are used for this purpose. The values obtained from both the machines were analysed statistically and correlated. Objectives: the aim of this study was to compare and correlate the values obtained on Architecti1000 fully automated analyser by Abbott Healthcare and EXL biochemistry analyser with LM by Siemens Healthcare. Correlation study by using statistical methods will help in suggesting the possibilities of using either method for estimating Tacrolimus levels interchangeably although the technology and principle in the two methods differ. Material and Methods: This comparative study was done on 83 samples obtained from post-transplant BMT patients to detect the tacrolimus level in the whole-blood on Architecti1000 and EXL autoanalyzers simultaneously between the periods of May 2017 to September 2017. Values obtained were analysed statistically to see correlation between the two methods and observe the bias if any. The study included the linearity ( 0.1 to 30.0$)$, limit of blank, limit of detection and limit of quantification for the tacrolimus estimation on both the analysers. Results: The study showed acceptable correlation between the two methods statistically. Passing Bablok regression analysis showed R - 0.911 (Fig. 1) and Bland Altman plot showed a minor bias of -0.69 (Fig. 2). Conclusions: The results obtained by both the methods showed good correlation with $\mathrm{R}$ value 0.911 hence either of the methods (Architect i1000\& EXL) may be used for detection of Tacrolimus levels in BMT patients. 


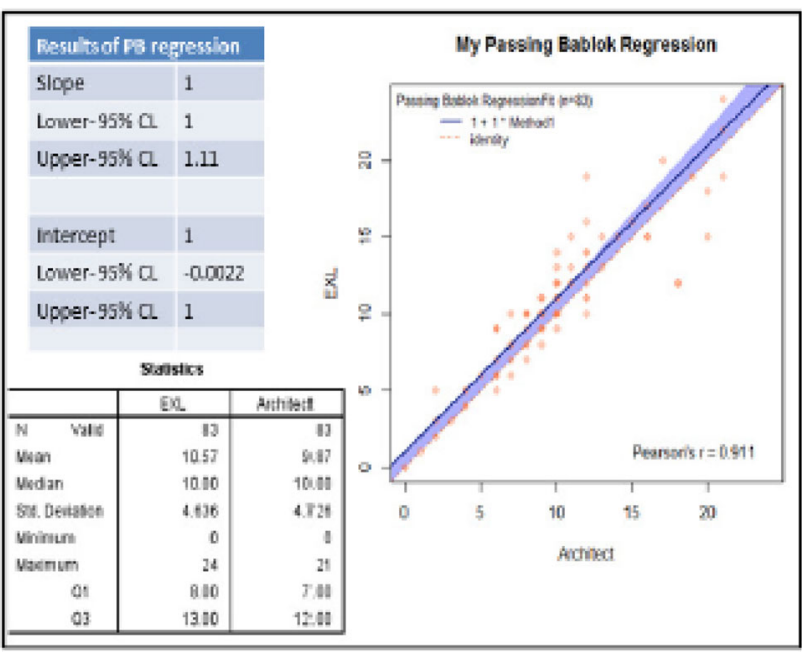

Figure 1 .

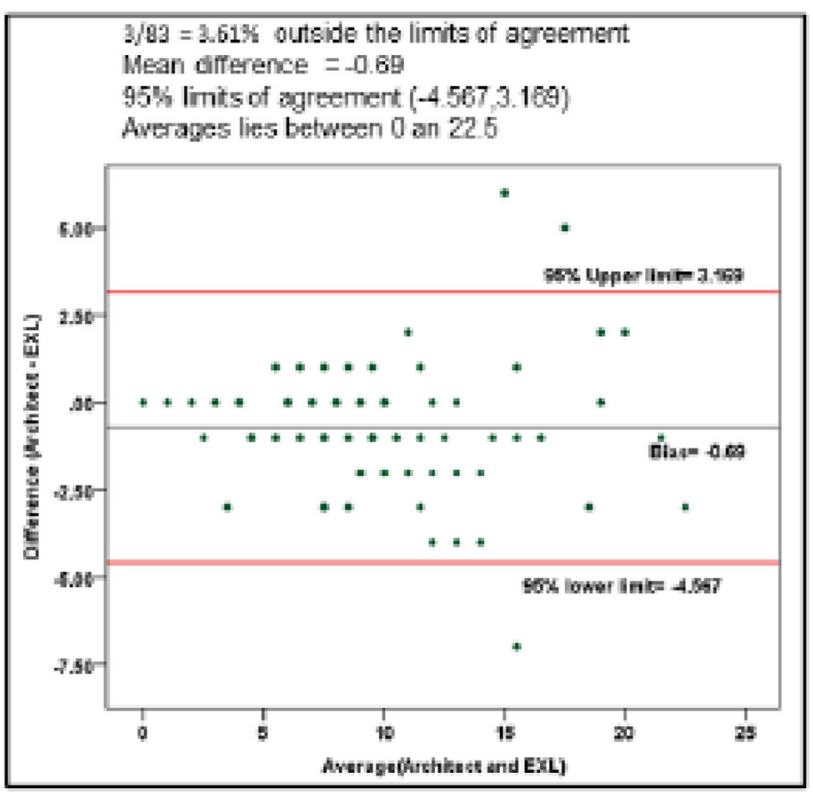

Figure 2 .

\section{P-SCT 05}

The Importance of Donor Specific Antibody on the Outcome of T-Cell Replete Haploidentical Hematopoietic Stem Cell Transplantation

Objectives: Haploidentical stem cell transplantation is considered to be an alternative option to cure hematological malignancies in absence of genoidentical donor which is available in only $30 \%$ of the recipients. Primary graft failure due to the presence of donor specific antibodies (DSA) against mismatched HLA between the donor and recipient is reported to be a major risk factor associated with the transplant outcome. This study explores the importance of DSA in the outcome of $\mathrm{T}$ cell replete haploidentical hematopoietic stem cell transplantation performed in a tertiary cancer care center in the eastern part of India. Materials and Methods: In the present study, the patients undergoing haploidentical transplantation were prospectively screened for the presence of HLA antibodies on Luminex platform using Lifecodes Lifescreen Delux Kit. The patients who were screened positive for anti-HLA antibodies (with a mean fluorescence intensity $>1000$ ), were further evaluated for the presence of DSA using Lifecodes Single Antigen Assay kit. Results: Anti-HLA antibodies were present in 15 of the 56 patients $(26.78 \%$ ) analyzed (both against Class I and Class II antigens); with a mean fluorescence intensity (MFI) varying from 1000 to 15000 . Further analysis by Single Antigen assay showed that 4 out of 15 patients (26.66\%) who had undergone haploidentical HSCT were positive for donor specific antibodies against their prospective donors. Among these four patients, graft rejection was reported in three patients; however one patient had stable engraftment in spite of the presence of donor specific antibodies. Conclusion: The data, though limited by the number of small cohort of patient, indicated that the presence of antiHLA sensitization correlates with graft failure and it should be taken into account before considering a HSCT from a HLA haplo-identical donor.

\section{P-SCT 06}

Antibiotic Sensitivity Pattern of Blood Culture Isolates in Bone Marrow Transplant Patients from a Tertiary Cancer Care Centre

Reshma Kamble\#, Vivek Bhat, Hemali Dethe, Nisha Pandey, Divya Rathish, Madhura Salvi

Email ID for Correspondence: kimayareshma75@gmail.com A.C.T.R.E.C., Tata Memorial Centre, Kharghar, Navi Mumbai

Introduction: Blood stream infections are a major cause of morbidity and mortality among Bone marrow transplant patients. Multidrug resistance among bacteria particularly Gram negative bacilli is a cause of great concern in recent times. Objectives: In this study we sought to determine the spectrum of bacterial blood stream infections in Bone marrow transplant patients and analyze the susceptibility patterns of the isolated organisms. Material and Methods: All blood culture samples received during the study period of one year from cases of suspected bacterial sepsis in stem cell transplant patients were processed in the BacT/Alert automated blood culture system in the microbiology laboratory. Bacteriological culture and sensitivity was performed following standard microbiological techniques by manual methods as well as by Vitek 2 compact automated analyzer. Susceptibility testing was performed and interpreted as per CLSI guidelines. Results: Of the 516 blood culture samples received from 74 patients from different sites (peripheral blood, Hickman catheter lumens), organisms were recovered from 21 patients (with a total of 63 positive blood cultures). These included Pseudomonas aeruginosa (2), Coagulase negative Staphylococci (11), Klebsiella pneumoniae (5), E. coli (2), and others (6). All Klebsiella pneumoniae isolates were resistant to $3^{\text {rd }}$ generation cephalosporins and imipenem and one isolate was resistant to colistin too. Both the Pseudomonas aeruginosa isolates were resistant to aminoglycosides, fluoroquinolones and the carbapenems. Conclusions: The study revealed that Gram-negative bacilli were more commonly associated with bloodstream infection than Gram positives. They showed a high degree of antibiotic resistance against aminoglycosides, cephalosporins and carbapenems. The poor activity of carbapenems against Klebsiella pneumoniae is alarming.

Keyword Antibiotic Sensitivity, blood culture 


\section{P-SCT 07}

\section{Outcomes of Hematopoietic Stem Cell Transplantation} in Primary Immunodeficiency Disorders

Vibhor Sharma ${ }^{\#}$, Anu Korula, Eunice Sindhuvi, Nisham P. N, Anup Devasia, Uday Kulkarni, Kavitha M.L, Aby Abraham, Vikram Mathews, Alok Srivastava and Biju George

Email ID for Correspondence: vibhor.tmh@gmail.com Christian Medical College, Vellore

Introduction: Hematopoietic stem cell transplant (HSCT) is required in a subset of patients with primary immunodeficiency disorders (PID). The use of donors other than fully matched siblings is now established practice world-wide; however data from India is sparse. Objectives: We studied the outcomes of patients who underwent allogeneic HSCT for primary immunodeficiency disorders in our institute. Material and Methods: This is a retrospective analysis of allogeneic HSCT carried out from January 1998 to August 2017 in patients with PID in a tertiary care centre in India. Data was retrieved from patient files and electronic medical records. Data was analyzed for patient and donor characteristics, sources of stem cells, conditioning chemotherapy received primary and secondary graft rejection, infectious complications, acute and chronic graft versus host disease (GVHD), event free survival (EFS), and overall survival (OS). Results: Thirty two patients with a median age 36 months (Range 6 months to 18 years) underwent allogeneic HSCT. The two most common disorders were Wiskott Aldrich syndrome $(\mathrm{n}=8)$ and severe combined immunodeficiency $(\mathrm{n}=7)$. Haploidentical transplants were carried out in $12(37.5 \%)$ patients, matched sibling donor (MSD) in $10(31.2 \%)$, matched family donor (MFD) and matched unrelated donor (MUD) in 5 (15.6\%) each. Stem cells were derived from peripheral blood (PBSC) in 19 (59.3\%) patients, bone marrow (BMSC) in $12(37.5 \%)$ and cord blood in one $(3.1 \%)$. Median CD34+ cell dose infused was $10.4 \times 10^{6} / \mathrm{kg}$. Median total nucleated cell (TNC) cell dose infused was $6.5 \times 10^{8} / \mathrm{kg}$. Conditioning regimes used were busulfan based in 9 $(28.1 \%)$ patients and treosulfan based in $22(68.8 \%)$. One patient did not receive conditioning. Six patients expired before day +28 due to infectious complications. Of the remaining patients, all except one engrafted. Grade 3-4 acute GVHD occurred in $8(25 \%)$ patients. Bacterial, viral and fungal infections were noted in $22(68.8 \%), 19(59.3 \%)$ and $11(34.3 \%)$ patients respectively in peritransplant period. Seven $(21.8 \%)$ patients had chronic GVHD, including one patient with extensive chronic GVHD. Of the 14 patients who expired, GVHD was the primary cause of death in six $(42.8 \%)$ patients and infections in eight $(57.1 \%)$. The median follow up was 314 days (range $12-5143$ days). The 2 year OS and EFS for the entire cohort was $51.3 \pm 10 \%$ and $48.7 \pm 9.8 \%$ respectively. At 2 years the OS in MSD, MUD/MFD and Haploidentical transplants was $88.9 \pm 10.5 \%, \quad 33.3 \pm 17.2 \%$ and $40.0 \pm 14.6 \%$ respectively $(\mathrm{p}=0.033)$, and the EFS was $88.9 \pm 10.5 \%, 33.3 \pm 17.2 \%$ and $33.3 \pm 13.6 \%$ respectively $(\mathrm{p}=0.022)$. Conclusions: Outcomes in matched sibling donor HSCT is comparable to data in the West, however there are significant challenges associated with haploidentical transplants in the Indian setting, with a significant mortality secondary to infections.

\section{P-TM 01}

Nucleic Acid Testing: A Screening Tool in Blood Banking; An Institutional Study in Odisha.

Dr. Deepak Kumar Dasmohapatra ${ }^{\#}$, Dr. Chitta Ranjan Prasad
Introduction: The aim of any blood transfusion is to save the life by issuing safe blood. For that purpose, Transfusion Transmitted Infections like HIV, HBV, HCV, Malaria and syphilis are screened for each unit of blood issued. To cut the window period of the three Viral diseases (HIV, HBV, HCV) NAT method is now being adopted in Blood Banks. Objectives: To find out added advantages and limitations of NAT method over ELISA for screening TTI. Materials and Methods: It is a Multicentric Study. All the ELISA negative samples were sent for NAT. The Study was carried out for a period of around nine months. Results: Some cases of sero-reactivity which were missed in the conventional ELISA were detected in the advanced NAT method. Conclusions: Even though NAT has an added advantage of reducing the window period in screening of TTI, INDIA being a devolping country we should focus more on procedure of Donor selection. Only the ELISA negative samples to be tested by NAT method to reduce the financial burden.

\section{P-TM 02}

Seroprevalence of Transfusion Transmissible Infections (TTIs) Among Blood Donors in a Tertiary Care Centre- A Comparison Between Voluntary and Replacement Donors

\section{Dr. Debasmita Konwar ${ }^{\#}$, Dr. (Mrs.) Adity Sharma}

Email ID for Correspondence: debasmitakonwar0985@gmail.com Assam Medical College and Hospital, Dibrugarh

Introduction: Blood transfusion has been used since 1930 for various indications. Safety of blood transfusion is of extreme importance in order to avoid any severe morbidity and mortality in the patient. Approximately $1 \%$ of transfusions are associated with adverse events like transfusion transmissible infections (TTIs). Outcomes of TTIs range from subclinical to life threatening manifestations. By screening donated blood units, we get a clue of the prevalence of those infections among donor populations and consequently the safety of collected donations. It also gives us an idea of the prevalence of the transfusion transmissible infections (TTIs) in the community. Objectives: To determine the seroprevalence of transfusion transmissible infections amongst voluntary and replacement blood donors at a tertiary care centre and to study the prevalence category wise among the different types of blood donors. Material and Methods: Before blood donation, "Donor Screening Forms" were filled out by every donor. Donation was taken after physical examination and estimation of $\mathrm{Hb} \%$, weight has been carried out. Blood donations taken in either blood donation camps or in blood bank, without remuneration were considered as voluntary donors. Replacement donors included donations given for a particular patient. Tests for TTIs were done using ELISA for anti-HIVantibodies, HBsAg and anti-HCV antibodies. Tests for Syphilis and Malaria were done using rapid card tests for RPR and Pv/Pf respectively. Results: Out of 21,386 total blood donors; 340 donors tested positive for any one aforementioned TTIs. HBsAg was the most commonly encountered TTI in our set up. The number of seropositive case in replacement donors was more in comparison to voluntary donors. This difference was statistically significant in case of HBV, HCV, HIV and syphilis $(\mathrm{p}<0.05)$ but not statistically significant $(p>0.05)$ in malaria. Conclusions: Seroprevalence was more in replacement donors than in voluntary donors, so it should be discouraged and voluntary donations be encouraged. Keywords Transfusion Transmissible Infections (TTIs), Human Immunodeficiency Virus (HIV), Hepatitis B Virus (HBV), Hepatitis C Virus (HCV), Syphilis, Malaria

Email ID for Correspondence: dkdm@rediffmail.com

Veer Surendra Sai Medical College, Burla 


\section{P-TM 03}

Granulocyte Transfusions in Severe Neutropenic Sepsis and Their Role in Combating Infections Due to Multi- Drug Resistant Organisms (MDRO)

\section{Akanksha Garg", Ashish Mishra, Manoj Singh, Anshul Gupta, Sanjeev, Soniya Nityanand}

Email ID for Correspondence: sadge85@gmail.com Department of Haematology, Sanjay Gandhi Post Graduate Institute of Medical Sciences, Lucknow

Introduction: Despite advances in antimicrobial and supportive therapy, bacterial and fungal infections still remain an important cause of mortality in patients of haematological malignancies and in recipients of hematopoietic stem cell transplants (HSCT) with treatment related neutropenia. One of the important reasons is due to emergence of infections by multi- drug resistant organisms (MDROs), and granulocyte transfusions (GTX) from healthy donors providing extrinsic neutrophils may lead to early clearance of index infection when antimicrobials fail to combat MDROs, and thus prevent mortality. Objectives: To study the efficacy and safety of GTX in combating infections due to MDROs. Material and Methods: We conducted a prospective, uncontrolled observational analysis of patients with different hematological disorders who received GTX for severe neutropenic sepsis. The study period was from August 2013 to August 2017. All patients with absolute Neutrophil Count $<0.5 \times 10^{9} / \mathrm{L}$ with life threatening sepsis not responsive to even $3^{\text {rd }}$ line antimicrobials and/ or having drug- resistant infections were included in the study.Granulocytes were collected from blood group matched healthy donors who were mobilized with G- CSF (@10mcg/kg SC) and dexamethasone ( $8 \mathrm{mg} \mathrm{IV}$ ), 12 hours prior to collection. Granulocytes were collected using Spectra OPTIA apheresis machine with a target collection of $50 \times 10^{9}$ granulocytes or higher. The product was transfused within 6 hours. The harvest data and patent's clinical details were recorded. Results: A total of 143 granulocyte collections were done for 66 infectious episodes (IEs) in 60 patients. Males constituted $68 \%$ of the total patients. Resolution of index infection after GTX was seen in 45/66 IEs (68.2\%) and the 30 day overall survival (OS) was $67.7 \%$. MDROs were observed in 47/66 IEs (71.2\%). Fungal infections were seen in 9/66 IEs $(13.6 \%)$. GTX were well tolerated and only 6 patients developed mild features of transfusion related acute lung injury (TRALI), and 1 patient demonstrated hypocalcemic tetany. Overall survival was better in patients who received early GTX therapy within 7 days of neutropenic sepsis in comparison to those who received after 7 days $(81.8 \%$ vs $54.5 \%, p=0.01)$. There was a trend for higher OS in those who received a GTX dose of $>50 \times 10^{9}$ granulocytes per IE in comparison to those who received a GTX dose less than this $(71.7 \%$ vs $60 \%, p=0.36$ ). The post GTX 6 hour increment in the TLC and ANC values were higher in the group which received a GTX dose $>50 \times 10^{9}$ granulocytes per IE $(2530 /$ cumm vs $1100 /$ cumm, $\mathrm{p}=0.01 ; 2018 /$ cumm vs $840 /$ cumm, $\mathrm{p}=0.02$, respectively). The mean durations of fever and neutropenia were shorter in patients who received early GTX within 7 days of the IE ( 8 days vs 19 days; $\mathrm{p}=0.001$ and 20 days vs 32 days, $\mathrm{p}=0.02$, respectively). Patients receiving $>3$ GTX had a significantly shorter mean duration of neutropenia in comparison to those who received $\leq 3$ GTX ( 25 days vs 31 days, $p=0.01$ ). Conclusions: In a country like India, where MDROs are rampant in haematological diseases, GTX play a vital role when other antimicrobial therapies fail. Our data shows that GTX led to resolution of index infection in $68 \%$ patients with MDROs.The benefits are more pronounced in patients who are given GTX early (within 7 days). Higher doses of GTX are well tolerated and lead to a trend for higher OS.GTX may bridge the gap till recovery of counts hence prolonging survival of patients.

\section{P-TM 04}

\section{Platelet Audit in Viral Thrombocytopenia in a Tertiary Care Teaching Hospital}

Rashmi B K ${ }^{\#}$, Vijaya B, Pallavi P, Puneet Jain

Email ID for Correspondence: drrashmibk@gmail.com Department of Transfusion Medicine, JSS Medical College, JSS University, Mysuru, Karnataka

Introduction: Viral fevers especially dengue fever has emerged as a major public health problem in the recent years. Platelets due to the short shelf life are frequently in scarcity in the blood bank, which recommends its usage very aptly. Hence this study was undertaken to assess the appropriateness of platelet transfusions in viral thrombocytopenia. Objectives: Our study objective was to assess the appropriateness of platelet transfusions in viral fever with thrombocytopenia during the study period. Materials and methods: A retrospective study was conducted in blood bank of a tertiary care teaching hospital in the month of May 2017. All patients with viral thrombocytopenia who received platelet transfusion formed the subjects of study. Patients clinical data, laboratory results, platelet count and platelet transfusions were analyzed. National Guidelines suggested by ministry of health and family welfare, Government of India was used to analyze the appropriateness of platelet transfusions. Results: During the study period 87 patients with viral thrombocytopenia received platelet transfusion. Males constituted $59.7 \%$ and females constituted $40.3 \%$. A total of 467 random donor platelets (RDP), and 18 single donor platelets (SDP) were transfused. Out of 467 units of RDP transfusions, $89.3 \%$ were prophylactic and $10.7 \%$ were therapeutic. Of the prophylactic platelet transfusions of RDPs, inappropriate transfusions were $49.6 \%$. Among 18 units of SDPs $61.1 \%$ were prophylactic platelet transfusions and $39.9 \%$ were therapeutic transfusions. Of the prophylactic platelet transfusions of SDPs inappropriate transfusions were $33.3 \%$. Conclusion: In our study, inappropriate platelet transfusion was $49.6 \%$ for RDPs and $33.3 \%$ for SDPs. In a developing country like India with limited resources, judicial use of platelet concentrates is a necessity. Therefore it is essential to strengthen the awareness of guidelines and transfusion practices in India.

\section{P-TM 05}

\section{Therapeutic Plasma Exchange (TPE) In Atypical Hemolytic Uremic Syndrome (HUS)}

Objectives: Atypical HUS is a life threatening condition and no single standard treatment for this condition. Several approaches are described and we have salvaged two advanced cases using TPE. Materials and Methods: A retrospective analysis of two atypical HUS patients treated with TPE. TPE was started within 24hours of presentation and continued daily or alternate days along with hemodialysis (HD). 1 to 1.5 plasma volume was exchanged per session and replaced by FFP. All laboratory profiles were analyzed to assess the response of TPE. Case -I: 14 years girl presented with hemolytic anemia, jaundice, thrombocytopenia and renal dysfunction. She was diagnosed as atypical HUS and TPE was started with systemic steroid. TPE was continued on alternate days along with hemodialysis. Initially patient responded well after 8 sessions of TPE and 3 sessions of hemodialysis. Platelet count and LDH became normal but creatinine remained high. Hemodialysis was continued twice per week and planned for renal transplant. Case-II: 10 years boy, presented with microangiopathic hemolysis, thrombocytopenia, renal impairment and diagnosed as atypical HUS. TPE was started 
within 24hours of presentation and continued daily with systemic steroid. Total 7 sessions of TPE were given with 2 sessions of hemodialysis. Patient responded after 5 sessions. Platelet count and creatinine became normal. LDH became half from presentation. Rituximab - weekly for 4 doses were given after completing initial 5 sessions of TPE. Result: Both cases were complete responders to TPE initialy and remission was persistent with additional HD and immunotherapy. Conclusion: TPE was lifesaving procedure in described cases. Early institution of TPE after diagnosis declines mortality and changed this fatal disease to an almost curable disease.

\section{P-TM 06}

Incidence of Minor Blood Group Antigens (Rh system) Amongst North Indian Blood Donors

Dr. Ishani Gupta ${ }^{\#}$, Dr. Gaurav Raturi, Dr. Aparna Bhardawaj, Dr. Rajnish Kumar

Email ID for Correspondence: dr.ishani90@gmail.com Shri Guru Ram Rai Institute of Medical \& Health Sciences

Introduction: Karl Landsteiner and Weiner discovered Rhesus (Rh) system in the year 1940. Presently there are more than 50 antigens in $\mathrm{Rh}$ blood group system but major ones are D, C, c, E and e. Patients requiring multiple blood transfusions develop alloantibody against these rare antigens. The most important irregular red blood cell alloantibodies in daily transfusion practice, in terms of frequency of occurrence, are directed towards the Rh (anti-D, -c, -E, -c and -e) and kell. Therefore, it is important to match the rare antigens before issuing blood to vulnerable recipients.

\section{Objectives:}

1. To find out the incidence of $\mathrm{RhD}, \mathrm{RhC}, \mathrm{Rhc}, \mathrm{RhE}$ and Rhe amongst the voluntary blood donors of Uttrakhand.

2. To compare the incidence of Rh subtypes with Asian population of the Subcontinent.

Material and Methods: A prospective cross-sectional non-interventional study was carried out on 500 healthy voluntary blood donors from January'16 to July'17 at our blood bank. Donors were grouped and typed for $\mathrm{ABO}$ and $\mathrm{Rh}$ major antigens using monoclonal blood grouping reagents. Chi-Square test was applied wherever required. Results: A total of 500 blood samples from voluntary blood donors were phenotyped. Among Rh antigens, e was the most common antigen $(98.2 \%)$, followed by D-94.2\%, C-88.6\%, c-54.8\% and E-18.6\% with DCe/DCe (36.2\%) being the most common phenotype and the least common phenotype is dce/dce and $\mathrm{dCE} / \mathrm{dCE}(0.2 \%)$. Conclusions: Database for antigen frequency to at least $\mathrm{Rh}$ blood group in local donors helps to provide antigen negative blood to patients with multiple alloantibodies, minimize alloimmunization rate and thereby improve blood safety.

\section{P-TM 07}

How Safe is Paediatric Peripheral Stem Cell Harvest Under Anaesthesia in the Day Care Setting? A Single Centre Experience

Anup J Devasia, Melvin Alex Abraham*, Chidambaram Sagadevan, Nisham PN, Kulkarni Uday Prakash, Anu Korula, Aby Abraham, Alok Srivastava, Vikram Mathews, Sajan Philip George*, Biju George.

Department of Haematology, Christian Medical College, Vellore; *Department of Anaesthesia, Christian Medical College, Vellore
Introduction: The use of cytokine mobilized peripheral blood stem cells (PBSC) for stem cell transplantation offers more rapid myeloid and platelet engraftment, and less early transplant related mortality and morbidity. This can be done easily in the out-patient setting if the donor is an adult, but becomes very difficult in children as they are unlikely to cooperate for the associated invasive procedures. In such instances, vascular access placement and peripheral stem cell harvest needs to be done under anaesthesia. Metabolic complications like hypocalcaemia, hypoglycaemia etc. due to citrate toxicity, volume shifts due to extra corporeal volume can occur during such procedures and hence is often challenging. The increase in the number of operative procedures makes it difficult to obtain a suitable time for a paediatric PBSC harvest in the operating room. The safety and efficacy of general anaesthesia outside the controlled operation room setting is quite challenging and demanding. We present our experience with PBSC harvest in 158 children (predominantly healthy sibling donors) under anaesthesia in the out-patient day care setting at our centre. Methodology: Paediatric donors who underwent peripheral blood stem cell harvest under anaesthesia in the Department of Hematology, Christian Medical College, Vellore, India between January 2009 to June 2017 were included in this retrospective analysis. Data collection was done from apheresis records and anaesthesia notes from the in-patient chart. All donors were seen in the pre- anaesthetic clinic and were cleared ahead of the procedure. After premedication with trichloryl or diazepam, the donors were induced with inhalational anaesthesia, airway was secured and intravenous (IV) anaesthesia was given. Intravenous access was obtained and stem cell harvest was done on COBE spectra ${ }^{\circledR}$ apheresis system. Vital signs were continuously monitored throughout the procedure. Results: A total of 158 children underwent 164 PBSC harvests during the study period. In 8 children, a repeat harvest had to be done for inadequate stem cell dose on the first harvest or repeat transplant for various reasons. Two were autologous stem cell transplants for acute promyelocytic leukemia. Donors were predominantly females with a median age of 5 years (1-12) and a median weight of $17.5 \mathrm{~kg}$ (9.4-51). The most common indication for transplant was Thalassemia major $(\mathrm{n}=113)$ followed by bone marrow failure syndromes $(\mathrm{n}=26)$. In $50 \%$ of the cases, induction of anaesthesia was by sevoflurane followed by total intravenous anaesthesia (TIVA) while in 32\% it was sevoflurane induction followed by sedation. Hudson mask (48.5\%) and LMA $(50 \%)$ were the most common modes of airway and all patients were ventilated in the spontaneous mode. Propofol was the most commonly used maintenance agent $(67 \%)$. There were no major complications except for acute pulmonary edema secondary to infusion of blood products requiring a short stay in ICU for one donor. The median CD34 cell dose harvested was $11.38 \times 10^{6}$ cells $/ \mathrm{kg}(0.55-49.40)$. All donors were discharged on the next day of harvest. No long term complications have been reported in any of these donors. Conclusion: Paediatric PBSC harvest can be safely done under anaesthesia with due precautions in the day care setting.

\section{P-TM 08}

Adverse Events in Plateletpheresis: Experience of a Hospital Based Transfusion Service in Northern India

Rajendra Chaudhary (Presenting author)

\section{Sanjay Gandhi Postgraduate Institute of Medical Sciences}

Introduction: Although plateletpheresis procedures are considered safe, adverse events related to these procedures are not uncommon. Apheresis procedures are commonly associated with citrate-related reactions and comprise of 30-40\% of all reactions. Other adverse events include hematoma, pain or swelling at the phlebotomy site, peripheral neuropathy, blood loss, hypertension, allergic reactions etc. We 
perform plateletpheresis using three apheresis machines, based on either continuous flow or intermittent flow technology. Here we presented our experience of donor reactions due to plateletpheresis. Materials and methods: Over a period 10 months, 351 plateletpheresis procedures performed on eligible donors. No prophylactic calcium supplementation was administered to any donors. Pre-donation hematological values of all donors, such as platelets (PLT), hemoglobin (Hb), hematocrit (Hct), WBC count were measured using automated cell counter. Any adverse event during or after plateletpheresis was reported to the incharge of apheresis unit and details of each reaction including its management were documented in the procedure register. Results: Out of total 351 plateletpheresis donors, $45(\%)$ suffered adverse reactions. Of these majority were related to citrate toxicity $(57.8 \%)$ followed by vasovagal reactions $(15.6 \%)$ and others. No significant difference was observed in the rate of reaction among various apheresis machines used. Conclusion: Apheresis procedures performed on cell separators are safe, with a low incidence of significant AEs. No significant difference was noted in AEs among the two cell separators studied. The rate of vascular injury (VI), Citrate reaction (CR), and Presyncopal/Syncopal (PS/S) in plateletpheresis was $1.6 \%(52 / 3,120)$, $0.96 \%(30 / 3,120)$, and $0.096 \%(03 / 3,120)$, respectively. We planned this prospective study to look into donor safety aspect by studying adverse events in normal healthy plateletpheresis donors. In this study we included 500 healthy, first-time $(n=301)$ and repeat $(n=199)$ plateletpheresis donors after informed consent. The plateletpheresis procedures were performed on TrimaAccel (5.1 version, GAMBRO BCT) and Amicus (3.2 version FENWAL) cell separators. The adverse events during procedure were recorded and classified according to their nature. In results we found a total of $18 \%(\mathrm{n}=90)$ adverse events were recorded in 500 plateletpheresis donors, of which $9 \%$ of were hypocalcaemia in nature followed by hematoma $(7.4 \%)$, vasovagal reaction $(0.8 \%)$ and kit related adverse events in $(0.8 \%)$. There was significant postprocedure drop in $\mathrm{Hb}$, Hct, platelet count of the donors $(\mathrm{p}<0.0001)$ whereas WBC count showed a statistically significant rise $(\mathrm{p}<0.0001)$. Divalent cations ( $\mathrm{iCa}+, \mathrm{TCa}+, \mathrm{TMg}+)$ also showed a statistically significant decline after donation $(\mathrm{p}<0.0001)$. However there were no statistically significance differences between adverse events in TrimaAccel (5.1 version, GAMBRO BCT) and Amicus (3.2 version FENWAL) cell separators. Donor reactions can adversely affect the voluntary donor recruitment strategies to increase the public awareness regarding constant need for blood and blood products. Commonly observed adverse events in plateletpheresis donors were hypocalcemia, hematoma formation and vasovagal reactions which can be prevented by pre-donation education of the donors and change of machine configuration.

\section{P-TM 09}

\section{Anti C Willis Antibody in Multitransfused Thalassemics: Report} of 3 Cases

\section{Shivali Sehgal ${ }^{\#}$, Sangeeta Pahuja, Geetika Sharma, Sunita Sharma}

Email ID for Correspondence: shivalisehgal@gmail.com Lady Hardinge Medical College, New Delhi

Introduction: Anti $\mathrm{C}$ Willis $\left(\right.$ Anti $\mathrm{C}^{\mathrm{w}}$ ) is an antibody against the the $\mathrm{C}^{\mathrm{w}}$ (Rh8) antigen, which is a low incidence antigen of the Rh system. $\mathrm{C}^{\mathrm{w}}$ results from a single amino acid change (Gln41 Arg) most often found on the $\mathrm{RhCe}$ protein. Production of Anti $\mathrm{C}^{\mathrm{w}}$ can occur in response to the presence of $\mathrm{C}^{\mathrm{w}}$ antigen on the membranes of donor red cells and is usually of the $\operatorname{IgG}$ type. This antibody may be seen in combination with other antibodies to red blood cells. Objectives: To report 3 cases of alloimmunization against $\mathrm{C}^{\mathrm{w}}$ antigen in multitransfused thalassemic patients.
Material and Methods: Antibody screening (using Biorad ID-DiaPanel) was done in all recipients before each transfusion. If positive, antibody identification (using Biorad ID-Diacell I-II-II Asia) was performed. Results: Out of 567 thalassemic patients registered in blood bank, anti $\mathrm{C}^{\mathrm{W}}$ was detected in three cases (Aged 4 years, 27 years and 30 years), giving an incidence of $0.53 \%$. Two of these patients (Aged 4 years and 30 years) had only anti $\mathrm{C}^{\mathrm{w}}$ whereas one patient had anti $\mathrm{C}^{\mathrm{w}}$ along with anti $\mathrm{E}$. Conclusions: Anti $\mathrm{C}^{\mathrm{w}}$ has been identified in individuals without known exposure to foreign RBCs (naturally occurring) and also after transfusion and pregnancy. Patients with anti $\mathrm{C}^{\mathrm{w}}$ should always receive $\mathrm{C}^{\mathrm{W}}$ negative packed cells after Indirect Antiglobulin test (IAT) cross match. Since $\mathrm{C}^{\mathrm{W}}$ is a low prevalence antigen, $\mathrm{C}^{\mathrm{w}}$ antigen negative blood is readily available. Physicians must be aware that occurrence of Anti $\mathrm{C}^{\mathrm{w}}$ can lead to serious transfusion complications. In addition, if Anti $C^{\mathrm{w}}$ is identified during pregnancy, titres should be monitored throughout in order to avoid Hemolytic disease of newborn.

\section{P-TM 10}

\section{Bacterial Contamination of Blood Components - Evaluation} and Prevention

\section{Lalita Jyotsna $\mathbf{P}^{\#}$, Shivali Sehgal, Sangeeta Pahuja, Sunita Sharma}

Email ID for Correspondence: plj.constantspring@gmail.com Lady Hardinge Medical College, New Delhi

Introduction: Testing for transfusion transmitted infections has reduced the rate of viral transmission amongst patients transfused with blood. However, the low but definite risk of bacterial contamination has emerged as the residual but major threat of transfusion transmitted disease. Objectives: To study the incidence of bacterial contamination of blood components and any associated morbidity and mortality, at a Regional blood transfusion centre (RBTC) attached with a tertiary care hospital. Material and Methods: Retrospective study conducted in RBTC, evaluating the data of blood bags randomly sent/ following transfusion reactions for sterility testing over a 2-year period. Results: A total of 154 blood components were sent for sterility testing during the period of the study including 52 units of whole blood, 85 units of red cell concentrate, 6 units of fresh frozen plasma, 93 units of platelets .2.54\% of the units (whole blood, PC and PRP) were positive for E.coli/Klebsiella/Acinetobacter. All units of red cell concentrate and fresh frozen plasma were sterile. None of blood bags received following transfusion reactions showed any growth. The probable cause for growth of gram negative bacteria could be lack of stringent precautions/delay while sending the samples for culture. It was ensured that there is minimal, if any, delay in sending the samples to the microbiology lab. "Diversion" that is discarding the first aliquot of donor blood removed was started. Conclusions: Knowledge of prevalence of bacterial contamination of blood transfusion and its causes is important in devising methods to prevent transfusion transmitted bacterial infections.

\section{P-TM 11}

Using Stem Cell Donors for Platelet Apheresis After Stem Cell Donation - A Feasible Strategy Following Allogeneic Stem Cell Transplantation

Himal Shah, Anu korula, Anup Devasia, Uday Kulkarni, Kavitha N Lakshmi, Nisham PN, Aby Abraham, Alok Srivastava, Vikram Mathews, Biju George

Department of Haematology, Christian Medical College, Vellore 
Introduction: Delayed platelet engraftment following allogeneic stem cell transplantation (HSCT) is one of the complications seen in the immediate post-transplant period. Infusion of single donor platelets (SDP) are associated with better platelet recovery compared to random donor platelets. We retrospectively analysed the impact of SDP transfusion obtained after platelet apheresis from stem cell donors following allogeneic HSCT. Objectives: To study the efficacy of transfusion of single donor platelets obtained from stem cell donors following allogeneic HSCT. Material and Methods: Stem cell donors underwent platelet apheresis 7-10 days after stem cell donation provided their platelet counts were $>1,00,000 /$ cumm. The apheresis product was infused over 1 or 2 days depending upon clinical status and platelet recovery. Data on HSCT including neutrophil and platelet engraftment were collected from individual records and the institutional database. Successful platelet engraftment was defined as a platelet count $>20,000$ without any further transfusion support. Results were analysed with Kruskal walis test and chi square test. Result: 166 allogeneic HSCT recipients with a median age of 23 year, who received SDPs from their respective stem cell donors were analysed. The median time to platelet engraftment was 11 days [range: 8 - 63]. Successful platelet engraftment was seen in 74 patients $(44.5 \%)$ while $92(55.6 \%)$ did not show any platelet recovery. We divided these patients in 3 different groups - Group A Those who showed increment of platelet more than 20000 with no further requirement of platelet support $(n=62)$ group B - those with platelet increment $>20000$ but required further platelet support $(\mathrm{n}=12)$ and Group $\mathrm{C}=$ Those who did not show platelet recovery beyond $20000(\mathrm{n}=92)$. A number of factors influencing platelet recovery including age, gender, donor platelet counts, disease status, Blood group disparity, HLA typing and intensity of conditioning regimen were studied. Presence of a HLA mismatch and use of myeloablative conditioning seemed to be the factors that adversely influenced platelet engraftment. There were no significant difference among these 3 groups in respect to age, gender, donor platelet count, blood group disparity or disease status. Conclusion: Platelet apheresis using HSCT stem cell donors is feasible and associated with adequate platelet engraftment in about $45 \%$ of patients. Other strategies need to be explored in refractory patients.

\section{P-TH 01}

\section{A Study on Haematological Manifestations in Pulmonary Tuberculosis Pre and Post Treatment (DOTS)}

\section{Dr. Md sarfaraj ${ }^{a}$, Prof B.Laskar ${ }^{a}$, Dr. Dhriti Sundar Das ${ }^{a^{*} \text {, }}$ Prof R K Hazarika ${ }^{b}$, Dr B N Mahanta ${ }^{a}$}

Dhriti Sundar Das (Corresponding author)

${ }^{a}$ Department of Medicine, Assam Medical College \& Hospital, Dibrugarh; ${ }^{\mathrm{b}}$ Department of Pathology, Assam Medical College \& Hospital, Dibrugarh

Introduction: Tuberculosis is a major public health problem in India. Haematological changes associated with tuberculosis have been incompletely investigated. There is no comprehensive study assessing the haematological abnormalities in these patients from this part of the country. In the present study, peripheral blood and bone marrow findings in patients with pulmonary tuberculosis were analysed. An attempt has also been made to assess the effect of antituberculosis therapy on the haematological abnormalities. Materials and methods: Fifty one patients with pulmonary tuberculosis were prospectively studied (from Sept'10 to Aug'11) to determine the various haematological manifestations in tuberculosis and the effect of antituberculosis therapy. All patients received standard antituberculosis treatment. They were subjected to a detailed hemogram including peripheral blood examination, which was repeated on completion of antituberculosis therapy. Bone marrow aspiration was also done in all patients before starting antituberculosis treatment. Results \& Observation: Microcytic hypochromic anaemia was the most common abnormality observed (54.9\%) followed by normocytic hypochromic $(45.1 \%)$. Other haematological abnormalities of the white blood cells include leucopenia (9.8\%), neutropenia (5.9\%), lymphocytopenia $(19.6 \%)$, monocytopenia $(52.9 \%$; $<<0.000)$, leucocytosis (15.7\%), neutrophilia $(15.7 \%)$ and lymphocytosis (3.9\%). Pancytopenia was observed in only 2 patients $(2.4 \%)$. Thrombocytopenia was rare. Bone marrow showed granulomas (1.96\%), AFB (1.96\%) and megaloblastosis. The hemogram improved with antituberculosis therapy in these patients. Conclusion: The present study suggest that tuberculosis can present with a spectrum of haematological manifestations. Prominent haematological abnormalities associated with tuberculosis patients improved substantially with DOTS.

\section{P-TH 02}

Hemophagocytic Lymphohistiocytosis (HLH) Induced MAHA and AKI in a Patient with HIV and Disseminated Tuberculosis: A Case Report

\section{Dr. Vamsidhar Veeranki", Dr. Molly Mary Thabah, Dr. Kadhiravan.T}

Email ID for Correspondence: vamsidharveeranki@gmail.com JIPMER

Introduction: Hemophagocytic lymphohistiocytosis (HLH) a hyperinflammatory state is a known entity in setting of HIV infection or in tuberculosis (TB) due to excessive activation of lymphocytes and macrophages. The prognosis of HLH in HIV positive patients is grave compared to the ones who are HIV negative. Case report: A 40-yearold male presented with fever for 4 months and altered sensorium for 10 days. Examination revealed lymphadenopathy and mild splenomegaly but no neck rigidity. Cytopenias and transaminitis were found on initial blood investigations. Peripheral smear showed schistocytes with thrombocytopenia - suggestive of microangiopathic hemolytic anemia (MAHA). Aspiration of the lymph node revealed necrotizing lymphadenitis with positive stain for Acid fast bacillus (AFB). Because of transaminitis he was stared on modified antituberculous therapy (ATT). On day 3 of hospital stay on day 3 his sensorium worsened, he also developed AKI with severe metabolic acidosis. Because of cytopenia HLH workup was done. Serum triglycerides and serum ferritin were elevated, and bone marrow aspirate confirmed hemophagocytosis. Patient was then started on intravenous dexamethasone to which there was a dramatic response with improvement in sensorium, AKI, acidosis, cytopenias and transaminitis. He was then given full dose 4 drug ATT (HREZ). Bone marrow biopsy revealed caseous granuloma confirming diagnosis of TB. Anti-retroviral therapy which was initially withheld because of AKI was also restarted. Patient completed 6-month ATT, at the end of which there was complete resolution of symptoms and lymphadenopathy. The CD4 count has improved from 64 at presentation to 124 at $2^{\text {nd }}$ week during follow up. Discussion: Incidence of HLH in HIV is nearly $20 \%$ but the mortality in case of HLH in HIV ranges from 70-80\%. Mortality increases with low CD4 count. The present case is a successfully treated case of HLH inspite of HIV infection and very low CD4 count. Good response could have been due to early empiric initiation ATT at the time of presentation and also to initiation of corticosteroids at the earliest suspicion of HLH. Early initiation of treatment of the underlying cause (TB in this case) might have accounted for successful recovery in this patient. The second interesting point of this case is though incidence of AKI in HLH ranges up to 62\%, MAHA as 
an etiology of AKI is rare in a case of HLH with an incidence of $<5 \%$ (case series by Florence et al., 2014). Resolution of both AKI and MAHA with corticosteroids in the present case supports the diagnosis of MAHA with TMA as cause of AKI. Persistent thrombocytopenia precluded from doing renal biopsy. Moreover patient has improved with steroids and AKI, metabolic acidosis also has improved. Conclusion: We report a case of HLH, MAHA with AKI due to disseminated TB in a HIV patient. Inspite of the reported high mortality of $60-70 \%$ of HLH in HIV, the early initiation of treatment for underlying precipitating infection (TB) and also high index of suspicion for HLH might have accounted for successful outcome of the patient in this case.

\section{P-TH 03}

To Study the Serum Levels of Pro-Inflammatory and AntiInflammatory Cytokines in Pediatric Tuberculosis

\section{Dr. Anjisha Ajayan\#, Dr. Anita Nangia, Dr. Varinder Singh, Dr. Sangeeta Pahuja Sindhwani}

Email ID for Correspondence: anjisha2006@yahoo.co.in Lady Hardinge Medical College

Introduction: Pediatric tuberculosis is a leading cause of morbidity and mortality in children with India accounting for $25 \%$ global annual incidence of 8.6 million cases. Cytokines, both pro-inflammatory and anti-inflammatory are key mediators of immunological response to tuberculosis at cellular level. The level of these cytokines may be a rapid, sensitive and specific biomarker for diagnosis and monitoring of tuberculosis in children. Objectives: To study the pre-treatment and post treatment profiles of pro and anti-inflammatory cytokines in newly diagnosed cases of pediatric tuberculosis and evaluate the sequential changes in cytokine levels with treatment in these patients. Material and Methods: The serum levels of pro-inflammatory (IP10, IFN gamma, TNF alpha) and anti-inflammatory cytokines (IL-10 and IL-4) were evaluated in 50 newly diagnosed cases of pediatric tuberculosis using ELISA on day 0, day 30, day 60 and day 180 (wherever possible) of antitubercular therapy. Results: Both proinflammatory and anti-inflammatory cytokines were raised significantly on day 0 in all cases. IP-10 was the most sensitive and specific proinflammatory cytokine which consistently declined with therapy and reached normal values by day 60 of treatment. IL-4, an anti-inflammatory cytokine showed a significant rise in levels with therapy upto day 60. IP-10 maintained normal levels even after 6 months of therapy while IL-4 levels remained high after 6 months of antitubercular therapy. Conclusions: IP-10 was the most consistent cytokine which could detect all cases of active tuberculosis and monitor response to antitubercular therapy and its levels were not affected by age group, gender, pulmonary or extrapulmonary presentation and single or multiple sites of involvement.

\section{P-WBCD 01}

\section{Chediak-Higashi syndrome- A case report}

Akanksha Singh", Rashmi Kushwaha, Sanjay Mishra, Geeta Yadav, Mili Jain, Ashutosh Kumar

Email ID for Correspondence: dr.akanksha.ald@gmail.com King George's Medical University, Lucknow

Introduction: Chediak-Higashi syndrome (CHS) is a rare autosomal recessive disease, characterized by partial oculocutaneous albinism, frequent pyogenic infections, and the presence of abnormal large granules in leukocytes and other granule containing cells. The abnormal granules are readily seen in blood and marrow granulocytes. Other clinical features include silvery hair, photophobia, nystagmus and hepatosplenomegaly. Objectives: Case presentation of ChediakHigashi syndrome in 4 year male. Material and Methods: Clinical workup, peripheral blood and bone marrow examination of patient. Results: A 4 yr male presented with recurrent fever and abdominal distension. There was history of consanguineous marriage of parents. On examination, pallor, silvery gray hair and hypopigmented patches over the face and the limbs were observed. Ultrasound revealed moderate hepatosplenomegaly with gross peritoneal collection. On peripheral blood smear examination, WBC and platelet counts were reduced with presence of large cohesive intracytoplasmic granules in neutrophils and lymphocytes. These abnormal granules were also observed in most granulocytic and few erythroid precursors on bone marrow examination. Conclusions: The presence of abnormal giant intracytoplasmic granules in neutrophils and their precursors are diagnostic of CHS. Allogeneic bone marrow transplant is the only possibly curative treatment, when done early, before the onset of accelerated phase. Hence, the early identification of the disease is essential by careful examination of the peripheral smear, so that bone marrow transplantation can be suggested at the earliest.

\section{P-WBCD 02}

\section{Lymphocyte Subset Analysis in Polyclonal Lymphocytosis \\ Dr Rajeev Sen\#, Dr Sakshi Dahiya, Dr Monika Gupta, Dr P S Ghalaut}

Email ID for Correspondence: drrajeevsen@gmail.com Pt B D Sharma PGIMS, Rohtak

Introduction: Abnormal increases or decreases in the lymphocyte count (lymphocytosis or lymphocytopenia) may reveal a transient infection or one of a number of other benign or malignant conditions. In absolute lymphocytosis, the total lymphocyte count is elevated. The study of these lymphocyte subsets can yield a lot of new information. Objectives: To study the subsets of lymphocytes in adult peripheral blood and correlate with various causes for polyclonal/ reactive lymphocytosis. Material and Methods: flow cytometry was performed on the BD FACS canto II machine (6 color, 2 laser) using peripheral blood in adult patients. In our study we have included 20 cases of lymphocytosis (absolute count $>3500 / \mathrm{mm}^{3}$ ). Results: A total of 20 consecutive cases were studied. Age range varied from 20-85 years. Male:female ratio was found to be $7: 4$. Out of the 20 cases, Six cases were reactive (polyclonal). Out of the remaining 14 cases, one was of monoclonal B cell lymphocytosis and 13 cases belonged to CLPDs (chronic lymphoproliferative disorders). This category included 10 cases of CLL and 3 cases of NHL. Further evaluation of $\mathrm{B}$ cell, $\mathrm{T}$ cell and $\mathrm{NK}$ cell percentages alongwith clonality assessment and CD4:CD8 ratio was done. Polyclonal cases were majorly showing Tcell proliferation and monoclonal displayed Bcell proliferation. Out of the 14 monoclonal cases, 4 cases showed lambda positivity and 3 showed kappa chain clonality. Further, CD4:CD8 ratio was reversed in 3 reactive cases and 6 cases of CLPDs. Conclusions: Lymphocytosis in the blood may fall in two broad groups. One, polyclonal lymphocytosis, which can be transient, reactive or benign. Causes for this category encompass a variety like Tuberculosis, viral infections like Infectious mononucleosis (EBV), other infections, drugs, stress, smoking, massive trauma, after bone marrow transplant and chemotherapy.etc. Second group of Monoclonal lymphocytosis occurs due to lymphproliferative disorders like CLL (chronic lymphoid leukemia), MBL (monoclonal B cell 
lymphocytosis), Hairy cell leukemia, Mantle zone lymphoma, Follicular lymphoma.etc All the polyclonal cases show a proliferation of $\mathrm{T}$ cells and CLPDs show increase in B cells with monoclonality.

\section{P-WBCD 03}

\section{Flow Cytometry in the Diagnosis of Severe Combined} Immunodeficiency

\section{Dr. S.Sri Gayathri MD, Dr Aruna Rajendiran MD, DM, Dr Malini T. MPhil PhD}

Objectives: To study the diagnostic role of Flowcytomery in the diagnosis of severe combined immunodeficiency based on Flowcytometric enumeration lymphocyte subsets in our hospital. Materials and Methods: The patients who presented with clinical features of SCID in department of Peadiatrics, Sri Ramachandra Medical college and for whom flowcytometric lymphocyte subset analysis was done, in department of Pathology were included in the study. Flowcytometry was done in BD FACS Calibur using two tubes. CD45 PerCP, CD19PE, CD3FITC, CD16+ 56 APC in one tube and CD45 PerCP, CD3FITC, CD4 PE, CD8APC in other tube. $200 \mathrm{uL}$ of Peripheral blood in EDTA as anticoagulant and Trucount beads were added in each tube. Minimum of 4000 events were captured. Analysis was done in Multiset software. $\mathrm{CD} 3+\mathrm{T}$ lymphocytes \%, Absolute CD3 + T lymphocytes count, CD4+ CD3+ T Helper cells, Absolute CD4+ CD3+ $\mathrm{T}$ Helper cells count, CD8+ CD3+ Suppressor T cells $\%$ and absolute count, CD19+ B lymphocytes \% and absolute CD19+ B lymphocytes count, CD16and or or CD56+ Natural killer cells $\%$ and absolute count were analysed for each patient. Results: Thirteen patients were enrolled in the study. They were in the age group of 2 months to 5 years of Age, The common presenting complaints were recurrent infections, fever and stunted growth. CD3+ T lymphocytes \% range from 16 to $72 \%$, Absolute CD3+ T lymphocytes count 342 to $8121 / \mathrm{uL}, \mathrm{CD} 4+\mathrm{CD} 3+\mathrm{T}$ Helper cells \% 9 to 57\%, Absolute CD4+ CD3+ T Helper cells count 181 to 4667/uL, CD8+ CD3 + Suppressor T cells $\% 6$ to $42 \%$ and absolute count 117 to $1275, \mathrm{CD} 19+\mathrm{B}$ lymphocytes $\% 7$ to $73 \%$ and absolute CD19+ B lymphocytes count 41 to 1984 , CD16and or or CD56+ Natural killer cells $\% 0$ to $48 \%$ and absolute Natural killer cell count 9 to $1528.3 / 13$ (23\%)patients had lower than normal CD3 + T lymphocytes and were the candidate for Bone marrow transplant. 4/13 (30\%) patients had lower than normal CD19+ B lymphocytes and 2/13 (15\%)patients had lower than normal NK cells, $2 / 13$ (15\%)patients had increase in T lymphocytes, significance of which is not known. Conclusion: Severe combined immunodeficiency is a rapidly fatal disease in which bone marrow transplantation is a life saving treatment. Flowcytometry is an effective tool in diagnosis of SCID and for guiding the selection of patients suitable for bone marrow transplantation. 